Supporting Information

\title{
Phosphination of Phenol Derivatives and Applications to Divergent Synthesis of Phosphine Ligands
}

Chenchen Li", Kezhuo Zhang\#, Minghao Zhang, Wu Zhang and Wanxiang Zhao* State Key Laboratory of Chemo/Biosensing and Chemometrics, College of Chemistry and

Chemical Engineering, Hunan University, Changsha, Hunan 410082, China.

E-mail : zhaowanxiang@hnu.edu.cn.

\section{Table of Contents}

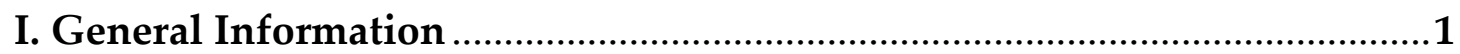

II. Synthesis and Characterization of Substrates...........................................

III. Synthesis and Characterization of Products .........................................21

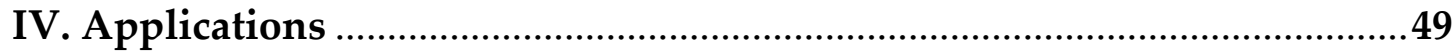

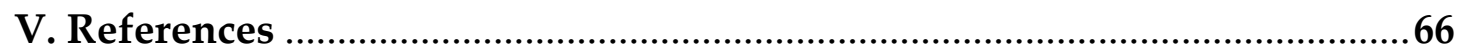

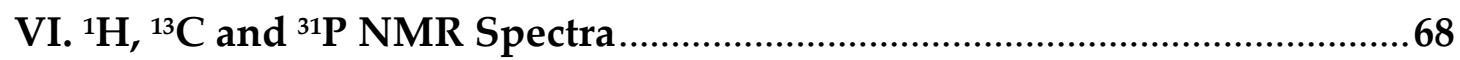




\section{General Information}

Unless otherwise noted, all reactions were conducted in an oven-dried vial with a magnetic stirrer under nitrogen atmosphere. Solvents were purified under nitrogen using a solvent purification system. Analytical thin layer chromatography (TLC) was performed using silica gel plates. Visualisation was by ultraviolet fluorescence, and/or phosphomolybdic acid, and/or $\mathrm{KMnO}_{4}$. Flash column chromatography was performed using EM Science (200-300 mesh) silica gel. ${ }^{1} \mathrm{H}-\mathrm{Nuclear}$ Magnetic Resonance ( $\left.{ }^{1} \mathrm{H}-\mathrm{NMR}\right),{ }^{13} \mathrm{C}$ Nuclear Magnetic Resonance $\left({ }^{13} \mathrm{C}-\mathrm{NMR}\right)$ spectra and ${ }^{31} \mathrm{P}-\mathrm{Nuclear}$ Magnetic Resonance ( $\left.{ }^{31} \mathrm{P}-\mathrm{NMR}\right)$ were recorded on Bruker $400 \mathrm{MHz}$ at $20{ }^{\circ} \mathrm{C}$ with $\mathrm{CDCl}_{3}$ or $\left(\mathrm{CD}_{3}\right)_{2} \mathrm{CO}$ as solvent. Chemical shifts $(\mathrm{ppm})$ are given relative to solvent: references for $\mathrm{CDCl}_{3}$ were $7.26 \mathrm{ppm}\left({ }^{1} \mathrm{H} \mathrm{NMR}\right)$ and $77.16 \mathrm{ppm}\left({ }^{13} \mathrm{C} \mathrm{NMR}\right)$; references for $\left(\mathrm{CD}_{3}\right)_{2} \mathrm{CO}$ were $2.05 \mathrm{ppm}\left({ }^{1} \mathrm{H} \mathrm{NMR}\right)$ and $29.84 \mathrm{ppm}\left({ }^{13} \mathrm{C} \mathrm{NMR}\right)$. The data are reported as follows: chemical shift (ppm), multiplicity ( $=$ singlet, $\mathrm{d}=$ doublet, $\mathrm{t}=$ triplet, $\mathrm{q}=$ quartet, $\mathrm{m}=$ multiplet, $\mathrm{br}=$ broad), coupling constant $J(\mathrm{~Hz})$, and integration. High resolution mass spectra were recorded on a Bruker Maxis System using Electron Spray Ionization (ESI). IR spectra were collected on a Spectrum BX FTIR from Perkin-Elmer and reported in unit of $\mathrm{cm}^{-1}$.

Note: These phosphines are generally bench stable, and a trace amount of oxidized product could be observed by ${ }^{31} \mathrm{P}$ NMR after a week. 
Table S1. Optimization of reaction conditions. ${ }^{a}$<smiles>CCOc1ccccc1C#N</smiles>

$1 \mathrm{a}$

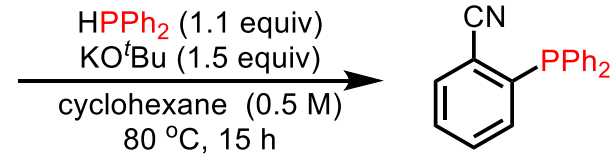

2a

$\begin{array}{lll}\text { Entry } & \text { Change from standard conditions } & \text { Yield }^{b}(\%) \\ 1 & \text { none } & 95 \\ 2 & \text { LiO }^{t} \mathrm{Bu} \text { instead of } \mathrm{KO}^{t} \mathrm{Bu} & 0 \\ 3 & \mathrm{NaO}^{t} \mathrm{Bu} \text { instead of } \mathrm{KO}^{t} \mathrm{Bu} & 41 \\ 4 & \mathrm{KOH} \text { instead of } \mathrm{KO}^{t} \mathrm{Bu} & 20 \\ 5 & \mathrm{KHMDS} \text { instead of KO} \mathrm{KO}^{t} \mathrm{Bu} & 5 \\ 6 & \mathrm{THF}^{\mathrm{instead}} \text { of cyclohexane } & 91 \\ 7 & \text { toluene instead of cyclohexane } & 89 \\ 8 & \text { dioxane instead of cyclohexane } & 94 \\ 9 & \text { DMF instead of cyclohexane } & 2\end{array}$<smiles>COc1ccccc1C#N</smiles><smiles>CCCOc1ccccc1C#N</smiles><smiles>CCCCOc1ccccc1C#N</smiles><smiles>CCCCOc1ccccc1C#N</smiles><smiles>N#Cc1ccccc1Oc1ccccc1</smiles>

1a-1, 55\%

1a-3, 85\%

1a-4, 93\%

1a-5, $99 \%$<smiles>[R]Oc1ccccc1C#N</smiles>

$32 \%^{c}$ 1a-6, $R=$ TBS, $16 \%$

1a-7, $R=A c$, trace

1a-8, $R=T f$, trace

1a-9, $R=$ Ts, trace<smiles>CSc1ccccc1C#N</smiles>
1a-10, 85\%<smiles>CN(c1ccccc1C#N)C(C)(Cl)Cl</smiles><smiles>CCOc1ccccc1P(c1ccccc1)c1ccccc1Pc1ccccc1</smiles>

$38 \%^{d}$<smiles>O=[N+]([O-])c1ccccc1Pc1ccccc1</smiles>

N.D. ${ }^{e}$

aStandard conditions: 1 (0.2 mmol), $\mathrm{HPPh}_{2}$ (1.1 equiv), $\mathrm{KO}^{t} \mathrm{Bu}$ (1.5 equiv), cyclohexane $(0.5 \mathrm{M}), 80{ }^{\circ} \mathrm{C}, 15 \mathrm{~h} .{ }^{b}$ The yields were determined by GC analysis using dodecane as the internal standard. ${ }^{c}$ Ethyl 2-ethoxybenzoate was used. ${ }^{d} 1$-Ethoxy-2-(ethylsulfonyl)benzene was used. ${ }^{e} 1$-Ethoxy-2-nitrobenzene was used. $\mathrm{KHMDS}=$ potassiumbis(trimethylsilyl)amide. $\mathrm{THF}=$ tetrahydrofuran . $\mathrm{DMF}=N, N$-dimethylformamide. 
Table S2. Optimization of reaction conditions for 2 aa.

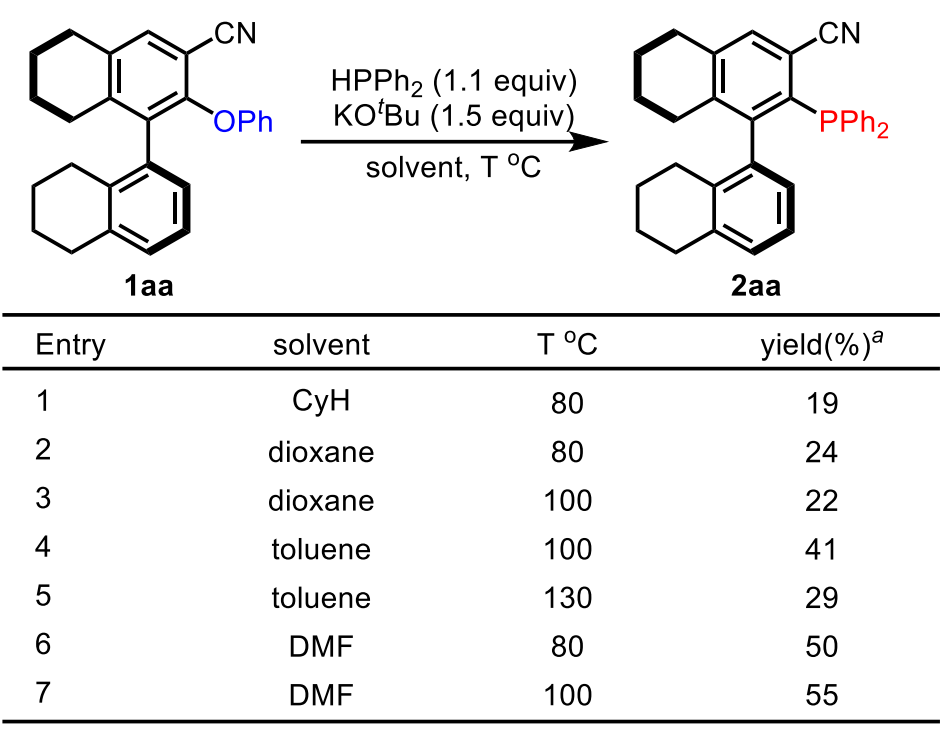

${ }^{a}$ All reactions performed on $0.20 \mathrm{mmol}$ scale, and the yields were isolated by silica gel column chromatography.

Table S3. Optimization of reaction conditions for $4 a$.

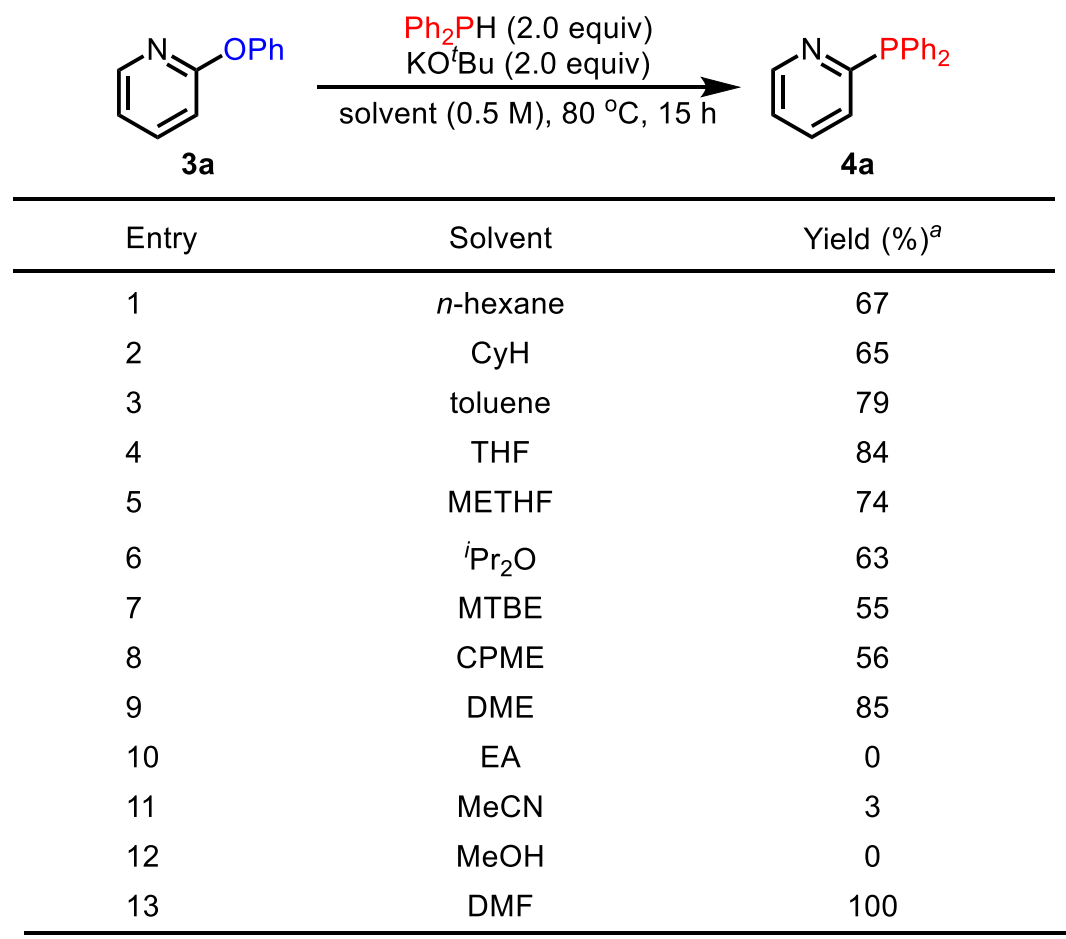

${ }^{a}$ All reactions performed on $0.20 \mathrm{mmol}$ scale and monitored by GC with dodecane as an internal standard. 


\section{Synthesis and Characterization of Substrates}

Table S1. Substrates used in the phosphination.<smiles>CCOc1ccccc1C#N</smiles>

$1 \mathrm{a}$<smiles>CCOc1ccc(C)cc1C#N</smiles>

1b<smiles>CCNc1ccc(OCC)c(C#N)c1</smiles>

1c<smiles>CCOc1ccc(OCC)c(C#N)c1</smiles>

1d<smiles>CCOc1ccc(/C=C/c2ccccc2)cc1C#N</smiles><smiles>CCOc1ccc(Cc2ccccc2)cc1C#N</smiles>

$1 f$<smiles>CCOc1ccc(Cl)cc1C#N</smiles>

19<smiles>CCOc1cc([N+](=O)[O-])ccc1C#N</smiles>

$1 \mathrm{~h}$<smiles>CCOc1cc(C(C)(C)C)ccc1C#N</smiles>

$1 \mathrm{i}$<smiles>COc1cc(C(OC)OC)ccc1C#N</smiles>

$1 \mathrm{j}$<smiles>CCOc1ccc(C#N)cc1</smiles>

$1 k$<smiles>CCOc1cc([N+](=O)[O-])cc(C)c1C#N</smiles>

$1 \mathrm{p}$<smiles>CCOc1cc2c(cc1N=O)CCC2</smiles>

1u<smiles>CCOc1ccc2c(ccc3ccccc32)c1C#N</smiles>

$1 y$<smiles>CCOc1ccc(C(C)(C)c2ccc(OCC)c(C#N)c2)cc1C#N</smiles>

1 ac<smiles>CCOc1c(C#N)cccc1CC</smiles>

11<smiles>CCCc1cccc(C#N)c1OCC</smiles>

$1 \mathrm{~m}$<smiles>CCOc1c(C#N)cccc1-c1ccccc1</smiles>

$1 \mathrm{n}$<smiles>CCOc1ccccc1-c1cccc(C#N)c1OCC</smiles>

10<smiles>CCOc1c(C#N)cc(C)cc1[N+](=O)[O-]</smiles>

19<smiles>CCOc1c(C)ccc(C)c1C#N</smiles>

$1 \mathrm{r}$<smiles>CCOc1c(C#N)ccc([N+](=O)[O-])c1[N+](=O)[O-]</smiles>

$1 \mathrm{~s}$<smiles>CCCc1cc(C#N)c(OCC)cc1N</smiles>

1t<smiles>CCOc1c(C#N)ccc2c1CCCC2</smiles><smiles>CCOc1c(C#N)cc2ccc3cccc4ccc1c2c34</smiles>

$1 z$<smiles>COc1ccc2ccccc2c1C#N</smiles>

$1 \mathrm{w}$<smiles>COc1c(C#N)ccc2ccccc12</smiles>

$1 \mathrm{x}$<smiles>N#Cc1ccc2c(c1-c1ccccc1)[C@@H]1CC[C@H]2C[C@@H]1c1ccccc1</smiles>

1 aa<smiles>c1ccc(Oc2ccccn2)cc1</smiles><smiles>c1ccc(Oc2ccncc2)cc1</smiles>

$3 b$

1a, 1k, $\mathbf{3 a}$ and $\mathbf{3 b}$ were purchased from Energy Chemicals Inc. and used as received. $\mathbf{1 w}$ and $\mathbf{1 x}$ were prepared according to the reported procedure. ${ }^{1}$ 


\section{General procedure A}

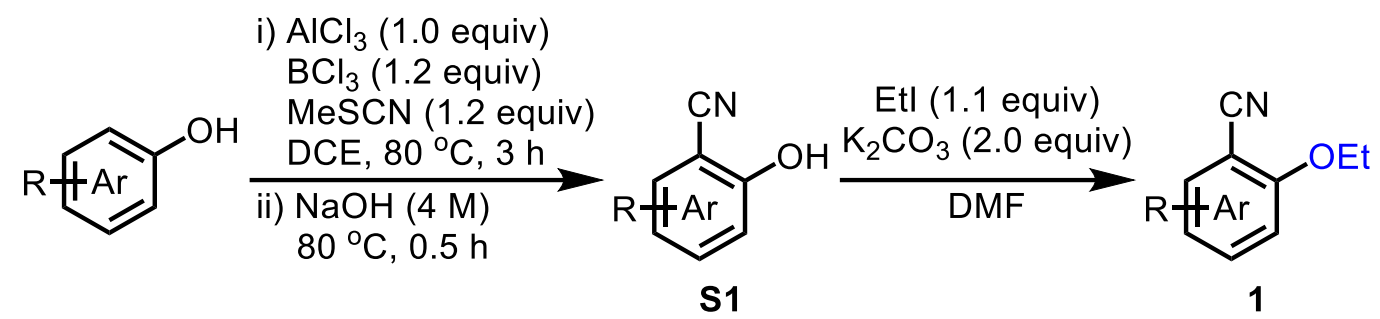

To a stirred suspension of phenol (10 mmol), MeSCN (12 mmol, 1.2 equiv) and $\mathrm{AlCl}_{3}\left(10 \mathrm{mmol}, 1.0\right.$ equiv) in DCE $(10 \mathrm{~mL})$ was added $\mathrm{BCl}_{3}(1.0 \mathrm{M}$ in dichloromethane, $12 \mathrm{~mL}, 12 \mathrm{mmol}, 1.2$ equiv) dropwise at $0{ }^{\circ} \mathrm{C}$. After stirred for $3 \mathrm{~h}$ at $80{ }^{\circ} \mathrm{C}$ in an oil bath, the reaction mixture was cooled to room temperature and quenched with $4.0 \mathrm{M} \mathrm{NaOH}(33 \mathrm{~mL})$, and the mixture was heated up to $80{ }^{\circ} \mathrm{C}$ and stirred for $0.5 \mathrm{~h}$. After cooled to room temperature, DCM was added to the reaction mixture. The aqueous layer was washed with DCM, acidified with $6 \mathrm{M} \mathrm{HCl}(30 \mathrm{~mL})$ and extracted with EtOAc. The combined organic phase was washed with water and brine, and dried over $\mathrm{Na}_{2} \mathrm{SO}_{4}$. The solvent was removed to afford S1, which was used without further purification. ${ }^{2}$

The $\mathbf{S 1}$ was dissolved in $\mathrm{DMF}$, and $\mathrm{K}_{2} \mathrm{CO}_{3}$ (2.0 equiv) and iodoethane (1.1 equiv) were then added at room temperature. Upon completion, $\mathrm{H}_{2} \mathrm{O}$ and EtOAc were added to the reaction mixture. The organic layer was washed with brine and water, dried over $\mathrm{Na}_{2} \mathrm{SO}_{4}$, filtered, and concentrated in vacuo. The resulting crude mixture was purified by silica gel column chromatography to provide the desired ethoxybenzonitrile $\mathbf{1}$.

\section{2-Ethoxy-5-methylbenzonitrile (1b)}<smiles>CCOc1ccc(C)cc1C#N</smiles>

1b was prepared as a colorless oil in $72 \%$ yield (1.16 g, eluent: petroleum ether $/$ EtOAc $=50: 1)$ following the general procedure A.

$\mathbf{R}_{f}=0.55$ (petroleum ether/EtOAc $=10 / 1$ )

${ }^{1} \mathrm{H}$ NMR (400 MHz, $\left.\mathrm{CDCl}_{3}\right) \delta 7.31-7.24(\mathrm{~m}, 2 \mathrm{H}), 6.82(\mathrm{~d}, J=8.4 \mathrm{~Hz}, 1 \mathrm{H}), 4.08$ $(\mathrm{q}, J=7.0 \mathrm{~Hz}, 2 \mathrm{H}), 2.26(\mathrm{~s}, 3 \mathrm{H}), 1.42(\mathrm{t}, J=7.0 \mathrm{~Hz}, 3 \mathrm{H})$. 
${ }^{13} \mathrm{C}$ NMR (100 MHz, $\left.\mathrm{CDCl}_{3}\right) \delta$ 158.7, 135.0, 133.7, 130.2, 116.8, 112.3, 101.7, 64.7, 20.1, 14.6.

HRMS (ESI-TOF) calcd for $\mathrm{C}_{10} \mathrm{H}_{12} \mathrm{NO}^{+}[\mathrm{M}+\mathrm{H}]^{+}: 162.0914$, found: 162.0914 .

IR (neat, cm$^{-1}$ ): 2984, 2929, 2225, 1502, 1475, 1291, 1262, 1160, 1123, 808.

\section{5-(Diethylamino)-2-ethoxybenzonitrile (1c)}

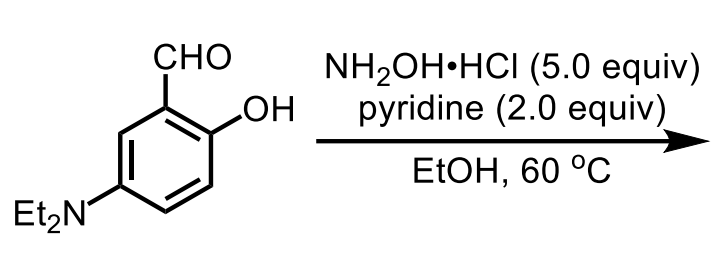

S2

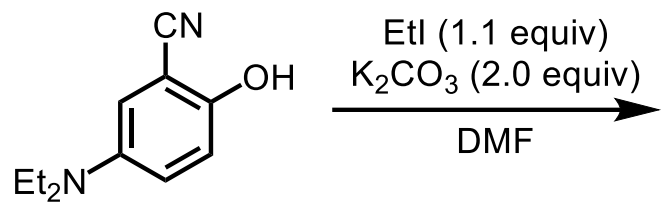

S4

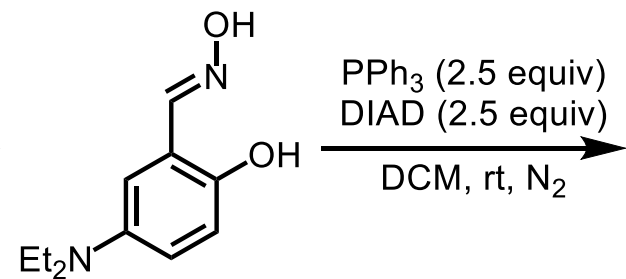

S3

To a solution of aldehyde $\mathbf{S 2}(10 \mathrm{mmol})$ in $\mathrm{EtOH}$ were added $\mathrm{NH}_{2} \mathrm{OH} \cdot \mathrm{HCl}$ (50 mmol) and pyridine $(20 \mathrm{mmol})$, and the resulting mixture was stirred at $60^{\circ} \mathrm{C}$ in an oil bath. Upon completion, the reaction mixture was concentrated and recrystallized to provide oxime $\mathbf{S} 3$.

To a solution of S3 and $\mathrm{PPh}_{3}$ (2.5 equiv) in anhydrous DCM was added DIAD (2.5 equiv) dropwise at room temperature under nitrogen. Upon completion, $0.1 \mathrm{M} \mathrm{NaOH}$ and DCM were added to the reaction mixture. The aqueous layer was washed with $\mathrm{DCM}$, and $1 \mathrm{M} \mathrm{HCl}$ and saturated $\mathrm{NH}_{4} \mathrm{Cl}$ were then added. The acidic aqueous was extracted with DCM. The combined organic phase was washed with water and brine, and dried over $\mathrm{Na}_{2} \mathrm{SO}_{4}$. The solvent was removed to afford salicylonitrile S4, which was used without further purification.

To a stirred suspension of $\mathbf{S} 4$ and $\mathrm{K}_{2} \mathrm{CO}_{3}$ (2.0 equiv) in DMF was added iodoethane (1.1 equiv) at room temperature. Upon completion, $\mathrm{H}_{2} \mathrm{O}$ and EtOAc were added to the reaction mixture. The organic layer were washed with brine and water, dried over $\mathrm{Na}_{2} \mathrm{SO}_{4}$, filtered, and concentrated in vacuo. The resulting crude mixture was purified by silica gel column chromatography (petroleum ether/EtOAc $=10 / 1)$ to afford $1 \mathrm{c}(1.14 \mathrm{~g}, 52 \%)$ as a white solid. 
$\mathbf{R}_{f}=0.20$ (petroleum ether $/$ EtOAc $=5 / 1$ )

${ }^{1} \mathrm{H}$ NMR (400 MHz, $\left.\mathrm{CDCl}_{3}\right) \delta 7.27(\mathrm{~d}, J=8.9 \mathrm{~Hz}, 1 \mathrm{H}), 6.20(\mathrm{dd}, J=8.9,2.3 \mathrm{~Hz}$, $1 \mathrm{H}), 6.06(\mathrm{~s}, 1 \mathrm{H}), 4.10(\mathrm{q}, J=7.0 \mathrm{~Hz}, 2 \mathrm{H}), 3.38(\mathrm{q}, J=7.1 \mathrm{~Hz}, 4 \mathrm{H}), 1.45(\mathrm{t}, J=7.0$ $\mathrm{Hz}, 3 \mathrm{H}), 1.19(\mathrm{t}, J=7.1 \mathrm{~Hz}, 6 \mathrm{H})$.

${ }^{13} \mathrm{C}$ NMR (100 MHz, $\left.\mathrm{CDCl}_{3}\right) \delta$ 162.3, 152.0, 134.4, 118.7, 104.0, 94.3, 87.1, 64.1, $44.7,14.6,12.4$.

HRMS (ESI-TOF) calcd for $\mathrm{C}_{13} \mathrm{H}_{19} \mathrm{~N}_{2} \mathrm{O}^{+}[\mathrm{M}+\mathrm{H}]^{+}:$219.1492, found: 219.1494 .

IR (neat, cm-1): 2975, 2207, 1604, 1549, 1518, 1355, 1271, 1229, 1118, 806, 788.

\section{2,5-Diethoxybenzonitrile (1d)}<smiles>CCOc1ccc(OCC)c(C#N)c1</smiles>

1d was prepared as a colorless oil in 69\% yield (1.32 g, eluent: petroleum ether $/$ EtOAc $=30: 1)$ following the general procedure A.

$\mathbf{R}_{f}=0.50$ (petroleum ether/EtOAc $=10 / 1$ )

${ }^{1} \mathrm{H}$ NMR (400 MHz, $\left.\mathrm{CDCl}_{3}\right) \delta 7.06-6.98(\mathrm{~m}, 2 \mathrm{H}), 6.85$ (d, J = 8.9 Hz, 1H), 4.07 $(\mathrm{q}, J=6.6 \mathrm{~Hz}, 2 \mathrm{H}), 3.95(\mathrm{q}, J=6.7 \mathrm{~Hz}, 2 \mathrm{H}), 1.43(\mathrm{t}, J=6.6 \mathrm{~Hz}, 3 \mathrm{H}), 1.36(\mathrm{t}, J=6.7$ $\mathrm{Hz}, 3 \mathrm{H})$.

${ }^{13} \mathrm{C}$ NMR (100 MHz, $\left.\mathrm{CDCl}_{3}\right) \delta$ 155.1, 152.5, 121.5, 118.2, 116.6, 113.9, 102.2, 65.2, 64.4, 14.8, 14.7 .

HRMS (ESI-TOF) calcd for $\mathrm{C}_{11} \mathrm{H}_{14} \mathrm{NO}_{2}{ }^{+}[\mathrm{M}+\mathrm{H}]^{+}:$192.1019, found: 192.1020 .

IR (neat, cm ${ }^{-1}$ ): 2982, 2227, 1500, 1474, 1393, 1282, 1229, 1161, 924, 808.

(E)-2-Ethoxy-5-styrylbenzonitrile (1e)

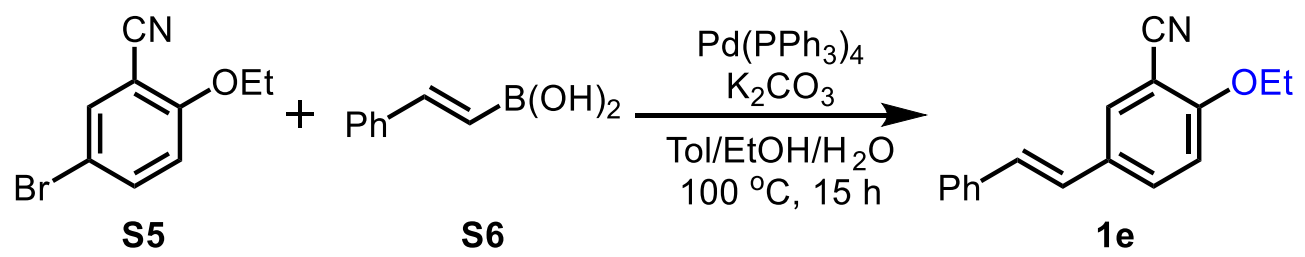

To a degassed suspension of S5 (565 mg, $2.5 \mathrm{mmol}), \mathrm{K}_{2} \mathrm{CO}_{3}(1.38 \mathrm{~g}, 10 \mathrm{mmol}$, 4.0 equiv) and $\mathrm{Pd}\left(\mathrm{PPh}_{3}\right)_{4}(72 \mathrm{mg}, 0.0625 \mathrm{mmol}, 0.025$ equiv) in toluene (12.5 $\mathrm{mL}) / \mathrm{EtOH}(5.0 \mathrm{~mL}) / \mathrm{H}_{2} \mathrm{O}(2.5 \mathrm{~mL})$ was added $\mathbf{S 6}$ (444 mg, $3 \mathrm{mmol}, 1.2$ equiv) under nitrogen, and the resulting mixture was stirred at $100{ }^{\circ} \mathrm{C}$ in an oil bath 
for $16 \mathrm{~h}$. Upon completion, $\mathrm{H}_{2} \mathrm{O}$ and DCM were added to the reaction mixture. The aqueous layer was extracted with DCM (20 mL x 3). The combined organic extracts were dried over $\mathrm{Na}_{2} \mathrm{SO}_{4}$, filtered, and concentrated in vacuo. The resulting crude mixture was purified by silica gel column chromatography (petroleum ether/EtOAc $=50 / 1)$ to afford 1 e $(0.43 \mathrm{~g}, 69 \%)$ as a white solid.

$\mathbf{R}_{f}=0.48$ (petroleum ether/EtOAc $=10 / 1$ )

${ }^{1} \mathrm{H}$ NMR (400 MHz, $\left.\mathrm{CDCl}_{3}\right) \delta 7.63-7.53(\mathrm{~m}, 2 \mathrm{H}), 7.44(\mathrm{~d}, J=7.5 \mathrm{~Hz}, 2 \mathrm{H}), 7.33$ $(\mathrm{t}, J=7.6 \mathrm{~Hz}, 2 \mathrm{H}), 7.24(\mathrm{t}, J=7.3 \mathrm{~Hz}, 1 \mathrm{H}), 6.92(\mathrm{~s}, 2 \mathrm{H}), 6.87(\mathrm{~d}, J=8.7 \mathrm{~Hz}, 1 \mathrm{H})$, $4.09(\mathrm{q}, J=7.1 \mathrm{~Hz}, 2 \mathrm{H}), 1.43(\mathrm{t}, J=7.0 \mathrm{~Hz}, 3 \mathrm{H})$.

${ }^{13} \mathrm{C}$ NMR (100 MHz, $\left.\mathrm{CDCl}_{3}\right) \delta$ 159.9, 136.7, 132.2, 131.4, 130.3, 128.8, 128.7, $127.9,126.5,125.9,116.5,112.5,102.3,64.9,14.5$.

HRMS (ESI-TOF) calcd for $\mathrm{C}_{17} \mathrm{H}_{16} \mathrm{NO}^{+}[\mathrm{M}+\mathrm{H}]^{+}:$250.1227, found: 250.1229 .

IR (neat, cm-1): 2927, 2227, 1500, 1264, 1123, 1039, 964, 811, 731, 703, 539, 510.

\section{2-Ethoxy-5-(4,4,5,5-tetramethyl-1,3,2-dioxaborolan-2-yl)benzonitrile (1f)}<smiles>CCOc1ccc(Br)cc1C#N</smiles>

S5

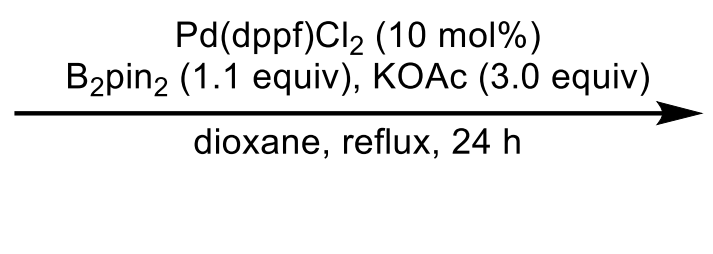<smiles>CCOc1ccc(Br)cc1C#N</smiles>

$1 f$

To a degassed suspension of S5 (2.26 g, $10 \mathrm{mmol})$, KOAc (2.94 g, $30 \mathrm{mmol}$, 3.0 equiv) and $\mathrm{Pd}(\mathrm{dppf}) \mathrm{Cl}_{2}$ (732 mg, $10 \mathrm{mmol}, 0.1$ equiv) in 1,4-dioxane (50 mL) was added bis(pinacolato)diboron $(2.79 \mathrm{~g}, 11 \mathrm{mmol}, 1.1 \mathrm{equiv})$ under nitrogen, and the resulting mixture was refluxed in an oil bath for $24 \mathrm{~h}$. Upon completion, the mixture was concentrated. DCM and $\mathrm{H}_{2} \mathrm{O}$ were added to the residue. The aqueous layer was extracted with DCM $(20 \mathrm{~mL} \times 3)$. The combined organic extracts were dried over $\mathrm{Na}_{2} \mathrm{SO}_{4}$, filtered, and concentrated in vacuo. The resulting crude mixture was purified by silica gel column chromatography (petroleum ether/EtOAc $=10 / 1)$ to afford $1 f(2.7 \mathrm{~g}, 99 \%)$ as a yellow oil.

$\mathbf{R}_{f}=0.50$ (petroleum ether/EtOAc $=10 / 1$ )

${ }^{1} \mathrm{H}$ NMR (400 MHz, $\left.\mathrm{CDCl}_{3}\right) \delta 7.98(\mathrm{~s}, 1 \mathrm{H}), 7.90(\mathrm{~d}, J=8.4 \mathrm{~Hz}, 1 \mathrm{H}), 6.91$ (d, $J=$ $8.5 \mathrm{~Hz}, 1 \mathrm{H}), 4.16(\mathrm{q}, J=6.9 \mathrm{~Hz}, 2 \mathrm{H}), 1.46(\mathrm{t}, J=7.1 \mathrm{~Hz}, 3 \mathrm{H}), 1.31(\mathrm{~s}, 12 \mathrm{H})$. 
${ }^{13} \mathrm{C}$ NMR (100 MHz, $\left.\mathrm{CDCl}_{3}\right) \delta 162.7,140.9,140.7,116.5,111.4,101.9,84.2,64.7$, $24.9,14.5$.

HRMS (ESI-TOF) calcd for $\mathrm{C}_{15} \mathrm{H}_{21} \mathrm{BNO}_{3}{ }^{+}[\mathrm{M}+\mathrm{H}]^{+}:$273.1645, found: 273.1647 .

IR (neat, cm-1): 2980, 2228, 1603, 1381, 1353, 1324, 1286, 1270, 1127, 964, 677.

\section{5-Chloro-2-ethoxybenzonitrile (1g)}<smiles>CCOc1ccc(Cl)cc1C#N</smiles>

$1 \mathrm{~g}$ was prepared as a white solid in 60\% yield (1.09 g, eluent: petroleum ether $/$ EtOAc $=50: 1)$ following the general procedure A.

$\mathbf{R}_{f}=0.43$ (petroleum ether/EtOAc $=10 / 1$ )

${ }^{1} \mathrm{H}$ NMR (400 MHz, $\left.\mathrm{CDCl}_{3}\right) \delta 7.49$ (s, 1H), $7.47-7.42(\mathrm{~m}, 1 \mathrm{H}), 6.89$ (d, J = 8.9 $\mathrm{Hz}, 1 \mathrm{H}), 4.13(\mathrm{q}, J=6.9 \mathrm{~Hz}, 2 \mathrm{H}), 1.47(\mathrm{t}, J=7.0 \mathrm{~Hz}, 3 \mathrm{H})$.

${ }^{13} \mathrm{C}$ NMR (100 MHz, $\left.\mathrm{CDCl}_{3}\right) \delta$ 159.4, 134.4, 133.1, 125.5, 115.3, 113.6, 103.4, 65.3, 14.5.

HRMS (ESI-TOF) calcd for $\mathrm{C}_{9} \mathrm{H}_{9} \mathrm{ClNO}^{+}[\mathrm{M}+\mathrm{H}]^{+}:$182.0367, found: 182.0368 .

IR (neat, cm$^{-1}$ ): 2986, 2231, 1491, 1471, 1396, 1282, 1257, 1135, 1035, 858, 810, 660.

\section{2-Ethoxy-4-methylbenzonitrile (1h)}<smiles>CCOc1cc(C)ccc1C#N</smiles>

1h was prepared as a white solid in 69\% yield (1.12 g, eluent: petroleum ether $/ \mathrm{EtOAc}=50: 1)$ following the reported procedure of cyanation ${ }^{3}$.

$\mathbf{R}_{f}=0.52$ (petroleum ether $/$ EtOAc $=10 / 1$ )

${ }^{1} \mathrm{H}$ NMR (400 MHz, $\left.\mathrm{CDCl}_{3}\right) \delta 7.36(\mathrm{~d}, J=7.8 \mathrm{~Hz}, 1 \mathrm{H}), 6.75(\mathrm{~d}, J=7.8 \mathrm{~Hz}, 1 \mathrm{H})$, $6.73(\mathrm{~s}, 1 \mathrm{H}), 4.09$ (q, J = 7.0 Hz, 2H), $2.36(\mathrm{~s}, 3 \mathrm{H}), 1.43(\mathrm{t}, J=7.0 \mathrm{~Hz}, 3 \mathrm{H})$.

${ }^{13} \mathrm{C}$ NMR (100 MHz, $\left.\mathrm{CDCl}_{3}\right) \delta$ 160.6, 145.6, 133.3, 121.5, 116.9, 112.9, 98.9, 64.5, $22.2,14.5$.

HRMS (ESI-TOF) calcd for $\mathrm{C}_{10} \mathrm{H}_{12} \mathrm{NO}^{+}[\mathrm{M}+\mathrm{H}]^{+}$: 162.0913, found: 162.0914 .

IR (neat, cm ${ }^{-1}$ ): 2939, 2218, 1604, 1506, 1301, 1258, 1172, 1039, 842, 808, 545. 


\section{4-(tert-Butyl)-2-ethoxybenzonitrile (1i)}<smiles>CCOc1cc(C(C)(C)C)ccc1C#N</smiles>

1i was prepared as a yellow oil in $65 \%$ yield (1.32 g, eluent: petroleum ether $/$ EtOAc $=50: 1)$ following the reported procedure of cyanation ${ }^{3}$.

$\mathbf{R}_{f}=0.54$ (petroleum ether/EtOAc $=10 / 1$ )

${ }^{1}$ H NMR (400 MHz, $\left.\mathrm{CDCl}_{3}\right) \delta 7.42(\mathrm{~d}, J=7.9 \mathrm{~Hz}, 1 \mathrm{H}), 6.98(\mathrm{~d}, J=8.1 \mathrm{~Hz}, 1 \mathrm{H})$, $6.94(\mathrm{~s}, 1 \mathrm{H}), 4.15(\mathrm{q}, J=7.2,6.7 \mathrm{~Hz}, 2 \mathrm{H}), 1.45(\mathrm{t}, J=6.9 \mathrm{~Hz}, 3 \mathrm{H}), 1.29(\mathrm{~s}, 9 \mathrm{H})$. ${ }^{13} \mathrm{C}$ NMR (100 MHz, $\left.\mathrm{CDCl}_{3}\right) \delta$ 160.6, 158.7, 133.2, 118.0, 116.9, 109.5, 99.0, 64.5, $35.5,31.0,14.6$.

HRMS (ESI-TOF) calcd for $\mathrm{C}_{13} \mathrm{H}_{18} \mathrm{NO}^{+}[\mathrm{M}+\mathrm{H}]^{+}:$: 204.1383, found: 204.1385.

IR (neat, cm ${ }^{-1}$ ): 2965, 2224, 1605, 1501, 1414, 1395, 1273, 1235, 1038, 962, 822.

\section{4-(Dimethoxymethyl)-2-methoxybenzonitrile (1j)}

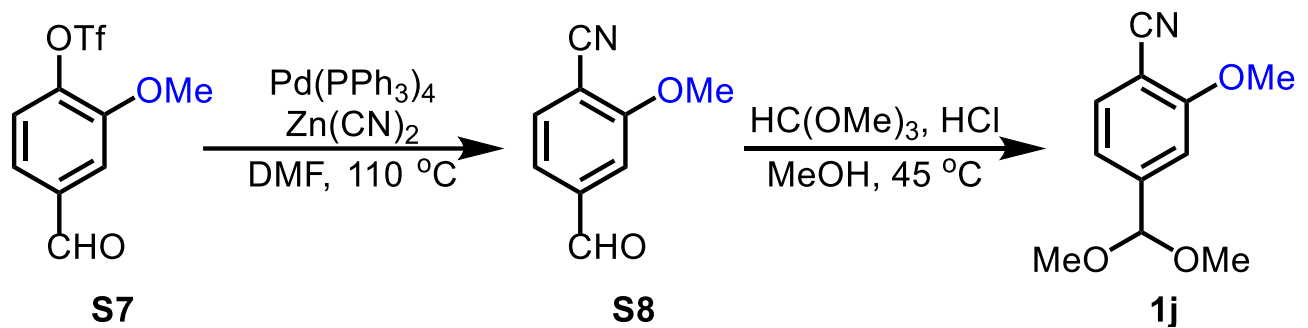

A mixture of $\mathrm{S}^{4}(2.84 \mathrm{~g}, 10 \mathrm{mmol}), \mathrm{Zn}(\mathrm{CN})_{2}(4.69 \mathrm{~g}, 40 \mathrm{mmol}, 4.0$ equiv) and $\mathrm{Pd}\left(\mathrm{PPh}_{3}\right)_{4}$ (578 mg, $0.5 \mathrm{mmol}, 0.05$ equiv) in DMF $(30 \mathrm{~mL})$ was stirred at $110{ }^{\circ} \mathrm{C}$ in an oil bath overnight. Upon completion, $\mathrm{H}_{2} \mathrm{O}$ and EtOAc were added to the reaction mixture. The aqueous layer was extracted with EtOAc $(20 \mathrm{~mL} \times 3)$. The combined organic extracts were washed with brine and water, dried over $\mathrm{Na}_{2} \mathrm{SO}_{4}$, filtered, and concentrated in vacuo. The resulting crude mixture was purified by silica gel column chromatography (petroleum ether/EtOAc $=10 / 1$ ) to afford S8 (0.66 g, 41\%).

To a stirred solution of $\mathbf{S 8}(0.64 \mathrm{~g}, 4 \mathrm{mmol})$ and $\mathrm{HCl}(1.2 \mathrm{mmol}, 0.3 \mathrm{equiv})$ in $\mathrm{MeOH}$ was added trimethylorthoformate $(2.6 \mathrm{~mL}, 24 \mathrm{mmol}, 6.0$ equiv), and the resulting mixture was stirred at $45{ }^{\circ} \mathrm{C}$ in an oil bath overnight. Saturated 
aqueous $\mathrm{Na}_{2} \mathrm{CO}_{3}$ and EtOAc were added to the reaction mixture. The combined organic extracts were dried over $\mathrm{Na}_{2} \mathrm{SO}_{4}$, filtered, and concentrated in vacuo to afford $\mathbf{1 j}(0.75 \mathrm{~g}, 91 \%)$ as a yellow oil, which was used without further purification.

${ }^{1} \mathrm{H}$ NMR (400 MHz, $\left.\left(\mathrm{CD}_{3}\right)_{2} \mathrm{CO}\right) \delta 7.69$ (d, J = 7.7 Hz, 1H), $7.27(\mathrm{~s}, 1 \mathrm{H}), 7.19$ (d, J $=7.7 \mathrm{~Hz}, 1 \mathrm{H}), 5.47(\mathrm{~s}, 1 \mathrm{H}), 4.02(\mathrm{~s}, 3 \mathrm{H}), 3.35(\mathrm{~s}, 6 \mathrm{H})$.

${ }^{13} \mathrm{C}$ NMR (100 MHz, (CD $\left.)_{2} \mathrm{CO}\right) \delta 162.1,146.7,134.3,120.2,116.7,110.9,102.8$, 102.1, 56.6, 53.1.

HRMS (ESI-TOF) calcd for $\mathrm{C}_{11} \mathrm{H}_{14} \mathrm{NO}_{3}{ }^{+}[\mathrm{M}+\mathrm{H}]^{+}:$208.0968, found: 208.0970.

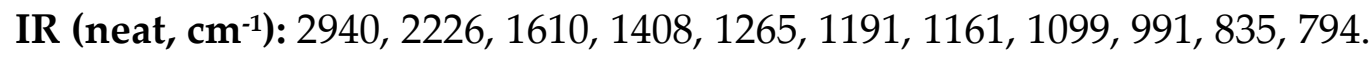

\section{2-Ethoxy-3-ethylbenzonitrile (11)}<smiles>CCOc1c(C#N)cccc1CC</smiles>

11 was prepared as a colorless oil in 75\% yield (1.31 g, eluent: petroleum ether $/$ EtOAc $=50: 1)$ following the general procedure A.

$\mathbf{R}_{f}=0.54$ (petroleum ether/EtOAc $=10 / 1$ )

${ }^{1} \mathrm{H}$ NMR (400 MHz, $\mathrm{CDCl}_{3}$ ) $\delta 7.43-7.36(\mathrm{~m}, 2 \mathrm{H}), 7.07(\mathrm{t}, J=8.1 \mathrm{~Hz}, 1 \mathrm{H}), 4.20$ $(\mathrm{q}, J=7.0 \mathrm{~Hz}, 2 \mathrm{H}), 2.67(\mathrm{q}, J=7.4 \mathrm{~Hz}, 2 \mathrm{H}), 1.46(\mathrm{t}, J=7.0 \mathrm{~Hz}, 3 \mathrm{H}), 1.20(\mathrm{t}, J=7.5$ $\mathrm{Hz}, 3 \mathrm{H})$.

${ }^{13} \mathrm{C}$ NMR (100 MHz, $\left.\mathrm{CDCl}_{3}\right) \delta$ 159.7, 138.5, 134.3, 131.4, 123.9, 117.3, 106.2, 70.9, 22.8, 15.8, 14.5 .

HRMS (ESI-TOF) calcd for $\mathrm{C}_{11} \mathrm{H}_{14} \mathrm{NO}^{+}[\mathrm{M}+\mathrm{H}]^{+}:$176.1070, found: 176.1071 .

IR (neat, cm-1): 2976, 2228, 1450, 1389, 1265, 1209, 1096, 1024, 907, 794, 756.

\section{2-Ethoxy-3-isopropylbenzonitrile (1m)}<smiles>CCCc1cccc(CC)c1OCC</smiles>

$1 \mathrm{~m}$ was prepared as a white solid in 76\% yield (1.44 g, eluent: petroleum ether $/$ EtOAc $=50: 1)$ following the general procedure A.

$\mathbf{R}_{f}=0.65$ (petroleum ether $/$ EtOAc $=10 / 1$ ) 
${ }^{1} \mathrm{H}$ NMR $\left(400 \mathrm{MHz}, \mathrm{CDCl}_{3}\right) \delta 7.46(\mathrm{~d}, J=7.8 \mathrm{~Hz}, 1 \mathrm{H}), 7.39(\mathrm{~d}, J=7.6 \mathrm{~Hz}, 1 \mathrm{H})$, $7.11(\mathrm{t}, J=7.6 \mathrm{~Hz}, 1 \mathrm{H}), 4.17(\mathrm{q}, J=6.8 \mathrm{~Hz}, 2 \mathrm{H}), 3.40-3.26(\mathrm{~m}, 1 \mathrm{H}), 1.47(\mathrm{t}, J=6.9$ $\mathrm{Hz}, 3 \mathrm{H}), 1.20(\mathrm{~d}, J=6.7 \mathrm{~Hz}, 6 \mathrm{H})$.

${ }^{13} \mathrm{C}$ NMR (100 MHz, $\left.\mathrm{CDCl}_{3}\right)$ o 158.9, 143.1, 131.6, 131.2, 124.2, 117.3, 106.3, 71.4, 26.6, 23.3, 15.8.

HRMS (ESI-TOF) calcd for $\mathrm{C}_{12} \mathrm{H}_{16} \mathrm{NO}^{+}[\mathrm{M}+\mathrm{H}]^{+}:$190.1227, found: 190.1227 .

IR (neat, cm-1): 2966, 2230, 1445, 1391, 1339, 1238, 1205, 1094, 1024, 908, 794, 757.

2-Ethoxy-[1,1'-biphenyl]-3-carbonitrile (1n)<smiles>CCOc1c(C#N)cccc1-c1ccccc1</smiles>

1n was prepared as a colorless oil in $45 \%$ yield ( $1.00 \mathrm{~g}$, eluent: petroleum ether $/ \mathrm{EtOAc}=50: 1)$ following the general procedure A.

$\mathbf{R}_{f}=0.60$ (petroleum ether $/ \mathrm{EtOAc}=10 / 1$ )

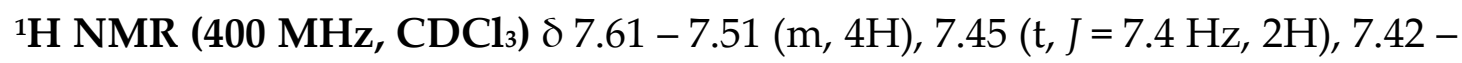
$7.37(\mathrm{~m}, 1 \mathrm{H}), 7.26-7.20(\mathrm{~m}, 1 \mathrm{H}), 3.80(\mathrm{q}, J=7.0 \mathrm{~Hz}, 2 \mathrm{H}), 1.20(\mathrm{t}, J=7.0 \mathrm{~Hz}, 3 \mathrm{H})$. ${ }^{13} \mathrm{C}$ NMR (100 MHz, $\mathrm{CDCl}_{3}$ ) $\delta$ 159.0, 136.8, 136.2, 135.8, 132.6, 129.0, 128.6, 128.1, 124.4, 116.9, 108.2, 70.7, 15.5 .

HRMS (ESI-TOF) calcd for $\mathrm{C}_{15} \mathrm{H}_{14} \mathrm{NO}^{+}[\mathrm{M}+\mathrm{H}]^{+}: 224.1070$, found: 224.1072 .

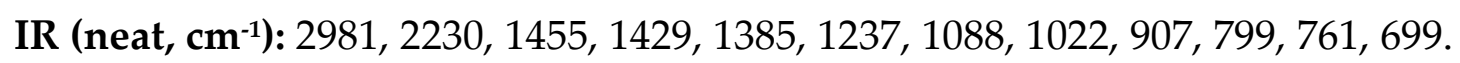

\section{2,2'-Diethoxy-[1,1'-biphenyl]-3-carbonitrile (10)}<smiles>N#Cc1cccc(-c2ccccc2O)c1O</smiles>

S9

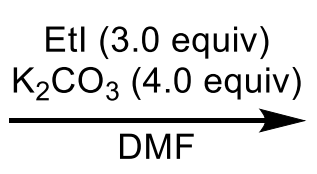<smiles>CCOc1ccccc1-c1cccc(C#N)c1OCC</smiles>

10

To a stirred suspension of $\mathbf{S 9}^{5}(1.06 \mathrm{~g}, 5 \mathrm{mmol})$ and $\mathrm{K}_{2} \mathrm{CO}_{3}(2.76 \mathrm{~g}, 20.0$ mmol, 4.0 equiv) in DMF was added iodoethane (1.2 mL, $15.0 \mathrm{mmol}, 3.0$ equiv) at room temperature. Upon completion, $\mathrm{H}_{2} \mathrm{O}$ and EtOAc were added to the 
reaction mixture. The organic layer was washed with brine and water, dried over $\mathrm{Na}_{2} \mathrm{SO}_{4}$, filtered, and concentrated in vacuo. The resulting crude mixture was purified by silica gel column chromatography $($ petroleum ether $/ \mathrm{EtOAc}=$ $50 / 1)$ to afford $10(0.84 \mathrm{~g}, 63 \%)$ as a colorless oil.

$\mathbf{R}_{f}=0.46$ (petroleum ether $/$ EtOAc $=10 / 1$ )

${ }^{1} \mathrm{H}$ NMR (400 MHz, $\left.\mathrm{CDCl}_{3}\right) \delta 7.41(\mathrm{t}, J=9.2 \mathrm{~Hz}, 2 \mathrm{H}), 7.23(\mathrm{t}, J=7.9 \mathrm{~Hz}, 1 \mathrm{H})$, $7.14(\mathrm{~d}, J=7.3 \mathrm{~Hz}, 1 \mathrm{H}), 7.05(\mathrm{t}, J=7.7 \mathrm{~Hz}, 1 \mathrm{H}), 6.94-6.82(\mathrm{~m}, 2 \mathrm{H}), 3.93(\mathrm{q}, J=$ $7.0 \mathrm{~Hz}, 2 \mathrm{H}), 3.70(\mathrm{q}, J=7.0 \mathrm{~Hz}, 2 \mathrm{H}), 1.17(\mathrm{t}, J=7.0 \mathrm{~Hz}, 3 \mathrm{H}), 1.01(\mathrm{t}, J=7.0 \mathrm{~Hz}$, $3 \mathrm{H})$.

${ }^{13} \mathrm{C}$ NMR (100 MHz, $\left.\mathrm{CDCl}_{3}\right) \delta$ 159.5, 156.0, 136.9, 133.2, 132.3, 131.1, 129.5, 126.0, 123.3, 120.4, 117.1, 112.2, 107.1, 70.2, 63.9, 15.4, 14.7.

HRMS (ESI-TOF) calcd for $\mathrm{C}_{17} \mathrm{H}_{18} \mathrm{NO}_{2}{ }^{+}[\mathrm{M}+\mathrm{H}]^{+}: 268.1332$, found: 268.1333 .

IR (neat, cm-1): 2927, 2228, 1435, 1387, 1265, 1235, 1080, 1043, 1023, 798, 751.

\section{2-Ethoxy-4,6-dimethylbenzonitrile (1p)}<smiles>CCOc1cc(C)cc(C)c1C#N</smiles>

1p was prepared as a white solid in $80 \%$ yield (1.40 g, eluent: petroleum ether $/ \mathrm{EtOAc}=50: 1)$ following the general procedure A.

$\mathbf{R}_{f}=0.52$ (petroleum ether $/$ EtOAc $=10 / 1$ )

${ }^{1} \mathrm{H}$ NMR (400 MHz, $\mathrm{CDCl}_{3}$ ) $\delta 6.64(\mathrm{~s}, 1 \mathrm{H}), 6.54(\mathrm{~s}, 1 \mathrm{H}), 4.08$ (q, J = $\left.7.1 \mathrm{~Hz}, 2 \mathrm{H}\right)$, $2.41(\mathrm{~s}, 3 \mathrm{H}), 2.32(\mathrm{~s}, 3 \mathrm{H}), 1.43(\mathrm{t}, J=6.9 \mathrm{~Hz}, 3 \mathrm{H})$.

${ }^{13} \mathrm{C}$ NMR (100 MHz, $\left.\mathrm{CDCl}_{3}\right) \delta$ 161.0, 144.6, 143.4, 122.9, 116.1, 110.0, 99.7, 64.5, 22.2, 20.4, 14.6 .

HRMS (ESI-TOF) calcd for $\mathrm{C}_{11} \mathrm{H}_{14} \mathrm{NO}^{+}[\mathrm{M}+\mathrm{H}]^{+}$: 176.1070, found: 176.1071 .

IR (neat, cm-1): 2982, 2220, 1607, 1573, 1443, 1317, 1144, 1114, 1090, 820.

\section{2-Ethoxy-3,5-dimethylbenzonitrile (1q)}<smiles>CCOc1c(C)cc(C)cc1C#N</smiles> 
1q was prepared as a white solid in 79\% yield (1.38 g, eluent: petroleum ether $/$ EtOAc $=50: 1)$ following the general procedure A.

$\mathbf{R}_{f}=0.50$ (petroleum ether/EtOAc $=10 / 1$ )

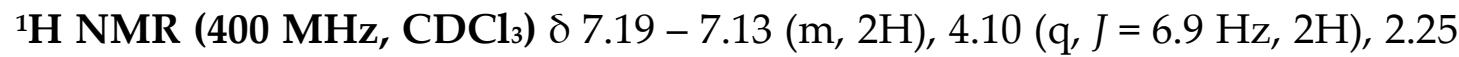
$(\mathrm{s}, 3 \mathrm{H}), 2.23(\mathrm{~s}, 3 \mathrm{H}), 1.42(\mathrm{t}, J=7.0 \mathrm{~Hz}, 3 \mathrm{H})$.

${ }^{13} \mathrm{C}$ NMR (100 MHz, $\left.\mathrm{CDCl}_{3}\right) \delta$ 157.7, 136.8, 133.5, 132.1, 131.1, 117.2, 106.0, 70.3, $20.4,16.0,15.7$.

HRMS (ESI-TOF) calcd for $\mathrm{C}_{11} \mathrm{H}_{14} \mathrm{NO}^{+}[\mathrm{M}+\mathrm{H}]^{+}$: 176.1070, found: 176.1072 .

IR (neat, cm-1): 2981, 2929, 2225, 1485, 1472, 1389, 1249, 1224, 1149, 1027, 896.

\section{2-Ethoxy-3,6-dimethylbenzonitrile (1r)}<smiles>CCOc1c(C)ccc(C)c1C#N</smiles>

1r was prepared as a colorless oil in 74\% yield (1.30 g, eluent: petroleum ether $/ \mathrm{EtOAc}=50: 1)$ following the general procedure A.

$\mathbf{R}_{f}=0.56$ (petroleum ether $/$ EtOAc $=10 / 1$ )

${ }^{1} \mathrm{H}$ NMR (400 MHz, $\left.\mathrm{CDCl}_{3}\right) \delta 7.24(\mathrm{~d}, J=7.9 \mathrm{~Hz}, 1 \mathrm{H}), 6.91(\mathrm{~d}, J=7.7 \mathrm{~Hz}, 1 \mathrm{H})$, $4.12(\mathrm{q}, J=7.1 \mathrm{~Hz}, 2 \mathrm{H}), 2.45(\mathrm{~s}, 3 \mathrm{H}), 2.24(\mathrm{~s}, 3 \mathrm{H}), 1.45(\mathrm{t}, J=7.1 \mathrm{~Hz}, 3 \mathrm{H})$.

${ }^{13} \mathrm{C}$ NMR (100 MHz, $\left.\mathrm{CDCl}_{3}\right) \delta 160.1,141.0,135.3,129.2,125.1,116.3,107.6,70.4$, 20.3, 15.8, 15.8 .

HRMS (ESI-TOF) calcd for $\mathrm{C}_{11} \mathrm{H}_{14} \mathrm{NO}^{+}[\mathrm{M}+\mathrm{H}]^{+}:$176.1070, found: 176.1072 .

IR (neat, cm ${ }^{-1}$ ): 2981, 2224, 1487, 1443, 1386, 1266, 1221, 1063, 1022, 815.

\section{2-Ethoxy-3,4-dimethylbenzonitrile (1s)}<smiles>CCOc1c(C#N)ccc(C)c1C</smiles>

1s was prepared as a yellow oil in $76 \%$ yield $(1.33 \mathrm{~g}$, eluent: petroleum ether $/ \mathrm{EtOAc}=50: 1)$ following the general procedure A.

$\mathbf{R}_{f}=0.56$ (petroleum ether $/$ EtOAc $=10 / 1$ )

${ }^{1} \mathrm{H}$ NMR (400 MHz, $\left.\mathrm{CDCl}_{3}\right) \delta 7.30(\mathrm{~d}, J=7.9 \mathrm{~Hz}, 1 \mathrm{H}), 6.95(\mathrm{~d}, J=7.8 \mathrm{~Hz}, 1 \mathrm{H})$, 
$4.11(\mathrm{q}, J=7.0 \mathrm{~Hz}, 2 \mathrm{H}), 2.31(\mathrm{~s}, 3 \mathrm{H}), 2.19(\mathrm{~s}, 3 \mathrm{H}), 1.46(\mathrm{t}, J=7.0 \mathrm{~Hz}, 3 \mathrm{H})$.

${ }^{13} \mathrm{C}$ NMR (100 MHz, $\left.\mathrm{CDCl}_{3}\right)$ o 159.7, 144.6, 131.3, 130.4, 125.6, 117.5, 104.1, 70.7, 20.8, 15.8, 12.3 .

HRMS (ESI-TOF) calcd for $\mathrm{C}_{11} \mathrm{H}_{14} \mathrm{NO}^{+}[\mathrm{M}+\mathrm{H}]^{+}:$176.1070, found: 176.1071 .

IR (neat, cm ${ }^{-1}$ ): 2981, 2229, 1599, 1451, 1385, 1255, 1220, 1089, 1025, 813.

\section{2-Ethoxy-5-isopropyl-4-methylbenzonitrile (1t)}<smiles>CCCc1cc(C#N)c(OCC)cc1C</smiles>

1t was prepared as a colorless oil in 57\% yield (1.12 g, eluent: petroleum ether $/$ EtOAc $=50: 1)$ following the reported procedure of cyanation ${ }^{3}$.

$\mathbf{R}_{f}=0.58$ (petroleum ether/EtOAc $=10 / 1$ )

${ }^{1} \mathrm{H}$ NMR (400 MHz, $\left.\mathrm{CDCl}_{3}\right) \delta 7.33(\mathrm{~s}, 1 \mathrm{H}), 6.70(\mathrm{~s}, 1 \mathrm{H}), 4.08(\mathrm{q}, J=7.1 \mathrm{~Hz}, 2 \mathrm{H})$, $3.07-2.97(\mathrm{~m}, 1 \mathrm{H}), 2.34(\mathrm{~s}, 3 \mathrm{H}), 1.41(\mathrm{t}, J=6.8 \mathrm{~Hz}, 3 \mathrm{H}), 1.15(\mathrm{~d}, J=7.1 \mathrm{~Hz}, 6 \mathrm{H})$. ${ }^{13} \mathrm{C}$ NMR (100 MHz, $\left.\mathrm{CDCl}_{3}\right) \delta$ 158.3, 142.7, 139.5, 129.9, 117.2, 114.1, 99.3, 64.5, 28.5, 23.1, 20.1, 14.6 .

HRMS (ESI-TOF) calcd for $\mathrm{C}_{13} \mathrm{H}_{18} \mathrm{NO}^{+}[\mathrm{M}+\mathrm{H}]^{+}:$204.1383, found: 204.1385.

IR (neat, cm-1): 2964, 2224, 1612, 1502, 1477, 1394, 1274, 1149, 1109, 913, 896.

6-Ethoxy-2,3-dihydro-1H-indene-5-carbonitrile (1u)<smiles>CCOc1cc2c(cc1C#N)CCC2</smiles>

1u was prepared as a white solid in 67\% yield (1.25 g, eluent: petroleum ether $/ \mathrm{EtOAc}=50: 1)$ following the reported procedure of cyanation ${ }^{3}$.

$\mathbf{R}_{f}=0.54$ (petroleum ether $/$ EtOAc $=10 / 1$ )

${ }^{1} \mathrm{H}$ NMR (400 MHz, $\left.\mathrm{CDCl}_{3}\right) \delta 7.31(\mathrm{~s}, 1 \mathrm{H}), 6.82(\mathrm{~s}, 1 \mathrm{H}), 4.10(\mathrm{q}, J=7.1 \mathrm{~Hz}, 2 \mathrm{H})$, $2.92(\mathrm{t}, J=7.6 \mathrm{~Hz}, 2 \mathrm{H}), 2.83(\mathrm{t}, J=7.5 \mathrm{~Hz}, 2 \mathrm{H}), 2.09(\mathrm{~m}, 2 \mathrm{H}), 1.45(\mathrm{t}, J=7.1 \mathrm{~Hz}$, $3 \mathrm{H})$.

${ }^{13} \mathrm{C}$ NMR (100 MHz, $\left.\mathrm{CDCl}_{3}\right) \delta$ 159.9, 152.0, 136.2, 128.6, 117.4, 108.6, 99.4, 64.7, $33.8,31.6,25.5,14.6$.

HRMS (ESI-TOF) calcd for $\mathrm{C}_{12} \mathrm{H}_{14} \mathrm{NO}^{+}[\mathrm{M}+\mathrm{H}]^{+}:$: 188.1070, found: 188.1071 . 
IR (neat, cm-1): 2941, 2223, 1616, 1488, 1474, 1420, 1395, 1324, 1274, 1170, 874.

\section{1-Ethoxy-5,6,7,8-tetrahydronaphthalene-2-carbonitrile (1v)}<smiles>CCOc1c(C#N)ccc2c1CCCC2</smiles>

1v was prepared as a yellow oil in 54\% yield (1.08 g, eluent: petroleum ether $/$ EtOAc $=50: 1)$ following the general procedure A.

$\mathbf{R}_{f}=0.50$ (petroleum ether/EtOAc $=10 / 1$ )

${ }^{1}$ H NMR (400 MHz, $\left.\mathrm{CDCl}_{3}\right) \delta 7.19(\mathrm{~d}, J=7.7 \mathrm{~Hz}, 1 \mathrm{H}), 6.77(\mathrm{~d}, J=7.9 \mathrm{~Hz}, 1 \mathrm{H})$, $4.10(\mathrm{q}, J=6.8 \mathrm{~Hz}, 2 \mathrm{H}), 2.71-2.61(\mathrm{~m}, 4 \mathrm{H}), 1.72-1.65(\mathrm{~m}, 4 \mathrm{H}), 1.37(\mathrm{t}, J=6.8$ $\mathrm{Hz}, 3 \mathrm{H})$.

${ }^{13} \mathrm{C}$ NMR (100 MHz, $\left.\mathrm{CDCl}_{3}\right) \delta$ 159.8, 145.1, 131.9, 129.9, 124.8, 117.6, 102.5, 70.0, $29.9,23.4,22.3,15.8$.

HRMS (ESI-TOF) calcd for $\mathrm{C}_{13} \mathrm{H}_{16} \mathrm{NO}^{+}[\mathrm{M}+\mathrm{H}]^{+}:$202.1227, found: 202.1228.

IR (neat, cm-1): 2934, 2226, 1599, 1432, 1387, 1329, 1237, 1223, 1076, 1022, 805.

\section{2-Ethoxyphenanthrene-1-carbonitrile (1y)}<smiles>CCOc1ccc2c(ccc3ccccc32)c1C#N</smiles>

1y was prepared as a white solid in 50\% yield (1.24 g, eluent: petroleum ether $/$ EtOAc $=50: 1)$ following the general procedure A.

$\mathbf{R}_{f}=0.42$ (petroleum ether/EtOAc $\left.=10 / 1\right)$

${ }^{1}$ H NMR (400 MHz, $\left.\mathbf{C D C l}_{3}\right) \delta 8.59(\mathrm{~d}, J=9.2 \mathrm{~Hz}, 1 \mathrm{H}), 8.38(\mathrm{~d}, J=8.3 \mathrm{~Hz}, 1 \mathrm{H})$, $7.92(\mathrm{~d}, J=9.0 \mathrm{~Hz}, 1 \mathrm{H}), 7.86-7.75(\mathrm{~m}, 2 \mathrm{H}), 7.61(\mathrm{t}, J=7.6 \mathrm{~Hz}, 1 \mathrm{H}), 7.55(\mathrm{t}, J=$ $7.3 \mathrm{~Hz}, 1 \mathrm{H}), 7.13(\mathrm{~d}, J=9.2 \mathrm{~Hz}, 1 \mathrm{H}), 4.22(\mathrm{q}, J=7.0 \mathrm{~Hz}, 2 \mathrm{H}), 1.52(\mathrm{t}, J=7.1 \mathrm{~Hz}$, $3 \mathrm{H})$.

${ }^{13} \mathrm{C}$ NMR (100 MHz, $\left.\mathrm{CDCl}_{3}\right) \delta 160.8,133.5,130.8,130.6,129.8,129.0,128.9$, 127.6, 126.6, 124.1, 122.5, 121.9, 115.9, 111.8, 97.2, 65.2, 14.8 .

HRMS (ESI-TOF) calcd for $\mathrm{C}_{17} \mathrm{H}_{14} \mathrm{NO}^{+}[\mathrm{M}+\mathrm{H}]^{+}$: 248.1070, found: 248.1072 .

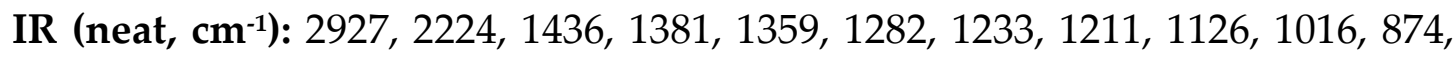
$841,827,686$. 


\section{1-Ethoxypyrene-2-carbonitrile (1z)}<smiles>CCOc1c(C#N)cc2ccc3cccc4ccc1c2c34</smiles>

$1 z$ was prepared as a yellow solid in $42 \%$ yield $(1.14 \mathrm{~g}$, purification with recrystallization, $n$-hexane /DCM) following the general procedure A.

$\mathbf{R}_{f}=0.50$ (petroleum ether/EtOAc $=10 / 1$ )

${ }^{1} \mathrm{H}$ NMR (400 MHz, $\mathrm{CDCl}_{3}$ ) $\delta 8.27$ (d, J = 9.1 Hz, 1H), 8.19 (s, 1H), $8.14(\mathrm{t}, J=$ $8.7 \mathrm{~Hz}, 2 \mathrm{H}), 8.09-8.00(\mathrm{~m}, 2 \mathrm{H}), 7.94(\mathrm{~d}, J=9.0 \mathrm{~Hz}, 1 \mathrm{H}), 7.84(\mathrm{~d}, J=9.0 \mathrm{~Hz}, 1 \mathrm{H})$, $4.50(\mathrm{q}, J=7.0 \mathrm{~Hz}, 2 \mathrm{H}), 1.64(\mathrm{t}, J=7.0 \mathrm{~Hz}, 3 \mathrm{H})$.

${ }^{13} \mathrm{C}$ NMR (100 MHz, $\left.\mathrm{CDCl}_{3}\right) \delta$ 155.2, 131.8, 131.7, 128.6, 128.2, 127.8, 127.7, $127.3,126.4,125.9,125.8,124.7,124.1,121.0,117.7,103.4,72.8,16.0$.

HRMS (ESI-TOF) calcd for $\mathrm{C}_{19} \mathrm{H}_{14} \mathrm{NO}^{+}[\mathrm{M}+\mathrm{H}]^{+}:$272.1070, found: 272.1071 .

IR (neat, cm-1): 2971, 2223, 1464, 1316, 1278, 1264, 1234, 1055, 822, 813, 749.

\section{Compound 1aa}

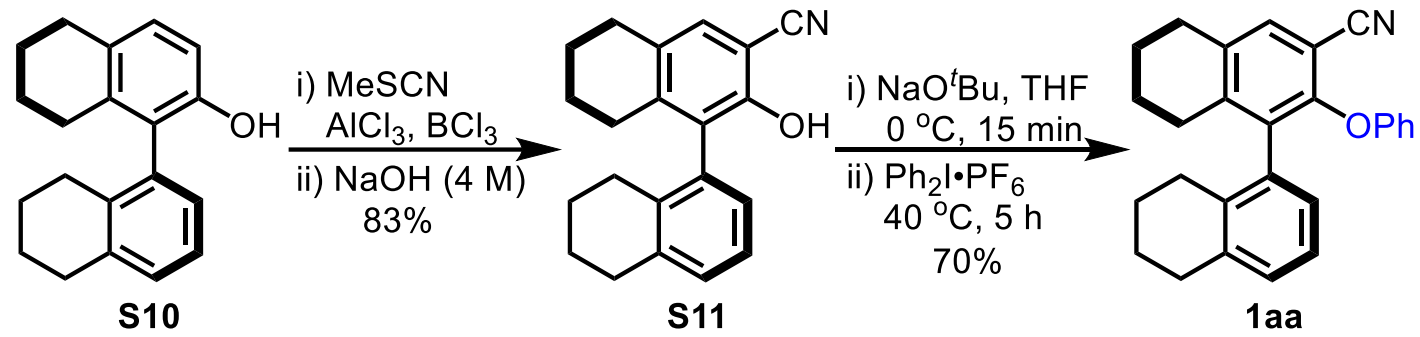

To a stirred suspension of phenol S10 5 (5 mol), MeSCN (6 mmol, 1.2 equiv) and $\mathrm{AlCl}_{3}$ (5 mmol, 1.0 equiv) in DCE $(5 \mathrm{~mL})$ was added $\mathrm{BCl}_{3}(1.0 \mathrm{M}$ in dichloromethane, $6 \mathrm{~mL}, 6 \mathrm{mmol}, 1.2$ equiv) slowly at $0{ }^{\circ} \mathrm{C}$. After stirred for $3 \mathrm{~h}$ at $80{ }^{\circ} \mathrm{C}$ in an oil bath, the reaction mixture was cooled to room temperature and quenched with $4.0 \mathrm{M} \mathrm{NaOH}(17 \mathrm{~mL})$, and the mixture was heated up to $80{ }^{\circ} \mathrm{C}$ and stirred for $0.5 \mathrm{~h}$. After cooling to room temperature, DCM was added to the reaction mixture. The aqueous layer was washed with DCM, acidified with $6 \mathrm{M} \mathrm{HCl}(15 \mathrm{~mL})$ and extracted with EtOAc. The combined organic phase was washed with water and brine, and dried over $\mathrm{Na}_{2} \mathrm{SO}_{4}$. The solvent was removed to afford S11 (1.26 g, 83\%), which was used without further purification. 
To a stirred solution of $\mathbf{S 1 1}$ in dry THF $(20 \mathrm{~mL})$ was added $\mathrm{NaO}^{t} \mathrm{Bu}(1.1$ equiv) at $0 \quad{ }^{\circ} \mathrm{C}$. After stirring for $15 \mathrm{~min}$, diphenyliodonium hexafluorophosphate (1.2 equiv) was added at $0{ }^{\circ} \mathrm{C}$, and the reaction mixture was stirred at $40{ }^{\circ} \mathrm{C}$ in an oil bath overnight. After cooling to room temperature, $\mathrm{H}_{2} \mathrm{O}$ and EtOAc were added to the reaction mixture. The aqueous layer was extracted with EtOAc $(20 \mathrm{~mL} \times 3)$. The combined organic extracts were washed brine and water, dried over $\mathrm{Na}_{2} \mathrm{SO}_{4}$, filtered, and concentrated in vacuo. The resulting crude mixture was purified by silica gel column chromatography (petroleum ether/EtOAc $=50 / 1)$ to afford 1aa $(1.10 \mathrm{~g}$, $70 \%)$ as a white solid.

$\mathbf{R}_{f}=0.52$ (petroleum ether $/ \mathrm{EtOAc}=10 / 1$ )

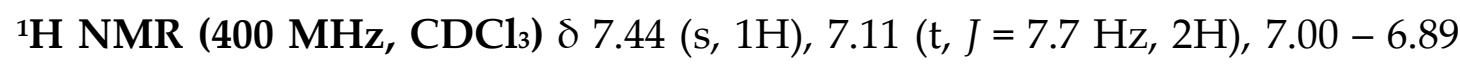
$(\mathrm{m}, 3 \mathrm{H}), 6.66-6.57(\mathrm{~m}, 3 \mathrm{H}), 2.90-2.83(\mathrm{~m}, 2 \mathrm{H}), 2.80-2.71(\mathrm{~m}, 2 \mathrm{H}), 2.50-2.39$ $(\mathrm{m}, 1 \mathrm{H}), 2.39-2.28(\mathrm{~m}, 1 \mathrm{H}), 2.26-2.09(\mathrm{~m}, 2 \mathrm{H}), 1.83-1.59(\mathrm{~m}, 8 \mathrm{H})$.

${ }^{13} \mathrm{C}$ NMR (100 MHz, $\left.\mathrm{CDCl}_{3}\right) \delta$ 158.0, 152.2, 144.1, 137.4, 136.4, 135.3, 135.1, 134.3, 132.8, 129.1, 128.8, 126.6, 125.1, 122.4, 116.6, 116.4, 105.2, 29.9, 29.4, 28.3, 27.0, 23.1, 22.9, 22.5, 22.3 .

HRMS (ESI-TOF) calcd for $\mathrm{C}_{27} \mathrm{H}_{26} \mathrm{NO}^{+}[\mathrm{M}+\mathrm{H}]^{+}: 380.2009$, found: 380.2011 .

IR (neat, cm-1): 3280, 2229, 1590, 1489, 1448, 1434, 1415, 1245, 1201, 781, 736, 688.

\section{Compound $1 \mathrm{ab}$}

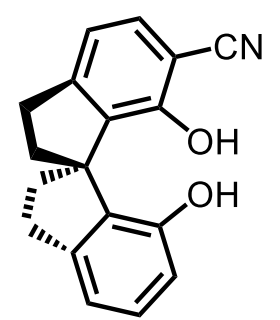

S12

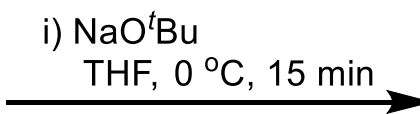

ii) $\mathrm{Ph}_{2} 1 \cdot \mathrm{PF}_{6}$ $40{ }^{\circ} \mathrm{C}, 5 \mathrm{~h}$

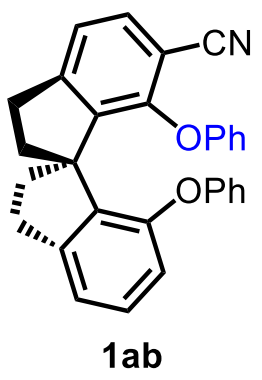

$1 \mathrm{ab}$

To a stirred solution of $\mathbf{S 1 2}{ }^{6}(1.39 \mathrm{~g}, 5 \mathrm{mmol})$ in dry THF $(20 \mathrm{~mL})$ was added $\mathrm{NaO}{ }^{\prime} \mathrm{Bu}\left(1.06 \mathrm{~g}, 11.0 \mathrm{mmol}, 2.2\right.$ equiv) at $0{ }^{\circ} \mathrm{C}$. After stirring for $15 \mathrm{~min}$, diphenyliodonium hexafluorophosphate $(5.11 \mathrm{~g}, 12.0 \mathrm{mmol}, 2.4$ equiv) was added at $0{ }^{\circ} \mathrm{C}$, and the reaction mixture was stirred at $40{ }^{\circ} \mathrm{C}$ in an oil bath overnight. After cooling to room temperature, $\mathrm{H}_{2} \mathrm{O}$ and EtOAc were added to the reaction mixture. The aqueous layer was extracted with EtOAc $(20 \mathrm{~mL} \times 3)$. The combined organic extracts were washed brine and water, dried over 
$\mathrm{Na}_{2} \mathrm{SO}_{4}$, filtered, and concentrated in vacuo. The resulting crude mixture was purified by silica gel column chromatography (petroleum ether/EtOAc $=30 / 1$ ) to afford $1 \mathrm{ab}(0.86 \mathrm{~g}, 40 \%)$ as a yellow oil.

$\mathbf{R}_{f}=0.25$ (petroleum ether/EtOAc $=10 / 1$ )

${ }^{1} \mathrm{H}$ NMR (400 MHz, $\left.\mathrm{CDCl}_{3}\right) \delta 7.50-7.37(\mathrm{~m}, 1 \mathrm{H}), 7.33-7.26(\mathrm{~m}, 2 \mathrm{H}), 7.20-$ $7.03(\mathrm{~m}, 5 \mathrm{H}), 7.02-6.95(\mathrm{~m}, 1 \mathrm{H}), 6.90-6.81(\mathrm{~m}, 3 \mathrm{H}), 6.61-6.49(\mathrm{~m}, 3 \mathrm{H}), 3.23-$ $3.05(\mathrm{~m}, 2 \mathrm{H}), 3.04-2.92(\mathrm{~m}, 1 \mathrm{H}), 2.90-2.76(\mathrm{~m}, 1 \mathrm{H}), 2.70-2.50(\mathrm{~m}, 1 \mathrm{H}), 2.35-$ $2.19(\mathrm{~m}, 3 \mathrm{H})$.

${ }^{13} \mathrm{C}$ NMR (100 MHz, $\left.\mathrm{CDCl}_{3}\right) \delta$ 157.7, 156.7, 153.6, 153.2, 153.0, 146.4, 143.5, $137.5,133.0,129.6,129.3,128.6,123.1,122.5,121.8,119.5,118.5,116.3,116.0$, $115.7,105.6,59.5,40.0,39.3,32.3,31.5$.

HRMS (ESI-TOF) calcd for $\mathrm{C}_{30} \mathrm{H}_{24} \mathrm{NO}_{2}{ }^{+}[\mathrm{M}+\mathrm{H}]^{+}: 430.1802$, found: 430.1800 .

IR (neat, cm$^{-1}$ ): 2931, 2227, 1591, 1581, 1489, 1465, 1435, 1243, 1165, 750, 690.

\section{Compound 1ac}<smiles>CCOc1ccc(C(C)(C)c2ccc(OCC)c(C#N)c2)cc1C#N</smiles>

1ac was prepared as a white solid in $62 \%$ yield $(2.07 \mathrm{~g}$, eluent: petroleum ether $/$ EtOAc $=15: 1)$ following the general procedure A.

$\mathbf{R}_{f}=0.22$ (petroleum ether $/$ EtOAc $=5 / 1$ )

${ }^{1}$ H NMR (400 MHz, $\left.\mathrm{CDCl}_{3}\right) \delta 7.39(\mathrm{~d}, J=2.5 \mathrm{~Hz}, 2 \mathrm{H}), 7.32(\mathrm{dd}, J=8.9,2.6 \mathrm{~Hz}$, $2 \mathrm{H}), 6.90(\mathrm{~d}, J=8.9 \mathrm{~Hz}, 2 \mathrm{H}), 4.14(\mathrm{q}, J=6.9 \mathrm{~Hz}, 4 \mathrm{H}), 1.64(\mathrm{~s}, 6 \mathrm{H}), 1.46(\mathrm{t}, J=7.0$ $\mathrm{Hz}, 6 \mathrm{H})$.

${ }^{13} \mathrm{C}$ NMR (100 MHz, $\left.\mathrm{CDCl}_{3}\right) \delta$ 158.9, 142.1, 132.8, 131.5, 116.7, 112.2, 101.5, 64.7, $41.6,30.5,14.5$.

HRMS (ESI-TOF) calcd for $\mathrm{C}_{21} \mathrm{H}_{23} \mathrm{~N}_{2} \mathrm{O}_{2}{ }^{+}[\mathrm{M}+\mathrm{H}]^{+}:$335.1754, found: 335.1755.

IR (neat, cm-1): 2978, 2929, 2226, 1606, 1500, 1474, 1394, 1287, 1153, 1037, 815.

\section{Compound 1ad}

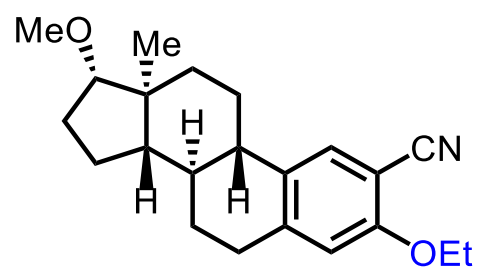


1ad was prepared as a white solid in $42 \%$ yield (1.42 g, eluent: petroleum ether $/ \mathrm{EtOAc}=30: 1)$ following the reported procedure of cyanation ${ }^{3}$.

$\mathbf{R}_{f}=0.32$ (petroleum ether $/$ EtOAc $=10 / 1$ )

${ }^{1} \mathrm{H}$ NMR (400 MHz, $\mathrm{CDCl}_{3}$ ) $\delta 7.40$ (s, 1H), 6.62 (s, 1H), 4.07 (q, J = 7.2 Hz, 2H), $3.36(\mathrm{~s}, 3 \mathrm{H}), 3.29(\mathrm{t}, J=8.5 \mathrm{~Hz}, 1 \mathrm{H}), 2.85(\mathrm{~s}, 2 \mathrm{H}), 2.25-1.99(\mathrm{~m}, 4 \mathrm{H}), 1.89-1.86$ $(\mathrm{m}, 1 \mathrm{H}), 1.70-1.63(\mathrm{~m}, 1 \mathrm{H}), 1.54-1.10(\mathrm{~m}, 10 \mathrm{H}), 0.76(\mathrm{~s}, 3 \mathrm{H})$.

${ }^{13} \mathrm{C}$ NMR (100 MHz, $\left.\mathrm{CDCl}_{3}\right) \delta$ 158.3, 144.3, 133.3, 130.7, 117.3, 112.4, 99.1, 90.6, 64.6, 58.0, 50.2, 43.4, 43.2, 38.2, 37.8, 30.4, 27.8, 26.8, 26.3, 23.0, 14.7, 11.6.

HRMS (ESI-TOF) calcd for $\mathrm{C}_{22} \mathrm{H}_{30} \mathrm{NO}_{2}{ }^{+}[\mathrm{M}+\mathrm{H}]^{+}: 340.2271$, found: 340.2273 .

IR (neat, cm-1): 2928, 2872, 2224, 1500, 1265, 1209, 1105, 1036, 733, 703. 


\section{Synthesis and Characterization of Products}

\section{General procedure B}<smiles>CCOc1ccc[R]#c1C#N</smiles>

1

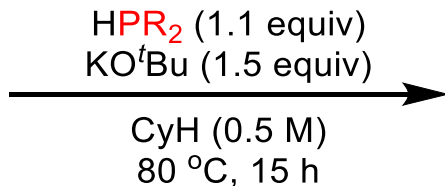

$80^{\circ} \mathrm{C}, 15 \mathrm{~h}$

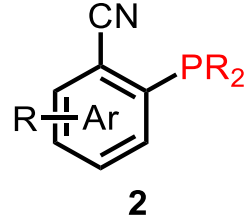

In a glovebox, to an oven-dried sealed tube were added $\mathrm{KO}^{t} \mathrm{Bu}(841.5 \mathrm{mg}$, $7.5 \mathrm{mmol}, 1.5$ equiv), $\mathrm{HPR}_{2}$ (5.5 mmol, 1.1 equiv), 2-ethoxyarylnitrile 1 (5.0 $\mathrm{mmol})$ and cyclohexane $(10 \mathrm{~mL})$ at room temperature, and the tube was then removed from the glovebox. The resulting mixture was stirred at $80{ }^{\circ} \mathrm{C}$ in an oil bath for $15 \mathrm{~h}$. After cooling to room temperature, the reaction mixture was diluted with DCM, filtered through a plug of silica gel, and concentrated in vacuo. The resulting crude mixture was purified by silica gel column chromatography to afford the corresponding product 2.

\section{General procedure C}<smiles>c1ccc(Oc2ccccn2)cc1</smiles>

3

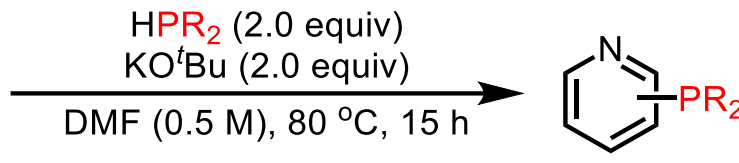

4

In a glovebox, to an oven-dried sealed tube were added $\mathrm{KO}^{t} \mathrm{Bu}(44.8 \mathrm{mg}, 0.4$ mmol, 2.0 equiv), $\mathrm{HPR}_{2}$ (0.4 mmol, 2.0 equiv), 2-ethoxyarylnitrile 3 (0.2 mmol) and DMF $(0.4 \mathrm{~mL})$ at room temperature, and the tube was then removed from the glovebox. The resulting mixture was stirred at $80^{\circ} \mathrm{C}$ in an oil bath for $15 \mathrm{~h}$. After cooling to room temperature, the reaction mixture was diluted with DCM, filtered through a plug of silica gel, and concentrated in vacuo. The resulting crude mixture was purified by silica gel column chromatography to afford the corresponding product 4 .

\section{2-(Diphenylphosphaneyl)benzonitrile (2a)}<smiles>N#Cc1ccccc1Pc1ccccc1</smiles>

2a was prepared as a white solid in $89 \%$ yield (1.28 g, eluent: petroleum ether $/$ EtOAc $=50: 1)$ from $1 \mathrm{a}(0.74 \mathrm{~g}, 5.0 \mathrm{mmol}), \mathrm{HPPh}_{2}(0.95 \mathrm{~mL}, 1.02 \mathrm{~g}, 5.5 \mathrm{mmol})$ and $\mathrm{KO}^{t} \mathrm{Bu}(0.84 \mathrm{~g}, 7.5 \mathrm{mmol})$ following the general procedure $\mathrm{B}$. 
$\mathbf{R}_{f}=0.50$ (petroleum ether $/$ EtOAc $=10 / 1$ )

${ }^{1} \mathrm{H}$ NMR $\left(400 \mathrm{MHz}, \mathrm{CDCl}_{3}\right) \delta 7.71(\mathrm{~d}, J=6.5 \mathrm{~Hz}, 1 \mathrm{H}), 7.48(\mathrm{t}, J=7.6 \mathrm{~Hz}, 1 \mathrm{H})$, $7.45-7.27(\mathrm{~m}, 11 \mathrm{H}), 7.05(\mathrm{dd}, J=7.8,3.3 \mathrm{~Hz}, 1 \mathrm{H})$.

${ }^{13} \mathrm{C}$ NMR (100 MHz, $\left.\mathrm{CDCl}_{3}\right) \delta 143.1(\mathrm{~d}, J=19.6 \mathrm{~Hz}), 134.7(\mathrm{~d}, J=10.2 \mathrm{~Hz})$, $134.1(\mathrm{~d}, J=20.4 \mathrm{~Hz}), 133.8(\mathrm{~d}, J=4.8 \mathrm{~Hz}), 133.5,132.5,129.5,129.0,128.9(\mathrm{~d}, J=$ $7.3 \mathrm{~Hz}), 117.9(\mathrm{~d}, J=32.8 \mathrm{~Hz}), 117.7(\mathrm{~d}, J=3.7 \mathrm{~Hz})$.

${ }^{31}$ P NMR (162 MHz, $\left.\mathrm{CDCl}_{3}\right) \delta$-8.6.

The characterization data are consistent with the literature reported data. ${ }^{7}$

\section{2-(Diphenylphosphaneyl)-5-methylbenzonitrile (2b)}<smiles>Cc1ccc(P)c(P)c1</smiles>

$\mathbf{2} \mathbf{b}$ was prepared as a white solid in 79\% yield ( $1.19 \mathrm{~g}$, eluent: petroleum ether $/$ EtOAc $=60: 1)$ from $1 \mathrm{~b}(0.81 \mathrm{~g}, 5.0 \mathrm{mmol}), \mathrm{HPPh}_{2}(0.95 \mathrm{~mL}, 1.02 \mathrm{~g}, 5.5 \mathrm{mmol})$ and $\mathrm{KO}^{\prime} \mathrm{Bu}(0.84 \mathrm{~g}, 7.5 \mathrm{mmol})$ following the general procedure $\mathrm{B}$.

$\mathbf{R}_{f}=0.55$ (petroleum ether $/ \mathrm{EtOAc}=10 / 1$ )

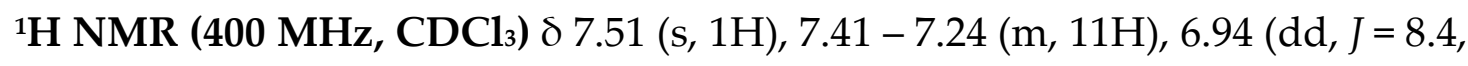
$3.3 \mathrm{~Hz}, 1 \mathrm{H}), 2.35(\mathrm{~s}, 3 \mathrm{H})$.

${ }^{13} \mathrm{C}$ NMR (100 MHz, $\left.\mathrm{CDCl}_{3}\right) \delta$ 139.5, 139.3 (d, $\left.J=18.3 \mathrm{~Hz}\right), 135.2(\mathrm{~d}, J=10.3$ $\mathrm{Hz}), 134.2(\mathrm{~d}, J=5.1 \mathrm{~Hz}), 133.9(\mathrm{~d}, J=20.2 \mathrm{~Hz}), 133.6(\mathrm{~d}, J=15.7 \mathrm{~Hz}), 129.3$, $128.9,128.8,118.0(\mathrm{~d}, J=37.2 \mathrm{~Hz}), 117.9,21.0$.

31P NMR (162 MHz, $\left.\mathrm{CDCl}_{3}\right) \delta$-9.6.

HRMS (ESI-TOF) calcd for $\mathrm{C}_{20} \mathrm{H}_{17} \mathrm{NP}^{+}[\mathrm{M}+\mathrm{H}]^{+}$: 302.1093, found: 302.1095 .

IR (neat, cm-1): 3053, 2225, 1596, 1479, 1465, 1434, 1276, 1233, 826, 743, 695.

\section{5-(Diethylamino)-2-(diphenylphosphaneyl)benzonitrile (2c)}<smiles>CCNc1ccc(P(=O)(c2ccccc2)c2ccccc2)c(C#N)c1</smiles>

2c was prepared as a white solid in $90 \%$ yield $(1.61 \mathrm{~g}$, eluent: petroleum ether $/$ EtOAc $=15: 1)$ from $1 \mathrm{c}(1.09 \mathrm{~g}, 5.0 \mathrm{mmol}), \mathrm{HPPh}_{2}(0.95 \mathrm{~mL}, 1.02 \mathrm{~g}, 5.5 \mathrm{mmol})$ and $\mathrm{KO}^{t} \mathrm{Bu}(0.84 \mathrm{~g}, 7.5 \mathrm{mmol})$ following the general procedure $\mathrm{B}$.

$\mathbf{R}_{f}=0.25$ (petroleum ether $/ \mathrm{EtOAc}=5 / 1$ ) 
${ }^{1} \mathrm{H}$ NMR (400 MHz, $\left.\mathrm{CDCl}_{3}\right) \delta 7.49(\mathrm{~s}, 1 \mathrm{H}), 7.36(\mathrm{~d}, J=7.4 \mathrm{~Hz}, 10 \mathrm{H}), 6.55(\mathrm{~d}, J=$ $8.5 \mathrm{~Hz}, 1 \mathrm{H}), 6.05(\mathrm{~s}, 1 \mathrm{H}), 3.15(\mathrm{q}, J=7.2 \mathrm{~Hz}, 4 \mathrm{H}), 0.96(\mathrm{t}, J=7.1 \mathrm{~Hz}, 6 \mathrm{H})$.

${ }^{13} \mathrm{C}$ NMR (100 MHz, $\left.\mathrm{CDCl}_{3}\right) \delta$ 149.7, $143.3(\mathrm{~d}, J=17.6 \mathrm{~Hz}), 135.4(\mathrm{~d}, J=10.8$ $\mathrm{Hz}), 135.1(\mathrm{~d}, J=5.8 \mathrm{~Hz}), 134.0(\mathrm{~d}, J=20.1 \mathrm{~Hz}), 129.2,128.7(\mathrm{~d}, J=7.1 \mathrm{~Hz}), 119.6$ $(\mathrm{d}, J=3.9 \mathrm{~Hz}), 116.2,110.7,101.7(\mathrm{~d}, J=31.2 \mathrm{~Hz}), 44.6,12.2$.

31P NMR (162 MHz, $\left.\mathrm{CDCl}_{3}\right) \delta-7.5$.

HRMS (ESI-TOF) calcd for $\mathrm{C}_{23} \mathrm{H}_{24} \mathrm{~N}_{2} \mathrm{P}^{+}[\mathrm{M}+\mathrm{H}]^{+}$: 359.1672, found: 359.1673 .

IR (neat, cm $\left.{ }^{-1}\right)$ : 2971, 2205, 1583, 1536, 1496, 1434, 1354, 1264, 743, 694.

\section{2-(Diphenylphosphaneyl)-5-ethoxybenzonitrile (2d)}<smiles>CCOc1ccc(P(c2ccccc2)c2ccccc2)c(C#N)c1</smiles>

2d was prepared as a white solid in $51 \%$ yield $(0.85 \mathrm{~g}$, eluent: petroleum ether $/$ EtOAc $=50: 1)$ from $1 d(0.96 \mathrm{~g}, 5.0 \mathrm{mmol}), \mathrm{HPPh}_{2}(0.95 \mathrm{~mL}, 1.02 \mathrm{~g}, 5.5 \mathrm{mmol})$ and $\mathrm{KO}^{t} \mathrm{Bu}(0.84 \mathrm{~g}, 7.5 \mathrm{mmol})$ following the general procedure $\mathrm{B}$.

$\mathbf{R}_{f}=0.45$ (petroleum ether/EtOAc $=10 / 1$ )

${ }^{1}$ H NMR (400 MHz, $\left.\mathrm{CDCl}_{3}\right) \delta 7.40-7.16(\mathrm{~m}, 11 \mathrm{H}), 7.03-6.92(\mathrm{~m}, 2 \mathrm{H}), 4.01$ (q, $J=7.1 \mathrm{~Hz}, 2 \mathrm{H}), 1.40(\mathrm{t}, J=7.1 \mathrm{~Hz}, 3 \mathrm{H})$.

${ }^{13} \mathrm{C}$ NMR (100 MHz, $\left.\mathrm{CDCl}_{3}\right) \delta$ 159.3, $135.6(\mathrm{~d}, J=10.4 \mathrm{~Hz}), 135.4,133.7$ (d, $J=$ $20.1 \mathrm{~Hz}), 132.8(\mathrm{~d}, J=16.8 \mathrm{~Hz}), 129.2,128.7(\mathrm{~d}, J=7.0 \mathrm{~Hz}), 119.5,119.4,119.1(\mathrm{~d}$, $J=6.0 \mathrm{~Hz}), 117.7(\mathrm{~d}, J=3.7 \mathrm{~Hz}), 64.1,14.6$.

${ }^{31 P}$ NMR (162 MHz, $\left.\mathrm{CDCl}_{3}\right) \delta-10.7$.

HRMS (ESI-TOF) calcd for $\mathrm{C}_{21} \mathrm{H}_{19} \mathrm{NOP}^{+}[\mathrm{M}+\mathrm{H}]^{+}$: 332.1199, found: 332.1200 .

IR (neat, cm$^{-1}$ ): 2985, 1593, 1555, 1467, 1435, 1301, 1274, 1238, 1058, 745, 696, 497.

(E)-2-(Diphenylphosphaneyl)-5-styrylbenzonitrile (2e)<smiles>N#Cc1cc(/C=C/c2ccccc2)ccc1P(c1ccccc1)c1ccccc1</smiles>

2e was prepared as a white solid in $79 \%$ yield $(0.31 \mathrm{~g}$, eluent: petroleum ether $/$ EtOAc $=50: 1)$ from 1 e $(0.25 \mathrm{~g}, 1.0 \mathrm{mmol}), \mathrm{HPPh}_{2}(0.19 \mathrm{~mL}, 0.20 \mathrm{~g}, 1.1 \mathrm{mmol})$ and $\mathrm{KO}^{t} \mathrm{Bu}(0.17 \mathrm{~g}, 1.5 \mathrm{mmol})$ following the general procedure $\mathrm{B}$. 
$\mathbf{R}_{f}=0.56$ (petroleum ether/EtOAc $=10 / 1$ )

${ }^{1} \mathrm{H}$ NMR (400 MHz, $\left.\mathrm{CDCl}_{3}\right) \delta 7.84(\mathrm{~s}, 1 \mathrm{H}), 7.58(\mathrm{~d}, J=8.2 \mathrm{~Hz}, 1 \mathrm{H}), 7.54(\mathrm{~d}, J=$ $7.7 \mathrm{~Hz}, 2 \mathrm{H}), 7.49-7.28(\mathrm{~m}, 13 \mathrm{H}), 7.18(\mathrm{~d}, J=16.3 \mathrm{~Hz}, 1 \mathrm{H}), 7.11-7.00(\mathrm{~m}, 2 \mathrm{H})$.

${ }^{13} \mathrm{C}$ NMR (100 MHz, $\left.\mathrm{CDCl}_{3}\right) \delta 141.3(\mathrm{~d}, J=19.4 \mathrm{~Hz}), 138.4,136.3,134.8(\mathrm{~d}, J=$ $10.3 \mathrm{~Hz}), 134.1,133.9,131.7,131.4(\mathrm{~d}, J=4.9 \mathrm{~Hz}), 130.1,129.5,128.94,128.87$ (d, $J=2.7 \mathrm{~Hz}), 128.6,126.9,125.8,118.4(\mathrm{~d}, J=33.3 \mathrm{~Hz}), 117.7(\mathrm{~d}, J=3.6 \mathrm{~Hz})$.

${ }^{31} \mathrm{P}$ NMR (162 MHz, $\left.\mathrm{CDCl}_{3}\right) \delta$-8.9.

HRMS (ESI-TOF) calcd for $\mathrm{C}_{27} \mathrm{H}_{21} \mathrm{NP}^{+}[\mathrm{M}+\mathrm{H}]^{+}$: 390.1406, found: 390.1407.

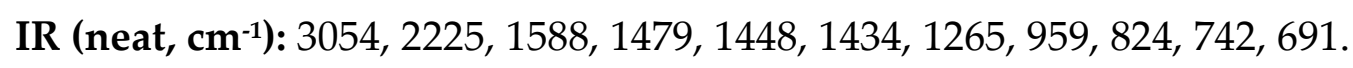

\section{2-(Diphenylphosphaneyl)-5-(4,4,5,5-tetramethyl-1,3,2-dioxaborolan-2-} yl)benzonitrile (2f)<smiles>N#Cc1cc(Cc2ccccc2)ccc1P</smiles>

2f was prepared as a white solid in 59\% yield (1.22 g, eluent: petroleum ether /EtOAc = 50:1) from 1f $(1.37 \mathrm{~g}, 5.0 \mathrm{mmol}) \mathrm{HPPh}_{2}(0.95 \mathrm{~mL}, 1.02 \mathrm{~g}, 5.5 \mathrm{mmol})$ and KHMDS (1.0 M in THF, 7.5 mL, $7.5 \mathrm{mmol}$ ) instead of $\mathrm{KO}^{t} \mathrm{Bu}$ following the general procedure $B$.

$\mathbf{R}_{f}=0.50$ (petroleum ether $/ \mathrm{EtOAc}=10 / 1$ )

${ }^{1} \mathrm{H}$ NMR (400 MHz, $\mathrm{CDCl}_{3}$ ) $\delta 8.15(\mathrm{~s}, 1 \mathrm{H}), 7.88(\mathrm{~d}, J=7.7 \mathrm{~Hz}, 1 \mathrm{H}), 7.44-7.28$ $(\mathrm{m}, 10 \mathrm{H}), 7.06(\mathrm{dd}, J=7.4,3.4 \mathrm{~Hz}, 1 \mathrm{H}), 1.35(\mathrm{~s}, 12 \mathrm{H})$.

${ }^{13} \mathrm{C}$ NMR (100 MHz, $\left.\mathrm{CDCl}_{3}\right) \delta 146.0$ (d, J = 20.4 Hz), 139.8 (d, J=4.5 Hz), 138.2, $134.5(\mathrm{~d}, J=10.1 \mathrm{~Hz}), 134.1(\mathrm{~d}, J=20.3 \mathrm{~Hz}), 132.6,129.5,128.9(\mathrm{~d}, J=7.4 \mathrm{~Hz})$, $117.6,117.4(\mathrm{~d}, J=37.4 \mathrm{~Hz}), 84.5,24.9$.

${ }^{31} \mathrm{P}$ NMR (162 MHz, $\left.\mathrm{CDCl}_{3}\right) \delta-8.1$.

HRMS (ESI-TOF) calcd for $\mathrm{C}_{25} \mathrm{H}_{26} \mathrm{BNO}_{2} \mathrm{P}^{+}[\mathrm{M}+\mathrm{H}]^{+}: 413.1825$, found: 413.1828 .

IR (neat, cm ${ }^{-1}$ ): 2979, 2222, 1595, 1386, 1353, 1324, 1140, 964, 849, 743, 695.

2,5-Bis(diphenylphosphaneyl)benzonitrile (2g)<smiles>CCOc1ccc(Cl)cc1C#N</smiles>

$1 \mathrm{~g}$

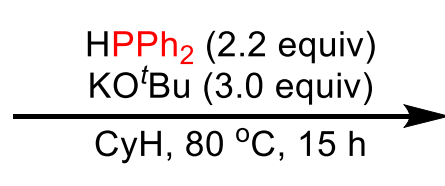

$\underset{\mathrm{CyH}, 80^{\circ} \mathrm{C}, 15 \mathrm{~h}}{\mathrm{KO}} \mathrm{Bu}(3.0$ equiv $)$<smiles>N#Cc1cc(Pc2ccccc2)ccc1Pc1ccccc1</smiles>

2g 
$2 \mathrm{~g}$ was prepared as a white solid in $92 \%$ yield $(0.43 \mathrm{~g}$, eluent: petroleum ether $/$ EtOAc $=50: 1)$ from $1 \mathrm{~g}(0.18 \mathrm{~g}, 1.0 \mathrm{mmol}), \mathrm{HPPh}_{2}(0.38 \mathrm{~mL}, 0.41 \mathrm{~g}, 2.2 \mathrm{mmol})$ and $\mathrm{KO}^{t} \mathrm{Bu}(0.34 \mathrm{~g}, 3.0 \mathrm{mmol})$ following the general procedure $\mathrm{B}$.

$\mathbf{R}_{f}=0.45$ (petroleum ether $/ \mathrm{EtOAc}=10 / 1$ )

${ }^{1} \mathrm{H}$ NMR $\left(400 \mathrm{MHz}, \mathrm{CDCl}_{3}\right) \delta 7.57(\mathrm{~d}, J=6.4 \mathrm{~Hz}, 1 \mathrm{H}), 7.44-7.30(\mathrm{~m}, 21 \mathrm{H}), 6.99$ $(\mathrm{d}, J=4.7 \mathrm{~Hz}, 1 \mathrm{H})$.

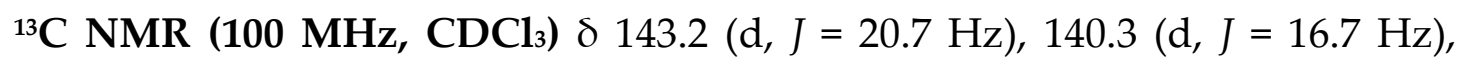
$137.8(\mathrm{dd}, J=18.4,4.4 \mathrm{~Hz}), 136.9(\mathrm{~d}, J=19.0 \mathrm{~Hz}), 135.3(\mathrm{~d}, J=10.5 \mathrm{~Hz}), 134.4(\mathrm{~d}$, $J=10.1 \mathrm{~Hz}), 134.3,134.1(\mathrm{~d}, J=1.5 \mathrm{~Hz}), 133.9,133.1(\mathrm{~d}, J=6.1 \mathrm{~Hz}), 129.6(\mathrm{~d}, J=$ $2.9 \mathrm{~Hz}), 129.0(\mathrm{~d}, J=1.4 \mathrm{~Hz}), 128.9(\mathrm{~d}, J=1.5 \mathrm{~Hz}), 118.0(\mathrm{dd}, J=32.6,6.0 \mathrm{~Hz})$, $117.6(\mathrm{~d}, J=3.5 \mathrm{~Hz})$.

${ }^{31}$ P NMR (162 MHz, $\left.\mathrm{CDCl}_{3}\right) \delta-5.9,-8.5$.

HRMS (ESI-TOF) calcd for $\mathrm{C}_{31} \mathrm{H}_{24} \mathrm{NP}_{2}{ }^{+}[\mathrm{M}+\mathrm{H}]^{+}: 472.1379$, found: 472.1381 .

IR (neat, cm ${ }^{-1}$ ): 3053, 2223, 1585, 1478, 1456, 1434, 1367, 1265, 999, 836, 740, 693.

2-(Diphenylphosphaneyl)-4-methylbenzonitrile (2h)<smiles>Cc1ccc(C#N)c(P)c1</smiles>

2h was prepared as a white solid in 65\% yield ( $0.98 \mathrm{~g}$, eluent: petroleum ether /EtOAc = 50:1) from $\mathbf{1 h}(0.81 \mathrm{~g}, 5.0 \mathrm{mmol}) \mathrm{HPPh}_{2}(0.95 \mathrm{~mL}, 1.02 \mathrm{~g}, 5.5 \mathrm{mmol})$ and $\mathrm{KO}^{t} \mathrm{Bu}(0.84 \mathrm{~g}, 7.5 \mathrm{mmol})$ following the general procedure $\mathrm{B}$.

$\mathbf{R}_{f}=0.50$ (petroleum ether/EtOAc $=10 / 1$ )

${ }^{1} \mathbf{H}$ NMR (400 MHz, $\left.\mathrm{CDCl}_{3}\right) \delta 7.60(\mathrm{~d}, J=8.0 \mathrm{~Hz}, 1 \mathrm{H}), 7.43-7.28(\mathrm{~m}, 10 \mathrm{H}), 7.22$ $(\mathrm{d}, J=7.9 \mathrm{~Hz}, 1 \mathrm{H}), 6.84(\mathrm{~s}, 1 \mathrm{H}), 2.29(\mathrm{~s}, 3 \mathrm{H})$.

${ }^{13} \mathrm{C}$ NMR (100 MHz, $\left.\mathrm{CDCl}_{3}\right) \delta$ 143.4, $142.7(\mathrm{~d}, J=19.5 \mathrm{~Hz}), 134.9(\mathrm{~d}, J=10.3$ $\mathrm{Hz}), 134.10$ (d, J = 20.4 Hz), 134.09, 133.8 (d, J = 5.1 Hz), 129.9, 129.4, 128.9 (d, J $=7.3 \mathrm{~Hz}), 118.0(\mathrm{~d}, J=4.0 \mathrm{~Hz}), 115.0(\mathrm{~d}, J=32.8 \mathrm{~Hz}), 22.1$.

${ }^{31} \mathrm{P}$ NMR (162 MHz, $\left.\mathrm{CDCl}_{3}\right) \delta-8.4$.

The characterization data are consistent with the literature reported data. ${ }^{8}$

4-(tert-Butyl)-2-(diphenylphosphaneyl)benzonitrile (2i) 
<smiles>CC(C)(C)c1ccc(C#N)c(P)c1</smiles>

$\mathbf{2 i}$ was prepared as a white solid in 59\% yield (1.01 g, eluent: petroleum ether $/$ EtOAc $=50: 1)$ from $1 \mathbf{i}(1.02 \mathrm{~g}, 5.0 \mathrm{mmol}) \mathrm{HPPh}_{2}(0.95 \mathrm{~mL}, 1.02 \mathrm{~g}, 5.5 \mathrm{mmol})$ and $\mathrm{KO}^{t} \mathrm{Bu}(0.84 \mathrm{~g}, 7.5 \mathrm{mmol})$ following the general procedure $\mathrm{B}$.

$\mathbf{R}_{f}=0.52$ (petroleum ether/EtOAc $=10 / 1$ )

${ }^{1} \mathrm{H}$ NMR (400 MHz, $\left.\mathrm{CDCl}_{3}\right) \delta 7.65(\mathrm{dd}, J=8.2,3.2 \mathrm{~Hz}, 1 \mathrm{H}), 7.44(\mathrm{~d}, J=8.2 \mathrm{~Hz}$, $2 \mathrm{H}), 7.43-7.31(\mathrm{~m}, 10 \mathrm{H}), 7.10(\mathrm{dd}, J=4.1,2.0 \mathrm{~Hz}, 1 \mathrm{H}), 1.18(\mathrm{~s}, 9 \mathrm{H})$.

${ }^{13} \mathrm{C}$ NMR (100 MHz, $\left.\mathrm{CDCl}_{3}\right) \delta 155.9,142.2(\mathrm{~d}, J=19.2 \mathrm{~Hz}), 134.9$ (d, $J=10.5$ $\mathrm{Hz}), 133.8(\mathrm{~d}, J=20.2 \mathrm{~Hz}), 133.4(\mathrm{~d}, J=4.9 \mathrm{~Hz}), 130.8,129.3,128.7(\mathrm{~d}, J=7.2 \mathrm{~Hz})$, $125.9,117.8(\mathrm{~d}, J=3.7 \mathrm{~Hz}), 114.7(\mathrm{~d}, J=32.2 \mathrm{~Hz}), 35.2,30.7$.

${ }^{31} \mathrm{P}$ NMR (162 $\left.\mathrm{MHz}, \mathrm{CDCl}_{3}\right) \delta-7.6$.

HRMS (ESI-TOF) calcd for $\mathrm{C}_{23} \mathrm{H}_{23} \mathrm{NP}^{+}[\mathrm{M}+\mathrm{H}]^{+}:$344.1563, found: 344.1562 .

IR (neat, cm-1): 2963, 2221, 1587, 1477, 1435, 1386, 1117, 1091, 836, 744, 695.

\section{4-(Dimethoxymethyl)-2-(diphenylphosphaneyl)benzonitrile (2j)}

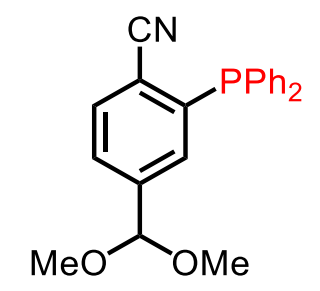

$2 \mathbf{j}$ was prepared as a white solid in $63 \%$ yield $(0.23 \mathrm{~g}$, eluent: petroleum ether $/$ EtOAc $=20: 1)$ from $1 \mathbf{j}(0.21 \mathrm{~g}, 1.0 \mathrm{mmol}), \mathrm{HPPh}_{2}(0.19 \mathrm{~mL}, 0.20 \mathrm{~g}, 1.1 \mathrm{mmol})$ and $\mathrm{KO}^{t} \mathrm{Bu}(0.17 \mathrm{~g}, 1.5 \mathrm{mmol})$ following the general procedure $\mathrm{B}$.

$\mathbf{R}_{f}=0.25$ (petroleum ether $/$ EtOAc $=10 / 1$ )

${ }^{1} \mathrm{H}$ NMR (400 MHz, $\left.\mathrm{CDCl}_{3}\right) \delta 7.72(\mathrm{~d}, J=8.2 \mathrm{~Hz}, 1 \mathrm{H}), 7.55(\mathrm{~d}, J=8.1 \mathrm{~Hz}, 1 \mathrm{H})$, $7.42-7.27(\mathrm{~m}, 10 \mathrm{H}), 7.14(\mathrm{~s}, 1 \mathrm{H}), 5.28(\mathrm{~s}, 1 \mathrm{H}), 3.22(\mathrm{~s}, 6 \mathrm{H})$.

${ }^{13} \mathrm{C}$ NMR (100 MHz, $\left.\mathrm{CDCl}_{3}\right) \delta 143.2(\mathrm{~d}, J=20.5 \mathrm{~Hz}), 142.7,134.6(\mathrm{~d}, J=10.1$ $\mathrm{Hz}), 134.0(\mathrm{~d}, J=20.2 \mathrm{~Hz}), 133.8(\mathrm{~d}, J=4.8 \mathrm{~Hz}), 131.9,129.5,128.9$ (d, $J=7.3 \mathrm{~Hz})$, $127.5,117.9(\mathrm{~d}, J=32.7 \mathrm{~Hz}), 117.6(\mathrm{~d}, J=3.9 \mathrm{~Hz}), 101.8,52.8$.

${ }^{31}$ P NMR (162 $\left.\mathrm{MHz} \mathrm{CDCl}_{3}\right) \delta-8.1$.

HRMS (ESI-TOF) calcd for $\mathrm{C}_{22} \mathrm{H}_{21} \mathrm{NO}_{2} \mathrm{P}^{+}[\mathrm{M}+\mathrm{H}]^{+}:$362.1305, found: 362.1304.

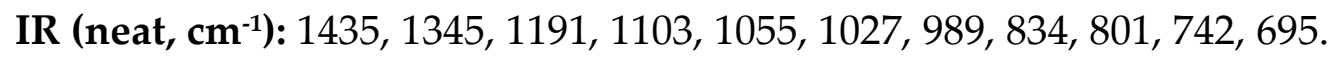




\section{4-(Diphenylphosphaneyl)benzonitrile (2k)}<smiles>N#Cc1ccc(P)cc1</smiles>

2k was prepared as a white solid in $49 \%$ yield ( $0.70 \mathrm{~g}$, eluent: petroleum ether $/$ EtOAc $=50: 1)$ from 1k $(0.74 \mathrm{~g}, 5.0 \mathrm{mmol}), \mathrm{HPPh}_{2}(0.95 \mathrm{~mL}, 1.02 \mathrm{~g}, 5.5 \mathrm{mmol})$ and $\mathrm{KO}^{t} \mathrm{Bu}(0.84 \mathrm{~g}, 7.5 \mathrm{mmol})$ following the general procedure $\mathrm{B}$.

$\mathbf{R}_{f}=0.36$ (petroleum ether $/$ EtOAc $=10 / 1$ )

${ }^{1} \mathrm{H}$ NMR (400 MHz, $\left.\mathrm{CDCl}_{3}\right) \delta 7.58(\mathrm{~d}, J=7.8 \mathrm{~Hz}, 2 \mathrm{H}), 7.47-7.29(\mathrm{~m}, 12 \mathrm{H})$.

${ }^{13} \mathrm{C}$ NMR (100 MHz, $\left.\mathrm{CDCl}_{3}\right) \delta 145.2(\mathrm{~d}, J=16.7 \mathrm{~Hz}), 135.5(\mathrm{~d}, J=10.5 \mathrm{~Hz})$, $134.1(\mathrm{~d}, J=20.2 \mathrm{~Hz}), 133.6$ (d, $J=18.5 \mathrm{~Hz}), 131.8$ (d, $J=6.1 \mathrm{~Hz}), 129.6,128.9$ (d, $J=7.5 \mathrm{~Hz}), 118.8,112.0$.

${ }^{31} \mathrm{P}$ NMR (162 MHz, $\left.\mathrm{CDCl}_{3}\right) \delta-4.3$.

The characterization data are consistent with the literature reported data. ${ }^{9}$

\section{2-(Diphenylphosphaneyl)-3-ethylbenzonitrile (21)}<smiles>CCc1cccc(C#N)c1Pc1ccccc1</smiles>

21 was prepared as a white solid in $61 \%$ yield $(0.96 \mathrm{~g}$, eluent: petroleum ether /EtOAc = 50:1) from 11 (0.88 g, $5.0 \mathrm{mmol}), \mathrm{HPPh}_{2}(0.95 \mathrm{~mL}, 1.02 \mathrm{~g}, 5.5 \mathrm{mmol})$ and $\mathrm{KO}^{t} \mathrm{Bu}(0.84 \mathrm{~g}, 7.5 \mathrm{mmol})$ following the general procedure $\mathrm{B}$.

$\mathbf{R}_{f}=0.38$ (petroleum ether/EtOAc $=10 / 1$ )

${ }^{1} \mathrm{H}$ NMR (400 MHz, $\left.\mathrm{CDCl}_{3}\right) \delta 7.61(\mathrm{~d}, J=7.5 \mathrm{~Hz}, 1 \mathrm{H}), 7.54-7.50(\mathrm{~m}, 1 \mathrm{H}), 7.46$ $(\mathrm{d}, J=7.8 \mathrm{~Hz}, 1 \mathrm{H}), 7.45-7.32(\mathrm{~m}, 10 \mathrm{H}), 3.00(\mathrm{q}, J=7.1 \mathrm{~Hz}, 2 \mathrm{H}), 1.06(\mathrm{t}, J=7.4$ $\mathrm{Hz}, 3 \mathrm{H})$.

${ }^{13} \mathrm{C}$ NMR (100 MHz, $\left.\mathrm{CDCl}_{3}\right) \delta 152.9(\mathrm{~d}, J=22.2 \mathrm{~Hz}), 137.3(\mathrm{~d}, J=25.6 \mathrm{~Hz})$, $134.6(\mathrm{~d}, J=12.1 \mathrm{~Hz}), 134.0(\mathrm{~d}, J=1.6 \mathrm{~Hz}), 133.5$ (d, $J=4.6 \mathrm{~Hz}), 132.7(\mathrm{~d}, J=19.2$ $\mathrm{Hz}), 130.3,128.7(\mathrm{~d}, J=2.6 \mathrm{~Hz}), 128.65,119.9$ (d, $J=8.4 \mathrm{~Hz}), 118.0(\mathrm{~d}, J=2.5 \mathrm{~Hz})$, $28.7(\mathrm{~d}, J=22.5 \mathrm{~Hz}), 16.1(\mathrm{~d}, J=3.1 \mathrm{~Hz})$.

${ }^{31}$ P NMR (162 MHz, $\left.\mathrm{CDCl}_{3}\right) \delta-12.8$.

HRMS (ESI-TOF) calcd for $\mathrm{C}_{21} \mathrm{H}_{19} \mathrm{NP}^{+}[\mathrm{M}+\mathrm{H}]^{+}$: 316.1250, found: 316.1248.

IR (neat, cm ${ }^{-1}$ ): 2964, 2223, 1584, 1481, 1450, 1434, 1027, 804, 736, 695. 


\section{2-(Diphenylphosphaneyl)-3-isopropylbenzonitrile (2m)}<smiles>N#Cc1cccc(P)c1P</smiles>

$2 \mathrm{~m}$ was prepared as a white solid in $56 \%$ yield ( $0.92 \mathrm{~g}$, eluent: petroleum ether /EtOAc = 50:1) from 1m (0.95 g, $5.0 \mathrm{mmol}), \mathrm{HPPh}_{2}(0.95 \mathrm{~mL}, 1.02 \mathrm{~g}, 5.5 \mathrm{mmol})$ and $\mathrm{KO}^{t} \mathrm{Bu}(0.84 \mathrm{~g}, 7.5 \mathrm{mmol})$ following the general procedure $\mathrm{B}$.

$\mathbf{R}_{f}=0.50$ (petroleum ether/EtOAc $=10 / 1$ )

${ }^{1} \mathrm{H}$ NMR (400 MHz, $\left.\mathrm{CDCl}_{3}\right) \delta 7.65-7.57(\mathrm{~m}, 2 \mathrm{H}), 7.50(\mathrm{t}, J=7.8 \mathrm{~Hz}, 1 \mathrm{H}), 7.47$ $7.30(\mathrm{~m}, 10 \mathrm{H}), 4.01-3.86(\mathrm{~m}, J=7.1 \mathrm{~Hz}, 1 \mathrm{H}), 1.07(\mathrm{~d}, J=6.9 \mathrm{~Hz}, 6 \mathrm{H})$.

${ }^{13} \mathrm{C}$ NMR (100 MHz, $\left.\mathrm{CDCl}_{3}\right) \delta 157.3$ (d, $\left.J=18.8 \mathrm{~Hz}\right), 136.8$ (d, $\left.J=24.6 \mathrm{~Hz}\right)$, $134.7(\mathrm{~d}, J=12.4 \mathrm{~Hz}), 133.8(\mathrm{~d}, J=2.5 \mathrm{~Hz}), 132.6(\mathrm{~d}, J=19.1 \mathrm{~Hz}), 130.6,130.4$ (d, $J=3.6 \mathrm{~Hz}), 128.7,128.6,120.0(\mathrm{~d}, J=11.8 \mathrm{~Hz}), 118.2,31.5(\mathrm{~d}, J=24.1 \mathrm{~Hz}), 23.9$. ${ }^{31}$ P NMR (162 MHz, $\left.\mathrm{CDCl}_{3}\right) \delta-13.20$.

HRMS (ESI-TOF) calcd for $\mathrm{C}_{21} \mathrm{H}_{19} \mathrm{NP}^{+}[\mathrm{M}+\mathrm{H}]^{+}:$330.1406, found: 330.1405 .

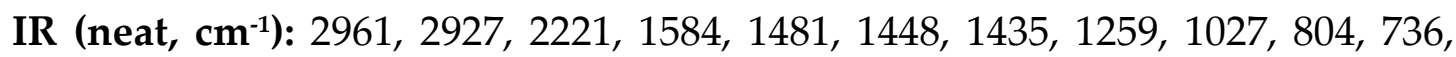
695.

\section{2-(Diphenylphosphaneyl)-[1,1'-biphenyl]-3-carbonitrile (2n)}<smiles>N#Cc1cccc(-c2ccccc2)c1P</smiles>

2n was prepared as a white solid in 56\% yield (1.01 g, eluent: petroleum ether /DCM = 10:1) from 1n (1.12 g, $5.0 \mathrm{mmol}), \mathrm{HPPh}_{2}(1.31 \mathrm{~mL}, 1.40 \mathrm{~g}, 7.5 \mathrm{mmol})$ and $\mathrm{KO}^{t} \mathrm{Bu}(0.84 \mathrm{~g}, 7.5 \mathrm{mmol})$ in toluene following the general procedure $\mathrm{B}$. $\mathbf{R}_{f}=0.40$ (petroleum ether/DCM $=2 / 1$ )

${ }^{1} \mathrm{H}$ NMR (400 MHz, $\left.\mathrm{CDCl}_{3}\right) \delta 7.68(\mathrm{~d}, J=7.6 \mathrm{~Hz}, 1 \mathrm{H}), 7.58-7.52(\mathrm{~m}, 1 \mathrm{H}), 7.47$ $(\mathrm{t}, J=7.6 \mathrm{~Hz}, 1 \mathrm{H}), 7.37-7.24(\mathrm{~m}, 13 \mathrm{H}), 7.12(\mathrm{~d}, J=7.4 \mathrm{~Hz}, 2 \mathrm{H})$.

${ }^{13} \mathrm{C}$ NMR (100 MHz, $\left.\mathrm{CDCl}_{3}\right) \delta 152.0$ (d, J = 33.1 Hz), 140.9 (d, J = 8.7 Hz), 138.4 $(\mathrm{d}, J=28.0 \mathrm{~Hz}), 135.5,135.1(\mathrm{~d}, J=13.3 \mathrm{~Hz}), 134.2(\mathrm{~d}, J=5.2 \mathrm{~Hz}), 133.0(\mathrm{~d}, J=$ $19.5 \mathrm{~Hz}), 129.7(\mathrm{~d}, J=3.9 \mathrm{~Hz}), 129.5,128.7,128.6(\mathrm{~d}, J=6.2 \mathrm{~Hz}), 127.94,127.86$, 
$119.2(\mathrm{~d}, J=2.6 \mathrm{~Hz}), 117.5$.

${ }^{31}$ P NMR (162 MHz, $\left.\mathrm{CDCl}_{3}\right) \delta-10.2$.

HRMS (ESI-TOF) calcd for $\mathrm{C}_{25} \mathrm{H}_{19} \mathrm{NP}^{+}[\mathrm{M}+\mathrm{H}]^{+}:$: 364.1250, found: 364.1249 .

IR (neat, cm-1): 2925, 2220, 1478, 1449, 1436, 1431, 1252, 1025, 810, 760, 742, 597.

2-(Diphenylphosphaneyl)-2'-ethoxy-[1,1'-biphenyl]-3-carbonitrile (2o)<smiles>CCOc1ccccc1-c1cccc(C#N)c1[PH](=O)c1ccccc1</smiles>

20 was prepared as a white solid in $43 \%$ yield ( $0.18 \mathrm{~g}$, eluent: petroleum ether $/$ EtOAc $=50: 1)$ from $10(0.27 \mathrm{~g}, 1.0 \mathrm{mmol}), \mathrm{HPPh}_{2}(0.19 \mathrm{~mL}, 0.20 \mathrm{~g}, 1.1 \mathrm{mmol})$ and KHMDS (1.0 M in THF, $1.5 \mathrm{~mL}, 1.5 \mathrm{mmol})$ instead of $\mathrm{KO}^{t} \mathrm{Bu}$ in methyl tert-butyl ether at room temperature for $24 \mathrm{~h}$ following the general procedure B.

$\mathbf{R}_{f}=0.40$ (petroleum ether $/$ EtOAc $=10 / 1$ )

${ }^{1} \mathrm{H}$ NMR (400 MHz, $\left.\mathrm{CDCl}_{3}\right) \delta 7.74(\mathrm{~d}, J=7.0 \mathrm{~Hz}, 1 \mathrm{H}), 7.58-7.50(\mathrm{~m}, 2 \mathrm{H}), 7.46$ $-7.30(\mathrm{~m}, 11 \mathrm{H}), 7.04(\mathrm{~d}, J=7.4 \mathrm{~Hz}, 1 \mathrm{H}), 7.00-6.89(\mathrm{~m}, 2 \mathrm{H}), 4.02-3.81(\mathrm{~m}, 2 \mathrm{H})$, $1.14(\mathrm{t}, J=7.0 \mathrm{~Hz}, 3 \mathrm{H})$.

${ }^{13} \mathrm{C}$ NMR (100 MHz, $\left.\mathrm{CDCl}_{3}\right) \delta 155.8$ (d, J = 1.8 Hz), 148.9 (d, $\left.J=36.1 \mathrm{~Hz}\right), 139.9$ $(\mathrm{d}, J=25.4 \mathrm{~Hz}), 135.6(\mathrm{~d}, J=13.6 \mathrm{~Hz}), 135.3,135.1,134.7$ (d, $J=6.2 \mathrm{~Hz}), 133.3$ (d, $J=20.3 \mathrm{~Hz}), 132.4(\mathrm{~d}, J=18.4 \mathrm{~Hz}), 131.1(\mathrm{~d}, J=2.9 \mathrm{~Hz}), 130.3(\mathrm{~d}, J=9.0 \mathrm{~Hz})$, 129.6, 129.5, 128.7, 128.4, 128.3 (d, $J=3.9 \mathrm{~Hz}), 128.2$ (d, $J=6.3 \mathrm{~Hz}), 119.9,118.5$ $(\mathrm{d}, J=3.6 \mathrm{~Hz}), 117.8,111.3,63.4,14.5$.

${ }^{31} \mathrm{P}$ NMR (162 MHz, $\left.\mathrm{CDCl}_{3}\right) \delta$-9.5.

HRMS (ESI-TOF) calcd for $\mathrm{C}_{27} \mathrm{H}_{23} \mathrm{NOP}^{+}[\mathrm{M}+\mathrm{H}]^{+}$: 408.1512, found: 408.1511 .

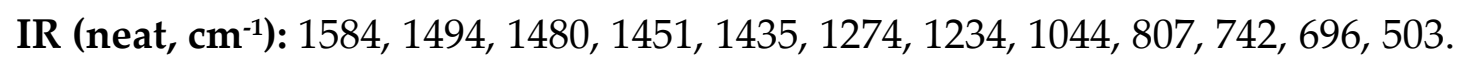

\section{2-(Diphenylphosphaneyl)-4,6-dimethylbenzonitrile (2p)}<smiles>Cc1cc(C)c(C#N)c(P)c1</smiles> 
$2 \mathbf{p}$ was prepared as a white solid in 94\% yield (1.48 g, eluent: petroleum ether /EtOAc = 50:1) from 1p $(0.88 \mathrm{~g}, 5.0 \mathrm{mmol}), \mathrm{HPPh}_{2}(0.95 \mathrm{~mL}, 1.02 \mathrm{~g}, 5.5 \mathrm{mmol})$ and $\mathrm{KO}^{t} \mathrm{Bu}(0.84 \mathrm{~g}, 7.5 \mathrm{mmol})$ following the general procedure $\mathrm{B}$.

$\mathbf{R}_{f}=0.52$ (petroleum ether/EtOAc $=10 / 1$ )

${ }^{1} \mathrm{H}$ NMR (400 MHz, $\left.\mathrm{CDCl}_{3}\right) \delta 7.38-7.31(\mathrm{~m}, 10 \mathrm{H}), 7.10(\mathrm{~s}, 1 \mathrm{H}), 6.66(\mathrm{~s}, 1 \mathrm{H})$, $2.52(\mathrm{~s}, 3 \mathrm{H}), 2.25(\mathrm{~s}, 3 \mathrm{H})$.

${ }^{13} \mathrm{C}$ NMR (100 MHz, $\left.\mathrm{CDCl}_{3}\right) \delta 143.1$ (d, $\left.J=5.4 \mathrm{~Hz}\right), 142.8$ (d, J = 9.4 Hz), 142.7 $(\mathrm{d}, J=9.4 \mathrm{~Hz}), 135.1(\mathrm{~d}, J=10.4 \mathrm{~Hz}), 134.0(\mathrm{~d}, J=20.2 \mathrm{~Hz}), 131.5,131.2,129.3$, $128.8(\mathrm{~d}, J=7.2 \mathrm{~Hz}), 117.0(\mathrm{~d}, J=4.3 \mathrm{~Hz}), 115.3(\mathrm{~d}, J=32.9 \mathrm{~Hz}), 21.9,20.7(\mathrm{~d}, J=$ $1.4 \mathrm{~Hz})$.

${ }^{31} \mathrm{P}$ NMR (162 $\left.\mathrm{MHz}, \mathrm{CDCl}_{3}\right) \delta-8.1$.

HRMS (ESI-TOF) calcd for $\mathrm{C}_{21} \mathrm{H}_{19} \mathrm{NP}^{+}[\mathrm{M}+\mathrm{H}]^{+}$: 316.1250, found: 316.1249.

IR (neat, cm-1): 2209, 1594, 1475, 1434, 1089, 1026, 861, 743, 694, 578, 496.

\section{2-(Diphenylphosphaneyl)-3,5-dimethylbenzonitrile (2q)}<smiles>Cc1cc(C)c(P)c(P)c1</smiles>

2q was prepared as a white solid in $76 \%$ yield (1.20 g, eluent: petroleum ether $/$ EtOAc $=50: 1)$ from $1 q(0.88 \mathrm{~g}, 5.0 \mathrm{mmol}), \mathrm{HPPh}_{2}(0.95 \mathrm{~mL}, 1.02 \mathrm{~g}, 5.5 \mathrm{mmol})$ and $\mathrm{KO}^{t} \mathrm{Bu}(0.84 \mathrm{~g}, 7.5 \mathrm{mmol})$ following the general procedure $\mathrm{B}$.

$\mathbf{R}_{f}=0.45$ (petroleum ether/EtOAc $=10 / 1$ )

${ }^{1}$ H NMR (400 MHz, $\left.\mathbf{C D C l}_{3}\right) \delta 7.41(\mathrm{~s}, 1 \mathrm{H}), 7.39-7.28(\mathrm{~m}, 10 \mathrm{H}), 7.22(\mathrm{~s}, 1 \mathrm{H})$, $2.34(\mathrm{~s}, 3 \mathrm{H}), 2.25(\mathrm{~s}, 3 \mathrm{H})$.

${ }^{13} \mathrm{C}$ NMR (100 MHz, $\left.\mathrm{CDCl}_{3}\right) \delta 146.0$ (d, J = 15.9 Hz), 140.5, 136.1 (d, J=3.3 Hz), $134.5(\mathrm{~d}, J=12.4 \mathrm{~Hz}), 134.3(\mathrm{~d}, J=23.1 \mathrm{~Hz}), 134.0(\mathrm{~d}, J=3.7 \mathrm{~Hz}), 132.6$ (d, $J=$ $19.1 \mathrm{~Hz}), 128.7,128.7(\mathrm{~d}, J=4.5 \mathrm{~Hz}), 120.1(\mathrm{~d}, J=19.4 \mathrm{~Hz}), 118.3(\mathrm{~d}, J=3.4 \mathrm{~Hz})$, $22.7(\mathrm{~d}, J=17.4 \mathrm{~Hz}), 20.9$.

31P NMR (162 MHz, $\left.\mathrm{CDCl}_{3}\right) \delta-10.2$.

HRMS (ESI-TOF) calcd for $\mathrm{C}_{21} \mathrm{H}_{19} \mathrm{NP}^{+}[\mathrm{M}+\mathrm{H}]^{+}:$316.1250, found: 316.1249 .

IR (neat, cm$^{-1}$ ): 2222, 1598, 1585, 1481, 1434, 1027, 864, 742, 695, 545.

\section{2-(Diphenylphosphaneyl)-3,6-dimethylbenzonitrile (2r)}


<smiles>Cc1ccc(C)c(P)c1P</smiles>

2r was prepared as a white solid in $83 \%$ yield $(1.31 \mathrm{~g}$, eluent: petroleum ether /EtOAc = 50:1) from 1r $(0.88 \mathrm{~g}, 5.0 \mathrm{mmol}), \mathrm{HPPh}_{2}(0.95 \mathrm{~mL}, 1.02 \mathrm{~g}, 5.5 \mathrm{mmol})$ and $\mathrm{KO}^{t} \mathrm{Bu}(0.84 \mathrm{~g}, 7.5 \mathrm{mmol})$ following the general procedure $\mathrm{B}$.

$\mathbf{R}_{f}=0.48$ (petroleum ether $/$ EtOAc $=10 / 1$ )

${ }^{1} \mathrm{H}$ NMR (400 MHz, $\mathrm{CDCl}_{3}$ ) $\delta 7.41-7.33$ (m, 10H), $7.31-7.29$ (m, 2H), 2.53 (s, $3 \mathrm{H}), 2.20(\mathrm{~s}, 3 \mathrm{H})$.

${ }^{13} \mathrm{C}$ NMR (100 MHz, $\left.\mathrm{CDCl}_{3}\right) \delta 143.2(\mathrm{~d}, J=13.2 \mathrm{~Hz}), 142.0$ (d, $\left.J=4.8 \mathrm{~Hz}\right), 137.6$ $(\mathrm{d}, J=24.0 \mathrm{~Hz}), 134.9(\mathrm{~d}, J=3.1 \mathrm{~Hz}), 134.4(\mathrm{~d}, J=12.3 \mathrm{~Hz}), 132.5(\mathrm{~d}, J=19.1 \mathrm{~Hz})$, 131.6, 128.73, $128.65(\mathrm{~d}, J=3.3 \mathrm{~Hz}), 120.7$ (d, $J=23.8 \mathrm{~Hz}), 117.04(\mathrm{~d}, J=4.0 \mathrm{~Hz})$, $22.5(\mathrm{~d}, J=15.2 \mathrm{~Hz}), 21.1$.

31P NMR (162 MHz, $\left.\mathrm{CDCl}_{3}\right) \delta-7.2$.

HRMS (ESI-TOF) calcd for $\mathrm{C}_{21} \mathrm{H}_{19} \mathrm{NP}^{+}[\mathrm{M}+\mathrm{H}]^{+}:$316.1250, found: 316.1249.

IR (neat, cm ${ }^{-1}$ ): 3053, 2216, 1584, 1481, 1459, 1435, 1027, 822, 744, 696, 481.

\section{2-(Diphenylphosphaneyl)-3,4-dimethylbenzonitrile (2s)}<smiles>COc1c(C)ccc(C#N)c1P</smiles>

2s was prepared as a white solid in $51 \%$ yield $(0.80 \mathrm{~g}$, eluent: petroleum ether /EtOAc $=50: 1)$ from $1 \mathrm{~s}(0.88 \mathrm{~g}, 5.0 \mathrm{mmol}), \mathrm{HPPh}_{2}(0.95 \mathrm{~mL}, 1.02 \mathrm{~g}, 5.5 \mathrm{mmol})$ and $\mathrm{KO}^{t} \mathrm{Bu}(0.84 \mathrm{~g}, 7.5 \mathrm{mmol})$ following the general procedure $\mathrm{B}$.

$\mathbf{R}_{f}=0.46$ (petroleum ether $/$ EtOAc $=10 / 1$ )

${ }^{1} \mathrm{H}$ NMR (400 MHz, $\left.\mathrm{CDCl}_{3}\right) \delta 7.53(\mathrm{~d}, J=7.9 \mathrm{~Hz}, 1 \mathrm{H}), 7.43-7.33(\mathrm{~m}, 10 \mathrm{H}), 7.31$ $(\mathrm{d}, J=8.1 \mathrm{~Hz}, 1 \mathrm{H}), 2.35(\mathrm{~s}, 3 \mathrm{H}), 2.30(\mathrm{~s}, 3 \mathrm{H})$.

${ }^{13} \mathrm{C}$ NMR (100 MHz, $\left.\mathrm{CDCl}_{3}\right) \delta 144.8(\mathrm{~d}, J=15.5 \mathrm{~Hz}), 143.0(\mathrm{~d}, J=3.0 \mathrm{~Hz}), 137.7$ $(\mathrm{d}, J=23.4 \mathrm{~Hz}), 134.7(\mathrm{~d}, J=12.4 \mathrm{~Hz}), 133.1(\mathrm{~d}, J=4.0 \mathrm{~Hz}), 132.6(\mathrm{~d}, J=19.1 \mathrm{~Hz})$, $131.9,128.7,128.7,118.5(\mathrm{~d}, J=3.6 \mathrm{~Hz}), 118.0(\mathrm{~d}, J=19.4 \mathrm{~Hz}), 21.6,19.2(\mathrm{~d}, J=$ $21.0 \mathrm{~Hz})$.

31P NMR (162 MHz, $\left.\mathrm{CDCl}_{3}\right) \delta$-8.2.

HRMS (ESI-TOF) calcd for $\mathrm{C}_{21} \mathrm{H}_{19} \mathrm{NP}^{+}[\mathrm{M}+\mathrm{H}]^{+}:$316.1250, found: 316.1250. 
IR (neat, cm ${ }^{-1}$ ): 2219, 1583, 1480, 1434, 1386, 999, 825, 743, 695, 593, 529, 510, 482.

2-(Diphenylphosphaneyl)-5-isopropyl-4-methylbenzonitrile (2t)<smiles>CCCc1cc(C#N)c(-c2ccccc2)cc1C</smiles>

$2 \mathbf{t}$ was prepared as a white solid in $74 \%$ yield $(1.27 \mathrm{~g}$, eluent: petroleum ether $/$ EtOAc $=50: 1)$ from $1 t(1.02 \mathrm{~g}, 5.0 \mathrm{mmol}) \mathrm{HPPh}_{2}(0.95 \mathrm{~mL}, 1.02 \mathrm{~g}, 5.5 \mathrm{mmol})$ and $\mathrm{KO}^{t} \mathrm{Bu}(0.84 \mathrm{~g}, 7.5 \mathrm{mmol})$ following the general procedure $\mathrm{B}$.

$\mathbf{R}_{f}=0.54$ (petroleum ether/EtOAc $=10 / 1$ )

${ }^{1}$ H NMR (400 MHz, $\left.\mathrm{CDCl}_{3}\right) \delta 7.59$ (d, J = 3.0 Hz, 1H), $7.42-7.29(\mathrm{~m}, 10 \mathrm{H}), 6.81$ $(\mathrm{d}, J=3.8 \mathrm{~Hz}, 1 \mathrm{H}), 3.18-3.05(\mathrm{~m}, 1 \mathrm{H}), 2.26(\mathrm{~s}, 3 \mathrm{H}), 1.24(\mathrm{~d}, J=6.8 \mathrm{~Hz}, 6 \mathrm{H})$.

${ }^{13} \mathrm{C}$ NMR (100 MHz, $\left.\mathrm{CDCl}_{3}\right) \delta$ 148.5, 140.7, $138.8(\mathrm{~d}, J=18.1 \mathrm{~Hz}), 135.4,135.3$ (d, $J=10.3 \mathrm{~Hz}), 134.0$ (d, $J=20.2 \mathrm{~Hz}), 130.6$ (d, $J=5.4 \mathrm{~Hz}), 129.3,128.8$ (d, J = 7.2 $\mathrm{Hz}), 118.4(\mathrm{~d}, J=3.9 \mathrm{~Hz}), 115.9(\mathrm{~d}, J=32.9 \mathrm{~Hz}), 29.2,22.9,19.8$.

31P NMR (162 MHz, $\left.\mathrm{CDCl}_{3}\right) \delta-9.4$.

HRMS (ESI-TOF) calcd for $\mathrm{C}_{23} \mathrm{H}_{23} \mathrm{NP}^{+}[\mathrm{M}+\mathrm{H}]^{+}:$344.1563, found: 344.1563.

IR (neat, cm-1): 2965, 2928, 2220, 1476, 1448, 1432, 1091, 1025, 904, 897, 744, 697.

6-(Diphenylphosphaneyl)-2,3-dihydro-1H-indene-5-carbonitrile (2u)<smiles>N#Cc1cc2c(cc1Pc1ccccc1)CCC2</smiles>

$2 \mathbf{u}$ was prepared as a white solid in 93\% yield (1.52 g, eluent: petroleum ether $/$ EtOAc $=50: 1)$ from $1 \mathbf{u}(0.94 \mathrm{~g}, 5.0 \mathrm{mmol}) \mathrm{HPPh}_{2}(0.95 \mathrm{~mL}, 1.02 \mathrm{~g}, 5.5 \mathrm{mmol})$ and $\mathrm{KO}^{t} \mathrm{Bu}(0.84 \mathrm{~g}, 7.5 \mathrm{mmol})$ following the general procedure $\mathrm{B}$.

$\mathbf{R}_{f}=0.52$ (petroleum ether $/$ EtOAc $=10 / 1$ )

${ }^{1}$ H NMR (400 MHz, $\left.\mathrm{CDCl}_{3}\right) \delta 7.56(\mathrm{~s}, 1 \mathrm{H}), 7.43-7.28(\mathrm{~m}, 10 \mathrm{H}), 6.92(\mathrm{~d}, J=3.4$ $\mathrm{Hz}, 1 \mathrm{H}), 2.94(\mathrm{t}, J=7.6 \mathrm{~Hz}, 2 \mathrm{H}), 2.84(\mathrm{t}, J=7.6 \mathrm{~Hz}, 2 \mathrm{H}), 2.14-2.04(\mathrm{~m}, 2 \mathrm{H})$.

${ }^{13} \mathrm{C}$ NMR (100 MHz, $\left.\mathrm{CDCl}_{3}\right) \delta$ 150.0, 145.9, $140.2(\mathrm{~d}, J=18.2 \mathrm{~Hz}), 135.3$ (d, $J=$ $10.6 \mathrm{~Hz}), 133.9$ (d, J = 20.1 Hz), 129.6 (d, J = 5.8 Hz), 129.5, 129.2, 128.8 (d, J = 7.1 $\mathrm{Hz}), 118.4(\mathrm{~d}, J=4.1 \mathrm{~Hz}), 115.8(\mathrm{~d}, J=34.7 \mathrm{~Hz}), 33.2,32.5,25.1$. 
${ }^{31}$ P NMR (162 MHz, $\mathrm{CDCl}_{3}$ ) $\delta-8.7$.

HRMS (ESI-TOF) calcd for $\mathrm{C}_{22} \mathrm{H}_{19} \mathrm{NP}^{+}[\mathrm{M}+\mathrm{H}]^{+}$: 328.1250, found: 328.1248.

IR (neat, cm-1): 2952, 2220, 1466, 1433, 1091, 886, 743, 694, 512, 500, 489.

1-(Diphenylphosphaneyl)-5,6,7,8-tetrahydronaphthalene-2-carbonitrile (2v)<smiles>N#Cc1ccc2c(c1P)CCCC2</smiles>

$2 \mathbf{v}$ was prepared as a white solid in $84 \%$ yield $(1.43 \mathrm{~g}$, eluent: petroleum ether $/$ EtOAc = 50:1) from $1 \mathbf{v}(1.01 \mathrm{~g}, 5.0 \mathrm{mmol}), \mathrm{HPPh}_{2}(0.95 \mathrm{~mL}, 1.02 \mathrm{~g}, 5.5 \mathrm{mmol})$ and $\mathrm{KO}^{t} \mathrm{Bu}(0.84 \mathrm{~g}, 7.5 \mathrm{mmol})$ following the general procedure $\mathrm{B}$.

$\mathbf{R}_{f}=0.42$ (petroleum ether $/$ EtOAc $=10 / 1$ )

${ }^{1} \mathrm{H}$ NMR (400 MHz, $\left.\mathrm{CDCl}_{3}\right) \delta 7.47(\mathrm{~d}, J=8.0 \mathrm{~Hz}, 1 \mathrm{H}), 7.42-7.28(\mathrm{~m}, 10 \mathrm{H}), 7.19$ $(\mathrm{d}, J=8.0 \mathrm{~Hz}, 1 \mathrm{H}), 2.88-2.75(\mathrm{~m}, 4 \mathrm{H}), 1.75-1.63(\mathrm{~m}, 4 \mathrm{H})$.

${ }^{13} \mathrm{C}$ NMR (100 MHz, $\left.\mathrm{CDCl}_{3}\right) \delta 145.1(\mathrm{~d}, J=18.3 \mathrm{~Hz}), 143.2(\mathrm{~d}, J=4.3 \mathrm{~Hz}), 138.0$ $(\mathrm{d}, J=24.5 \mathrm{~Hz}), 134.4(\mathrm{~d}, J=11.7 \mathrm{~Hz}), 132.92(\mathrm{~d}, J=19.4 \mathrm{~Hz}), 132.86(\mathrm{~d}, J=2.8$ $\mathrm{Hz}), 131.3,128.8,128.7(\mathrm{~d}, J=6.4 \mathrm{~Hz}), 118.3(\mathrm{~d}, J=2.7 \mathrm{~Hz}), 116.8(\mathrm{~d}, J=12.9 \mathrm{~Hz})$, 30.6, $29.5(\mathrm{~d}, J=23.9 \mathrm{~Hz}), 22.9(\mathrm{~d}, J=3.6 \mathrm{~Hz}), 22.0$.

${ }^{31 P}$ NMR (162 MHz, $\left.\mathrm{CDCl}_{3}\right) \delta$-11.6.

HRMS (ESI-TOF) calcd for $\mathrm{C}_{23} \mathrm{H}_{21} \mathrm{NP}^{+}[\mathrm{M}+\mathrm{H}]^{+}$: 342.1406, found: 342.1406 .

IR (neat, cm-1): 2933, 2219, 1583, 1480, 1456, 1434, 816, 743, 696, 509, 483.

\section{2-(Diphenylphosphaneyl)-1-naphthonitrile (2w)}<smiles>N#Cc1c(P)ccc2ccccc12</smiles>

$2 \mathbf{w}$ was prepared as a white solid in $96 \%$ yield (1.62 g, eluent: petroleum ether $/$ EtOAc $=50: 1)$ from $1 w(0.92 \mathrm{~g}, 5.0 \mathrm{mmol}), \mathrm{HPPh}_{2}(0.95 \mathrm{~mL}, 1.02 \mathrm{~g}, 5.5 \mathrm{mmol})$ and $\mathrm{KO}^{t} \mathrm{Bu}(0.84 \mathrm{~g}, 7.5 \mathrm{mmol})$ at room temperature following the general procedure B.

$\mathbf{R}_{f}=0.50$ (petroleum ether $/ \mathrm{EtOAc}=10 / 1$ )

${ }^{1} \mathrm{H}$ NMR (400 MHz, $\left.\mathrm{CDCl}_{3}\right) \delta 8.31(\mathrm{~d}, J=8.4 \mathrm{~Hz}, 1 \mathrm{H}), 7.94(\mathrm{~d}, J=8.3 \mathrm{~Hz}, 1 \mathrm{H})$, $7.89(\mathrm{~d}, J=8.3 \mathrm{~Hz}, 1 \mathrm{H}), 7.70(\mathrm{t}, J=7.8 \mathrm{~Hz}, 1 \mathrm{H}), 7.62(\mathrm{t}, J=7.6 \mathrm{~Hz}, 1 \mathrm{H}), 7.43-$ $7.34(\mathrm{~m}, 10 \mathrm{H}), 7.21-7.16(\mathrm{~m}, 1 \mathrm{H})$.

${ }^{13} \mathrm{C}$ NMR (100 MHz, $\left.\mathrm{CDCl}_{3}\right) \delta 143.5(\mathrm{~d}, J=20.8 \mathrm{~Hz}), 135.2(\mathrm{~d}, J=10.7 \mathrm{~Hz})$, 
$134.0(\mathrm{~d}, J=20.2 \mathrm{~Hz}), 133.3(\mathrm{~d}, J=6.3 \mathrm{~Hz}), 132.7,132.5,129.5,128.99,128.97$, 128.90, 128.6, 128.1, $125.4(\mathrm{~d}, J=1.7 \mathrm{~Hz}), 116.7(\mathrm{~d}, J=19.9 \mathrm{~Hz}), 116.5(\mathrm{~d}, J=10.8$ $\mathrm{Hz})$.

31P NMR (162 MHz, $\left.\mathrm{CDCl}_{3}\right) \delta$ - -7.1.

HRMS (ESI-TOF) calcd for $\mathrm{C}_{23} \mathrm{H}_{17} \mathrm{NP}^{+}[\mathrm{M}+\mathrm{H}]^{+}: 338.1093$, found: 338.1092.

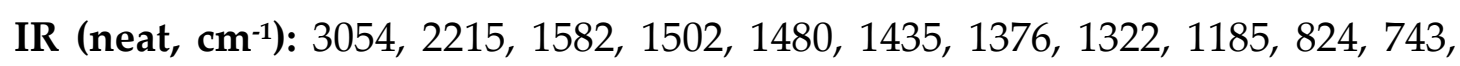
694.

\section{1-(Diphenylphosphaneyl)-2-naphthonitrile (2x)}<smiles>N#Cc1ccc2ccccc2c1-c1ccccc1</smiles>

$2 \mathbf{x}$ was prepared as a yellow solid in $85 \%$ yield ( $1.43 \mathrm{~g}$, eluent: petroleum ether /EtOAc = 50:1) from 1x $(0.92 \mathrm{~g}, 5.0 \mathrm{mmol}), \mathrm{HPPh}_{2}(0.95 \mathrm{~mL}, 1.02 \mathrm{~g}, 5.5 \mathrm{mmol})$ and $\mathrm{KO}^{t} \mathrm{Bu}(0.84 \mathrm{~g}, 7.5 \mathrm{mmol})$ at room temperature following the general procedure $\mathrm{B}$.

$\mathbf{R}_{f}=0.26$ (petroleum ether $/$ EtOAc $=10 / 1$ )

${ }^{1} \mathrm{H}$ NMR (400 MHz, CDCl $)$ \& $8.54(\mathrm{~d}, J=7.6 \mathrm{~Hz}, 1 \mathrm{H}), 8.01(\mathrm{~d}, J=8.4 \mathrm{~Hz}, 1 \mathrm{H})$, $7.91(\mathrm{~d}, J=8.1 \mathrm{~Hz}, 1 \mathrm{H}), 7.73(\mathrm{~d}, J=8.4 \mathrm{~Hz}, 1 \mathrm{H}), 7.59(\mathrm{t}, J=7.4 \mathrm{~Hz}, 1 \mathrm{H}), 7.50-$ $7.41(\mathrm{~m}, 5 \mathrm{H}), 7.38-7.32(\mathrm{~m}, 6 \mathrm{H})$.

${ }^{13} \mathrm{C}$ NMR (100 MHz, CDCl $)$ ) $140.1(\mathrm{~d}, J=28.7 \mathrm{~Hz}), 135.3(\mathrm{~d}, J=10.9 \mathrm{~Hz})$, $134.9(\mathrm{~d}, J=2.5 \mathrm{~Hz}), 134.3(\mathrm{~d}, J=11.8 \mathrm{~Hz}), 132.7$ (d, J = 19.3 Hz), 131.7, $129.2(\mathrm{~d}$, $J=4.9 \mathrm{~Hz}), 129.1,128.9(\mathrm{~d}, J=7.2 \mathrm{~Hz}), 128.8,128.7,128.4(\mathrm{~d}, J=20.1 \mathrm{~Hz}), 127.7$, $119.1(\mathrm{~d}, J=23.0 \mathrm{~Hz}), 118.6(\mathrm{~d}, J=4.6 \mathrm{~Hz})$.

31P NMR (162 MHz, $\left.\mathrm{CDCl}_{3}\right) \delta$ - 10.7 .

HRMS (ESI-TOF) calcd for $\mathrm{C}_{23} \mathrm{H}_{17} \mathrm{NP}^{+}[\mathrm{M}+\mathrm{H}]^{+}$: 338.1093, found: 338.1093 .

IR (neat, cm-1): 3054, 2220, 1585, 1499, 1480, 1434, 1264, 1027, 999, 820, 782, 693.

\section{2-(Diphenylphosphaneyl)phenanthrene-1-carbonitrile (2y)}<smiles>N#Cc1c(-c2ccccc2)ccc2c1ccc1ccccc12</smiles>

2y was prepared as a white solid in $81 \%$ yield $(0.32 \mathrm{~g}$, purification with recrystallization, $n$-hexane/DCM) from $1 y$ (0.25 g, $1.0 \mathrm{mmol}), \mathrm{HPPh}_{2}(0.19 \mathrm{~mL}$, 
$0.20 \mathrm{~g}, 1.1 \mathrm{mmol})$ and $\mathrm{KO}{ }^{t} \mathrm{Bu}(0.17 \mathrm{~g}, 1.5 \mathrm{mmol})$ following the general procedure B.

$\mathbf{R}_{f}=0.56$ (petroleum ether/EtOAc $=10 / 1$ )

${ }^{1} \mathrm{H}$ NMR (400 MHz, $\left.\mathrm{CDCl}_{3}\right) \delta 8.74(\mathrm{~d}, J=8.6 \mathrm{~Hz}, 1 \mathrm{H}), 8.58(\mathrm{~d}, J=7.6 \mathrm{~Hz}, 1 \mathrm{H})$, $8.20(\mathrm{~d}, J=9.1 \mathrm{~Hz}, 1 \mathrm{H}), 7.93(\mathrm{~d}, J=8.3 \mathrm{~Hz}, 2 \mathrm{H}), 7.74-7.64(\mathrm{~m}, 2 \mathrm{H}), 7.47-7.36$ $(\mathrm{m}, 10 \mathrm{H}), 7.32(\mathrm{~d}, J=8.7 \mathrm{~Hz}, 1 \mathrm{H})$.

${ }^{13} \mathrm{C}$ NMR (100 MHz, $\left.\mathrm{CDCl}_{3}\right) \delta 143.0$ (d, $\left.J=19.9 \mathrm{~Hz}\right), 135.2$ (d, $\left.J=10.5 \mathrm{~Hz}\right)$, $134.1(\mathrm{~d}, J=20.2 \mathrm{~Hz}), 133.1(\mathrm{~d}, J=5.7 \mathrm{~Hz}), 132.2,130.5,130.4,130.0,129.6,129.5$, 129.03, $128.97(\mathrm{~d}, J=7.3 \mathrm{~Hz}), 128.0,127.8,126.9,123.2(\mathrm{~d}, J=1.6 \mathrm{~Hz}), 122.7$, $116.8(\mathrm{~d}, J=4.3 \mathrm{~Hz}), 116.6(\mathrm{~d}, J=34.4 \mathrm{~Hz})$.

31P NMR (162 MHz, $\left.\mathrm{CDCl}_{3}\right) \delta$-7.7.

HRMS (ESI-TOF) calcd for $\mathrm{C}_{27} \mathrm{H}_{19} \mathrm{NP}^{+}[\mathrm{M}+\mathrm{H}]^{+}:$388.1250, found: 388.1251.

IR (neat, cm-1): 3054, 2216, 1480, 1434, 1093, 820, 744, 722, 695, 672, 587, 492.

\section{1-(Diphenylphosphaneyl)pyrene-2-carbonitrile (2z)}<smiles>N#CC1=CC2=C(P)c3ccc4c1c3C(=CC=C4)C=C2</smiles>

$2 \mathbf{z}$ was prepared as a yellow solid in $78 \%$ yield $(0.64 \mathrm{~g}$, purification with recrystallization, $n$-hexane/DCM) from $1 z(0.54 \mathrm{~g}, 2.0 \mathrm{mmol}), \mathrm{HPPh}_{2}(0.38 \mathrm{~mL}$, $0.41 \mathrm{~g}, 2.2 \mathrm{mmol})$ and $\mathrm{KO} \mathrm{Bu}(0.34 \mathrm{~g}, 3.0 \mathrm{mmol})$ following the general procedure B.

$\mathbf{R}_{f}=0.42$ (petroleum ether/EtOAc $=10 / 1$ )

${ }^{1} \mathrm{H}$ NMR (400 MHz, $\left.\mathrm{CDCl}_{3}\right) \delta 8.79(\mathrm{dd}, J=9.4,4.4 \mathrm{~Hz}, 1 \mathrm{H}), 8.55(\mathrm{~d}, J=1.4 \mathrm{~Hz}$, $1 \mathrm{H}), 8.27(\mathrm{dd}, J=7.7,1.1 \mathrm{~Hz}, 1 \mathrm{H}), 8.25-8.20(\mathrm{~m}, 2 \mathrm{H}), 8.13-8.06(\mathrm{~m}, 2 \mathrm{H}), 8.04$ $(\mathrm{d}, J=9.4 \mathrm{~Hz}, 1 \mathrm{H}), 7.53-7.45(\mathrm{~m}, 4 \mathrm{H}), 7.39-7.31(\mathrm{~m}, 6 \mathrm{H})$.

${ }^{13} \mathrm{C}$ NMR (100 MHz, $\left.\mathrm{CDCl}_{3}\right) \delta 136.1(\mathrm{~d}, J=11.8 \mathrm{~Hz}), 135.0(\mathrm{~d}, J=12.0 \mathrm{~Hz})$, 132.7, 132.6, 132.5, 132.3, 131.9, 131.1, $130.8(\mathrm{~d}, J=5.0 \mathrm{~Hz}), 130.4,129.3(\mathrm{~d}, J=$ $1.8 \mathrm{~Hz}), 128.82(\mathrm{~d}, J=6.2 \mathrm{~Hz}), 128.75,127.8,126.8(\mathrm{~d}, J=5.5 \mathrm{~Hz}), 126.62(\mathrm{~d}, J=$ 22.4 Hz), 126.60, 126.55, 124.0, 119.1 (d, J = 4.0 Hz), $118.1(\mathrm{~d}, J=22.9 \mathrm{~Hz})$.

31P NMR (162 MHz, $\left.\mathrm{CDCl}_{3}\right) \delta-11.7$.

HRMS (ESI-TOF) calcd for $\mathrm{C}_{29} \mathrm{H}_{19} \mathrm{NP}^{+}[\mathrm{M}+\mathrm{H}]^{+}:$412.1250, found: 412.1251 .

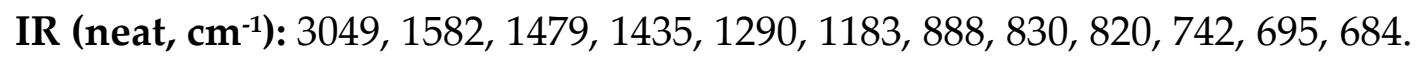




\section{Compound 2aa}

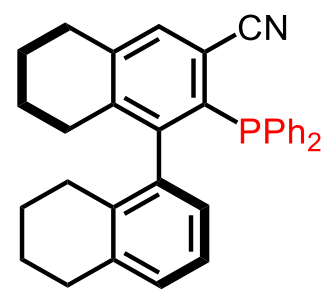

2aa was prepared as a white solid in $55 \%$ yield ( $0.26 \mathrm{~g}$, eluent: petroleum ether /EtOAc = 50:1) from 1aa $(0.43 \mathrm{~g}, 1.0 \mathrm{mmol}), \mathrm{HPPh}_{2}(0.19 \mathrm{~mL}, 0.20 \mathrm{~g}, 1.1 \mathrm{mmol})$ and $\mathrm{KO}^{t} \mathrm{Bu}(0.17 \mathrm{~g}, 1.5 \mathrm{mmol})$ in $\mathrm{N}, \mathrm{N}$-dimethylformamide at $100{ }^{\circ} \mathrm{C}$ following the general procedure B.

$\mathbf{R}_{f}=0.48$ (petroleum ether $/$ EtOAc $=10 / 1$ )

${ }^{1}$ H NMR (400 MHz, CDCl $_{3}$ ) $\delta 7.49$ (s, 1H), $7.40-7.27$ (m, 10H), 7.13 (d, J = 7.5 $\mathrm{Hz}, 1 \mathrm{H}), 7.06(\mathrm{t}, J=7.5 \mathrm{~Hz}, 1 \mathrm{H}), 6.56(\mathrm{~d}, J=7.3 \mathrm{~Hz}, 1 \mathrm{H}), 2.91-2.82(\mathrm{~m}, 4 \mathrm{H})$, $2.38-2.23(\mathrm{~m}, 2 \mathrm{H}), 2.23-2.12(\mathrm{~m}, 2 \mathrm{H}), 1.84-1.69(\mathrm{~m}, 6 \mathrm{H}), 1.69-1.61(\mathrm{~m}, 2 \mathrm{H})$. ${ }^{13} \mathrm{C}$ NMR (100 MHz, $\left.\mathrm{CDCl}_{3}\right) \delta 150.9$ (d, J = 36.0 Hz), 141.1 (d, J = 6.5 Hz), 140.0, $139.2(\mathrm{~d}, J=9.5 \mathrm{~Hz}), 137.6,136.4,136.2(\mathrm{~d}, J=14.3 \mathrm{~Hz}), 135.4(\mathrm{~d}, J=21.7 \mathrm{~Hz})$, $135.1(\mathrm{~d}, J=13.9 \mathrm{~Hz}), 134.4(\mathrm{~d}, J=2.4 \mathrm{~Hz}), 133.6(\mathrm{~d}, J=20.2 \mathrm{~Hz}), 132.5(\mathrm{~d}, J=$ $19.1 \mathrm{~Hz}), 128.9,128.7,128.43(\mathrm{~d}, J=1.9 \mathrm{~Hz}), 128.37$ (d, J = 3.2 Hz), 128.3, 127.1 $(\mathrm{d}, J=3.7 \mathrm{~Hz}), 125.3,118.0,115.1(\mathrm{~d}, J=3.8 \mathrm{~Hz}), 29.9,29.7,28.5(\mathrm{~d}, J=2.6 \mathrm{~Hz})$, $27.9(\mathrm{~d}, J=2.9 \mathrm{~Hz}), 23.1,22.9,22.8,22.2$.

${ }^{31} \mathrm{P}$ NMR (162 MHz, $\left.\mathrm{CDCl}_{3}\right) \delta$-11.5.

HRMS (ESI-TOF) calcd for $\mathrm{C}_{33} \mathrm{H}_{31} \mathrm{NP}^{+}[\mathrm{M}+\mathrm{H}]^{+}:$472.2189, found: 472.2189 .

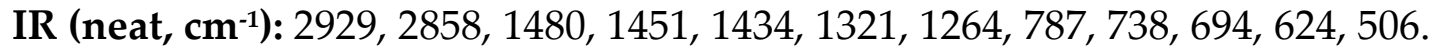

Ee: 96\%. Enantiomeric excess was determined by chiral HPLC (Daicel Chiralcel OD-H column, $n$-hexane $/ i$-propanol $=98.5 / 1.5$, flow rate $0.6 \mathrm{~mL} / \mathrm{min}$, detection at $220 \mathrm{~nm}$ ), $t_{1}=15.7 \mathrm{~min}$ (minor), $t_{2}=16.8 \mathrm{~min}$ (major).

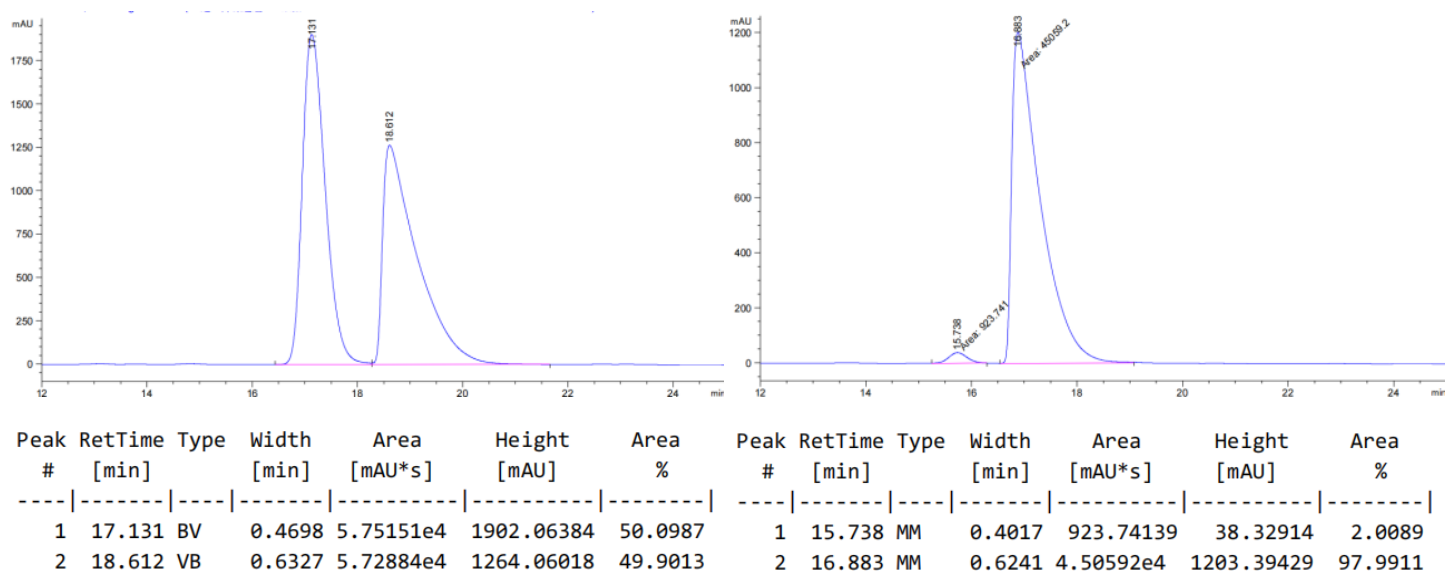




\section{Compound 2ab}

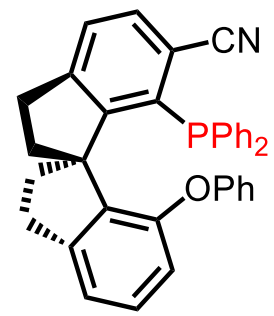

2ab was prepared as a white solid in $85 \%$ yield $(98.0 \mathrm{mg}$, eluent: petroleum ether $/$ EtOAc $=40: 1)$ from $1 \mathrm{ab}(85.9 \mathrm{mg}, 0.2 \mathrm{mmol}), \mathrm{HPPh}_{2}(41.0 \mathrm{mg}, 0.22$ $\mathrm{mmol})$ and $\mathrm{KO}^{t} \mathrm{Bu}(33.6 \mathrm{mg}, 0.3 \mathrm{mmol})$ following the general procedure $\mathrm{B}$.

$\mathbf{R}_{f}=0.20$ (petroleum ether $/$ EtOAc $=10 / 1$ )

${ }^{1}$ H NMR (400 MHz, $\left.\mathrm{CDCl}_{3}\right) \delta 7.58(\mathrm{~d}, J=7.7 \mathrm{~Hz}, 1 \mathrm{H}), 7.35-7.26(\mathrm{~m}, 9 \mathrm{H}), 7.21$ - $7.16(\mathrm{~m}, 4 \mathrm{H}), 7.05-6.97(\mathrm{~m}, 3 \mathrm{H}), 6.56(\mathrm{~d}, J=8.0 \mathrm{~Hz}, 2 \mathrm{H}), 6.24(\mathrm{~d}, J=7.6 \mathrm{~Hz}$, $1 \mathrm{H}), 3.30-3.13(\mathrm{~m}, 2 \mathrm{H}), 3.12-2.95(\mathrm{~m}, 2 \mathrm{H}), 2.73-2.63(\mathrm{~m}, 1 \mathrm{H}), 2.49-2.36(\mathrm{~m}$, $2 \mathrm{H}), 2.37-2.29(\mathrm{~m}, 1 \mathrm{H})$.

${ }^{13} \mathrm{C}$ NMR (100 MHz, $\left.\mathrm{CDCl}_{3}\right) \delta 158.9$ (d, J=27.7 Hz), 156.6, 152.9 (d, J=2.0 Hz), $149.1(\mathrm{~d}, J=8.8 \mathrm{~Hz}), 146.5(\mathrm{~d}, J=4.0 \mathrm{~Hz}), 140.6(\mathrm{~d}, J=4.2 \mathrm{~Hz}), 137.0(\mathrm{~d}, J=14.7$ $\mathrm{Hz}), 135.6,135.2$ (d, $J=34.5 \mathrm{~Hz}), 134.0$ (d, $J=14.3 \mathrm{~Hz}), 133.7$ (d, $J=20.8 \mathrm{~Hz})$, $132.3(\mathrm{~d}, J=18.7 \mathrm{~Hz}), 129.5,128.6,128.50,128.47,128.42,128.14,128.07,126.3$, 123.0, 119.4, 118.8, $117.7(\mathrm{~d}, J=5.7 \mathrm{~Hz}), 115.9,62.2(\mathrm{~d}, J=5.9 \mathrm{~Hz}), 40.9(\mathrm{~d}, J=7.6$ $\mathrm{Hz}), 39.8,31.7(\mathrm{~d}, J=3.2 \mathrm{~Hz}), 31.4$.

${ }^{31}$ P NMR (162 MHz, $\left.\mathrm{CDCl}_{3}\right) \delta-19.6$.

HRMS (ESI-TOF) calcd for $\mathrm{C}_{36} \mathrm{H}_{29} \mathrm{NOP}^{+}[\mathrm{M}+\mathrm{H}]^{+}: 522.1982$, found: 522.1984 .

IR (neat, cm-1): 2954, 2926, 1580, 1489, 1465, 1434, 1244, 1211, 740, 693.

Ee: 95\%. Enantiomeric excess was determined by chiral HPLC (Daicel Chiralpak AD-H column, $n$-hexane/ $i$-propanol $=98 / 2$, flow rate $1.0 \mathrm{~mL} / \mathrm{min}$, detection at $220 \mathrm{~nm}$ ), $t_{1}=8.6 \mathrm{~min}$ (major), $t_{2}=9.4 \mathrm{~min}$ (minor).

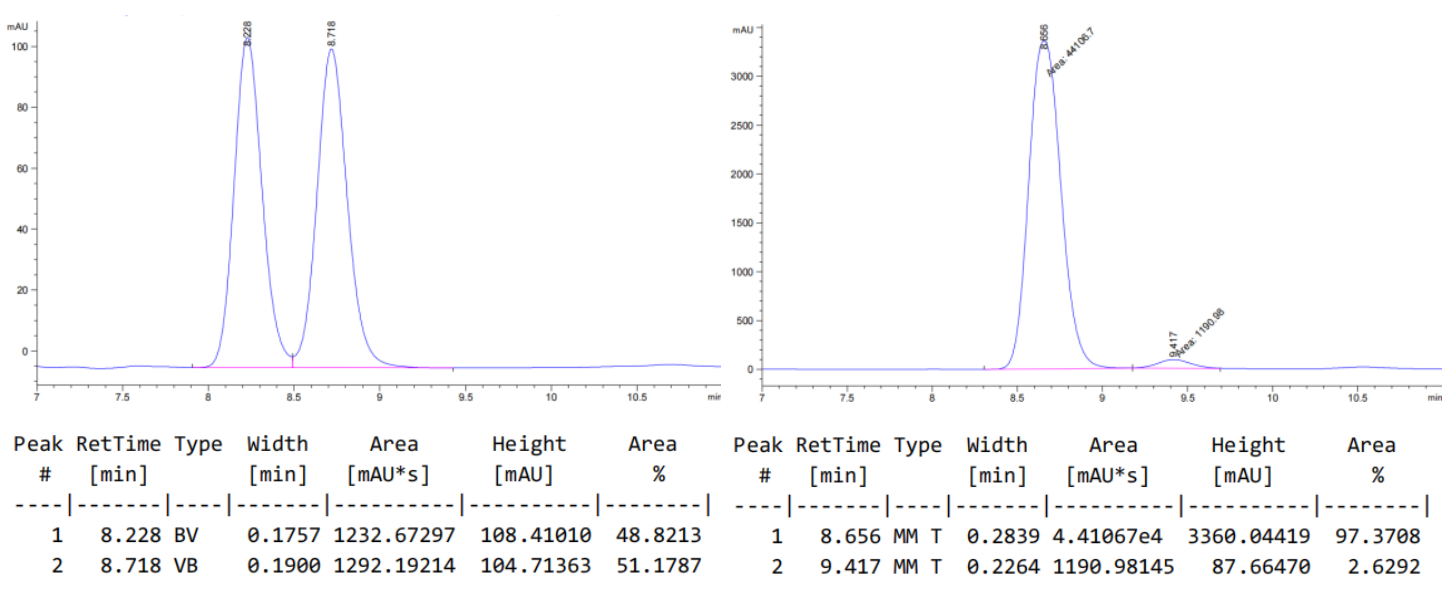




\section{Compound 2ac}<smiles>CC(C)(c1ccc(P)c(C#N)c1)c1ccc(P)c(C#N)c1</smiles>

2ac was prepared as a white solid in $83 \%$ yield $(2.55 \mathrm{~g}$, purification with recrystallization, Et $\left.{ }_{2} \mathrm{O} / \mathrm{DCM}\right)$ from $1 \mathrm{ac}(1.67 \mathrm{~g}, 5.0 \mathrm{mmol}), \mathrm{HPPh}_{2}(1.91 \mathrm{~mL}$, $2.05 \mathrm{~g}, 11.0 \mathrm{mmol})$ and $\mathrm{KO}^{t} \mathrm{Bu}(1.68 \mathrm{~g}, 15.0 \mathrm{mmol})$ following the general procedure $B$.

$\mathbf{R}_{f}=0.25$ (petroleum ether $/ \mathrm{EtOAc}=5 / 1$ )

${ }^{1}$ H NMR (400 MHz, $\left.\mathrm{CDCl}_{3}\right) \delta 7.63$ - 7.59 (m, 2H), 7.47 - 7.30 (m, 22H), 7.01 $(\mathrm{dd}, J=8.2,3.3 \mathrm{~Hz}, 2 \mathrm{H}), 1.72(\mathrm{~s}, 6 \mathrm{H})$.

${ }^{13} \mathrm{C}$ NMR (100 MHz, $\left.\mathrm{CDCl}_{3}\right) \delta$ 150.3, $140.9(\mathrm{~d}, J=19.8 \mathrm{~Hz}), 134.7$ (d, $J=10.1$ $\mathrm{Hz}), 134.1(\mathrm{~d}, J=20.4 \mathrm{~Hz}), 133.7,132.0(\mathrm{~d}, J=4.7 \mathrm{~Hz}), 131.0,129.6,128.9(\mathrm{~d}, J=$ $7.4 \mathrm{~Hz}), 118.1(\mathrm{~d}, J=32.7 \mathrm{~Hz}), 117.8(\mathrm{~d}, J=3.6 \mathrm{~Hz}), 43.0,30.2$.

${ }^{31} \mathrm{P}$ NMR (162 MHz, $\left.\mathrm{CDCl}_{3}\right) \delta-9.3$.

HRMS (ESI-TOF) calcd for $\mathrm{C}_{41} \mathrm{H}_{33} \mathrm{~N}_{2} \mathrm{P}_{2}{ }^{+}[\mathrm{M}+\mathrm{H}]^{+}:$: 615.2114, found: 615.2116.

IR (neat, cm cm: $^{-1}$ 2971, 2222, 1473, 1434, 1391, 1266, 1092, 897, 735, 694, 661.

\section{Compound 2ad}

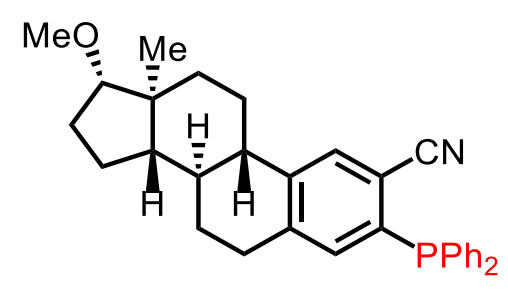

2ad was prepared as a white solid in $73 \%$ yield $(0.35 \mathrm{~g}$, eluent: petroleum ether $/$ EtOAc $=50: 1)$ from 1 ad $(0.34 \mathrm{~g}, 1.0 \mathrm{mmol}), \mathrm{HPPh}_{2}(0.19 \mathrm{~mL}, 0.20 \mathrm{~g}, 1.1$ $\mathrm{mmol})$ and $\mathrm{KO}^{t} \mathrm{Bu}(0.17 \mathrm{~g}, 1.5 \mathrm{mmol})$ following the general procedure $\mathrm{B}$.

$\mathbf{R}_{f}=0.32$ (petroleum ether $/$ EtOAc $=10 / 1$ )

${ }^{1}$ H NMR (400 MHz, $\left.\mathrm{CDCl}_{3}\right) \delta 7.63(\mathrm{~s}, 1 \mathrm{H}), 7.40$ - $7.27(\mathrm{~m}, 10 \mathrm{H}), 6.78-6.72(\mathrm{~m}$, $1 \mathrm{H}), 3.38(\mathrm{~s}, 3 \mathrm{H}), 3.33(\mathrm{t}, J=8.3 \mathrm{~Hz}, 1 \mathrm{H}), 2.78-2.66(\mathrm{~m}, 2 \mathrm{H}), 2.32-2.17(\mathrm{~m}, 2 \mathrm{H})$, $2.14-2.01(\mathrm{~m}, 2 \mathrm{H}), 1.91-1.81(\mathrm{~m}, 1 \mathrm{H}), 1.75-1.62(\mathrm{~m}, 1 \mathrm{H}), 1.62-1.45(\mathrm{~m}, 2 \mathrm{H})$, $1.47-1.36(\mathrm{~m}, 2 \mathrm{H}), 1.39-1.28(\mathrm{~m}, 2 \mathrm{H}), 1.26-1.14(\mathrm{~m}, 1 \mathrm{H}), 0.80(\mathrm{~s}, 3 \mathrm{H})$.

${ }^{13} \mathrm{C}$ NMR (100 MHz, $\left.\mathrm{CDCl}_{3}\right) \delta 142.2(\mathrm{~d}, J=25.4 \mathrm{~Hz}), 138.6(\mathrm{~d}, J=18.2 \mathrm{~Hz})$, $135.4-134.9(\mathrm{~m}), 134.1,133.9(\mathrm{~d}, J=2.9 \mathrm{~Hz}), 133.7(\mathrm{~d}, J=2.8 \mathrm{~Hz}), 131.0(\mathrm{~d}, J=$ 
$5.2 \mathrm{~Hz}), 129.2(\mathrm{~d}, J=1.6 \mathrm{~Hz}), 128.7(\mathrm{~d}, J=7.1 \mathrm{~Hz}), 118.3(\mathrm{~d}, J=3.7 \mathrm{~Hz}), 115.0(\mathrm{~d}$, $J=32.9 \mathrm{~Hz}), 90.4,57.9,50.2,43.9,43.1,37.71,37.69,29.7,27.7,26.6,25.9,23.0$, 11.5 .

31P NMR (162 MHz, $\left.\mathrm{CDCl}_{3}\right) \delta$-9.6.

HRMS (ESI-TOF) calcd for $\mathrm{C}_{32} \mathrm{H}_{35} \mathrm{NOP}^{+}[\mathrm{M}+\mathrm{H}]^{+}: 480.2451$, found: 480.2451 .

IR (neat, cm-1): 2927, 1473, 1434, 1265, 1135, 1103, 735, 695.

\section{2-(Di-p-tolylphosphaneyl)benzonitrile (2ae)}<smiles>Cc1ccccc1P(c1ccc([N+](=O)[O-])cc1)c1ccc([N+](=O)[O-])cc1</smiles>

2ae was prepared as a white solid in $86 \%$ yield ( $54.3 \mathrm{mg}$, eluent: petroleum ether $/$ EtOAc $=20: 1)$ from 1a $(29.4 \mathrm{mg}, 0.2 \mathrm{mmol}), \mathrm{HP}\left(4-\mathrm{MeC}_{6} \mathrm{H}_{4}\right)_{2}(47.1 \mathrm{mg}$, $0.22 \mathrm{mmol})$ and $\mathrm{KO} \mathrm{Bu}^{\mathrm{B} u}(33.7 \mathrm{mg}, 0.3 \mathrm{mmol})$ following the general procedure $\mathrm{B}$. $\mathbf{R}_{f}=0.45$ (petroleum ether $/ \mathrm{EtOAc}=10 / 1$ )

${ }^{1} \mathrm{H}$ NMR (400 MHz, CDCl $)$ ) $7.71-7.67(\mathrm{~m}, 1 \mathrm{H}), 7.49$ - $7.44(\mathrm{~m}, 1 \mathrm{H}), 7.42-$ $7.36(\mathrm{~m}, 1 \mathrm{H}), 7.25-7.17(\mathrm{~m}, 8 \mathrm{H}), 7.08-7.03(\mathrm{~m}, 1 \mathrm{H}), 2.37(\mathrm{~s}, 6 \mathrm{H})$.

${ }^{13} \mathrm{C}$ NMR (100 MHz, $\left.\mathrm{CDCl}_{3}\right) \delta 143.9(\mathrm{~d}, J=19.8 \mathrm{~Hz}), 139.5,134.1(\mathrm{~d}, J=20.5$ $\mathrm{Hz}), 133.7(\mathrm{~d}, J=4.6 \mathrm{~Hz}), 133.3,132.4,131.3(\mathrm{~d}, J=8.7 \mathrm{~Hz}), 129.7(\mathrm{~d}, J=7.6 \mathrm{~Hz})$, 128.7, $117.8(\mathrm{~d}, J=3.7 \mathrm{~Hz}), 117.7(\mathrm{~d}, J=32.7 \mathrm{~Hz}), 21.5$.

31P NMR (162 MHz, $\left.\mathrm{CDCl}_{3}\right) \delta$ - 10.1 .

The characterization data are consistent with the literature reported data. ${ }^{10}$

\section{2-(Bis(4-(tert-butyl)phenyl)phosphaneyl)benzonitrile (2af)}<smiles>Cc1ccccc1P(c1ccc(Br)cc1)c1ccc(Br)cc1</smiles>

2af was prepared as a white solid in 70\% yield $(56.1 \mathrm{mg}$, eluent: petroleum 
ether $/ \mathrm{EtOAc}=20: 1)$ from $1 \mathrm{a}(29.4 \mathrm{mg}, 0.2 \mathrm{mmol}), \mathrm{HP}\left(4-{ }^{-} \mathrm{BuC}_{6} \mathrm{H}_{4}\right)_{2}(65.6 \mathrm{mg}$, $0.22 \mathrm{mmol})$ and $\mathrm{KO}^{\star} \mathrm{Bu}(33.7 \mathrm{mg}, 0.3 \mathrm{mmol})$ following the general procedure $\mathrm{B}$. $\mathbf{R}_{f}=0.42$ (petroleum ether $/$ EtOAc $=10 / 1$ )

${ }^{1} \mathrm{H}$ NMR $\left(400 \mathrm{MHz}, \mathrm{CDCl}_{3}\right) \delta 7.71-7.66(\mathrm{~m}, 1 \mathrm{H}), 7.49-7.44(\mathrm{~m}, 1 \mathrm{H}), 7.42-$ $7.36(\mathrm{~m}, 5 \mathrm{H}), 7.27-7.21(\mathrm{~m}, 4 \mathrm{H}), 7.11-7.06(\mathrm{~m}, 1 \mathrm{H}), 1.32(\mathrm{~s}, 18 \mathrm{H})$.

${ }^{13} \mathrm{C}$ NMR (100 MHz, $\left.\mathrm{CDCl}_{3}\right) \delta$ 152.5, 143.9 (d, $\left.J=19.8 \mathrm{~Hz}\right), 133.9$ (d, $J=20.3$ $\mathrm{Hz}), 133.7(\mathrm{~d}, J=4.7 \mathrm{~Hz}), 133.5,132.4,131.3(\mathrm{~d}, J=8.5 \mathrm{~Hz}), 128.7,125.9(\mathrm{~d}, J=$ $7.4 \mathrm{~Hz}), 117.92(\mathrm{~d}, J=33.2 \mathrm{~Hz}), 117.86(\mathrm{~d}, J=3.7 \mathrm{~Hz}), 34.8,31.3$.

${ }^{31} \mathrm{P}$ NMR (162 MHz, $\left.\mathrm{CDCl}_{3}\right) \delta$-11.0.

HRMS (ESI-TOF) calcd for $\mathrm{C}_{27} \mathrm{H}_{31} \mathrm{NP}^{+}[\mathrm{M}+\mathrm{H}]^{+}: 400.2189$, found: 400.2191 .

IR (neat, cm ${ }^{-1}$ ): 2962, 1494, 1461, 1389, 1268, 1084, 826, 763, 559, 492.

\section{2-(Bis(4-(dimethylamino)phenyl)phosphaneyl)benzonitrile (2ag)}<smiles>Cc1ccccc1P(c1ccc(N(C)C)cc1)c1ccc(N(C)C)cc1</smiles>

2ag was prepared as a yellow solid in $82 \%$ yield $(61.3 \mathrm{mg}$, eluent: petroleum ether $/ \mathrm{EtOAc}=5: 1)$ from $1 \mathrm{a}(29.4 \mathrm{mg}, 0.2 \mathrm{mmol}), \mathrm{HP}\left(4-\mathrm{NMe}_{2} \mathrm{C}_{6} \mathrm{H}_{4}\right)_{2}(59.9 \mathrm{mg}$, $0.22 \mathrm{mmol})$ and $\mathrm{KO}^{\mathrm{B} u}(33.7 \mathrm{mg}, 0.3 \mathrm{mmol})$ following the general procedure $\mathrm{B}$. $\mathbf{R}_{f}=0.22$ (petroleum ether $/$ EtOAc $=5 / 1$ )

${ }^{1} \mathrm{H}$ NMR $\left(400 \mathrm{MHz}, \mathrm{CDCl}_{3}\right) \delta 7.66-7.60(\mathrm{~m}, 1 \mathrm{H}), 7.45-7.39(\mathrm{~m}, 1 \mathrm{H}), 7.35-$ $7.28(\mathrm{~m}, 1 \mathrm{H}), 7.26-7.17(\mathrm{~m}, 4 \mathrm{H}), 7.12-7.07(\mathrm{~m}, 1 \mathrm{H}), 6.74-6.67(\mathrm{~m}, 4 \mathrm{H}), 2.97(\mathrm{~s}$, $12 \mathrm{H})$.

${ }^{13} \mathrm{C}$ NMR (100 MHz, $\left.\mathrm{CDCl}_{3}\right) \delta$ 150.9, $146.3(\mathrm{~d}, J=20.2 \mathrm{~Hz}), 135.4(\mathrm{~d}, J=21.8$ Hz), $133.6(\mathrm{~d}, J=4.1 \mathrm{~Hz}), 132.8,132.0,127.9,120.2(\mathrm{~d}, J=4.0 \mathrm{~Hz}), 117.9$ (d, $J=$ $3.3 \mathrm{~Hz}), 117.0(\mathrm{~d}, J=30.8 \mathrm{~Hz}), 112.4(\mathrm{~d}, J=8.4 \mathrm{~Hz}), 40.2$.

${ }^{31}$ P NMR (162 MHz, $\left.\mathrm{CDCl}_{3}\right) \delta$-11.8.

HRMS (ESI-TOF) calcd for $\mathrm{C}_{23} \mathrm{H}_{25} \mathrm{~N}_{3} \mathrm{P}^{+}[\mathrm{M}+\mathrm{H}]^{+}: 374.1781$, found: 374.1780 .

IR (neat, cm-1): 1595, 1508, 1444, 1356, 1199, 1098, 810, 762.

\section{2-(Bis(4-methoxyphenyl)phosphaneyl)benzonitrile (2ah)}




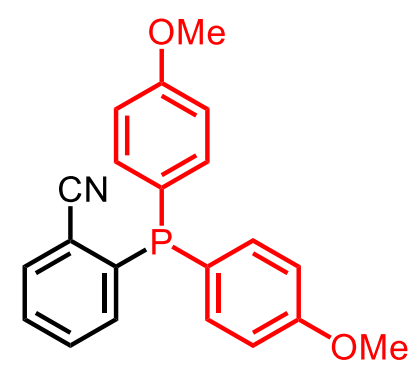

2ah was prepared as a white solid in $92 \%$ yield $(64.1 \mathrm{mg}$, eluent: petroleum ether $/ \mathrm{EtOAc}=5: 1)$ from $1 \mathrm{a}(29.4 \mathrm{mg}, 0.2 \mathrm{mmol}), \mathrm{HP}\left(4-\mathrm{OMeC}_{6} \mathrm{H}_{4}\right)_{2}(54.2 \mathrm{mg}$, $0.22 \mathrm{mmol})$ and $\mathrm{KO}^{\mathrm{B}} \mathrm{Bu}(33.7 \mathrm{mg}, 0.3 \mathrm{mmol})$ following the general procedure $\mathrm{B}$. $\mathbf{R}_{f}=0.24$ (petroleum ether $/$ EtOAc $=5 / 1$ )

${ }^{1} \mathrm{H}$ NMR (400 MHz, $\left.\mathrm{CDCl}_{3}\right) \delta 7.69-7.64(\mathrm{~m}, 1 \mathrm{H}), 7.48-7.42(\mathrm{~m}, 1 \mathrm{H}), 7.40-$ $7.34(\mathrm{~m}, 1 \mathrm{H}), 7.30-7.22(\mathrm{~m}, 4 \mathrm{H}), 7.04-7.00(\mathrm{~m}, 1 \mathrm{H}), 6.95-6.87(\mathrm{~m}, 4 \mathrm{H}), 3.81(\mathrm{~s}$, $6 \mathrm{H})$.

${ }^{13}$ C NMR (100 MHz, $\left.\mathrm{CDCl}_{3}\right) \delta$ 160.7, $144.5(\mathrm{~d}, J=19.7 \mathrm{~Hz}), 135.7(\mathrm{~d}, J=21.9$ $\mathrm{Hz}), 133.8(\mathrm{~d}, J=4.4 \mathrm{~Hz}), 132.9,132.3,128.5,125.7$ (d, $J=7.0 \mathrm{~Hz}), 117.7$ (d, $J=$ $3.6 \mathrm{~Hz}), 117.3(\mathrm{~d}, J=31.5 \mathrm{~Hz}), 114.6(\mathrm{~d}, J=8.4 \mathrm{~Hz}), 55.3$.

${ }^{31} \mathrm{P}$ NMR (162 MHz, $\left.\mathrm{CDCl}_{3}\right) \delta$-11.4.

The characterization data are consistent with the literature reported data. ${ }^{11}$

\section{2-(Bis(3,5-di-tert-butylphenyl)phosphaneyl)benzonitrile (2ai)}<smiles>Cc1ccccc1B(c1cc(Br)cc(C(C)(C)C)c1)c1cc(C(=O)c2ccccc2)cc(C(C)(C)C)c1</smiles>

2ai was prepared as a white solid in $97 \%$ yield $(99.3 \mathrm{mg}$, eluent: petroleum ether $/ \mathrm{EtOAc}=20: 1)$ from $1 \mathrm{a}(29.4 \mathrm{mg}, 0.2 \mathrm{mmol}), \mathrm{HP}\left(3,5-{ }^{-} \mathrm{BuC}_{6} \mathrm{H}_{3}\right) 2(90.3 \mathrm{mg}$, $0.22 \mathrm{mmol})$ and $\mathrm{KO}^{\mathrm{t}} \mathrm{Bu}(33.7 \mathrm{mg}, 0.3 \mathrm{mmol})$ following the general procedure $\mathrm{B}$. $\mathbf{R}_{f}=0.56$ (petroleum ether $/$ EtOAc $=10 / 1$ )

${ }^{1} \mathrm{H}$ NMR $\left(400 \mathrm{MHz}, \mathrm{CDCl}_{3}\right) \delta 7.73-7.69(\mathrm{~m}, 1 \mathrm{H}), 7.52-7.46(\mathrm{~m}, 1 \mathrm{H}), 7.46-$ $7.42(\mathrm{~m}, 2 \mathrm{H}), 7.42-7.38(\mathrm{~m}, 1 \mathrm{H}), 7.18-7.12(\mathrm{~m}, 4 \mathrm{H}), 7.11-7.07(\mathrm{~m}, 1 \mathrm{H}), 1.26(\mathrm{~s}$, $36 \mathrm{H})$.

${ }^{13} \mathrm{C}$ NMR (100 MHz, $\mathrm{CDCl}_{3}$ ) \& 150.9 (d, $\left.J=7.0 \mathrm{~Hz}\right), 144.6$ (d, $\left.J=20.7 \mathrm{~Hz}\right), 134.0$ $(\mathrm{d}, J=8.7 \mathrm{~Hz}), 133.6(\mathrm{~d}, J=4.9 \mathrm{~Hz}), 133.4,132.1,128.7,128.3(\mathrm{~d}, J=20.6 \mathrm{~Hz})$, 
123.2, $118.1(\mathrm{~d}, J=32.9 \mathrm{~Hz}), 117.9(\mathrm{~d}, J=3.6 \mathrm{~Hz}), 35.0,31.5$.

${ }^{31}$ P NMR (162 $\left.\mathbf{M H z}, \mathrm{CDCl}_{3}\right) \delta-6.3$.

The characterization data are consistent with the literature reported data. ${ }^{12}$

\section{2-(Di(naphthalen-2-yl)phosphaneyl)benzonitrile (2aj)}<smiles>Cc1ccccc1P(c1ccc2ccccc2c1)c1ccc2ccccc2c1</smiles>

2aj was prepared as a white solid in 95\% yield (73.6 mg, eluent: petroleum ether $/$ EtOAc $=20: 1)$ from $1 \mathrm{a}(29.4 \mathrm{mg}, 0.2 \mathrm{mmol})$, di(naphthalen-2$\mathrm{yl})$ phosphane $(63.0 \mathrm{mg}, 0.22 \mathrm{mmol})$ and $\mathrm{KO} \mathrm{Bu}^{\circ}(33.7 \mathrm{mg}, 0.3 \mathrm{mmol})$ following the general procedure $B$.

$\mathbf{R}_{f}=0.34$ (petroleum ether $/$ EtOAc $=10 / 1$ )

${ }^{1} \mathrm{H}$ NMR (400 MHz, $\left.\mathrm{CDCl}_{3}\right) \delta 7.93(\mathrm{~d}, J=9.2 \mathrm{~Hz}, 2 \mathrm{H}), 7.87(\mathrm{~d}, J=8.2 \mathrm{~Hz}, 4 \mathrm{H})$, $7.81-7.74(\mathrm{~m}, 3 \mathrm{H}), 7.58-7.40(\mathrm{~m}, 8 \mathrm{H}), 7.23-7.17(\mathrm{~m}, 1 \mathrm{H})$.

${ }^{13} \mathrm{C}$ NMR (100 MHz, $\left.\mathrm{CDCl}_{3}\right) \delta 142.9$ (d, $\left.J=19.8 \mathrm{~Hz}\right), 135.0$ (d, $\left.J=23.8 \mathrm{~Hz}\right)$, $133.9(\mathrm{~d}, J=5.0 \mathrm{~Hz}), 133.7(\mathrm{~d}, J=1.7 \mathrm{~Hz}), 133.4(\mathrm{~d}, J=8.8 \mathrm{~Hz}), 132.6,132.1(\mathrm{~d}, J=$ $10.3 \mathrm{~Hz}), 129.9,129.8,129.1,128.5(\mathrm{~d}, J=6.9 \mathrm{~Hz}), 128.3,127.8,127.2,126.6,118.2$ $(\mathrm{d}, J=33.4 \mathrm{~Hz}), 117.8(\mathrm{~d}, J=3.9 \mathrm{~Hz})$.

31P NMR (162 MHz, $\left.\mathrm{CDCl}_{3}\right) \delta-7.7$.

HRMS (ESI-TOF) calcd for $\mathrm{C}_{27} \mathrm{H}_{19} \mathrm{NP}^{+}[\mathrm{M}+\mathrm{H}]^{+}$: 388.1250, found: 388.1252.

IR (neat, cm-1): 1587, 1498, 1460, 1435, 1270, 1134, 1078, 858, 815, 762, 703, 638.

\section{(1-Isocyanonaphthalen-2-yl)diisopropylphosphine oxide (2ak)}

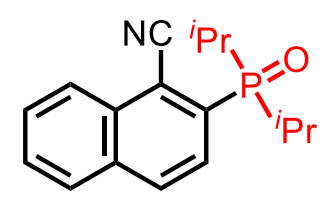

In a glovebox, to an oven-dried sealed tube were added KHMDS (1.0 $\mathrm{M}$ in $\mathrm{THF}$, 7.5 mL, $7.5 \mathrm{mmol}), \mathrm{HP}^{i} \mathrm{Pr} 2$ (0.65 g, $\left.5.5 \mathrm{mmol}\right)$, 2-methoxy-1naphthonitrile $\mathbf{1 w}(0.92 \mathrm{~g}, 5.0 \mathrm{mmol})$ and cyclohexane $(10 \mathrm{~mL})$ at room 
temperature, and the tube was removed from glovebox. The resulting mixture was stirred at $80^{\circ} \mathrm{C}$ in an oil bath for $15 \mathrm{~h}$. After cooling to room temperature, the reaction mixture was diluted with DCM, filtered through a plug of silica gel. After removal of solvents under reduced pressure, the residue was dissolved in THF $\left(10 \mathrm{~mL}\right.$ ), then $\mathrm{H}_{2} \mathrm{O}_{2}$ (5.0 equiv, $30 \%$ in water) was added dropwise and stirred at room temperature. Upon completion, the resulting mixture was quenched by sat. $\mathrm{NaHCO}_{3}$ solution and sat. $\mathrm{Na}_{2} \mathrm{~S}_{2} \mathrm{O}_{3}$ solution. The aqueous layer was washed with DCM. The combined organic layers were dried over $\mathrm{Na}_{2} \mathrm{SO}_{4}$, filtered, and concentrated in vacuo. The resulting crude mixture was purified by silica gel column chromatography (petroleum ether $/$ EtOAc $=5: 1$ to $2: 1)$ to afford the corresponding phosphine oxide $2 \mathbf{a k}(0.68 \mathrm{~g}$, $47 \%)$ as a yellow oil.

$\mathbf{R}_{f}=0.30$ (petroleum ether $/$ EtOAc $=1 / 1$ )

${ }^{1} \mathrm{H}$ NMR (400 MHz, $\left.\mathrm{CDCl}_{3}\right)$ \& 8.29 (d, J = 8.4 Hz, 1H), $8.23-8.12(\mathrm{~m}, 2 \mathrm{H}), 7.92$ $(\mathrm{d}, J=8.1 \mathrm{~Hz}, 1 \mathrm{H}), 7.69(\mathrm{t}, J=7.5 \mathrm{~Hz}, 1 \mathrm{H}), 7.64(\mathrm{~d}, J=7.6 \mathrm{~Hz}, 1 \mathrm{H}), 2.80-2.66(\mathrm{~m}$, $J=7.1 \mathrm{~Hz}, 2 \mathrm{H}), 1.28(\mathrm{dd}, J=15.4,7.1 \mathrm{~Hz}, 6 \mathrm{H}), 1.00(\mathrm{dd}, J=16.6,7.1 \mathrm{~Hz}, 6 \mathrm{H})$.

${ }^{13} \mathrm{C}$ NMR (100 MHz, CDCl $\left.{ }_{3}\right) \delta 136.3(\mathrm{~d}, J=72.1 \mathrm{~Hz}), 133.8$ (d, $\left.J=2.0 \mathrm{~Hz}\right), 132.9$ $(\mathrm{d}, J=8.2 \mathrm{~Hz}), 132.6(\mathrm{~d}, J=8.8 \mathrm{~Hz}), 129.3,129.0(\mathrm{~d}, J=4.8 \mathrm{~Hz}), 128.9,128.7$, 125.7, $117.3(\mathrm{~d}, J=4.6 \mathrm{~Hz}), 111.2(\mathrm{~d}, J=5.1 \mathrm{~Hz}), 26.7(\mathrm{~d}, J=66.0 \mathrm{~Hz}), 16.7(\mathrm{~d}, J=$ $3.0 \mathrm{~Hz}), 15.8(\mathrm{~d}, J=3.7 \mathrm{~Hz})$.

31P NMR (162 MHz, $\left.\mathrm{CDCl}_{3}\right) \delta 52.4$.

HRMS (ESI-TOF) calcd for $\mathrm{C}_{17} \mathrm{H}_{21} \mathrm{NOP}^{+}[\mathrm{M}+\mathrm{H}]^{+}:$: 286.1356, found: 286.1355 .

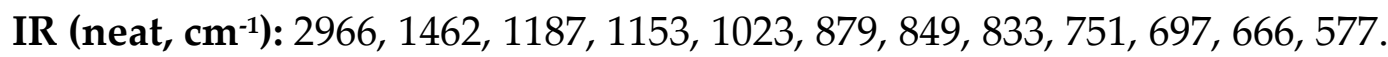

\section{Dicyclohexyl(1-isocyanonaphthalen-2-yl)phosphane (2al)}<smiles>N#Cc1c(P(C2CCCCC2)C2CCCCC2)ccc2ccccc12</smiles>

2al was prepared as a yellow oil in $69 \%$ yield $(0.24 \mathrm{~g}$, eluent: petroleum ether $/$ EtOAc $=80: 1)$ from $1 \mathbf{w}(0.18 \mathrm{~g}, 1.0 \mathrm{mmol}), \mathrm{HPCy}_{2}(0.22 \mathrm{~g}, 1.1 \mathrm{mmol})$ and KHMDS (1.0 M in THF, $1.5 \mathrm{~mL}, 1.5 \mathrm{mmol})$ instead of $\mathrm{KO}^{t} \mathrm{Bu}$ following the general procedure $\mathrm{B}$.

$\mathbf{R}_{f}=0.52$ (petroleum ether $/ \mathrm{EtOAc}=20 / 1$ ) 
${ }^{1} \mathrm{H}$ NMR (400 MHz, $\left.\mathrm{CDCl}_{3}\right) \delta 8.33(\mathrm{~d}, J=8.4 \mathrm{~Hz}, 1 \mathrm{H}), 8.00(\mathrm{~d}, J=8.5 \mathrm{~Hz}, 1 \mathrm{H})$, $7.91(\mathrm{~d}, J=8.1 \mathrm{~Hz}, 1 \mathrm{H}), 7.70(\mathrm{t}, J=7.7 \mathrm{~Hz}, 1 \mathrm{H}), 7.63(\mathrm{~d}, J=8.1 \mathrm{~Hz}, 2 \mathrm{H}), 2.14-$ $1.96(\mathrm{~m}, 4 \mathrm{H}), 1.80(\mathrm{~d}, J=13.1 \mathrm{~Hz}, 2 \mathrm{H}), 1.70-1.60(\mathrm{~m}, 2 \mathrm{H}), 1.55(\mathrm{~d}, J=13.3 \mathrm{~Hz}$, 2H), $1.37-1.04(\mathrm{~m}, 12 \mathrm{H})$.

${ }^{13} \mathrm{C}$ NMR (100 MHz, $\left.\mathrm{CDCl}_{3}\right) \delta 141.9$ (d, J=27.5 Hz), 133.2 (d, J= $\left.7.3 \mathrm{~Hz}\right), 132.8$, 131.2, 128.6, 128.4, 128.3, 127.9, 125.7, 119.5 (d, $J=35.6 \mathrm{~Hz}), 117.3$ (d, J=4.5 Hz), $33.7(\mathrm{~d}, J=13.7 \mathrm{~Hz}), 30.2(\mathrm{~d}, J=17.3 \mathrm{~Hz}), 29.1(\mathrm{~d}, J=7.4 \mathrm{~Hz}), 27.1$ (d, $J=12.7$ $\mathrm{Hz}), 26.9(\mathrm{~d}, J=7.4 \mathrm{~Hz}), 26.2$.

${ }^{31 P}$ NMR (162 MHz, $\left.\mathrm{CDCl}_{3}\right) \delta 0.0$.

HRMS (ESI-TOF) calcd for $\mathrm{C}_{23} \mathrm{H}_{29} \mathrm{NP}^{+}[\mathrm{M}+\mathrm{H}]^{+}$: 350.2032, found: 350.2031.

IR (neat, cm-1): 2924, 2851, 1448, 1263, 1082, 1021, 820, 801, 782, 736, 696, 453.

\section{Compound 2am}

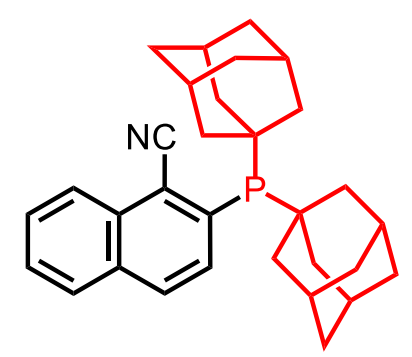

2am was prepared as a yellow solid in $53 \%$ yield $(0.48 \mathrm{~g}$, eluent: petroleum ether $/$ EtOAc $=80: 1)$ from $1 w(0.37 \mathrm{~g}, 2.0 \mathrm{mmol}), \mathrm{HPAd}_{2}(0.67 \mathrm{~g}, 2.2 \mathrm{mmol})$ and KHMDS (1.0 M in THF, $3.0 \mathrm{~mL}, 3.0 \mathrm{mmol})$ instead of $\mathrm{KO}^{t} \mathrm{Bu}$ following the general procedure $B$.

$\mathbf{R}_{f}=0.60$ (petroleum ether/EtOAc $=20 / 1$ )

${ }^{1} \mathrm{H}$ NMR (400 MHz, $\left.\mathrm{CDCl}_{3}\right) \delta 8.36(\mathrm{~d}, J=8.3 \mathrm{~Hz}, 1 \mathrm{H}), 7.98(\mathrm{~s}, 2 \mathrm{H}), 7.93(\mathrm{~d}, J=$ $8.2 \mathrm{~Hz}, 1 \mathrm{H}), 7.72-7.60(\mathrm{~m}, 2 \mathrm{H}), 2.12-2.02(\mathrm{~m}, 6 \mathrm{H}), 2.00-1.88(\mathrm{~m}, 12 \mathrm{H}), 1.71-$ $1.62(\mathrm{~m}, 12 \mathrm{H})$.

${ }^{13} \mathrm{C}$ NMR (100 MHz, $\left.\mathrm{CDCl}_{3}\right) \delta 141.7$ (d, J = 32.1 Hz), 133.4 (d, J = 9.2 Hz), 132.8, $131.6(\mathrm{~d}, J=2.6 \mathrm{~Hz}), 129.9,128.5,128.3,128.0,126.1,121.6(\mathrm{~d}, J=42.0 \mathrm{~Hz}), 117.7$ $(\mathrm{d}, J=5.0 \mathrm{~Hz}), 41.8(\mathrm{~d}, J=12.4 \mathrm{~Hz}), 37.8(\mathrm{~d}, J=23.8 \mathrm{~Hz}), 36.9,28.9(\mathrm{~d}, J=8.7 \mathrm{~Hz})$. ${ }^{31} \mathrm{P}$ NMR (162 MHz, $\left.\mathrm{CDCl}_{3}\right) \delta 34.0$.

HRMS (ESI-TOF) calcd for $\mathrm{C}_{31} \mathrm{H}_{37} \mathrm{NP}^{+}[\mathrm{M}+\mathrm{H}]^{+}:$454.2658, found: 454.2659.

IR (neat, cm-1): 2899, 2847, 1448, 1343, 1301, 1264, 824, 734, 695, 667, 454. 
<smiles>c1ccc(P(c2ccccc2)c2ccccn2)cc1</smiles>

4a was prepared as a white solid in $93 \%$ yield $(48.9 \mathrm{mg}$, eluent: petroleum ether $/ \mathrm{EtOAc}=30: 1$ to 20:1) from 3a $(34.2 \mathrm{mg}, 0.2 \mathrm{mmol})$, HPPh2 (74.5 mg, 0.4 $\mathrm{mmol})$ and $\mathrm{KO}^{t} \mathrm{Bu}(44.9 \mathrm{mg}, 0.4 \mathrm{mmol})$ following the general procedure $\mathrm{C}$.

$\mathbf{R}_{f}=0.20$ (petroleum ether $/$ EtOAc $=10 / 1$ )

${ }^{1} \mathrm{H}$ NMR (400 MHz, $\left.\mathrm{CDCl}_{3}\right) \delta 8.75$ - $8.70(\mathrm{~m}, 1 \mathrm{H}), 7.58-7.52(\mathrm{~m}, 1 \mathrm{H}), 7.44-$ $7.33(\mathrm{~m}, 10 \mathrm{H}), 7.20-7.14(\mathrm{~m}, 1 \mathrm{H}), 7.11-7.06(\mathrm{~m}, 1 \mathrm{H})$.

${ }^{13} \mathrm{C}$ NMR (100 MHz, $\left.\mathrm{CDCl}_{3}\right) \delta 164.1$ (d, J = 4.1 Hz), 150.4 (d, J = 12.7 Hz), 136.3 $(\mathrm{d}, J=10.7 \mathrm{~Hz}), 135.9$ (d, $J=2.2 \mathrm{~Hz}), 134.3(\mathrm{~d}, J=19.7 \mathrm{~Hz}), 129.2,128.7$ (d, J = 7.2 $\mathrm{Hz}), 127.9(\mathrm{~d}, J=15.5 \mathrm{~Hz}), 122.3$.

${ }^{31} \mathrm{P}$ NMR (162 MHz, $\left.\mathrm{CDCl}_{3}\right) \delta-4.0$.

The characterization data are consistent with the literature reported data. ${ }^{13}$

2-(Bis(4-(tert-butyl)phenyl)phosphaneyl)pyridine (4b)<smiles>CC(C)(C)c1ccc(P(c2ccc(Br)cc2)c2ccccn2)cc1</smiles>

4b was prepared as a white solid in $88 \%$ yield $(66.1 \mathrm{mg}$, eluent: petroleum ether $/ \mathrm{EtOAc}=30: 1$ to 20:1) from 3a $(34.2 \mathrm{mg}, 0.2 \mathrm{mmol}), \mathrm{HP}\left(4-{ }^{-} \mathrm{BuC}_{6} \mathrm{H}_{4}\right)_{2}$ (119.4 mg, $0.4 \mathrm{mmol})$ and $\mathrm{KO}^{\mathrm{t}} \mathrm{Bu}(44.9 \mathrm{mg}, 0.4 \mathrm{mmol})$ following the general procedure $\mathrm{C}$.

$\mathbf{R}_{f}=0.25$ (petroleum ether $/$ EtOAc $=10 / 1$ )

${ }^{1}$ H NMR (400 MHz, $\left.\mathrm{CDCl}_{3}\right) \delta 8.73$ - $8.68(\mathrm{~m}, 1 \mathrm{H}), 7.57$ - $7.51(\mathrm{~m}, 1 \mathrm{H}), 7.40$ $7.30(\mathrm{~m}, 8 \mathrm{H}), 7.16-7.12(\mathrm{~m}, 1 \mathrm{H}), 7.12-7.08(\mathrm{~m}, 1 \mathrm{H}), 1.31(\mathrm{~s}, 18 \mathrm{H})$.

${ }^{13} \mathrm{C}$ NMR (100 MHz, $\left.\mathrm{CDCl}_{3}\right) \delta 164.9$ (d, J = 4.3 Hz), 152.2, $150.4(\mathrm{~d}, J=12.5 \mathrm{~Hz})$, $135.7(\mathrm{~d}, J=2.2 \mathrm{~Hz}), 134.2(\mathrm{~d}, J=20.0 \mathrm{~Hz}), 132.9$ (d, $J=9.0 \mathrm{~Hz}), 127.8$ (d, $J=15.3$ $\mathrm{Hz}), 125.8(\mathrm{~d}, J=7.4 \mathrm{~Hz}), 122.0,34.8,31.4$.

${ }^{31} \mathrm{P}$ NMR (162 $\left.\mathbf{M H z}, \mathrm{CDCl}_{3}\right) \delta-6.1$. 
HRMS (ESI-TOF) calcd for $\mathrm{C}_{25} \mathrm{H}_{31} \mathrm{NP}^{+}[\mathrm{M}+\mathrm{H}]^{+}:$376.2189, found: 376.2191 .

IR (neat, cm$^{-1}$ ): 2960, 1571, 1446, 1419, 1267, 1083, 824, 764, 744, 558.

\section{4,4'-(Pyridin-2-ylphosphanediyl)bis( $N, N$-dimethylaniline) (4c)}<smiles>CN(C)c1ccc(P(c2ccc(N(C)C)cc2)c2ccccn2)cc1</smiles>

4c was prepared as a yellow solid in $92 \%$ yield $(64.3 \mathrm{mg}$, eluent: petroleum ether $/ \mathrm{EtOAc}=5: 1)$ from $3 \mathrm{a}(34.2 \mathrm{mg}, 0.2 \mathrm{mmol}), \mathrm{HP}\left(4-\mathrm{NMe}_{2} \mathrm{C}_{6} \mathrm{H}_{4}\right)_{2}(108.9 \mathrm{mg}$, $0.4 \mathrm{mmol})$ and $\mathrm{KO}^{t} \mathrm{Bu}(44.9 \mathrm{mg}, 0.4 \mathrm{mmol})$ following the general procedure $\mathrm{C}$. $\mathbf{R}_{f}=0.25$ (petroleum ether $/$ EtOAc $=2 / 1$ )

${ }^{1} \mathrm{H}$ NMR (400 MHz, $\left.\mathrm{CDCl}_{3}\right) \delta 8.71-8.63(\mathrm{~m}, 1 \mathrm{H}), 7.53-7.46(\mathrm{~m}, 1 \mathrm{H}), 7.34-$ $7.27(\mathrm{~m}, 4 \mathrm{H}), 7.13-7.04(\mathrm{~m}, 2 \mathrm{H}), 6.74-6.67(\mathrm{~m}, 4 \mathrm{H}), 2.95(\mathrm{~s}, 12 \mathrm{H})$.

${ }^{13} \mathrm{C}$ NMR (100 MHz, $\left.\mathrm{CDCl}_{3}\right) \delta 166.7$ (d, J=3.9 Hz), 150.9, 150.0 (d, J=11.8 Hz), 135.5, 135.3, $127.2(\mathrm{~d}, J=14.6 \mathrm{~Hz}), 122.0(\mathrm{~d}, J=4.5 \mathrm{~Hz}), 121.4,112.4(\mathrm{~d}, J=8.2$ $\mathrm{Hz}), 40.3$.

${ }^{31} \mathrm{P}$ NMR (162 $\left.\mathrm{MHz}, \mathrm{CDCl}_{3}\right) \delta-7.1$.

HRMS (ESI-TOF) calcd for $\mathrm{C}_{21} \mathrm{H}_{25} \mathrm{~N}_{3} \mathrm{P}^{+}[\mathrm{M}+\mathrm{H}]^{+}$: 350.1781, found: 350.1778 .

IR (neat, cm ${ }^{-1}$ ): 1594, 1570, 1508, 1445, 1354, 1199, 1098, 809, 762, 529.

\section{2-(Bis(3,5-di-tert-butylphenyl)phosphaneyl)pyridine (4d)}<smiles>CC(C)(C)c1cc(P(c2cc(C(C)(C)C)cc(C(C)(C)C)c2)c2ccccn2)cc(C(C)(C)C)c1</smiles>

4d was prepared as a white solid in $86 \%$ yield $(84.0 \mathrm{mg}$, eluent: petroleum ether $/ \mathrm{EtOAc}=30: 1$ to 20:1) from $3 \mathbf{a}(34.2 \mathrm{mg}, 0.2 \mathrm{mmol}), \mathrm{HP}\left(3,5-{ }^{-} \mathrm{BuC}_{6} \mathrm{H}_{3}\right)_{2}$ (164.2 mg, $0.4 \mathrm{mmol})$ and $\mathrm{KO}^{t} \mathrm{Bu}(44.9 \mathrm{mg}, 0.4 \mathrm{mmol})$ following the general procedure $\mathrm{C}$.

$\mathbf{R}_{f}=0.30$ (petroleum ether $/$ EtOAc $=10 / 1$ ) 
${ }^{1} \mathbf{H}$ NMR (400 MHz, $\left.\mathrm{CDCl}_{3}\right) \delta 8.73-8.68(\mathrm{~m}, 1 \mathrm{H}), 7.57-7.51(\mathrm{~m}, 1 \mathrm{H}), 7.40(\mathrm{t}, J$ $=1.9 \mathrm{~Hz}, 2 \mathrm{H}), 7.22(\mathrm{dd}, J=8.4,1.9 \mathrm{~Hz}, 4 \mathrm{H}), 7.16-7.11(\mathrm{~m}, 1 \mathrm{H}), 7.07-7.03(\mathrm{~m}$, $1 \mathrm{H}), 1.25(\mathrm{~s}, 36 \mathrm{H})$.

${ }^{13} \mathrm{C}$ NMR (100 MHz, $\left.\mathrm{CDCl}_{3}\right) \delta 165.8$ (d, $\left.J=4.3 \mathrm{~Hz}\right), 150.7$ (d, $\left.J=7.1 \mathrm{~Hz}\right), 150.2$ $(\mathrm{d}, J=13.2 \mathrm{~Hz}), 135.5,135.2(\mathrm{~d}, J=9.1 \mathrm{~Hz}), 128.7(\mathrm{~d}, J=20.2 \mathrm{~Hz}), 127.4(\mathrm{~d}, J=$ $12.5 \mathrm{~Hz}), 123.1,121.9,35.0,31.5$.

${ }^{31 P}$ NMR (162 MHz, $\left.\mathrm{CDCl}_{3}\right) \delta-1.3$.

HRMS (ESI-TOF) calcd for $\mathrm{C}_{33} \mathrm{H}_{47} \mathrm{NP}^{+}[\mathrm{M}+\mathrm{H}]^{+}: 488.3441$, found: 488.3448 .

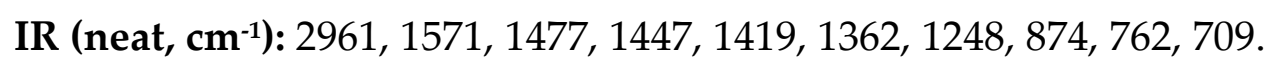

\section{4-(Diphenylphosphaneyl)pyridine (4e)}<smiles>c1ccc(P(c2ccccc2)c2ccncc2)cc1</smiles>

4e was prepared as a white solid in $93 \%$ yield $(48.9 \mathrm{mg}$, eluent: petroleum ether $/ \mathrm{EtOAc}=10: 1)$ from $3 \mathbf{b}(34.2 \mathrm{mg}, 0.2 \mathrm{mmol}), \mathrm{HPPh}_{2}(74.5 \mathrm{mg}, 0.4 \mathrm{mmol})$ and $\mathrm{KO}^{t} \mathrm{Bu}(44.9 \mathrm{mg}, 0.4 \mathrm{mmol})$ following the general procedure $\mathrm{C}$.

$\mathbf{R}_{f}=0.20$ (petroleum ether $/$ EtOAc $=5 / 1$ )

${ }^{1} \mathbf{H}$ NMR (400 MHz, $\left.\mathrm{CDCl}_{3}\right) \delta 8.54-8.48(\mathrm{~m}, 2 \mathrm{H}), 7.43-7.31$ (m, 10H), 7.14 $7.07(\mathrm{~m}, 2 \mathrm{H})$.

${ }^{13} \mathrm{C}$ NMR (100 MHz, $\left.\mathrm{CDCl}_{3}\right) \delta 149.3$ (d, J = 4.3 Hz), $149.0(\mathrm{~d}, J=17.9 \mathrm{~Hz}), 135.0$ $(\mathrm{d}, J=10.1 \mathrm{~Hz}), 134.3(\mathrm{~d}, J=20.5 \mathrm{~Hz}), 129.6,128.9(\mathrm{~d}, J=7.6 \mathrm{~Hz}), 127.3(\mathrm{~d}, J=$ $15.1 \mathrm{~Hz})$.

31P NMR (162 MHz, $\left.\mathrm{CDCl}_{3}\right) \delta-7.0$.

The characterization data are consistent with the literature reported data. ${ }^{14}$

\section{4-(Bis(4-(tert-butyl)phenyl)phosphaneyl)pyridine (4f)}<smiles>CC(C)(C)c1ccc(P(c2ccncc2)c2ccc(Br)cc2)cc1</smiles> 
4f was prepared as a white solid in $81 \%$ yield $(61.1 \mathrm{mg}$, eluent: petroleum ether $/ \mathrm{EtOAc}=10: 1)$ from $3 \mathbf{b}(34.2 \mathrm{mg}, 0.2 \mathrm{mmol}), \mathrm{HP}\left(4-{ }^{-} \mathrm{BuC}_{6} \mathrm{H}_{4}\right)_{2}(119.4 \mathrm{mg}$, $0.4 \mathrm{mmol})$ and $\mathrm{KO}^{t} \mathrm{Bu}(44.9 \mathrm{mg}, 0.4 \mathrm{mmol})$ following the general procedure $\mathrm{C}$. $\mathbf{R}_{f}=0.25$ (petroleum ether $/$ EtOAc $=5 / 1$ )

${ }^{1} \mathrm{H}$ NMR (400 MHz, $\left.\mathrm{CDCl}_{3}\right) \delta 8.51$ - $8.46(\mathrm{~m}, 2 \mathrm{H}), 7.42-7.37$ (m, 4H), $7.34-$ $7.27(\mathrm{~m}, 4 \mathrm{H}), 7.14-7.08(\mathrm{~m}, 2 \mathrm{H}), 1.32(\mathrm{~s}, 18 \mathrm{H})$.

${ }^{13} \mathrm{C}$ NMR (100 MHz, $\left.\mathrm{CDCl}_{3}\right) \delta$ 152.8, $150.0(\mathrm{~d}, J=17.5 \mathrm{~Hz}), 149.2(\mathrm{~d}, J=4.1 \mathrm{~Hz})$, $134.1(\mathrm{~d}, J=20.6 \mathrm{~Hz}), 131.6(\mathrm{~d}, J=8.3 \mathrm{~Hz}), 127.2(\mathrm{~d}, J=14.8 \mathrm{~Hz}), 125.9$ (d, J = 7.8 $\mathrm{Hz}), 34.8,31.3$.

${ }^{31} \mathrm{P}$ NMR (162 $\left.\mathrm{MHz}, \mathrm{CDCl}_{3}\right) \delta-9.3$.

HRMS (ESI-TOF) calcd for $\mathrm{C}_{25} \mathrm{H}_{31} \mathrm{NP}^{+}[\mathrm{M}+\mathrm{H}]^{+}: 376.2189$, found: 376.2184 .

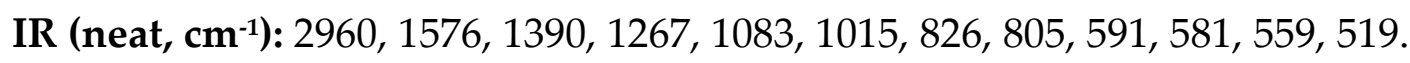




\section{Applications}

\section{Gram-scale reaction.}<smiles>CCOc1ccccc1C#N</smiles>

$1 \mathrm{a}(80 \mathrm{mmol})$

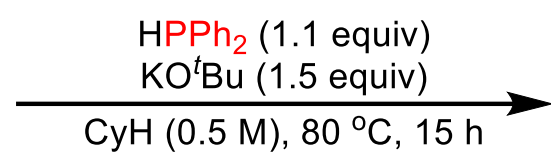

$\mathrm{CyH}(0.5 \mathrm{M}), 80^{\circ} \mathrm{C}, 15 \mathrm{~h}$<smiles>N#Cc1ccccc1P</smiles>

2a, $19.3 \mathrm{~g}, 84 \%$

In a glovebox, to an oven-dried sealed tube were added $\mathrm{KO}^{t} \mathrm{Bu}(13.5 \mathrm{~g}, 120.0$ mmol, 1.5 equiv), HPPh2 (15.3 mL, $16.4 \mathrm{~g}$, $88.0 \mathrm{mmol}, 1.1$ equiv), 2ethoxybenzonitrile 1a $(11.8 \mathrm{~g}, 80 \mathrm{mmol})$ and cyclohexane $(160 \mathrm{~mL})$ at room temperature, and the tube was then removed from the glovebox. The resulting mixture was stirred at $80{ }^{\circ} \mathrm{C}$ in an oil bath for $15 \mathrm{~h}$. After cooling to room temperature, the reaction mixture was diluted with DCM, filtered through a plug of silica gel, and concentrated in vacuo. The resulting crude mixture was purified by recrystallization ( $n$-hexane/DCM) to afford 2a (19.3 g, $84 \%)$.

\section{Synthesis of chiral ligands.}

\section{General procedure D}

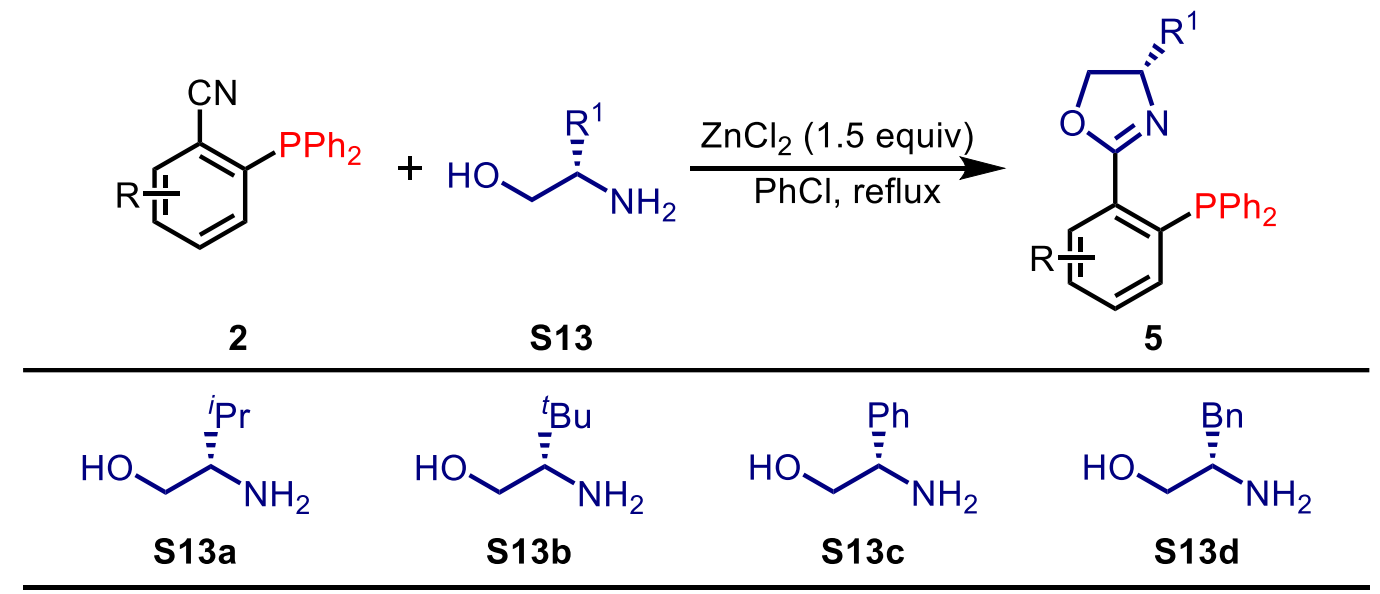

A mixture of 2-(diphenylphosphaneyl)benzonitrile 2 (1.0 mmol), 2aminoethanol S13 (1.5 mmol, 1.5 equiv), and $\mathrm{ZnCl}_{2}(204 \mathrm{mg}, 1.5 \mathrm{mmol}, 1.5$ equiv) in chlorobenzene $(8 \mathrm{~mL})$ was stirred under reflux in an oil bath for $6 \mathrm{~d}$. After cooling to room temperature, 2,2'-bipyridine (234 mg, $1.5 \mathrm{mmol}, 1.5$ equiv) and chloroform were added to the reaction mixture, and the resulting mixture was stirred for $1.5 \mathrm{~h}$. The suspension was filtered through a plug of silica gel and washed with chloroform. After removal of solvents under 
reduced pressure, the residue was purified by silica gel flash chromatography to give the PHOX ligand 5.

\section{General procedure E}

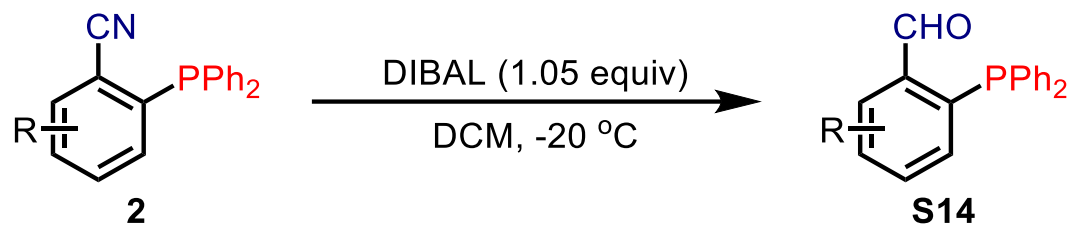

To a solution of 2-(diphenylphosphaneyl)benzonitrile $2(5.0 \mathrm{mmol})$ in DCM (25 mL) was added DIBAL-H (5.25 mL, 1.0 M in hexane, $5.25 \mathrm{mmol}, 1.05$ equiv) dropwise at $-20{ }^{\circ} \mathrm{C}$ under nitrogen. After stirring for $4 \mathrm{~h}$ at $-20{ }^{\circ} \mathrm{C}$, the resulting solution was quenched carefully with $\mathrm{MeOH}$. DCM and potassium sodium tartrate aqueous were added to the reaction mixture, and the resulting mixture was stirred vigorously for $2 \mathrm{~h}$. The aqueous layer was washed with DCM. The organic extract was dried over $\mathrm{MgSO}_{4}$, filtered, and concentrated in vacuo. The resulting crude mixture was purified by silica gel column chromatography to afford 2-(diphenylphosphaneyl)-benzaldehyde S14.

\section{General procedure F}

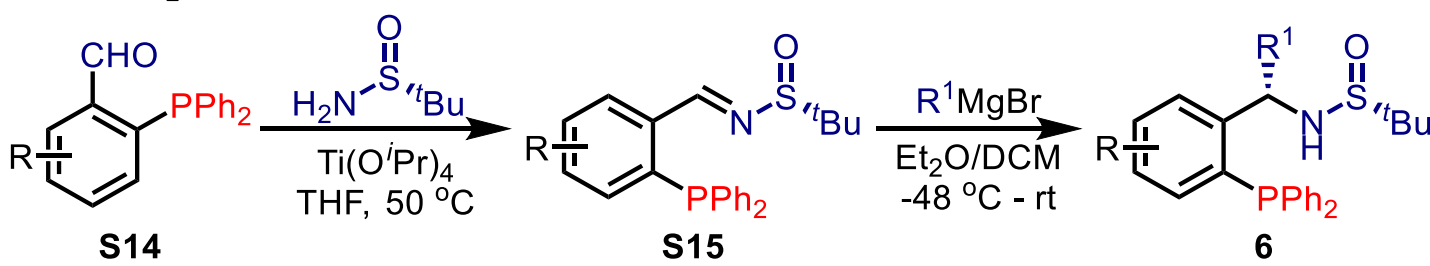

To a solution of 2-(diphenylphosphaneyl)-benzaldehyde S14 (0.5 mmol) and $(R)-(+)-2$-methyl-2-propanesulfinamide $(0.75 \mathrm{mmol}, 1.5$ equiv) in THF (2 $\mathrm{mL})$ was added $\mathrm{Ti}\left(\mathrm{O}^{i} \mathrm{Pr}\right)_{4}(1.0 \mathrm{mmol}, 2.0$ equiv), and the reaction solution was stirred at $50{ }^{\circ} \mathrm{C}$ in an oil bath overnight. After cooling to room temperature, EtOAc and brine were added to the reaction solution, and the resulting mixture was stirred vigorously. The resulting suspension was filtered through a plug of Celite and the filter cake was washed with EtOAc. The organic extract was dried over $\mathrm{MgSO}_{4}$, filtered, and concentrated in vacuo. The resulting crude mixture was purified by silica gel column chromatography to afford S15.

To a solution of S15 in DCM was added Grignard reagent (2.0 equiv) in 
$\mathrm{Et}_{2} \mathrm{O}$ at $-48{ }^{\circ} \mathrm{C}$. After stirring for $6 \mathrm{~h}$ at $-48{ }^{\circ} \mathrm{C}$, the resulting solution was warmed to room temperature with stirring overnight. The reaction mixture was quenched by $\mathrm{NH}_{4} \mathrm{Cl}$ aq. The aqueous layer was extracted with EtOAc. The combined organic layers were dried over $\mathrm{Na}_{2} \mathrm{SO}_{4}$, filtered, concentrated. The crude mixture was purified by flash chromatography to afford 6 .

\section{General procedure G}
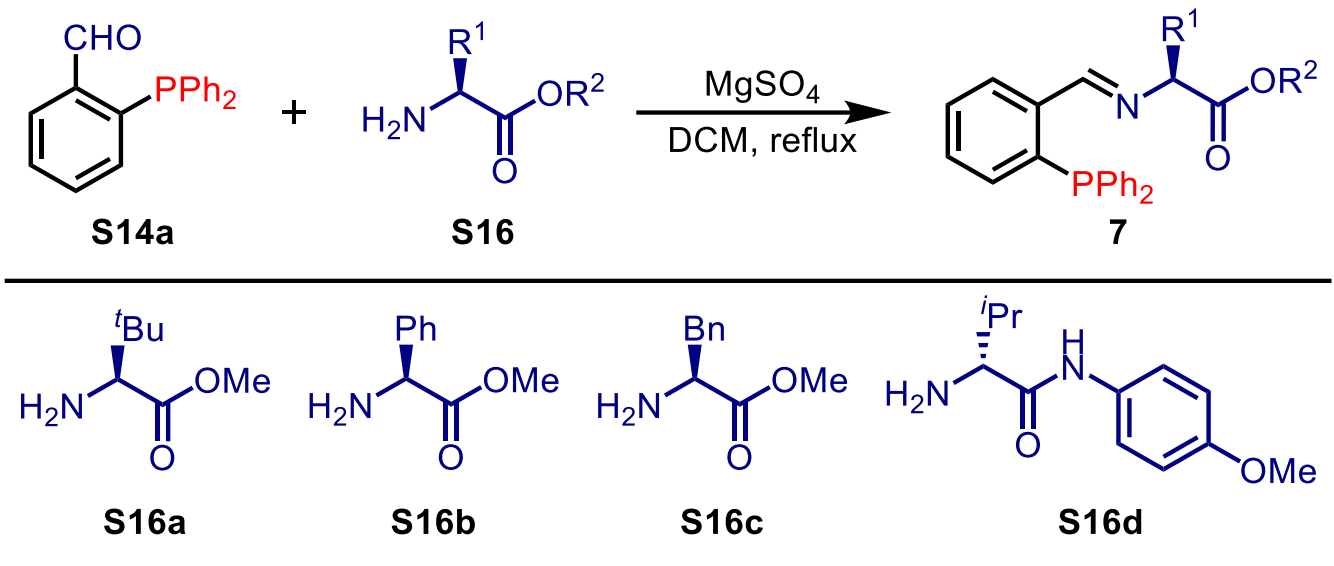

A mixture of 2-(diphenylphosphaneyl)-benzaldehyde S14a (0.5 mmol), primary amine S16 (0.6 mmol, 1.2 equiv) and $\mathrm{MgSO}_{4}(96 \mathrm{mg}, 0.8 \mathrm{mmol}, 1.6$ equiv) in DCM (2 mL) was stirred under reflux in an oil bath overnight. After cooling to room temperature, the suspension was filtered through a plug of Celite, and the filter cake was washed with DCM. After removal of solvents under reduced pressure, the residue was purified by silica gel flash chromatography (PE/EA, 3\% Et $3 \mathrm{~N})$ to afford 7.

(S)-2-(2-(Diphenylphosphaneyl)phenyl)-4-isopropyl-4,5-dihydrooxazole (5a)

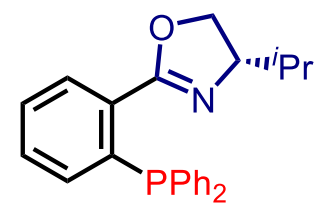

5a was prepared as a white solid in $65 \%$ yield (242 $\mathrm{mg}$, eluent: petroleum ether $/ \mathrm{DCM}=2: 1$ to petroleum ether $/$ EtOAc $=5: 1)$ from $2 \mathrm{a}(287 \mathrm{mg}, 1.0 \mathrm{mmol})$, S13a (155 mg, $1.5 \mathrm{mmol})$ following the general procedure D.

$\mathbf{R}_{f}=0.49$ (petroleum ether/DCM = 2/1)

${ }^{1} \mathrm{H}$ NMR (400 MHz, $\left.\mathrm{CDCl}_{3}\right) \delta 7.95(\mathrm{~d}, J=7.5 \mathrm{~Hz}, 1 \mathrm{H}), 7.40-7.22(\mathrm{~m}, 12 \mathrm{H}), 6.92$ $(\mathrm{d}, J=7.5 \mathrm{~Hz}, 1 \mathrm{H}), 4.22-4.10(\mathrm{~m}, 1 \mathrm{H}), 3.96-3.81(\mathrm{~m}, 2 \mathrm{H}), 1.59-1.45(\mathrm{~m}, 1 \mathrm{H})$, $0.85(\mathrm{~d}, J=6.7 \mathrm{~Hz}, 3 \mathrm{H}), 0.75(\mathrm{~d}, J=6.7 \mathrm{~Hz}, 3 \mathrm{H})$. 
${ }^{13} \mathrm{C}$ NMR (100 MHz, $\left.\mathrm{CDCl}_{3}\right) \delta$ 162.7, $138.8(\mathrm{~d}, J=25.8 \mathrm{~Hz}), 138.3(\mathrm{~d}, J=12.1$ $\mathrm{Hz}), 138.1(\mathrm{~d}, J=10.2 \mathrm{~Hz}), 134.2(\mathrm{~d}, J=21.1 \mathrm{~Hz}), 133.8,133.6(\mathrm{~d}, J=20.5 \mathrm{~Hz})$, $131.8(\mathrm{~d}, J=19.2 \mathrm{~Hz}), 130.2,129.7,128.4(\mathrm{~d}, J=11.6 \mathrm{~Hz}), 128.25,128.20,128.1$, $127.9,73.1,69.9,32.7,18.8,18.4$.

31P NMR (162 $\left.\mathrm{MHz} \mathrm{CDCl}_{3}\right) \delta$-5.5.

The characterization data are consistent with the literature reported data. ${ }^{15}$ (S)-4-(tert-Butyl)-2-(2-(diphenylphosphaneyl)phenyl)-4,5-dihydrooxazole (5b)<smiles>CCC(C)[C@H]1COC(c2ccccc2P)=N1</smiles>

5b was prepared as a white solid in $73 \%$ yield $(281 \mathrm{mg}$, eluent: petroleum ether $/ \mathrm{DCM}=2: 1$ to petroleum ether $/ \mathrm{EtOAc}=5: 1)$ from $2 \mathrm{a}(287 \mathrm{mg}, 1.0 \mathrm{mmol})$, $\mathrm{S} 13 \mathbf{b}$ (176 mg, $1.5 \mathrm{mmol})$ following the general procedure D.

$\mathbf{R}_{f}=0.52$ (petroleum ether/DCM = 2/1)

${ }^{1} \mathrm{H}$ NMR (400 MHz, $\left.\mathrm{CDCl}_{3}\right) \delta 7.99$ (d, J = 7.0 Hz, 1H), $7.40-7.26(\mathrm{~m}, 12 \mathrm{H}), 6.95$ - $6.90(\mathrm{~m}, 1 \mathrm{H}), 4.16-4.08(\mathrm{~m}, 1 \mathrm{H}), 4.08-4.00(\mathrm{~m}, 1 \mathrm{H}), 3.96-3.89(\mathrm{~m}, 1 \mathrm{H}), 0.78$ (s, 9H).

${ }^{13} \mathrm{C}$ NMR (100 MHz, $\left.\mathrm{CDCl}_{3}\right) \delta 162.6$ (d, J = 2.8 Hz), 138.9 (d, $\left.J=25.7 \mathrm{~Hz}\right), 138.6$ $(\mathrm{d}, J=12.5 \mathrm{~Hz}), 138.4(\mathrm{~d}, J=10.0 \mathrm{~Hz}), 134.3(\mathrm{~d}, J=21.0 \mathrm{~Hz}), 134.1,133.6(\mathrm{~d}, J=$ $20.2 \mathrm{~Hz}), 132.0(\mathrm{~d}, J=20.1 \mathrm{~Hz}), 130.3,129.8$ (d, $J=3.0 \mathrm{~Hz}), 128.5(\mathrm{~d}, J=7.9 \mathrm{~Hz})$, $128.3(\mathrm{~d}, J=5.0 \mathrm{~Hz}), 128.2,128.0,76.8,68.3,33.6,25.8$.

${ }^{31} \mathrm{P}$ NMR (162 $\left.\mathbf{M H z}, \mathrm{CDCl}_{3}\right) \delta-6.0$.

The characterization data are consistent with the literature reported data. ${ }^{16}$

(S)-2-(2-(Diphenylphosphaneyl)phenyl)-4-phenyl-4,5-dihydrooxazole (5c)<smiles>Pc1cccc(C2=N[C@H](c3ccccc3)CO2)c1P</smiles>

5c was prepared as a yellow oil in 54\% yield (218 mg, eluent: petroleum ether $/ \mathrm{DCM}=2: 1$ to petroleum ether $/$ EtOAc $=5: 1)$ from $2 \mathrm{a}(287 \mathrm{mg}, 1.0 \mathrm{mmol}), \mathbf{S 1 3 c}$ (206 $\mathrm{mg}, 1.5 \mathrm{mmol}$ ) following the general procedure D.

$\mathbf{R}_{f}=0.45$ (petroleum ether/DCM $=2 / 1$ )

${ }^{1} \mathrm{H}$ NMR (400 MHz, $\left.\mathrm{CDCl}_{3}\right) \delta 8.12(\mathrm{dd}, J=8.0,3.5 \mathrm{~Hz}, 1 \mathrm{H}), 7.49-7.35(\mathrm{~m}, 12 \mathrm{H})$, 
$7.32-7.25(\mathrm{~m}, 3 \mathrm{H}), 7.09-6.99(\mathrm{~m}, 3 \mathrm{H}), 5.33(\mathrm{t}, J=9.6 \mathrm{~Hz}, 1 \mathrm{H}), 4.63(\mathrm{t}, J=9.2 \mathrm{~Hz}$, $1 \mathrm{H}), 4.02(\mathrm{t}, J=8.7 \mathrm{~Hz}, 1 \mathrm{H})$.

${ }^{13} \mathrm{C}$ NMR (100 MHz, $\left.\mathrm{CDCl}_{3}\right) \delta 164.5$ (d, J = 2.7 Hz), 142.0, 139.1 (d, J=25.8 Hz), $138.0(\mathrm{~d}, J=12.5 \mathrm{~Hz}), 137.9(\mathrm{~d}, J=10.1 \mathrm{~Hz}), 134.3(\mathrm{~d}, J=21.1 \mathrm{~Hz}), 133.85(\mathrm{~d}, J=$ $20.2 \mathrm{~Hz}), 133.81(\mathrm{~d}, J=1.4 \mathrm{~Hz}), 131.4(\mathrm{~d}, J=19.3 \mathrm{~Hz}), 130.6,130.2(\mathrm{~d}, J=3.0 \mathrm{~Hz})$, $128.6(\mathrm{~d}, J=11.9 \mathrm{~Hz}), 128.5,128.45,128.38,128.35,128.0,127.1,126.6,74.3,70.1$. 31P NMR (162 MHz, $\left.\mathrm{CDCl}_{3}\right) \delta$-5.4.

The characterization data are consistent with the literature reported data. ${ }^{17}$

(S)-4-Benzyl-2-(2-(diphenylphosphaneyl)phenyl)-4,5-dihydrooxazole (5d)<smiles>Pc1cccc(C2=N[C@H](Cc3ccccc3)CO2)c1P</smiles>

$\mathbf{5 d}$ was prepared as a yellow oil in $65 \%$ yield $(274 \mathrm{mg}$, eluent: petroleum ether $/ \mathrm{DCM}=2: 1$ to petroleum ether $/$ EtOAc $=5: 1)$ from $2 \mathbf{a}(287 \mathrm{mg}, 1.0 \mathrm{mmol})$, S13d (227 $\mathrm{mg}, 1.5 \mathrm{mmol})$ following the general procedure D.

$\mathbf{R}_{f}=0.49$ (petroleum ether $/ \mathrm{DCM}=2 / 1$ )

${ }^{1} \mathbf{H}$ NMR (400 MHz, $\left.\mathrm{CDCl}_{3}\right) \delta 7.98(\mathrm{~d}, J=5.0 \mathrm{~Hz}, 1 \mathrm{H}), 7.51-7.34(\mathrm{~m}, 11 \mathrm{H}), 7.35$ $-7.27(\mathrm{~m}, 3 \mathrm{H}), 7.27-7.21(\mathrm{~m}, 1 \mathrm{H}), 7.19-7.13(\mathrm{~m}, 2 \mathrm{H}), 7.00(\mathrm{dd}, J=7.7,4.4 \mathrm{~Hz}$, $1 \mathrm{H}), 4.50-4.39(\mathrm{~m}, 1 \mathrm{H}), 4.10(\mathrm{t}, J=8.8 \mathrm{~Hz}, 1 \mathrm{H}), 3.86(\mathrm{t}, J=7.9 \mathrm{~Hz}, 1 \mathrm{H}), 3.02(\mathrm{dd}$, $J=13.9,5.2 \mathrm{~Hz}, 1 \mathrm{H}), 2.24(\mathrm{dd}, J=13.9,9.0 \mathrm{~Hz}, 1 \mathrm{H})$.

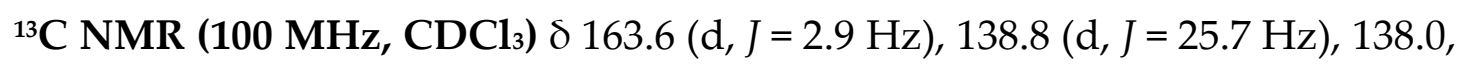
$137.9(\mathrm{~d}, J=11.5 \mathrm{~Hz}), 134.2(\mathrm{~d}, J=21.3 \mathrm{~Hz}), 133.7$ (d, $J=20.7 \mathrm{~Hz}), 133.5$ (d, $J=$ $2.2 \mathrm{~Hz}), 131.5(\mathrm{~d}, J=18.2 \mathrm{~Hz}), 130.3,129.8(\mathrm{~d}, J=2.6 \mathrm{~Hz}), 128.9,128.6,128.41$, $128.39,128.32,128.28,128.24,127.8,126.2,71.3,67.9,41.0$.

31P NMR (162 MHz, $\left.\mathrm{CDCl}_{3}\right) \delta-4.8$.

The characterization data are consistent with the literature reported data. ${ }^{18}$

(S)-4-(tert-Butyl)-2-(6-(diphenylphosphaneyl)-2,3-dihydro-1H-inden-5-yl)4,5-dihydrooxazole (5e)<smiles>CC(C)(C)[C@H]1COC(c2cc3c(cc2P)CCC3)=N1</smiles>

5e was prepared as a white solid in $77 \%$ yield $(328 \mathrm{mg}$, eluent: petroleum 
ether $/ \mathrm{DCM}=2: 1$ to petroleum ether $/ \mathrm{EtOAc}=5: 1)$ from $2 \mathbf{u}(327 \mathrm{mg}, 1.0 \mathrm{mmol})$, $\mathrm{S} 13 \mathrm{~b}$ (176 mg, $1.5 \mathrm{mmol})$ following the general procedure D.

$\mathbf{R}_{f}=0.50$ (petroleum ether/DCM $=2 / 1$ )

${ }^{1} \mathbf{H}$ NMR (400 MHz, $\left.\mathrm{CDCl}_{3}\right) \delta 7.83(\mathrm{~d}, J=3.5 \mathrm{~Hz}, 1 \mathrm{H}), 7.33-7.30(\mathrm{~m}, 5 \mathrm{H}), 7.30$ $-7.26(\mathrm{~m}, 5 \mathrm{H}), 6.75(\mathrm{~d}, J=4.0 \mathrm{~Hz}, 1 \mathrm{H}), 4.08(\mathrm{dd}, J=10.1,8.4 \mathrm{~Hz}, 1 \mathrm{H}), 4.01(\mathrm{t}, J=$ $8.3 \mathrm{~Hz}, 1 \mathrm{H}), 3.89$ (dd, J = 10.2, $8.0 \mathrm{~Hz}, 1 \mathrm{H}), 2.93(\mathrm{t}, J=7.4 \mathrm{~Hz}, 2 \mathrm{H}), 2.76(\mathrm{t}, J=7.4$ $\mathrm{Hz}, 2 \mathrm{H}), 2.08-2.00(\mathrm{~m}, 2 \mathrm{H}), 0.75(\mathrm{~s}, 9 \mathrm{H})$.

${ }^{13} \mathrm{C}$ NMR (100 MHz, $\left.\mathrm{CDCl}_{3}\right) \delta$ 163.4, 147.1, 144.7, 139.1 (d, J = $\left.13.0 \mathrm{~Hz}\right), 138.9$ $(\mathrm{d}, J=10.2 \mathrm{~Hz}), 136.2(\mathrm{~d}, J=24.2 \mathrm{~Hz}), 134.4(\mathrm{~d}, J=20.9 \mathrm{~Hz}), 133.7(\mathrm{~d}, J=20.1$ $\mathrm{Hz}), 130.3(\mathrm{~d}, J=21.3 \mathrm{~Hz}), 130.0,128.43,128.42,128.3(\mathrm{~d}, J=6.4 \mathrm{~Hz}), 128.2(\mathrm{~d}, J$ $=6.5 \mathrm{~Hz}), 126.0(\mathrm{~d}, J=4.0 \mathrm{~Hz}), 76.7,68.3,33.7,33.0,32.6,25.9,25.3$.

${ }^{31} \mathrm{P}$ NMR (162 $\left.\mathrm{MHz}, \mathrm{CDCl}_{3}\right) \delta-5.8$.

HRMS (ESI-TOF) calcd for $\mathrm{C}_{28} \mathrm{H}_{31} \mathrm{NOP}^{+}[\mathrm{M}+\mathrm{H}]^{+}$: 428.2138 , found: 428.2139 .

IR (neat, cm-1): 2951, 1652, 1477, 1433, 1353, 1084, 1027, 1001, 979, 742, 696.

\section{Compound $5 f$}

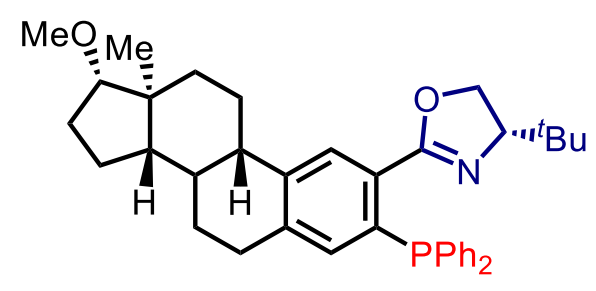

5f was prepared as a white solid in $61 \%$ yield $(580 \mathrm{mg}$, eluent: DCM to petroleum ether $/$ EtOAc $=1: 1)$ from $2 \mathbf{a d}(479 \mathrm{mg}, 1.0 \mathrm{mmol}), \mathbf{S 1 3 b}(176 \mathrm{mg}, 1.5$ $\mathrm{mmol}$ ) following the general procedure D.

$\mathbf{R}_{f}=0.50(\mathrm{DCM})$

${ }^{1} \mathrm{H}$ NMR (400 MHz, $\left.\mathrm{CDCl}_{3}\right) \delta 7.90(\mathrm{~d}, J=3.7 \mathrm{~Hz}, 1 \mathrm{H}), 7.34-7.26(\mathrm{~m}, 10 \mathrm{H}), 6.56$ $(\mathrm{d}, J=4.3 \mathrm{~Hz}, 1 \mathrm{H}), 4.10(\mathrm{dd}, J=10.1,8.3 \mathrm{~Hz}, 1 \mathrm{H}), 4.00(\mathrm{t}, J=8.3 \mathrm{~Hz}, 1 \mathrm{H}), 3.90$ $(\mathrm{dd}, J=10.0,8.3 \mathrm{~Hz}, 1 \mathrm{H}), 3.40(\mathrm{~s}, 3 \mathrm{H}), 3.34(\mathrm{t}, J=8.3 \mathrm{~Hz}, 1 \mathrm{H}), 2.74-2.57(\mathrm{~m}$, $2 \mathrm{H}), 2.47-2.38(\mathrm{~m}, 1 \mathrm{H}), 2.31-2.20(\mathrm{~m}, 1 \mathrm{H}), 2.14-2.04(\mathrm{~m}, 2 \mathrm{H}), 1.87-1.80(\mathrm{~m}$, $1 \mathrm{H}), 1.76-1.61(\mathrm{~m}, 2 \mathrm{H}), 1.60-1.50(\mathrm{~m}, 1 \mathrm{H}), 1.48-1.37(\mathrm{~m}, 2 \mathrm{H}), 1.36-1.18(\mathrm{~m}$, $3 \mathrm{H}), 0.83(\mathrm{~s}, 3 \mathrm{H}), 0.74(\mathrm{~s}, 9 \mathrm{H})$.

${ }^{13} \mathrm{C}$ NMR (100 MHz, $\left.\mathrm{CDCl}_{3}\right) \delta$ 163.0, 140.6, 139.4, 139.1 (d, $\left.J=12.8 \mathrm{~Hz}\right), 138.8$ $(\mathrm{d}, J=10.1 \mathrm{~Hz}), 135.3(\mathrm{~d}, J=24.0 \mathrm{~Hz}), 135.0,134.4(\mathrm{~d}, J=20.9 \mathrm{~Hz}), 133.7(\mathrm{~d}, J=$ $20.2 \mathrm{~Hz}), 129.2(\mathrm{~d}, J=19.3 \mathrm{~Hz}), 128.42,128.37,128.28(\mathrm{~d}, J=4.1 \mathrm{~Hz}), 128.18(\mathrm{~d}, J$ $=3.2 \mathrm{~Hz}), 127.1,90.8,76.8,68.2,58.0,50.5,44.3,43.3$, 38.2, 38.0, 33.7, 29.5, 27.8, 
27.0, 26.2, 25.9, 23.1, 11.6.

${ }^{31} \mathrm{P}$ NMR (162 $\left.\mathrm{MHz}, \mathrm{CDCl}_{3}\right) \delta-6.9$.

HRMS (ESI-TOF) calcd for $\mathrm{C}_{38} \mathrm{H}_{47} \mathrm{NO}_{2} \mathrm{P}^{+}[\mathrm{M}+\mathrm{H}]^{+}$: 428.2138 , found: 428.2139 .

IR (neat, cm-1): 2929, 2867, 1652, 1434, 1098, 986, 740, 696.

\section{2-(Diphenylphosphaneyl)benzaldehyde (S14a)}<smiles>O=Cc1ccccc1P</smiles>

S14a was prepared as a yellow solid in $63 \%$ yield $(0.91 \mathrm{~g}$, eluent: petroleum ether $/$ EtOAc $=50: 1)$ from $2 \mathrm{a}(1.44 \mathrm{~g}, 5.0 \mathrm{mmol})$ and DIBAL $(5.25 \mathrm{~mL}, 1.0 \mathrm{M}$ in hexane, $5.25 \mathrm{mmol}, 1.05$ equiv) following the general procedure E.

$\mathbf{R}_{f}=0.55$ (petroleum ether $/$ EtOAc $=10 / 1$ )

${ }^{1} \mathrm{H}$ NMR (400 MHz, $\mathrm{CDCl}_{3}$ ) $\delta 10.53$ (d, J = 5.3 Hz, 1H), $8.05-7.94$ (m, 1H), 7.53 $-7.44(\mathrm{~m}, 2 \mathrm{H}), 7.40-7.28(\mathrm{~m}, 10 \mathrm{H}), 7.04-6.95(\mathrm{~m}, 1 \mathrm{H})$.

${ }^{13} \mathrm{C}$ NMR (100 MHz, $\left.\mathrm{CDCl}_{3}\right) \delta 191.7$ (d, $\left.J=19.1 \mathrm{~Hz}\right), 141.2$ (d, $\left.J=26.5 \mathrm{~Hz}\right)$, $138.5(\mathrm{~d}, J=14.6 \mathrm{~Hz}), 136.2(\mathrm{~d}, J=9.6 \mathrm{~Hz}), 134.1(\mathrm{~d}, J=20.5 \mathrm{~Hz}), 133.9,133.7$, $130.8(\mathrm{~d}, J=3.8 \mathrm{~Hz}), 129.2,128.9,128.8(\mathrm{~d}, J=7.4 \mathrm{~Hz})$.

31P NMR (162 MHz, $\left.\mathrm{CDCl}_{3}\right) \delta$-11.6.

The characterization data are consistent with the literature reported data. ${ }^{19}$

\section{2-(Diphenylphosphaneyl)benzaldehyde (S14b)}<smiles>O=Cc1cc2c(cc1P(c1ccccc1)c1ccccc1)CCC2</smiles>

S14b was prepared as a yellow solid in 55\% yield $(0.18 \mathrm{~g}$, eluent: petroleum ether $/$ EtOAc $=50: 1)$ from $2 \mathbf{u}(0.33 \mathrm{~g}, 1.0 \mathrm{mmol})$ and DIBAL $(1.05 \mathrm{~mL}, 1.0 \mathrm{M}$ in hexane, $1.05 \mathrm{mmol}, 1.05$ equiv) following the general procedure E.

$\mathbf{R}_{f}=0.56$ (petroleum ether/EtOAc $=10 / 1$ )

${ }^{1} \mathrm{H}$ NMR (400 MHz, $\left.\mathrm{CDCl}_{3}\right) \delta 10.56(\mathrm{~d}, J=5.9 \mathrm{~Hz}, 1 \mathrm{H}), 7.88(\mathrm{~d}, J=3.6 \mathrm{~Hz}, 1 \mathrm{H})$, $7.40-7.28(\mathrm{~m}, 10 \mathrm{H}), 6.87(\mathrm{~d}, J=4.6 \mathrm{~Hz}, 1 \mathrm{H}), 2.98(\mathrm{t}, J=7.5 \mathrm{~Hz}, 2 \mathrm{H}), 2.83(\mathrm{t}, J=$ $7.5 \mathrm{~Hz}, 2 \mathrm{H}), 2.14-2.03(\mathrm{~m}, 2 \mathrm{H})$.

${ }^{13} \mathrm{C}$ NMR (100 MHz, $\left.\mathrm{CDCl}_{3}\right) \delta 191.6(\mathrm{~d}, J=22.2 \mathrm{~Hz}), 151.3,145.7,139.0(\mathrm{~d}, J=$ $25.2 \mathrm{~Hz}), 137.4(\mathrm{~d}, J=15.0 \mathrm{~Hz}), 136.6(\mathrm{~d}, J=10.0 \mathrm{~Hz}), 134.0(\mathrm{~d}, J=20.3 \mathrm{~Hz})$, 129.7, 128.9, $128.7(\mathrm{~d}, J=7.1 \mathrm{~Hz}), 126.2(\mathrm{~d}, J=4.7 \mathrm{~Hz}), 33.3,32.4$, 25.2. 
31P NMR (162 MHz, $\mathrm{CDCl}_{3}$ ) $\delta$-12.1.

HRMS (ESI-TOF) calcd for $\mathrm{C}_{22} \mathrm{H}_{20} \mathrm{OP}^{+}[\mathrm{M}+\mathrm{H}]^{+}$: 331.1247, found: 331.1246.

IR (neat, cm-1): 2925, 1684, 1597, 1464, 1433, 742, 695, 510, 493, 453.

(R)-N-((S)-(2-(Diphenylphosphaneyl)phenyl)(phenyl)methyl)-2-methylpro pane-2-sulfinamide (6a)<smiles>CC(C)(C)S(=O)N[C@H](c1ccccc1)c1ccccc1P</smiles>

6a was prepared as a white solid in $76 \%$ yield (179 $\mathrm{mg}$, eluent: petroleum ether $/$ EtOAc $=5: 1)$ from S14a $(145 \mathrm{mg}, 0.5 \mathrm{mmol})$, $(R)-(+)-2-$ methyl-2propanesulfinamide (91 mg, $0.75 \mathrm{mmol}), \mathrm{Ti}\left(\mathrm{O}^{i} \mathrm{Pr}\right)_{4}(0.3 \mathrm{~mL}, 1.0 \mathrm{mmol})$, $\mathrm{PhMgBr}\left(1.0 \mathrm{~mL}, 1.0 \mathrm{M}\right.$ in $\left.\mathrm{Et}_{2} \mathrm{O}, 1.0 \mathrm{mmol}\right)$ following the general procedure $\mathrm{F}$. $\mathbf{R}_{f}=0.42$ (petroleum ether $/ \mathrm{EtOAc}=2 / 1$ )

${ }^{1} \mathrm{H}$ NMR (400 MHz, $\left.\mathrm{CDCl}_{3}\right) \delta 7.72(\mathrm{dd}, J=8.0,4.3 \mathrm{~Hz}, 1 \mathrm{H}), 7.46-7.40(\mathrm{~m}, 1 \mathrm{H})$, $7.36-7.30(\mathrm{~m}, 5 \mathrm{H}), 7.29-7.18(\mathrm{~m}, 6 \mathrm{H}), 7.18-7.09(\mathrm{~m}, 6 \mathrm{H}), 6.74(\mathrm{dd}, J=8.9,3.7$ $\mathrm{Hz}, 1 \mathrm{H}), 4.00(\mathrm{~d}, J=3.9 \mathrm{~Hz}, 1 \mathrm{H}), 1.25(\mathrm{~s}, 9 \mathrm{H})$.

${ }^{13} \mathrm{C}$ NMR (100 MHz, $\left.\mathrm{CDCl}_{3}\right) \delta 146.7$ (d, $\left.J=24.3 \mathrm{~Hz}\right), 141.9,136.9$ (d, $J=10.8$ $\mathrm{Hz}), 135.9$ (d, J = 7.1 Hz), 135.8 (d, $J=1.6 \mathrm{~Hz}), 134.8$ (d, J=1.7 Hz), 133.7 (d, J = $13.8 \mathrm{~Hz}), 133.5(\mathrm{~d}, J=13.5 \mathrm{~Hz}), 129.2,128.4(\mathrm{~d}, J=5.6 \mathrm{~Hz}), 128.35(\mathrm{~d}, J=2.2 \mathrm{~Hz})$, 128.28, 128.22, 128.15, $127.8(\mathrm{~d}, J=1.5 \mathrm{~Hz}), 127.6,127.2,60.0(\mathrm{~d}, J=27.4 \mathrm{~Hz})$, $55.9,22.6$.

${ }^{31}$ P NMR (162 MHz, $\left.\mathrm{CDCl}_{3}\right) \delta-18.2$.

The characterization data are consistent with the literature reported data. ${ }^{20}$

(R)-N-((S)-(2-(Diphenylphosphaneyl)phenyl)(4-methoxyphenyl)methyl)-2methylpropane-2-sulfinamide (6b)<smiles>COc1ccc([C@H](NS(=O)CC(C)(C)C)c2ccccc2P)cc1</smiles>

6b was prepared as a white solid in $81 \%$ yield (203 $\mathrm{mg}$, eluent: petroleum ether $/$ EtOAc $=5: 1)$ from S14a $(145 \mathrm{mg}, 0.5 \mathrm{mmol})$, $(R)-(+)-2-$ methyl-2- 
propanesulfinamide $(91 \mathrm{mg}, 0.75 \mathrm{mmol}), \mathrm{Ti}\left(\mathrm{O}^{2} \mathrm{Pr}\right)_{4}(0.3 \mathrm{~mL}, 1.0 \mathrm{mmol}), 4$ $\mathrm{MeOC}_{6} \mathrm{H}_{4} \mathrm{MgBr}\left(1.0 \mathrm{~mL}, 1.0 \mathrm{M}\right.$ in $\left.\mathrm{Et}_{2} \mathrm{O}, 1.0 \mathrm{mmol}\right)$ following the general procedure $\mathrm{F}$.

$\mathbf{R}_{f}=0.38$ (petroleum ether $/$ EtOAc $=2 / 1$ )

${ }^{1} \mathrm{H}$ NMR $\left(400 \mathrm{MHz}, \mathrm{CDCl}_{3}\right) \delta 7.73(\mathrm{dd}, J=7.9,4.4 \mathrm{~Hz}, 1 \mathrm{H}), 7.43(\mathrm{t}, J=7.7 \mathrm{~Hz}$, $1 \mathrm{H}), 7.36-7.14(\mathrm{~m}, 10 \mathrm{H}), 7.14-7.04(\mathrm{~m}, 5 \mathrm{H}), 6.68-6.60(\mathrm{~m}, 3 \mathrm{H}), 3.89(\mathrm{~s}, 1 \mathrm{H})$, $3.70(\mathrm{~s}, 3 \mathrm{H}), 1.25(\mathrm{~s}, 9 \mathrm{H})$.

${ }^{13} \mathrm{C}$ NMR (100 MHz, $\left.\mathrm{CDCl}_{3}\right) \delta$ 158.6, $146.9(\mathrm{~d}, J=23.8 \mathrm{~Hz}), 137.0(\mathrm{~d}, J=10.8$ $\mathrm{Hz}), 135.8(\mathrm{~d}, J=4.5 \mathrm{~Hz}), 135.7(\mathrm{~d}, J=10.5 \mathrm{~Hz}), 134.9,134.1,133.8(\mathrm{~d}, J=14.6$ $\mathrm{Hz}), 133.6(\mathrm{~d}, J=14.2 \mathrm{~Hz}), 129.3,129.2,128.4(\mathrm{~d}, J=3.8 \mathrm{~Hz}), 128.3(\mathrm{~d}, J=7.5 \mathrm{~Hz})$, $128.2(\mathrm{~d}, J=7.0 \mathrm{~Hz}), 128.0(\mathrm{~d}, J=5.6 \mathrm{~Hz}), 127.5,113.7,59.5(\mathrm{~d}, J=27.1 \mathrm{~Hz}), 55.9$, 55.1, 22.7.

${ }^{31}$ P NMR (162 MHz, $\left.\mathrm{CDCl}_{3}\right) \delta-18.1$.

The characterization data are consistent with the literature reported data. ${ }^{20}$

(R)-N-((S)-(6-(Diphenylphosphaneyl)-2,3-dihydro-1H-inden-5-yl)(phenyl) methyl)-2-methylpropane-2-sulfinamide (6c)<smiles></smiles>

6c was prepared as a white solid in $86 \%$ yield $(220 \mathrm{mg}$, eluent: petroleum ether $/$ EtOAc $=5: 1)$ from S14b $(165 \mathrm{mg}, 0.5 \mathrm{mmol})$, (R)-(+)-2-methyl-2propanesulfinamide $(91 \mathrm{mg}, 0.75 \mathrm{mmol}), \mathrm{Ti}(\mathrm{O} P r)_{4}(0.3 \mathrm{~mL}, 1.0 \mathrm{mmol})$, $\mathrm{PhMgBr}\left(1.0 \mathrm{~mL}, 1.0 \mathrm{M}\right.$ in $\left.\mathrm{Et}_{2} \mathrm{O}, 1.0 \mathrm{mmol}\right)$ following the general procedure $\mathrm{F}$. $\mathbf{R}_{f}=0.45$ (petroleum ether $/$ EtOAc $=2 / 1$ )

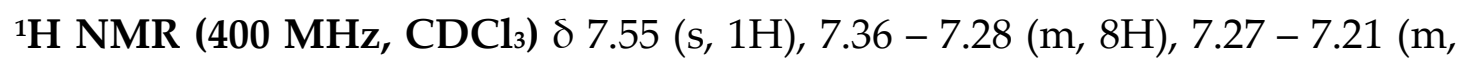
2H), $7.20-7.10(\mathrm{~m}, 5 \mathrm{H}), 6.99(\mathrm{~s}, 1 \mathrm{H}), 6.76(\mathrm{~d}, J=9.0 \mathrm{~Hz}, 1 \mathrm{H}), 4.00(\mathrm{~s}, 1 \mathrm{H}), 2.95(\mathrm{t}$, $J=7.6 \mathrm{~Hz}, 2 \mathrm{H}), 2.80(\mathrm{t}, J=7.6 \mathrm{~Hz}, 2 \mathrm{H}), 2.12-2.03(\mathrm{~m}, 2 \mathrm{H}), 1.27(\mathrm{~s}, 9 \mathrm{H})$.

${ }^{13} \mathrm{C}$ NMR (100 MHz, $\left.\mathrm{CDCl}_{3}\right) \delta$ 146.1, $145.0(\mathrm{~d}, J=25.5 \mathrm{~Hz}), 143.9,142.6,137.6$ (d, $J=11.0 \mathrm{~Hz}), 136.6(\mathrm{~d}, J=10.1 \mathrm{~Hz}), 133.7(\mathrm{~d}, J=9.3 \mathrm{~Hz}), 133.5(\mathrm{~d}, J=9.2 \mathrm{~Hz})$, $133.0(\mathrm{~d}, J=13.9 \mathrm{~Hz}), 130.7,128.4(\mathrm{~d}, J=6.8 \mathrm{~Hz}), 128.3,128.2,128.2,127.8(\mathrm{~d}, J=$ $1.5 \mathrm{~Hz}), 127.1,124.3(\mathrm{~d}, J=6.4 \mathrm{~Hz}), 60.1(\mathrm{~d}, J=28.7 \mathrm{~Hz}), 56.0,33.0,32.5,25.2$, 22.7.

31P NMR (162 MHz, $\left.\mathrm{CDCl}_{3}\right) \delta$ - 18.0. 
HRMS (ESI-TOF) calcd for $\mathrm{C}_{32} \mathrm{H}_{35} \mathrm{NOPS}^{+}[\mathrm{M}+\mathrm{H}]^{+}: 512.2172$, found: 512.2174.

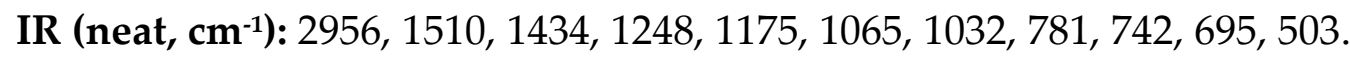

Methyl (S,E)-2-((2-(diphenylphosphaneyl)benzylidene)amino)-3,3-dimethyl butanoate $(7 \mathbf{a})$<smiles>CC(=O)[C@H](/N=C/c1ccccc1P)C(C)(C)C</smiles>

7a was prepared as a yellow oil in 93\% yield (194 mg, eluent: petroleum ether $/$ EtOAc $\left.=10: 1,3 \% \mathrm{Et}_{3} \mathrm{~N}\right)$ from S14a $(145 \mathrm{mg}, 0.5 \mathrm{mmol})$, S16a $(87 \mathrm{mg}, 0.6$ $\mathrm{mmol}), \mathrm{MgSO}_{4}(96 \mathrm{mg}, 0.8 \mathrm{mmol}$ ) following the general procedure $\mathrm{G}$.

$\mathbf{R}_{f}=0.45$ (petroleum ether/EtOAc/Et $3 \mathrm{~N}=5 / 1,3 \% \mathrm{Et}_{3} \mathrm{~N}$ )

${ }^{1} \mathrm{H}$ NMR (400 MHz, $\left.\mathrm{CDCl}_{3}\right) \delta 8.94(\mathrm{~d}, J=4.9 \mathrm{~Hz}, 1 \mathrm{H}), 8.20(\mathrm{dd}, J=7.8,3.9 \mathrm{~Hz}$, $1 \mathrm{H}), 7.42(\mathrm{t}, J=7.6 \mathrm{~Hz}, 1 \mathrm{H}), 7.38-7.27(\mathrm{~m}, 11 \mathrm{H}), 6.92(\mathrm{t}, J=6.3 \mathrm{~Hz}, 1 \mathrm{H}), 3.63(\mathrm{~s}$, $4 \mathrm{H}), 1.34(\mathrm{~d}, \mathrm{~J}=6.3 \mathrm{~Hz}, 1 \mathrm{H}), 0.95(\mathrm{~s}, 9 \mathrm{H})$.

${ }^{13} \mathrm{C}$ NMR (100 MHz, $\left.\mathrm{CDCl}_{3}\right) \delta$ 171.5, $161.4(\mathrm{~d}, J=23.5 \mathrm{~Hz}), 139.0(\mathrm{~d}, J=17.3$ $\mathrm{Hz}), 137.7(\mathrm{~d}, J=19.2 \mathrm{~Hz}), 136.1(\mathrm{~d}, J=9.6 \mathrm{~Hz}), 136.0(\mathrm{~d}, J=9.9 \mathrm{~Hz}), 134.2(\mathrm{~d}, J=$ $4.8 \mathrm{~Hz}), 134.0(\mathrm{~d}, J=4.8 \mathrm{~Hz}), 133.0,130.6,128.92,128.88,128.86,128.65(\mathrm{~d}, J=$ $1.4 \mathrm{~Hz}), 128.57(\mathrm{~d}, J=1.3 \mathrm{~Hz}), 127.9(\mathrm{~d}, J=4.2 \mathrm{~Hz}), 82.9,51.3,35.1,26.7$.

31P NMR (162 MHz, $\left.\mathrm{CDCl}_{3}\right) \delta$-14.2.

The characterization data are consistent with the literature reported data. ${ }^{21}$

Methyl (S,E)-2-((2-(diphenylphosphaneyl)benzylidene)amino)-2-phenyl acetate $\mathbf{( 7 b )}$<smiles>CC(=O)[C@H](/N=C/c1ccccc1Pc1ccccc1)c1ccccc1</smiles>

7b was prepared as a yellow solid in 91\% yield (199 $\mathrm{mg}$, eluent: petroleum ether $\left./ \mathrm{EtOAc}=10: 1,3 \% \mathrm{Et}_{3} \mathrm{~N}\right)$ from S14a $(145 \mathrm{mg}, 0.5 \mathrm{mmol}), \mathbf{S 1 6 b}(99 \mathrm{mg}, 0.6$ $\mathrm{mmol}), \mathrm{MgSO}_{4}(96 \mathrm{mg}, 0.8 \mathrm{mmol}$ ) following the general procedure $\mathrm{G}$.

$\mathbf{R}_{f}=0.42$ (petroleum ether $/ \mathrm{EtOAc}=5 / 1,3 \% \mathrm{Et}_{3} \mathrm{~N}$ )

${ }^{1} \mathrm{H}$ NMR (400 MHz, $\left.\mathbf{C D C l}_{3}\right) \delta 9.09(\mathrm{~d}, J=5.1 \mathrm{~Hz}, 1 \mathrm{H}), 8.26(\mathrm{dd}, J=7.9,3.9 \mathrm{~Hz}$, $1 \mathrm{H}), 7.46(\mathrm{t}, J=7.6 \mathrm{~Hz}, 1 \mathrm{H}), 7.43-7.30(\mathrm{~m}, 16 \mathrm{H}), 7.02-6.95(\mathrm{~m}, 1 \mathrm{H}), 5.19$ (s, $1 \mathrm{H}), 3.70$ (s, 3H). 
${ }^{13} \mathrm{C}$ NMR (100 MHz, $\left.\mathrm{CDCl}_{3}\right) \delta$ 171.3, $162.4(\mathrm{~d}, J=22.8 \mathrm{~Hz}), 139.0(\mathrm{~d}, J=17.3$ $\mathrm{Hz}), 138.0(\mathrm{~d}, J=19.8 \mathrm{~Hz}), 137.8,136.3$ (d, $J=2.9 \mathrm{~Hz}), 136.2(\mathrm{~d}, J=2.6 \mathrm{~Hz}), 134.1$ $(\mathrm{d}, J=6.9 \mathrm{~Hz}), 133.9(\mathrm{~d}, J=6.9 \mathrm{~Hz}), 133.3,130.9,128.94,128.86,128.65(\mathrm{~d}, J=2.6$ $\mathrm{Hz}), 128.59,128.57,128.2(\mathrm{~d}, J=4.3 \mathrm{~Hz}), 127.9,127.8,76.4,52.3$.

${ }^{31 P}$ NMR (162 MHz, $\left.\mathrm{CDCl}_{3}\right) \delta$-14.2.

The characterization data are consistent with the literature reported data. ${ }^{22}$

Methyl (S,E)-2-((2-(diphenylphosphaneyl)benzylidene)amino)-3-phenylpro panoate $(7 \mathrm{c})$<smiles>CC(=O)[C@H](Cc1ccccc1)/N=C/c1ccccc1Pc1ccccc1</smiles>

7c was prepared as a yellow solid in 90\% yield (203 mg, eluent: petroleum ether $\left./ \mathrm{EtOAc}=10: 1,3 \% \mathrm{Et}_{3} \mathrm{~N}\right)$ from S14a (145 mg, $\left.0.5 \mathrm{mmol}\right), \mathrm{S16}$ (108 mg, 0.6 $\mathrm{mmol}), \mathrm{MgSO}_{4}(96 \mathrm{mg}, 0.8 \mathrm{mmol}$ ) following the general procedure $\mathrm{G}$.

$\mathbf{R}_{f}=0.39$ (petroleum ether/EtOAc $=5 / 1,3 \% \mathrm{Et}_{3} \mathrm{~N}$ )

${ }^{1} \mathbf{H}$ NMR (400 MHz, $\left.\mathrm{CDCl}_{3}\right) \delta 8.79(\mathrm{~d}, J=5.3 \mathrm{~Hz}, 1 \mathrm{H}), 8.08(\mathrm{dd}, J=7.7,3.9 \mathrm{~Hz}$, $1 \mathrm{H}), 7.46-7.29(\mathrm{~m}, 12 \mathrm{H}), 7.26-7.20(\mathrm{~m}, 2 \mathrm{H}), 7.20-7.13(\mathrm{~m}, 3 \mathrm{H}), 7.00-6.93(\mathrm{~m}$, $1 \mathrm{H}), 4.19(\mathrm{t}, J=7.1 \mathrm{~Hz}, 1 \mathrm{H}), 3.64(\mathrm{~s}, 3 \mathrm{H}), 3.30(\mathrm{~d}, J=6.0 \mathrm{~Hz}, 1 \mathrm{H}), 2.99(\mathrm{dd}, J=$ 13.6, $8.1 \mathrm{~Hz}, 1 \mathrm{H})$.

${ }^{13} \mathrm{C}$ NMR (100 MHz, $\left.\mathrm{CDCl}_{3}\right) \delta$ 171.7, $162.4(\mathrm{~d}, J=22.5 \mathrm{~Hz}), 139.0(\mathrm{~d}, J=17.7$ $\mathrm{Hz}), 137.9(\mathrm{~d}, J=20.0 \mathrm{~Hz}), 137.3,136.4(\mathrm{~d}, J=10.0 \mathrm{~Hz}), 134.1(\mathrm{~d}, J=3.3 \mathrm{~Hz})$, $133.9(\mathrm{~d}, J=3.1 \mathrm{~Hz}), 133.4,130.7,129.5,128.9,128.8(\mathrm{~d}, J=2.6 \mathrm{~Hz}), 128.64(\mathrm{~d}, J=$ $3.2 \mathrm{~Hz}), 128.58(\mathrm{~d}, J=3.1 \mathrm{~Hz}), 128.3,128.0(\mathrm{~d}, J=4.3 \mathrm{~Hz}), 126.5,74.7,52.1,39.8$. ${ }^{31 P}$ NMR (162 MHz, $\left.\mathrm{CDCl}_{3}\right) \delta$-14.6.

The characterization data are consistent with the literature reported data. ${ }^{22}$

(R,E)-2-((2-(Diphenylphosphaneyl)benzylidene)amino)- $N$-(4-methoxyphen yl)-3-methylbutanamide (7d)<smiles>COc1ccc(NC(=O)[C@H](N=Cc2ccccc2-c2ccccc2)C(C)C)cc1</smiles>

$7 \mathbf{d}$ was prepared as a yellow solid in $95 \%$ yield $(235 \mathrm{mg}$, eluent: petroleum ether $\left./ \mathrm{EtOAc}=8: 1,3 \% \mathrm{Et}_{3} \mathrm{~N}\right)$ from S14a $(145 \mathrm{mg}, 0.5 \mathrm{mmol}), \mathbf{S 1 6 d}(133 \mathrm{mg}, 0.6$ 
$\mathrm{mmol}), \mathrm{MgSO}_{4}(96 \mathrm{mg}, 0.8 \mathrm{mmol}$ ) following the general procedure $\mathrm{G}$.

$\mathbf{R}_{f}=0.22$ (petroleum ether $/ \mathrm{EtOAc}=5 / 1,3 \% \mathrm{Et}_{3} \mathrm{~N}$ )

${ }^{1} \mathrm{H}$ NMR (400 MHz, $\mathrm{CDCl}_{3}$ ) $\delta 9.63(\mathrm{~d}, J=5.3 \mathrm{~Hz}, 1 \mathrm{H}), 8.49$ (s, 1H), $7.84-7.72$ $(\mathrm{m}, 3 \mathrm{H}), 7.51(\mathrm{t}, J=7.6 \mathrm{~Hz}, 1 \mathrm{H}), 7.44-7.21(\mathrm{~m}, 11 \mathrm{H}), 7.03(\mathrm{t}, J=6.3 \mathrm{~Hz}, 1 \mathrm{H})$, $6.92(\mathrm{~d}, J=7.9 \mathrm{~Hz}, 2 \mathrm{H}), 3.80(\mathrm{~s}, 3 \mathrm{H}), 3.79-3.76(\mathrm{~m}, 1 \mathrm{H}), 2.34-2.23(\mathrm{~m}, 1 \mathrm{H})$, $0.79(\mathrm{~d}, J=6.4 \mathrm{~Hz}, 3 \mathrm{H}), 0.48(\mathrm{~d}, J=6.4 \mathrm{~Hz}, 3 \mathrm{H})$.

${ }^{13} \mathrm{C}$ NMR (100 MHz, $\left.\mathrm{CDCl}_{3}\right) \delta$ 170.1, $162.3(\mathrm{~d}, J=3.6 \mathrm{~Hz}), 156.0,138.2(\mathrm{~d}, J=$ $15.9 \mathrm{~Hz}), 137.7(\mathrm{~d}, J=11.7 \mathrm{~Hz}), 136.7(\mathrm{~d}, J=20.9 \mathrm{~Hz}), 136.5(\mathrm{~d}, J=2.6 \mathrm{~Hz}), 134.7$, $134.5(\mathrm{~d}, J=20.3 \mathrm{~Hz}), 132.9(\mathrm{~d}, J=18.8 \mathrm{~Hz}), 132.2(\mathrm{~d}, J=4.6 \mathrm{~Hz}), 131.7,130.4$, 129.0, 128.72, 128.66, 128.5, $128.4(\mathrm{~d}, J=7.5 \mathrm{~Hz}), 121.3(\mathrm{~d}, J=2.7 \mathrm{~Hz}), 114.0,81.2$, $55.3,32.7,19.3,16.7$.

31P NMR (162 MHz, $\left.\mathrm{CDCl}_{3}\right) \delta-9.1$.

The characterization data are consistent with the literature reported data. ${ }^{23}$

(1E,1'E)-N,N'-((1R,2R)-Cyclohexane-1,2-diyl)bis(1-(2-(diphenylphosphaneyl) phenyl)methanimine) (7e)

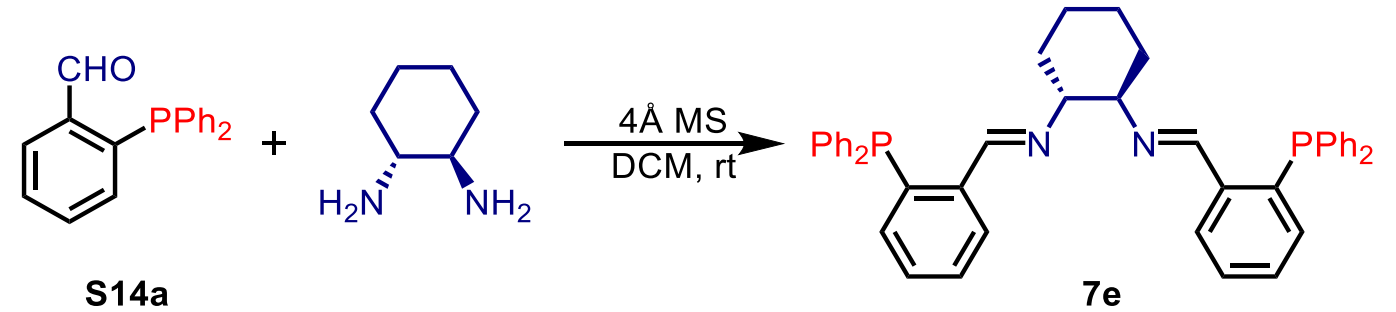

A mixture of S14a (1.0 mmol), (1R,2R)-(-)-1,2-diaminocyclohexane (57 mg, $0.5 \mathrm{mmol})$ and $4 \AA$ molecular sieve $(100 \mathrm{mg})$ in DCM $(4 \mathrm{~mL})$ was stirred at room temperature overnight. The suspension was filtered through a plug of Celite, and the filter cake was washed with DCM. After removal of solvents under reduced pressure to afford 7 e (326 $\mathrm{mg}, 99 \%)$ as a pale yellow solid.

${ }^{1} \mathbf{H}$ NMR (400 MHz, $\left.\mathrm{CDCl}_{3}\right) \delta 8.76(\mathrm{~d}, J=4.2 \mathrm{~Hz}, 2 \mathrm{H}), 7.86-7.78(\mathrm{~m}, 2 \mathrm{H}), 7.40$ $-7.24(\mathrm{~m}, 24 \mathrm{H}), 6.91-6.84(\mathrm{~m}, 2 \mathrm{H}), 3.25-3.13(\mathrm{~m}, 2 \mathrm{H}), 1.78-1.66(\mathrm{~m}, 2 \mathrm{H})$, $1.61-1.48(\mathrm{~m}, 2 \mathrm{H}), 1.48-1.39(\mathrm{~m}, 2 \mathrm{H}), 1.38-1.26(\mathrm{~m}, 2 \mathrm{H})$.

$\left.{ }^{13} \mathrm{C} \mathrm{NMR} \mathrm{(100} \mathrm{MHz,} \mathrm{CDCl}_{3}\right) \delta 159.4(\mathrm{~d}, J=18.9 \mathrm{~Hz}), 140.2(\mathrm{~d}, J=18.0 \mathrm{~Hz})$, $137.26(\mathrm{~d}, J=16.5 \mathrm{~Hz}), 137.24,137.1(\mathrm{~d}, J=13.6 \mathrm{~Hz}), 134.1(\mathrm{~d}, J=14.9 \mathrm{~Hz}), 133.9$ $(\mathrm{d}, J=14.9 \mathrm{~Hz}), 133.4,129.8,128.9,128.7,128.66,128.59,128.50(\mathrm{~d}, J=2.9 \mathrm{~Hz})$, $128.2(\mathrm{~d}, J=4.6 \mathrm{~Hz}), 73.7,32.6,24.4$.

${ }^{31}$ P NMR (162 MHz, $\left.\mathrm{CDCl}_{3}\right) \delta$-13.6.

The characterization data are consistent with the literature reported data. ${ }^{24}$ 


\section{Applications of chiral ligands.}

(a) Enantioselective Tsuji enol-carbonate allylation. ${ }^{25}$

A mixture of $\mathrm{Pd}_{2}(\mathrm{dba})_{3}(22.9 \mathrm{mg}, 0.025 \mathrm{mmol}, 0.025$ equiv) and PHOX ligand 5 (0.0625 mmol, 0.0625 equiv) in THF $(30 \mathrm{~mL})$ was stirred at $10{ }^{\circ} \mathrm{C}$ for $30 \mathrm{~min}$, at which time allyl enol carbonate $8(196.2 \mathrm{mg}, 1.0 \mathrm{mmol})$ was added by syringe in one portion. When the reaction was complete by TLC, the reaction mixture was evaporated under reduced pressure and the residue was purified by silica gel flash chromatography to afford ketone $9^{25}$ (184 mg, 92\%; $186 \mathrm{mg}$, 93\%; $194 \mathrm{mg}, 97 \%)$.<smiles>C=CCOC(=O)OC1=C(C)CCc2ccccc21</smiles>

8

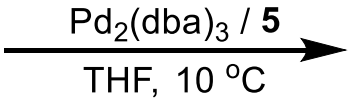

THF, $10^{\circ} \mathrm{C}$

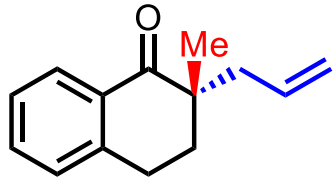

9

\begin{tabular}{cccc}
\hline entry & ligand $\mathbf{5}$ & yield (\%) & ee (\%) \\
\hline 1 & $\mathbf{5 b}$ & 92 & 86 \\
2 & $\mathbf{5 e}$ & 93 & 88 \\
3 & $\mathbf{5 f}$ & 97 & 87 \\
$\ldots$
\end{tabular}

${ }^{1}$ H NMR (400 MHz, $\left.\mathrm{CDCl}_{3}\right) \delta 8.04(\mathrm{~d}, J=7.8 \mathrm{~Hz}, 1 \mathrm{H}), 7.45(\mathrm{t}, J=7.4 \mathrm{~Hz}, 1 \mathrm{H})$, $7.30(\mathrm{t}, J=7.5 \mathrm{~Hz}, 1 \mathrm{H}), 7.22(\mathrm{~d}, J=7.6 \mathrm{~Hz}, 1 \mathrm{H}), 5.85-5.71(\mathrm{~m}, 1 \mathrm{H}), 5.10(\mathrm{~s}, 1 \mathrm{H})$, $5.08-5.02(\mathrm{~m}, 1 \mathrm{H}), 3.04-2.91(\mathrm{~m}, 2 \mathrm{H}), 2.46(\mathrm{dd}, J=13.8,7.3 \mathrm{~Hz}, 1 \mathrm{H}), 2.27(\mathrm{dd}$, $J=13.8,7.5 \mathrm{~Hz}, 1 \mathrm{H}), 2.11-2.04(\mathrm{~m}, 1 \mathrm{H}), 1.94-1.85(\mathrm{~m}, 1 \mathrm{H}), 1.19(\mathrm{~s}, 3 \mathrm{H})$.

${ }^{13} \mathrm{C}$ NMR (100 MHz, $\left.\mathrm{CDCl}_{3}\right) \delta$ 202.2, 143.4, 134.1, 133.2, 131.7, 128.8, 128.1, $126.7,118.3,44.7,41.2,33.4,25.5,22.0$.

Enantiomeric excess was determined by chiral HPLC (Daicel Chiralcel OD-H column, $n$-hexane/i-propanol $=99.9: 0.1$, flow rate $0.7 \mathrm{~mL} / \mathrm{min}$, detection at 254 $\mathrm{nm})$.

The characterization data are consistent with the literature reported data. ${ }^{25}$ 

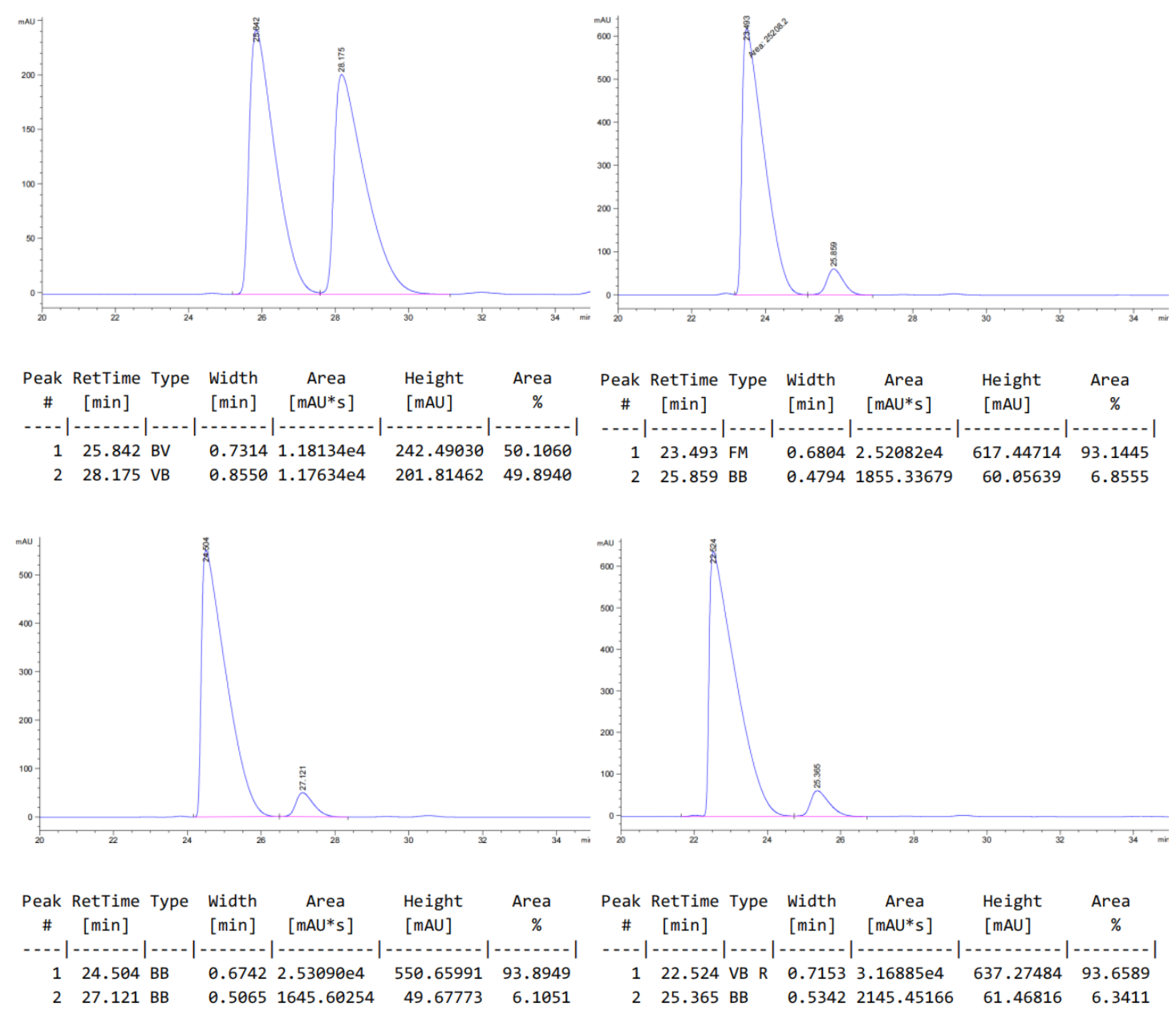

(b) Enantioselective gold(I)-catalyzed cycloaddition reaction..$^{20}$<smiles>CC(=O)C(C#CC(C)(C)C)=Cc1ccccc1</smiles>

10

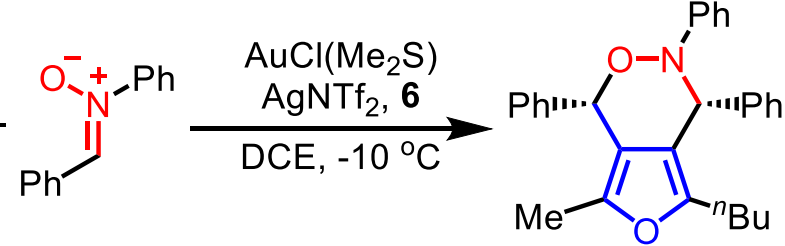

11
12

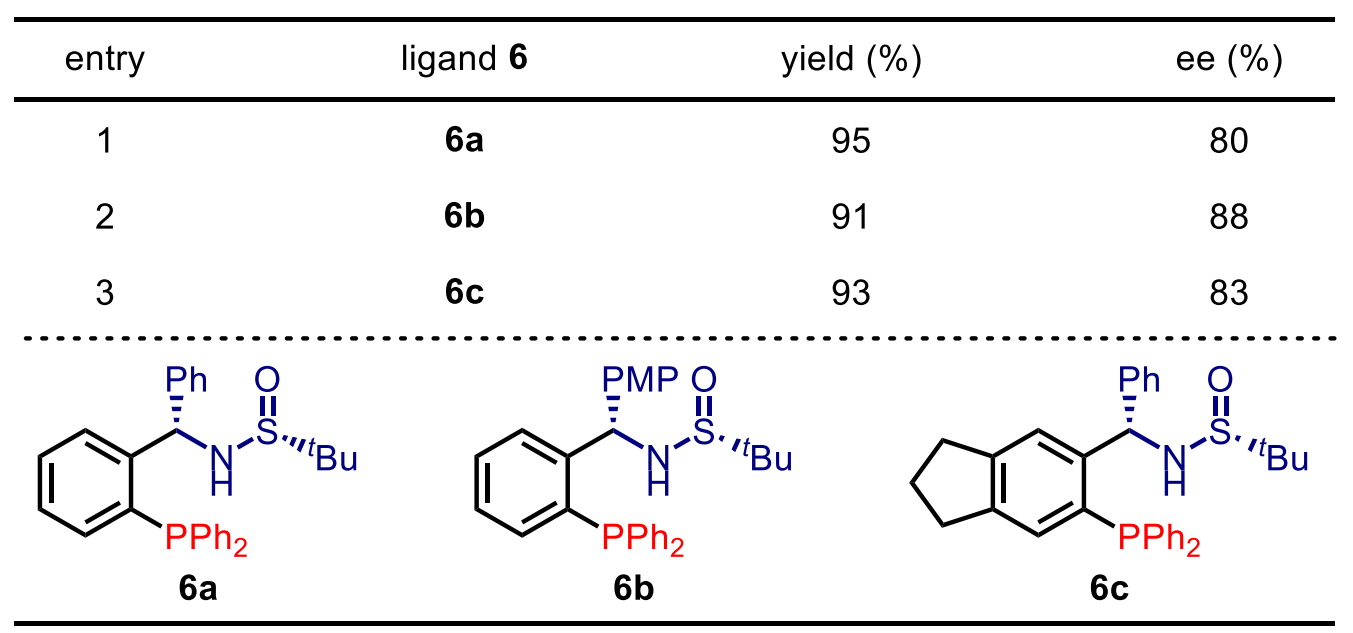


A mixture of [ $\mathrm{Me} 2 \mathrm{SAuCl}](5.9 \mathrm{mg}, 0.02 \mathrm{mmol})$ and $6(0.022 \mathrm{mmol})$ in $\mathrm{CH}_{2} \mathrm{Cl}_{2}$ $(1.0 \mathrm{~mL})$ was stirred at room temperature for $2 \mathrm{~h}$, then the solvent was removed in vacuum. A solution of $\operatorname{AgNTf}_{2}(7.8 \mathrm{mg}, 0.02 \mathrm{mmol})$ in DCE (1.0 $\mathrm{mL}$ ) was added to the residue, and the mixture was stirred at $-10{ }^{\circ} \mathrm{C}$ for 15 min. A solution of $10(90.4 \mathrm{mg}, 0.40 \mathrm{mmol})$ and $11(86.7 \mathrm{mg}, 0.44 \mathrm{mmol})$ in DCE $(3.0 \mathrm{~mL})$ was transferred to the above catalyst solution and the resulting mixture was stirred at $-10{ }^{\circ} \mathrm{C}$. When the reaction was complete by TLC, the reaction mixture was evaporated under reduced pressure and the residue was purified by silica gel flash chromatography to afford $\mathbf{1 2}^{20}$ (161 mg, 95\%; 155 $\mathrm{mg}, 91 \% ; 158 \mathrm{mg}, 93 \%)$.

${ }^{1} \mathrm{H}$ NMR (400 MHz, $\left.\mathrm{CDCl}_{3}\right) \delta 7.61-7.55(\mathrm{~m}, 2 \mathrm{H}), 7.48$ - 7.41 (m, 3H), 7.35 $7.29(\mathrm{~m}, 2 \mathrm{H}), 7.24-7.16(\mathrm{~m}, 5 \mathrm{H}), 7.08-7.02(\mathrm{~m}, 2 \mathrm{H}), 6.94-6.88(\mathrm{~m}, 1 \mathrm{H}), 5.99(\mathrm{~s}$, $1 \mathrm{H}), 5.71(\mathrm{~s}, 1 \mathrm{H}), 2.40(\mathrm{t}, J=7.6 \mathrm{~Hz}, 2 \mathrm{H}), 1.81(\mathrm{~s}, 3 \mathrm{H}), 1.55-1.42(\mathrm{~m}, 1 \mathrm{H}), 1.42-$ $1.33(\mathrm{~m}, 1 \mathrm{H}), 1.28-1.18(\mathrm{~m}, 2 \mathrm{H}), 0.83(\mathrm{t}, J=7.3 \mathrm{~Hz}, 3 \mathrm{H})$.

${ }^{13} \mathrm{C}$ NMR (100 MHz, $\left.\mathrm{CDCl}_{3}\right) \delta$ 148.6, 148.2, 143.3, 139.3, 138.1, 129.5, 129.4, $129.0,128.64,128.60,127.8,127.5,122.2,117.9,117.4,117.3,79.2,62.6,30.2$, 26.5, $22.4,13.9,12.6$.

Enantiomeric excess was determined by chiral HPLC (Daicel Chiralpak AD$\mathrm{H}$ column, $n$-hexane $/ i$-propanol $=90 / 10$, flow rate $0.8 \mathrm{~mL} / \mathrm{min}$, detection at 230 $\mathrm{nm})$.

The characterization data are consistent with the literature reported data. ${ }^{20}$
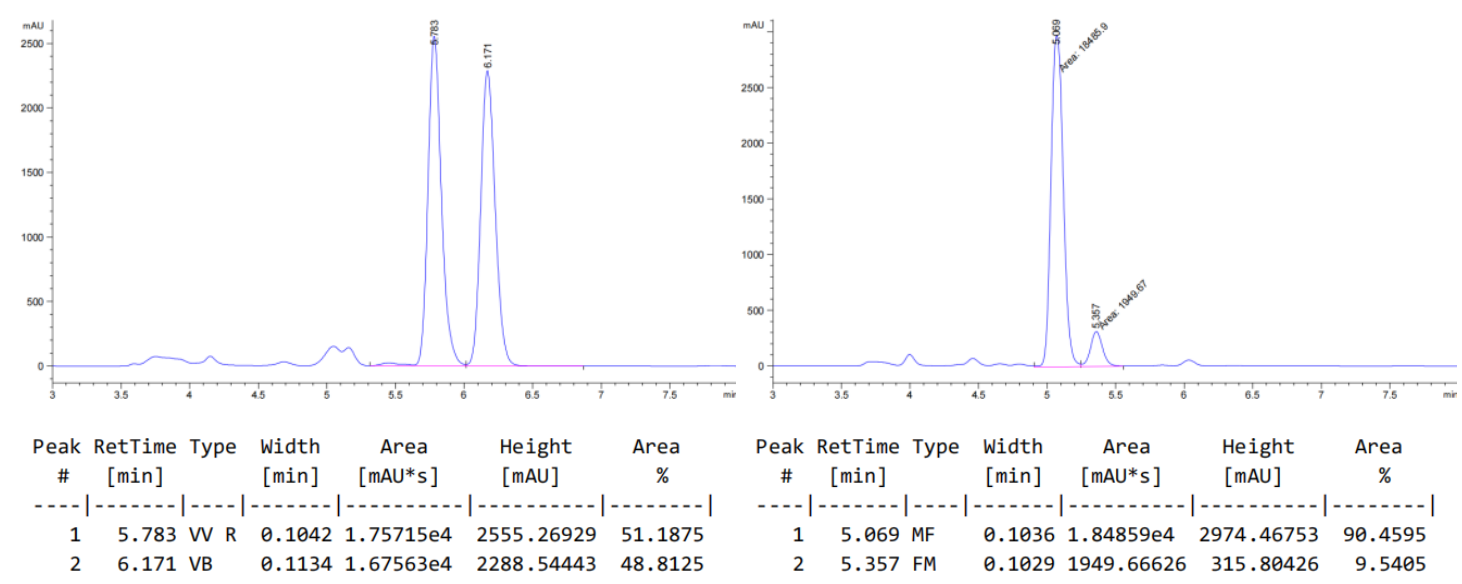

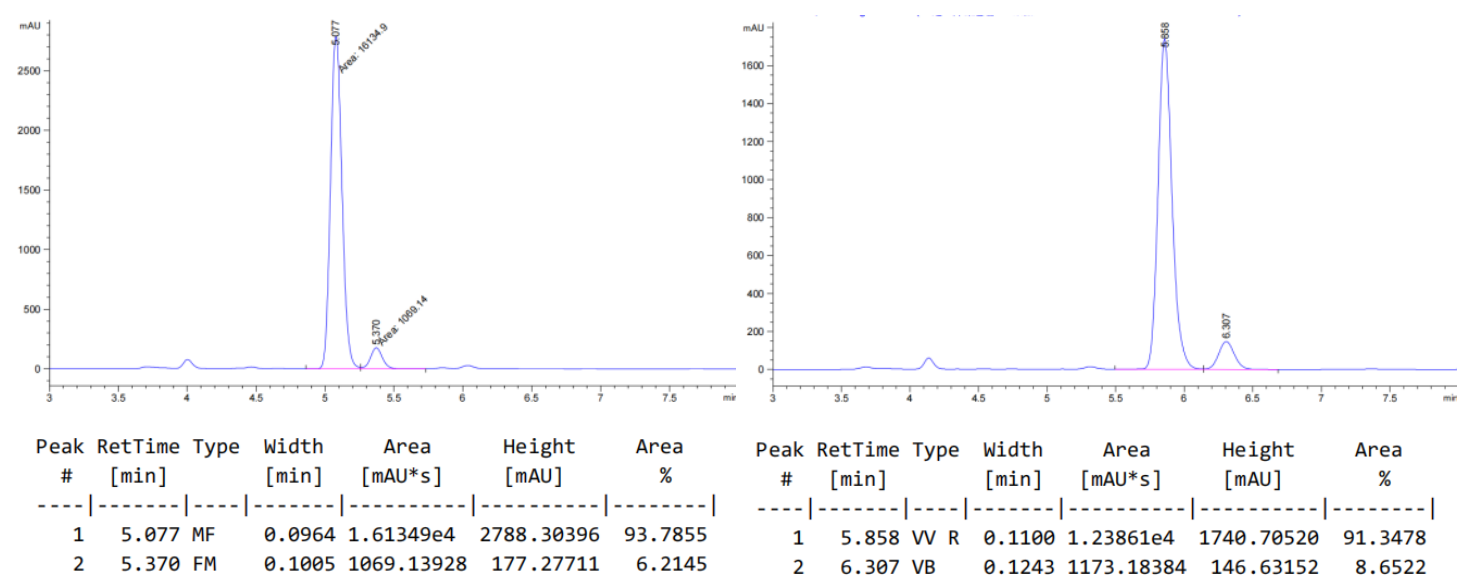

(c) Asymmetric hydrogenation. ${ }^{26}$

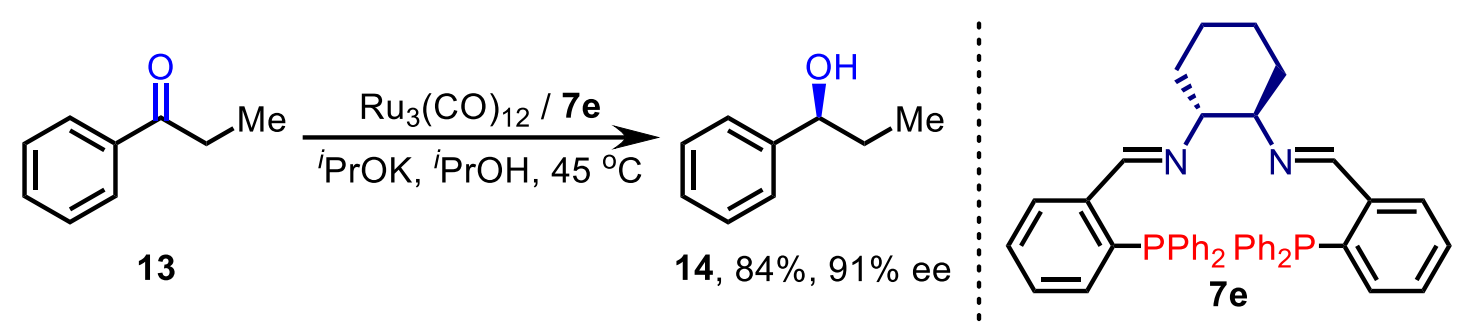

A mixture of ${ }^{i} \operatorname{PrOK}\left(5 \% \mathrm{w} / \mathrm{v}\right.$ in $\left.{ }^{i} \mathrm{PrOH}, 0.04 \mathrm{~mL}\right)$ and $\mathrm{Ru}_{3}(\mathrm{CO}){ }_{12}(1.3 \mathrm{mg}$, $0.002 \mathrm{mmol})$ in ${ }^{\mathrm{PrOH}}(4.0 \mathrm{~mL})$ was stirred at room temperature for $10 \mathrm{~min}$, at which time the chiral ligand $7 \mathrm{e}(1.3 \mathrm{mg}, 0.002 \mathrm{mmol})$ was added, and the reaction mixture stirred at $45^{\circ} \mathrm{C}$ in an oil bath for $1 \mathrm{~h}$. The propiophenone 13 (0.4 mmol, $53.7 \mathrm{mg}$ ) was added to the above catalyst solution and the resulting mixture was stirred at $45{ }^{\circ} \mathrm{C}$ for $5 \mathrm{~h}$. The reaction mixture was diluted with $n$-hexane and filtered through a plug of silica gel. After removal of solvents under reduced pressure, the residue was purified by silica gel flash chromatography to afford 14 (46 mg, 84\%).

${ }^{1}$ H NMR (400 MHz, $\left.\mathrm{CDCl}_{3}\right) \delta 7.37-7.22(\mathrm{~m}, 5 \mathrm{H}), 4.52(\mathrm{t}, J=6.6 \mathrm{~Hz}, 1 \mathrm{H}), 2.62$ (s, 1H), $1.85-1.64(\mathrm{~m}, 2 \mathrm{H}), 0.89(\mathrm{t}, J=7.5 \mathrm{~Hz}, 3 \mathrm{H})$.

${ }^{13} \mathrm{C}$ NMR (100 MHz, $\left.\mathrm{CDCl}_{3}\right) \delta$ 144.7, 128.3, 127.4, 126.0, 75.9, 31.9, 10.2.

Enantiomeric excess was determined by chiral HPLC (Daicel Chiralcel OD-H column, $n$-hexane $/ i$-propanol $=95 / 5$, flow rate $1.0 \mathrm{~mL} / \mathrm{min}$, detection at 220 $\mathrm{nm})$.

The characterization data are consistent with the literature reported data. ${ }^{26}$ 

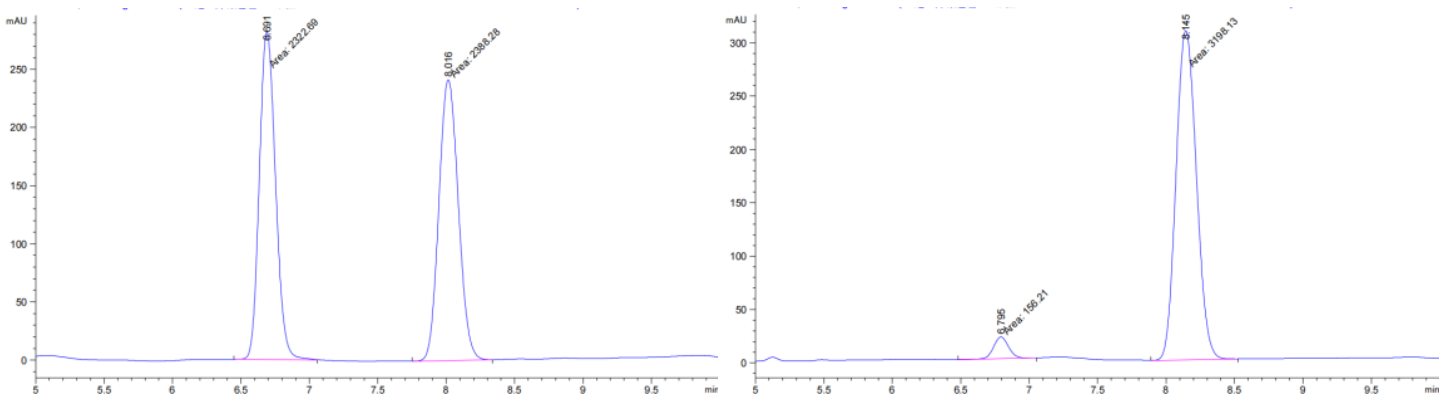

\# [min] Area
$[\mathrm{min}]$

Height

Area

Peak RetTime Type Width Area

Height [min] [mAU*s] [mAU]

Area

-

$\begin{array}{lllllll}1 & 6.691 & \text { MM } & 0.1372 & 2322.69312 & 282.05991 & 49.3038\end{array}$ \# [min] [0.1275 156.21022

$\begin{array}{lllllll}2 & 8.016 & \text { MM } & 0.1647 & 2388.28467 & 241.61252 & 50.6962\end{array}$

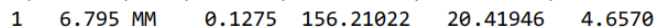

$\begin{array}{llllll}2 & 8.145 \text { MM } & 0.1727 & 3198.13013 & 308.67474 & 95.3430\end{array}$ 


\section{References}

1. Ouchaou, K.; Georgin, D.; Taran, F. Synlett 2010, 2010, 2083-2086.

2. Adachi, M.; Sugasawa, T. Synth. Commun. 1990, 20, 71-84.

3. Zhang, W.; Yang, W.; Zhao, W. Org. Biomol. Chem. 2020, 18, 4604-4609.

4. Speicher, A.; Groh, M.; Hennrich, M.; Huynh, A.-M. Eur. J. Org. Chem. 2010, 2010, 6760-6778.

5. Li, R.; Li, C.; Yang, W.; Zhao, W. Synthesis 2021, 53, 1605-1618.

6. Zhang, W.; Yang, W.; Zhao, W. J. Org. Chem. 2020, 85, 8702-8713.

7. Damian, K.; Clarke, M. L.; Cobley, C. J. Appl. Organometal. Chem. 2009, 23, 272-276.

8. Ren, W.; Zuo, Q.-M.; Yang, S.-D. Synlett 2019, 30, 1719-1724.

9. Kwong, F. Y.; Lai, C. W.; Yu, M.; Chan, K. S. Tetrahedron 2004, 60, 5635-5645.

10. Zhang, L.; Yang, F.; Tao, G.; Qiu, L.; Duan, Z.; Mathey, F. Eur. J. Inorg. Chem. 2017, 2017, 2355-2362.

11. Ma, B.; Ding, Z.; Liu, J.; He, Y.; Fan, Q.-H. Chem. Asian J. 2013, 8, 1101-1104.

12. Pang, X.; Zhao, Z. Z.; Wei, X. X.; Qi, L.; Xu, G. L.; Duan, J.; Liu, X. Y.; Shu, X. Z. J. Am. Chem. Soc. 2021, 143, 4536-4542.

13. Steinhoff, P.; Steinbock, R.; Friedrich, A.; Schieweck, B. G.; Cremer, C.; Truong, K. N.; Tauchert, M. E. Dalton Trans 2018, 47, 10439-10442.

14. Yu, R.; Chen, X.; Wang, Z. Tetrahedron Lett. 2016, 57, 3404-3406.

15. García-Yebra, C.; Janssen, J. P.; Rominger, F.; Helmchen, G. Organometallics 2004, 23, 5459-5470.

16. Tani, K.; Behenna, D. C.; McFadden, R. M.; Stoltz, B. M. Org. Lett. 2007, 9, 2529-2531.

17. Sedinkin, S. L.; Rath, N. P.; Bauer, E. B. J. Organomet. Chem. 2008, 693, 30813091.

18. Xi, T.; Mei, Y.; Lu, Z. Org. Lett. 2015, 17, 5939-5941.

19. Zhao, Y.; Snieckus, V. Org. Lett. 2014, 16, 390-393.

20. Zhang, Z.-M.; Chen, P.; Li, W.; Niu, Y.; Zhao, X.-L.; Zhang, J. Angew. Chem. Int. Ed. 2014, 53, 4350-4354.

21. Wencel, J.; Laurent, I.; Toupet, L.; Crévisy, C.; Mauduit, M. Organometallics 2010, 29, 1530-1533.

22. Schreiner, B.; Robl, C.; Wagner-Schuh, B.; Beck, W. Z. Naturforsch. 2010, 65, 503-510. 
23. Carswell, E. L.; Snapper, M. L.; Hoveyda, A. H. Angew. Chem. Int. Ed. 2006, $45,7230-7233$.

24. Stoop, R. M.; Bachmann, S.; Valentini, M.; Mezzetti, A. Organometallics 2000, 19, 4117-4126.

25. Behenna, D. C.; Stoltz, B. M. J. Am. Chem. Soc. 2004, 126, 15044-15045.

26. Li, Y. Y.; Chen, J. S.; Yang, C. B.; Dong, Z. R.; Li, B. Z.; Zhang, H.; Gao, J. X.; Ikariya, T. Chem. Res. Chin. Univ. 2004, 20, 180-184. 
VI. ${ }^{1} \mathrm{H},{ }^{13} \mathrm{C}$ and ${ }^{31} \mathrm{P}$ NMR Spectra
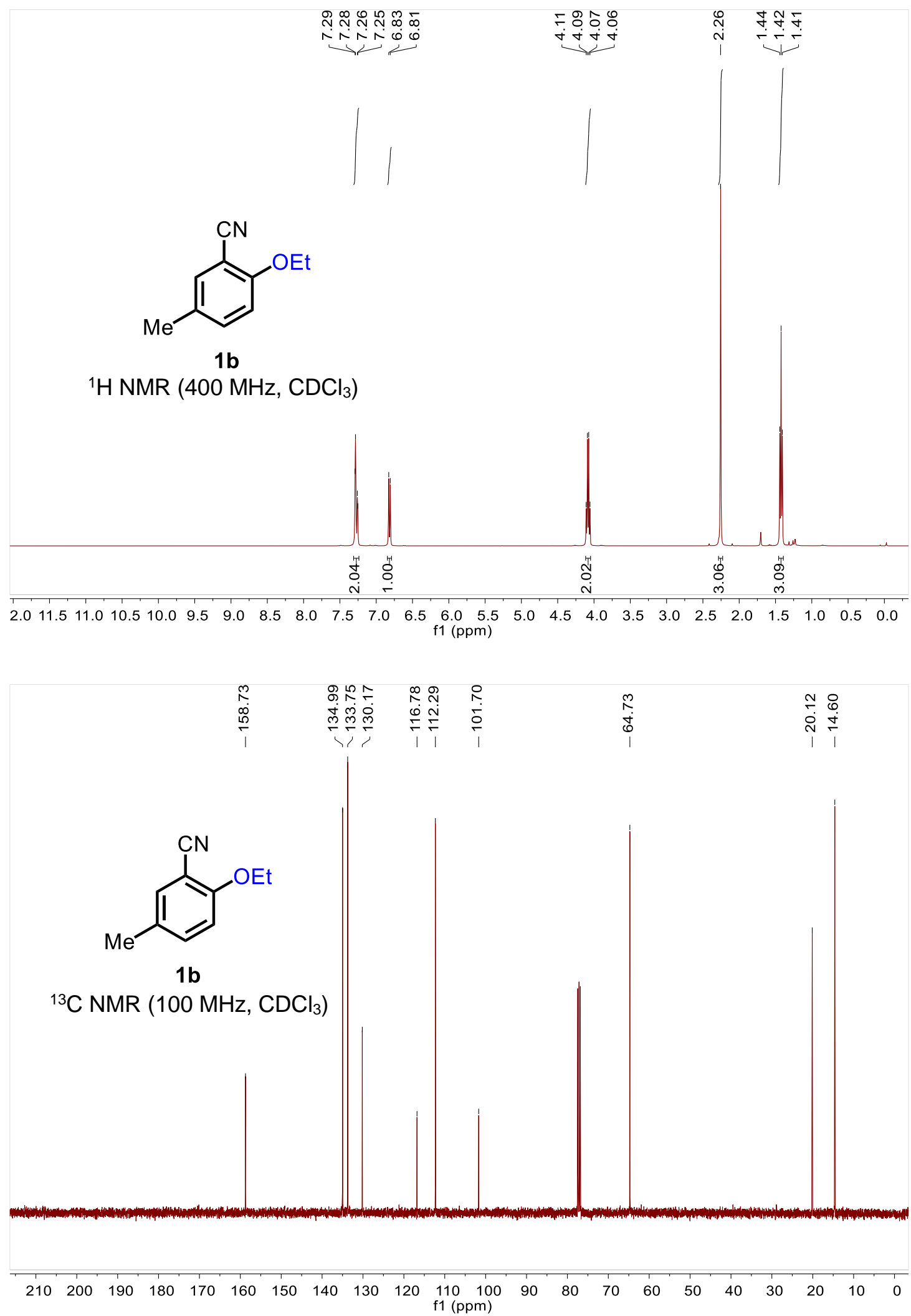

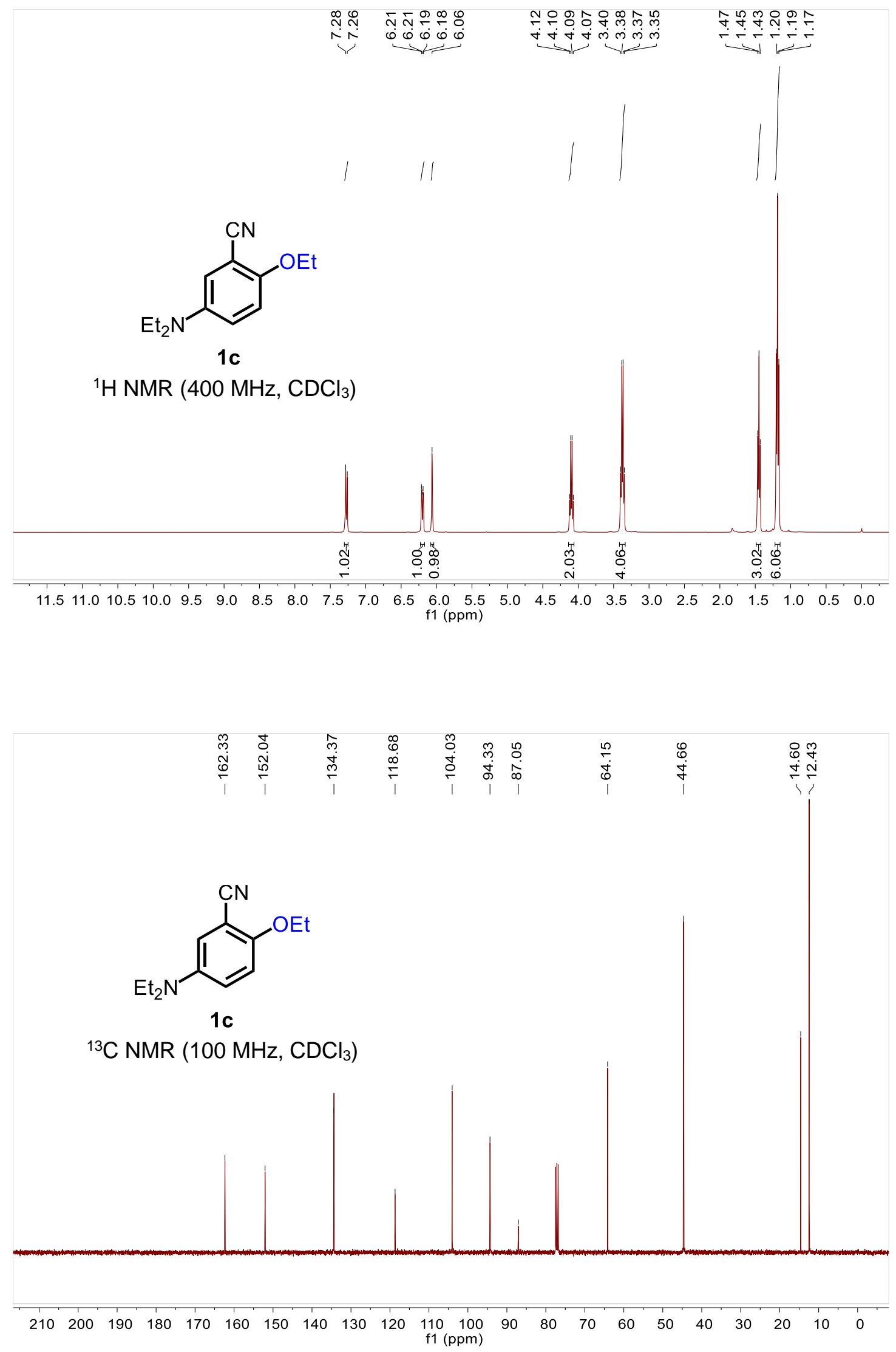

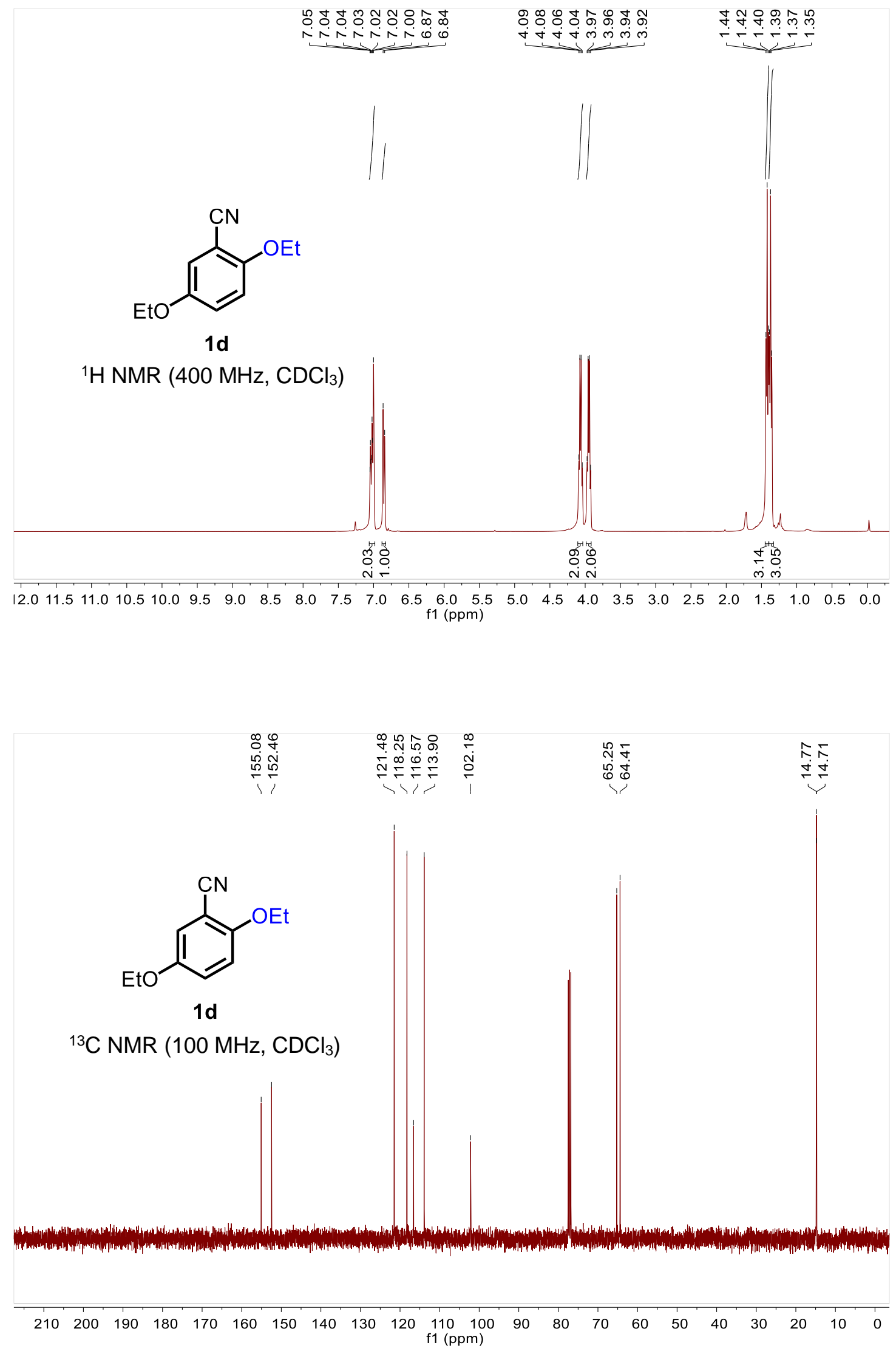

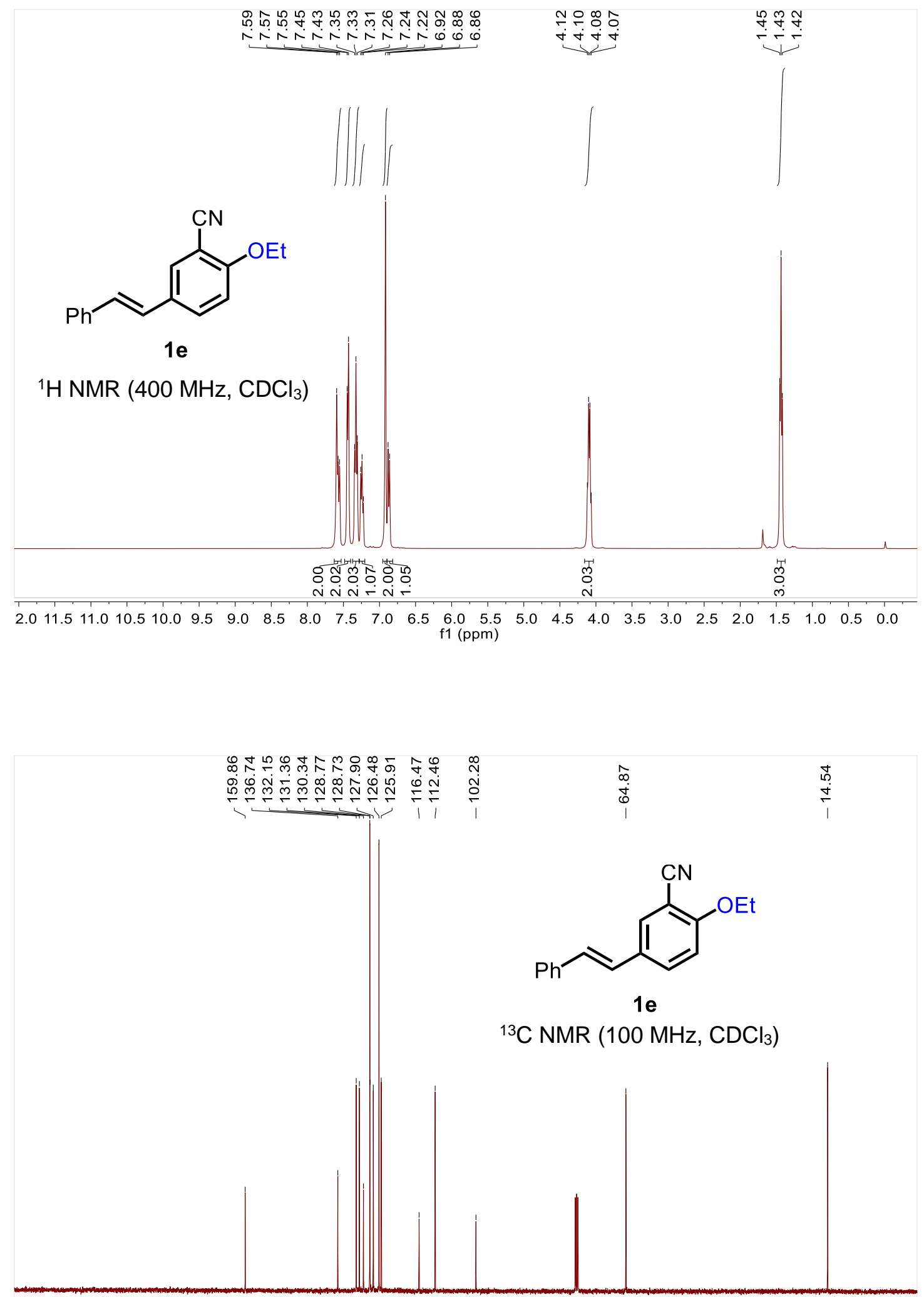

$\begin{array}{lllllllllllllllllllll}210 & 200 & 190 & 180 & 170 & 160 & 150 & 140 & 130 & 120 & \begin{array}{c}110 \\ \mathrm{f} 1(\mathrm{ppm})\end{array} & \begin{array}{l}100 \\ (120\end{array} & 80 & 70 & 60 & 50 & 40 & 30 & 20 & 10 & 0\end{array}$ 


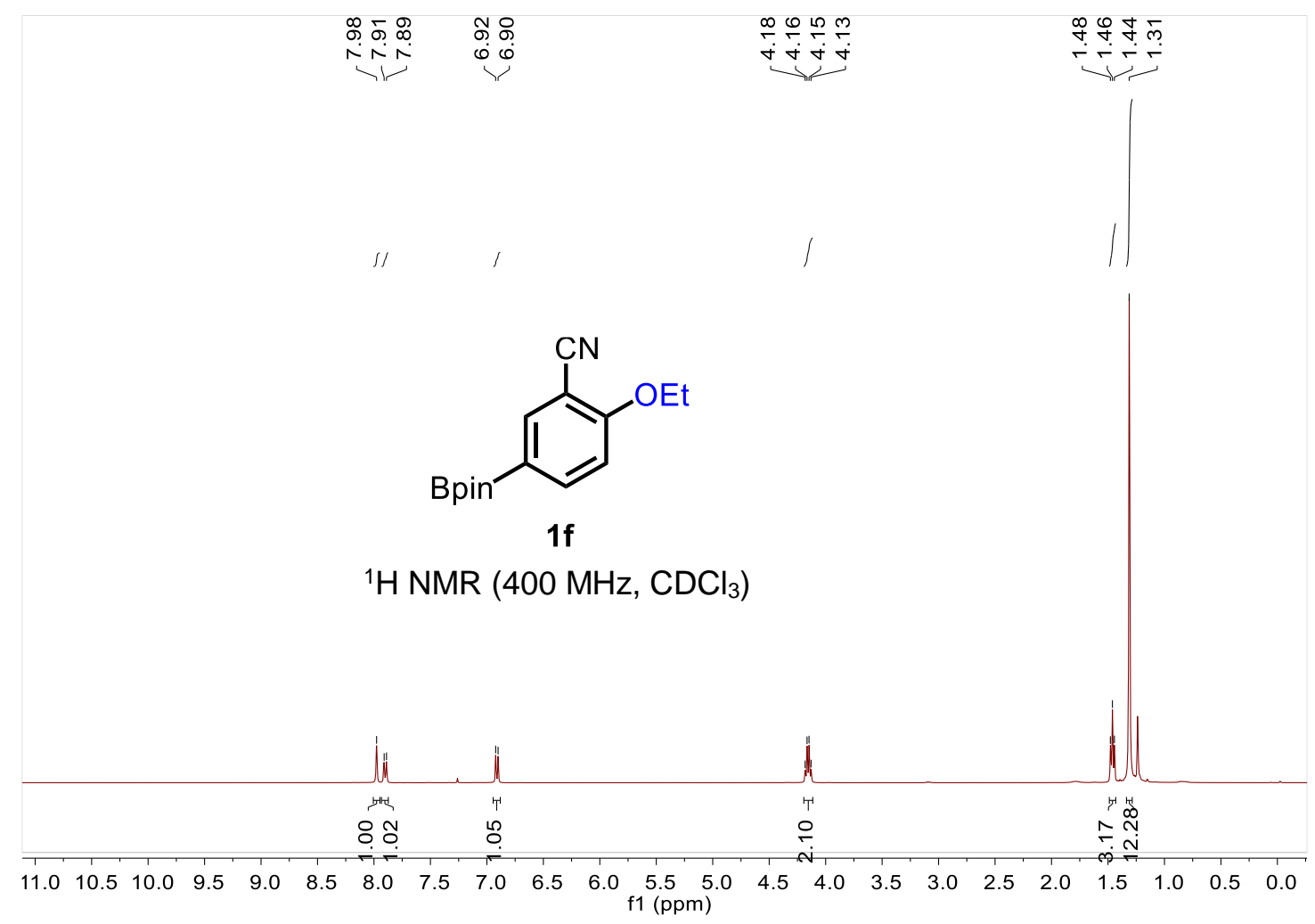

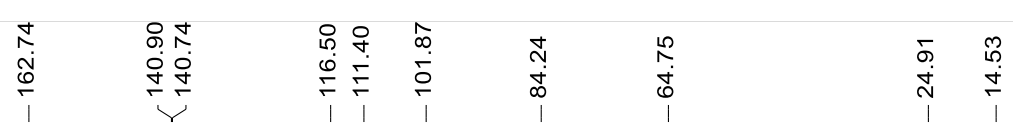<smiles>CCOc1ccc(Cc2ccccc2)cc1C#N</smiles>

${ }^{13} \mathrm{C}$ NMR $\left(100 \mathrm{MHz}, \mathrm{CDCl}_{3}\right)$

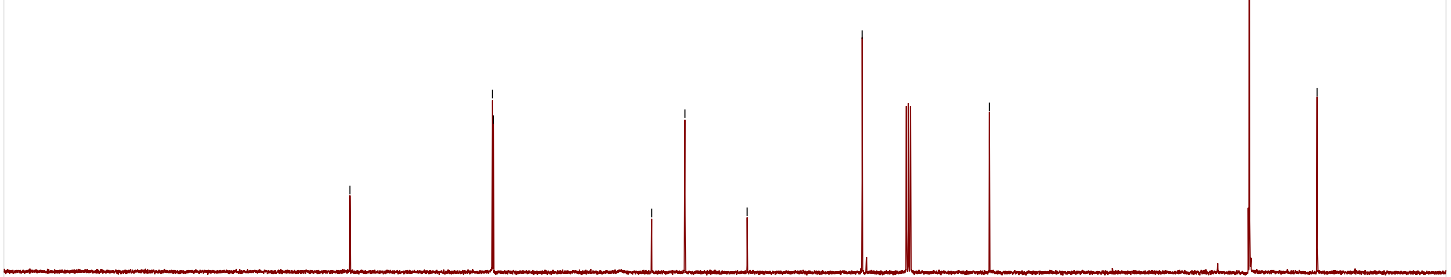

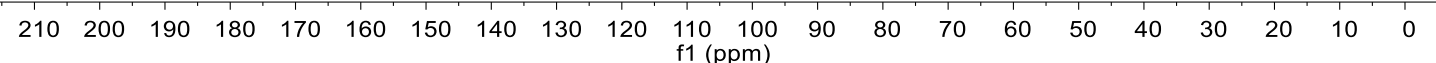



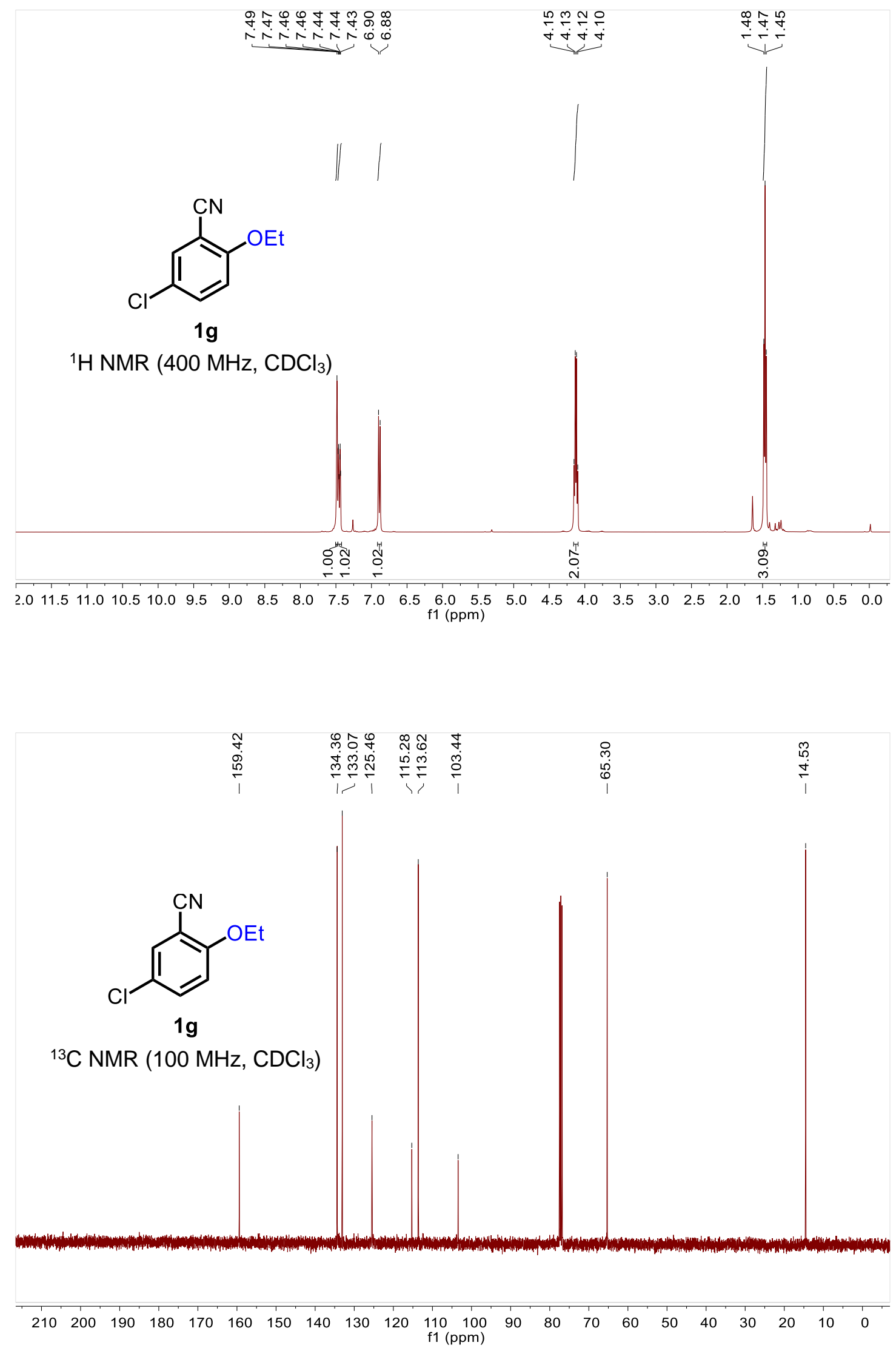

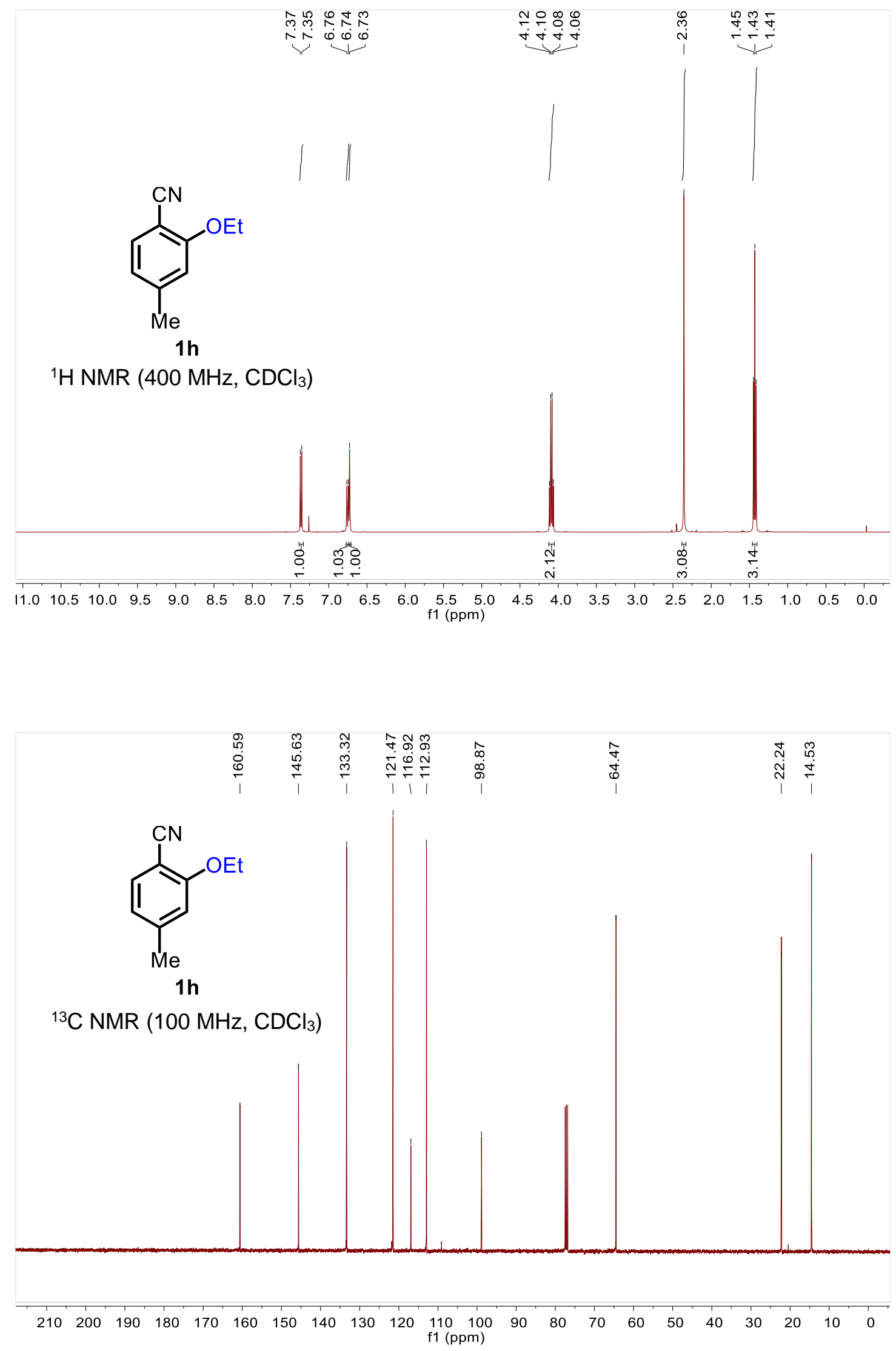


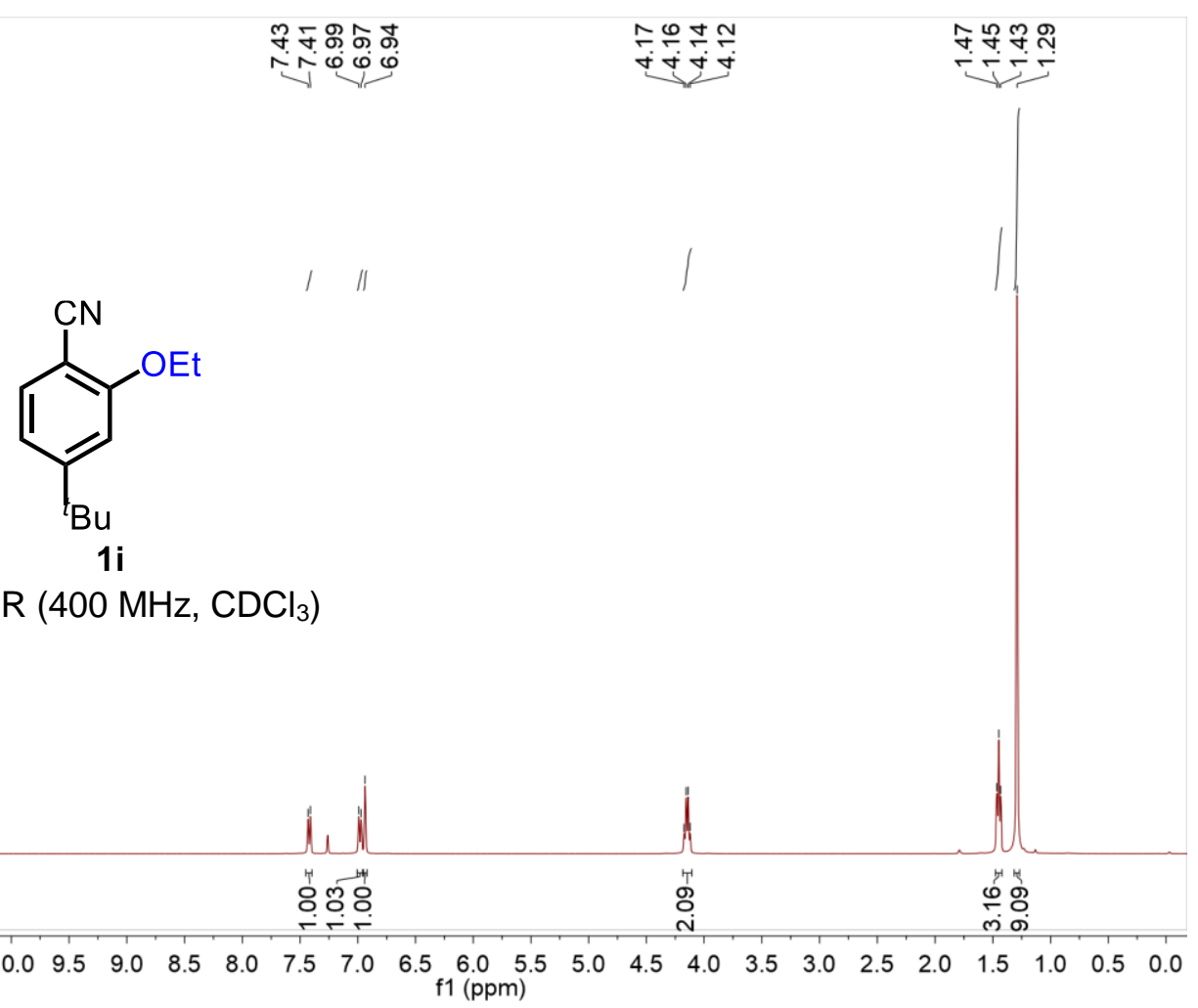

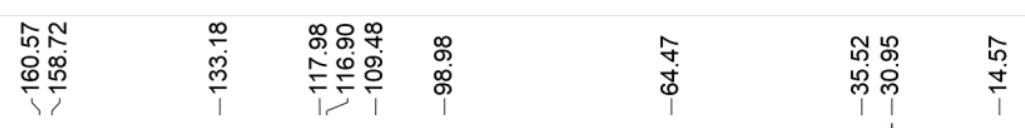

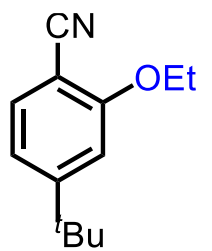

$1 \mathrm{i}$

${ }^{13} \mathrm{C} \operatorname{NMR}\left(100 \mathrm{MHz}, \mathrm{CDCl}_{3}\right)$

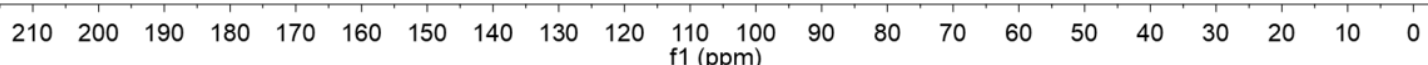




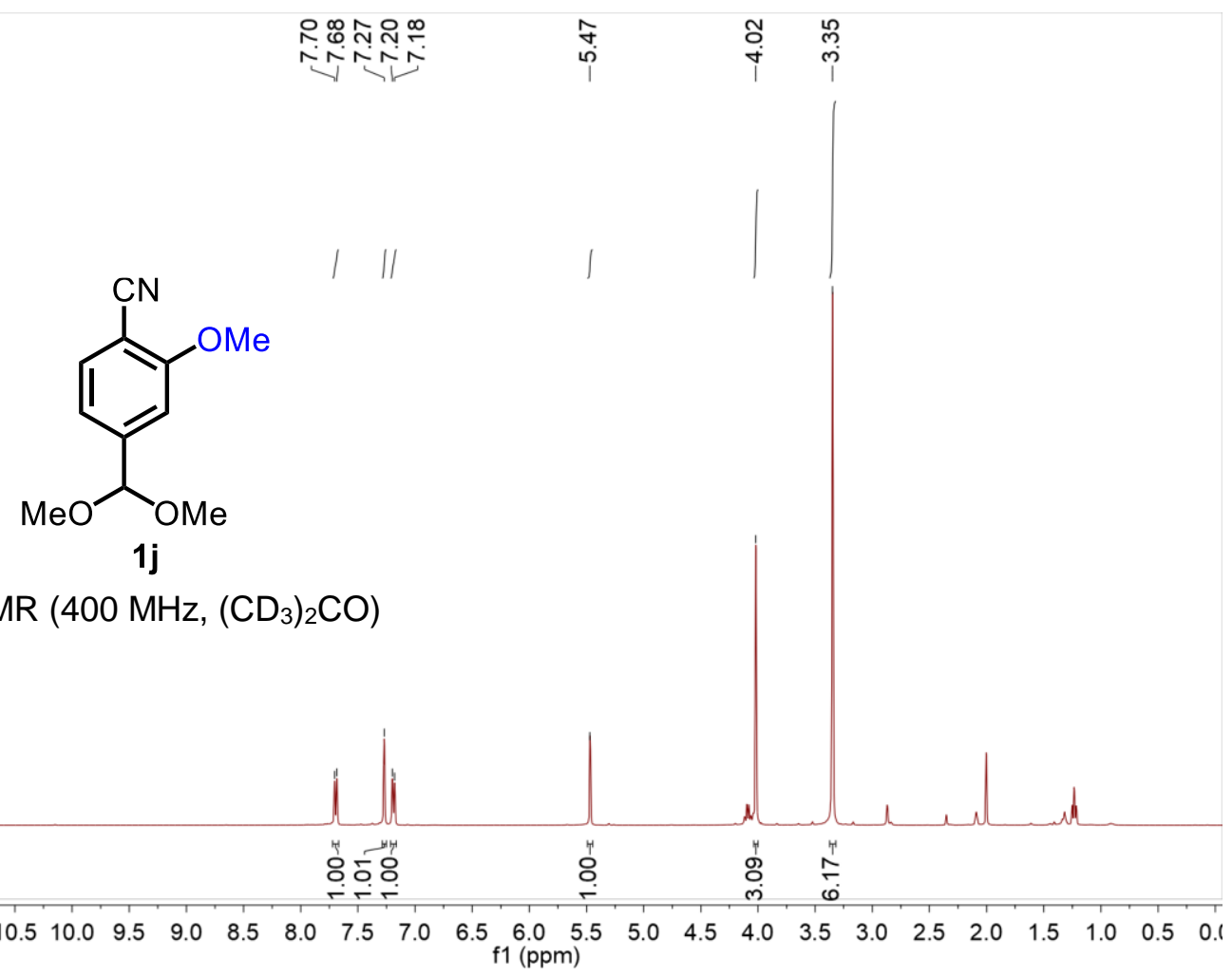

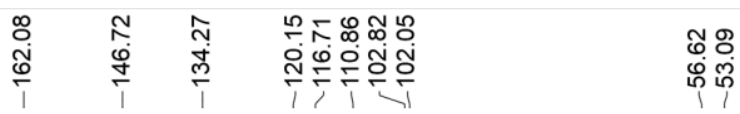

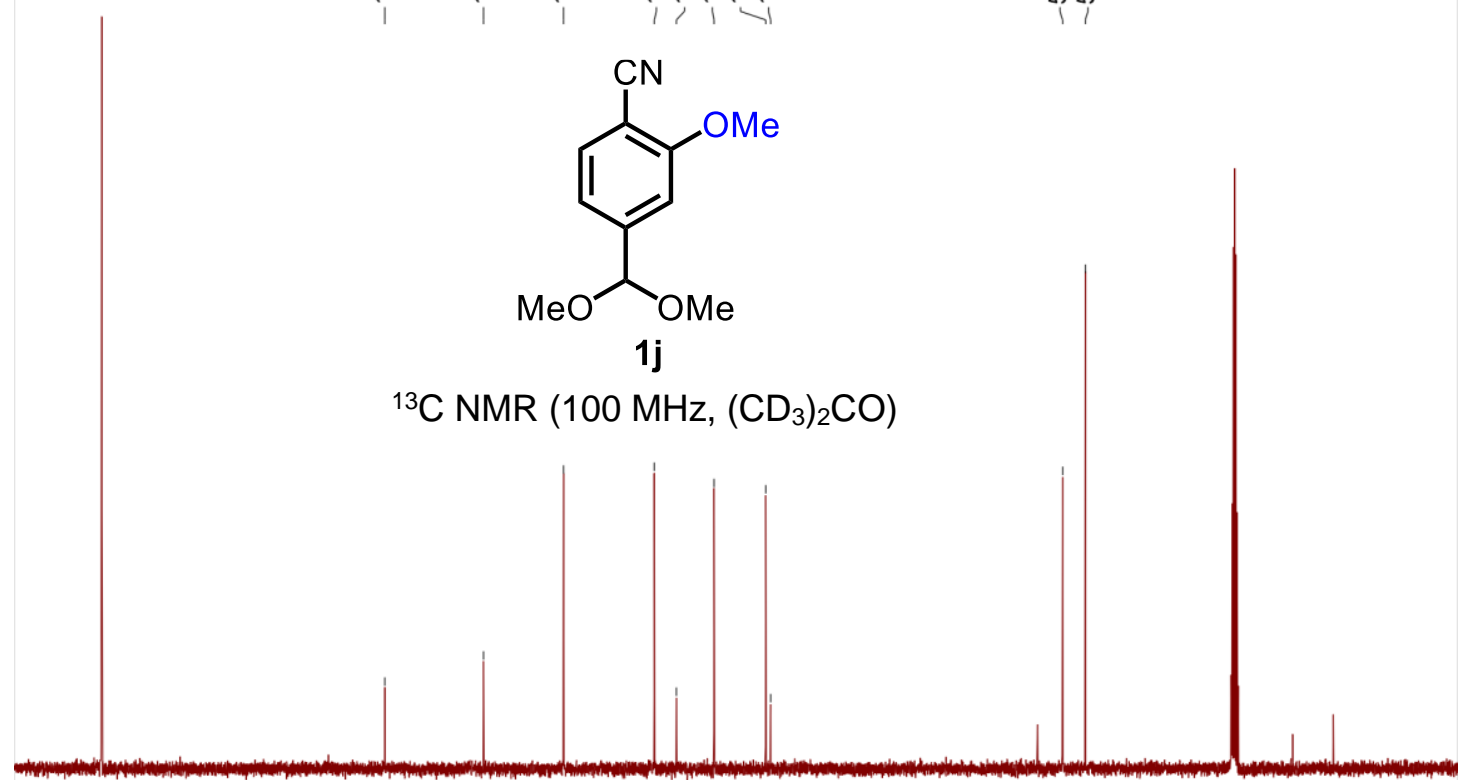

$\begin{array}{llllllllllllllllllllll}210 & 200 & 190 & 180 & 170 & 160 & 150 & 140 & 130 & 120 \begin{array}{c}110 \\ \mathrm{f} 1(\mathrm{ppm})\end{array} & 90 & 80 & 70 & 60 & 50 & 40 & 30 & 20 & 10 & 0\end{array}$ 

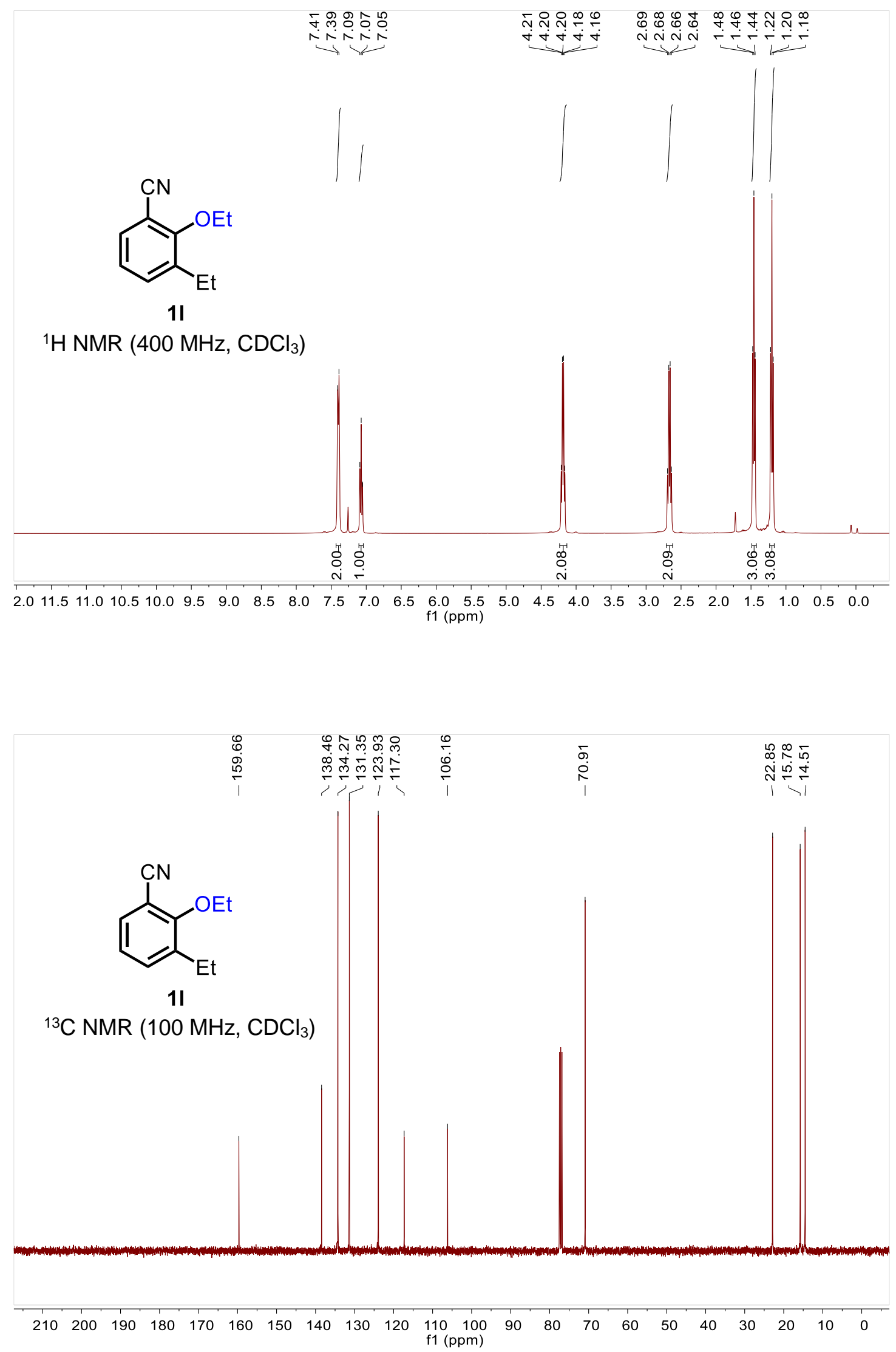

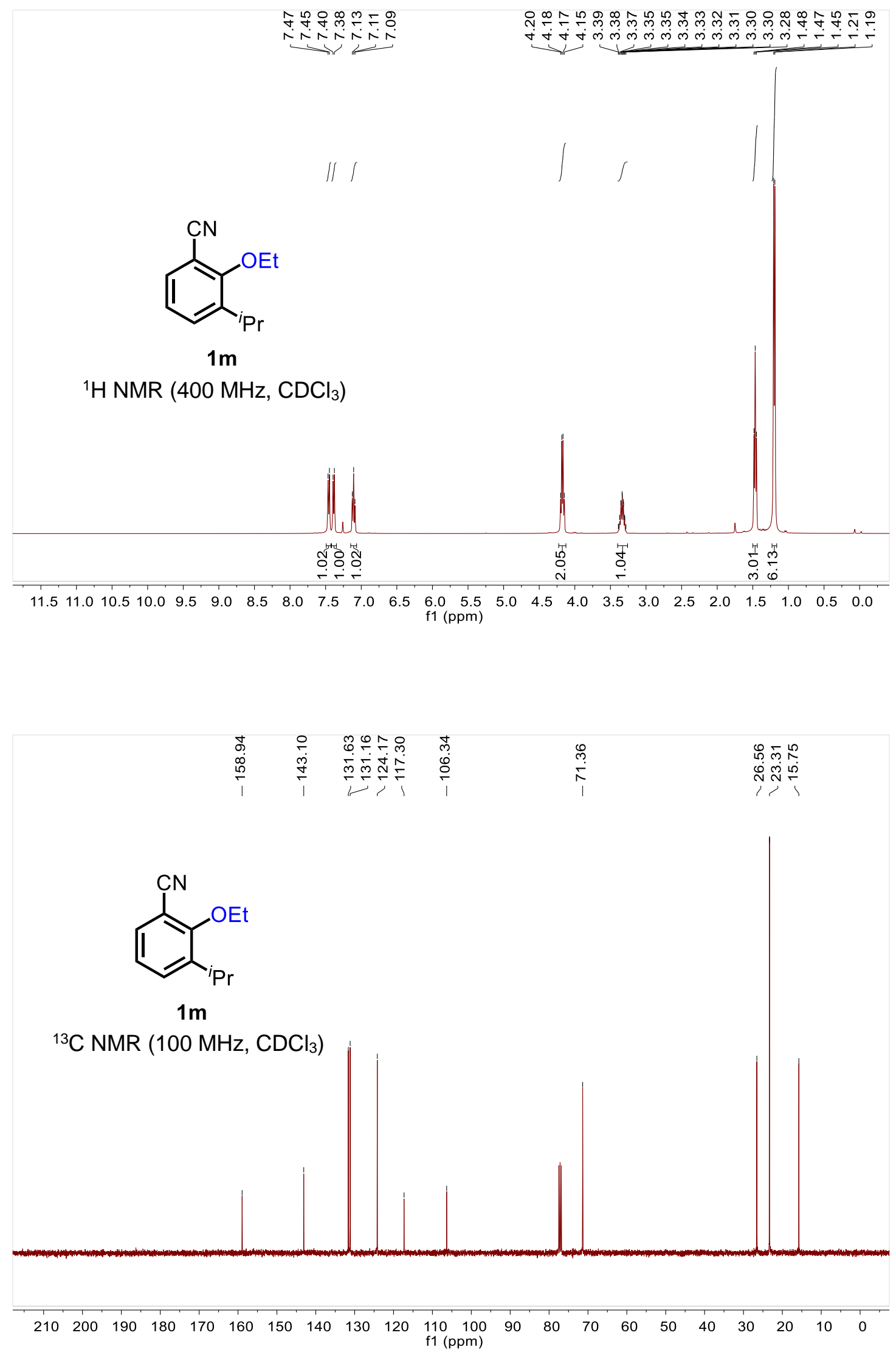

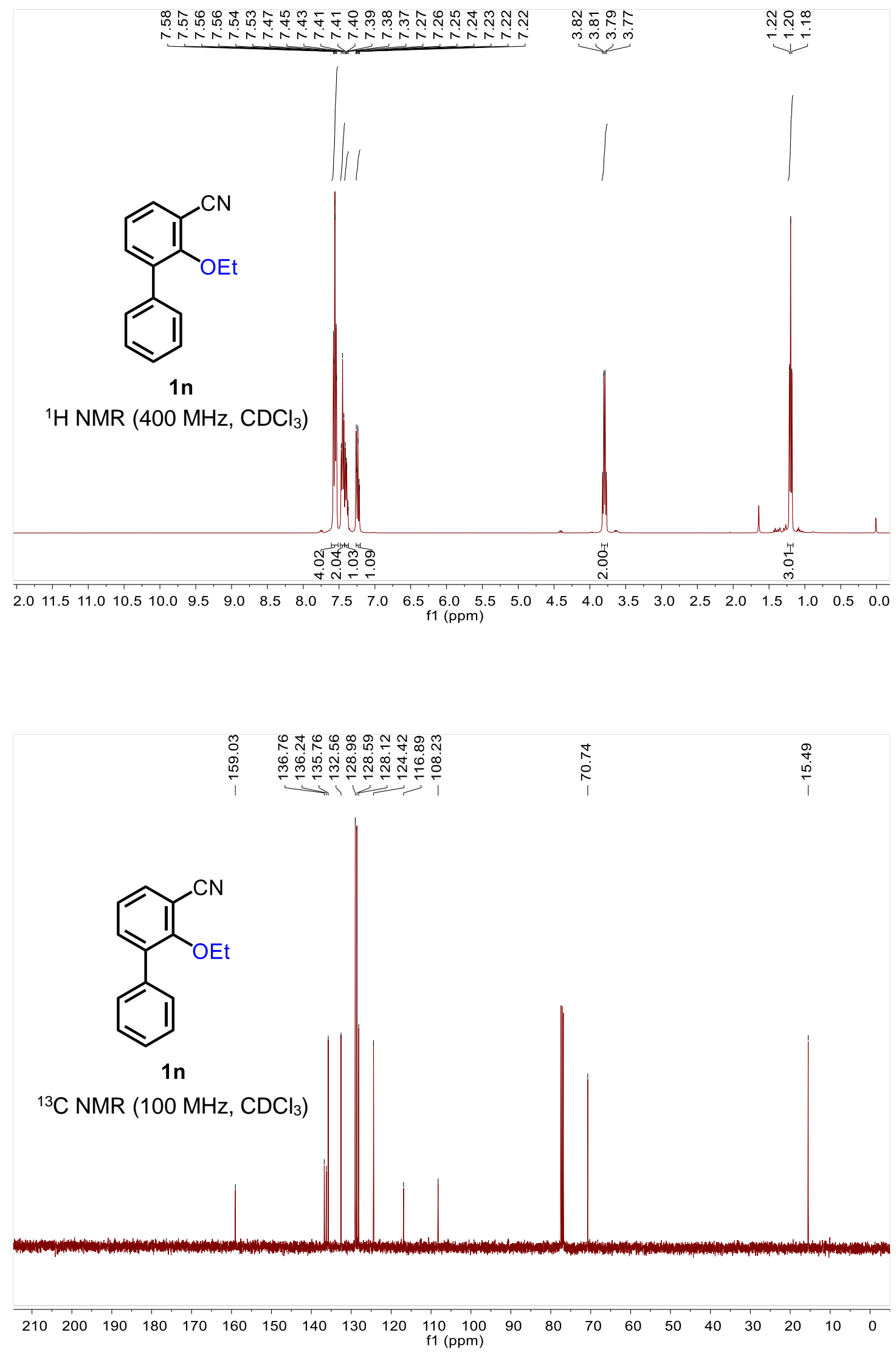

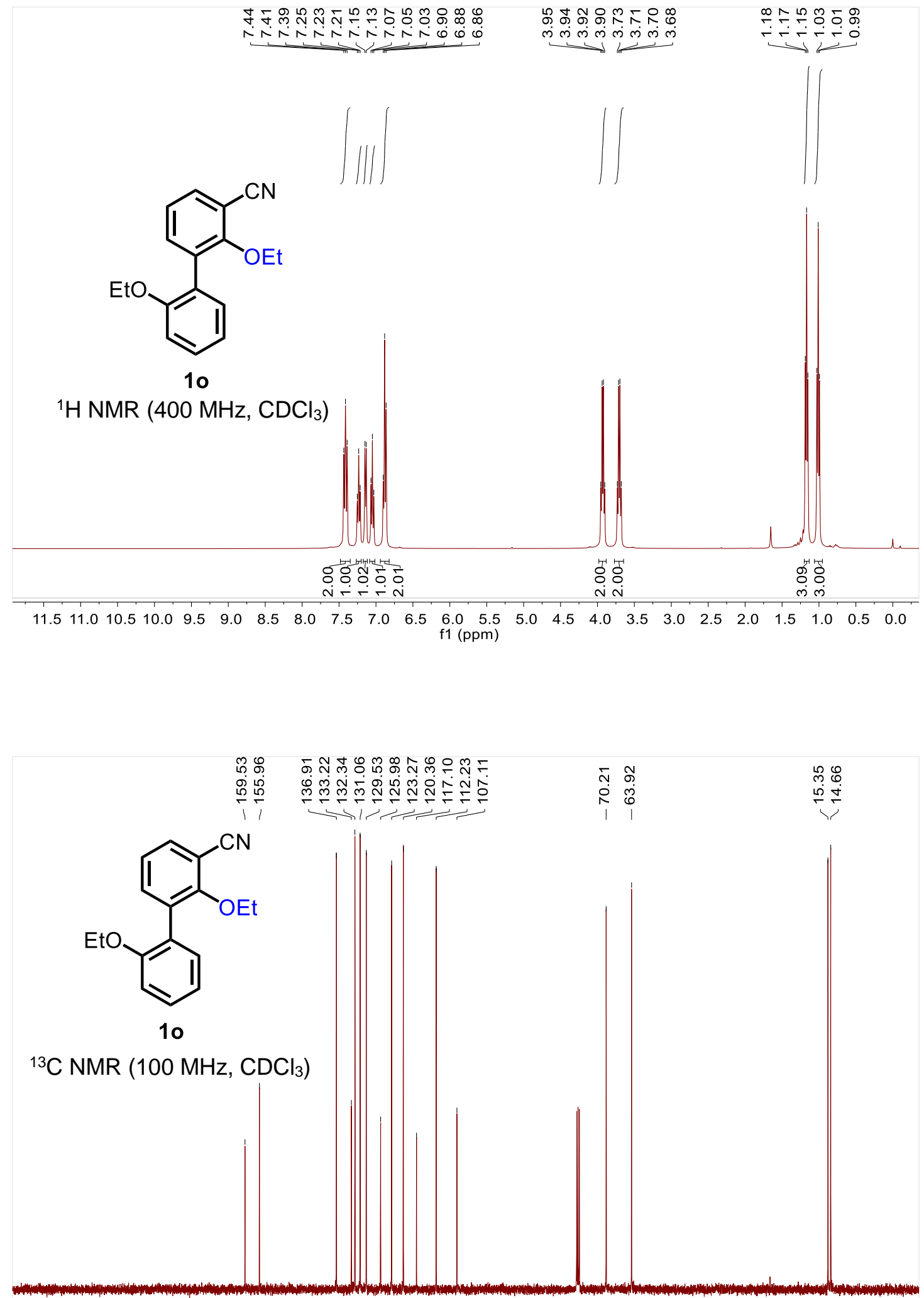

$\begin{array}{lllllllllllllllllllll}210 & 200 & 190 & 180 & 170 & 160 & 150 & 140 & 130 & 120 & \begin{array}{c}110 \\ \mathrm{f} 1(\mathrm{ppm})\end{array} & \begin{array}{l}100 \\ (\mathrm{ppo})\end{array} & 80 & 70 & 60 & 50 & 40 & 30 & 20 & 10 & 0\end{array}$ 

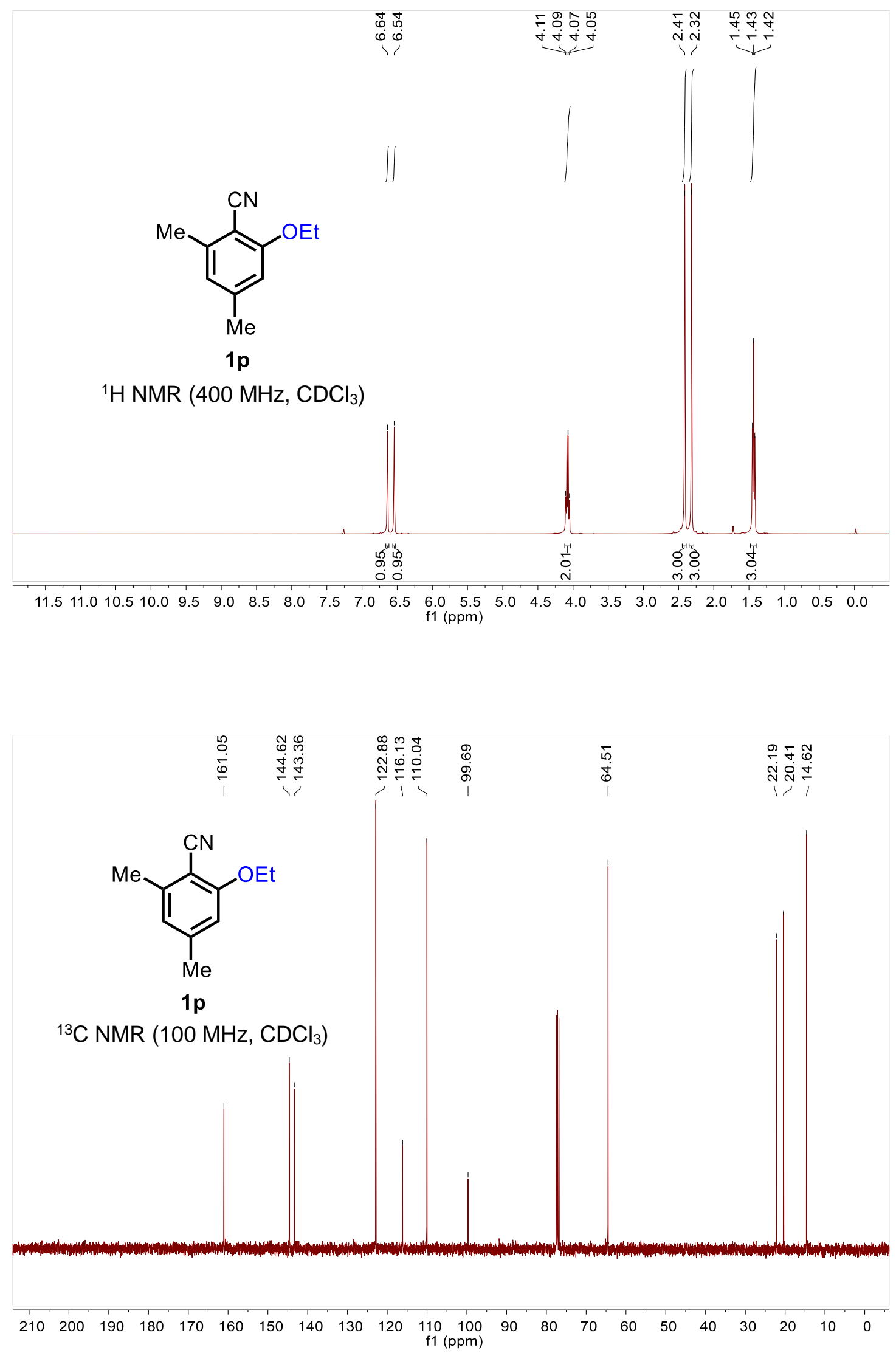

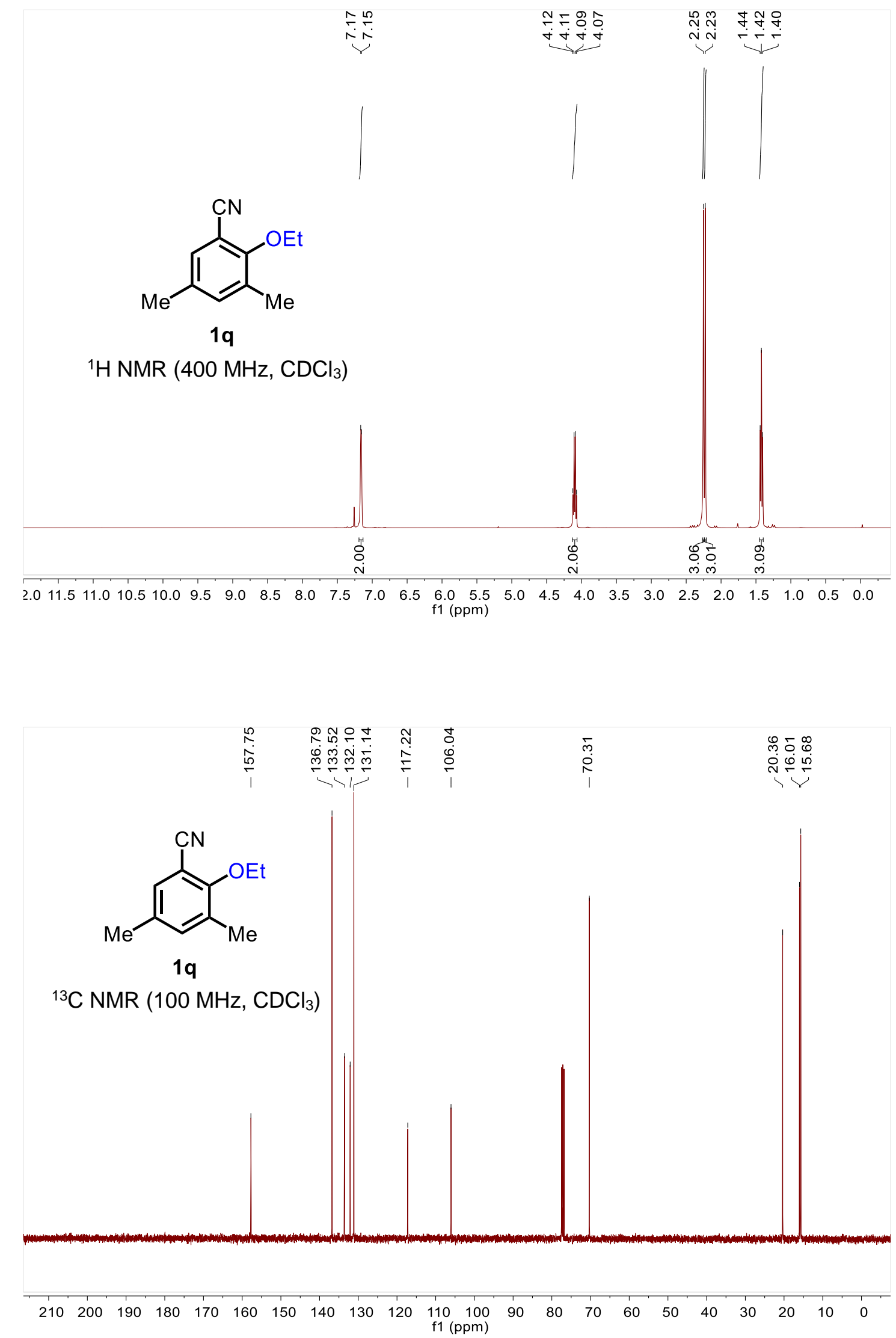

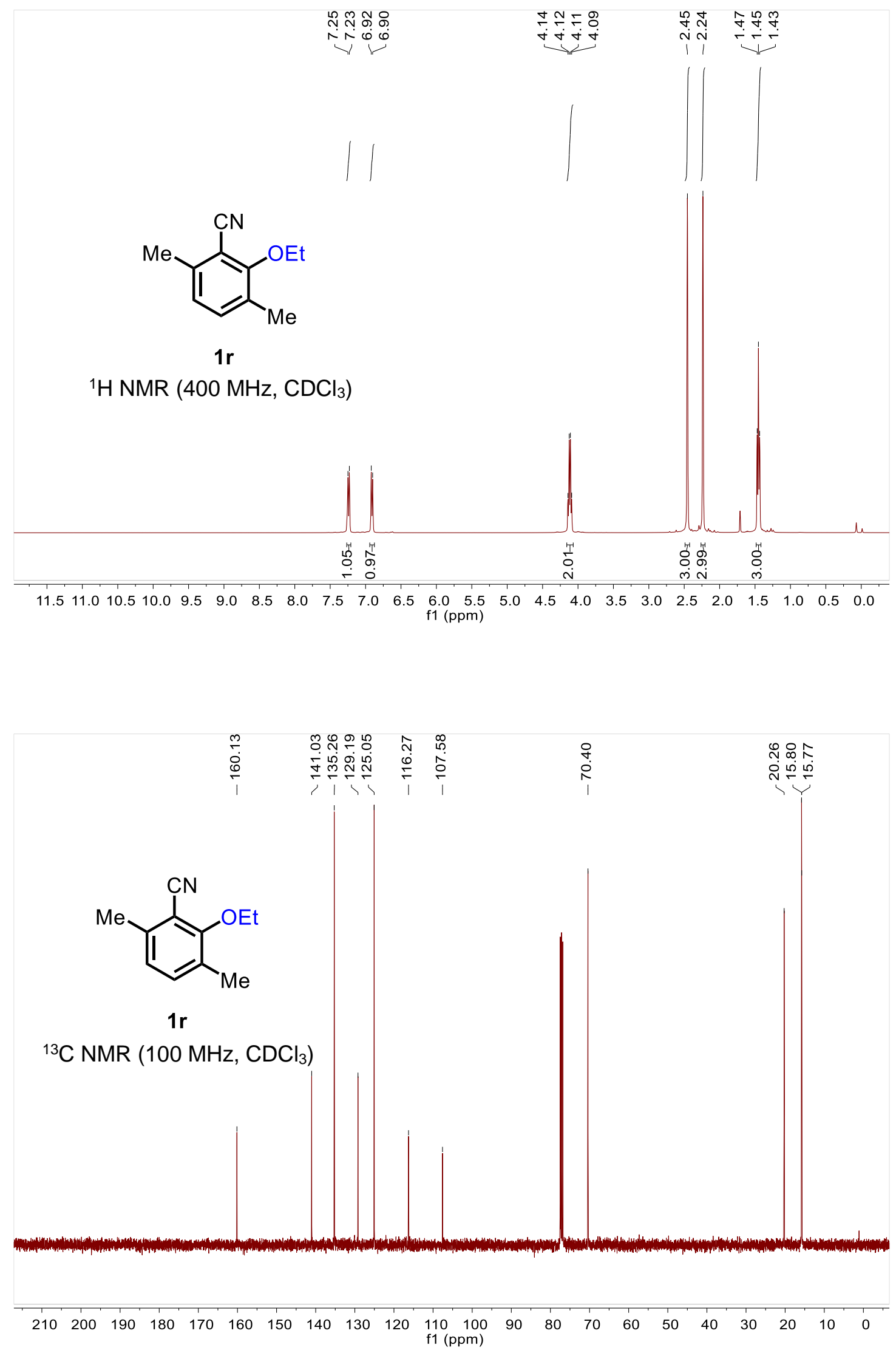

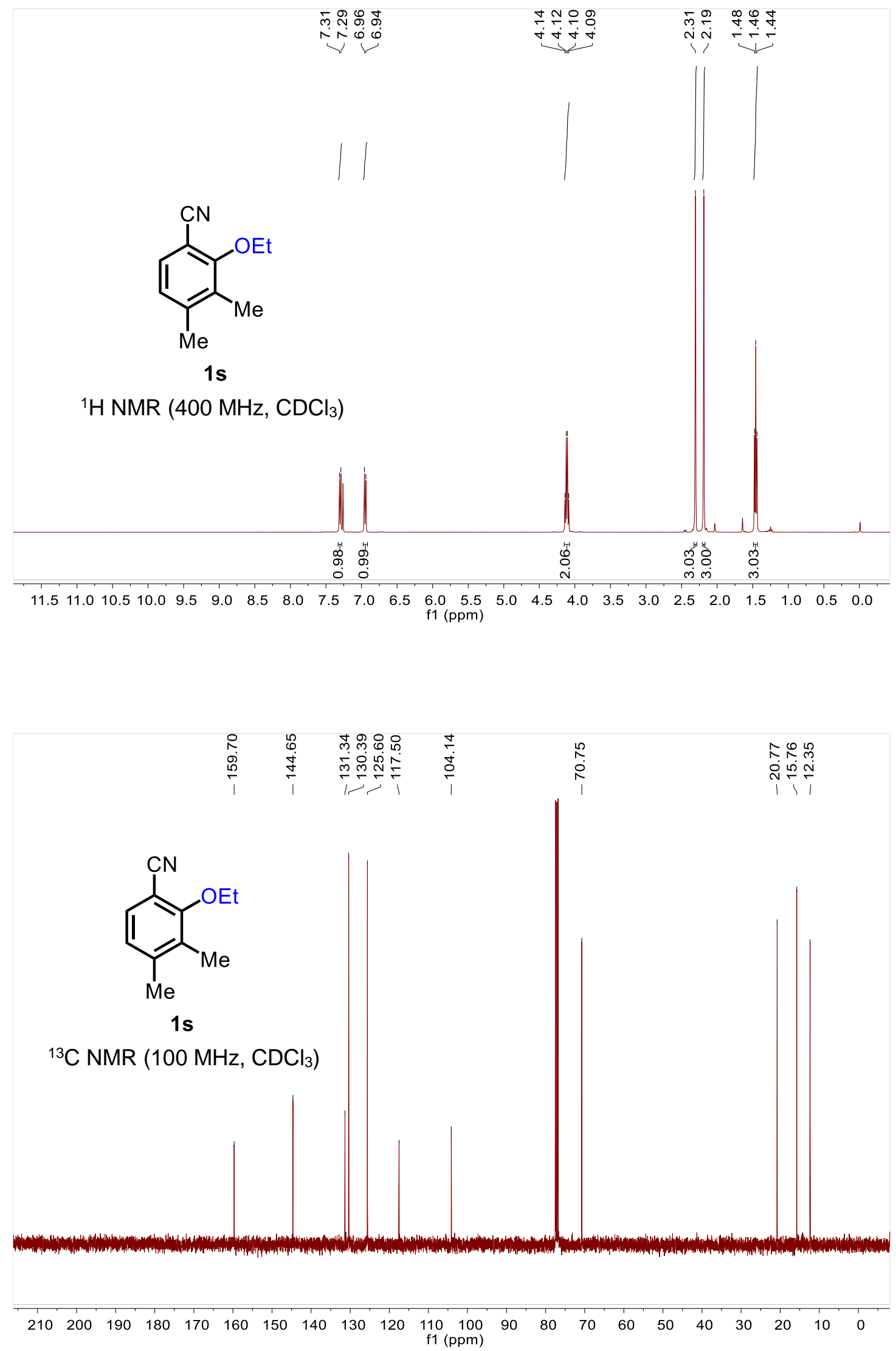


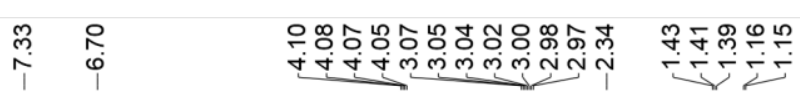<smiles>CCCc1cc(C#N)c(OCC)cc1C</smiles>

$1 \mathrm{t}$

${ }^{1} \mathrm{H} \mathrm{NMR}\left(400 \mathrm{MHz}, \mathrm{CDCl}_{3}\right)$

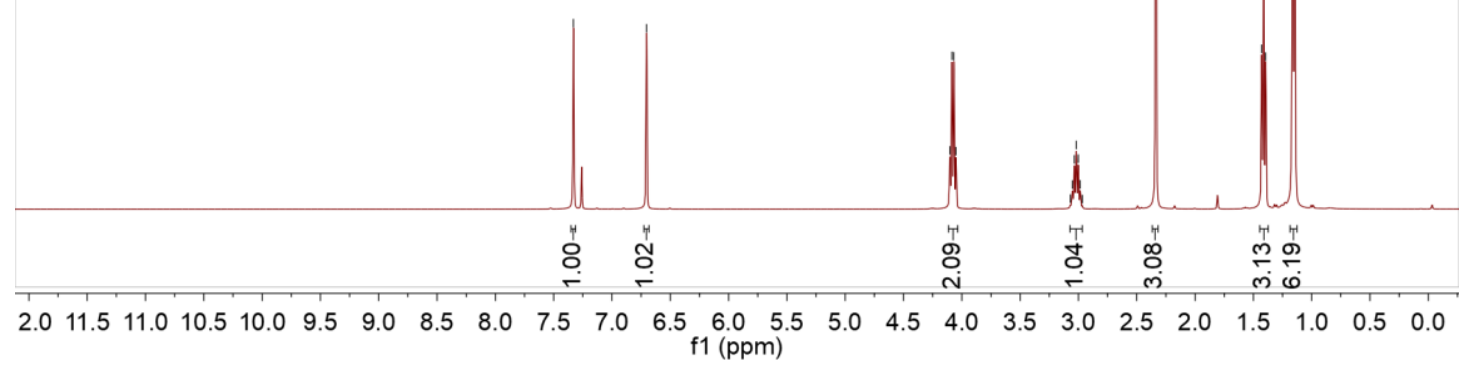

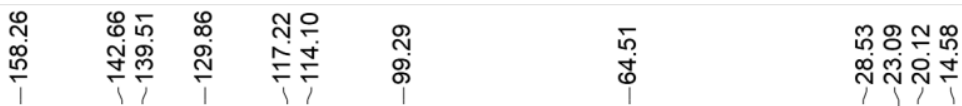<smiles>CCOc1cc(C)c(C(C)C)cc1C#N</smiles>

$1 \mathbf{t}$

${ }^{13} \mathrm{C} \mathrm{NMR}\left(100 \mathrm{MHz}, \mathrm{CDCl}_{3}\right)$

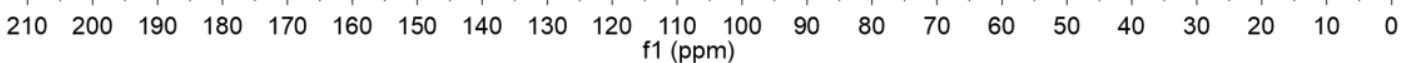



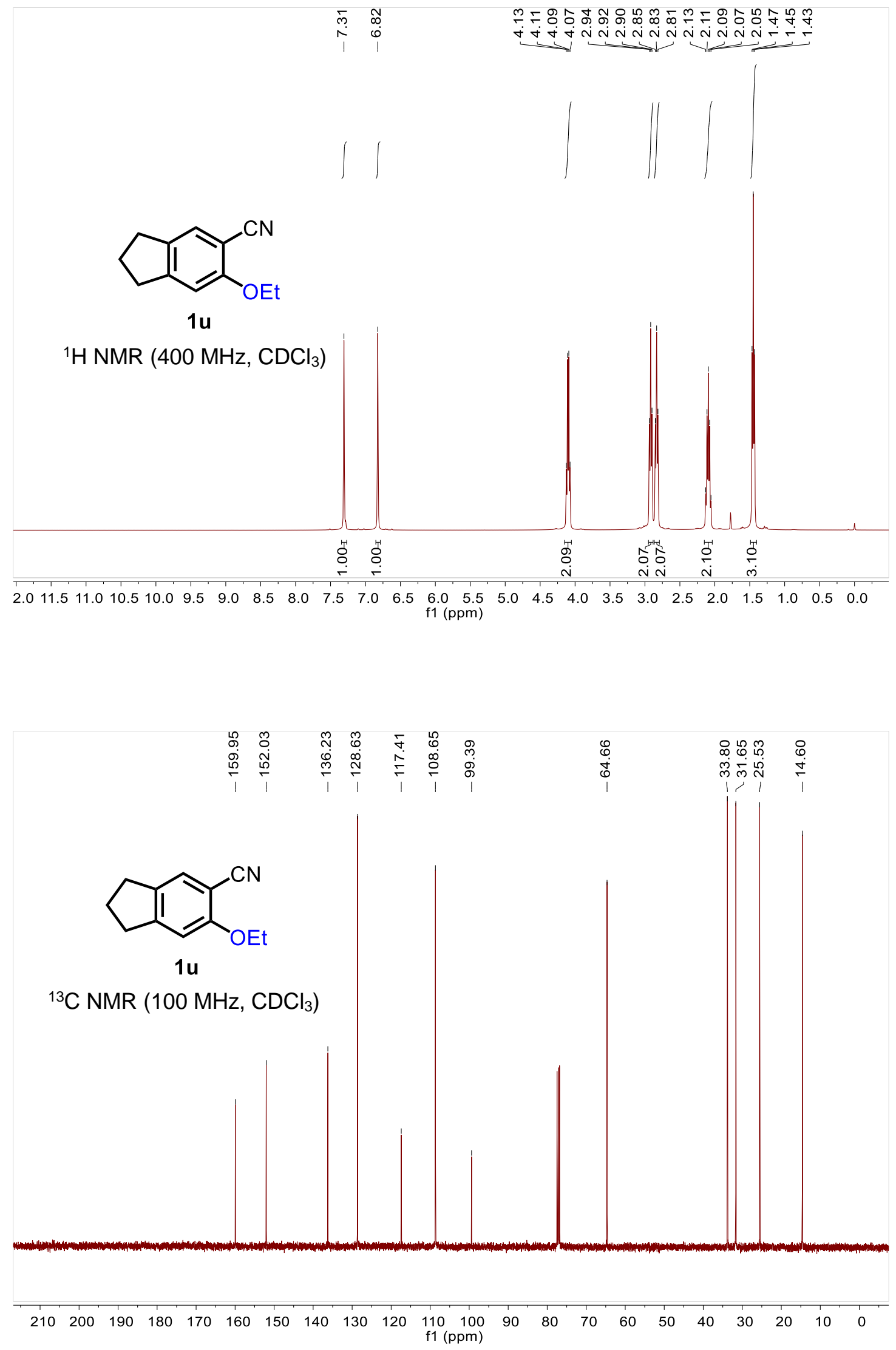

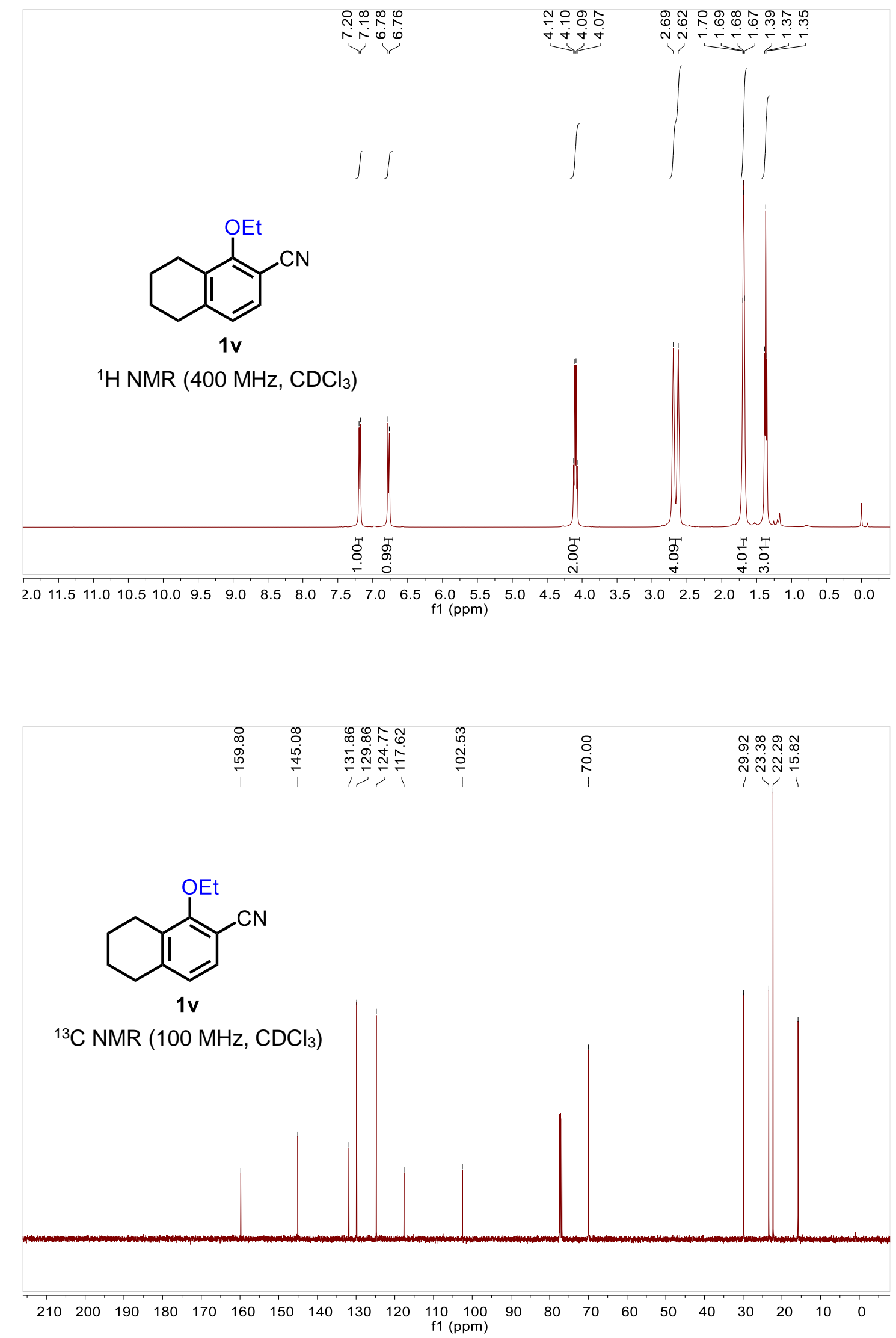
<smiles>CCOc1ccc2c(ccc3ccccc32)c1C#N</smiles><smiles>C1=CC2CCC(C=C1)C2</smiles>

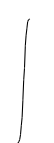

$1 \mathrm{y}$

${ }^{1} \mathrm{H}$ NMR $\left(400 \mathrm{MHz}, \mathrm{CDCl}_{3}\right)$
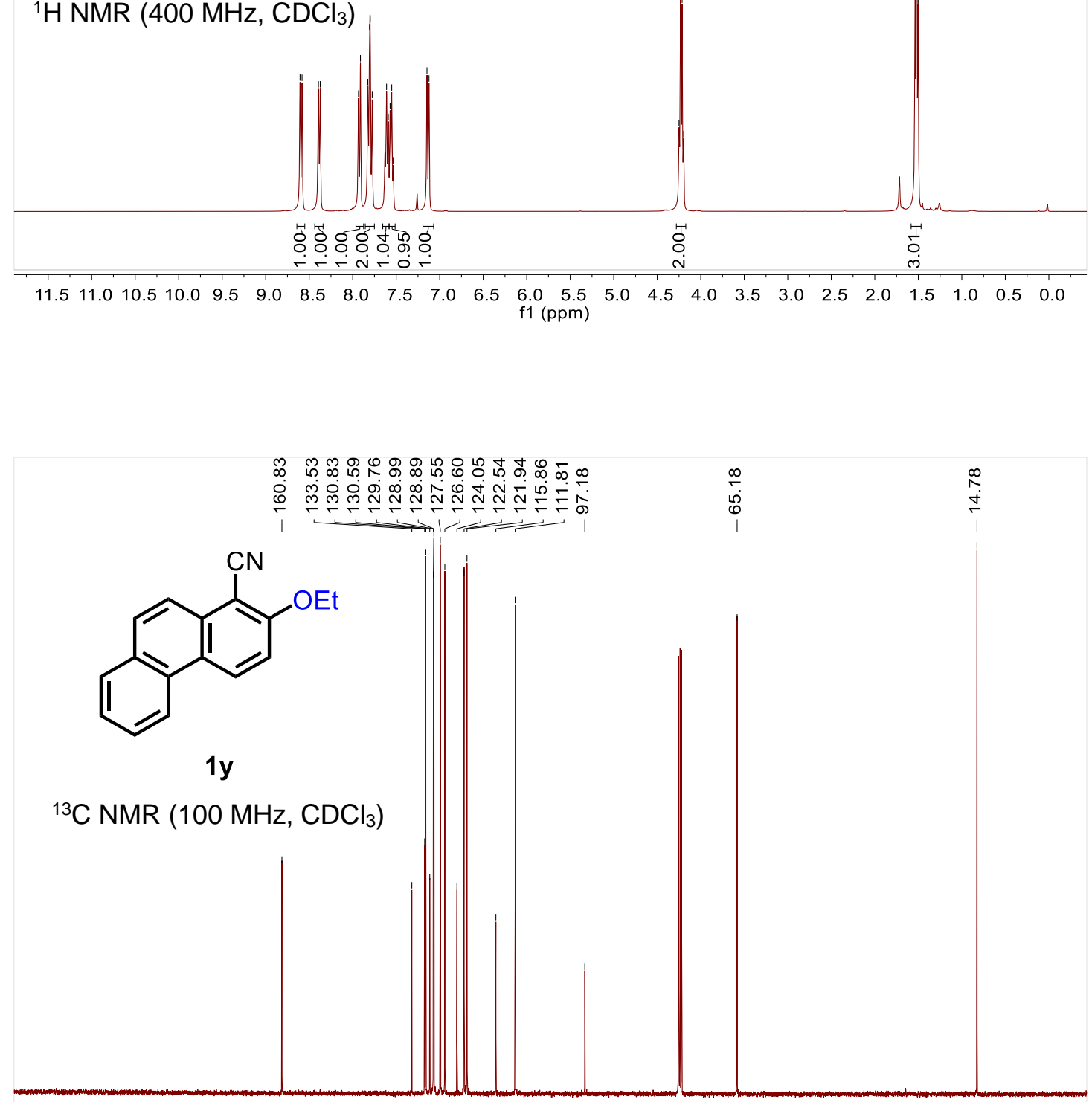

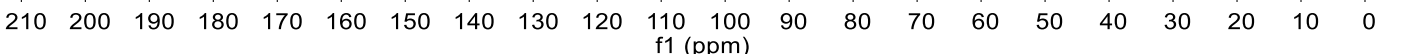


<smiles>C#CC#CCc1cc2ccc3cccc4ccc(c1OCC)c2c34</smiles>

$1 z$

${ }^{1} \mathrm{H}$ NMR $\left(400 \mathrm{MHz}, \mathrm{CDCl}_{3}\right)$

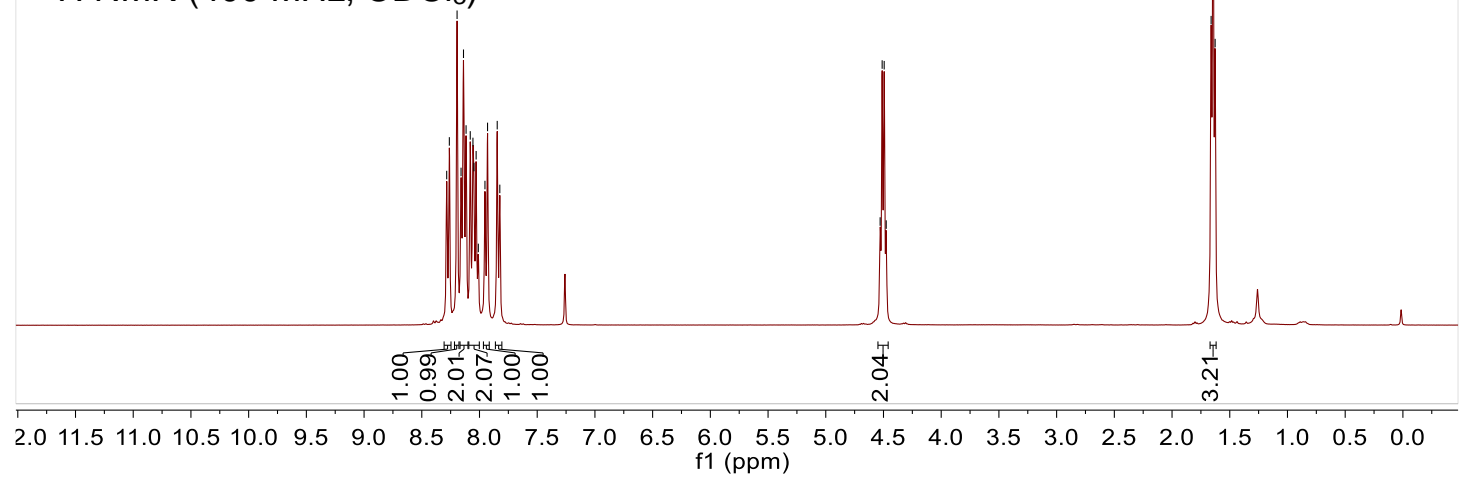

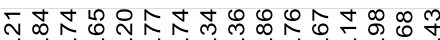

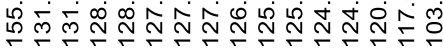
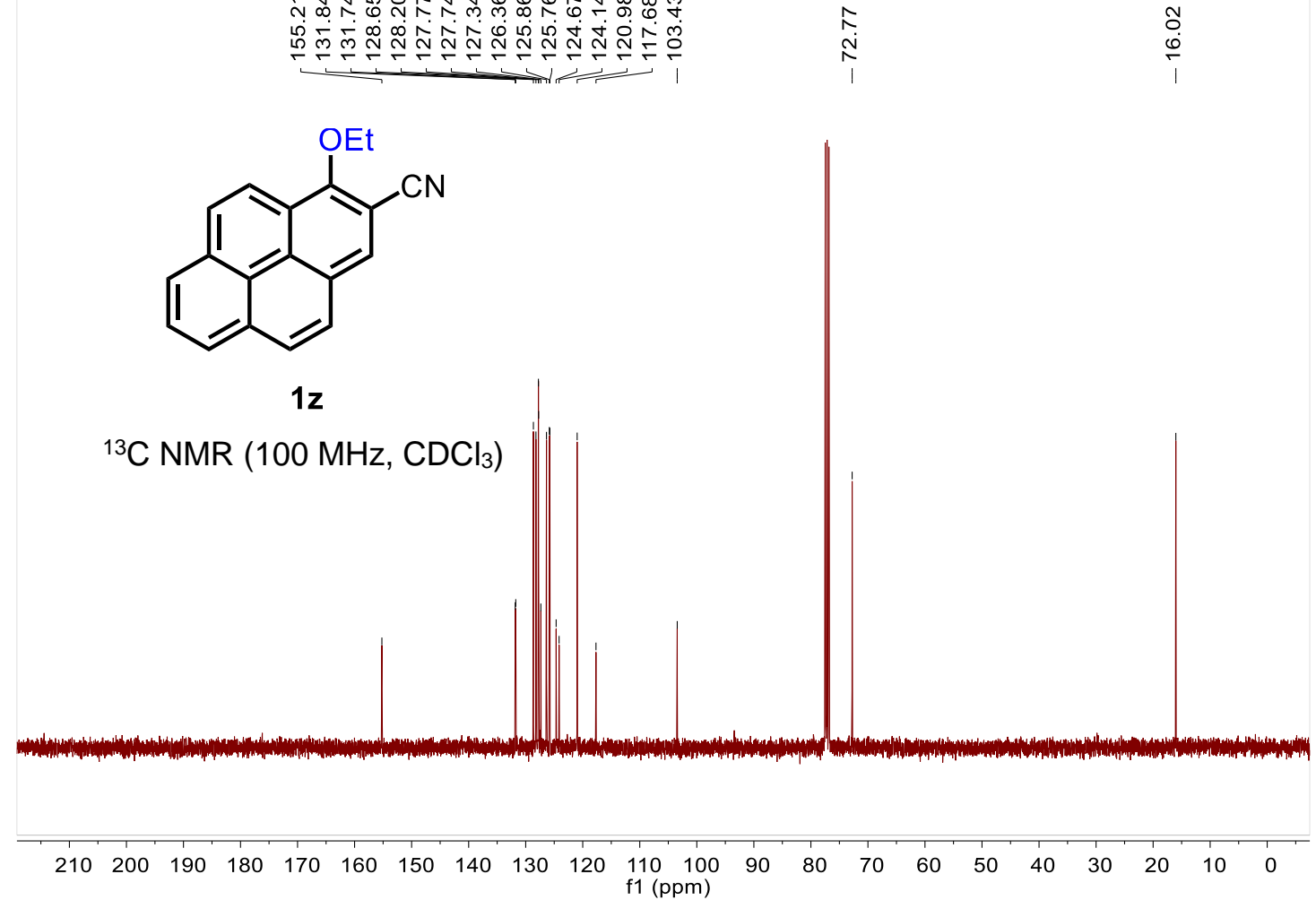

$1 z$

${ }^{13} \mathrm{C}$ NMR $\left(100 \mathrm{MHz}, \mathrm{CDCl}_{3}\right)$

$\begin{array}{llllllllllll}210 & 200 & 190 & 180 & 170 & 160 & 150 & 140 & 130 & 120 & \begin{array}{l}110 \\ \mathrm{f} 1\end{array}(\mathrm{ppm})\end{array}$ 


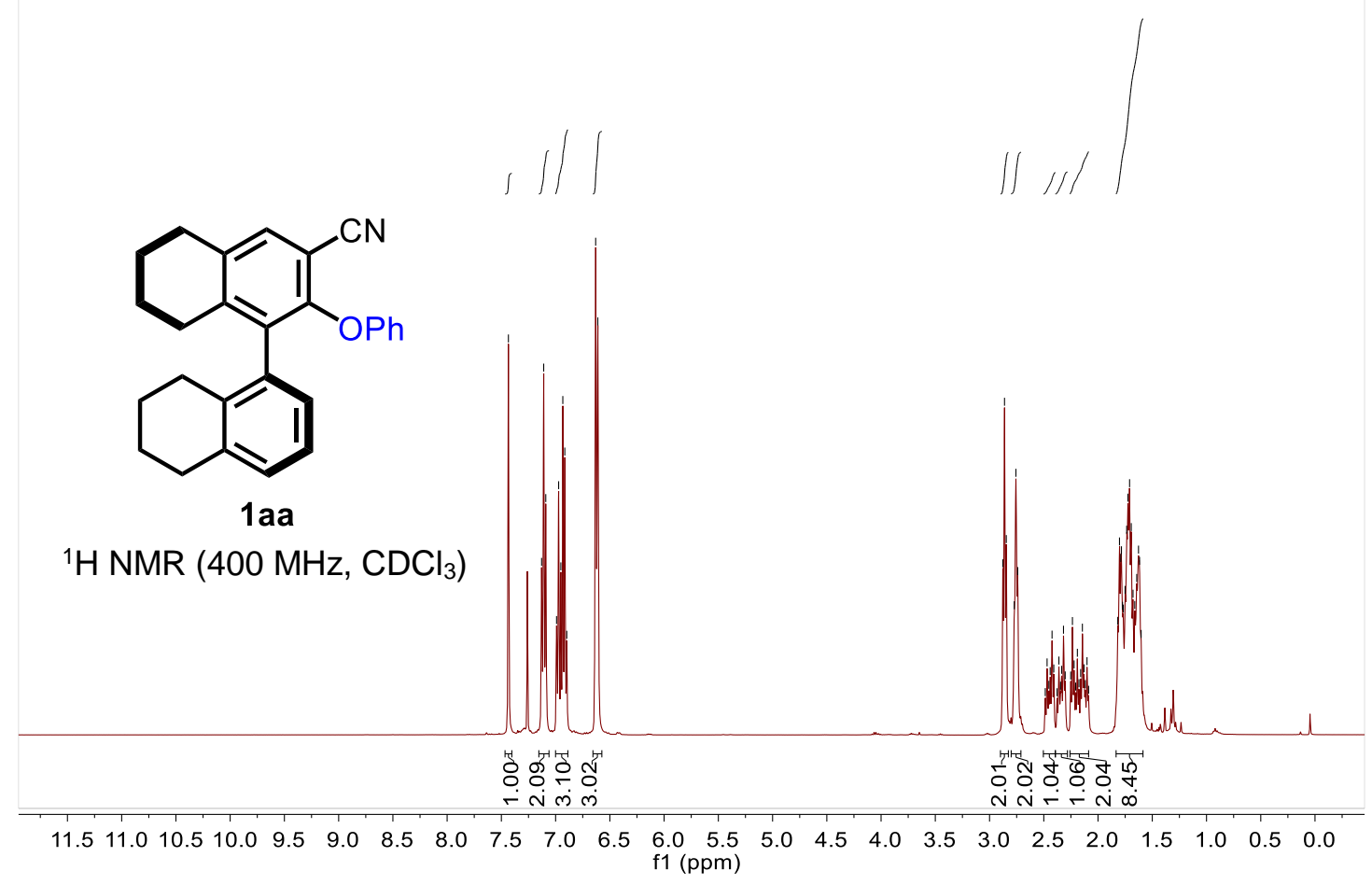

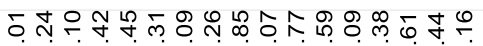

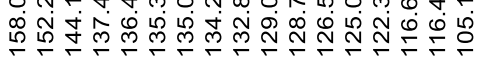

œ岗

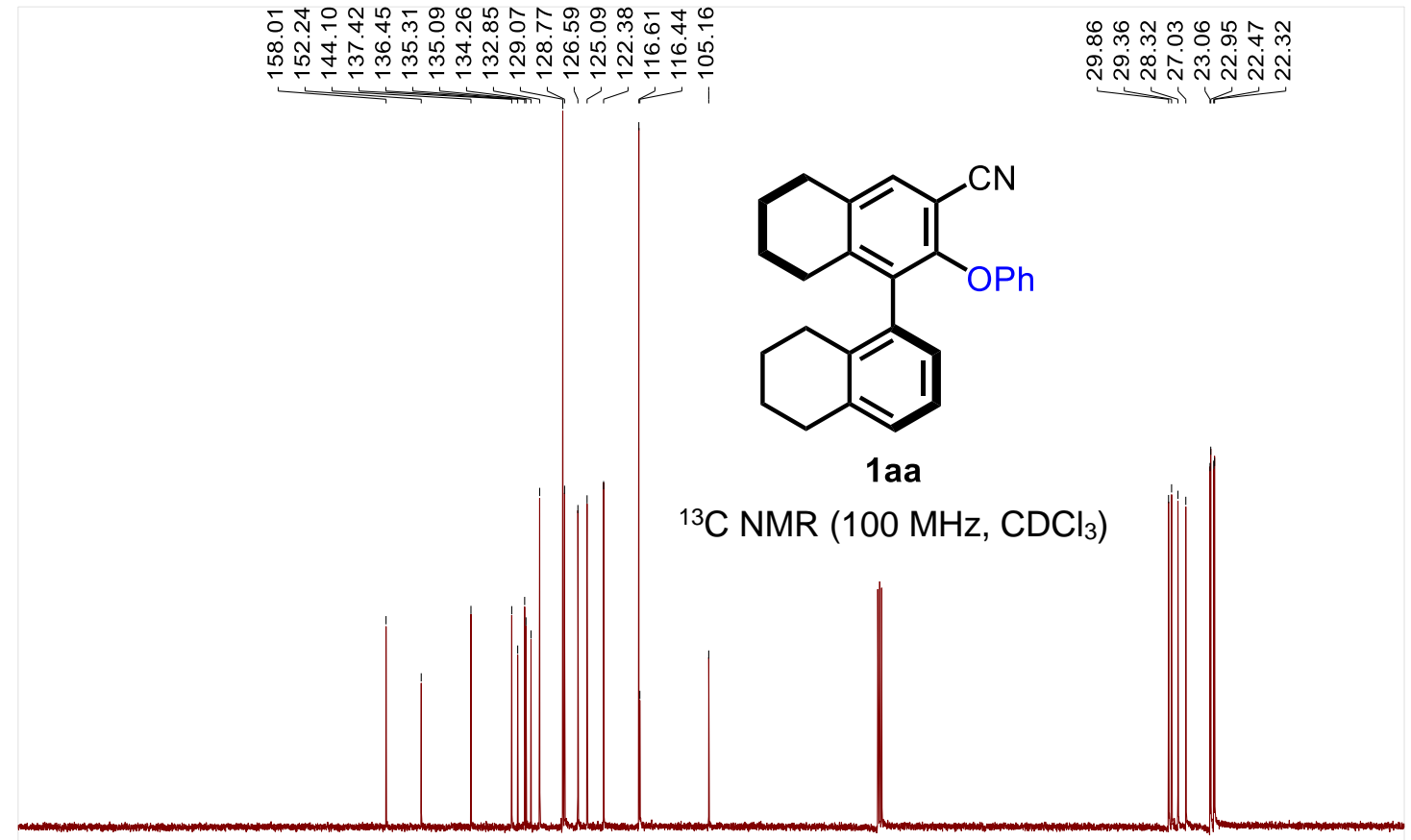

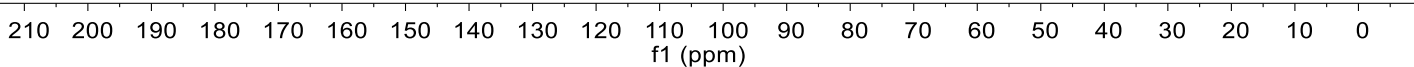




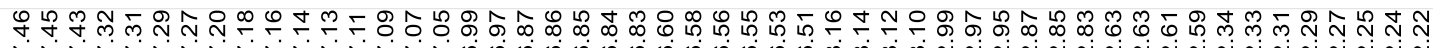

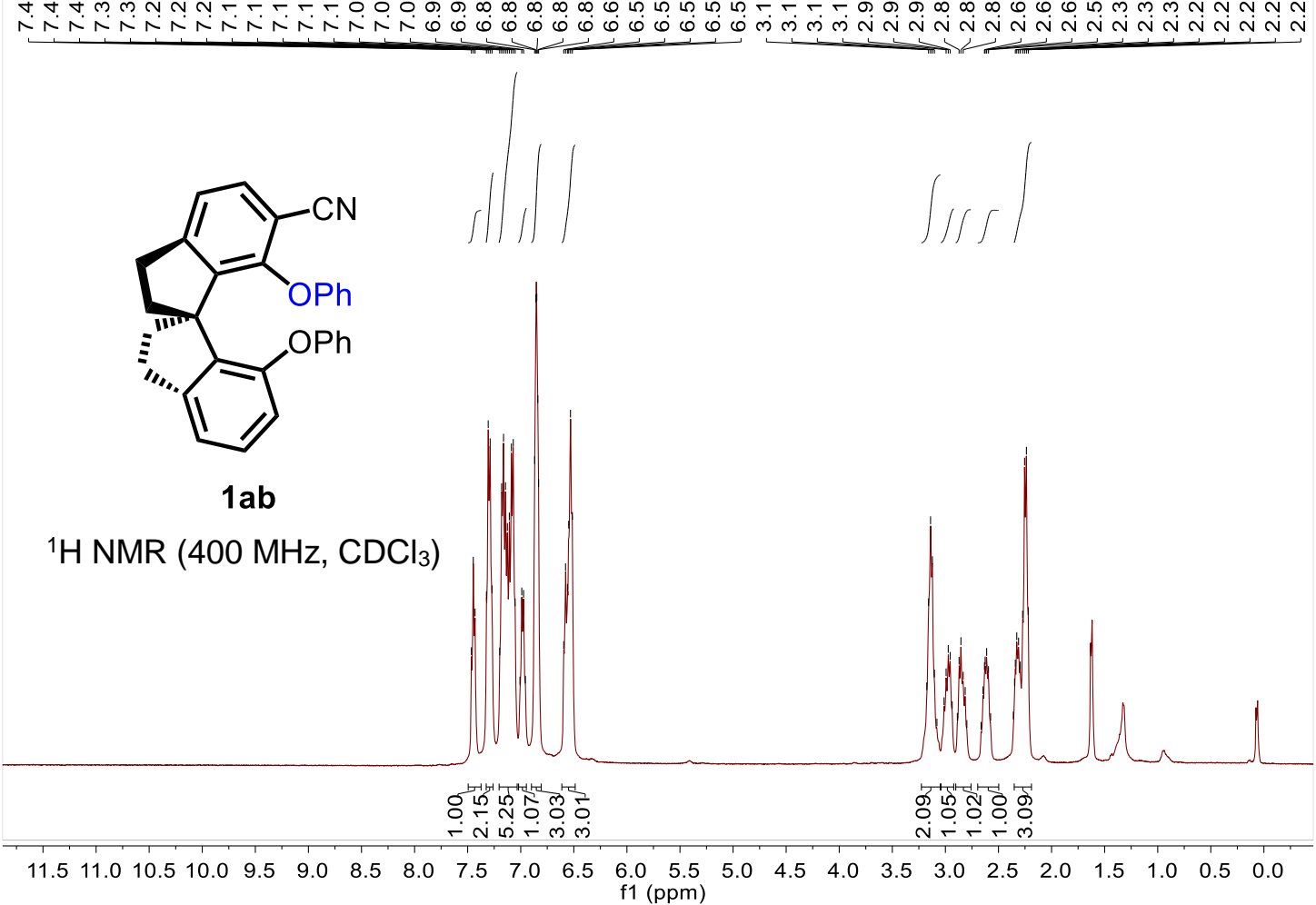

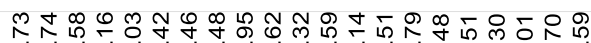

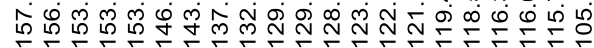

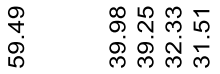

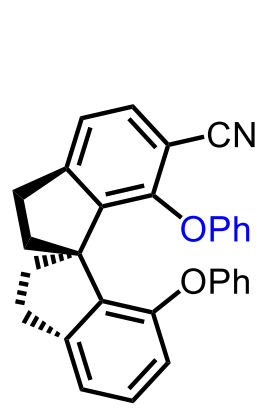

$1 \mathrm{ab}$

${ }^{13} \mathrm{C} \mathrm{NMR}\left(100 \mathrm{MHz}, \mathrm{CDCl}_{3}\right)$

$\begin{array}{lllllllllllllllllllllll}210 & 200 & 190 & 180 & 170 & 160 & 150 & 140 & 130 & 120 & \begin{array}{c}110 \\ \mathrm{f} 1\end{array}\left(\begin{array}{ll}100 \\ (\mathrm{ppm})\end{array}\right. & 90 & 80 & 70 & 60 & 50 & 40 & 30 & 20 & 10 & 0 & -10\end{array}$ 

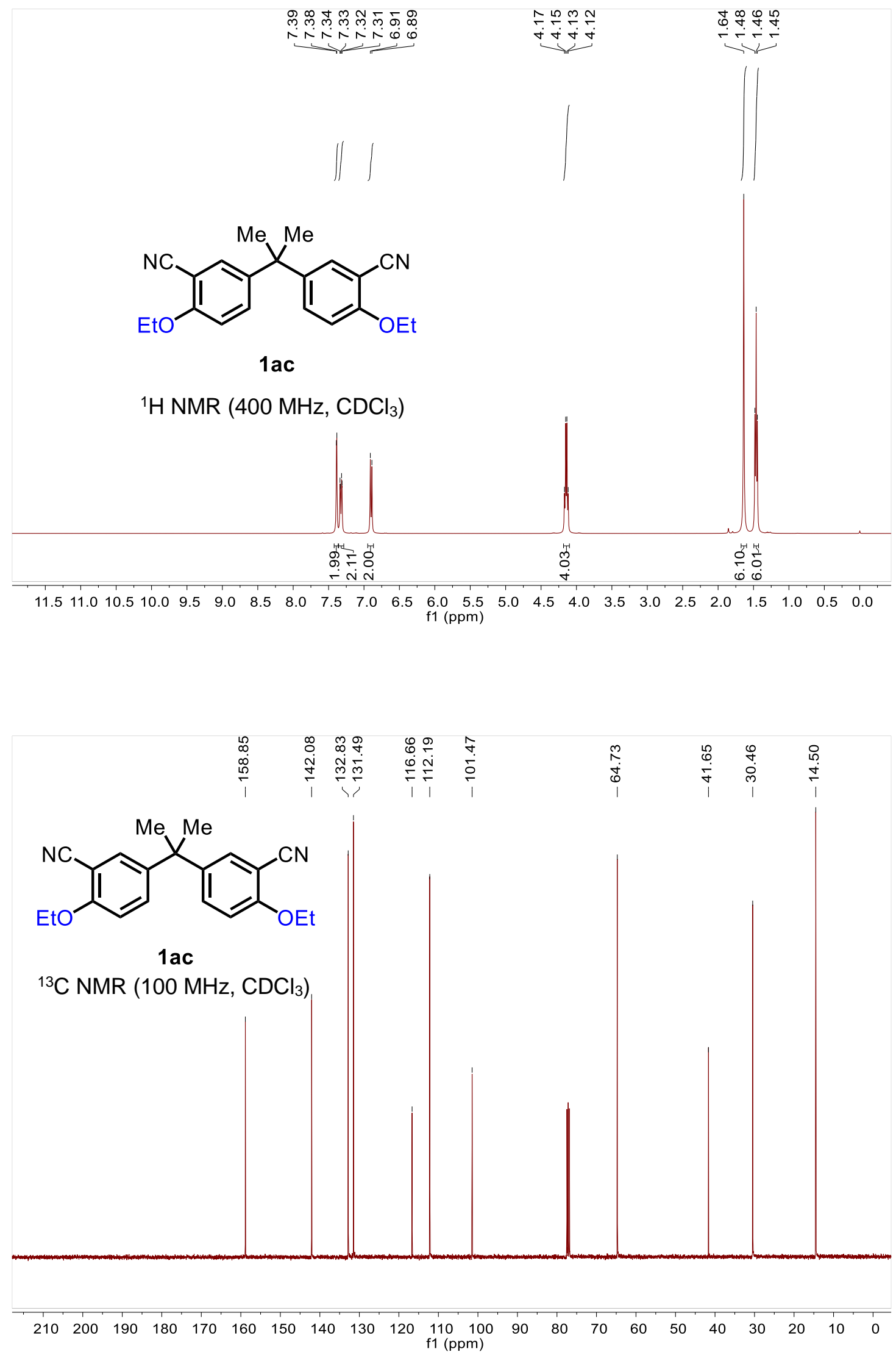
<smiles>CCOc1cc2c(cc1C#N)[C@]1(C)CC[C@]3(C)[C@@H](OC)CC[C@H]3[C@@H]1CC2</smiles>

$1 \mathrm{ad}$

${ }^{1} \mathrm{H}$ NMR $\left(400 \mathrm{MHz}, \mathrm{CDCl}_{3}\right)$

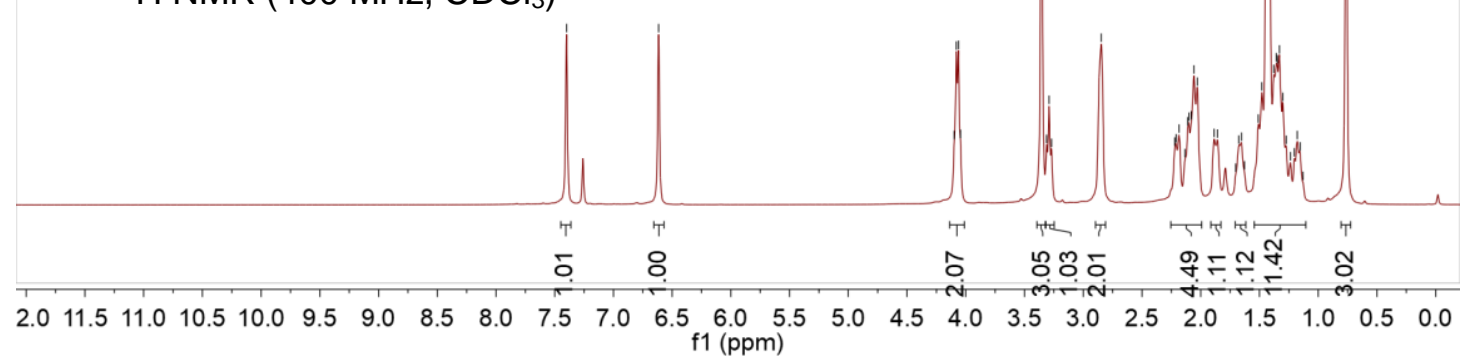

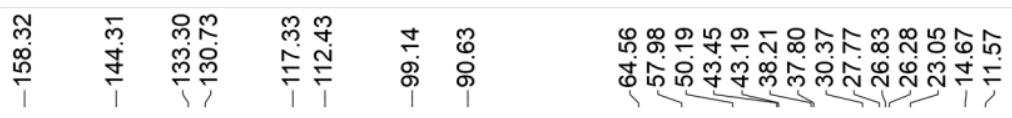

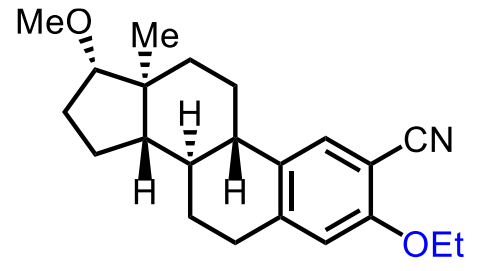

$1 \mathrm{ad}$

${ }^{13} \mathrm{C}$ NMR $\left(100 \mathrm{MHz}, \mathrm{CDCl}_{3}\right)$

$\begin{array}{lllllllllllllllllllllll}210 & 200 & 190 & 180 & 170 & 160 & 150 & 140 & 130 & 120 \begin{array}{c}110 \\ \mathrm{f} 1(\mathrm{ppm})\end{array} & 100 & 90 & 80 & 70 & 60 & 50 & 40 & 30 & 20 & 10 & 0\end{array}$ 


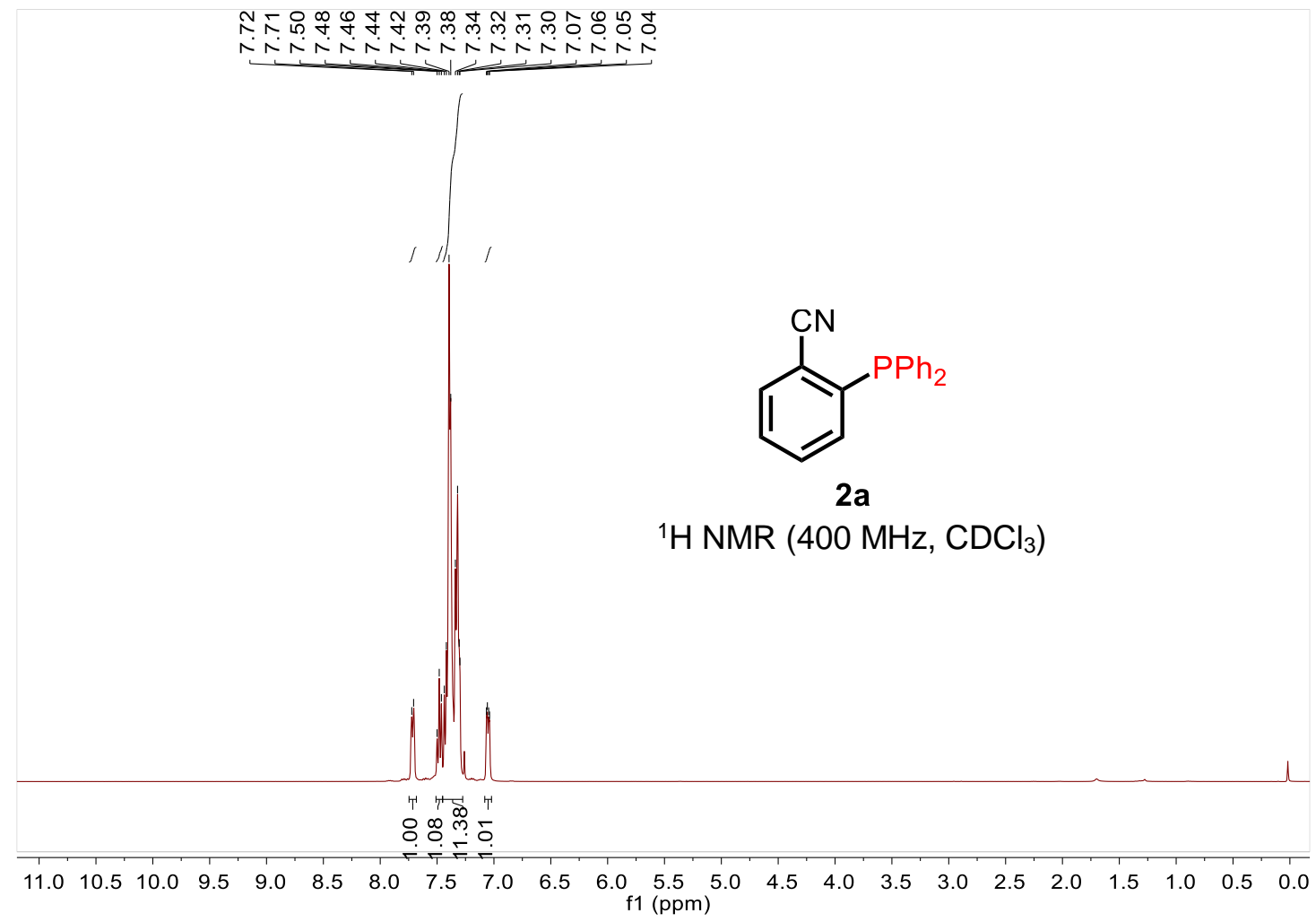

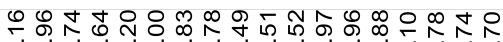

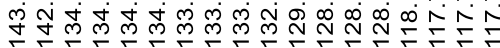

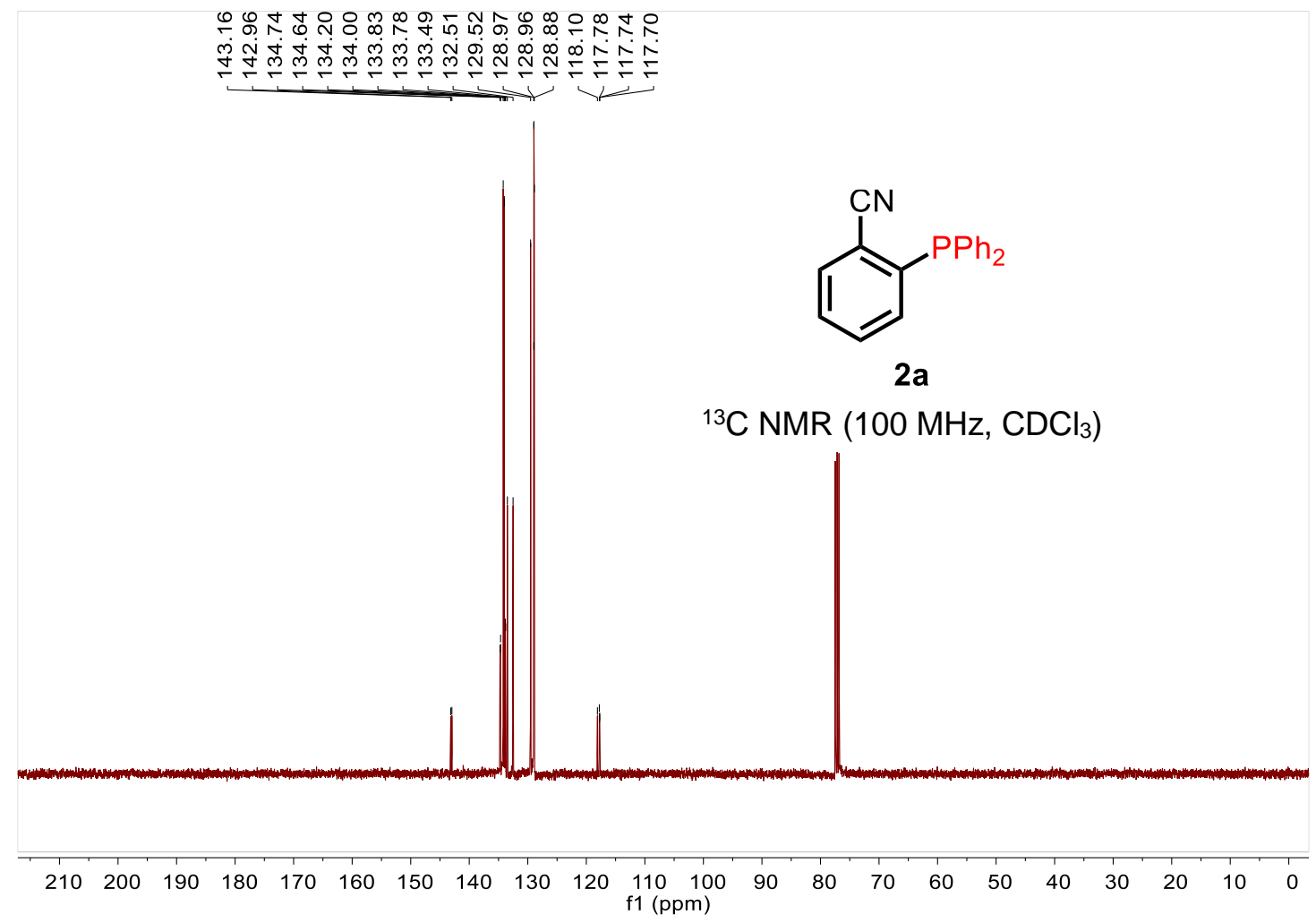


<smiles>N#Cc1ccccc1P</smiles>

$2 a$

${ }^{31} \mathrm{P}$ NMR (162 MHz, $\mathrm{CDCl}_{3}$ )

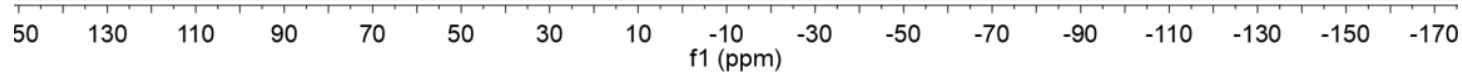

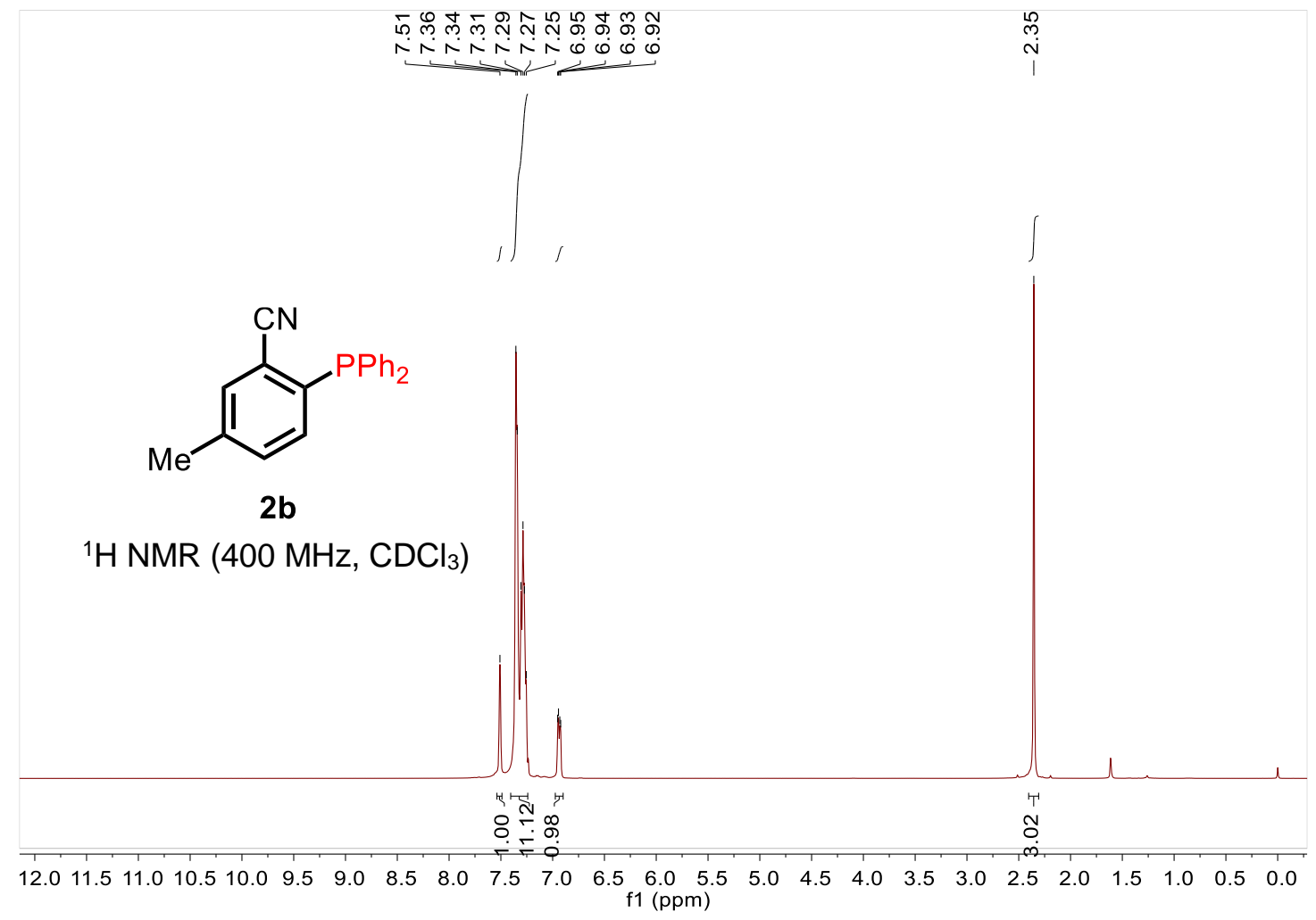




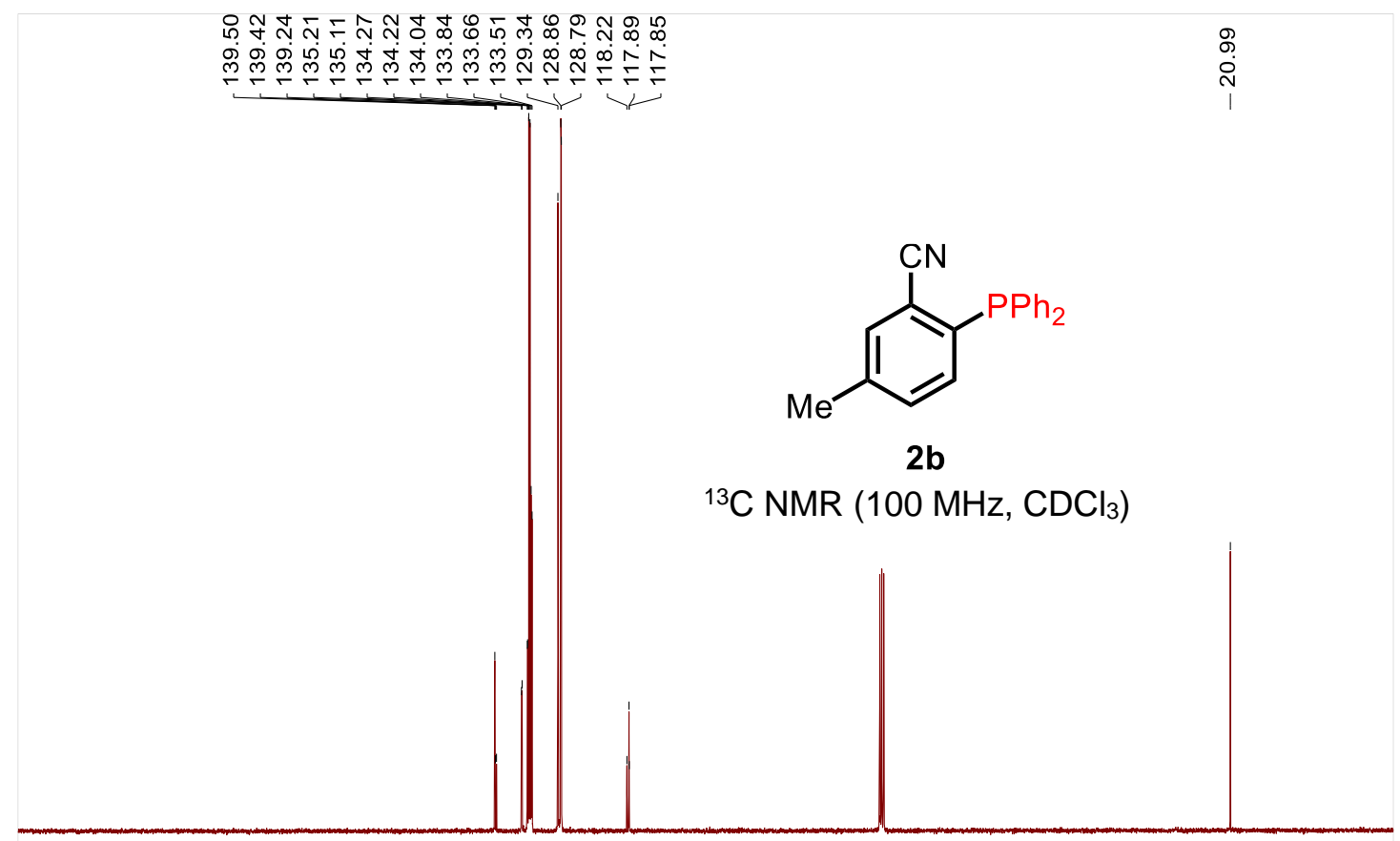

$\begin{array}{lllllllllllllllllllll}210 & 200 & 190 & 180 & 170 & 160 & 150 & 140 & 130 & 120 & \begin{array}{l}110 \\ \mathrm{f} 1(\mathrm{ppm})\end{array} & 90 & 80 & 70 & 60 & 50 & 40 & 30 & 20 & 10 & 0\end{array}$

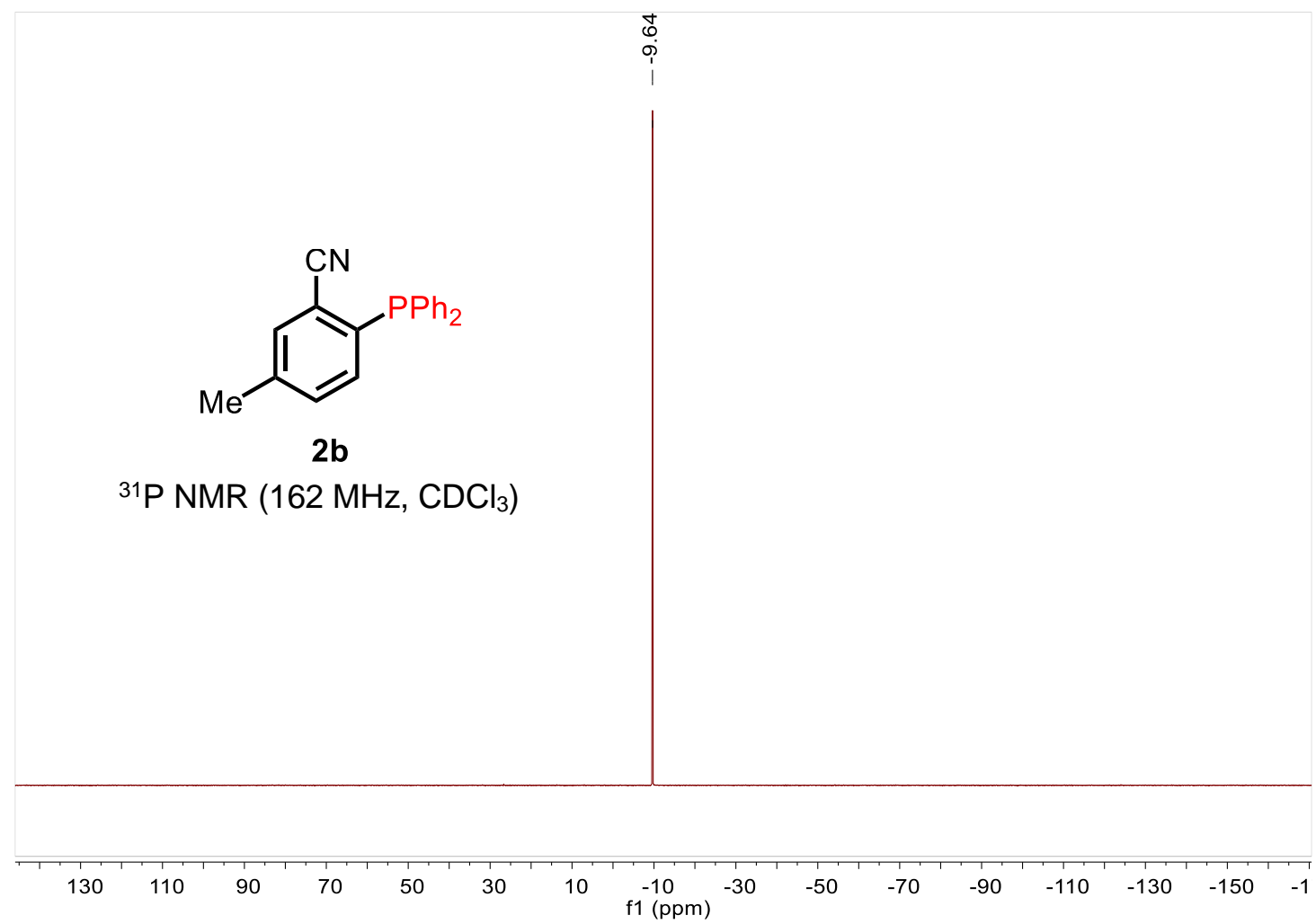



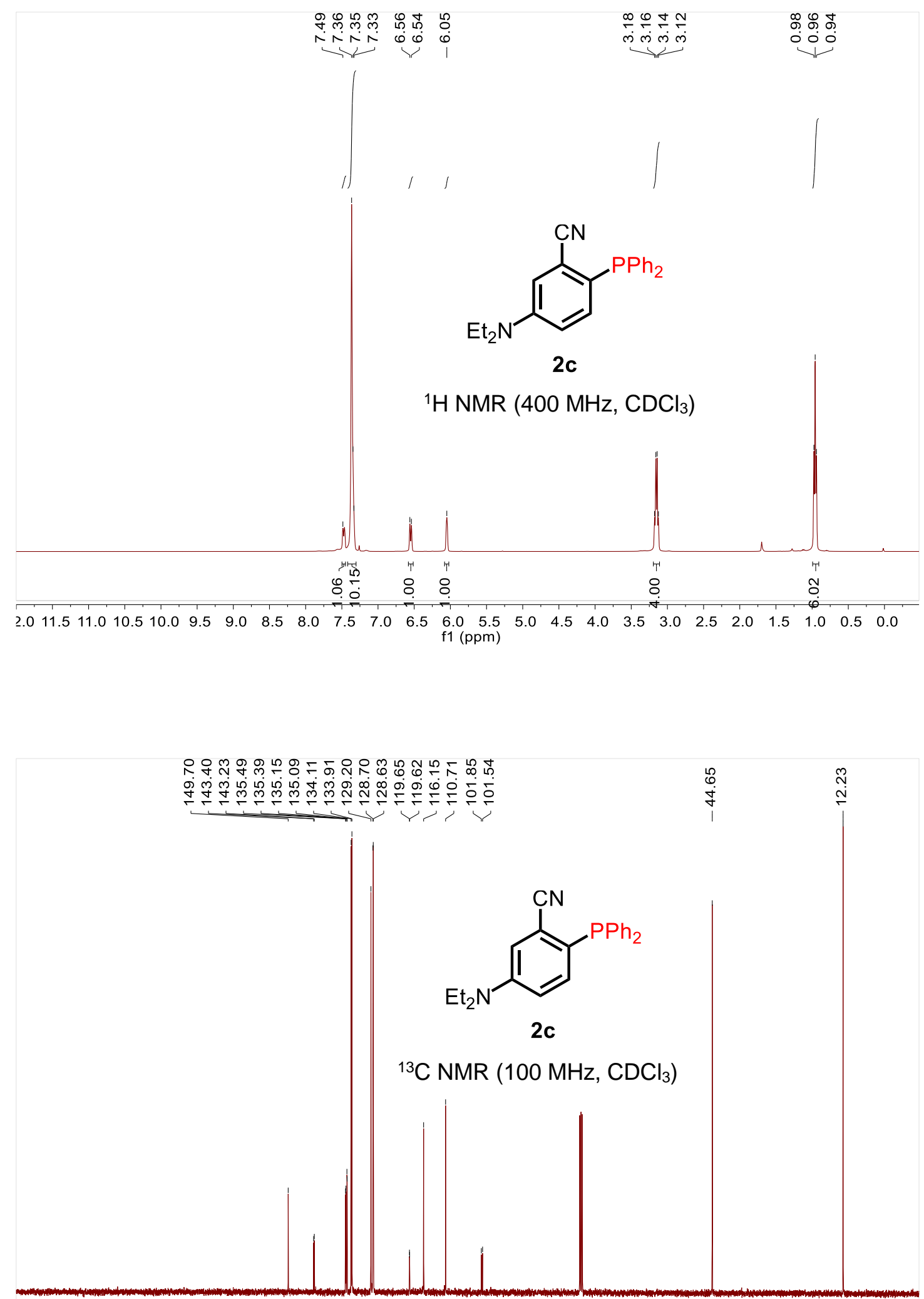

$\begin{array}{lllllllllllllllllllll}210 & 200 & 190 & 180 & 170 & 160 & 150 & 140 & 130 & 120 & \begin{array}{l}110 \\ \mathrm{f} 1(\mathrm{ppm})\end{array} & \begin{array}{l}100 \\ (120\end{array} & 80 & 70 & 60 & 50 & 40 & 30 & 20 & 10 & 0\end{array}$ 


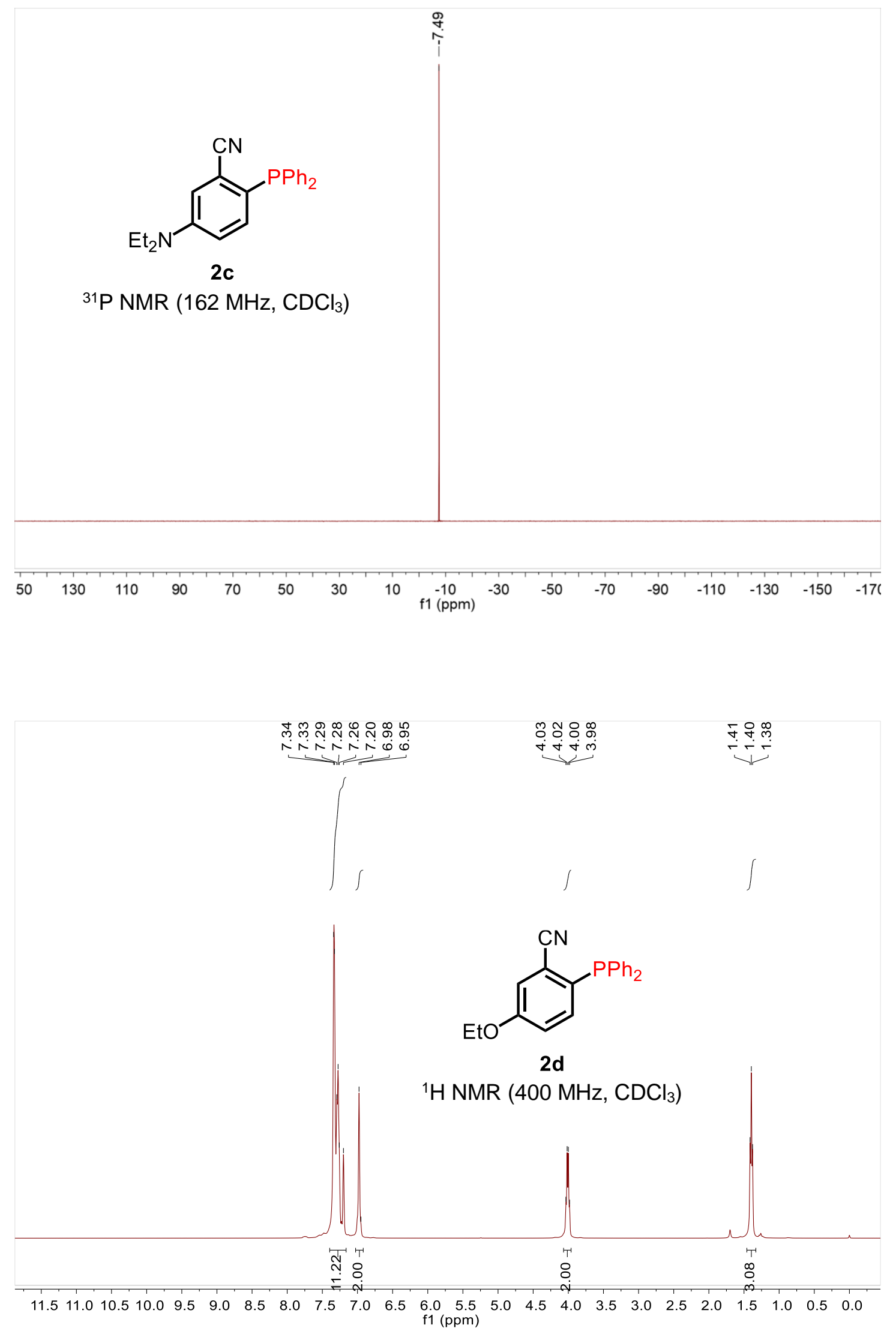




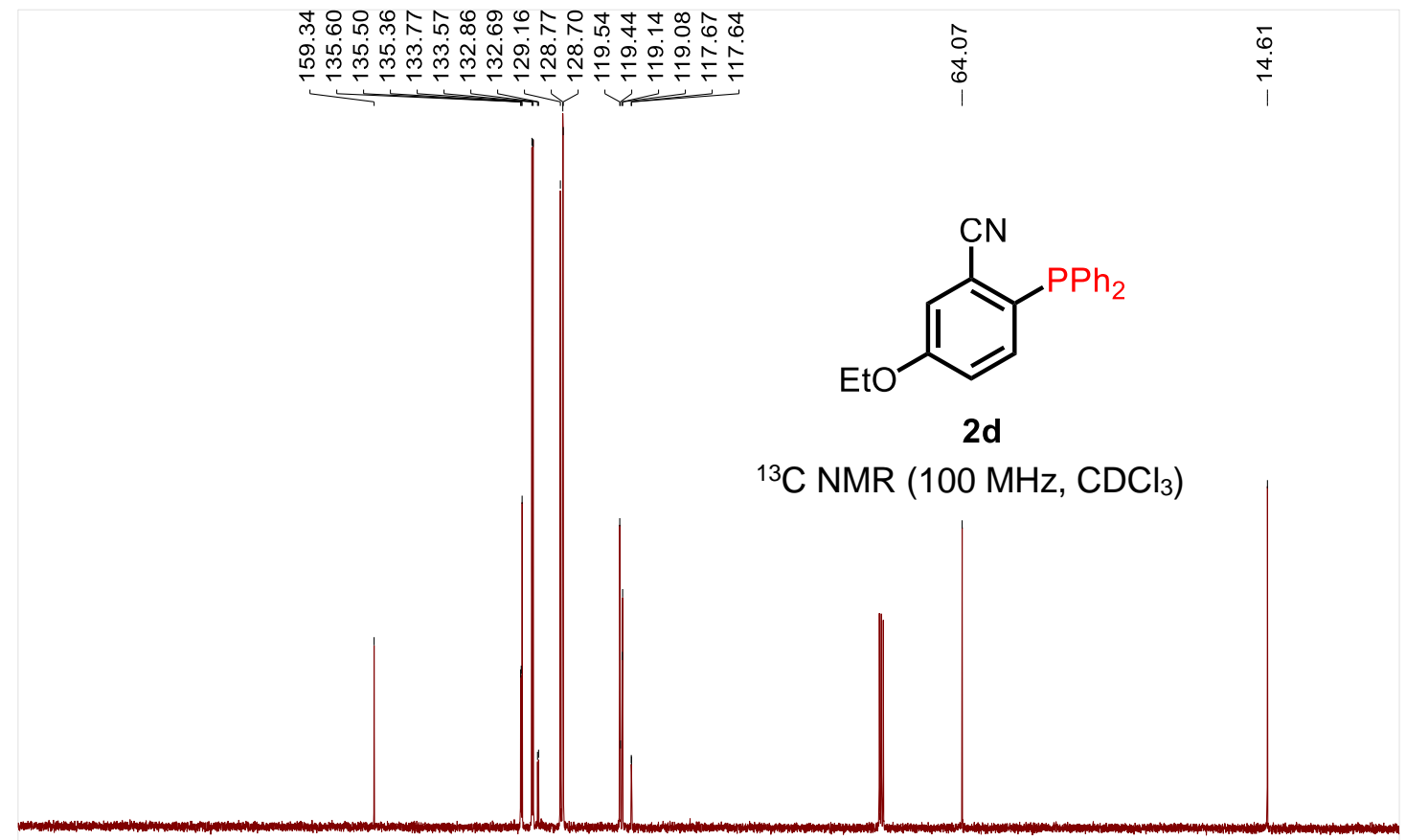

$\begin{array}{lllllllllllllllllllll}210 & 200 & 190 & 180 & 170 & 160 & 150 & 140 & 130 & 120 & \begin{array}{c}110 \\ \mathrm{f} 1(\mathrm{ppm})\end{array} & 90 & 80 & 70 & 60 & 50 & 40 & 30 & 20 & 10 & 0\end{array}$<smiles>CCOc1ccc(-c2ccccc2)c(C#N)c1</smiles>

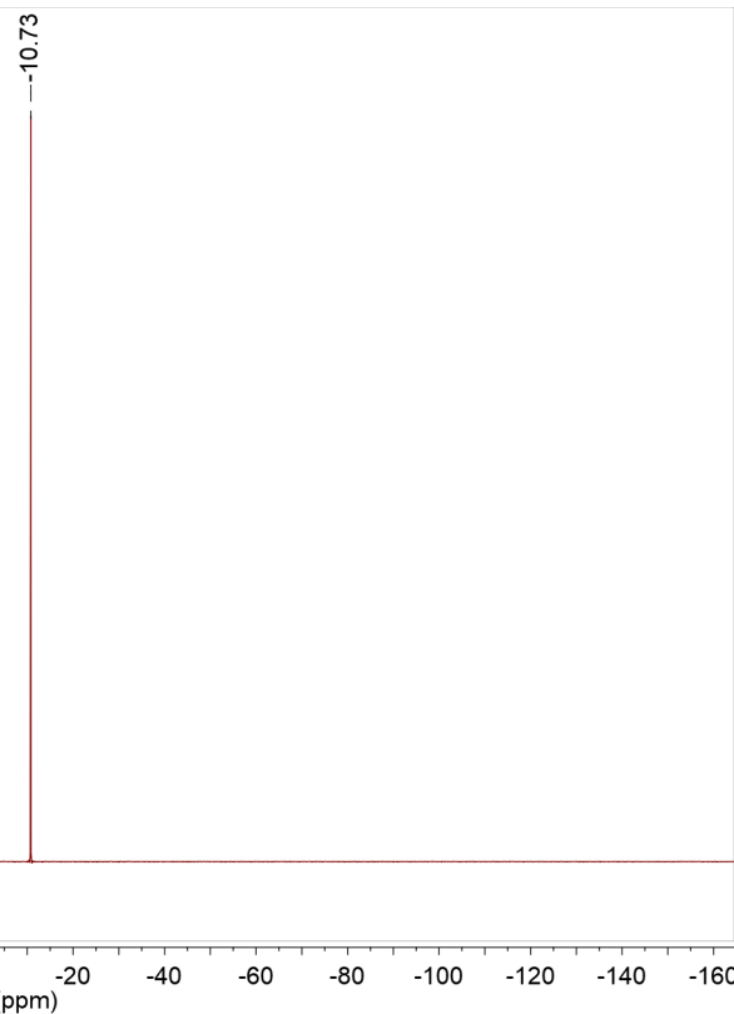




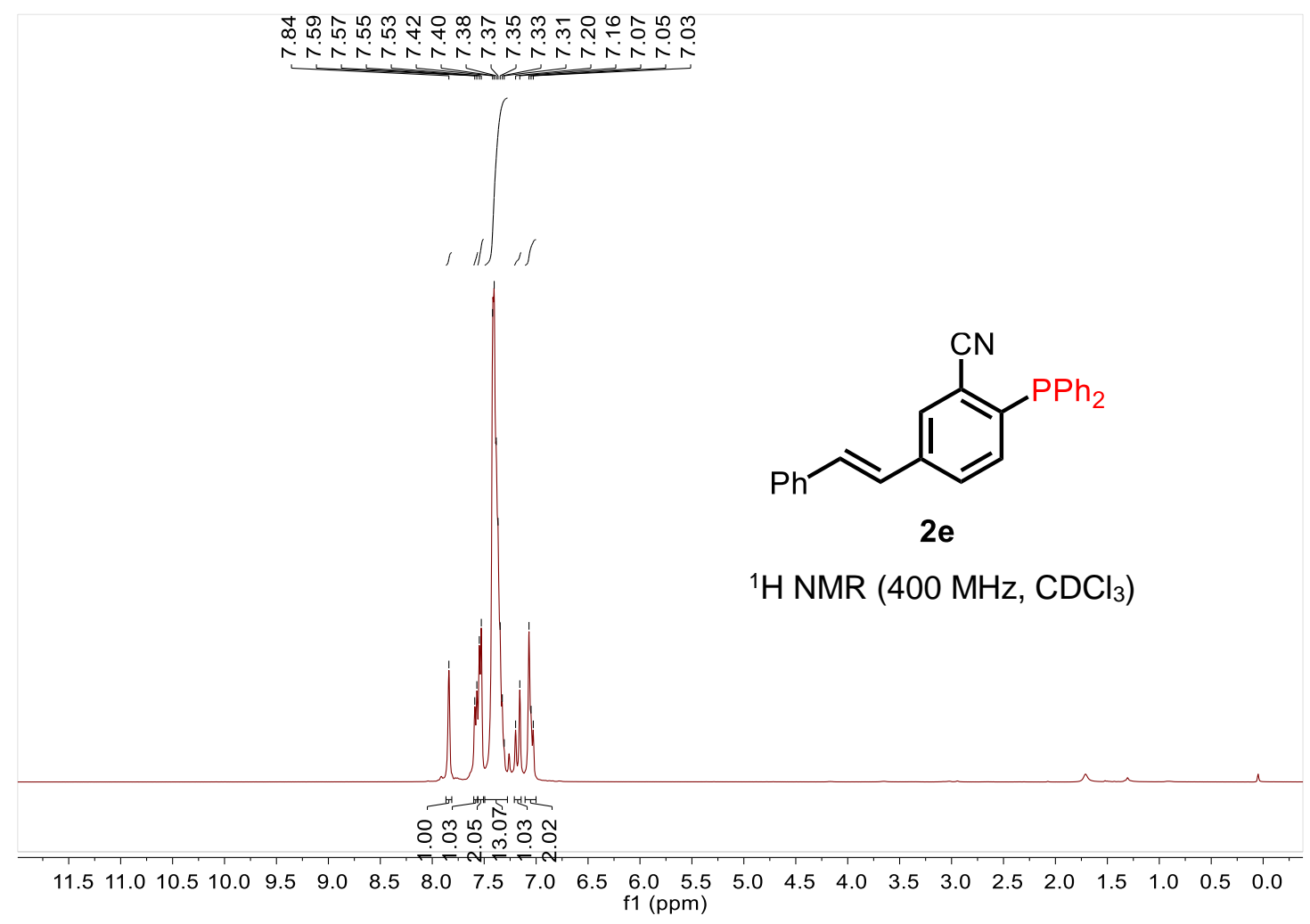

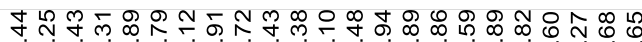

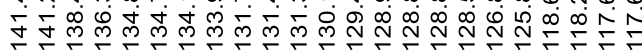

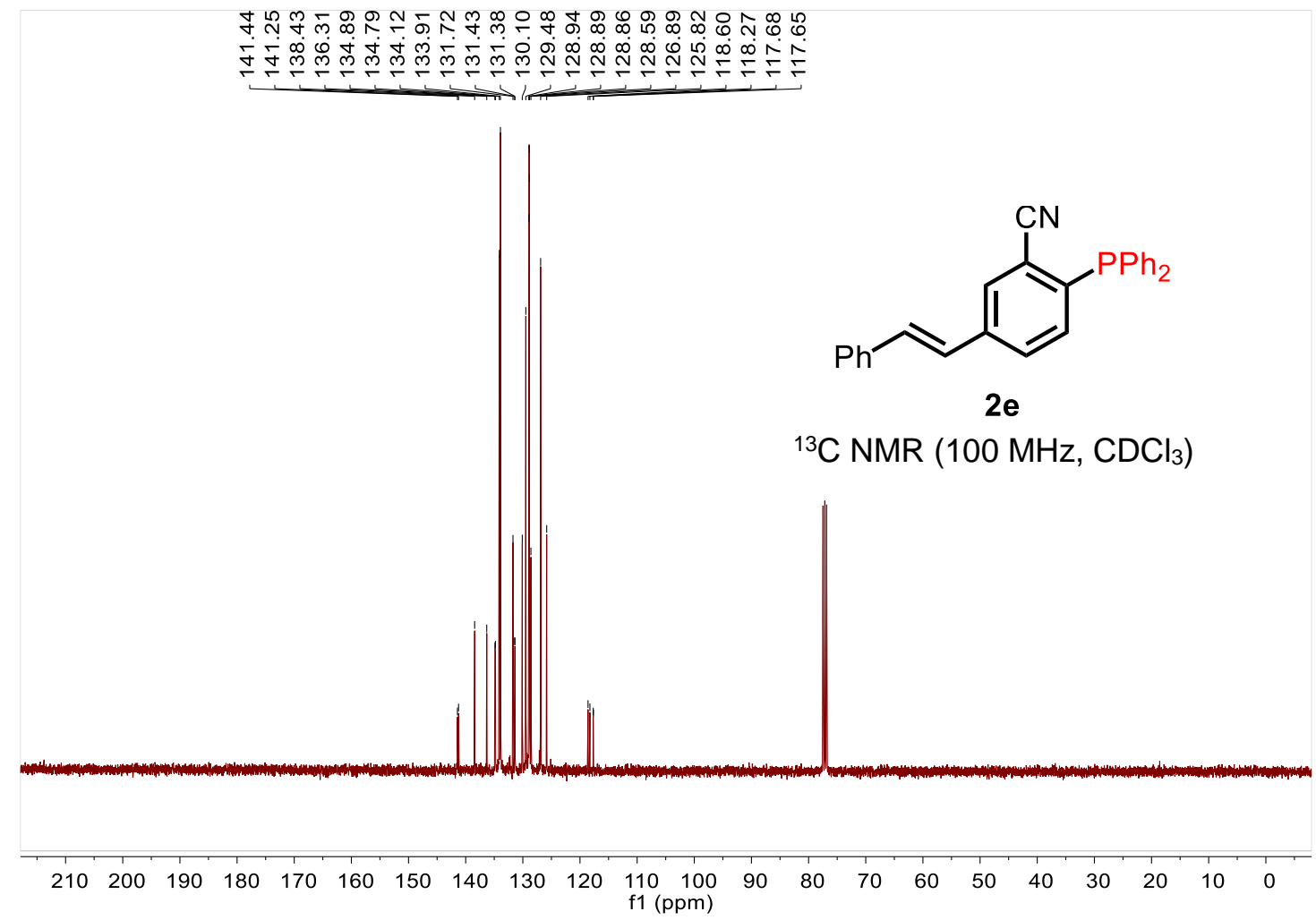




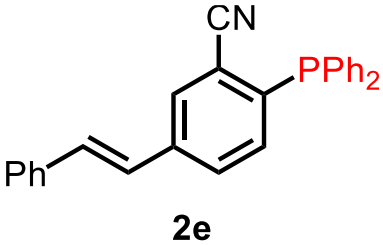

${ }^{31} \mathrm{P}$ NMR $\left(162 \mathrm{MHz}, \mathrm{CDCl}_{3}\right)$

$\begin{array}{lllllllllllllllll}50 & 130 & 110 & 90 & 70 & 50 & 30 & 10 & \begin{array}{l}-10 \\ \mathrm{f} 1(\mathrm{ppm})\end{array} & -30 & -50 & -70 & -90 & -110 & -130 & -150 & -170\end{array}$

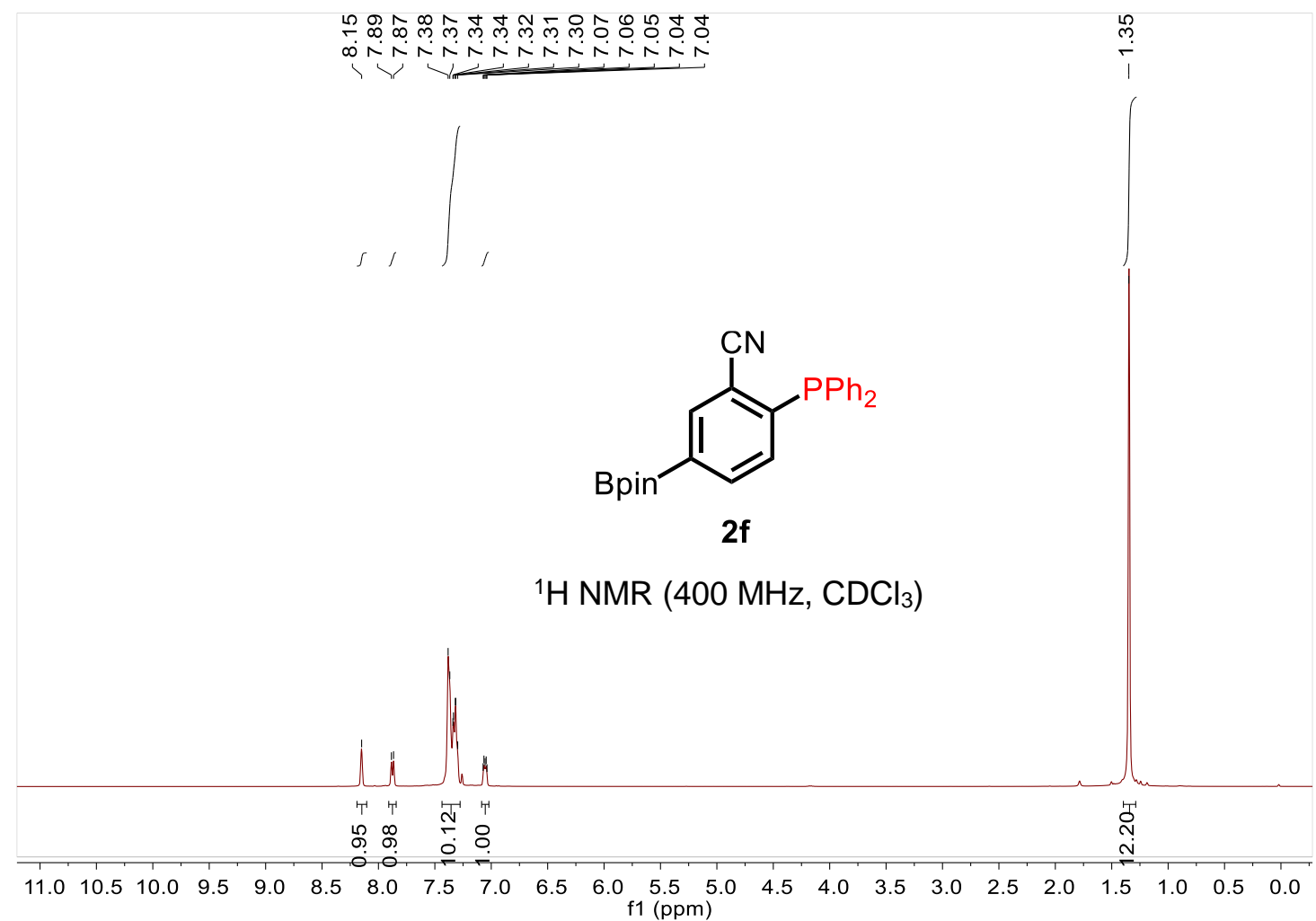




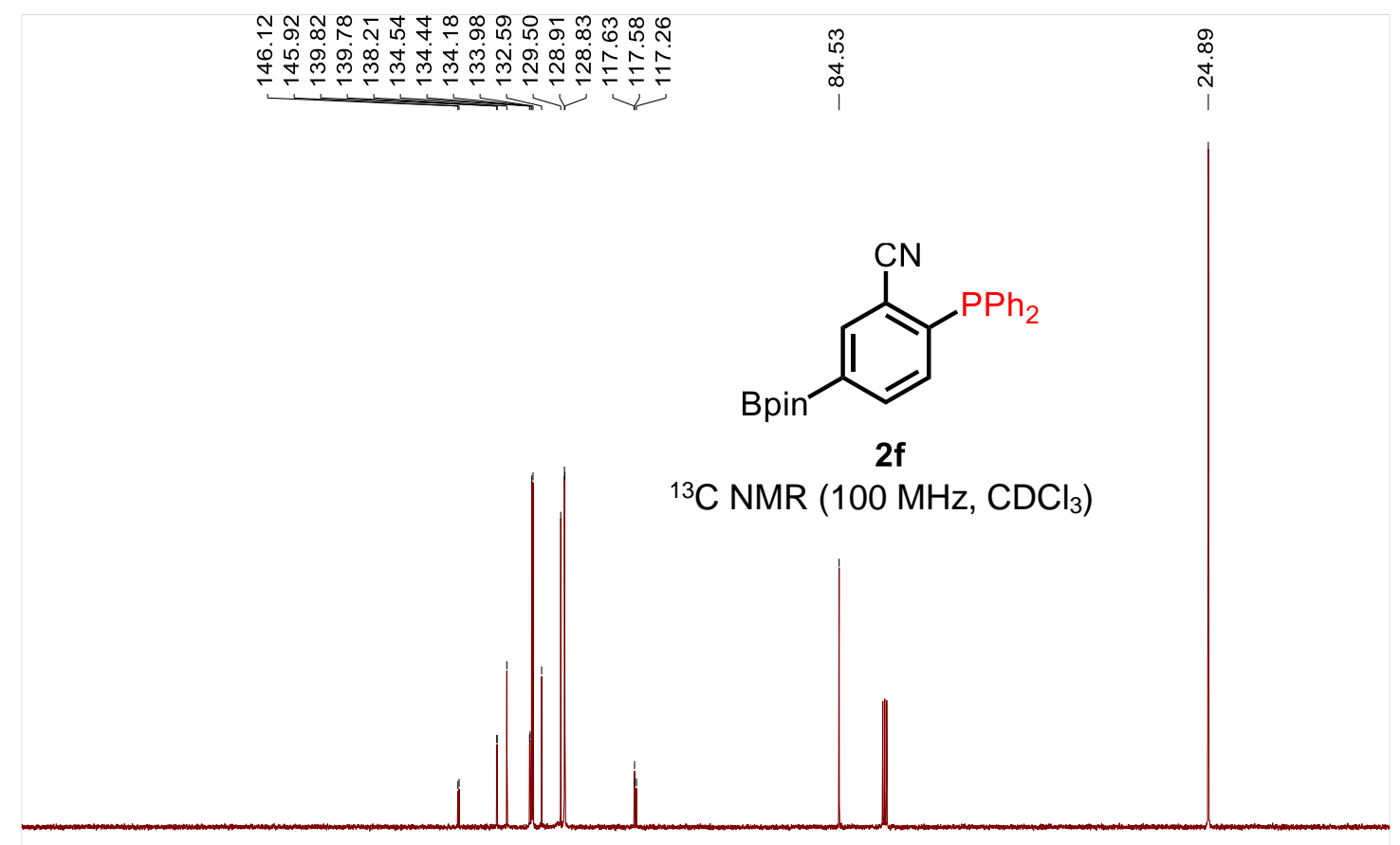

$\begin{array}{llllllllllllllllllllllll}210 & 200 & 190 & 180 & 170 & 160 & 150 & 140 & 130 & 120 & \begin{array}{c}110 \\ \mathrm{f} 1(\mathrm{ppm})\end{array} & 100 & 90 & 70 & 60 & 50 & 40 & 30 & 20 & 10 & 0\end{array}$

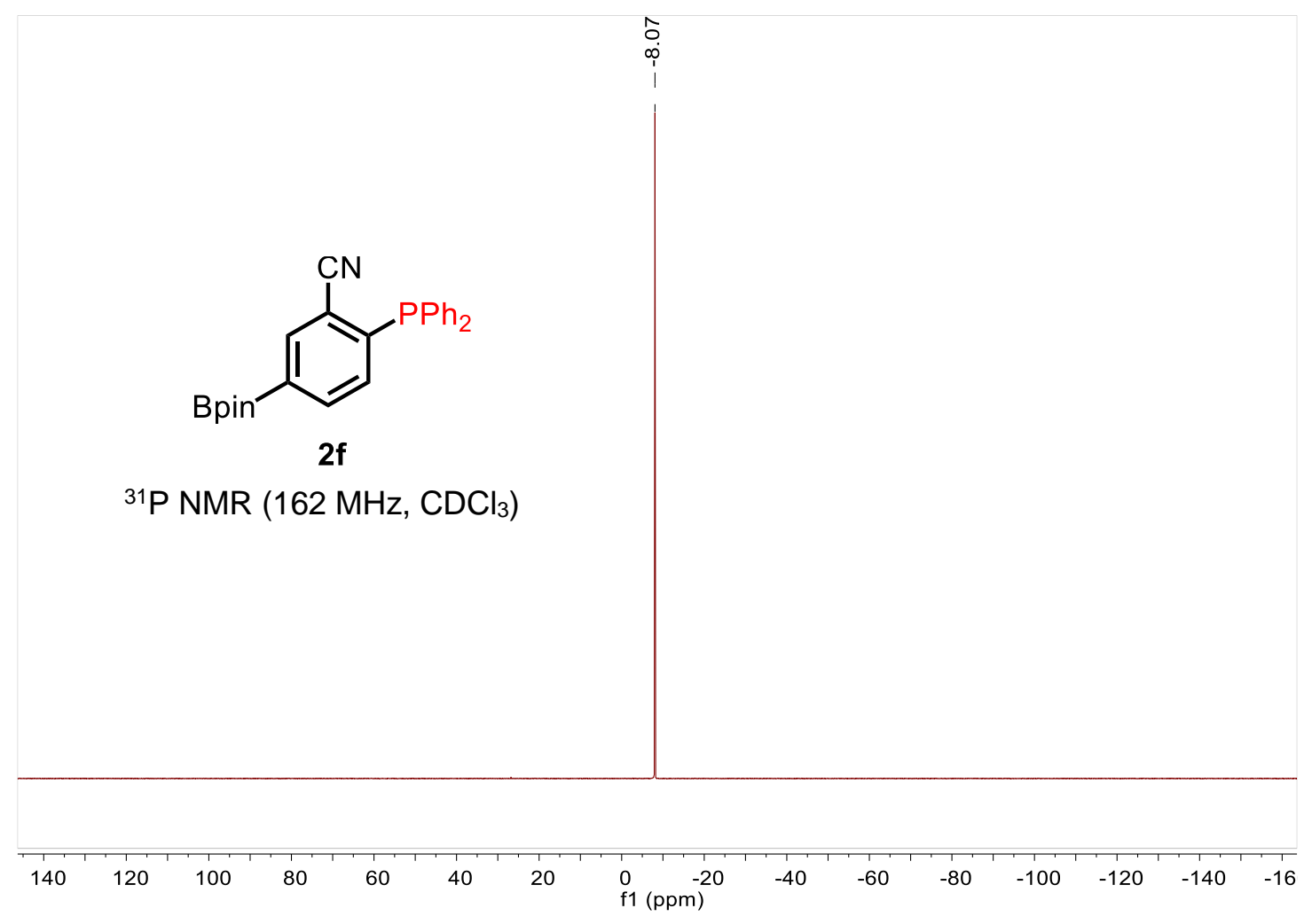




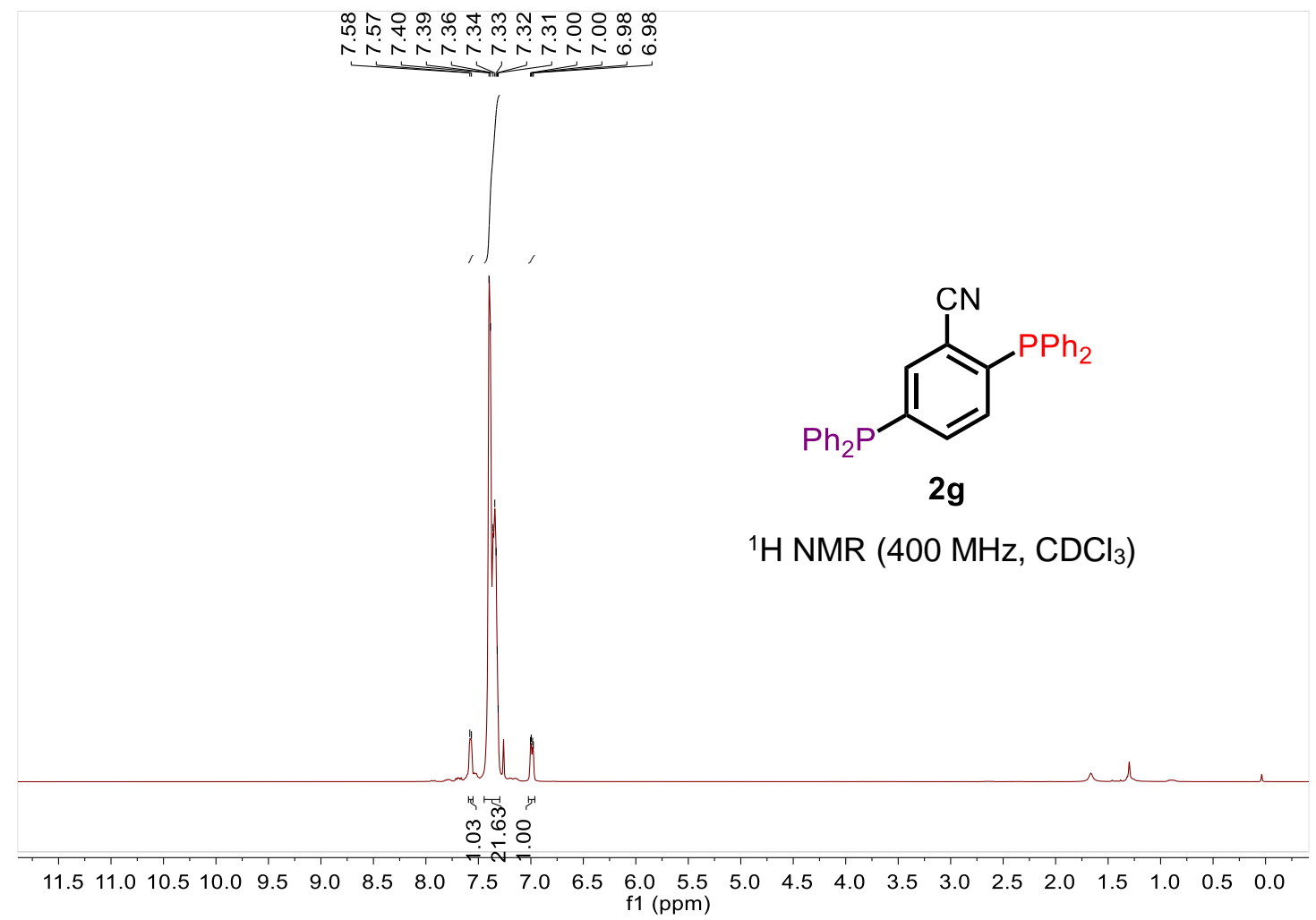

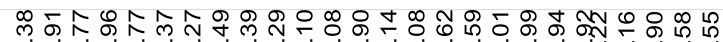

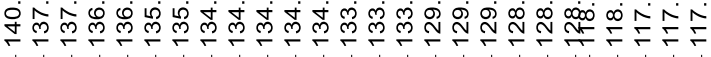

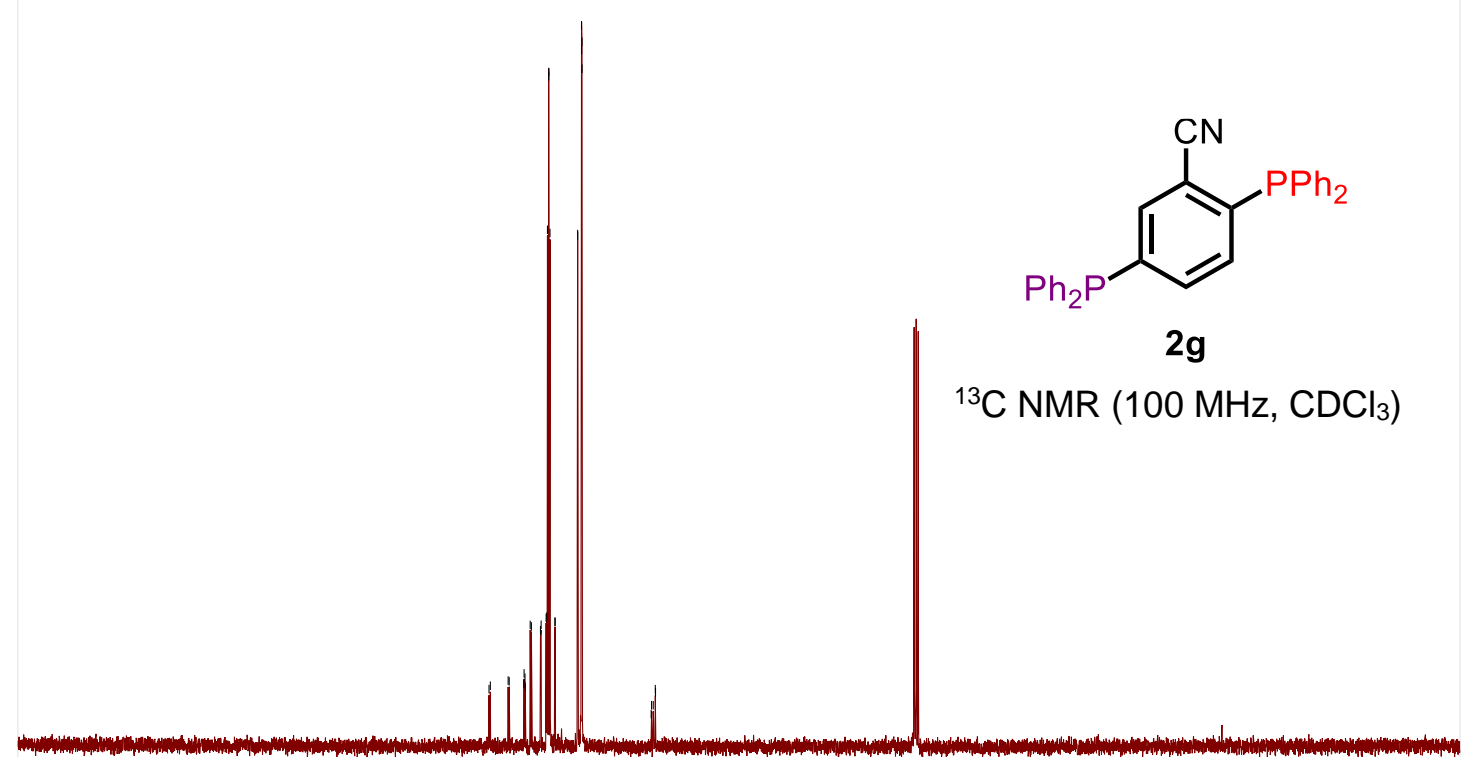

$\begin{array}{lllllllllllllllllllll}210 & 200 & 190 & 180 & 170 & 160 & 150 & 140 & 130 & 120 & \begin{array}{r}110 \\ \mathrm{f} 1(\mathrm{ppm})\end{array} & 100 & 80 & 70 & 60 & 50 & 40 & 30 & 20 & 10 & 0\end{array}$ 


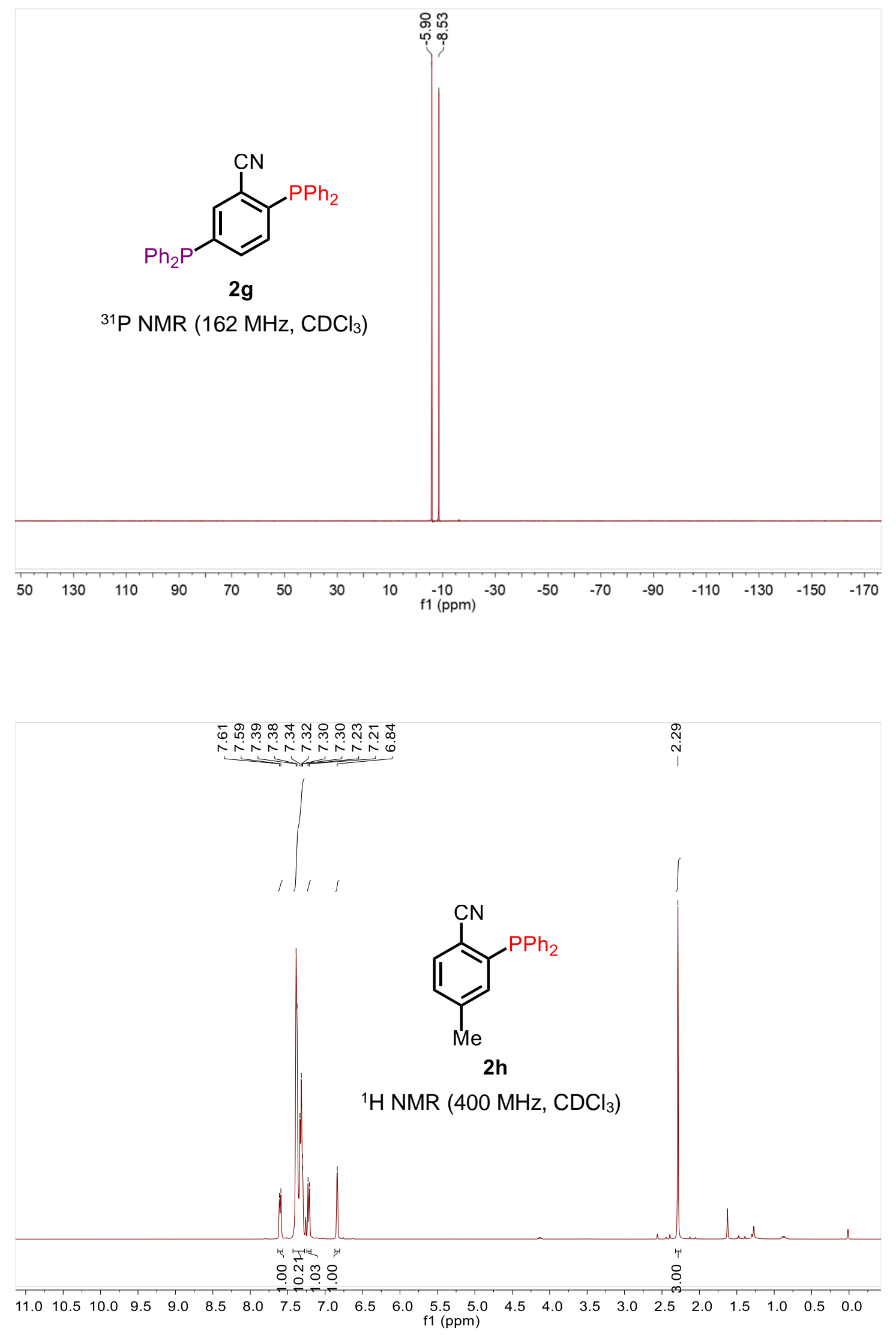




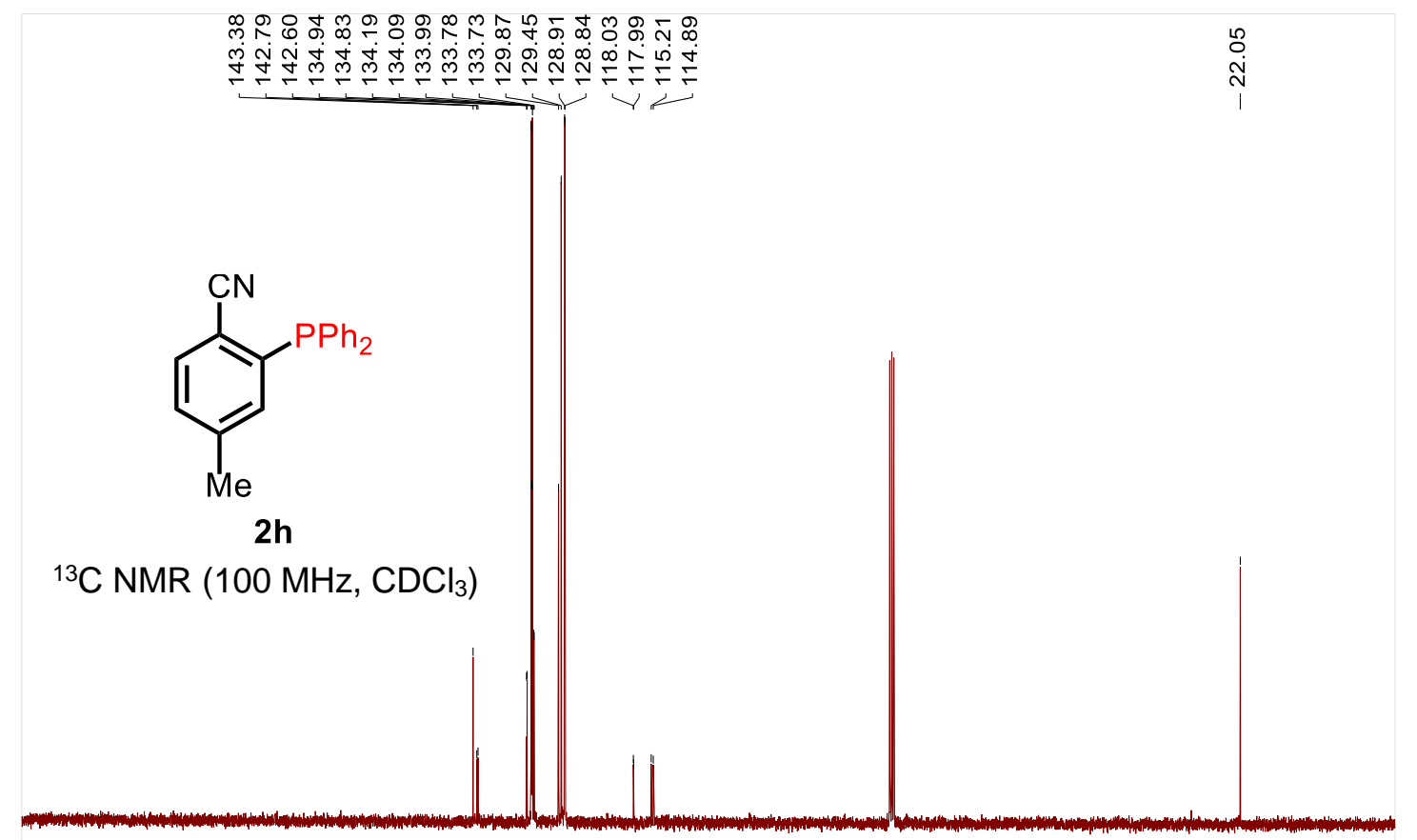

$\begin{array}{llllllllllllllllllllllllllllll}210 & 200 & 190 & 180 & 170 & 160 & 150 & 140 & 130 & 120 & \begin{array}{c}110 \\ \mathrm{f} 1(\mathrm{ppm})\end{array} & 100 & 90 & 80 & 70 & 60 & 50 & 40 & 30 & 20 & 10 & 0\end{array}$

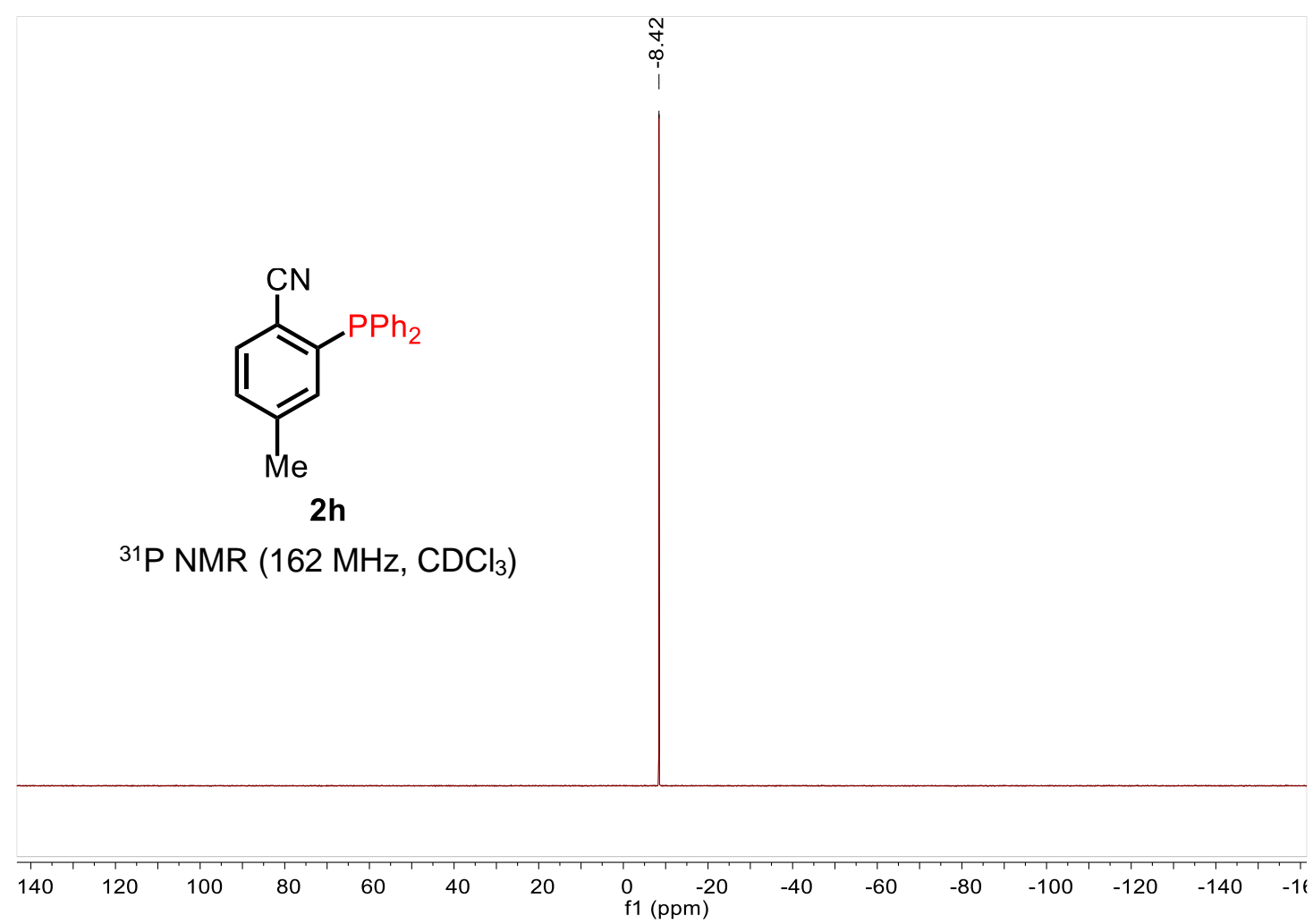



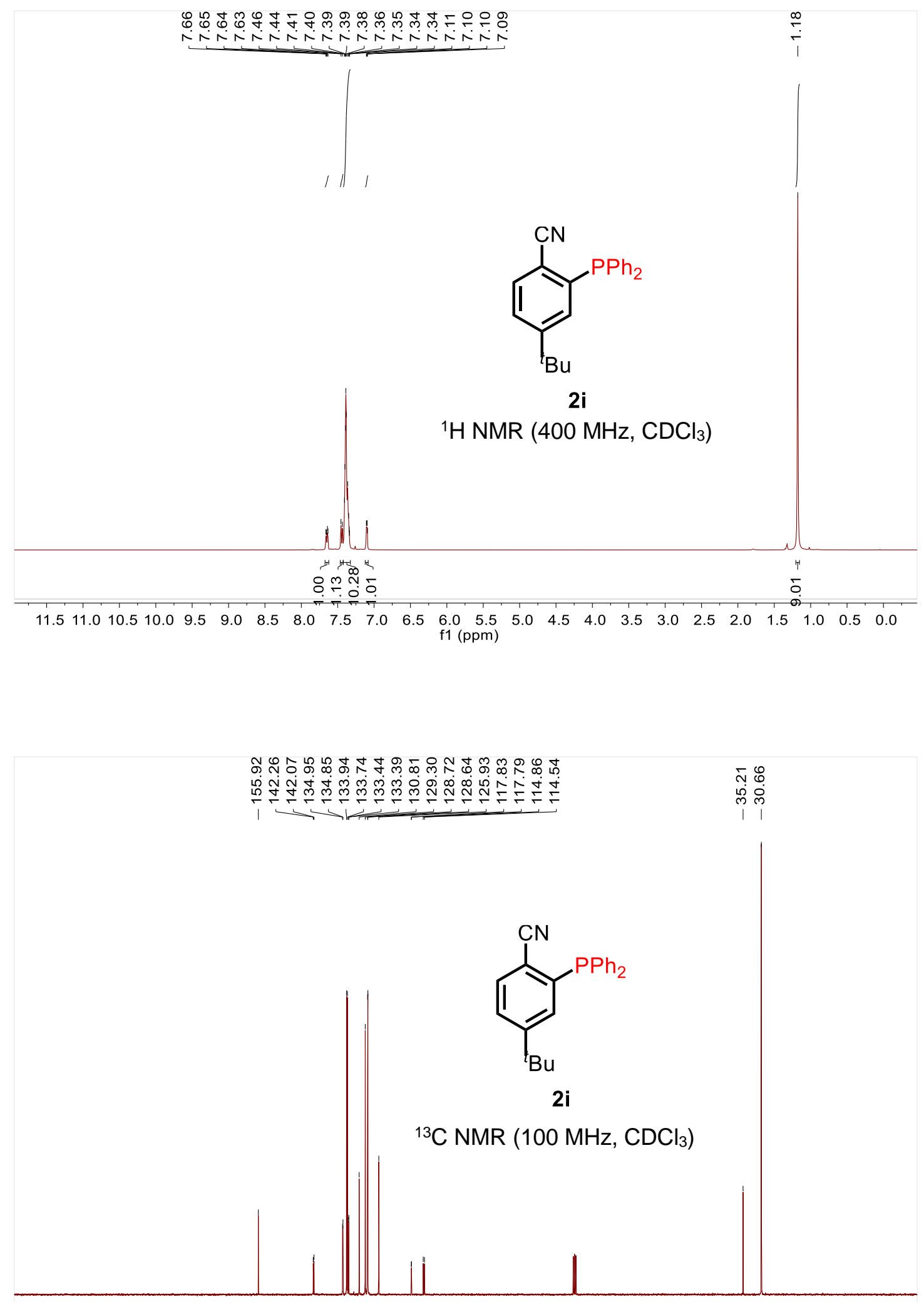

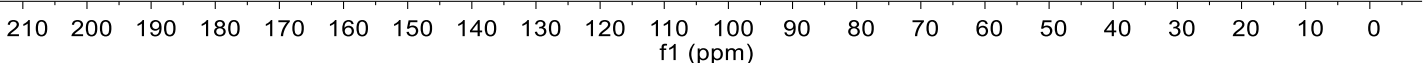




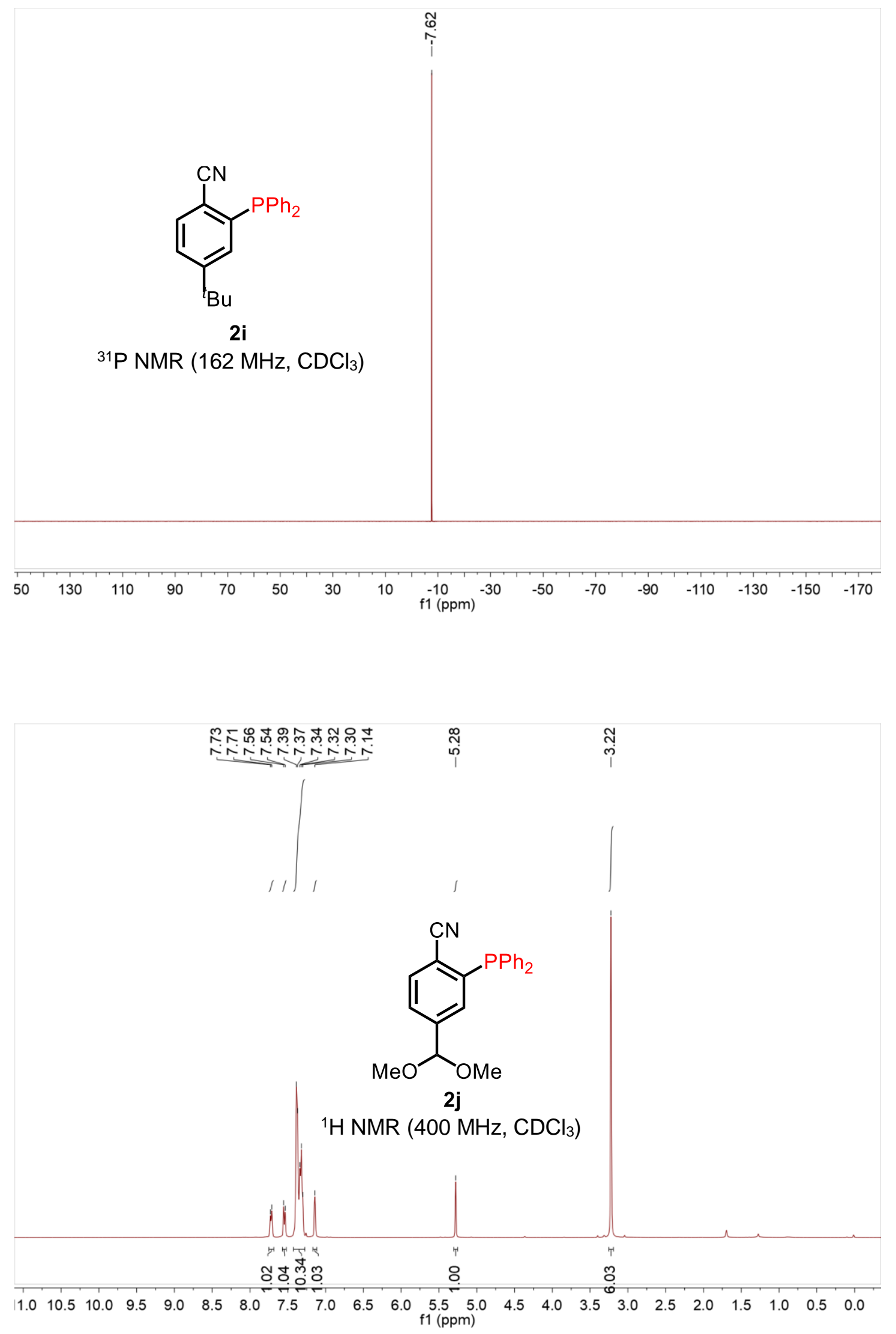




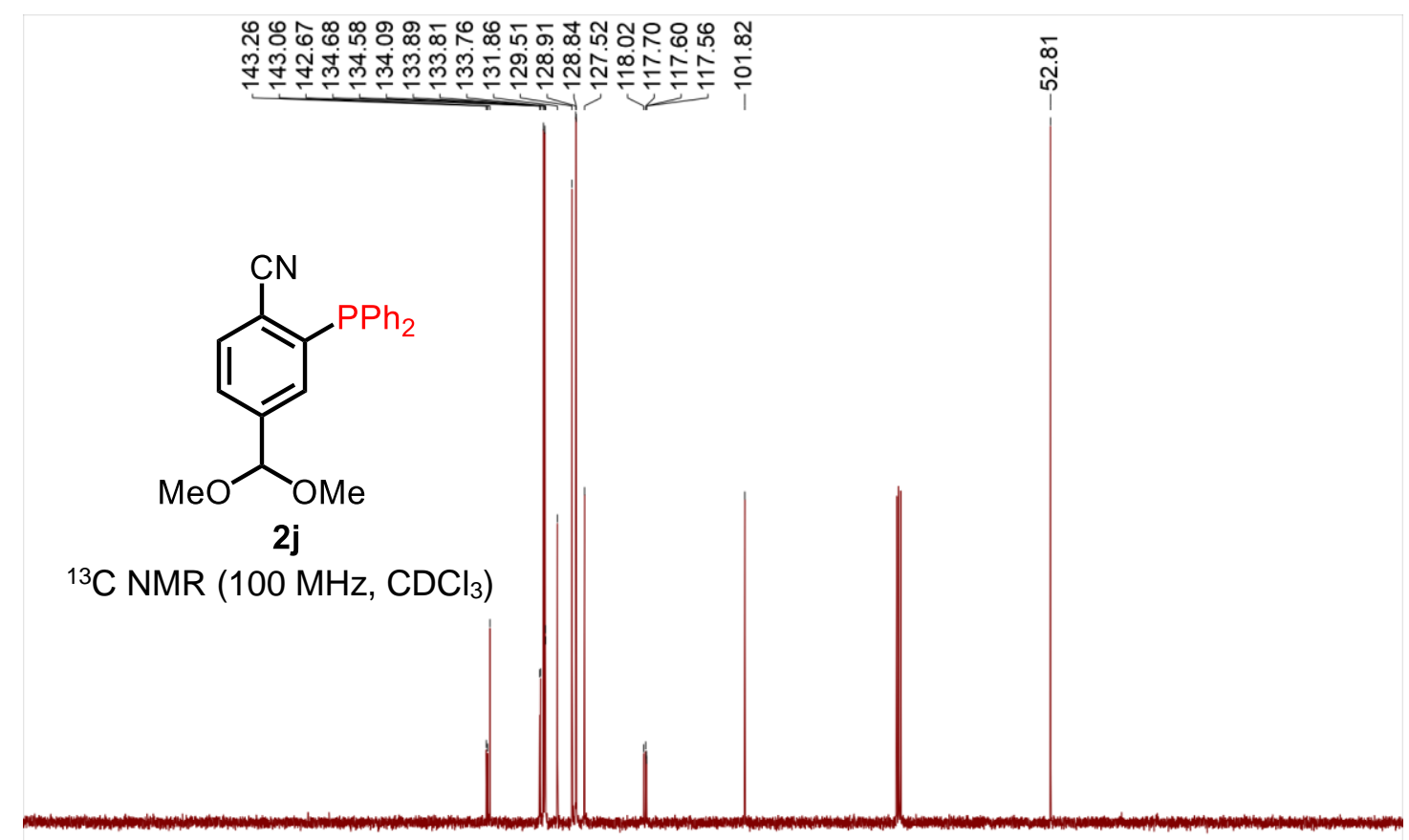

$\begin{array}{llllllllllllllllllllll}210 & 200 & 190 & 180 & 170 & 160 & 150 & 140 & 130 & 120 & \begin{array}{c}110 \\ \mathrm{f} 1(\mathrm{ppm})\end{array} & 90 & 80 & 70 & 60 & 50 & 40 & 30 & 20 & 10 & 0\end{array}$<smiles>COC(OC)c1ccc(C#N)c(P)c1</smiles>

2j

${ }^{31} \mathrm{P}$ NMR $\left(162 \mathrm{MHz}, \mathrm{CDCl}_{3}\right)$ 


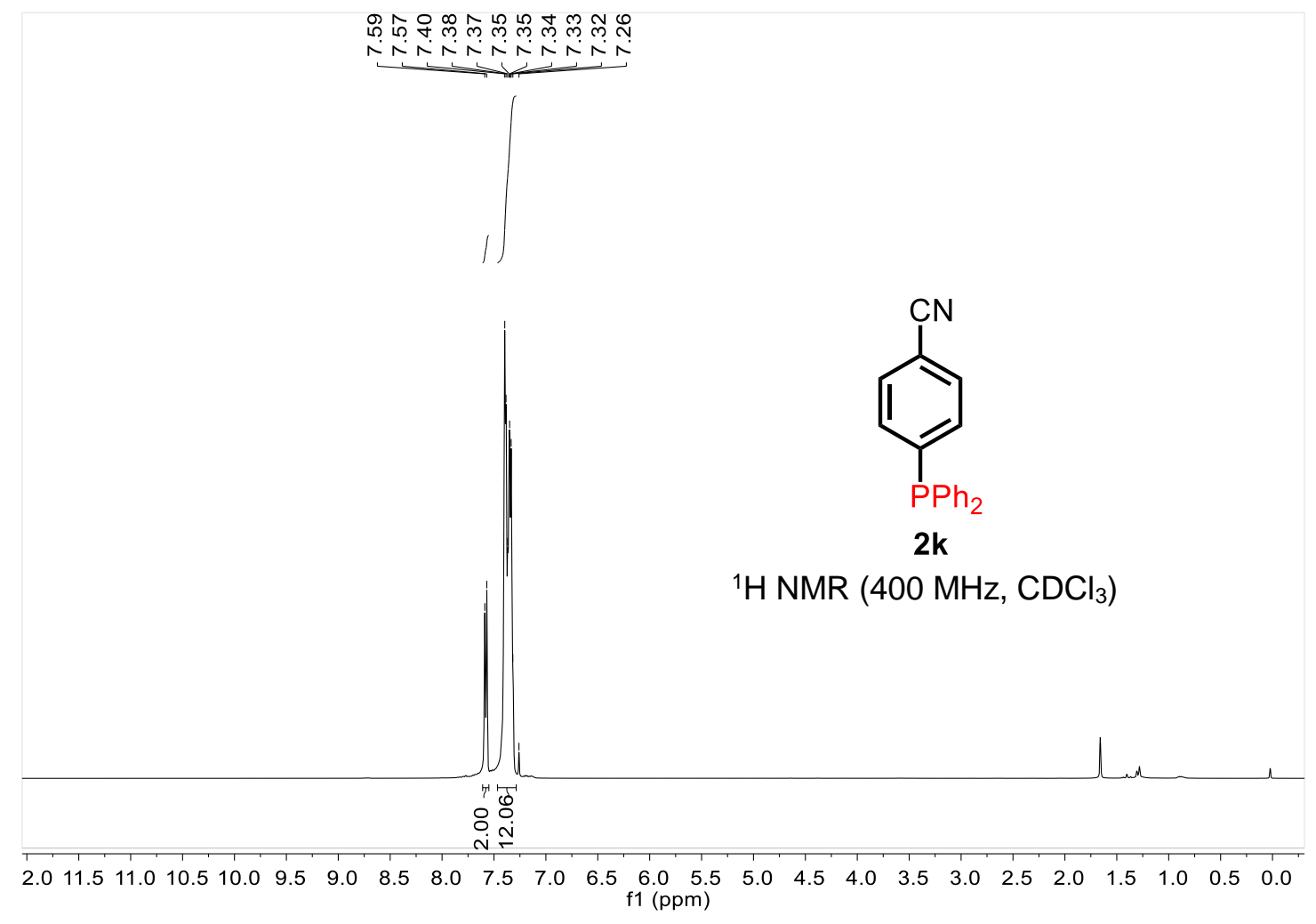

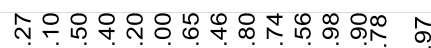

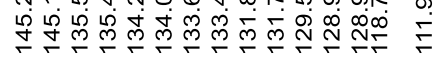

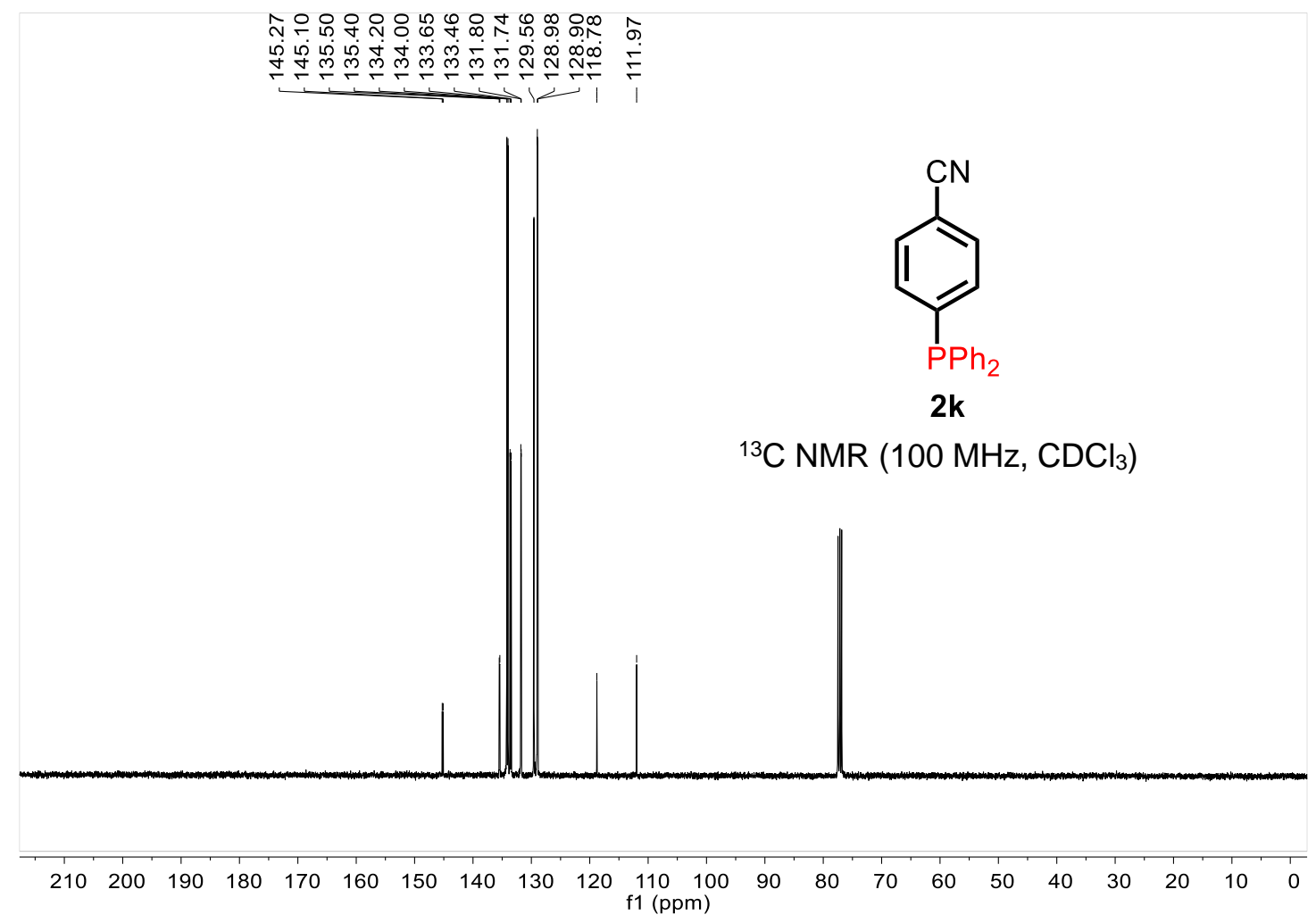


<smiles>N#Cc1ccc(P)cc1</smiles>

2k

${ }^{31} \mathrm{P} \mathrm{NMR}\left(162 \mathrm{MHz}, \mathrm{CDCl}_{3}\right)$

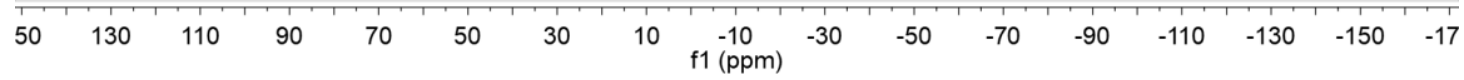

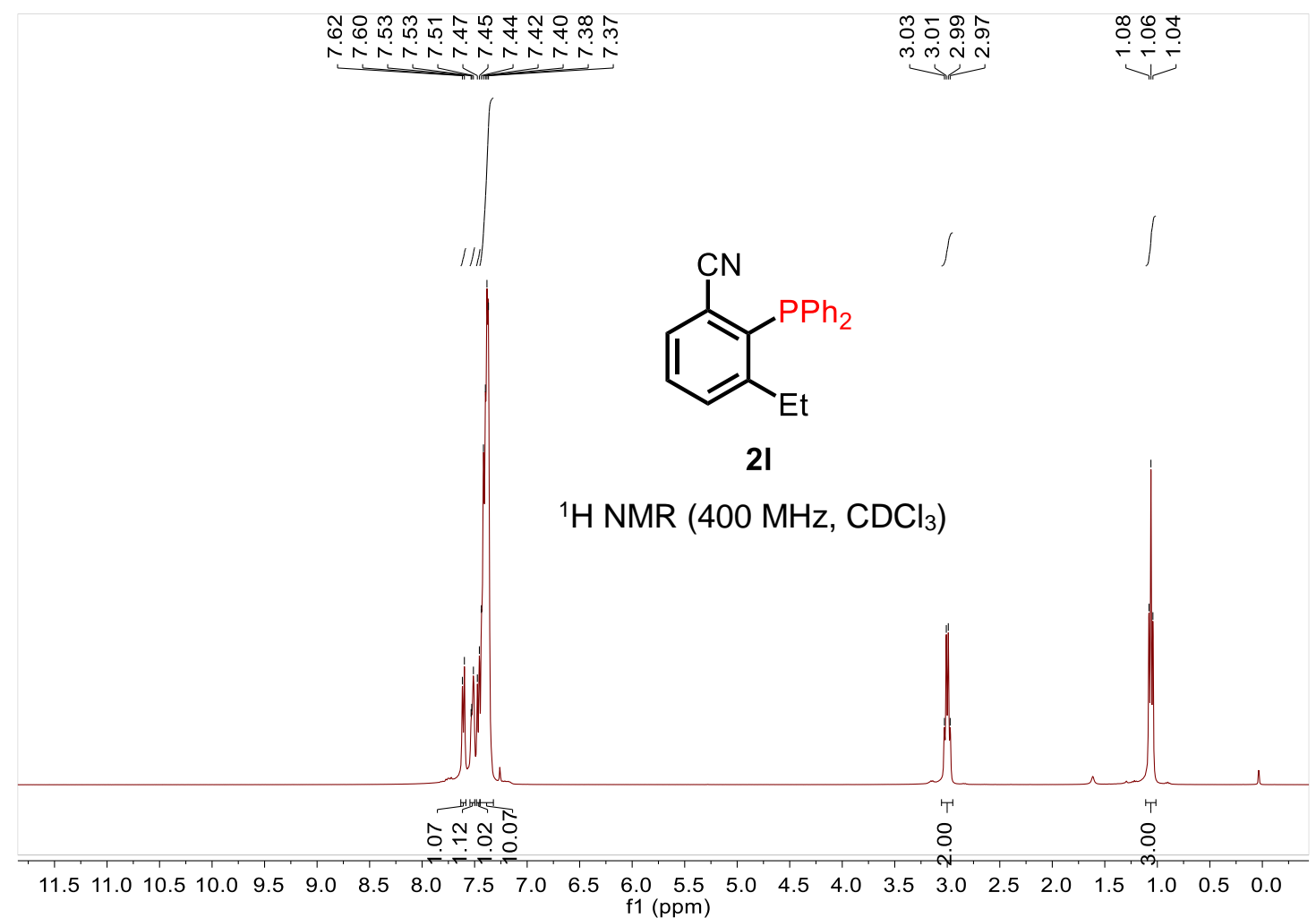




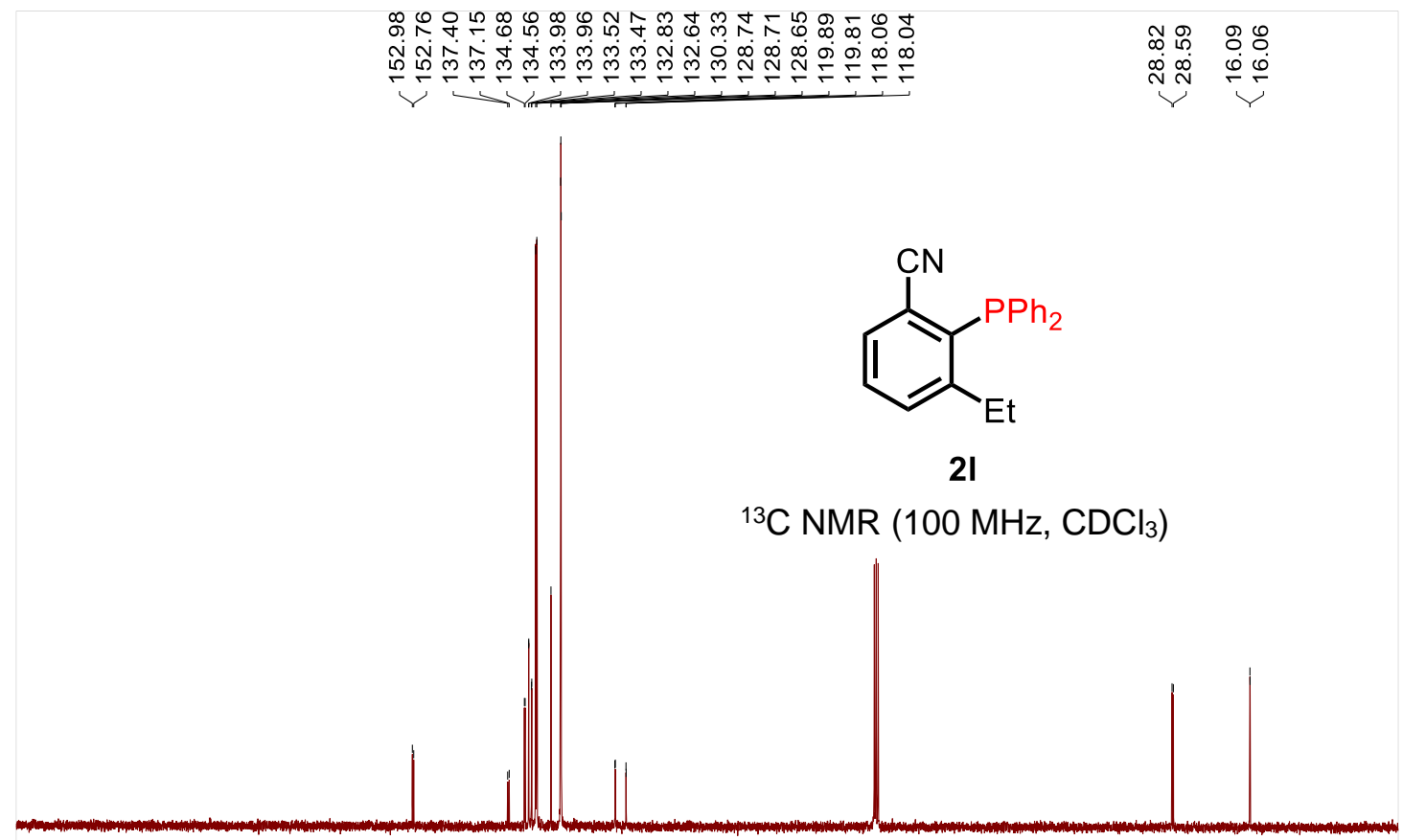

$\begin{array}{llllllllllllllllllllll}210 & 200 & 190 & 180 & 170 & 160 & 150 & 140 & 130 & 120 & \begin{array}{c}110 \\ \mathrm{f} 1(\mathrm{ppm})\end{array} & 90 & 80 & 70 & 60 & 50 & 40 & 30 & 20 & 10 & 0\end{array}$<smiles>CCc1cccc(C#N)c1Pc1ccccc1</smiles>

2I

${ }^{31} \mathrm{P}$ NMR (162 MHz, $\left.\mathrm{CDCl}_{3}\right)$

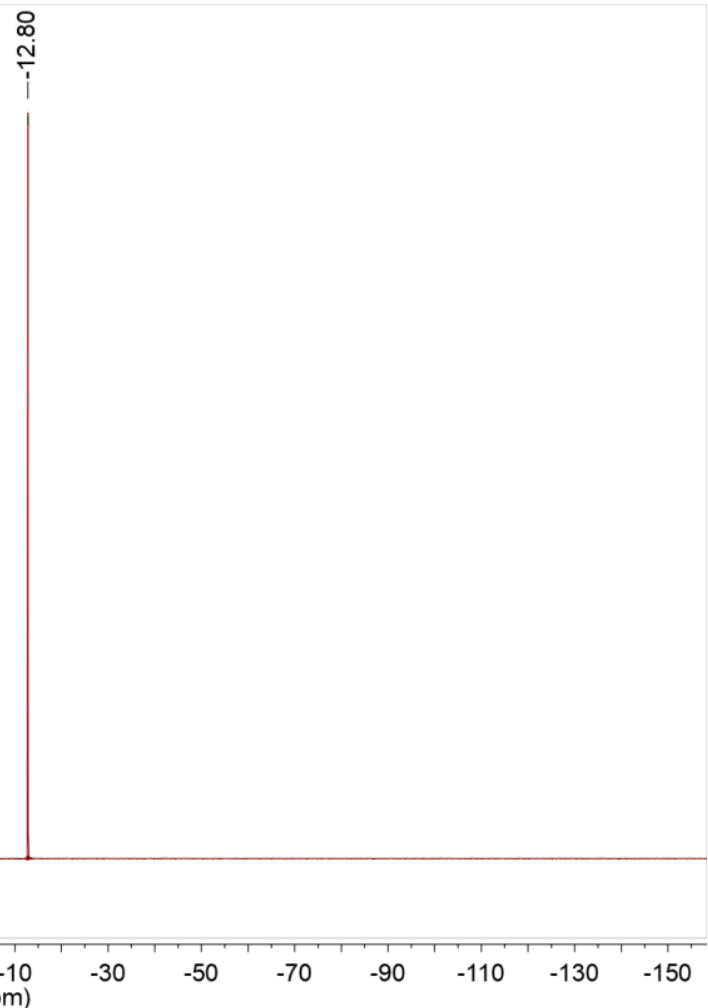




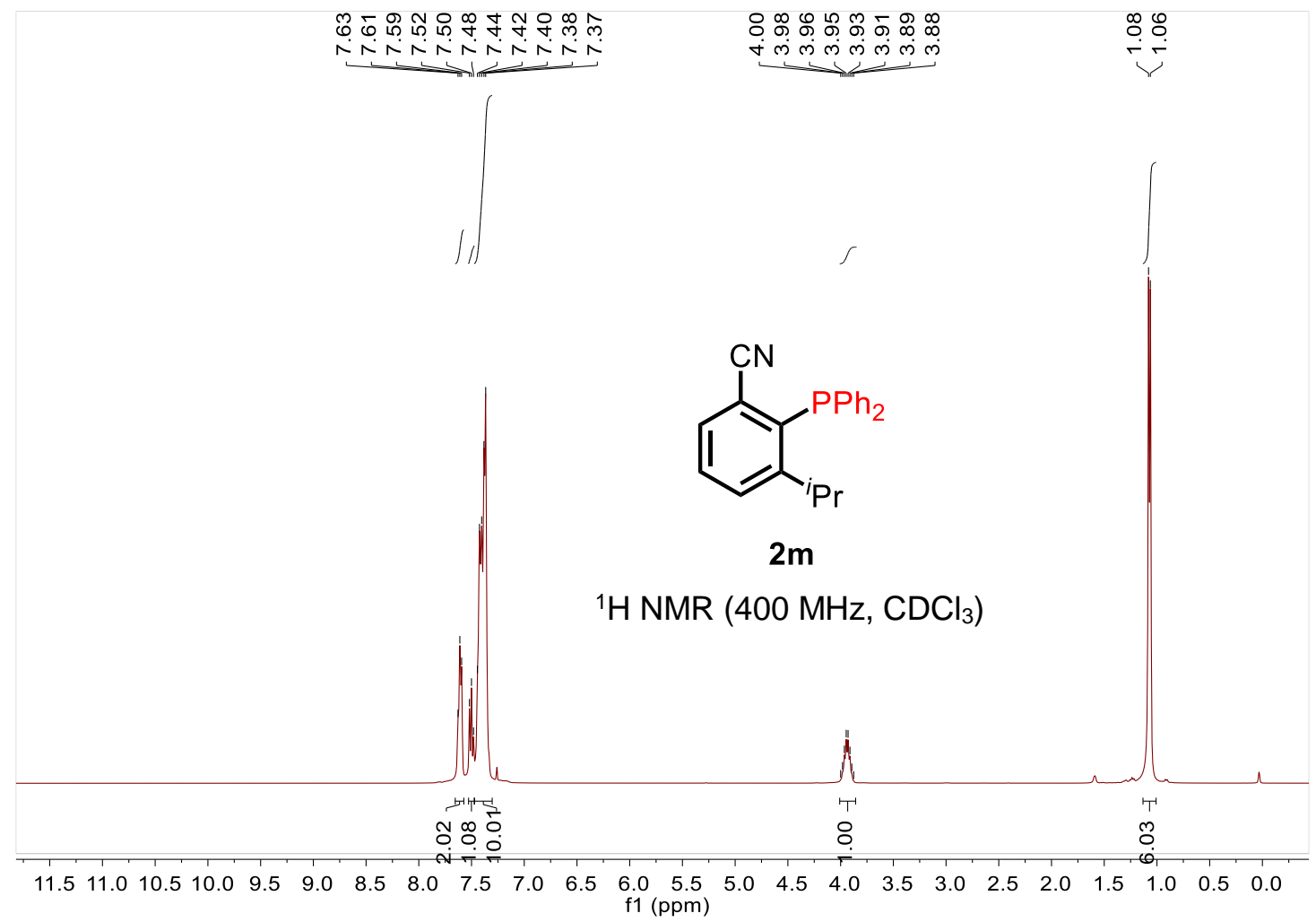

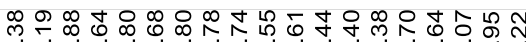

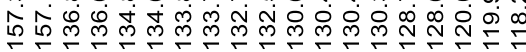

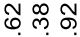

घं்<smiles>CCCc1cccc(C#N)c1P</smiles>

$2 m$

${ }^{13} \mathrm{C} \mathrm{NMR}\left(100 \mathrm{MHz}, \mathrm{CDCl}_{3}\right)$

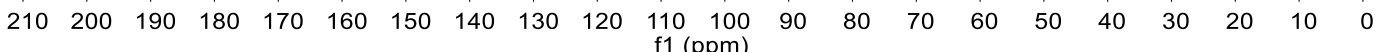




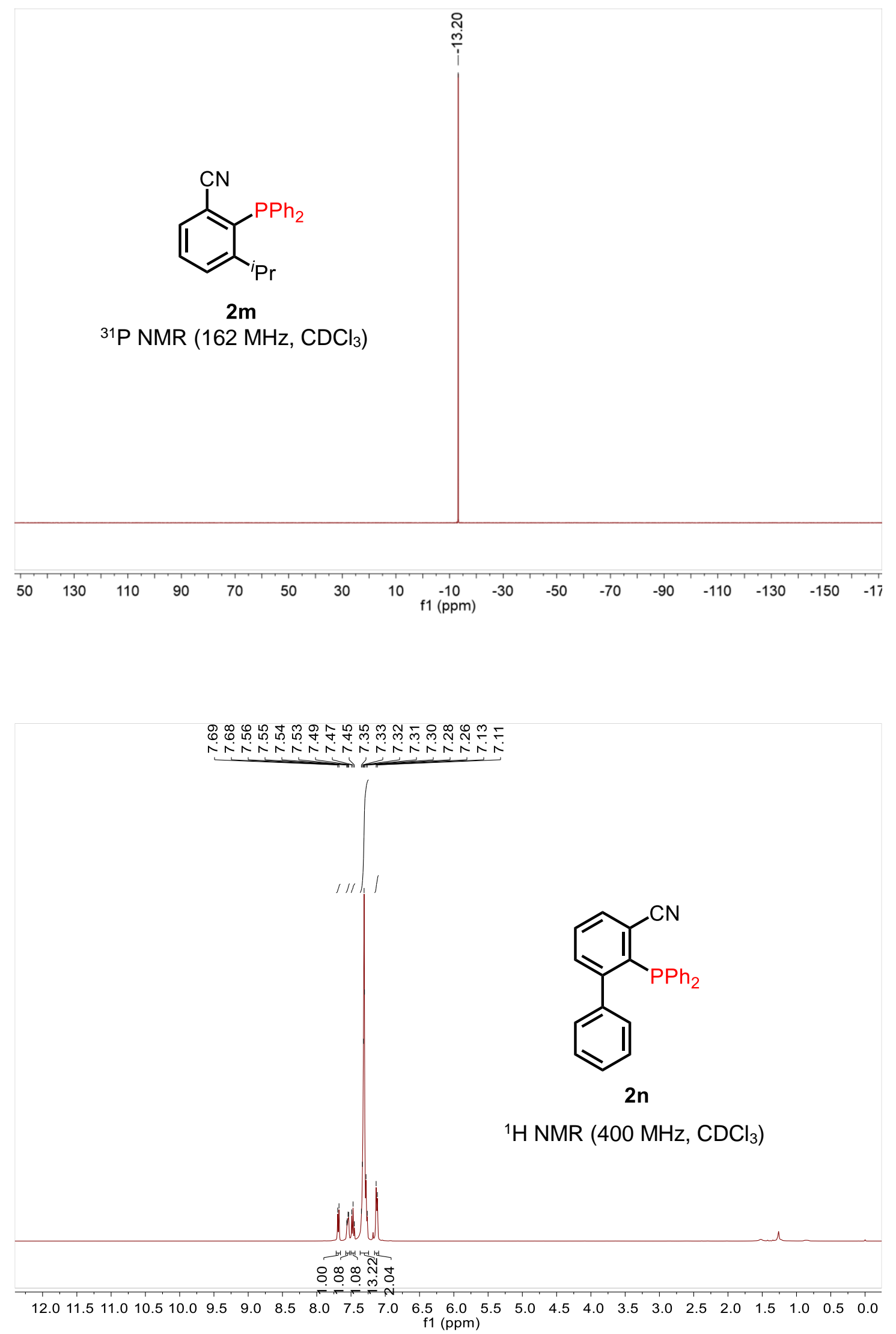




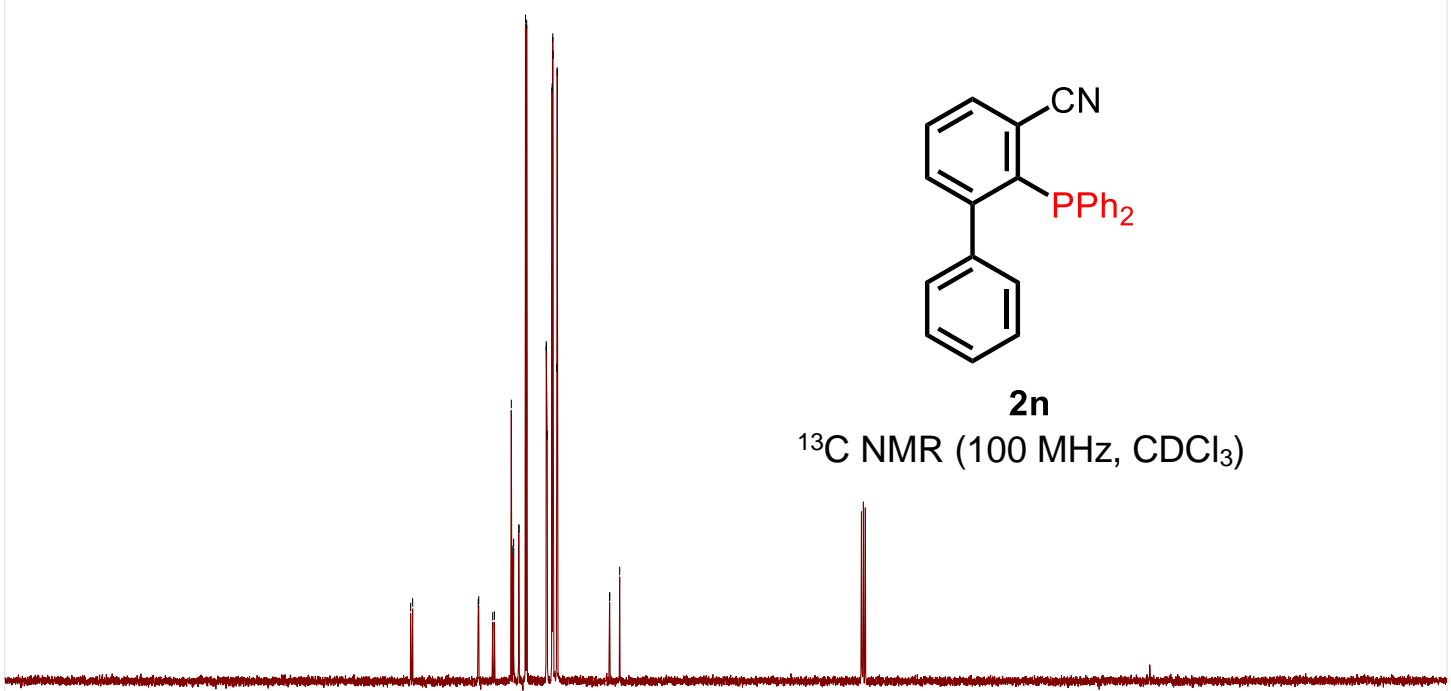<smiles>N#Cc1cccc(-c2ccccc2)c1P</smiles>

2n

${ }^{31} \mathrm{P}$ NMR $\left(162 \mathrm{MHz}, \mathrm{CDCl}_{3}\right)$ 


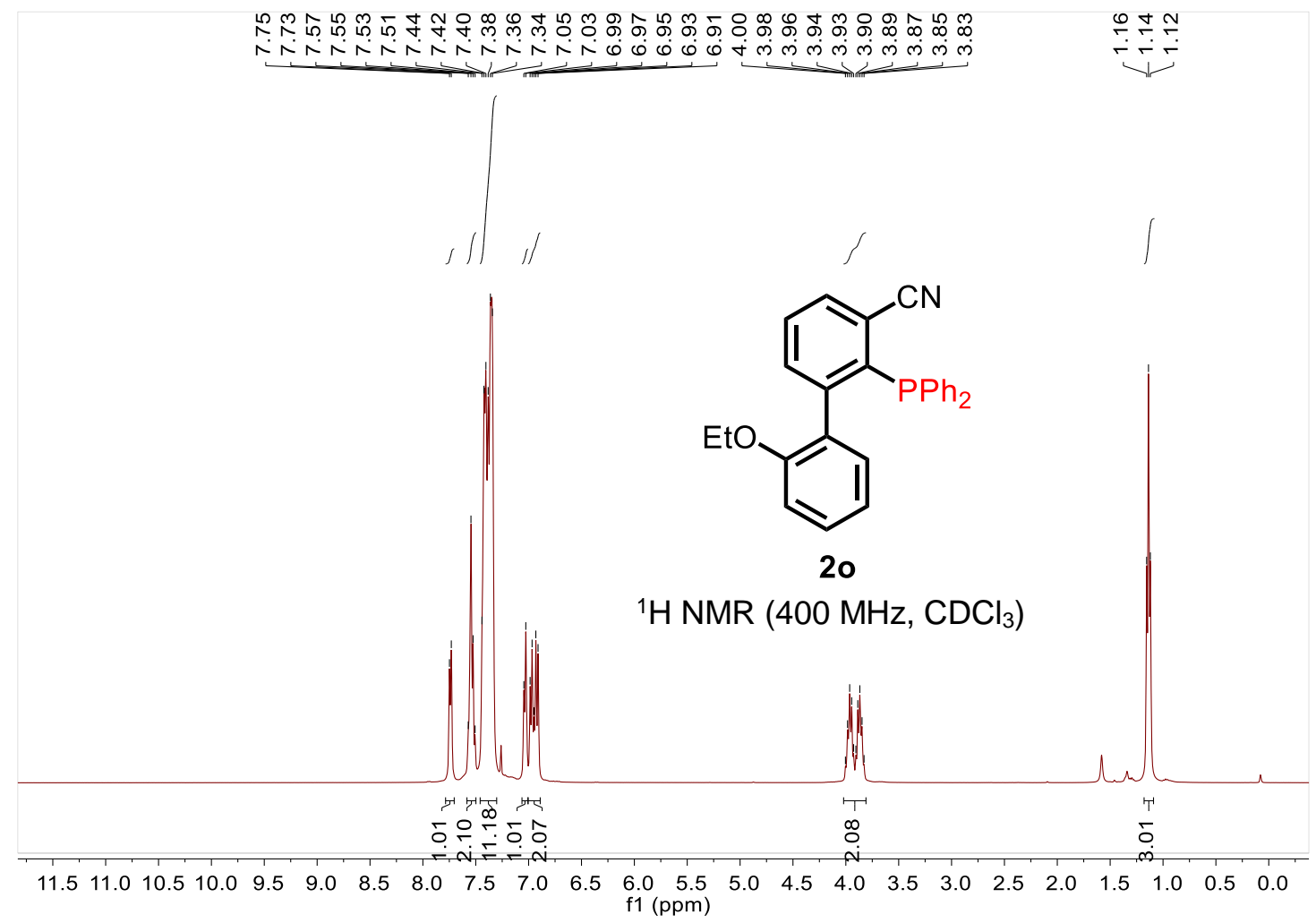

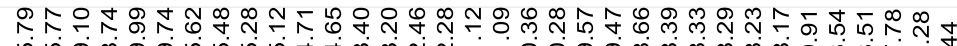

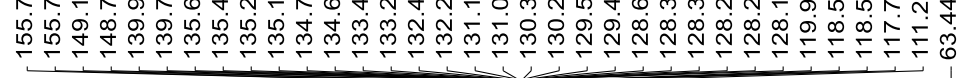

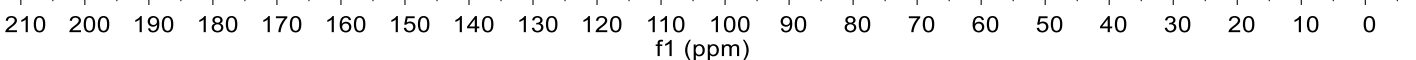




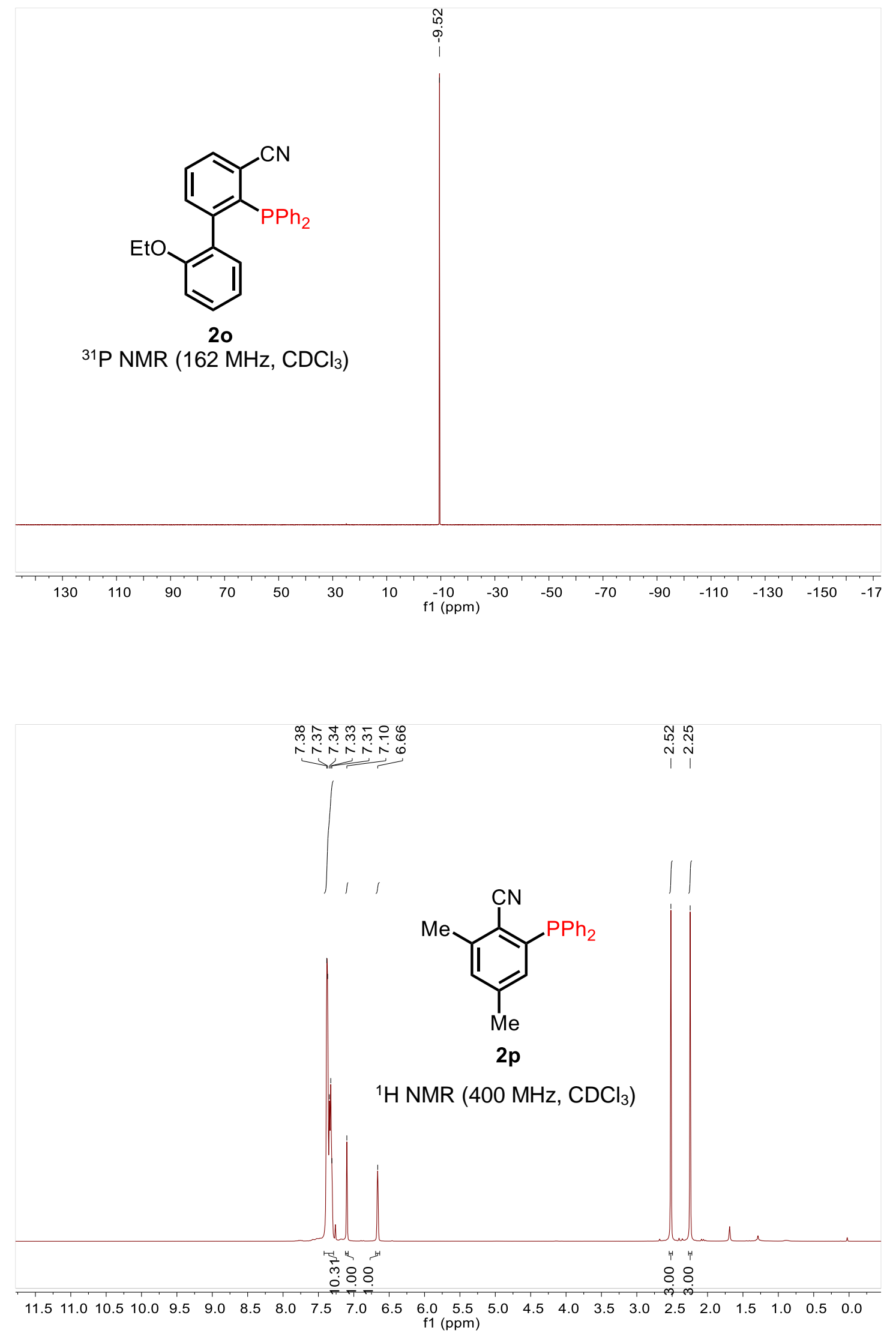




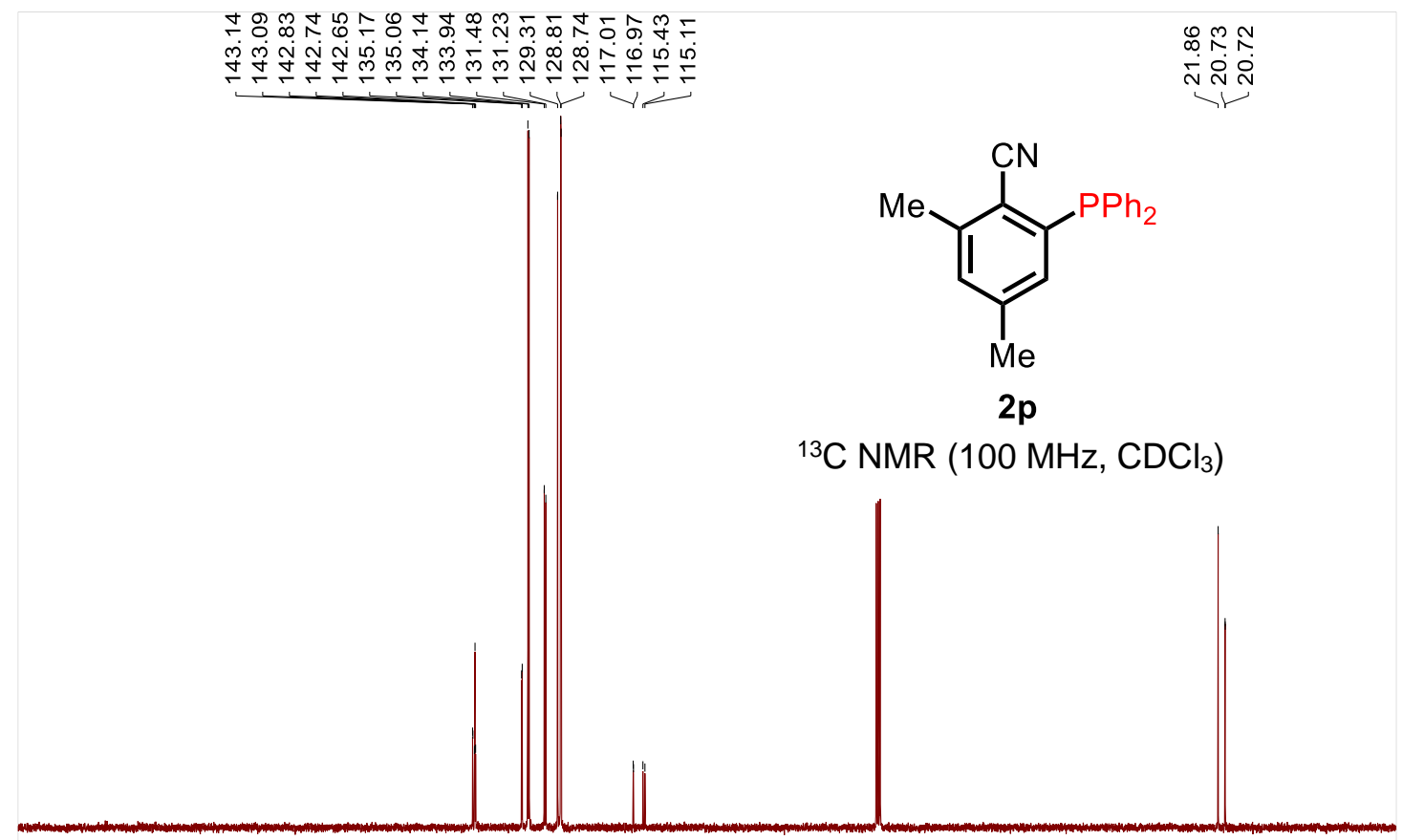

$\begin{array}{lllllllllllllllllllll}210 & 200 & 190 & 180 & 170 & 160 & 150 & 140 & 130 & 120 & \begin{array}{c}110 \\ \mathrm{f} 1(\mathrm{ppm})\end{array} & 90 & 80 & 70 & 60 & 50 & 40 & 30 & 20 & 10 & 0\end{array}$

$\stackrel{+}{\circ}$<smiles>Cc1cc(C)c(C#N)c(P)c1</smiles>

${ }^{31} \mathrm{P}$ NMR $\left(162 \mathrm{MHz}, \mathrm{CDCl}_{3}\right)$

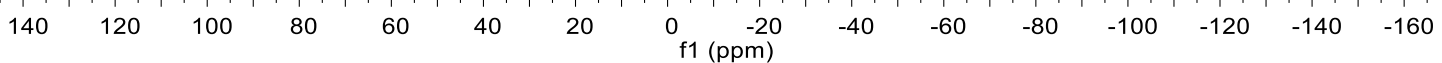




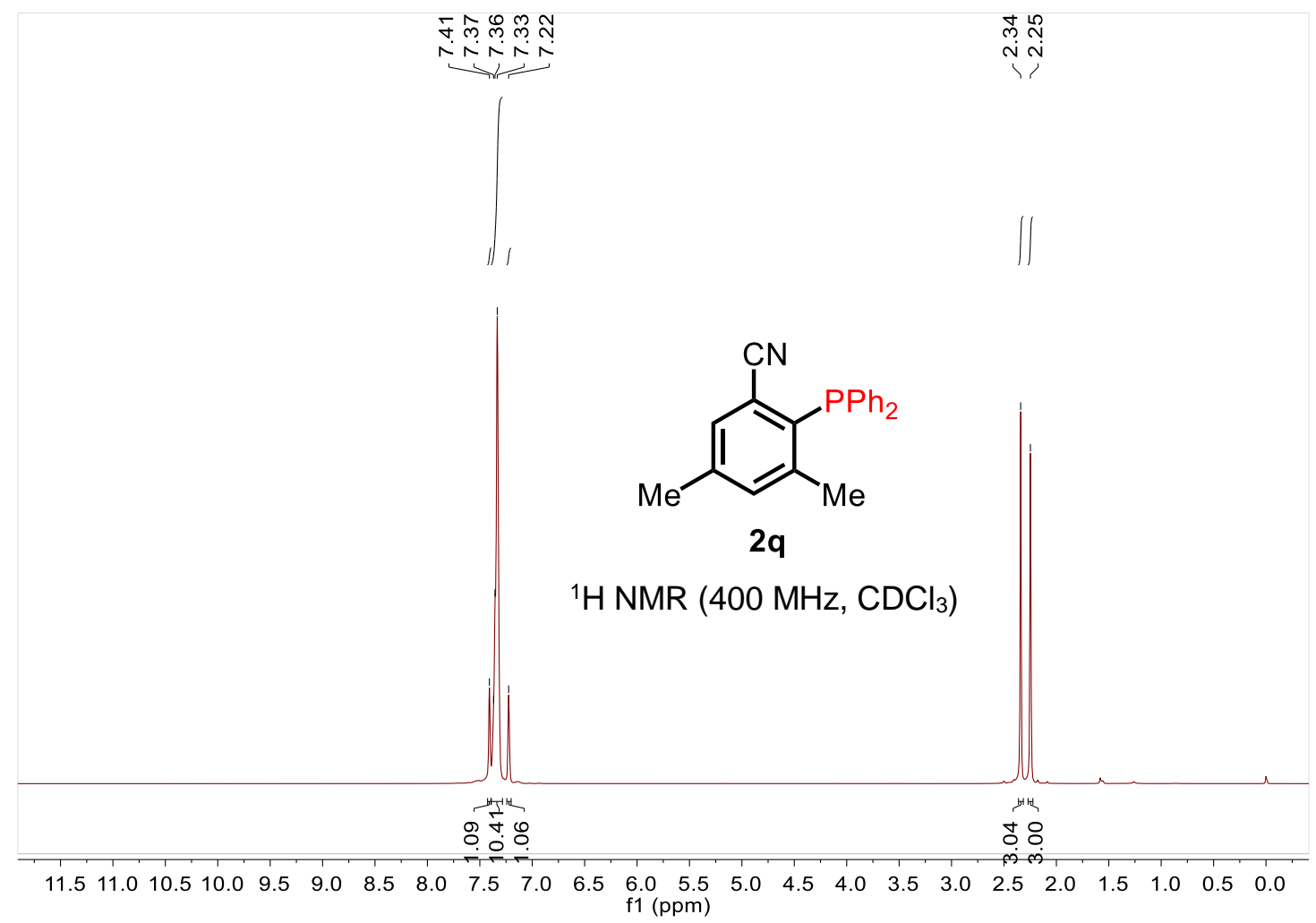

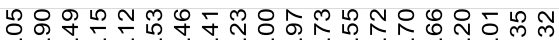

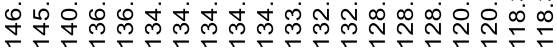

م. 赵象

ลู่ ำ

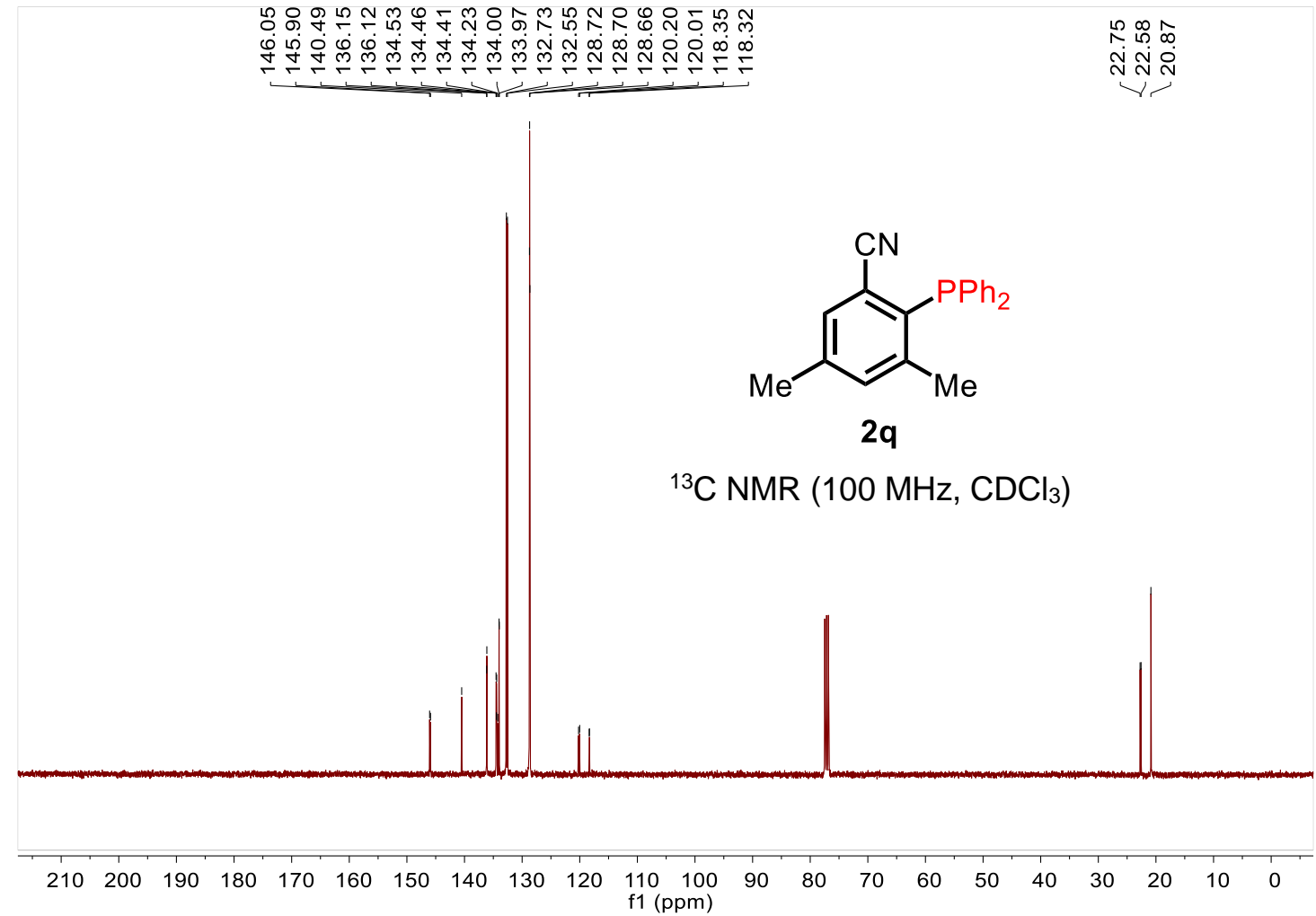




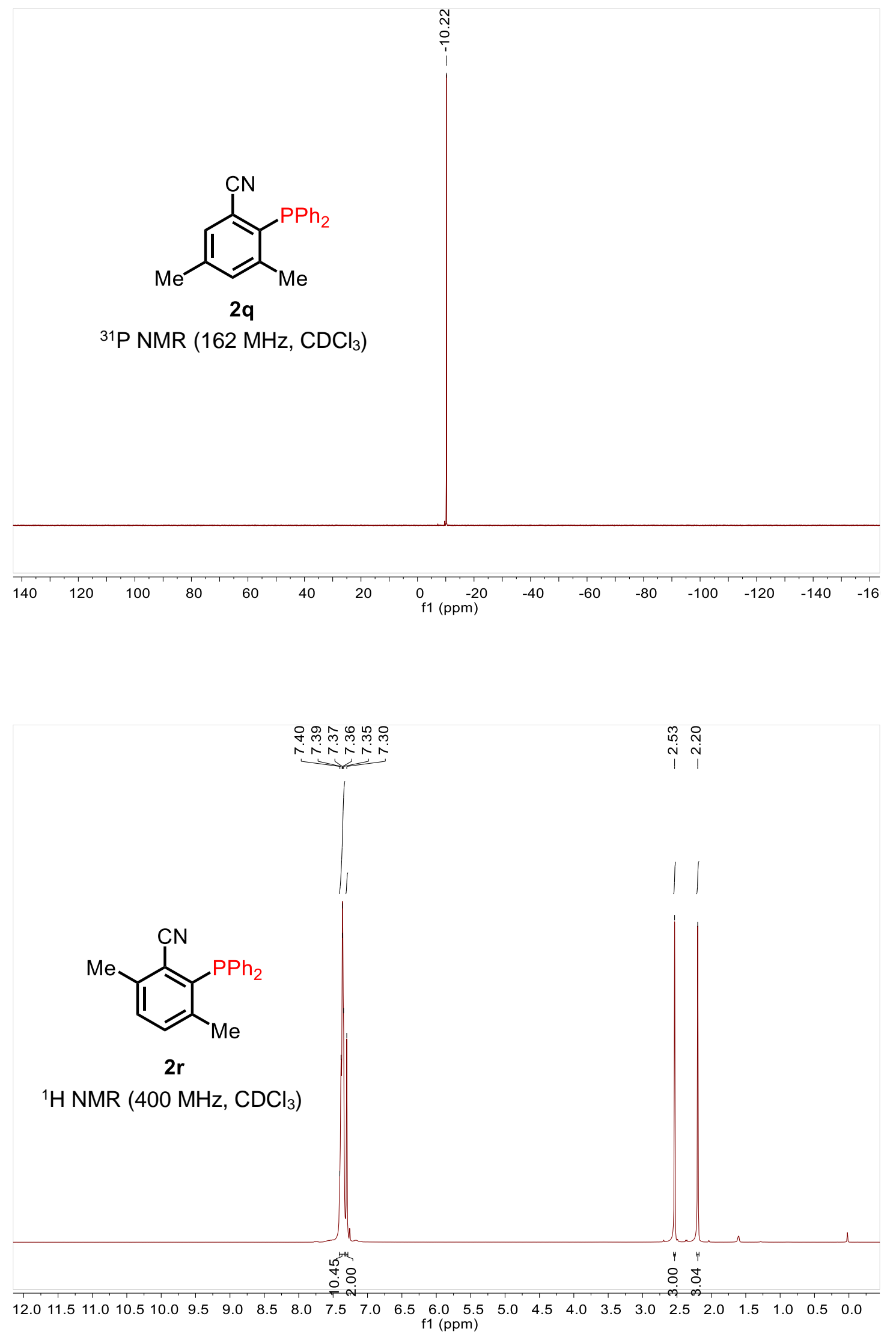




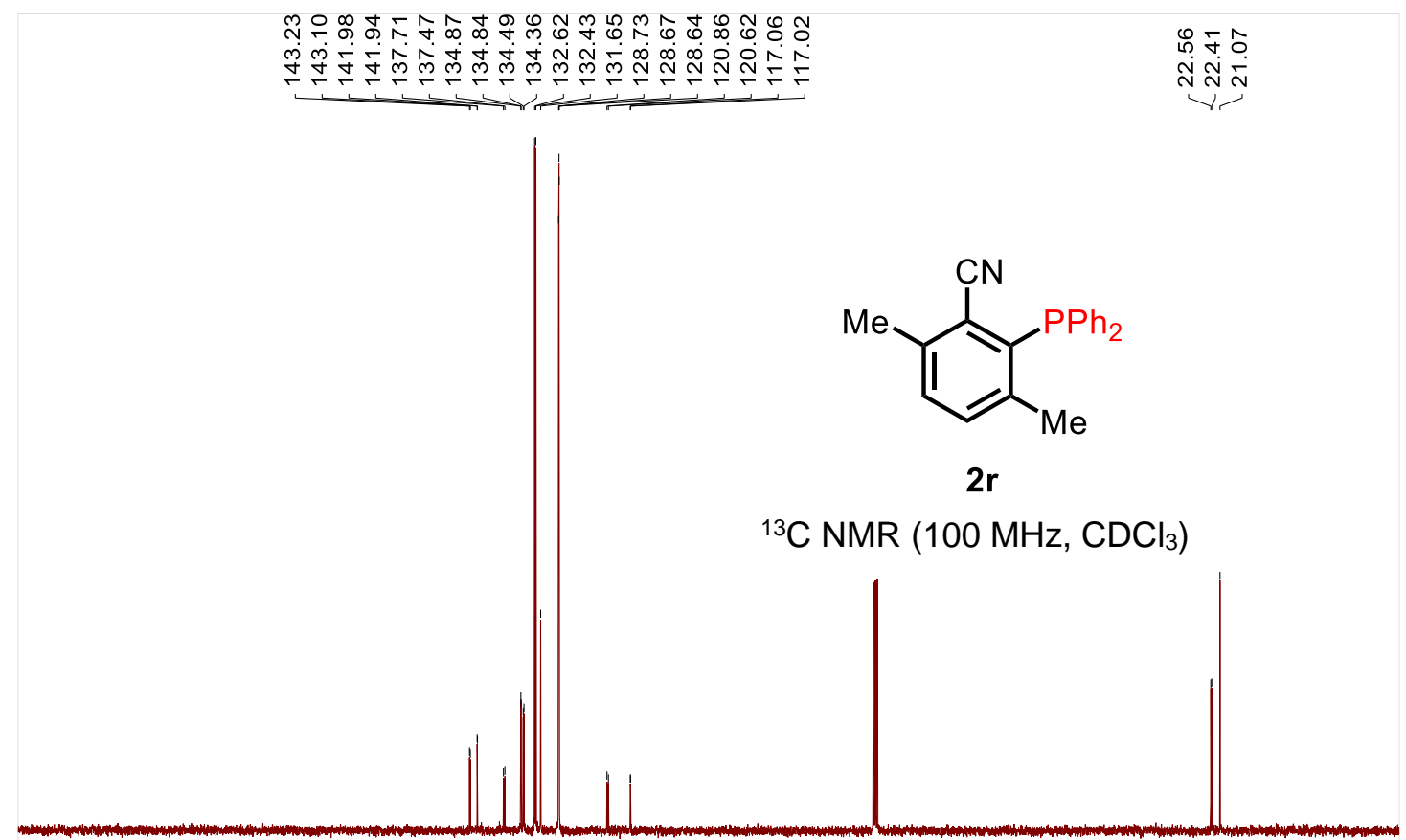

$\begin{array}{rrrrrrrrrrrrrrrrrrrrrr}210 & 200 & 190 & 180 & 170 & 160 & 150 & 140 & 130 & 120 & \begin{array}{c}110 \\ \mathrm{f} 1(\mathrm{ppm})\end{array} & 90 & 80 & 70 & 60 & 50 & 40 & 30 & 20 & 10 & 0\end{array}$
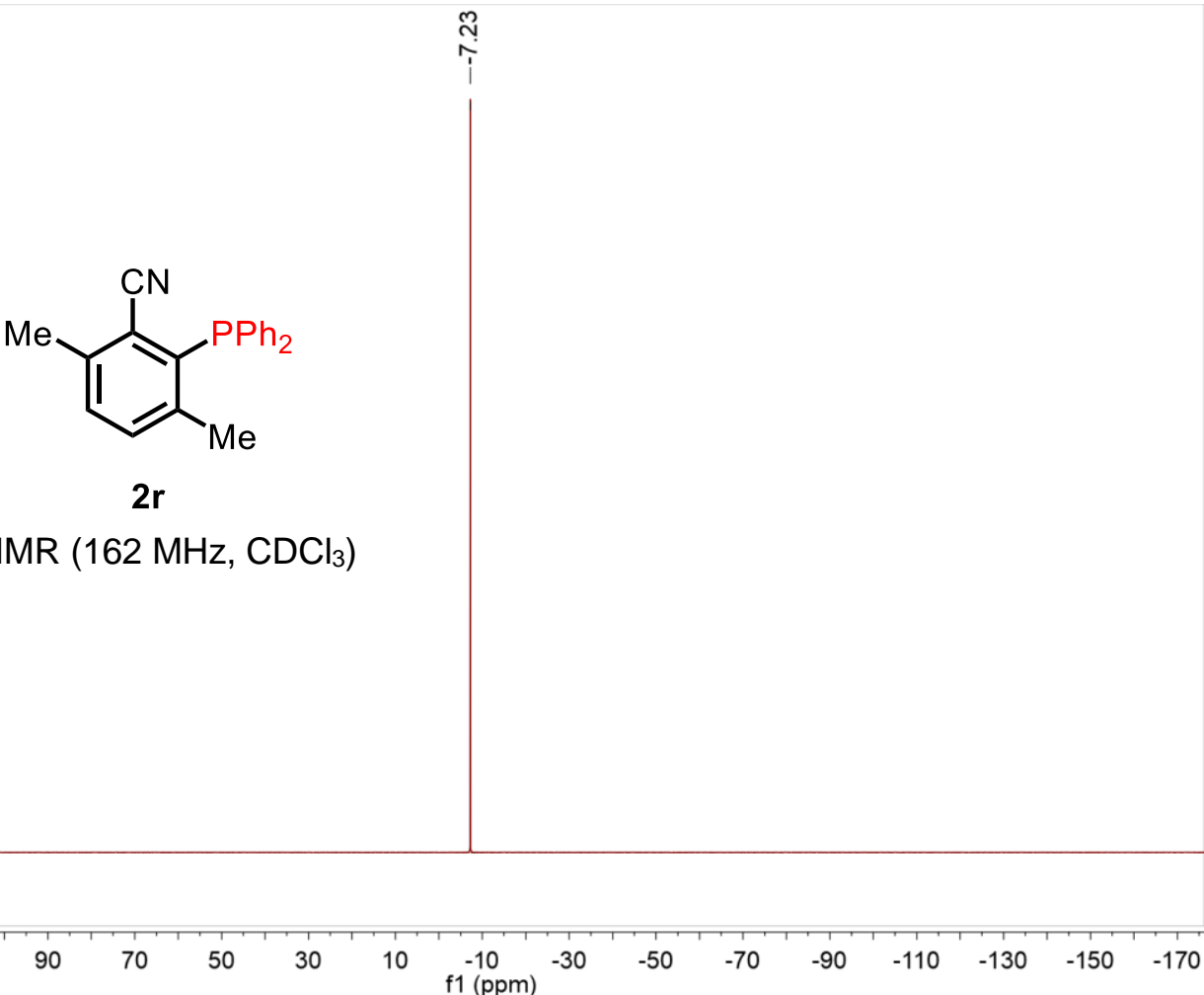


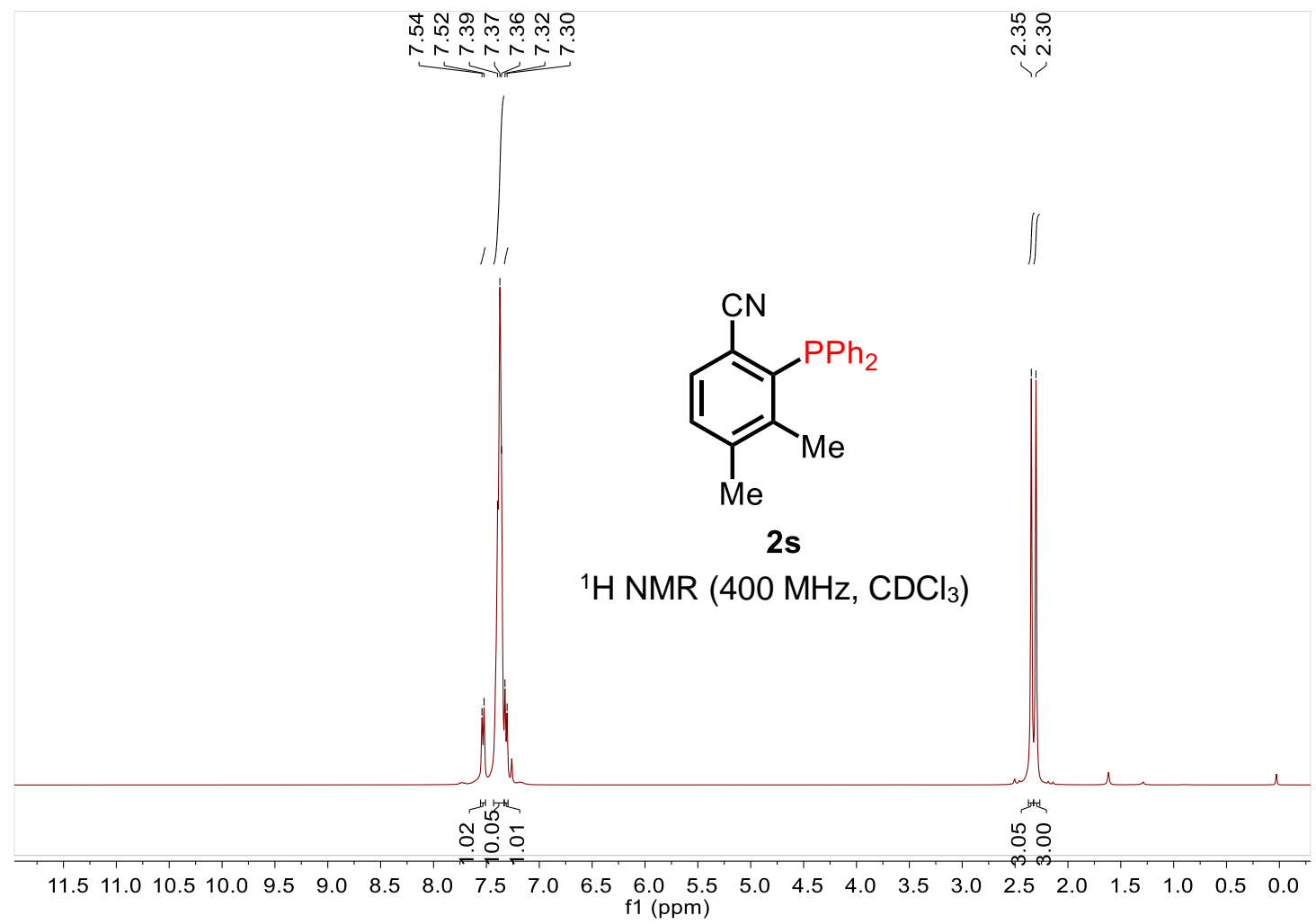

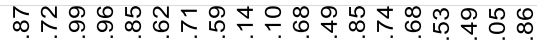

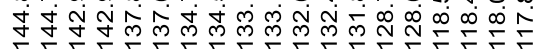

เุ่ กุะ

$\dot{\sim} \dot{\circ} \dot{\circ}$

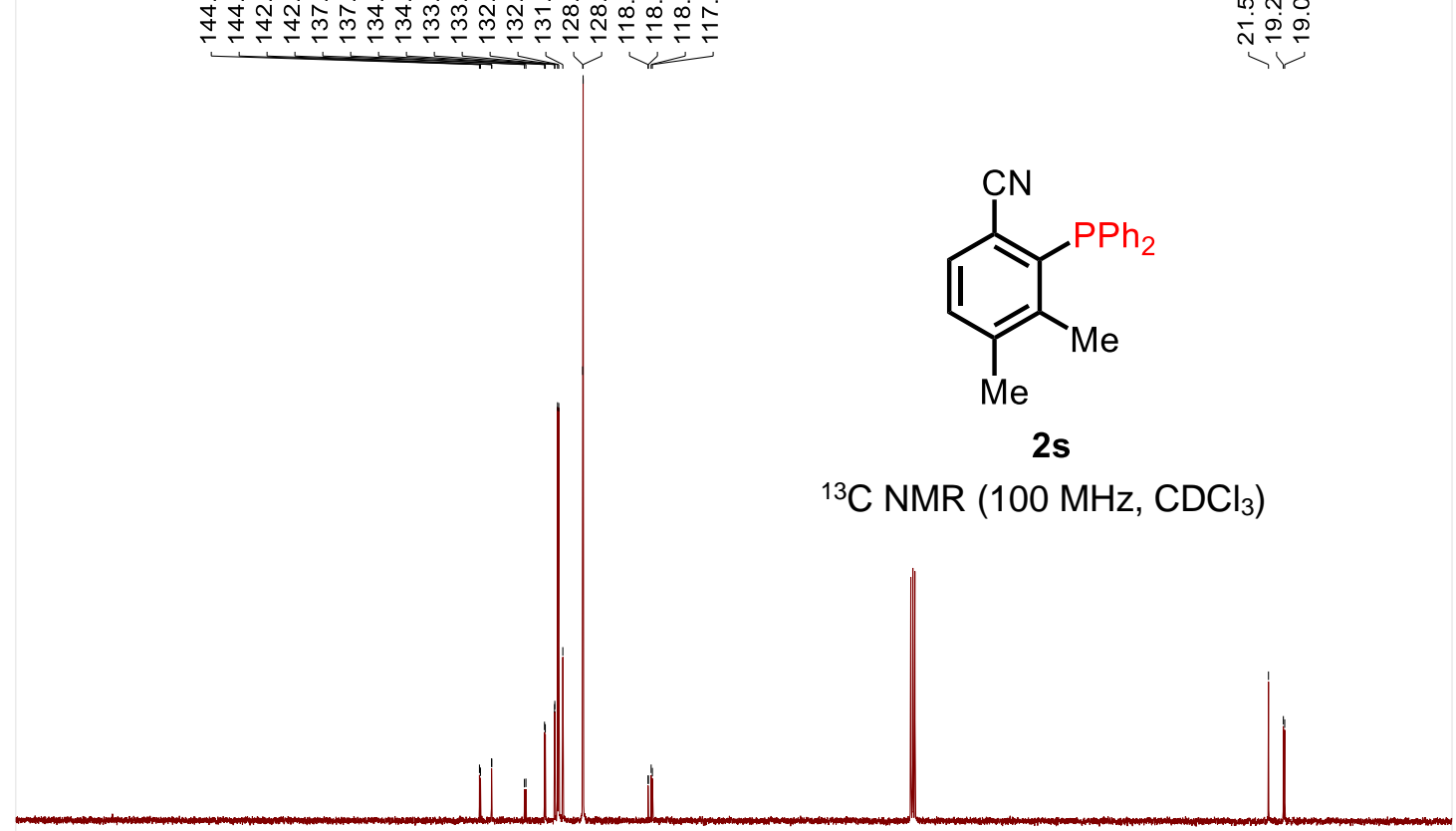

$\begin{array}{lllllllllllllllllllllllllllll}210 & 200 & 190 & 180 & 170 & 160 & 150 & 140 & 130 & 120 & 110 & 100 & 90 & 80 & 70 & 60 & 50 & 40 & 30 & 20 & 10 & 0\end{array}$ 


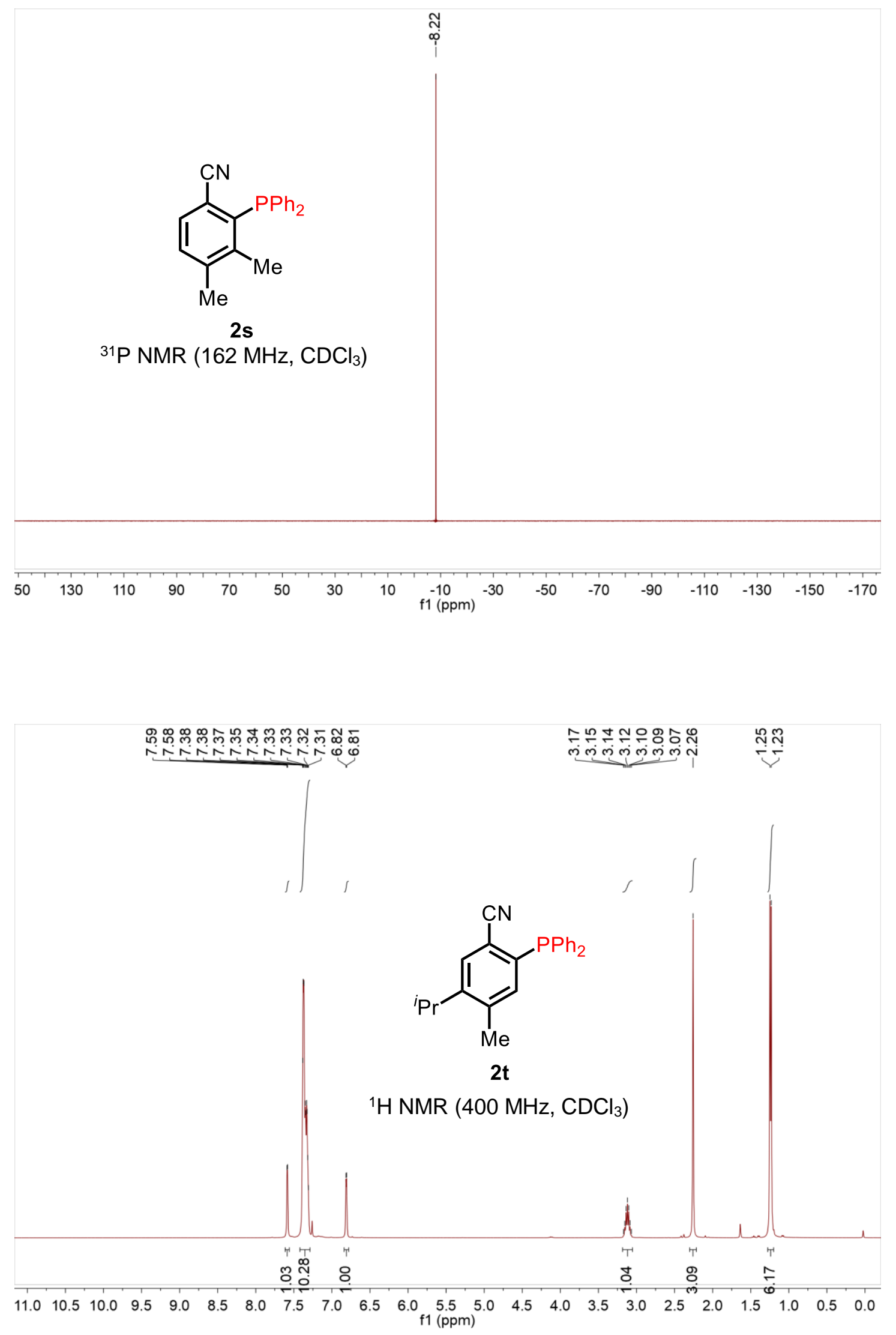




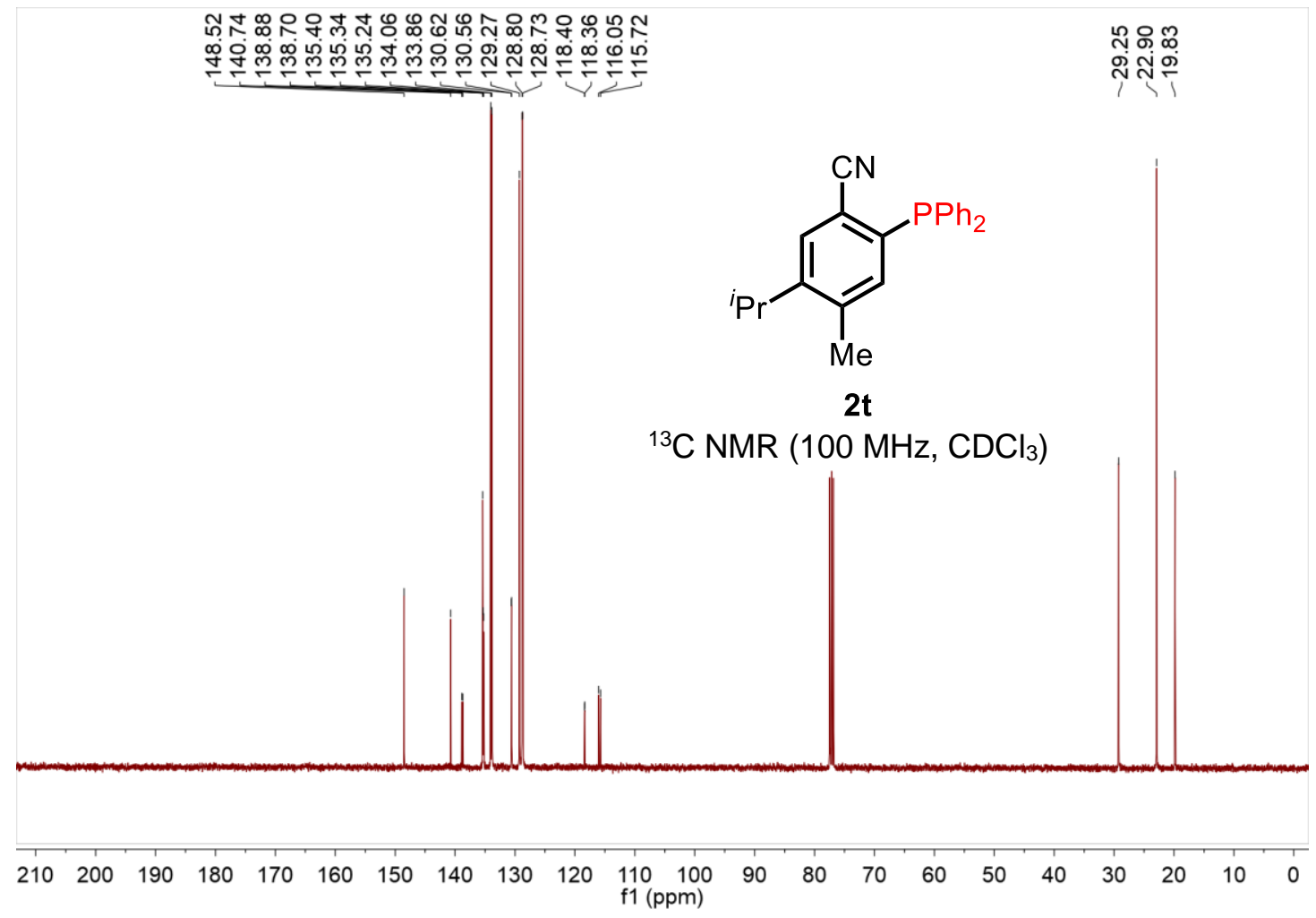

ฺุ<smiles>CCCc1cc(C#N)c(Pc2ccccc2)cc1C</smiles>

2t

${ }^{31} \mathrm{P} \mathrm{NMR}\left(162 \mathrm{MHz}, \mathrm{CDCl}_{3}\right)$

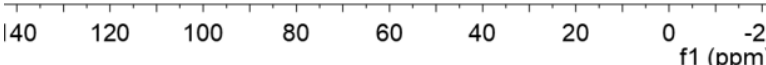

$\begin{array}{lllllll}-40 & -60 & -80 & -100 & -120 & -140 & -160\end{array}$ 


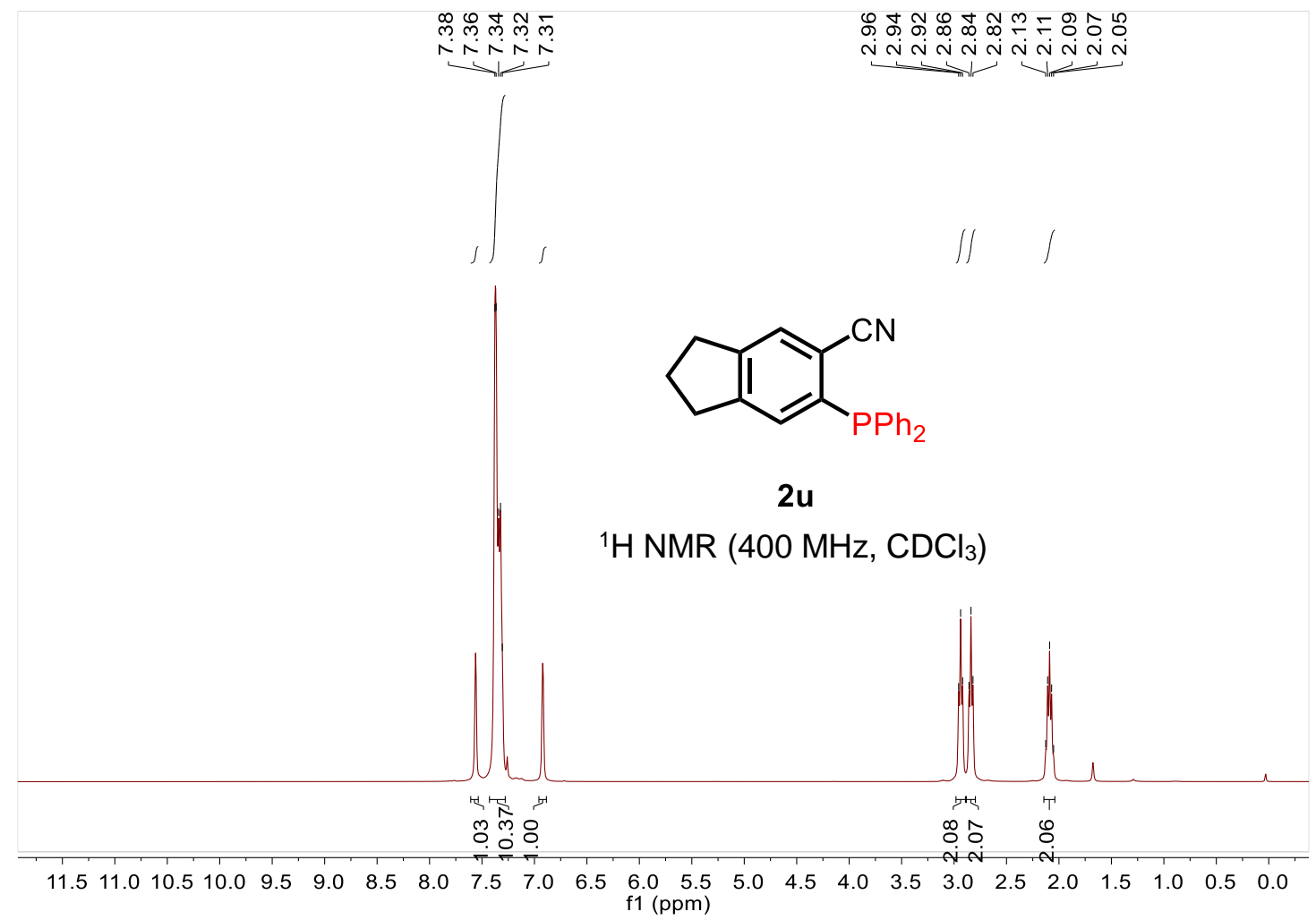

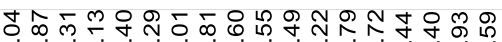

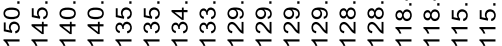

กุ่

mृ

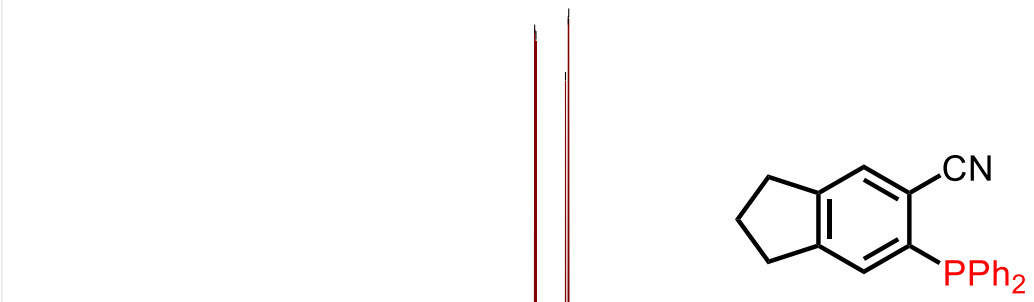

$2 u$

$\left.{ }^{13} \mathrm{C} \mathrm{NMR} \mathrm{(100} \mathrm{MHz,} \mathrm{CDCl}_{3}\right)$

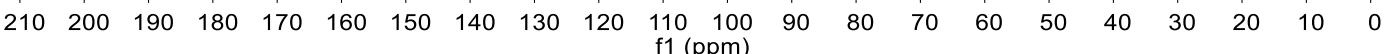




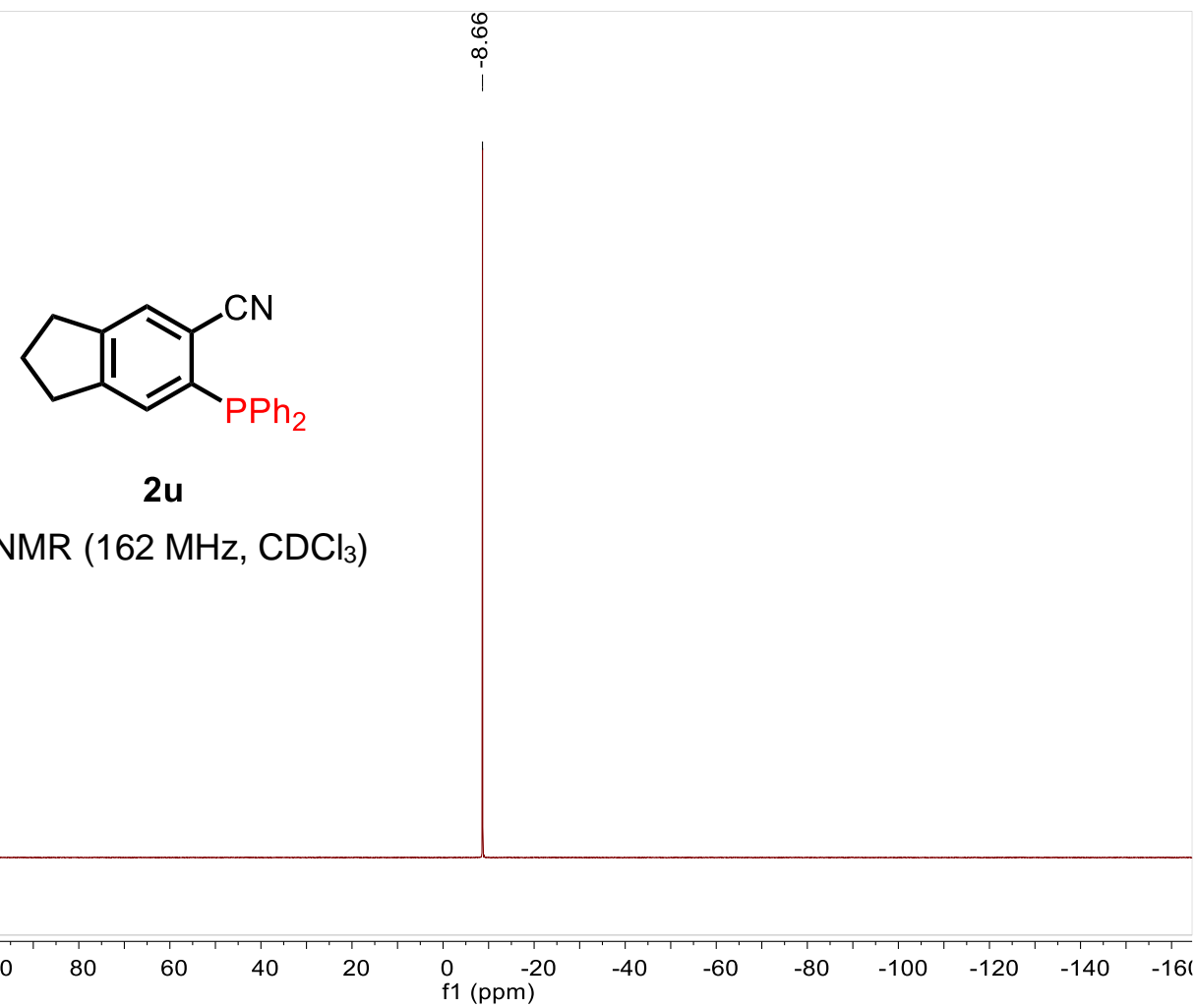

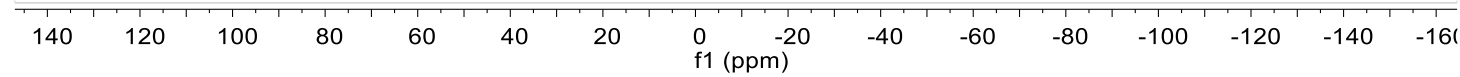

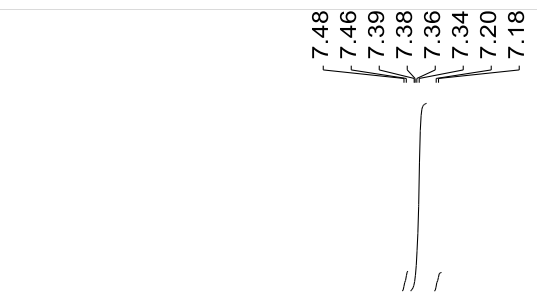

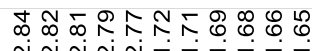

ก n n n n<smiles>N#Cc1ccc2c(c1P(P)c1ccccc1)CCCC2</smiles>

2v

${ }^{1} \mathrm{H} \mathrm{NMR}\left(400 \mathrm{MHz}, \mathrm{CDCl}_{3}\right)$

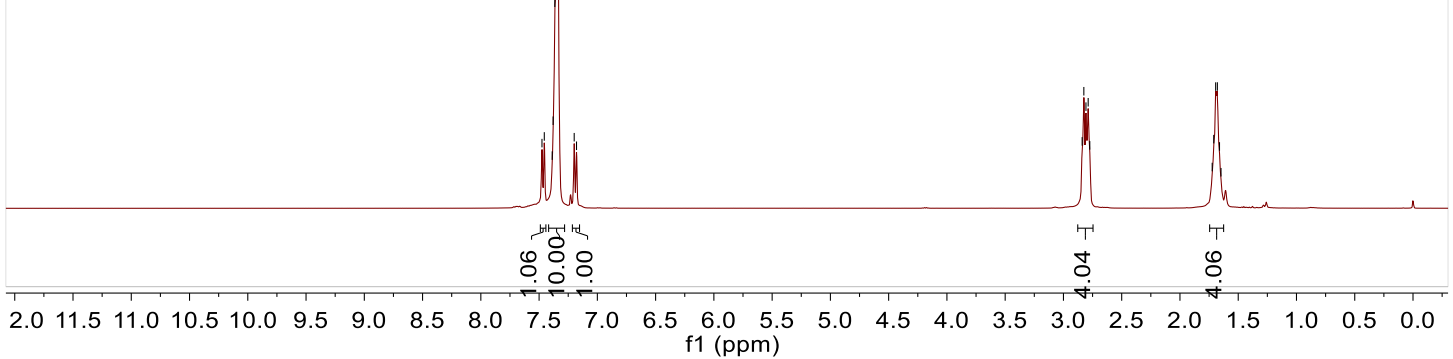




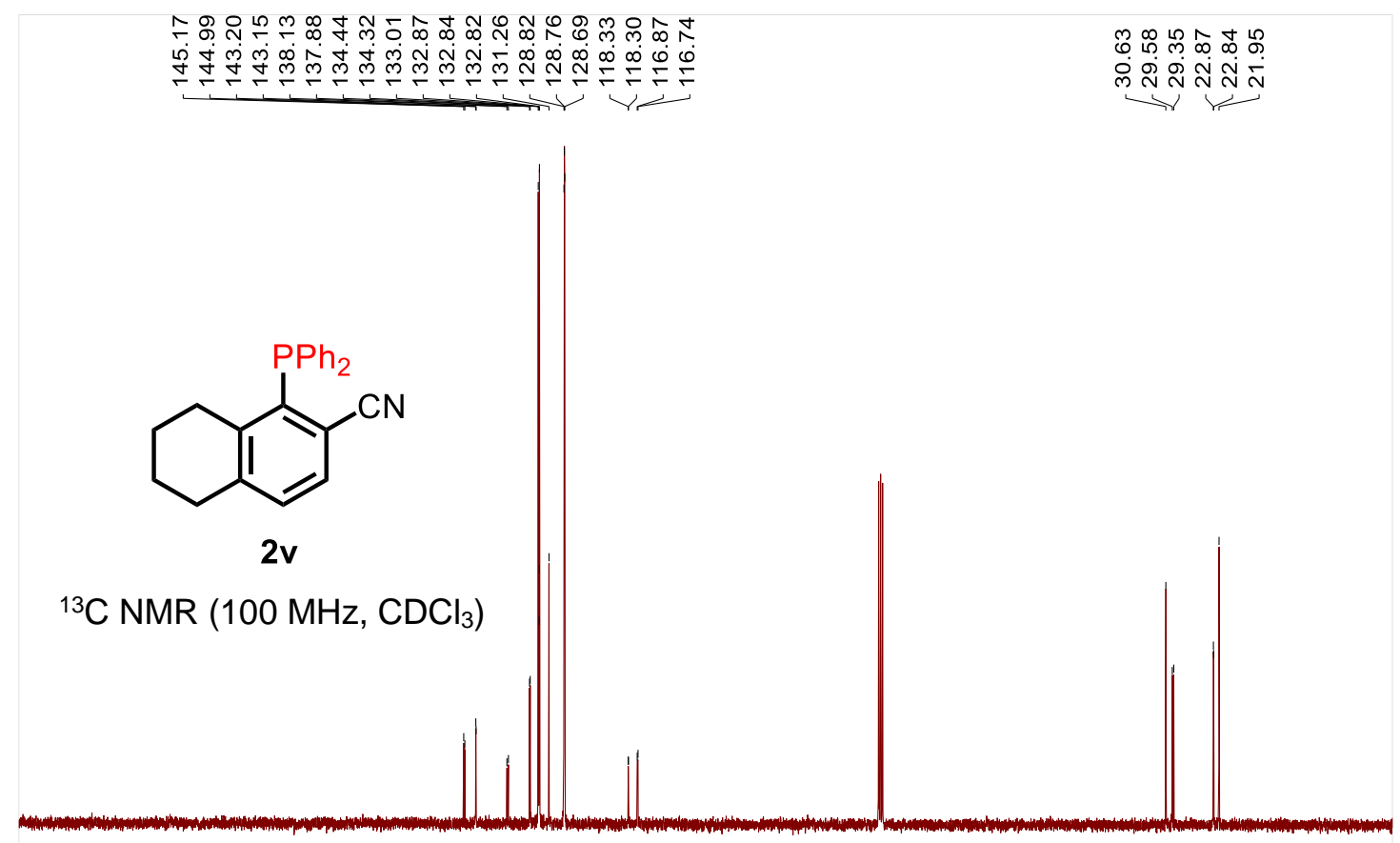

$\begin{array}{llllllllllllllllllllll}210 & 200 & 190 & 180 & 170 & 160 & 150 & 140 & 130 & 120 & \begin{array}{l}110 \\ \mathrm{f} 1(\mathrm{ppm})\end{array} & 90 & 80 & 70 & 60 & 50 & 40 & 30 & 20 & 10 & 0\end{array}$

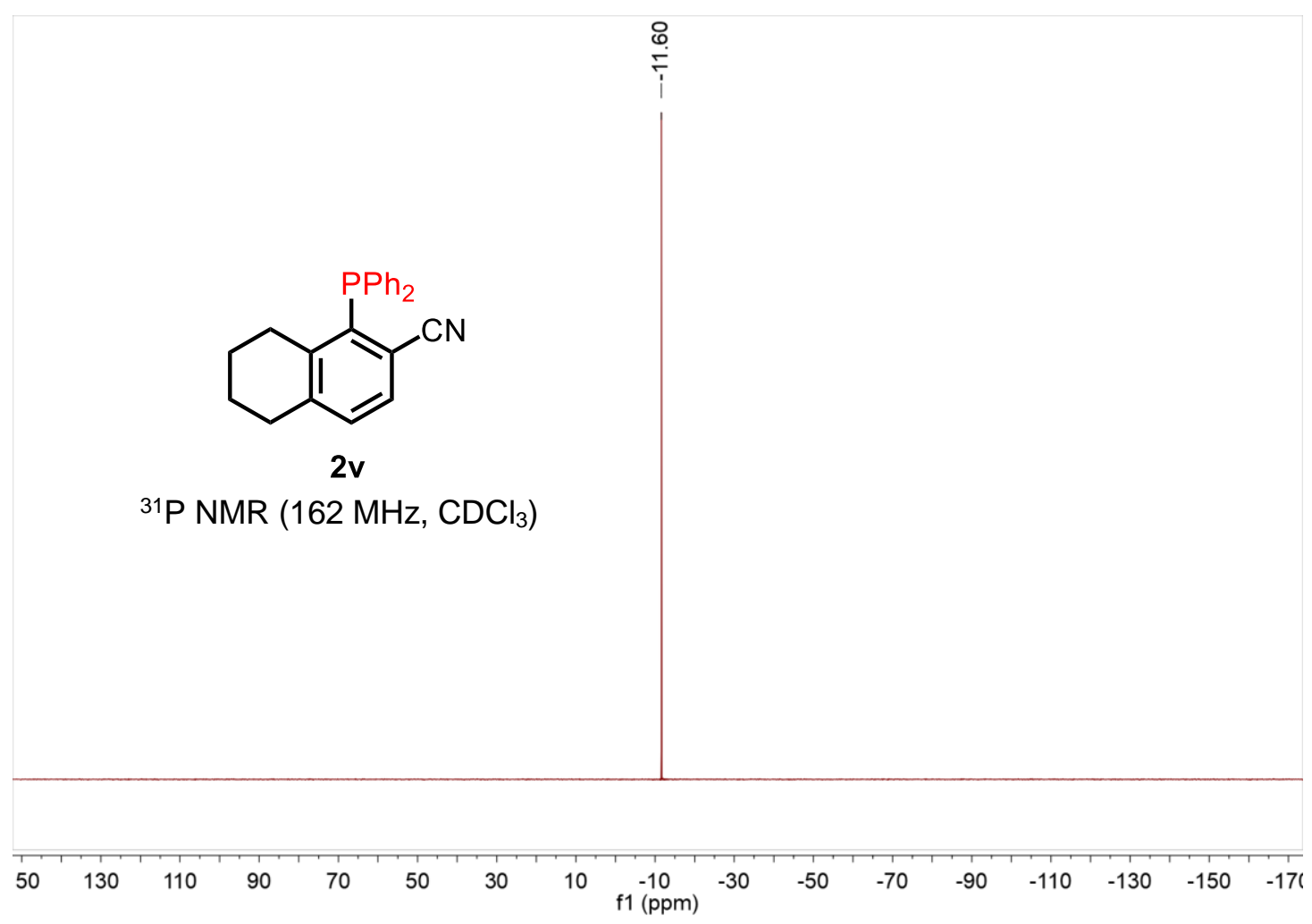



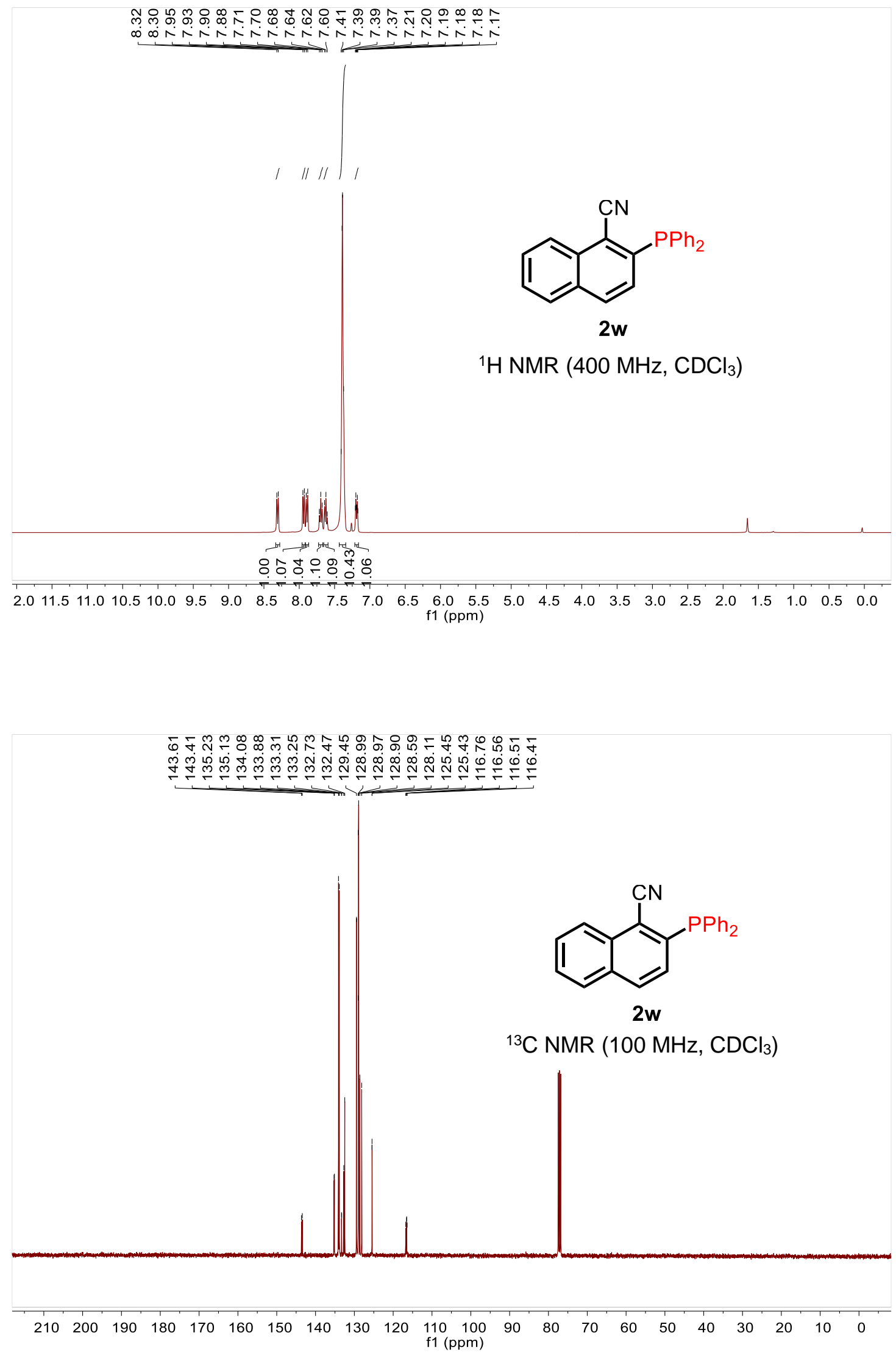

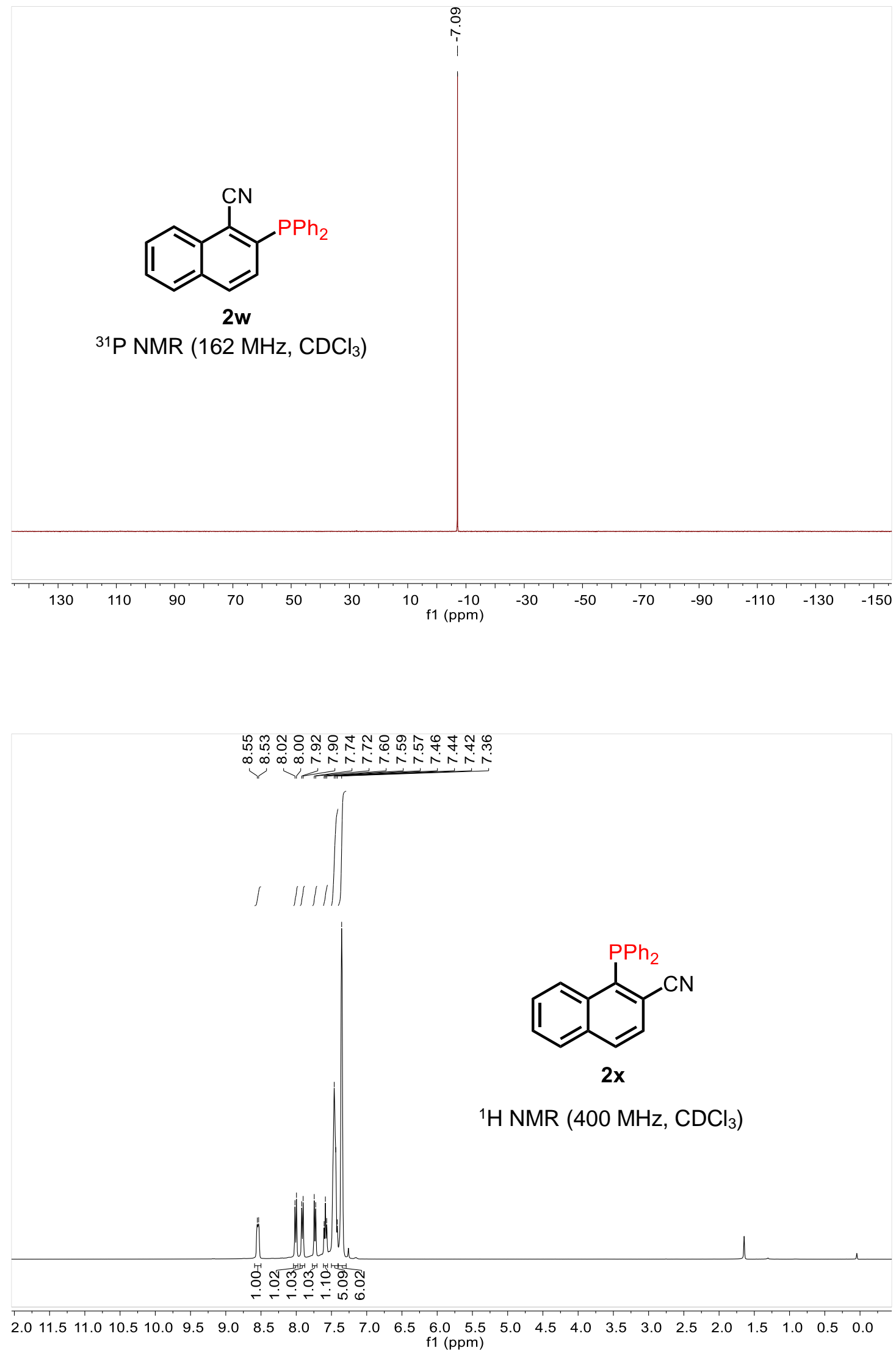


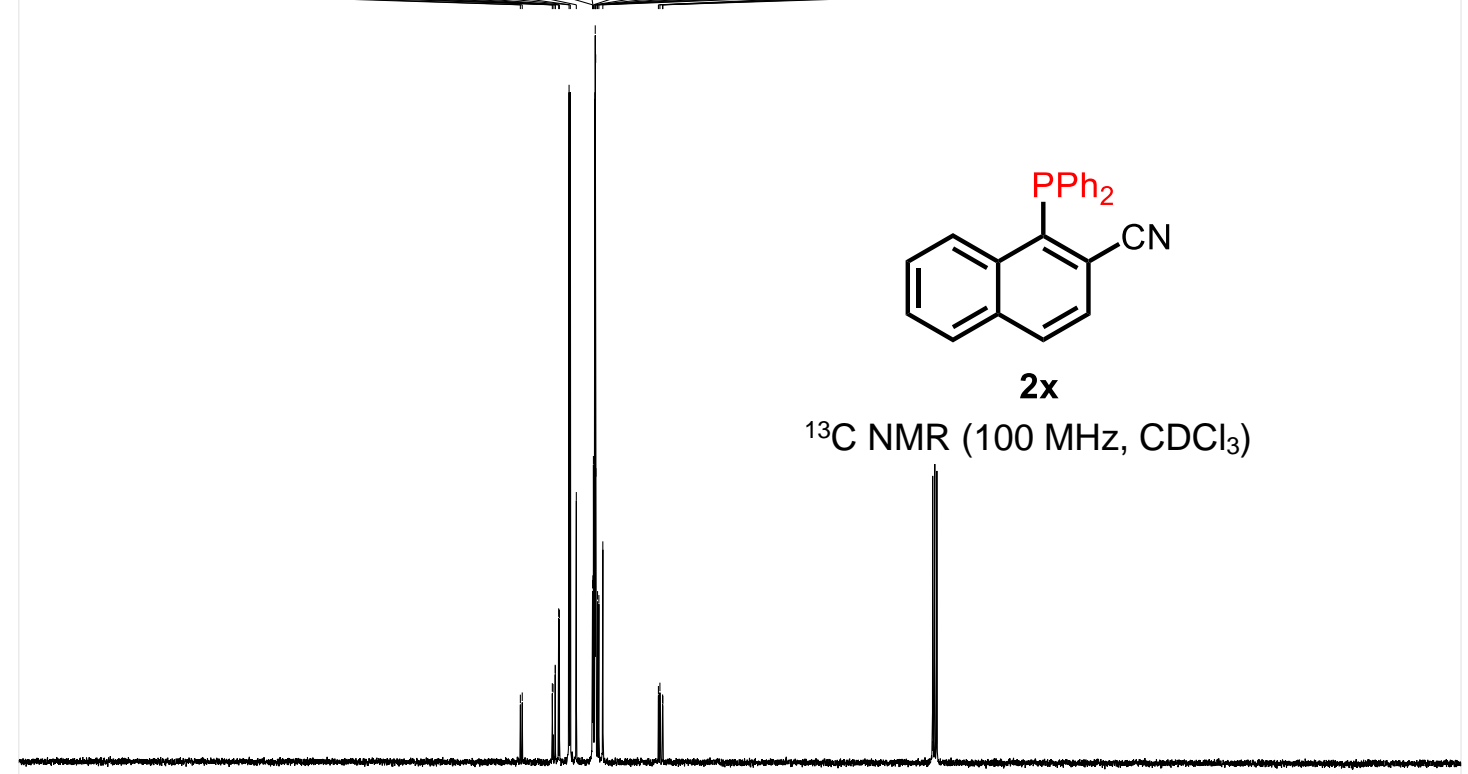

$\begin{array}{lllllllllllllllllllll}210 & 200 & 190 & 180 & 170 & 160 & 150 & 140 & 130 & 120 & \begin{array}{c}110 \\ \mathrm{f} 1(\mathrm{ppm})\end{array} & 90 & 80 & 70 & 60 & 50 & 40 & 30 & 20 & 10 & 0\end{array}$

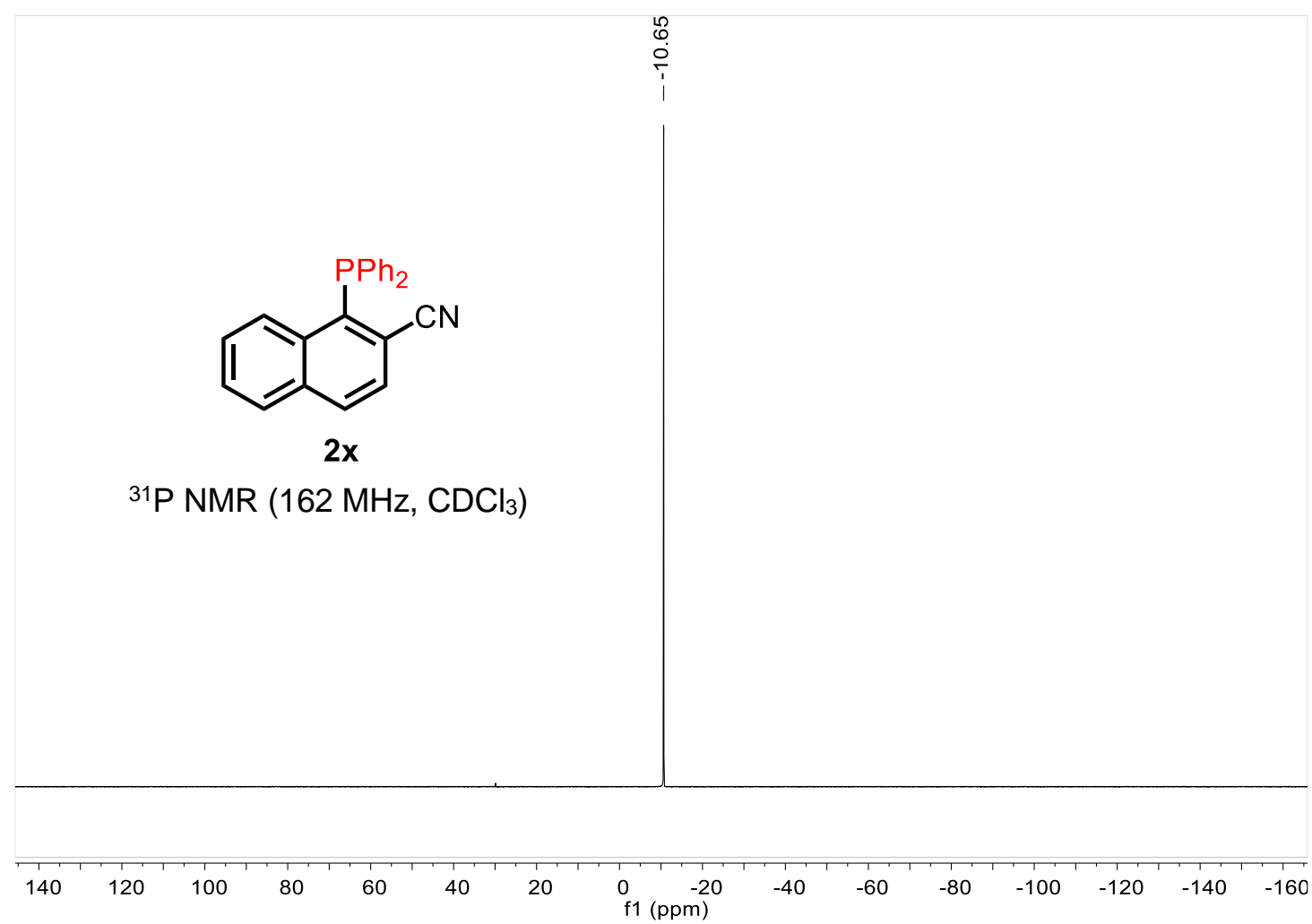




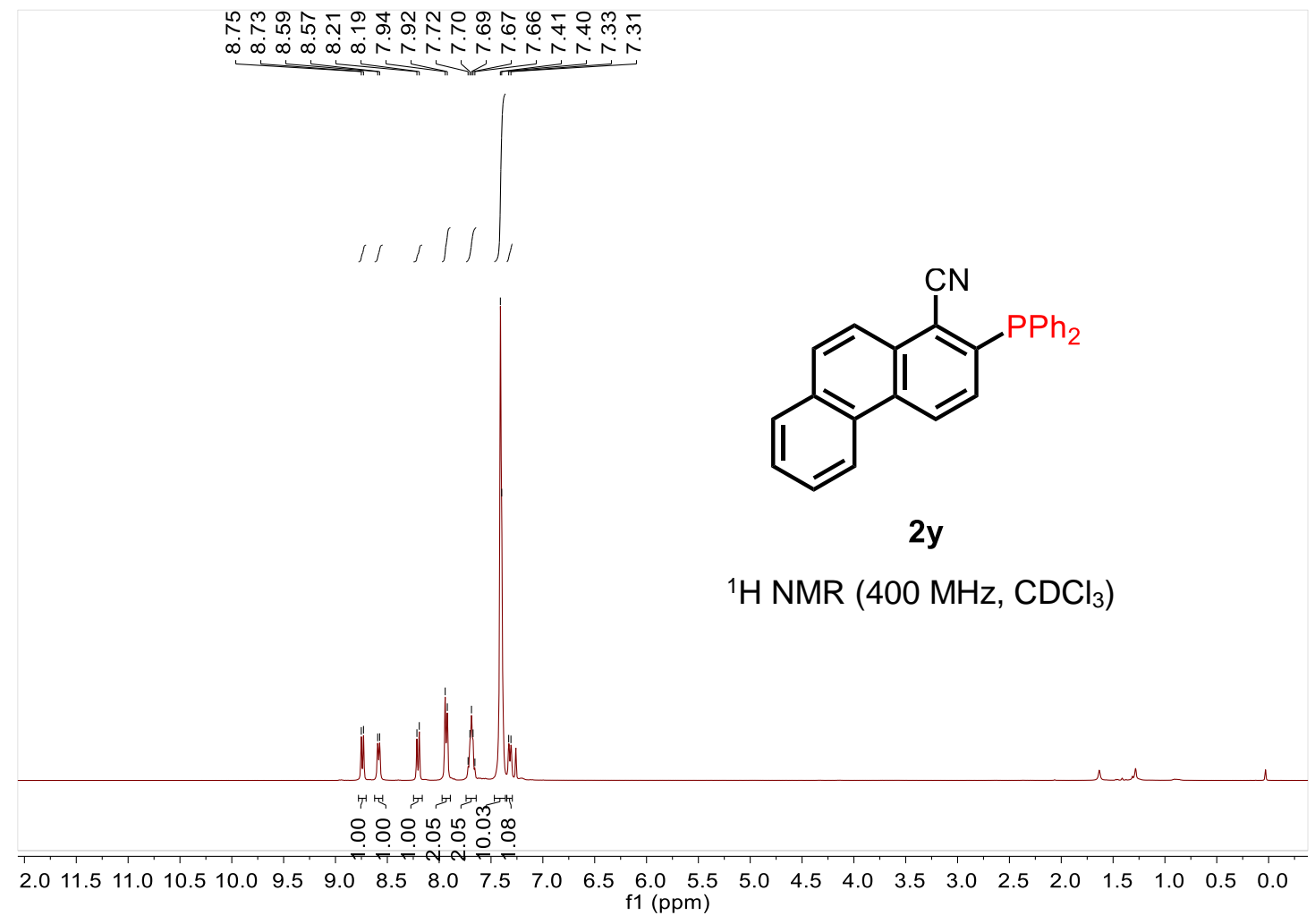

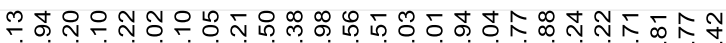

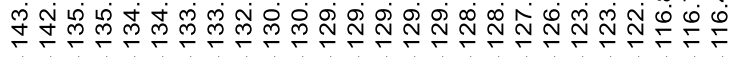

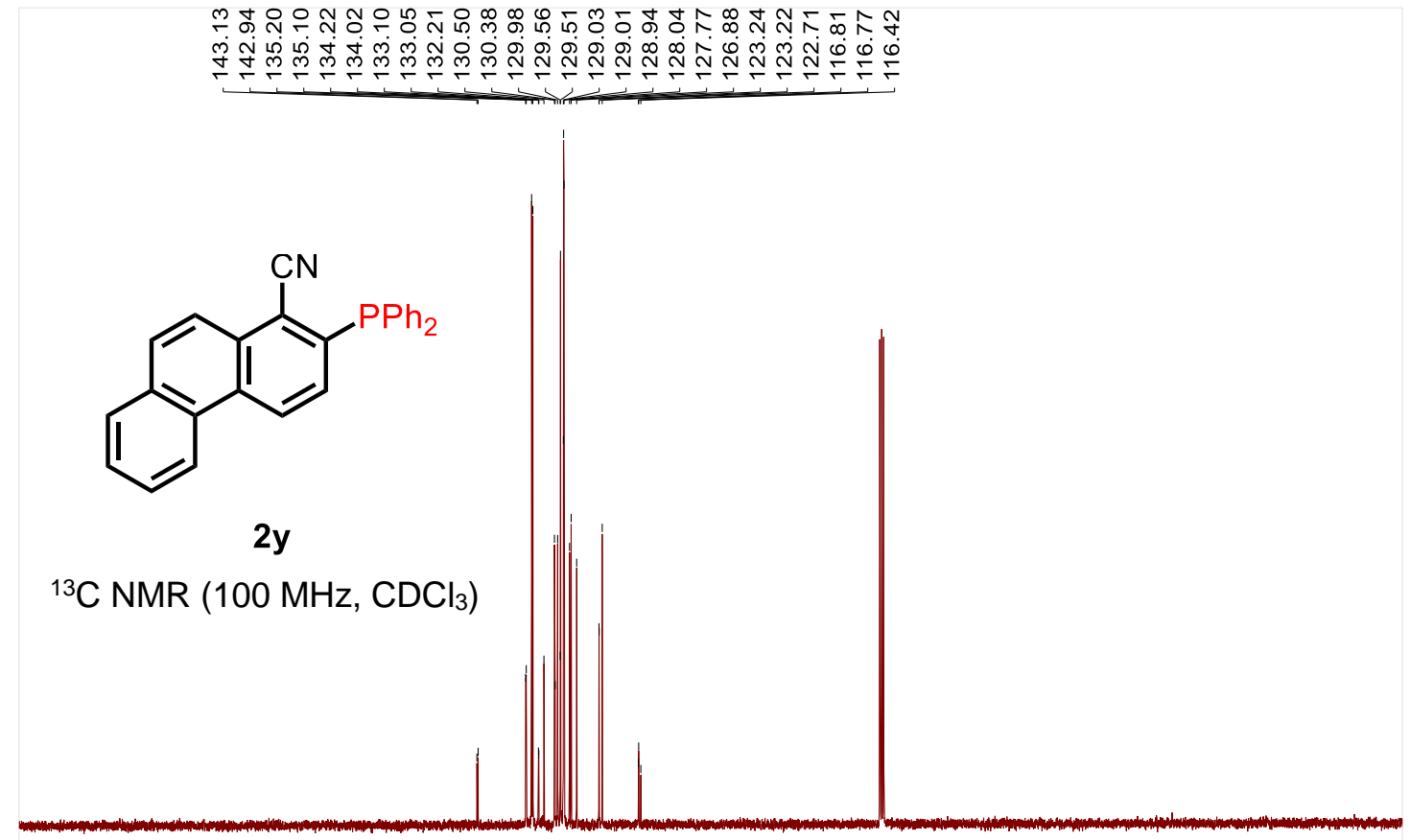

$\begin{array}{lllllllllllllllllllll}210 & 200 & 190 & 180 & 170 & 160 & 150 & 140 & 130 & 120 & \begin{array}{c}110 \\ \mathrm{f} 1(\mathrm{ppm})\end{array} & \begin{array}{l}100 \\ (120\end{array} & 80 & 70 & 60 & 50 & 40 & 30 & 20 & 10 & 0\end{array}$ 


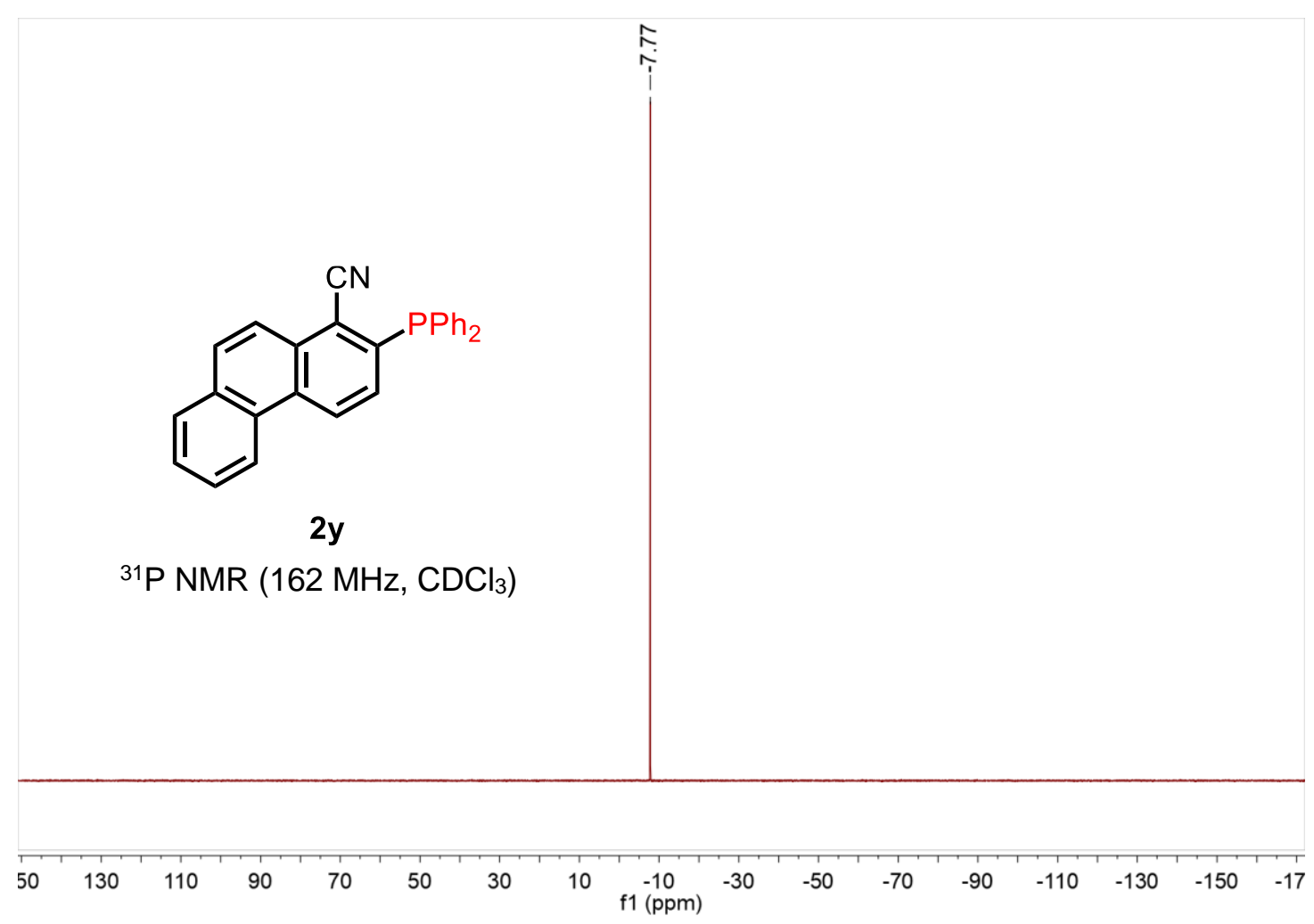

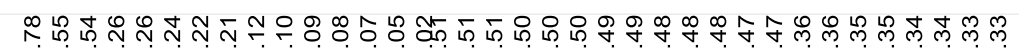

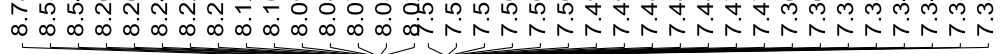

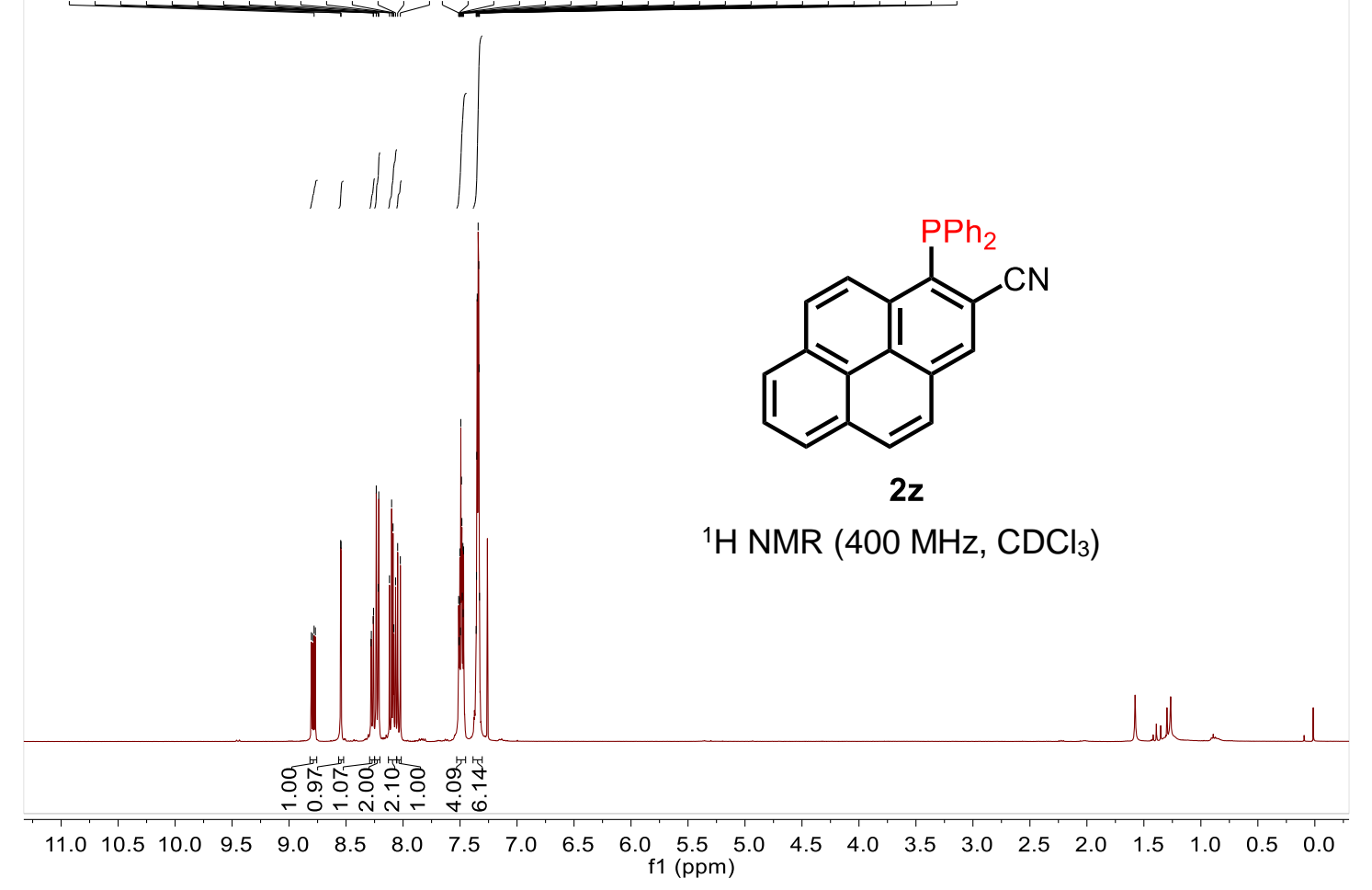




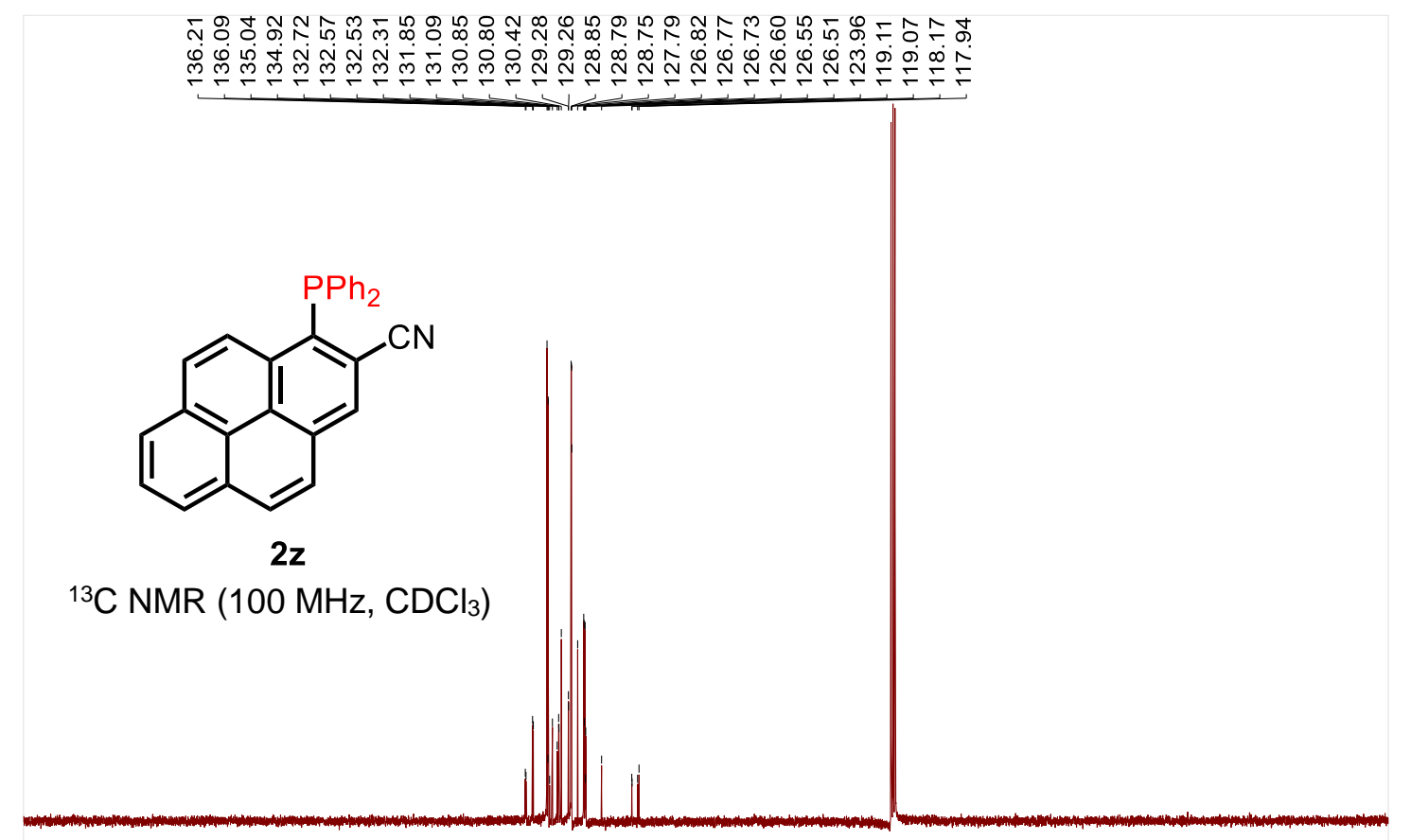

$\begin{array}{llllllllllllllllllllll}210 & 200 & 190 & 180 & 170 & 160 & 150 & 140 & 130 & 120 & \begin{array}{c}110 \\ \mathrm{f} 1(\mathrm{ppm})\end{array} & 90 & 80 & 70 & 60 & 50 & 40 & 30 & 20 & 10 & 0\end{array}$

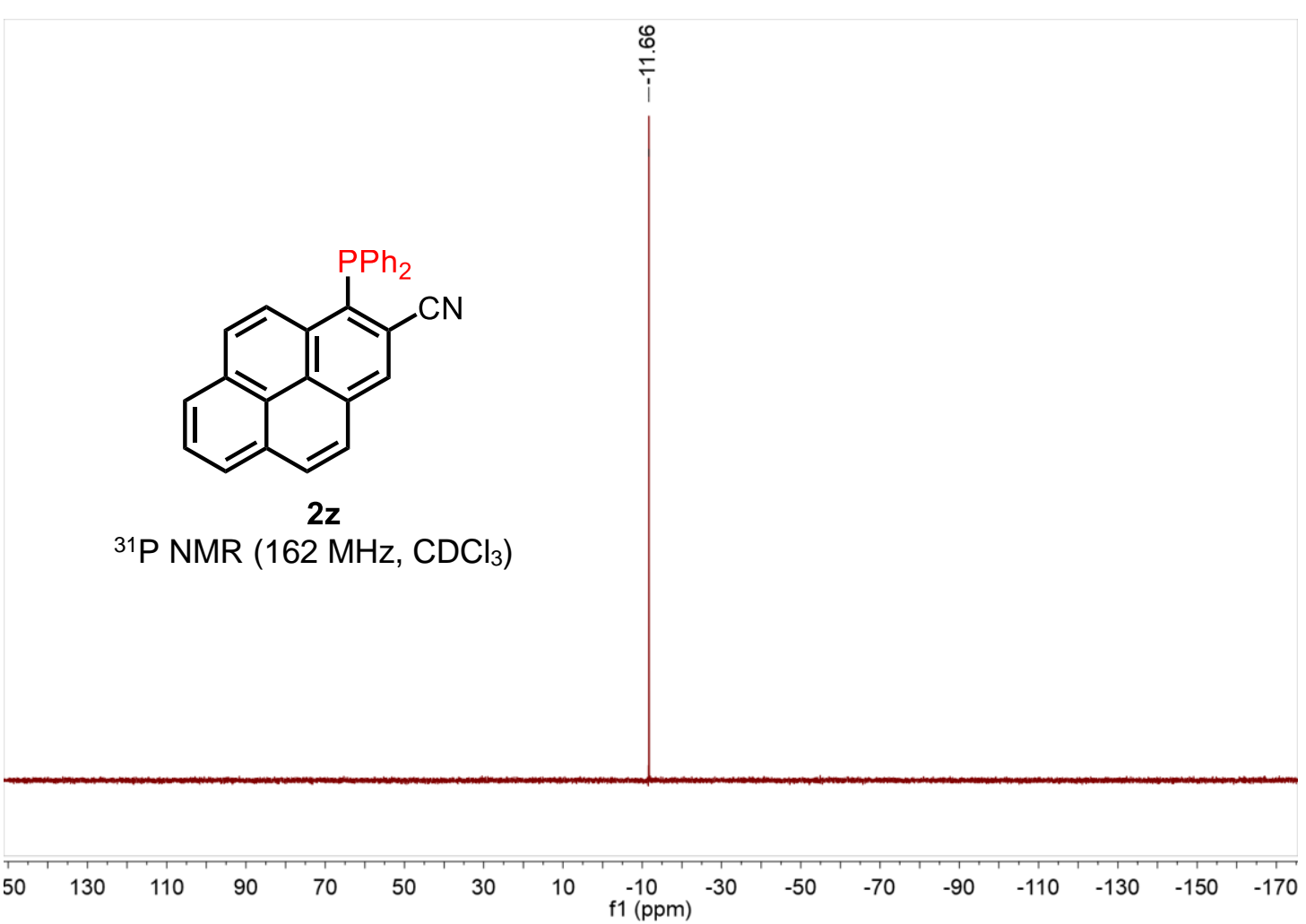



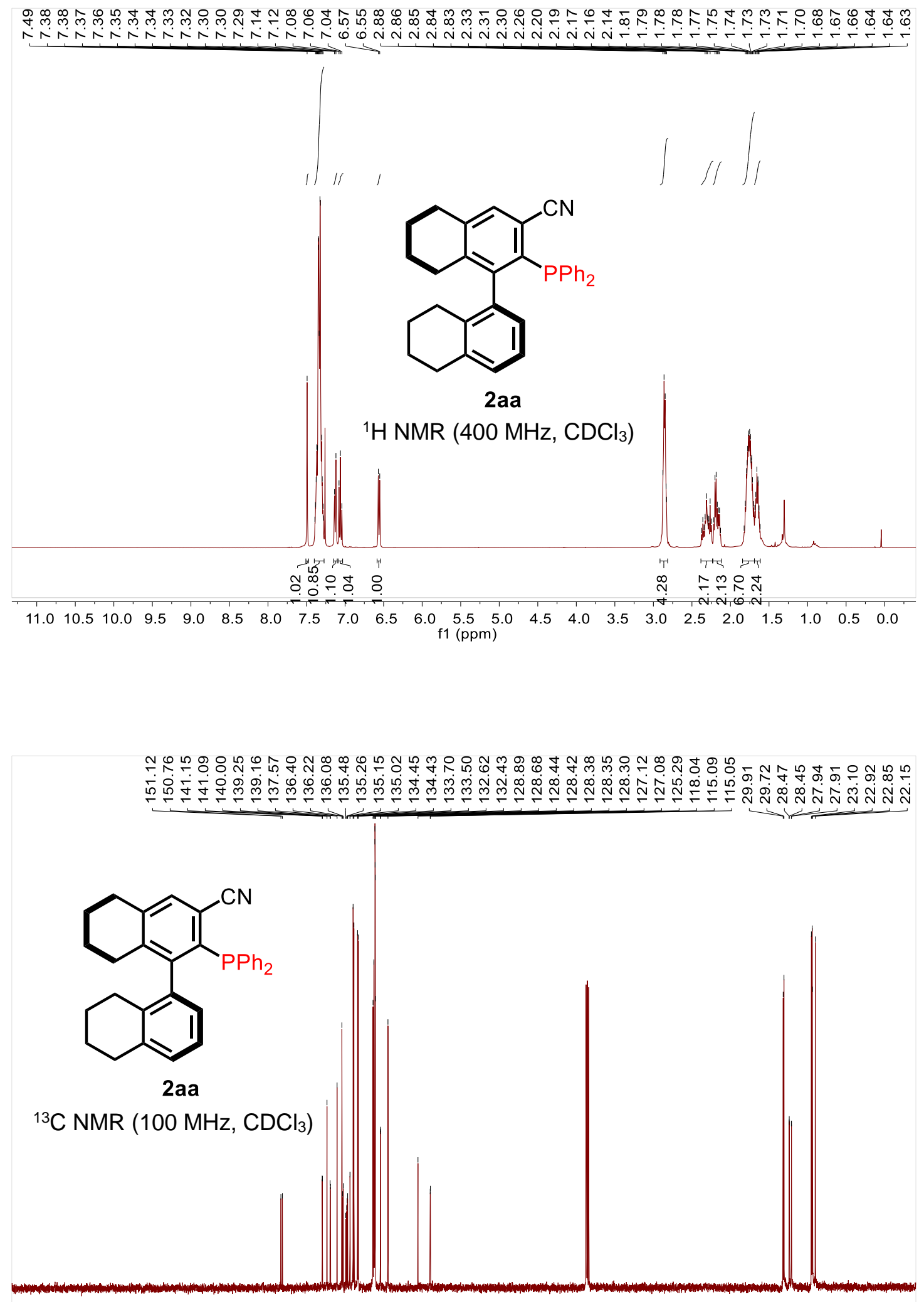

$\begin{array}{lllllllllllllllllllll}210 & 200 & 190 & 180 & 170 & 160 & 150 & 140 & 130 & 120 & \begin{array}{c}110 \\ \mathrm{f} 1(\mathrm{ppm})\end{array} & 90 & 80 & 70 & 60 & 50 & 40 & 30 & 20 & 10 & 0\end{array}$ 


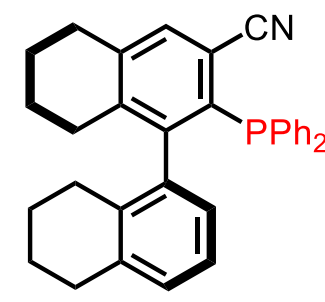

2aa

${ }^{31} \mathrm{P}$ NMR $\left(162 \mathrm{MHz}, \mathrm{CDCl}_{3}\right)$

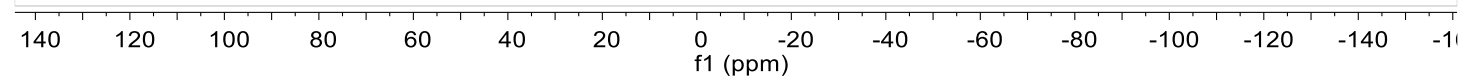

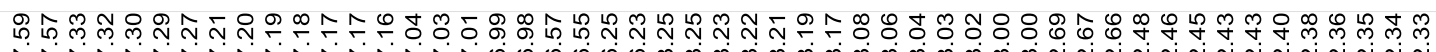

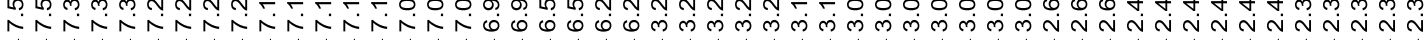

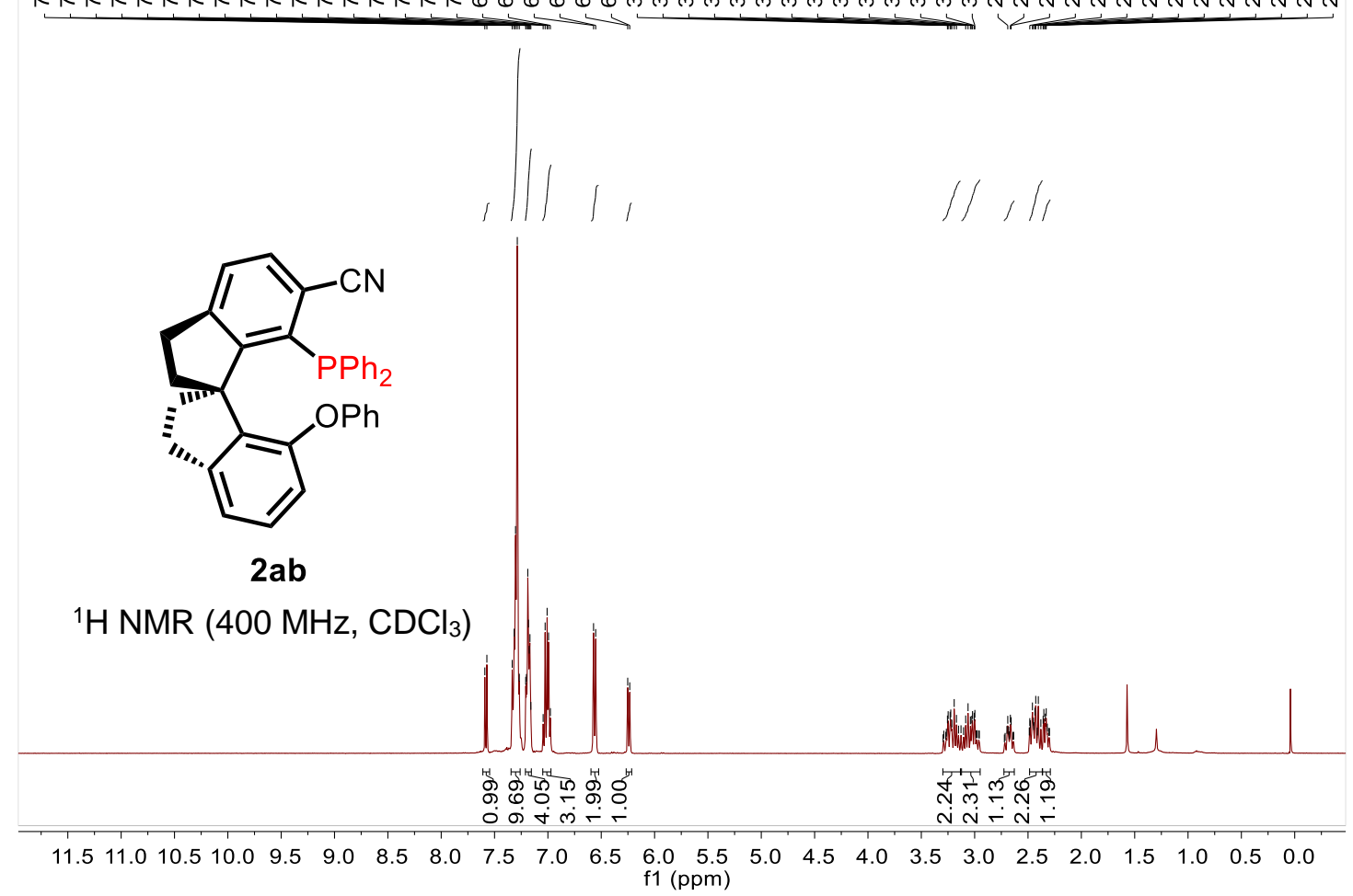




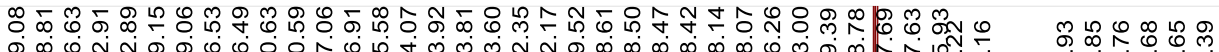

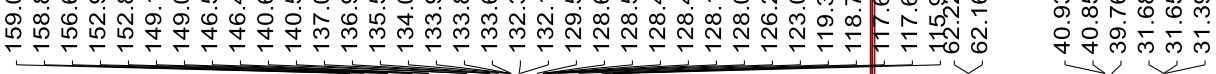<smiles>N#Cc1ccc2c(c1P)[C@H]1c3c(cccc3Oc3ccccc3)CC21</smiles>

$2 a b$

${ }^{13} \mathrm{C} \mathrm{NMR}\left(100 \mathrm{MHz}, \mathrm{CDCl}_{3}\right)$

$\begin{array}{llllllllllllllllllllllllll}210 & 200 & 190 & 180 & 170 & 160 & 150 & 140 & 130 & 120 & \begin{array}{r}110 \\ \mathrm{f} 1(\mathrm{ppm})\end{array} & 90 & 80 & 70 & 60 & 50 & 40 & 30 & 20 & 10 & 0\end{array}$

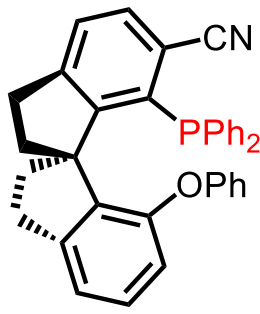

$2 \mathrm{ab}$

${ }^{31} \mathrm{P} \mathrm{NMR}\left(162 \mathrm{MHz}, \mathrm{CDCl}_{3}\right)$

$\begin{array}{lllllllllllllllll}140 & 120 & 100 & 80 & 60 & 40 & 20 & 0 & -20 & -40 & -60 & -80 & -100 & -120 & -140 & -161\end{array}$




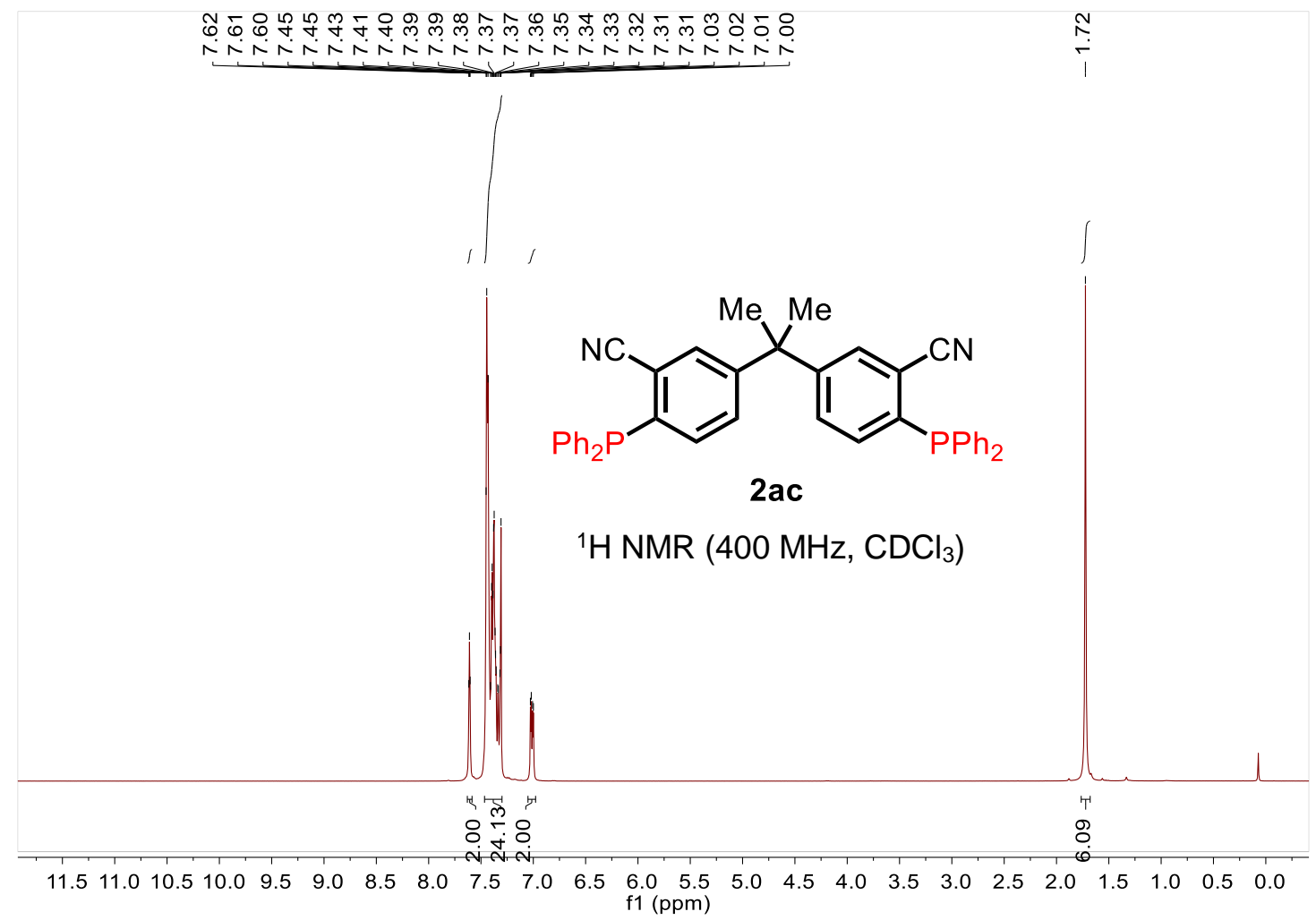

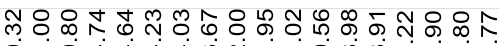

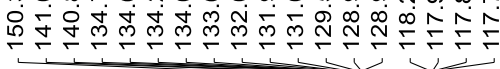

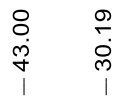

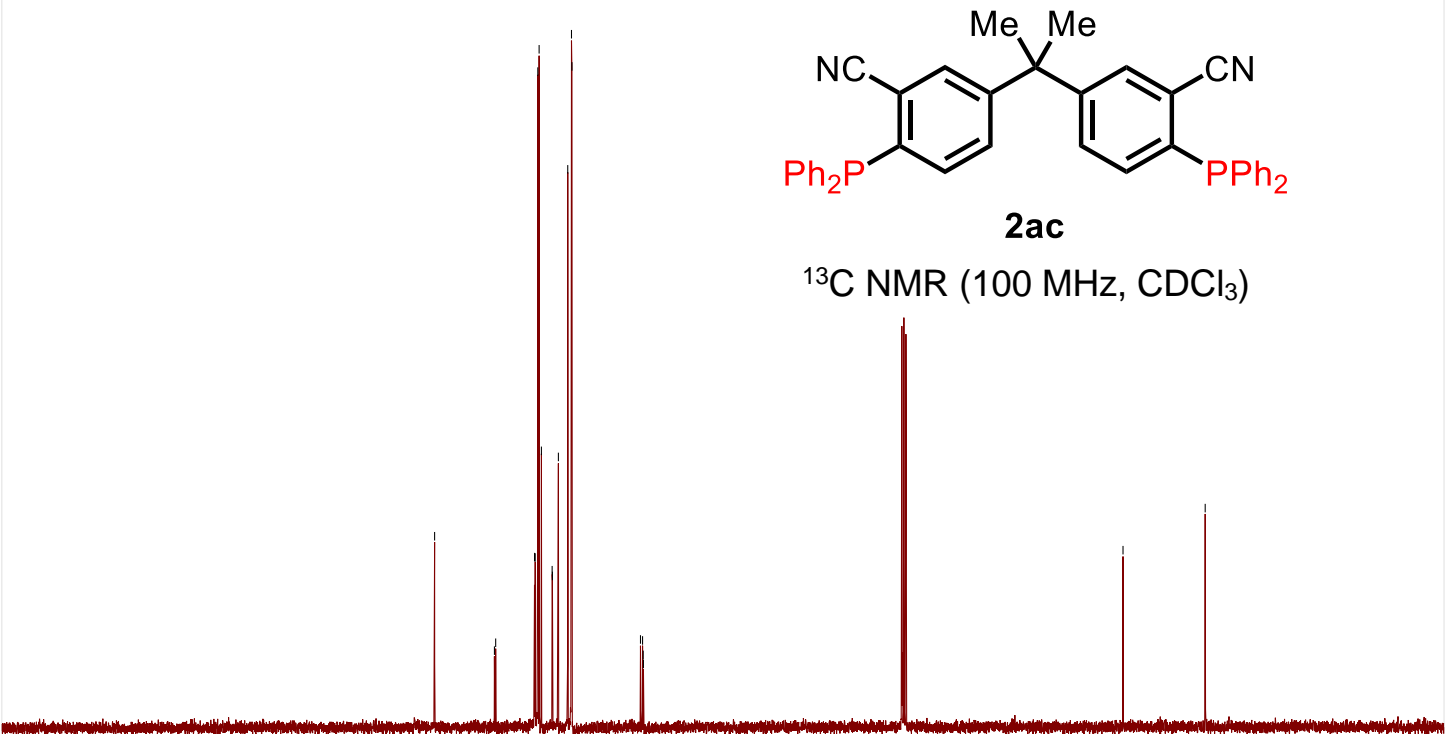

$\begin{array}{lllllllllllllllllllll}210 & 200 & 190 & 180 & 170 & 160 & 150 & 140 & 130 & 120 & \begin{array}{c}110 \\ \mathrm{f} 1(\mathrm{ppm})\end{array} & 90 & 80 & 70 & 60 & 50 & 40 & 30 & 20 & 10 & 0\end{array}$ 


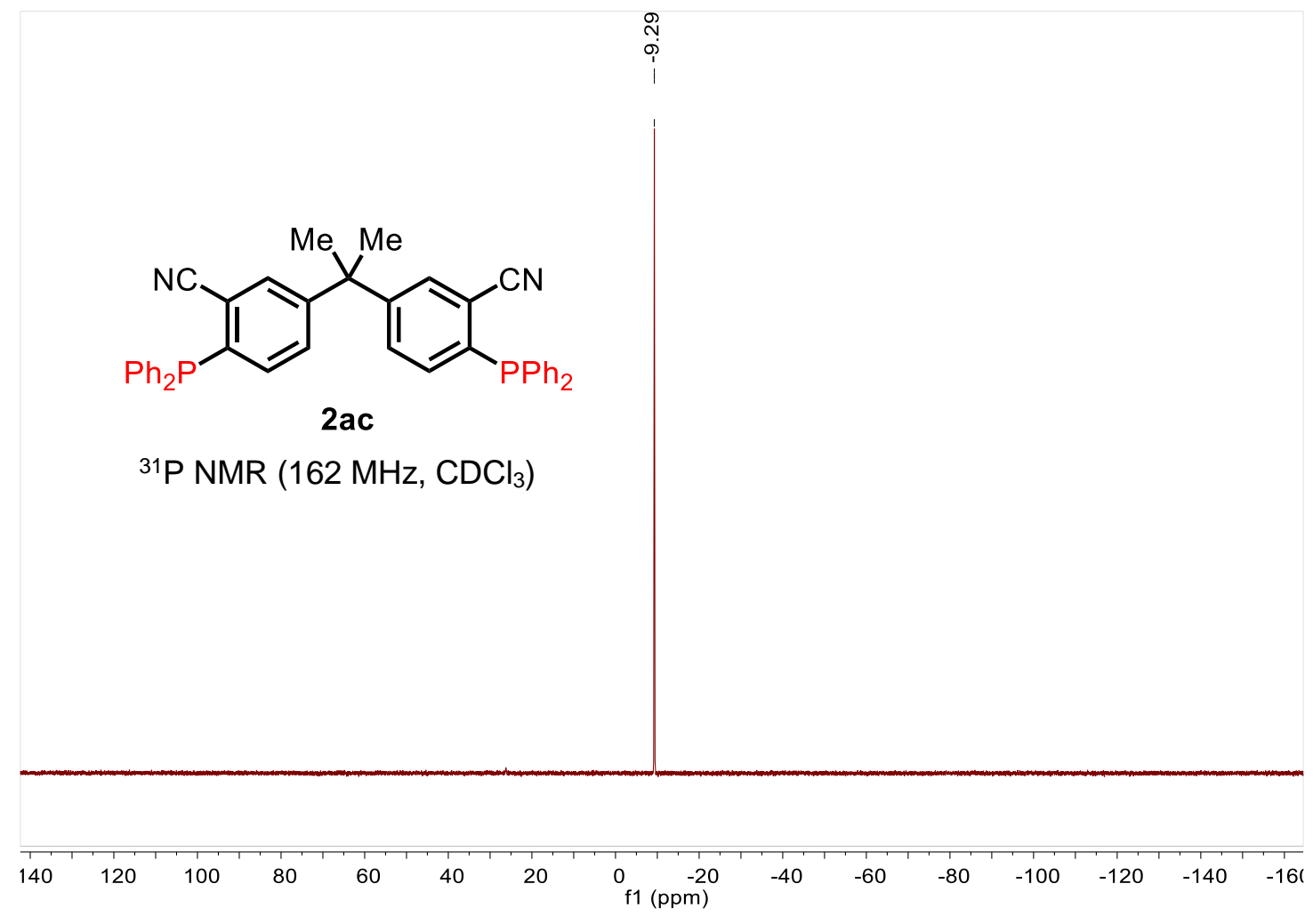

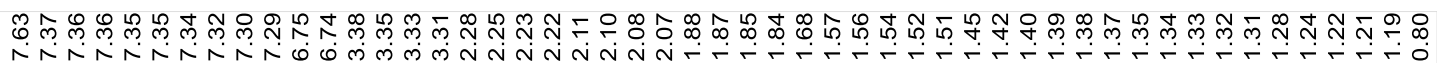

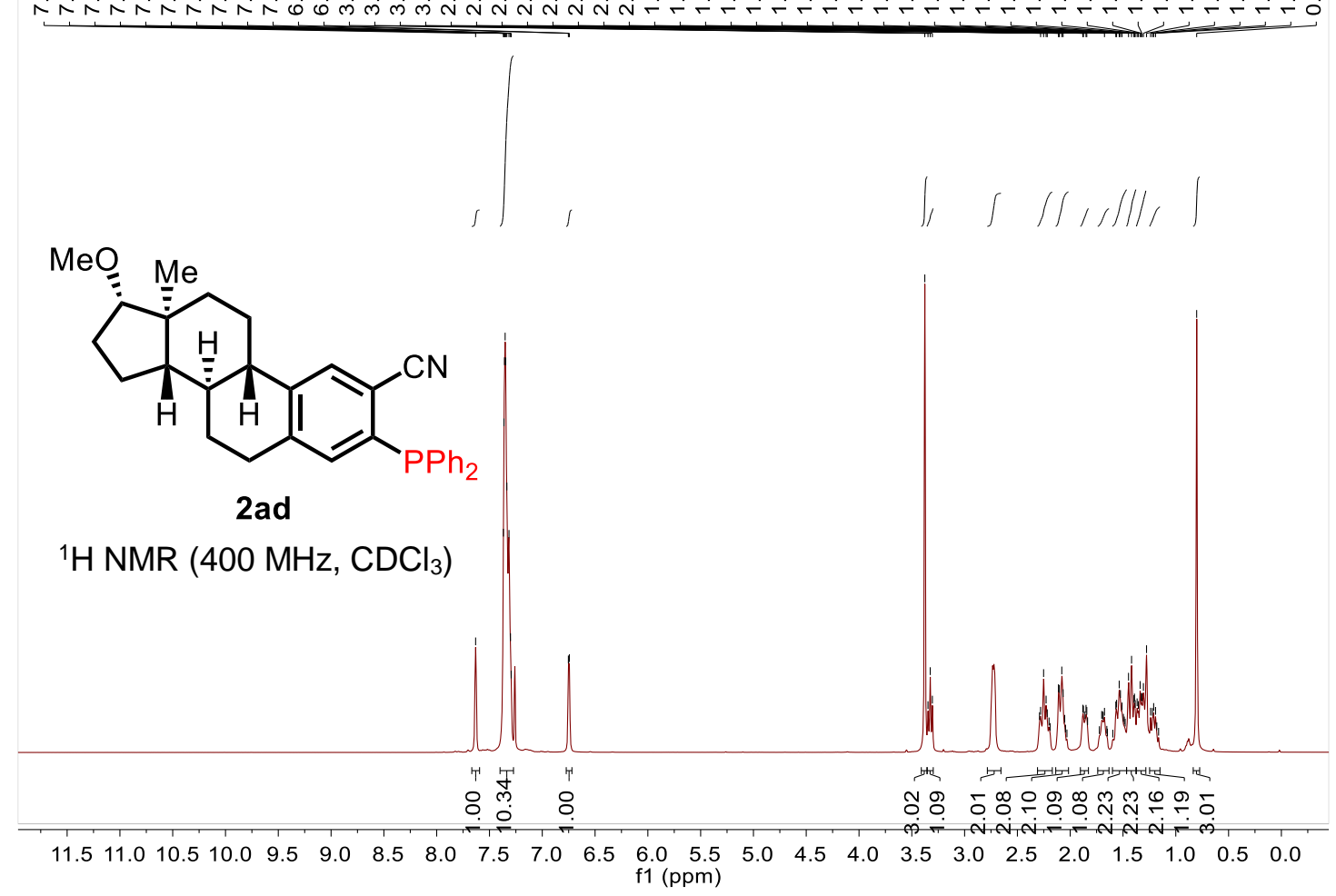




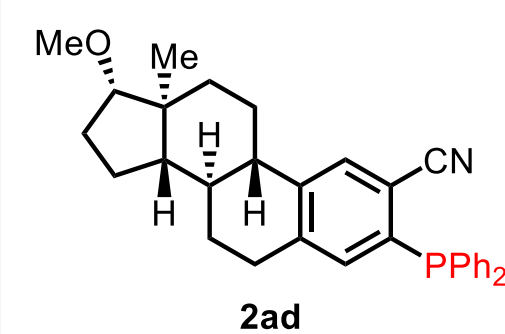

${ }^{13} \mathrm{C} \mathrm{NMR}\left(100 \mathrm{MHz}, \mathrm{CDCl}_{3}\right)$

$\begin{array}{lllllllllllllllllllll}210 & 200 & 190 & 180 & 170 & 160 & 150 & 140 & 130 & 120 & \begin{array}{c}110 \\ \mathrm{f} 1(\mathrm{ppm})\end{array} & 100 & 80 & 70 & 60 & 50 & 40 & 30 & 20 & 10 & 0\end{array}$

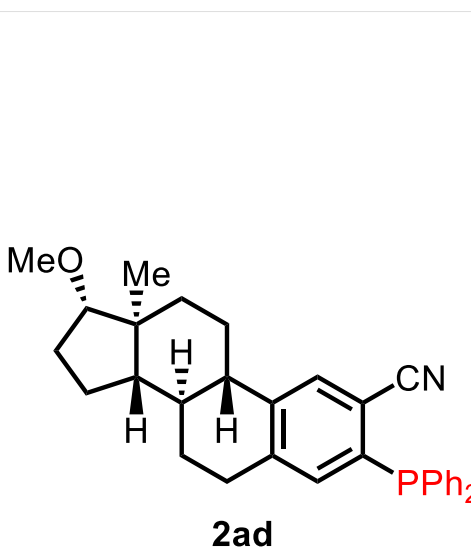

${ }^{31} \mathrm{P} \mathrm{NMR}\left(162 \mathrm{MHz}, \mathrm{CDCl}_{3}\right)$ 

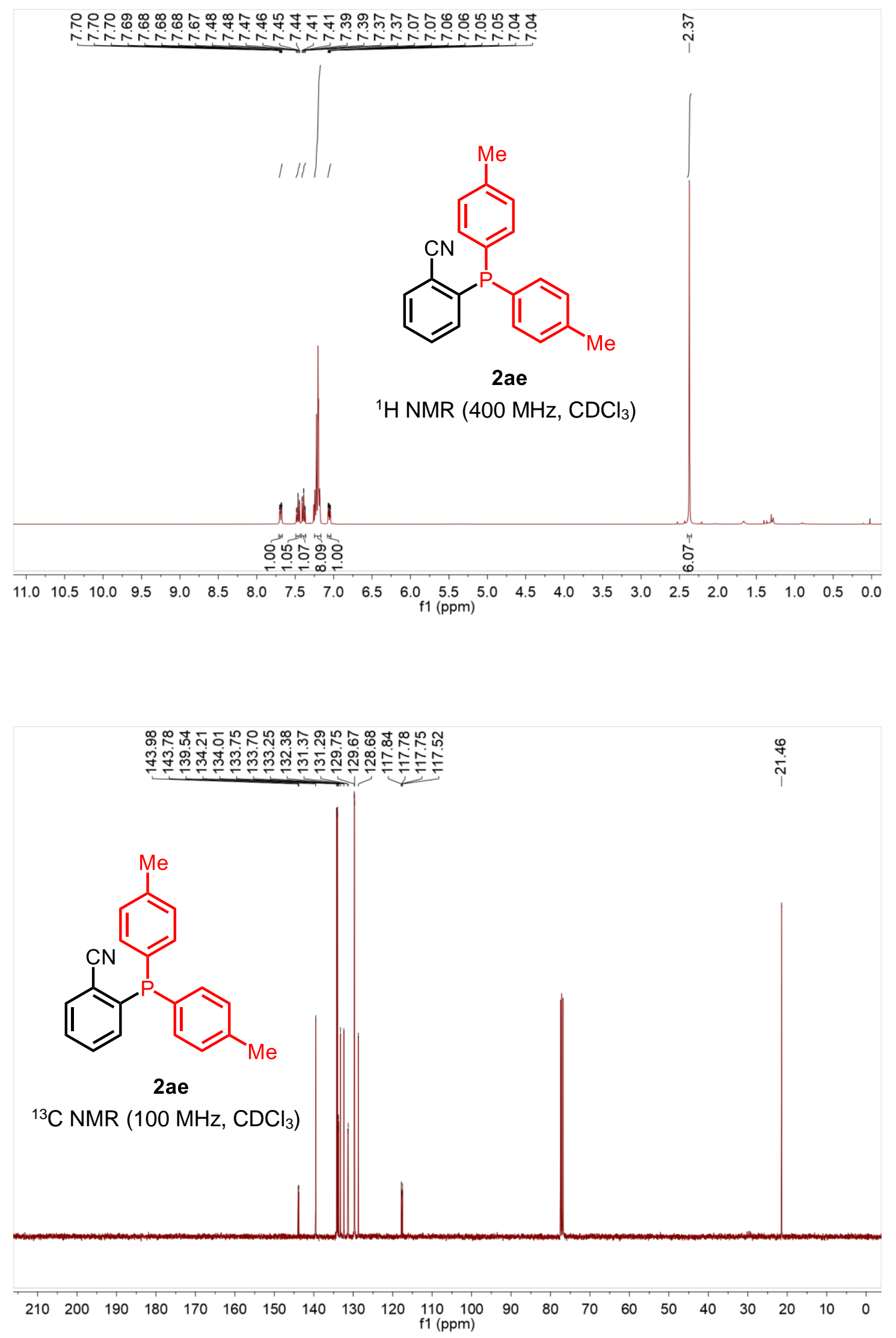


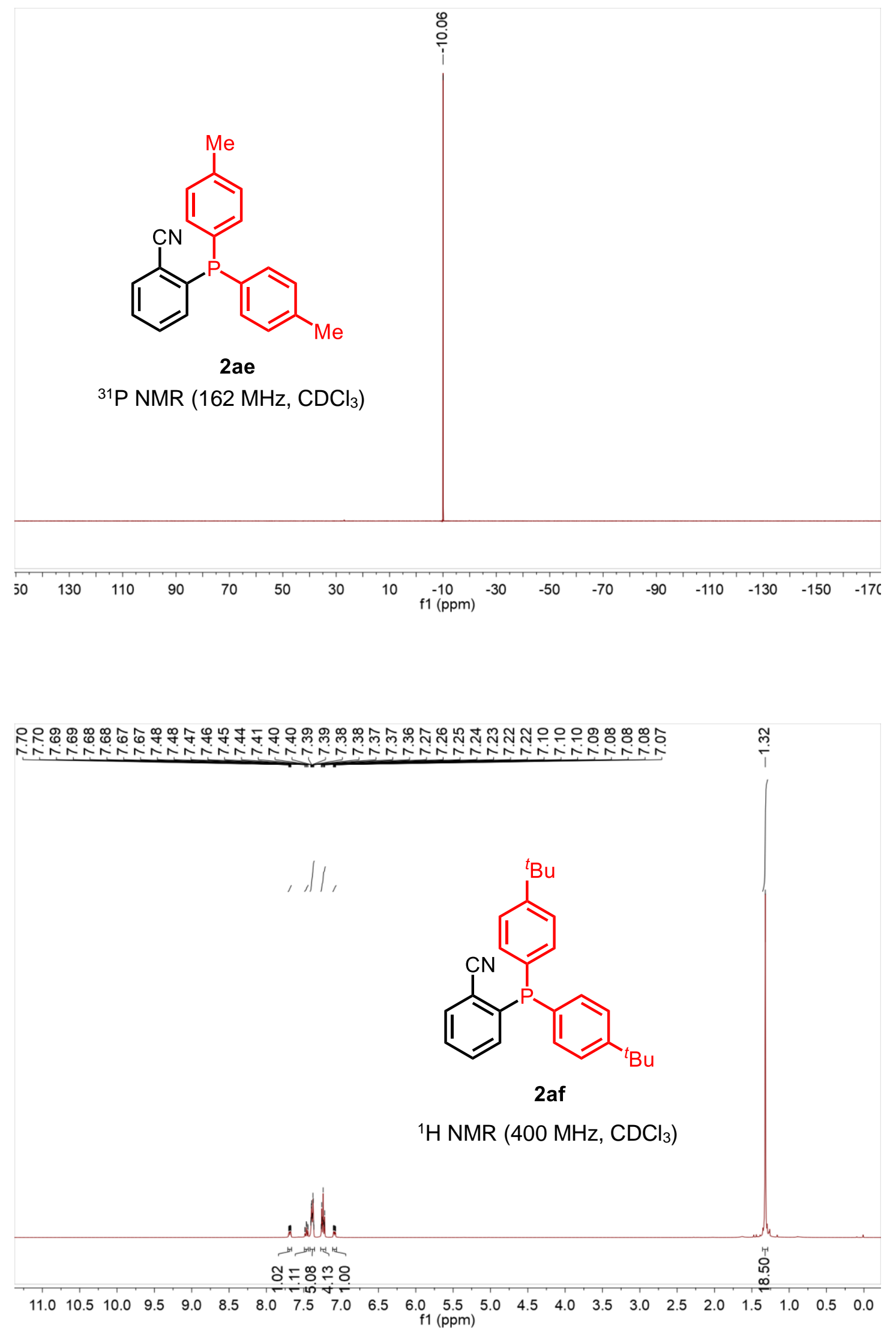


ำ

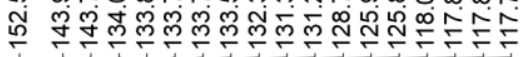<smiles>Cc1ccccc1P(c1ccc(Br)cc1)c1ccc(C(C)(C)C)cc1</smiles>

2af

${ }^{13} \mathrm{C}$ NMR $\left(100 \mathrm{MHz}, \mathrm{CDCl}_{3}\right)$

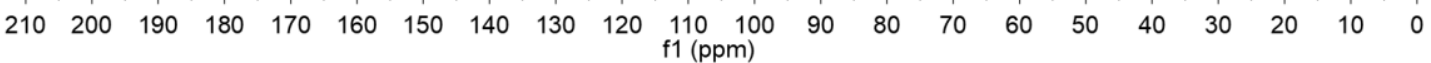

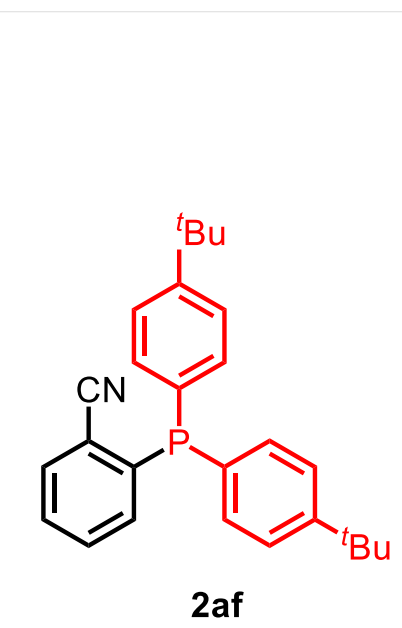

$\stackrel{\text { ஜํ }}{\stackrel{0}{+}}$

${ }^{31} \mathrm{P}$ NMR (162 MHz, $\mathrm{CDCl}_{3}$ ) 
<smiles>Cc1ccccc1P(c1ccc(N(C)C)cc1)c1ccc(N(C)C)cc1</smiles>

2ag

${ }^{1} \mathrm{H}$ NMR $\left(400 \mathrm{MHz}, \mathrm{CDCl}_{3}\right)$

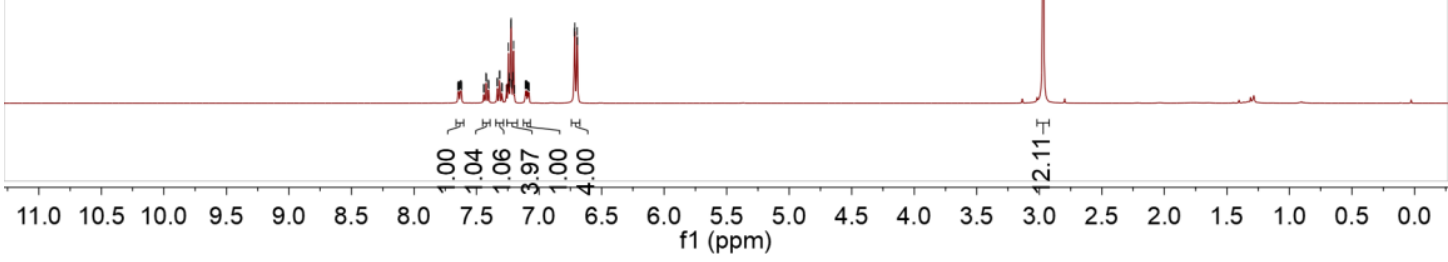<smiles>Cc1ccc(P(c2ccc(N(C)C)cc2)c2ccccc2C)cc1</smiles>

2ag

${ }^{13} \mathrm{C}$ NMR $\left(100 \mathrm{MHz}, \mathrm{CDCl}_{3}\right)$

$\begin{array}{llllllllllll}210 & 200 & 190 & 180 & 170 & 160 & 150 & 140 & 130 & 120 & \begin{array}{l}110 \\ \mathrm{f} 1(\mathrm{ppm})\end{array}\end{array}$ 


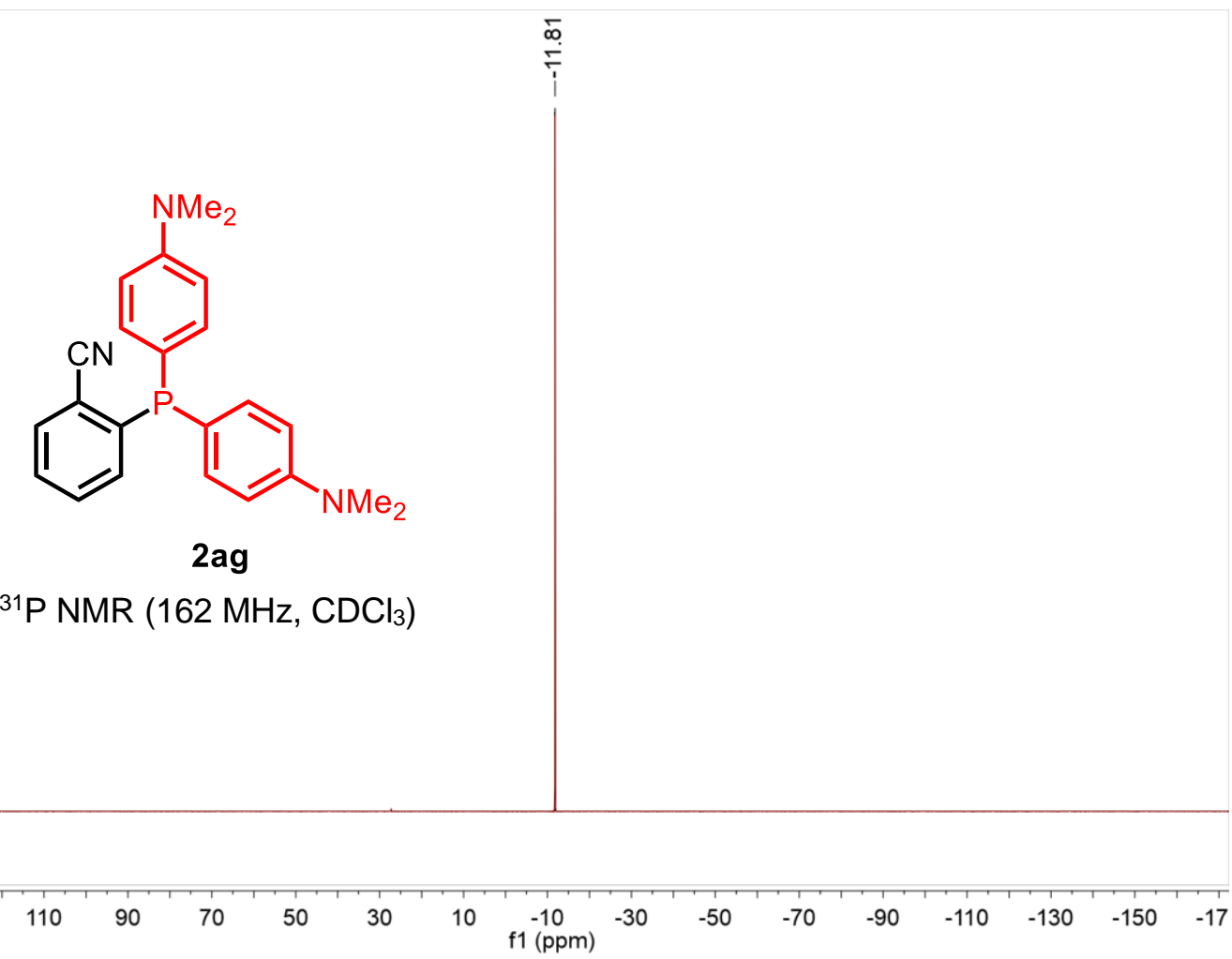

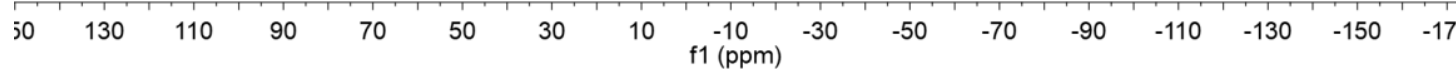

œ

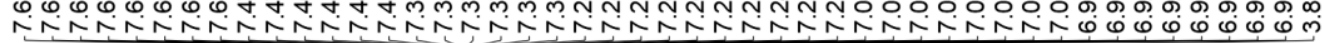<smiles>COc1ccc(P(c2ccc(OC)cc2)c2ccccc2C)cc1</smiles>

2ah

${ }^{1} \mathrm{H} \mathrm{NMR}\left(400 \mathrm{MHz}, \mathrm{CDCl}_{3}\right)$ 


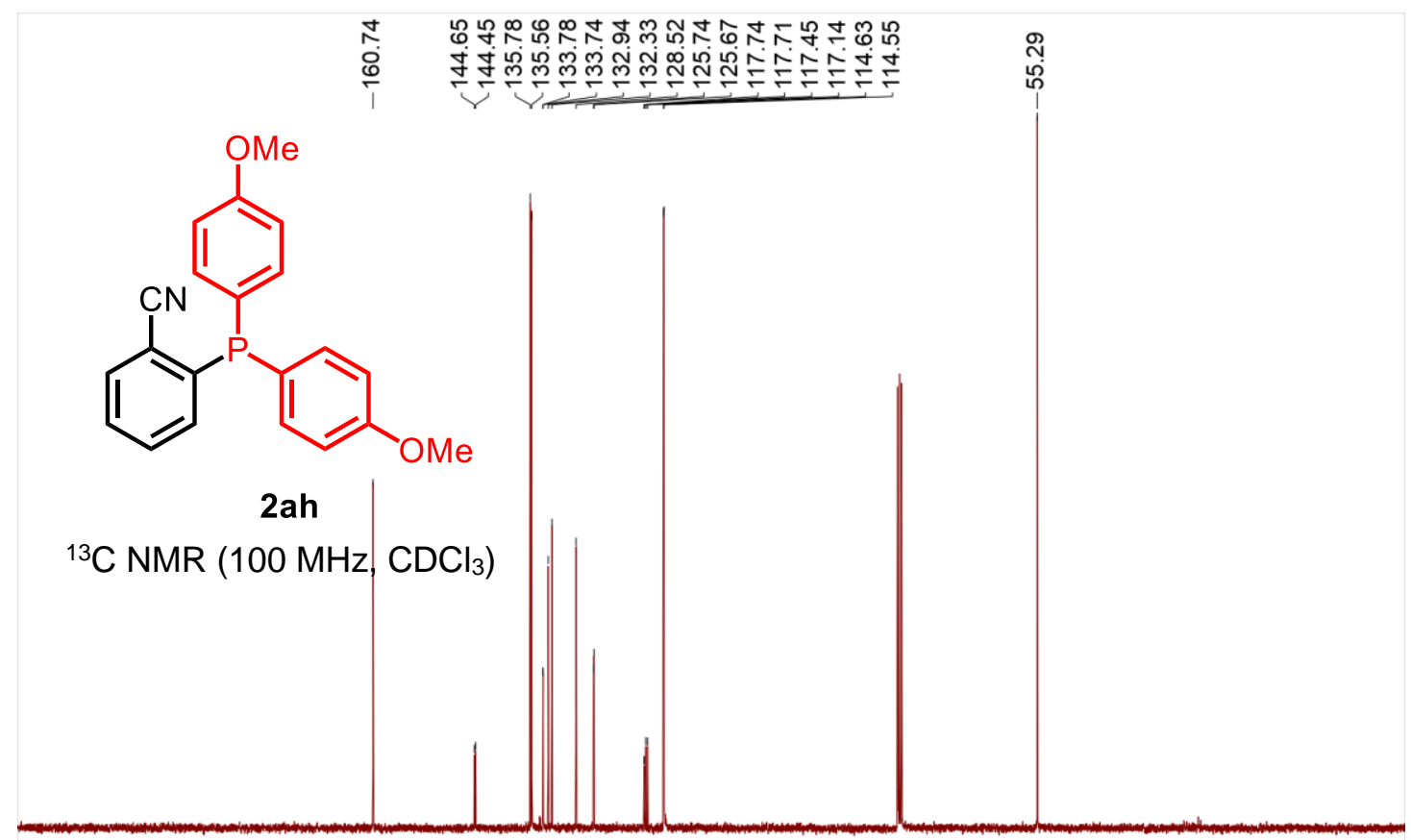

$\begin{array}{llllllllllllllllllllll}210 & 200 & 190 & 180 & 170 & 160 & 150 & 140 & 130 & 120 & \begin{array}{c}110 \\ \mathrm{f} 1(\mathrm{ppm})\end{array} & 90 & 80 & 70 & 60 & 50 & 40 & 30 & 20 & 10 & 0\end{array}$

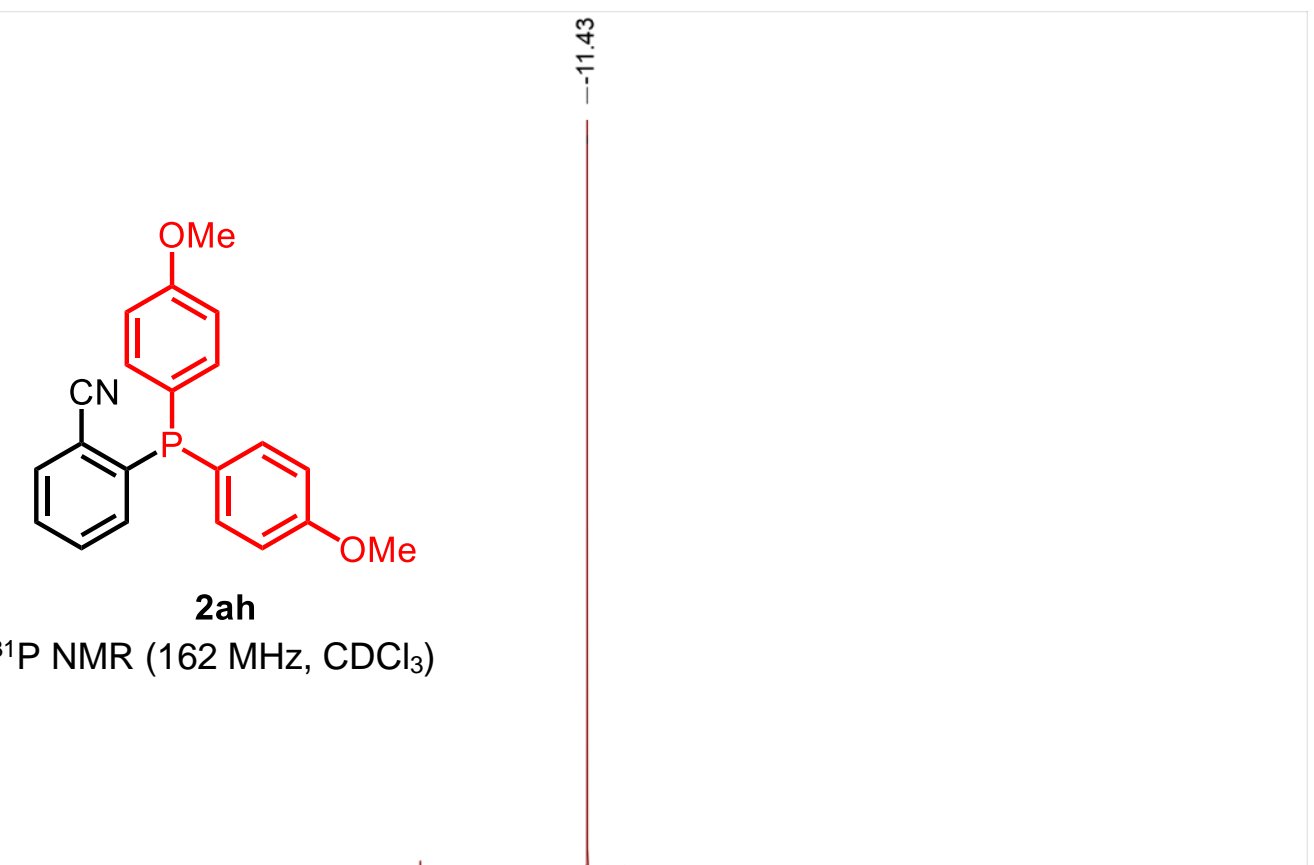

$\begin{array}{lllllllllllllllllll}50 & 130 & 110 & 90 & 70 & 50 & 30 & 10 & \begin{array}{c}-10 \\ \mathrm{f} 1(\mathrm{ppm})\end{array} & -30 & -50 & -70 & -90 & -110 & -130 & -150 & -17 \mathrm{C}\end{array}$ 
<smiles>Cc1ccccc1P(c1cc(C(C)(C)C)cc(C(C)(C)C)c1)c1cc(C(C)(C)C)cc(C(C)(C)C)c1</smiles>

2ai

${ }^{1} \mathrm{H} \mathrm{NMR}\left(400 \mathrm{MHz}, \mathrm{CDCl}_{3}\right)$

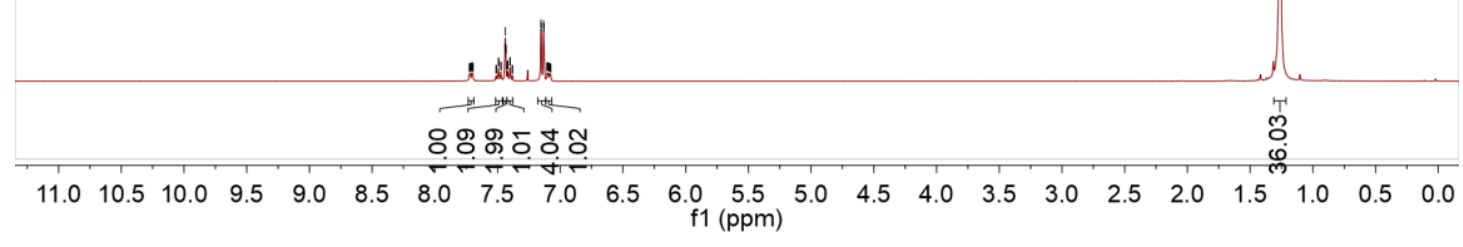<smiles>Cc1ccccc1P(c1cc(C(C)(C)C)cc(C(C)(C)C)c1)c1cc(C(C)(C)C)cc(C(C)(C)C)c1</smiles>

2ai

${ }^{13} \mathrm{C}$ NMR $\left(100 \mathrm{MHz}, \mathrm{CDCl}_{3}\right)$

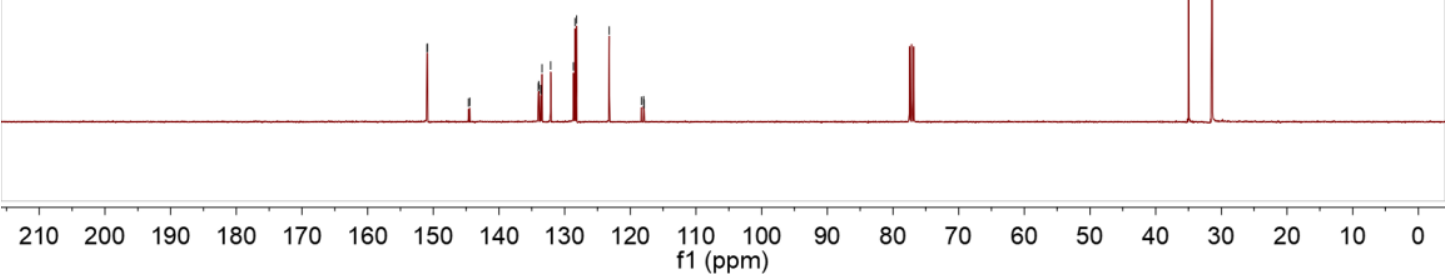




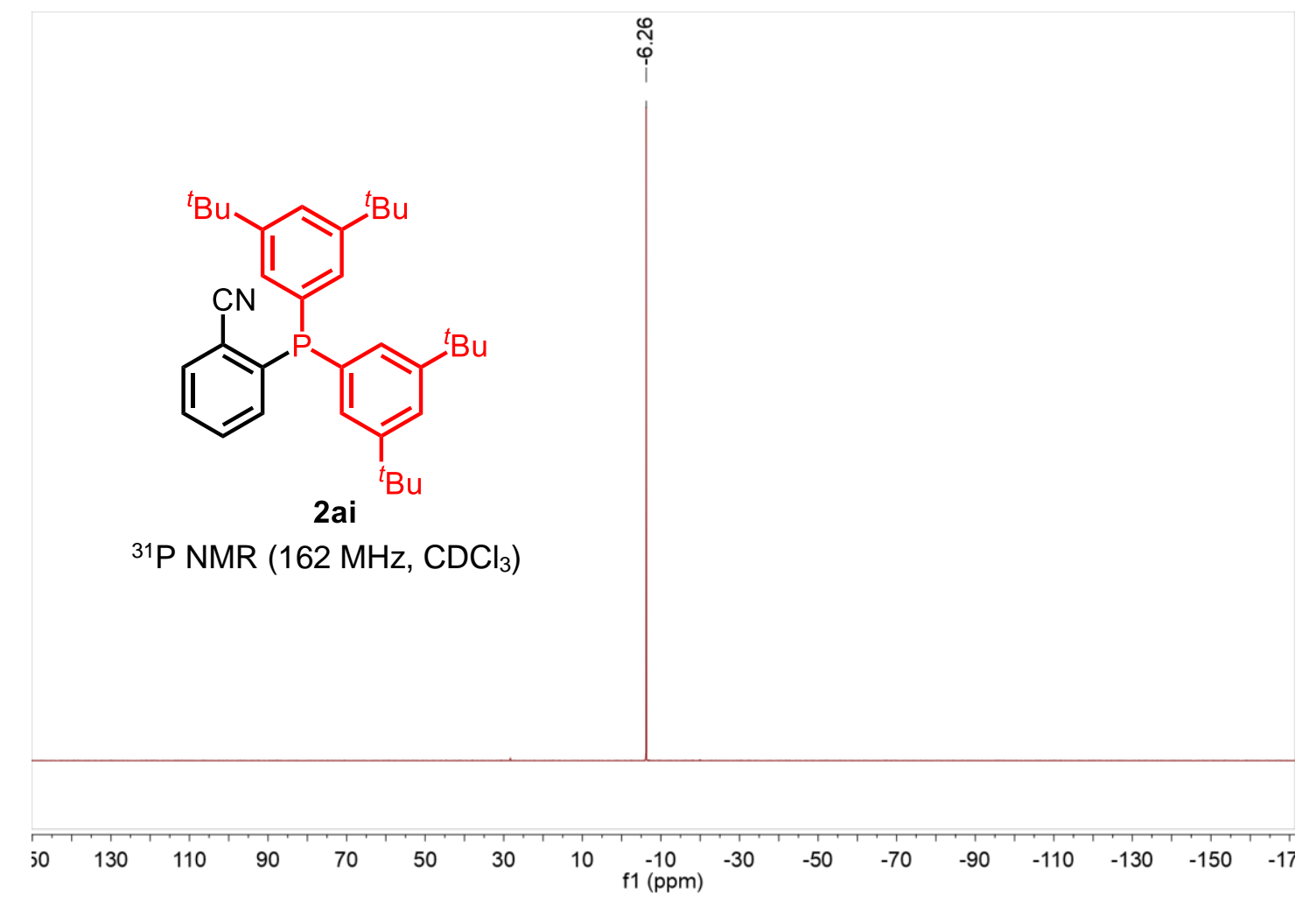

古

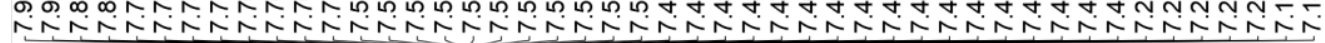

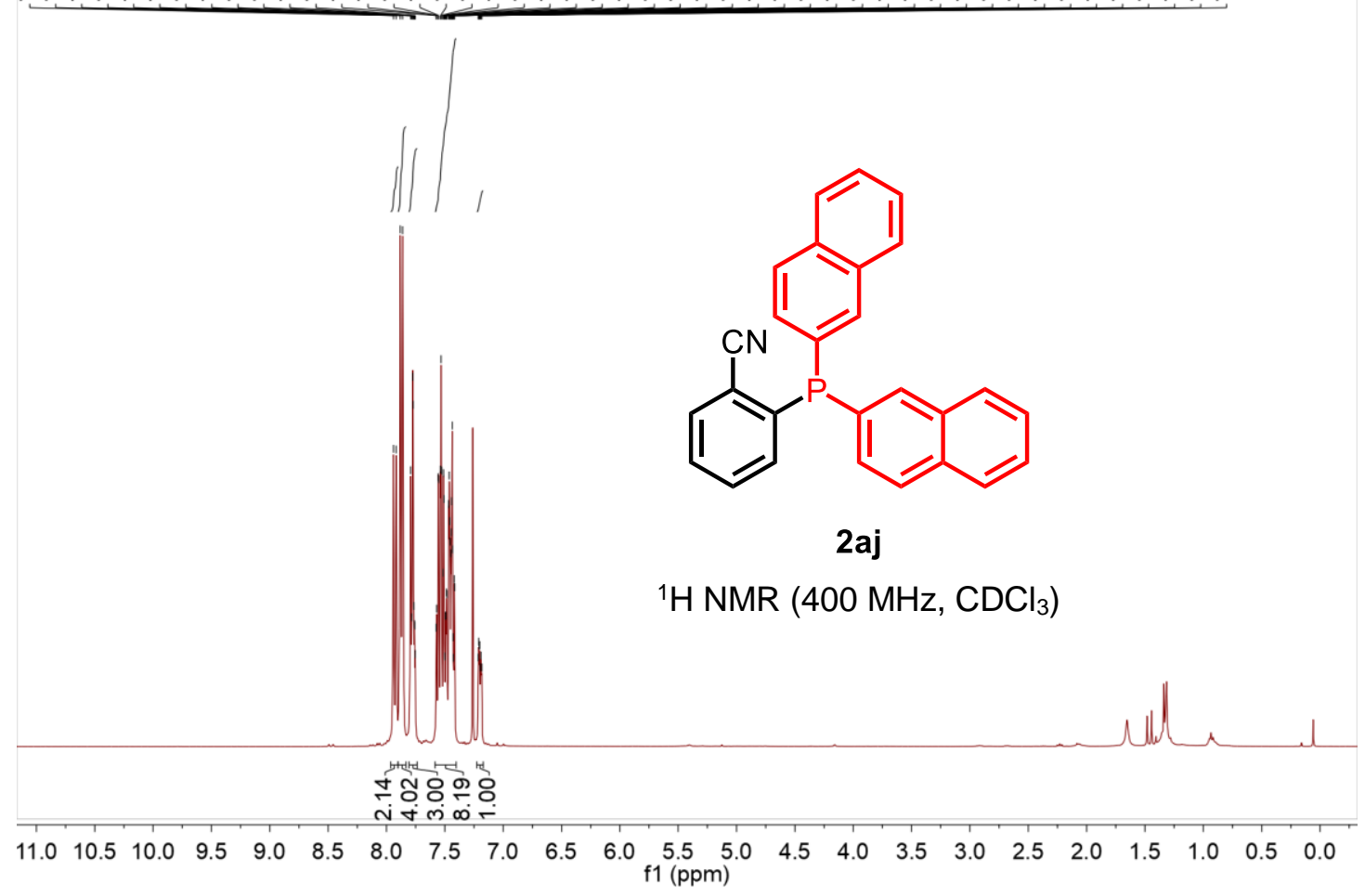




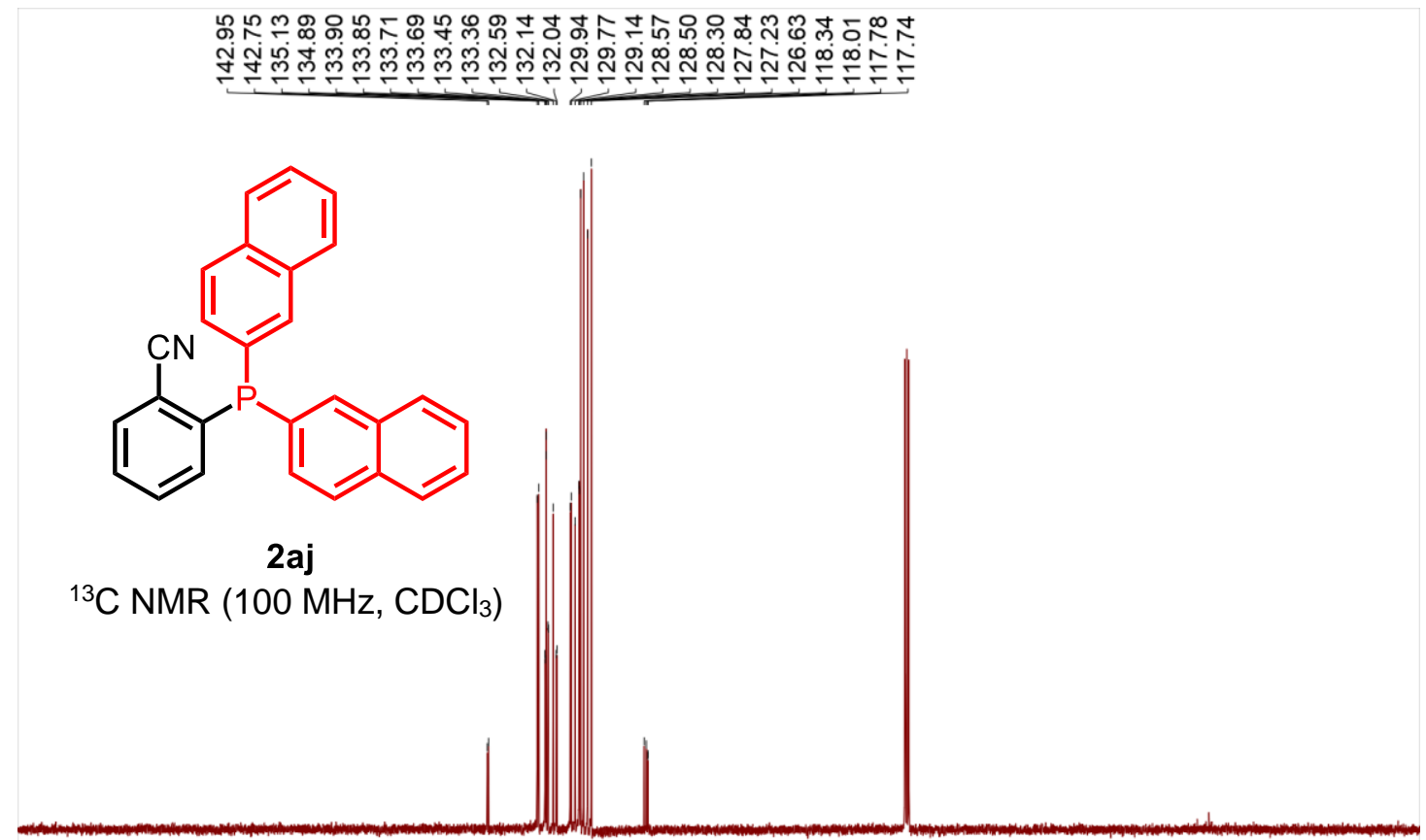

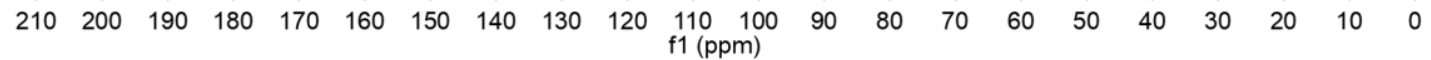

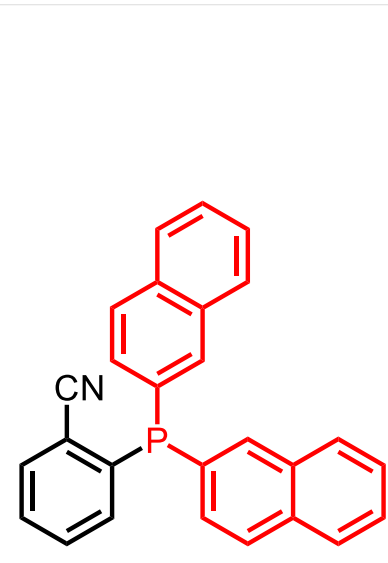

2aj

${ }^{31} \mathrm{P}$ NMR $\left(162 \mathrm{MHz}, \mathrm{CDCl}_{3}\right)$

$\begin{array}{lllllllllllllllllll}50 & 130 & 110 & 90 & 70 & 50 & 30 & 10 & -10 & -30 & -50 & -70 & -90 & -110 & -130 & -150 & -170\end{array}$ 

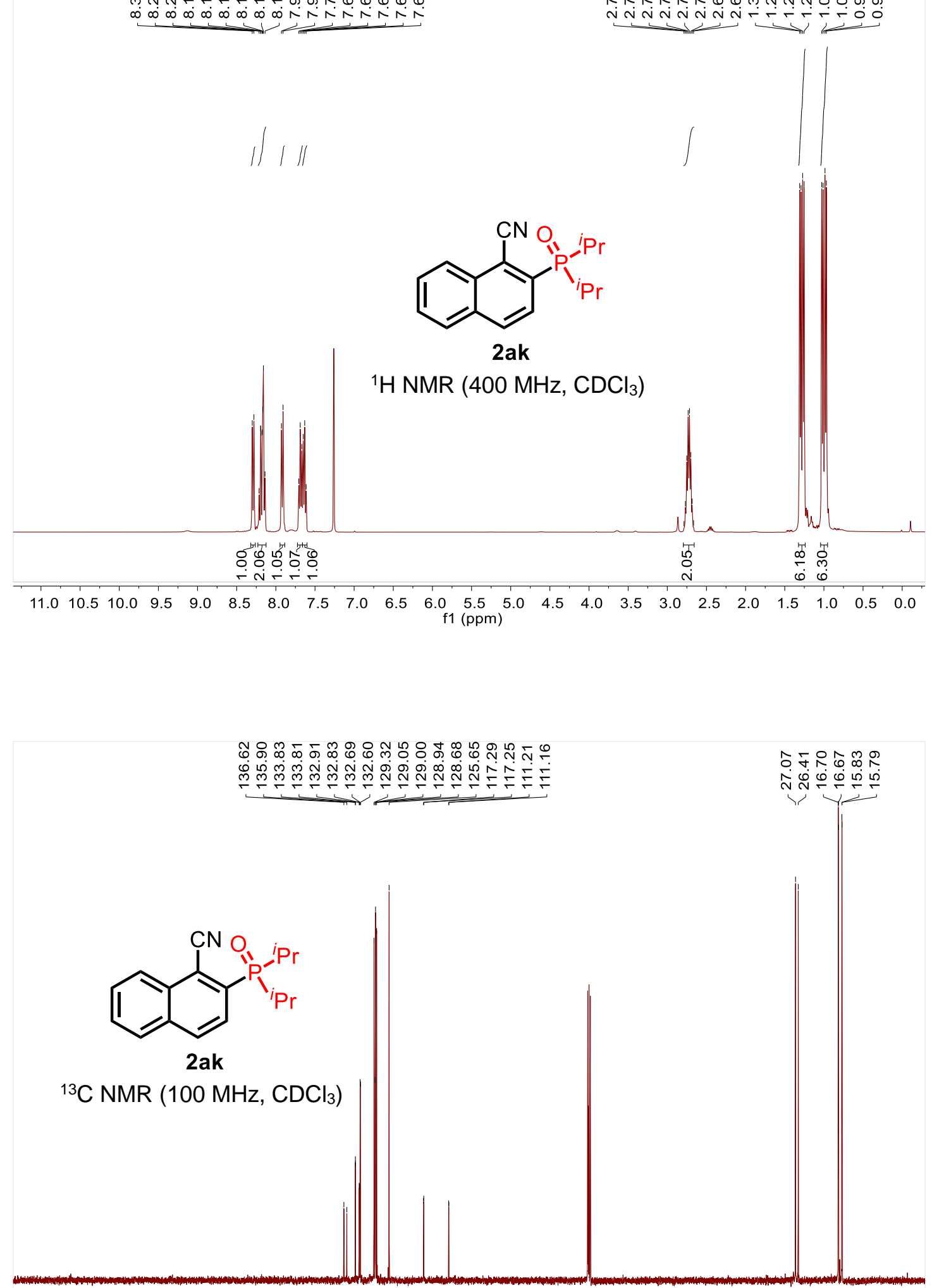

$\begin{array}{llllllllllllllllllllll}210 & 200 & 190 & 180 & 170 & 160 & 150 & 140 & 130 & 120 & \begin{array}{c}110 \\ \mathrm{f} 1(\mathrm{pmm})\end{array} & 90 & 80 & 70 & 60 & 50 & 40 & 30 & 20 & 10 & 0\end{array}$ 


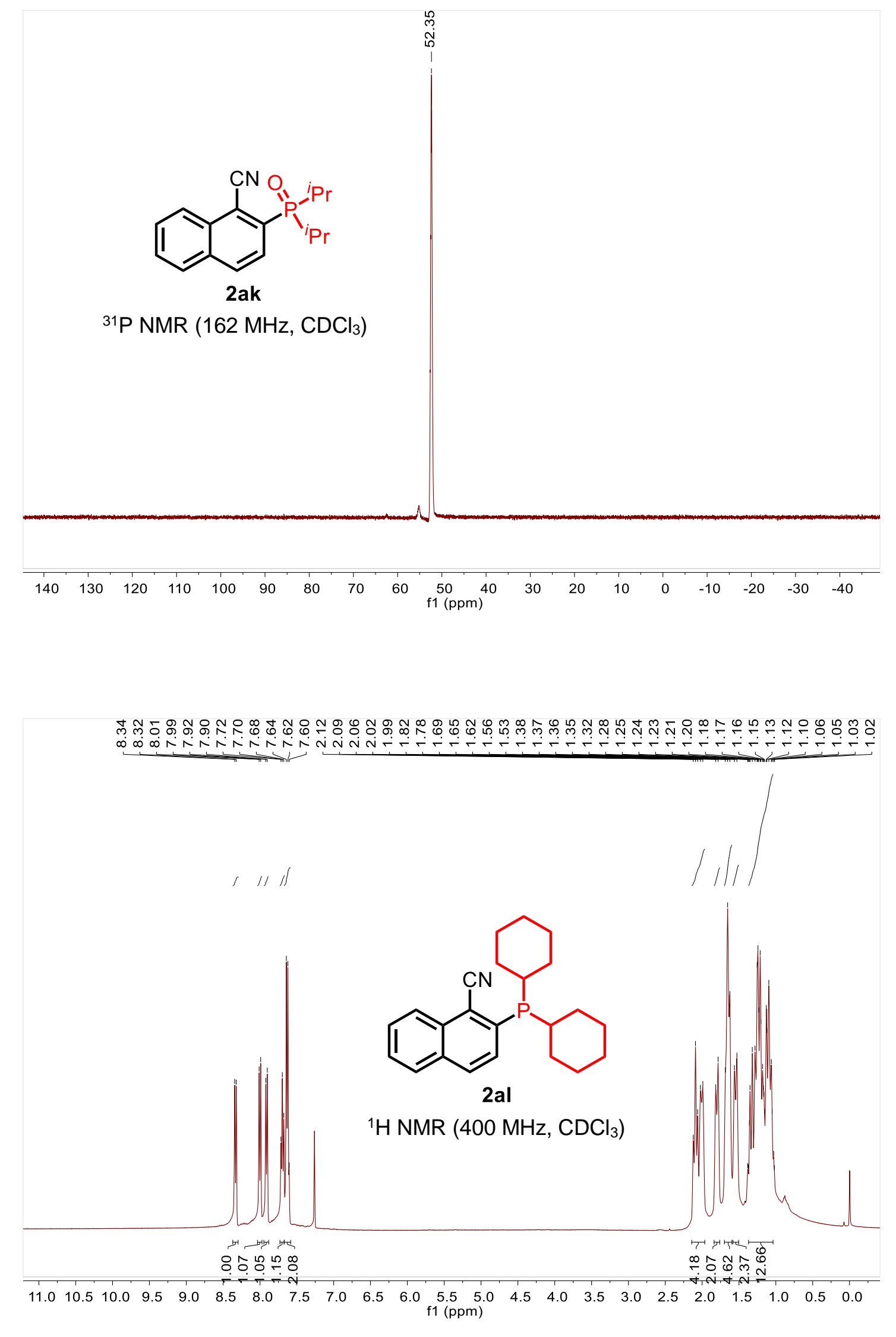




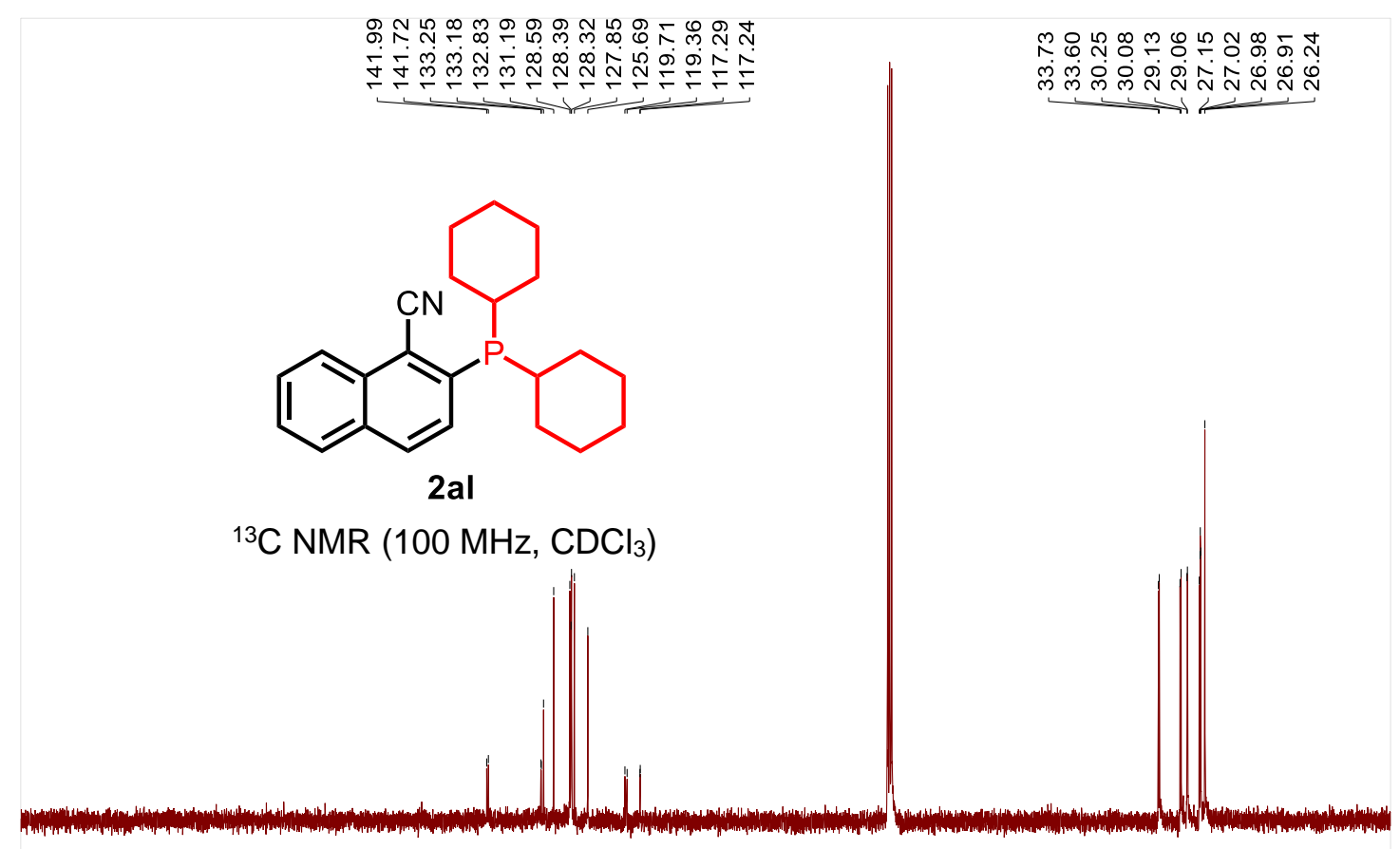

$\begin{array}{lllllllllllllllllllllllll}210 & 200 & 190 & 180 & 170 & 160 & 150 & 140 & 130 & 120 & \begin{array}{c}110 \\ \mathrm{f} 1(\mathrm{ppm})\end{array} & 100 & 80 & 70 & 60 & 50 & 40 & 30 & 20 & 10 & 0\end{array}$

$\stackrel{\substack{0 \\ i}}{i}$<smiles>N#Cc1c(P(C2CCCCC2)C2CCCCC2)ccc2ccccc12</smiles>

2al

${ }^{31} \mathrm{P}$ NMR $\left(162 \mathrm{MHz}, \mathrm{CDCl}_{3}\right)$

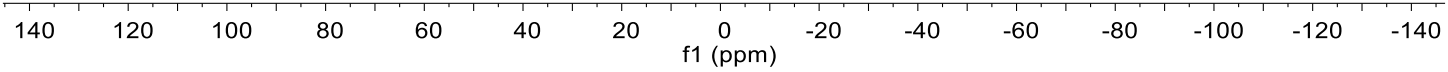



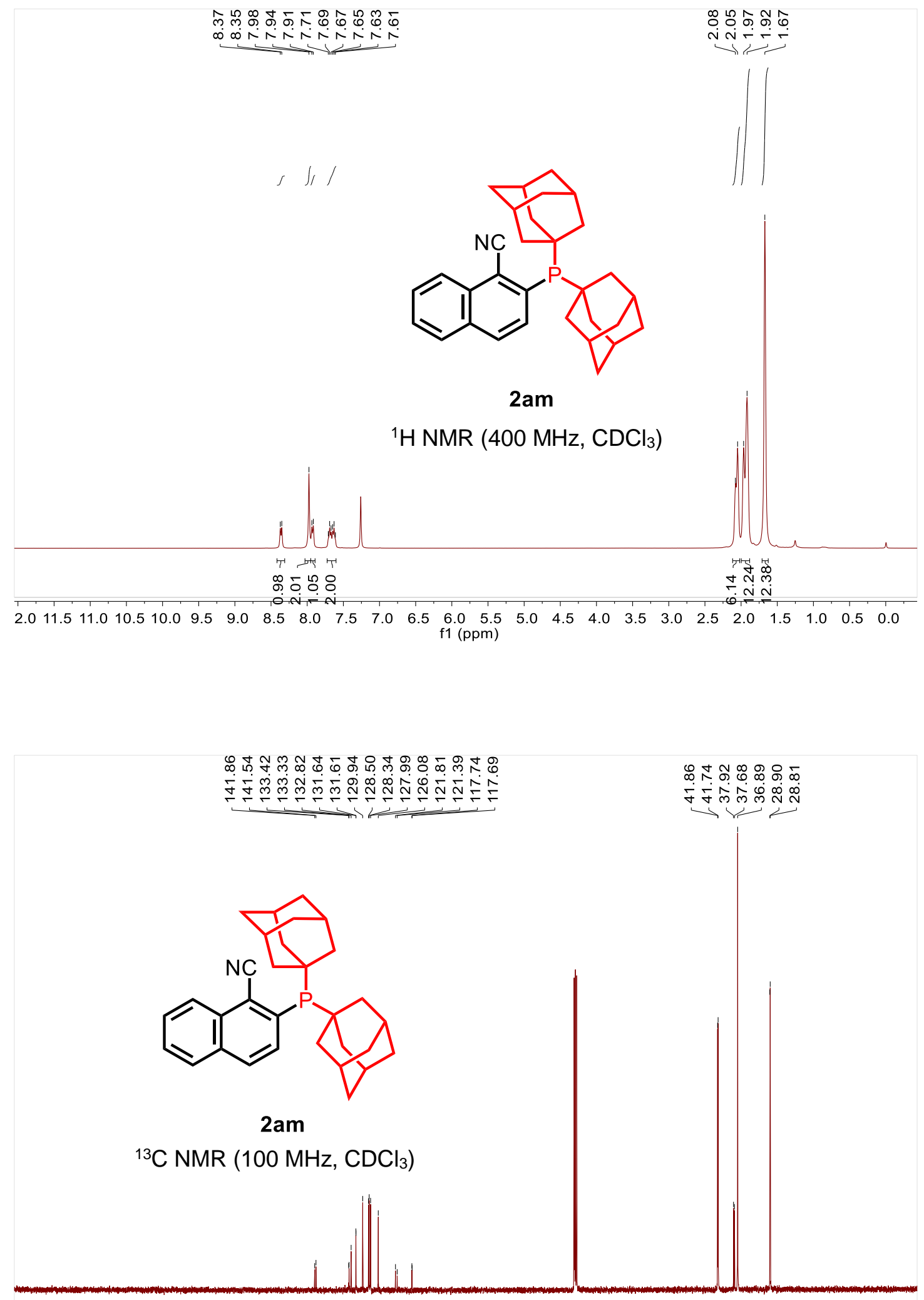

$\begin{array}{lllllllllllllllllllll}210 & 200 & 190 & 180 & 170 & 160 & 150 & 140 & 130 & 120 & \begin{array}{r}110 \\ \mathrm{f} 1(\mathrm{ppm})\end{array} & 100 & 80 & 70 & 60 & 50 & 40 & 30 & 20 & 10 & 0\end{array}$ 


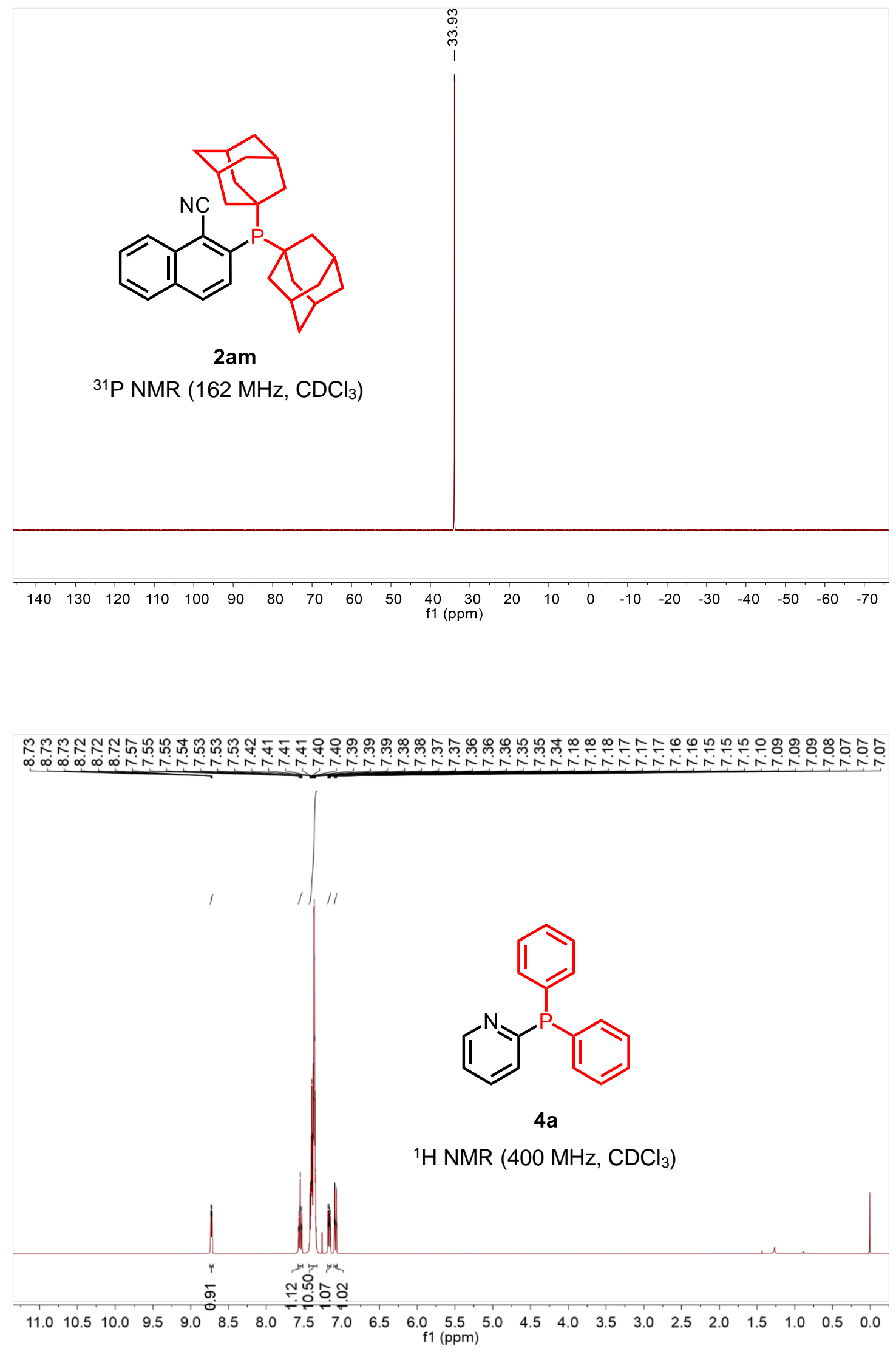




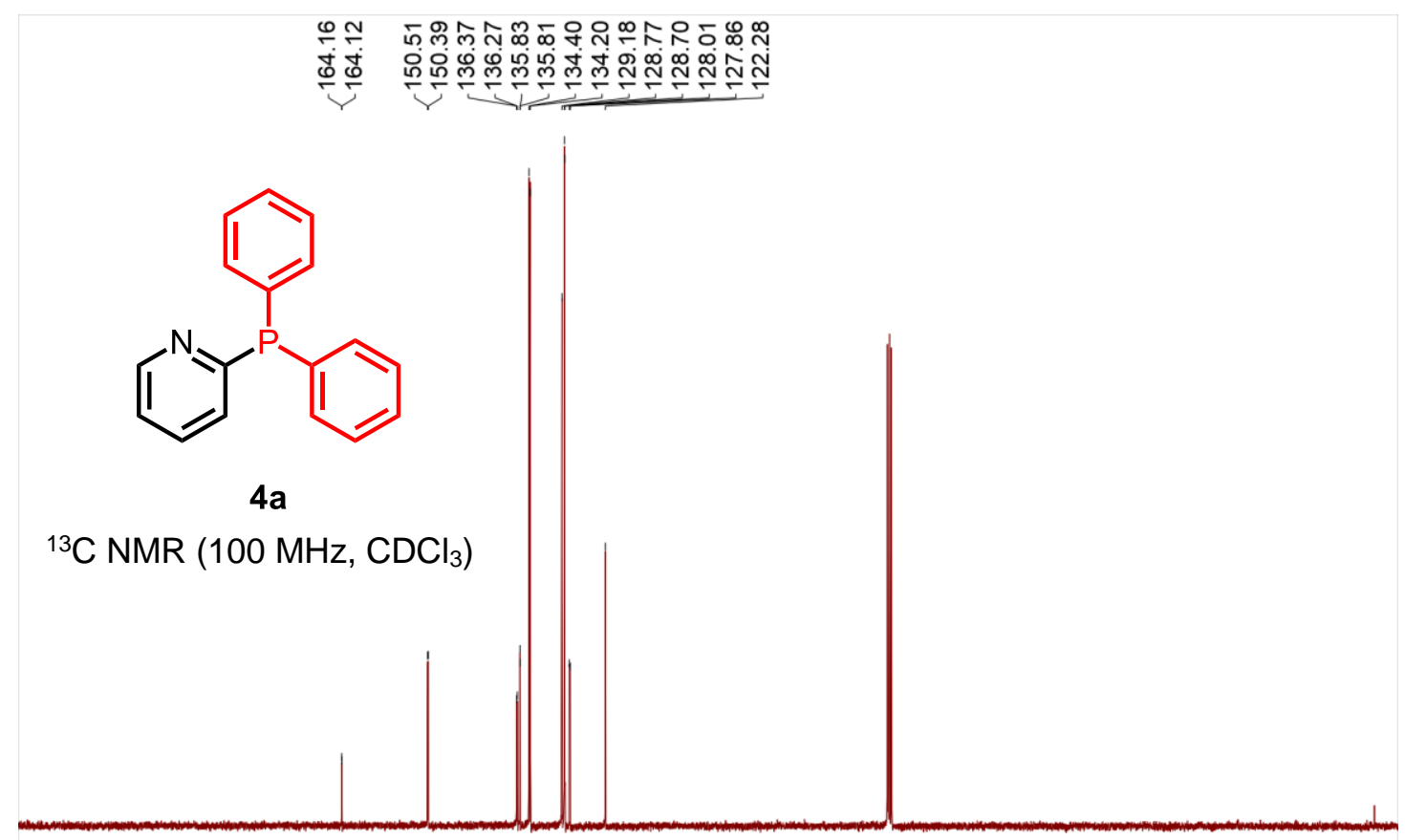

$\begin{array}{lllllllllllllllllllll}210 & 200 & 190 & 180 & 170 & 160 & 150 & 140 & 130 & 120 & \begin{array}{c}110 \\ \mathrm{f} 1(\mathrm{ppm})\end{array} & 90 & 80 & 70 & 60 & 50 & 40 & 30 & 20 & 10 & 0\end{array}$

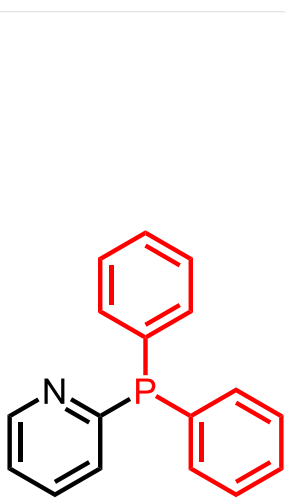

ஜு

4a

${ }^{31} \mathrm{P}$ NMR $\left(162 \mathrm{MHz}, \mathrm{CDCl}_{3}\right)$ 
<smiles>CC(C)(C)c1ccc(P(c2ccc(Br)cc2)c2ccccn2)cc1</smiles>

4b

${ }^{1} \mathrm{H}$ NMR $\left(400 \mathrm{MHz}, \mathrm{CDCl}_{3}\right)$

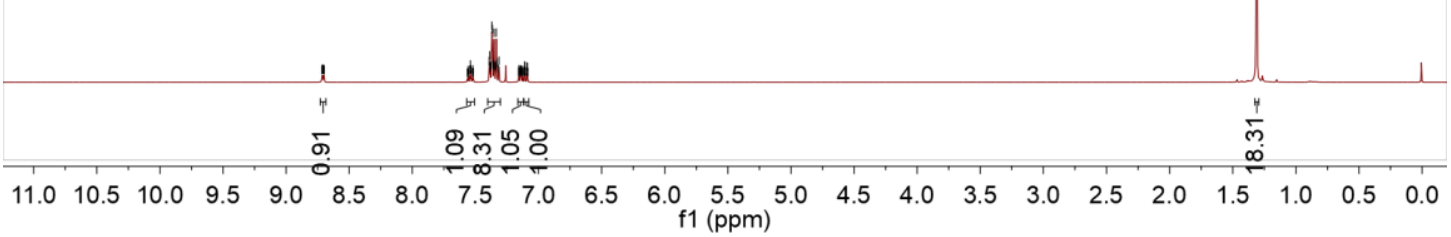<smiles>CC(C)(C)c1ccc(P(c2ccc(C(C)(C)C)cc2)c2ccccn2)cc1</smiles>

4b

${ }^{13} \mathrm{C}$ NMR $\left(100 \mathrm{MHz}, \mathrm{CDCl}_{3}\right)$

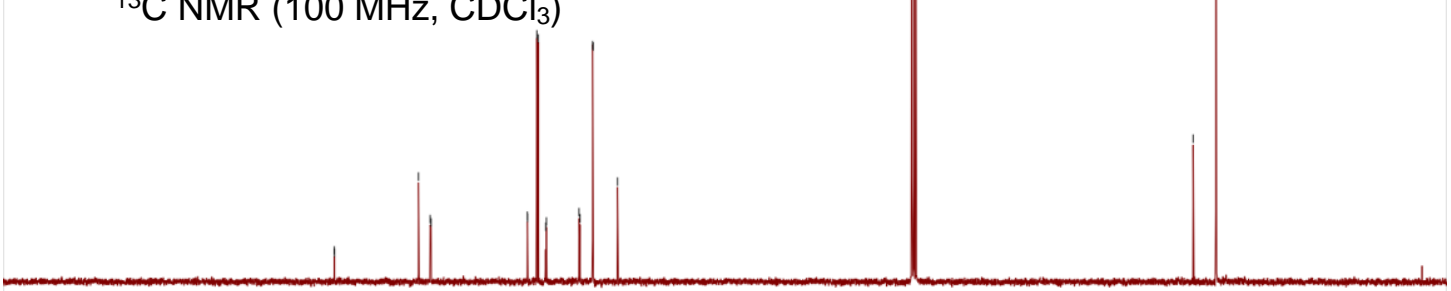

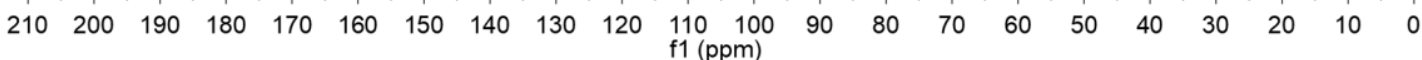




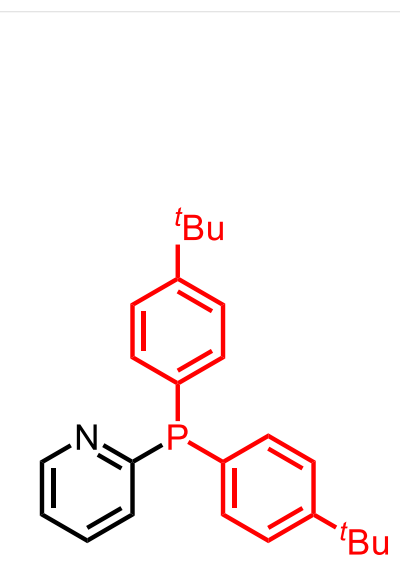

4b

${ }^{31} \mathrm{P}$ NMR (162 MHz, $\left.\mathrm{CDCl}_{3}\right)$

$\begin{array}{lllllllllllllllllll}50 & 130 & 110 & 90 & 70 & 50 & 30 & 10 & \begin{array}{c}-10 \\ \mathrm{f} 1(\mathrm{ppm})\end{array} & -30 & -50 & -70 & -90 & -110 & -130 & -150 & -11\end{array}$

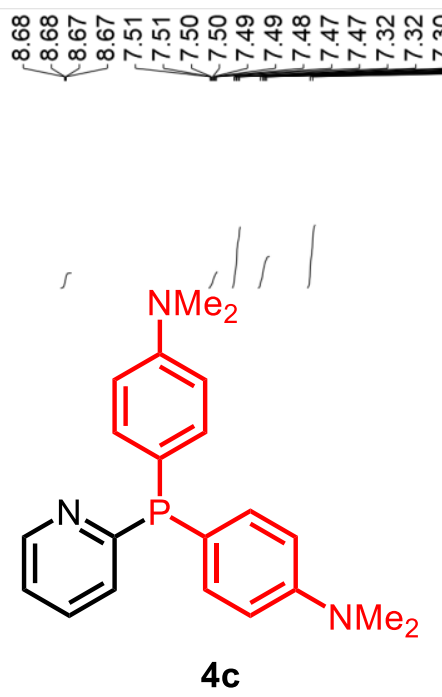

${ }^{1} \mathrm{H}$ NMR $\left(400 \mathrm{MHz}, \mathrm{CDCl}_{3}\right)$

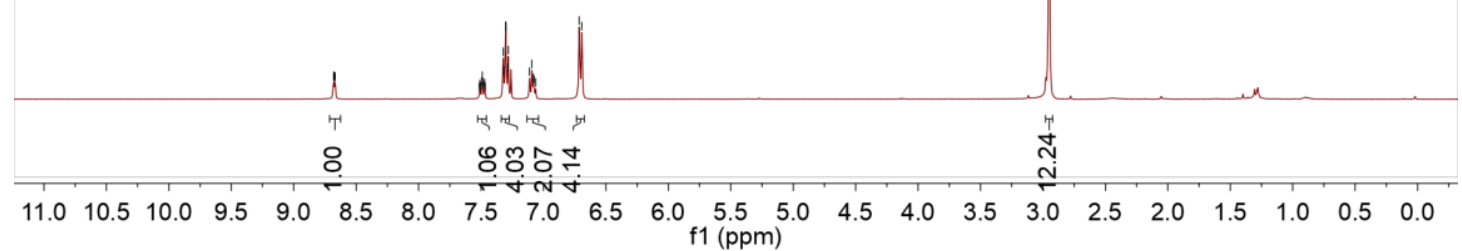



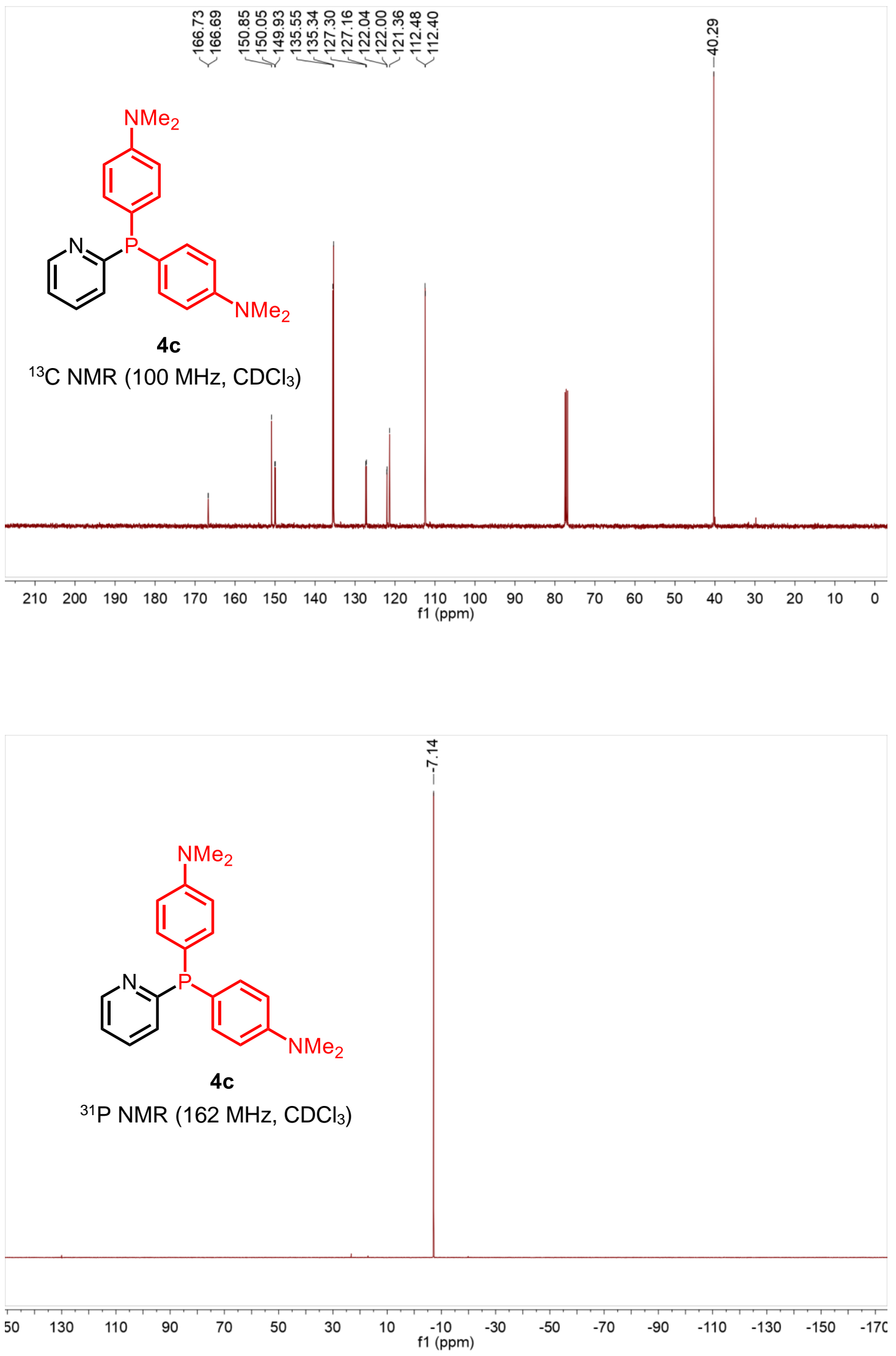

156 
$, 1,1$<smiles>CC(C)(C)c1cc(P(c2cc(C(C)(C)C)cc(C(C)(C)C)c2)c2ccccn2)cc(C(C)(C)C)c1</smiles>

4d

${ }^{1} \mathrm{H}$ NMR $\left(400 \mathrm{MHz}, \mathrm{CDCl}_{3}\right)$

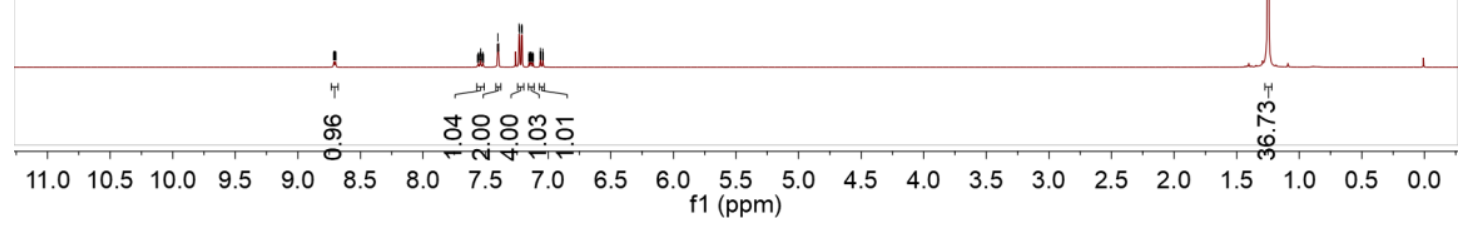

$\div$<smiles>CC(C)(C)c1cc(P(c2cc(C(C)(C)C)cc(C(C)(C)C)c2)c2ccccn2)cc(C(C)(C)C)c1</smiles>

4d

${ }^{13} \mathrm{C}$ NMR $\left(100 \mathrm{MHz}, \mathrm{CDCl}_{3}\right)$

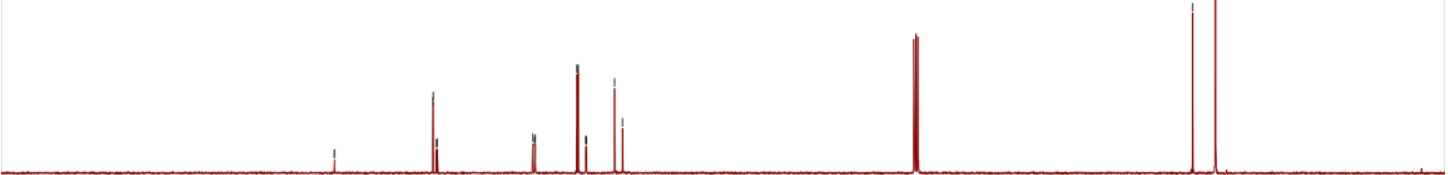

$\begin{array}{llllllllllllllllllllllll}210 & 200 & 190 & 180 & 170 & 160 & 150 & 140 & 130 & 120 & \begin{array}{c}110 \\ \mathrm{f} 1(\mathrm{ppm})\end{array} & 100 & 90 & 80 & 70 & 60 & 50 & 40 & 30 & 20 & 10 & 0\end{array}$ 


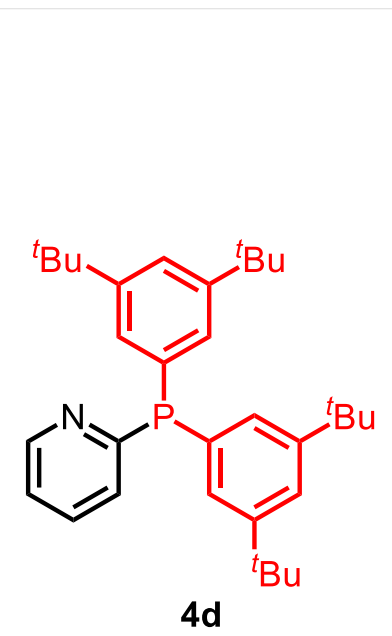

${ }^{31} \mathrm{P}$ NMR $\left(162 \mathrm{MHz}, \mathrm{CDCl}_{3}\right)$

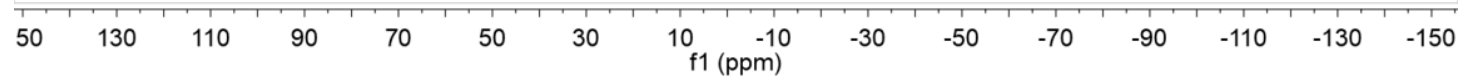

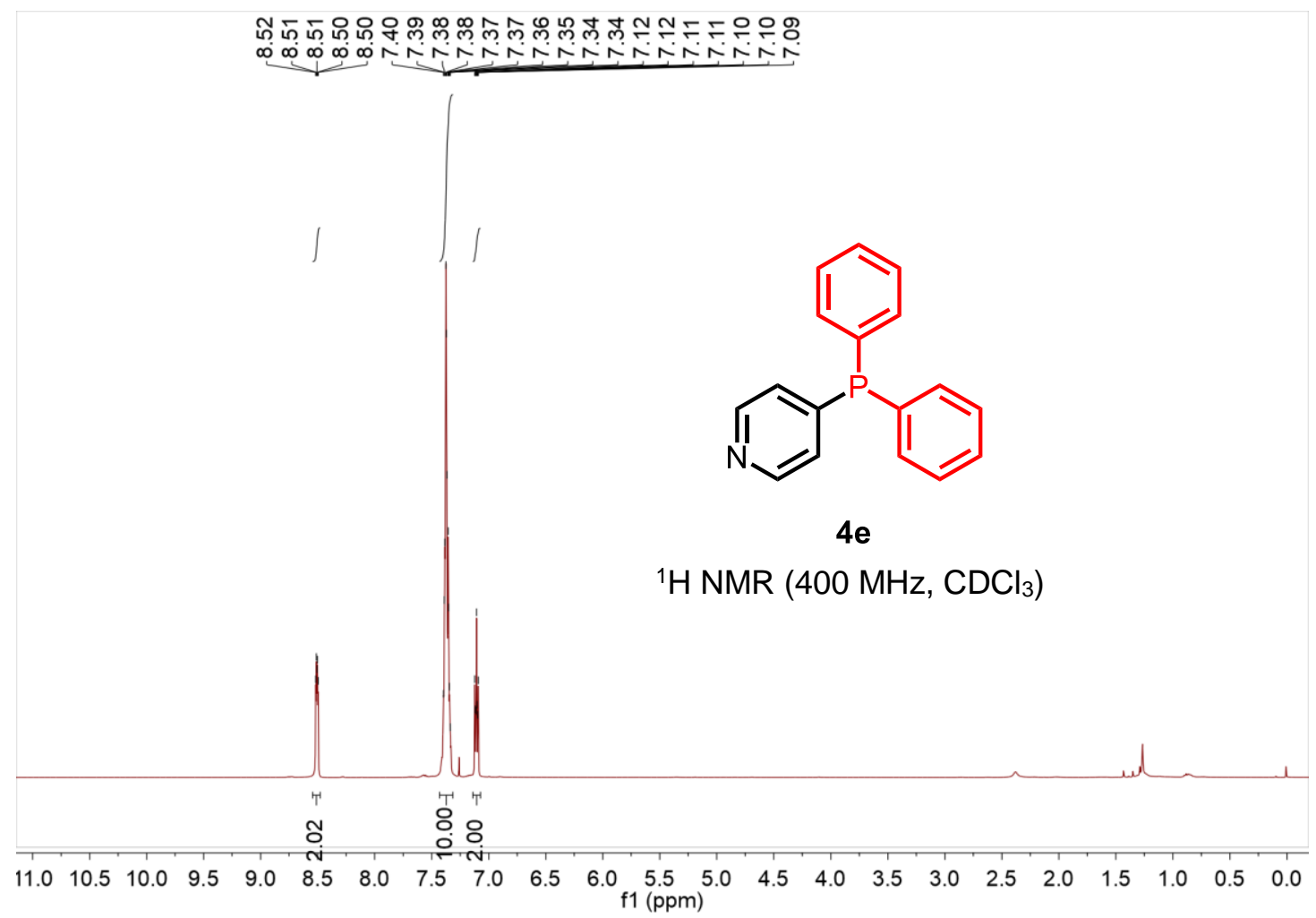




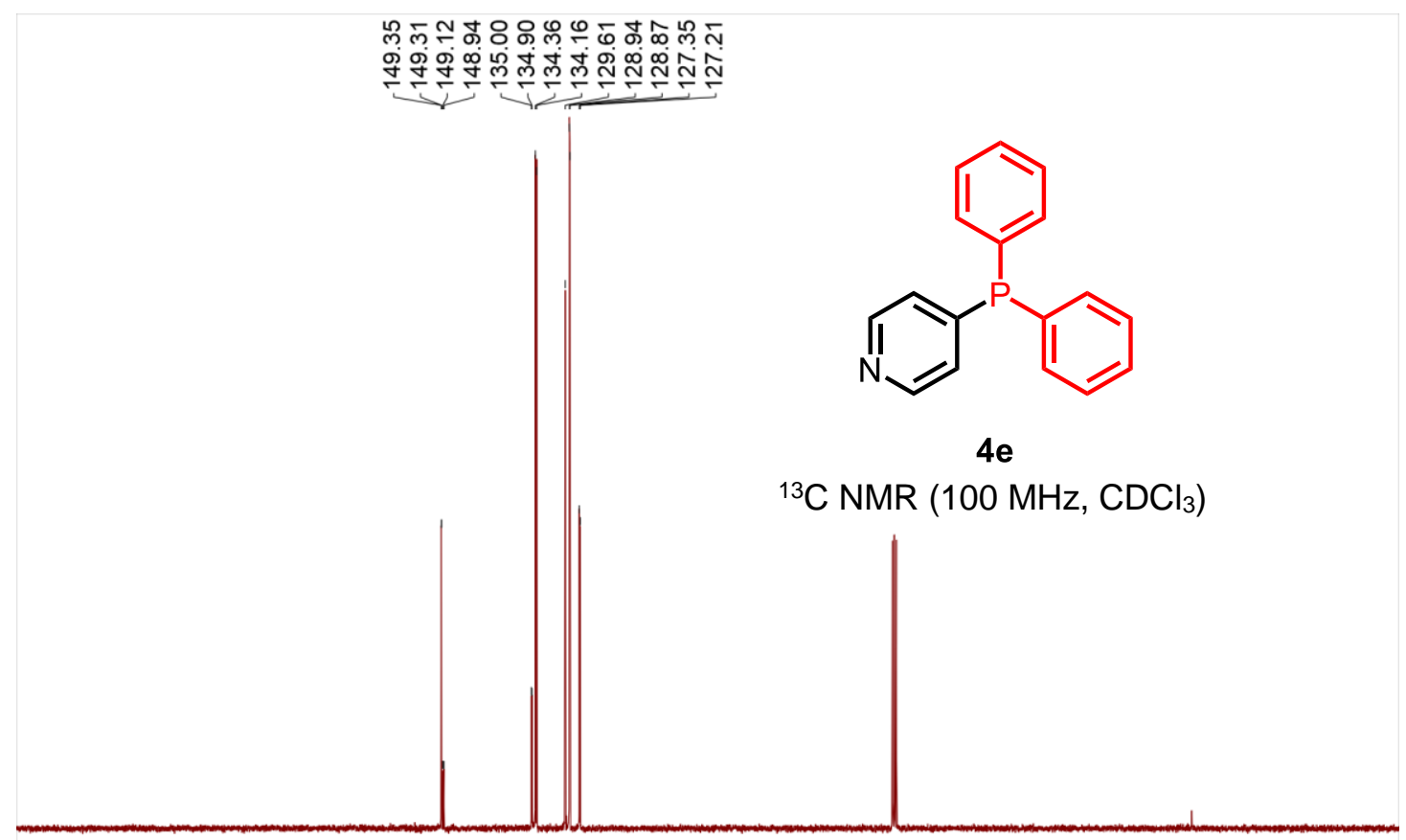

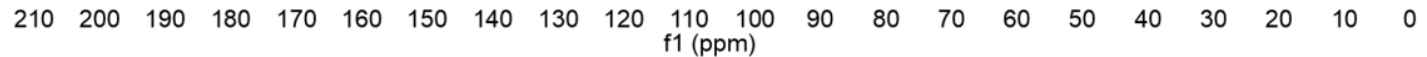

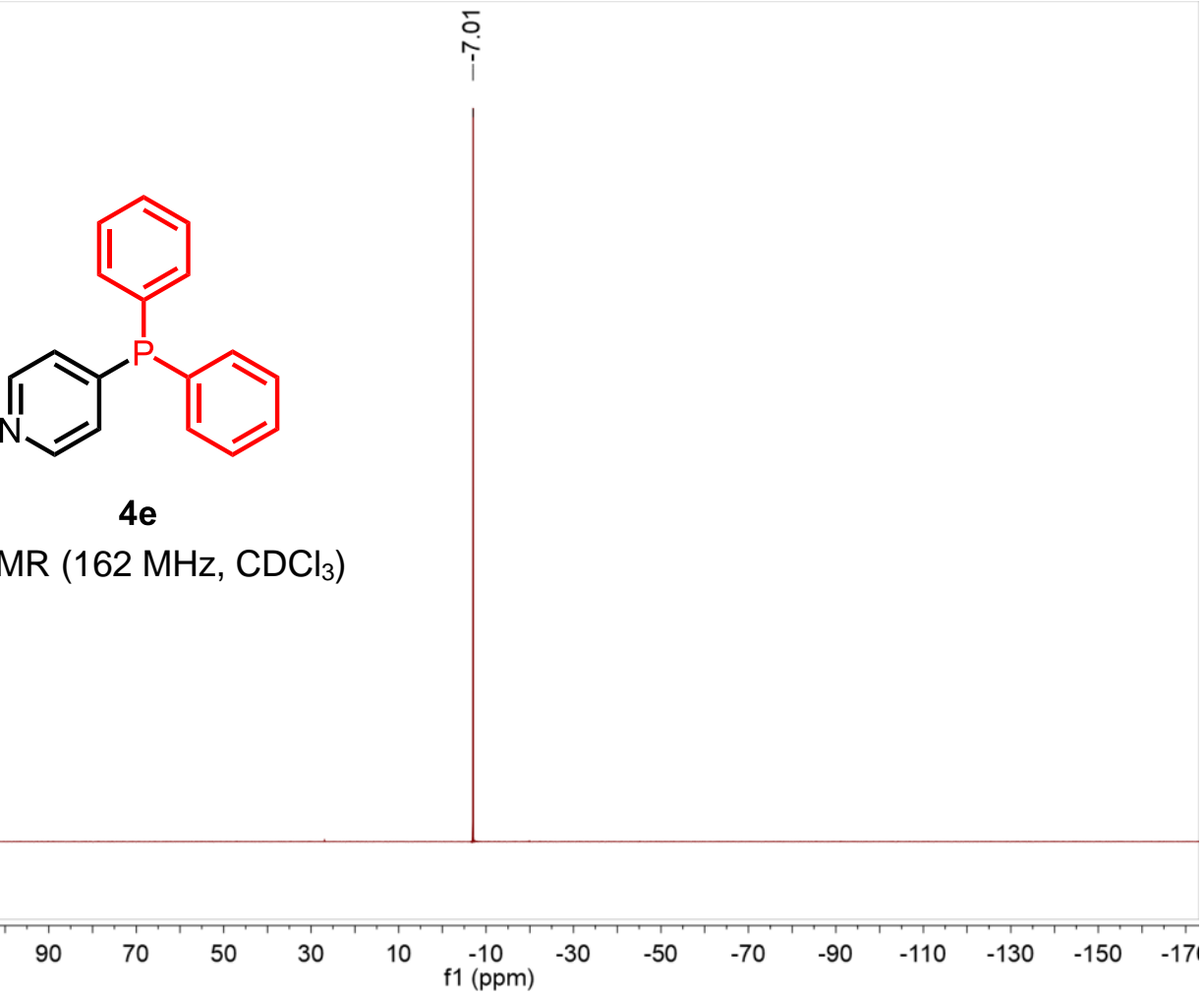


II,<smiles>CC(C)(C)c1ccc(P(c2ccncc2)c2ccc(Br)cc2)cc1</smiles>

$4 f$

${ }^{1} \mathrm{H} \mathrm{NMR}\left(400 \mathrm{MHz}, \mathrm{CDCl}_{3}\right)$

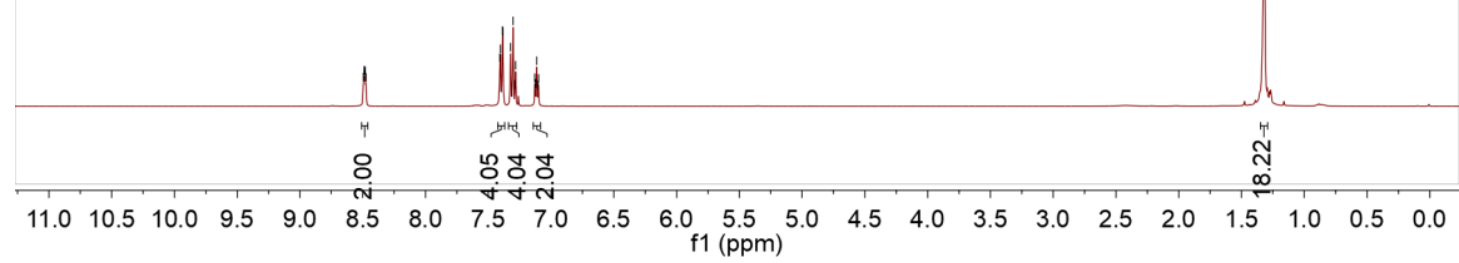

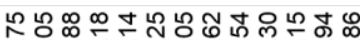

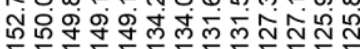<smiles>CC(C)(C)c1ccc(P(c2ccncc2)c2ccc(Br)cc2)cc1</smiles>

4f

${ }^{13} \mathrm{C} \mathrm{NMR}\left(100 \mathrm{MHz}, \mathrm{CDCl}_{3}\right)$

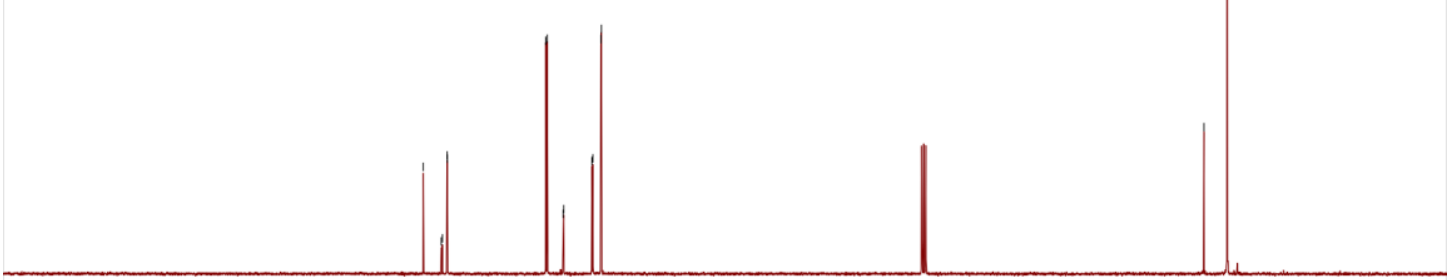

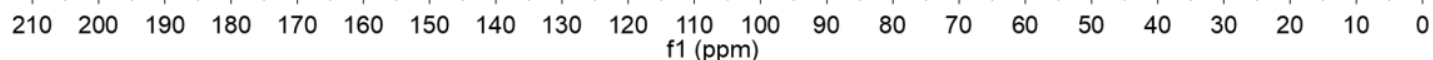




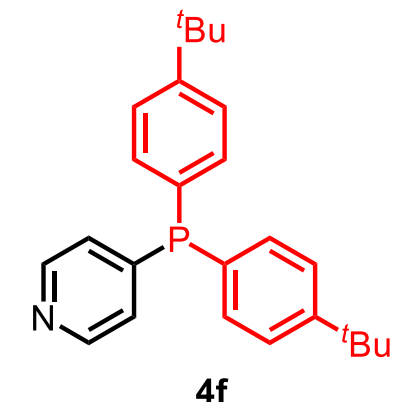

${ }^{31} \mathrm{P} \mathrm{NMR}\left(162 \mathrm{MHz}, \mathrm{CDCl}_{3}\right)$

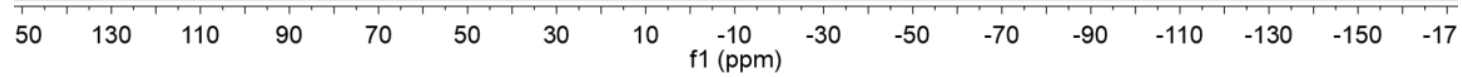

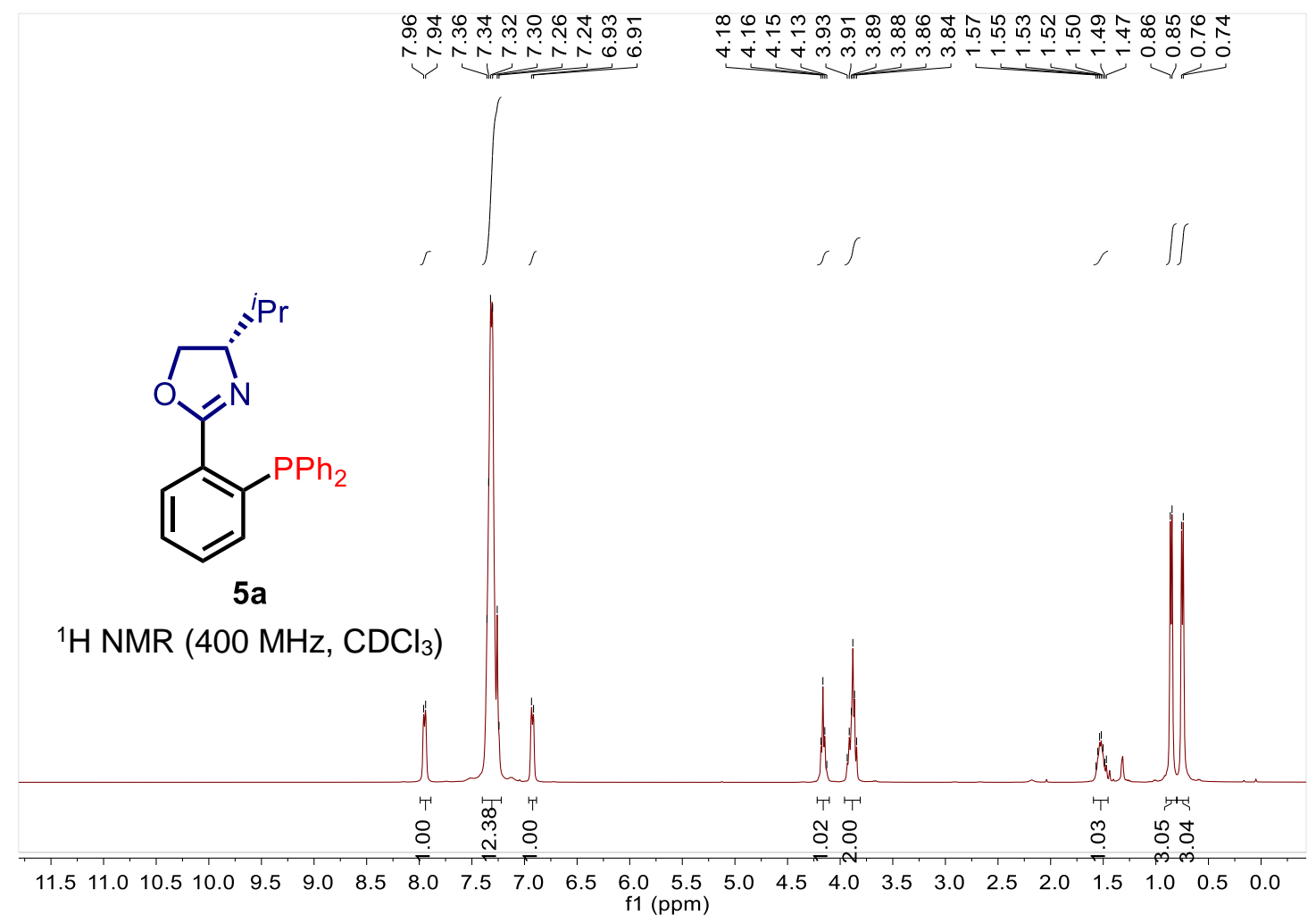




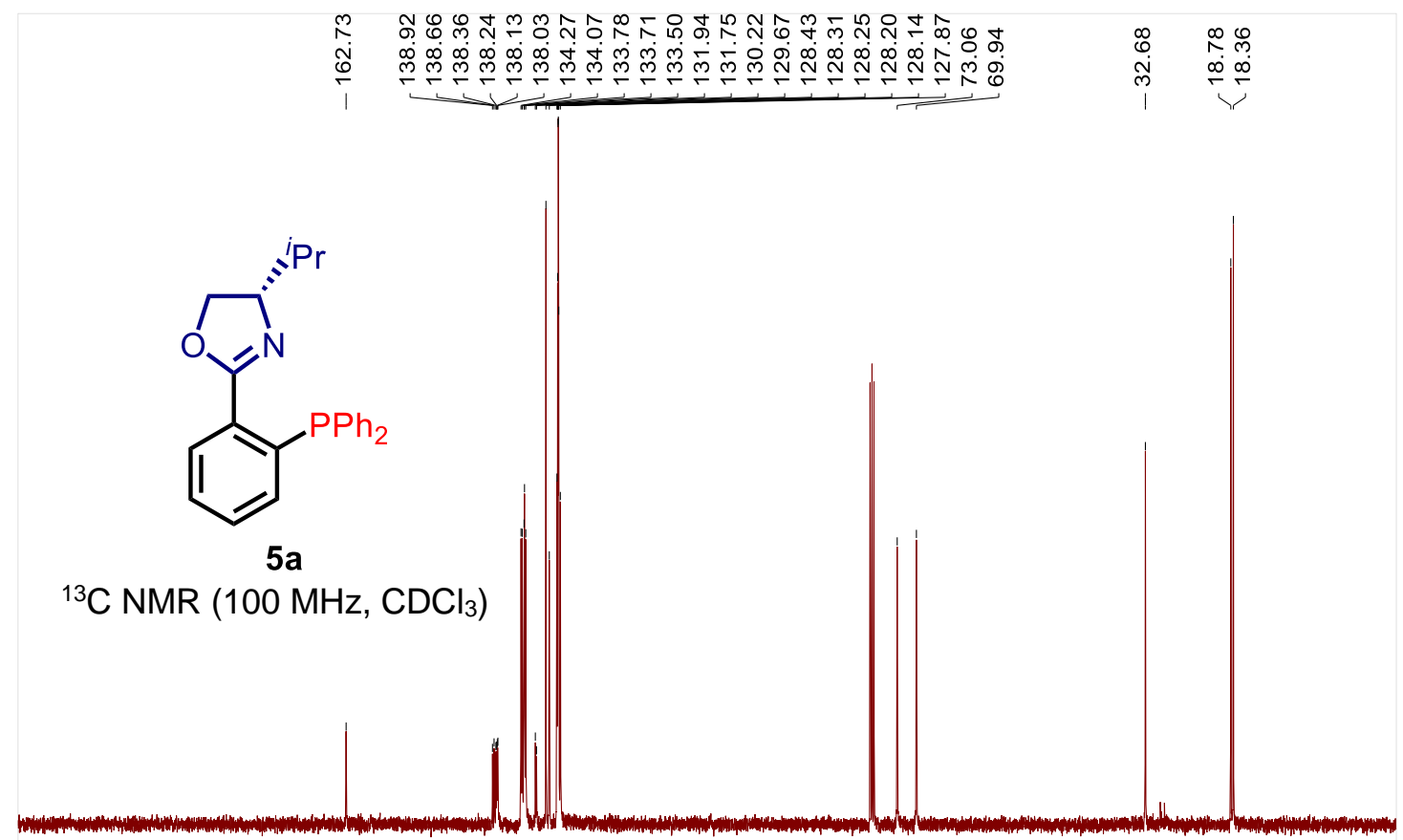

$\begin{array}{rlllllllllllllllllllll}210 & 200 & 190 & 180 & 170 & 160 & 150 & 140 & 130 & 120 & \begin{array}{c}110 \\ \mathrm{f} 1(\mathrm{ppm})\end{array} & 90 & 80 & 70 & 60 & 50 & 40 & 30 & 20 & 10 & 0\end{array}$

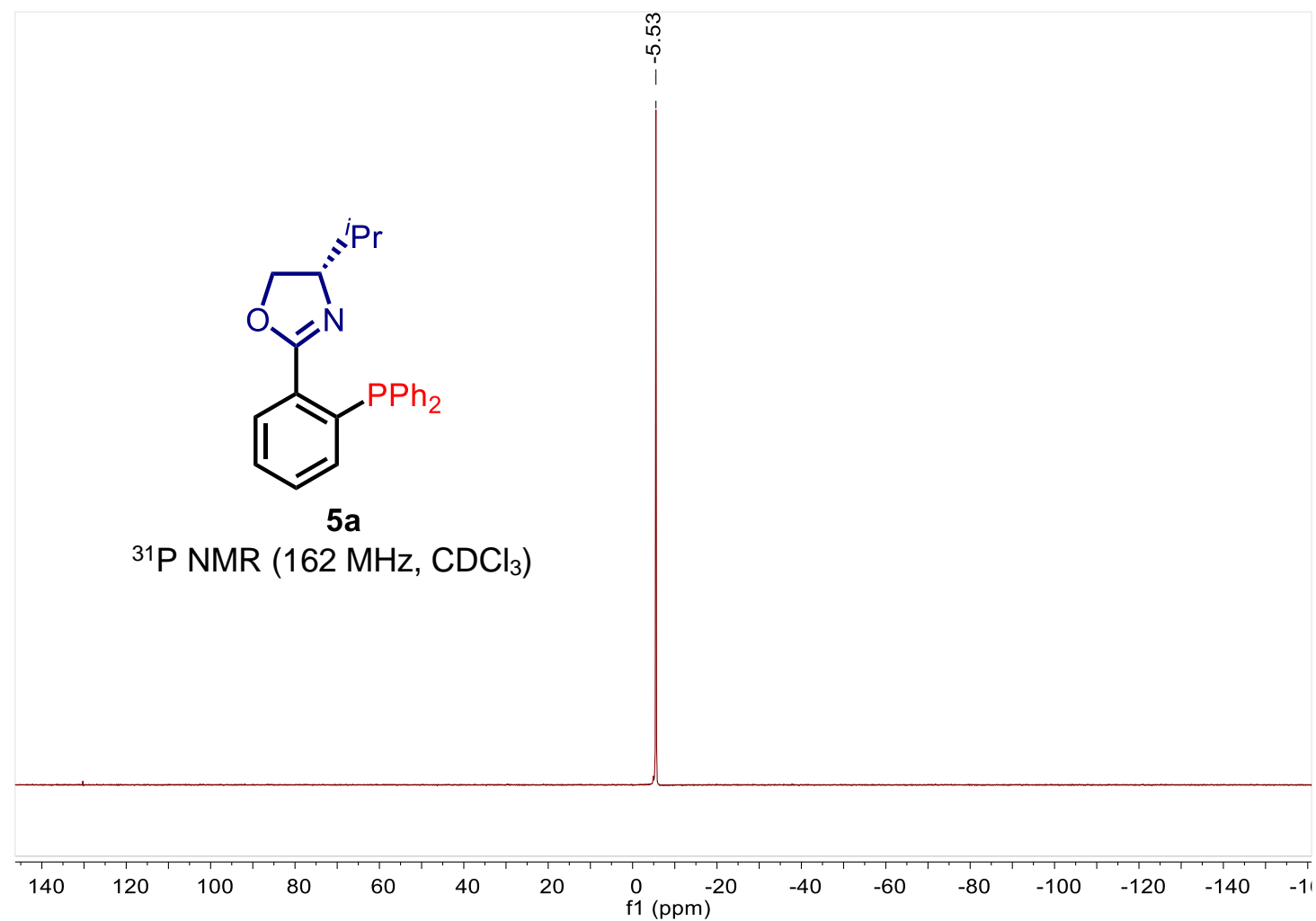



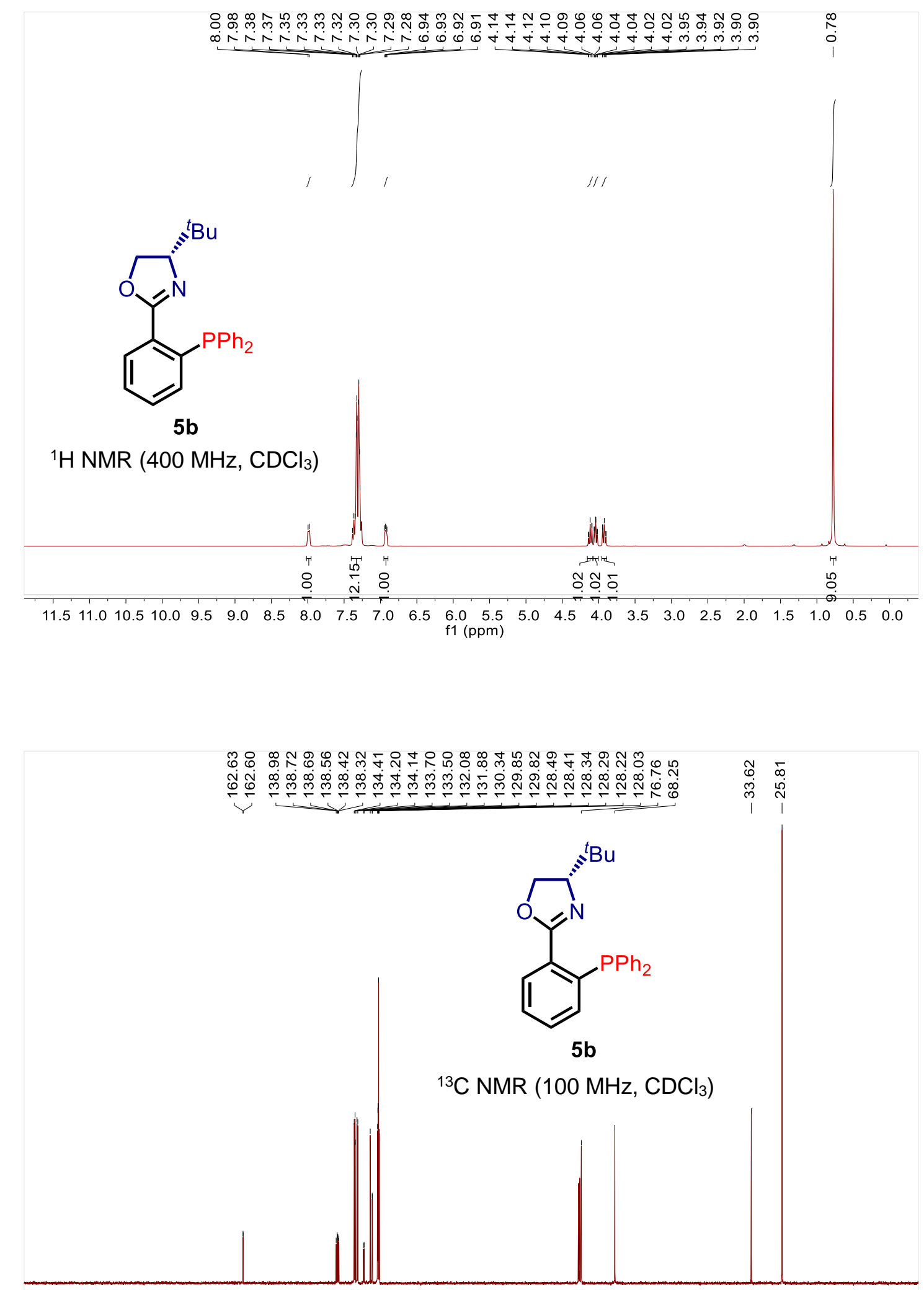

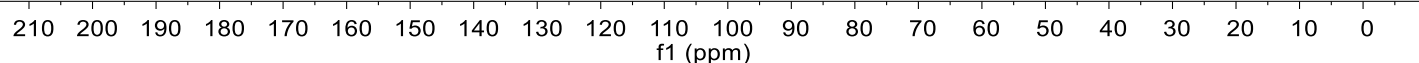


<smiles>CC(C)(C)[C@H]1COC(c2ccccc2P)=N1</smiles>

$5 b$

${ }^{31} \mathrm{P}$ NMR (162 MHz, $\left.\mathrm{CDCl}_{3}\right)$

$\begin{array}{lllllllllllllll}130 & 110 & 90 & 70 & 50 & 30 & 10 & -10 & -30 & -50 & -70 & -90 & -110 & -130 & -150\end{array}$

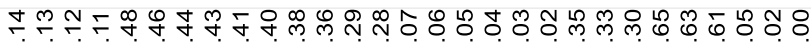

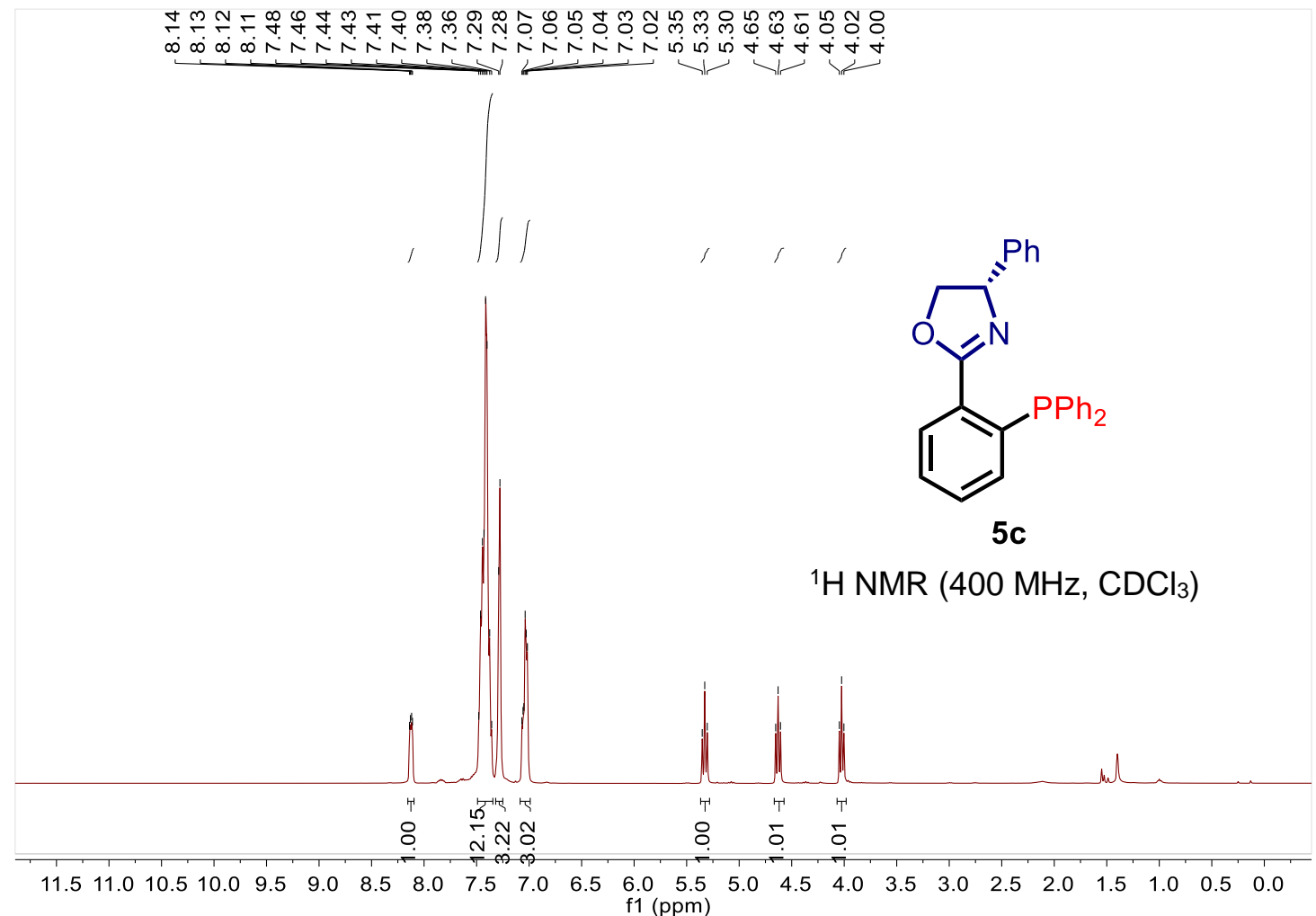




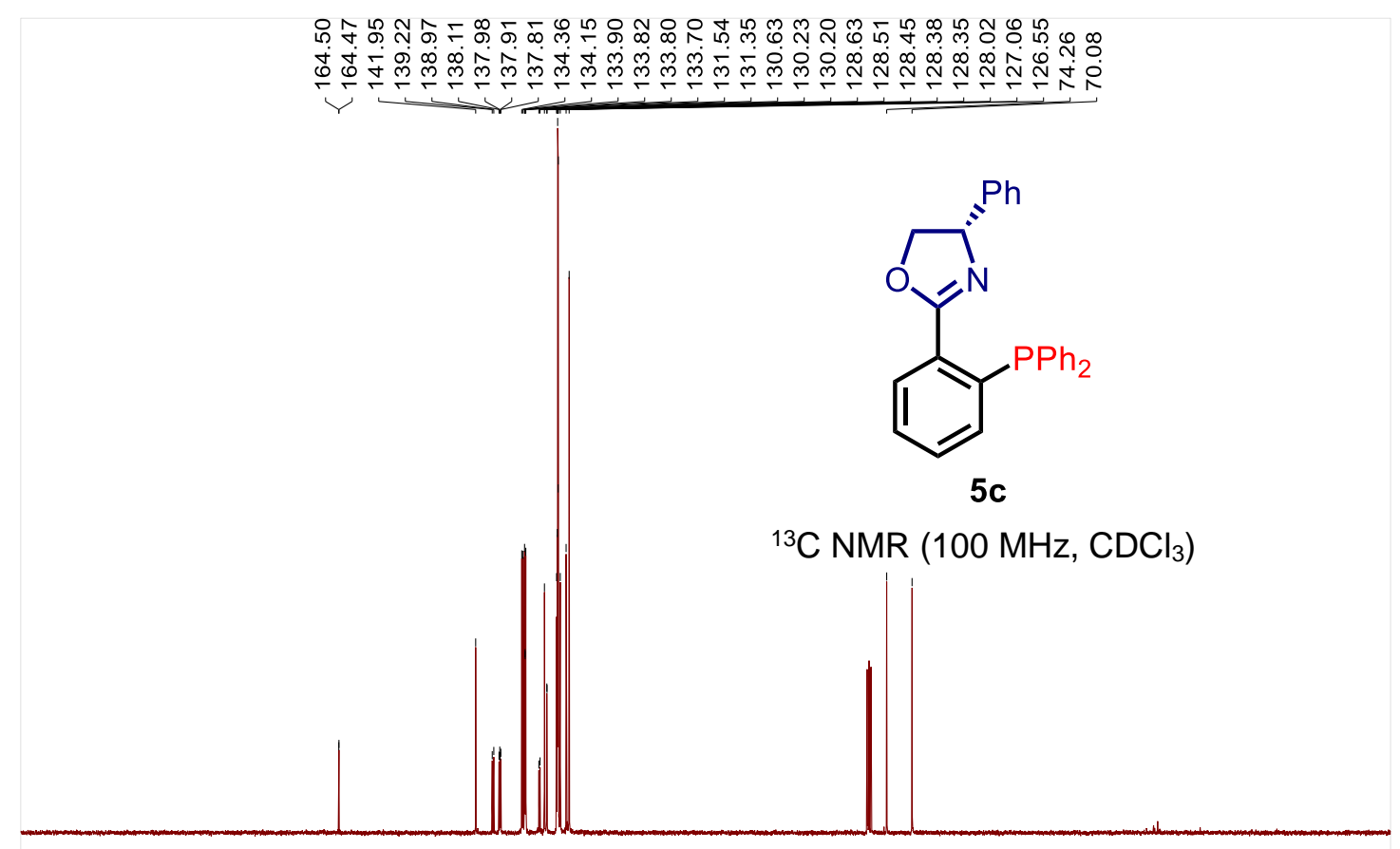

$\begin{array}{llllllllllllllllllllll}210 & 200 & 190 & 180 & 170 & 160 & 150 & 140 & 130 & 120 & 110 & 100 & 90 & 80 & 70 & 60 & 50 & 40 & 30 & 20 & 10 & 0\end{array}$

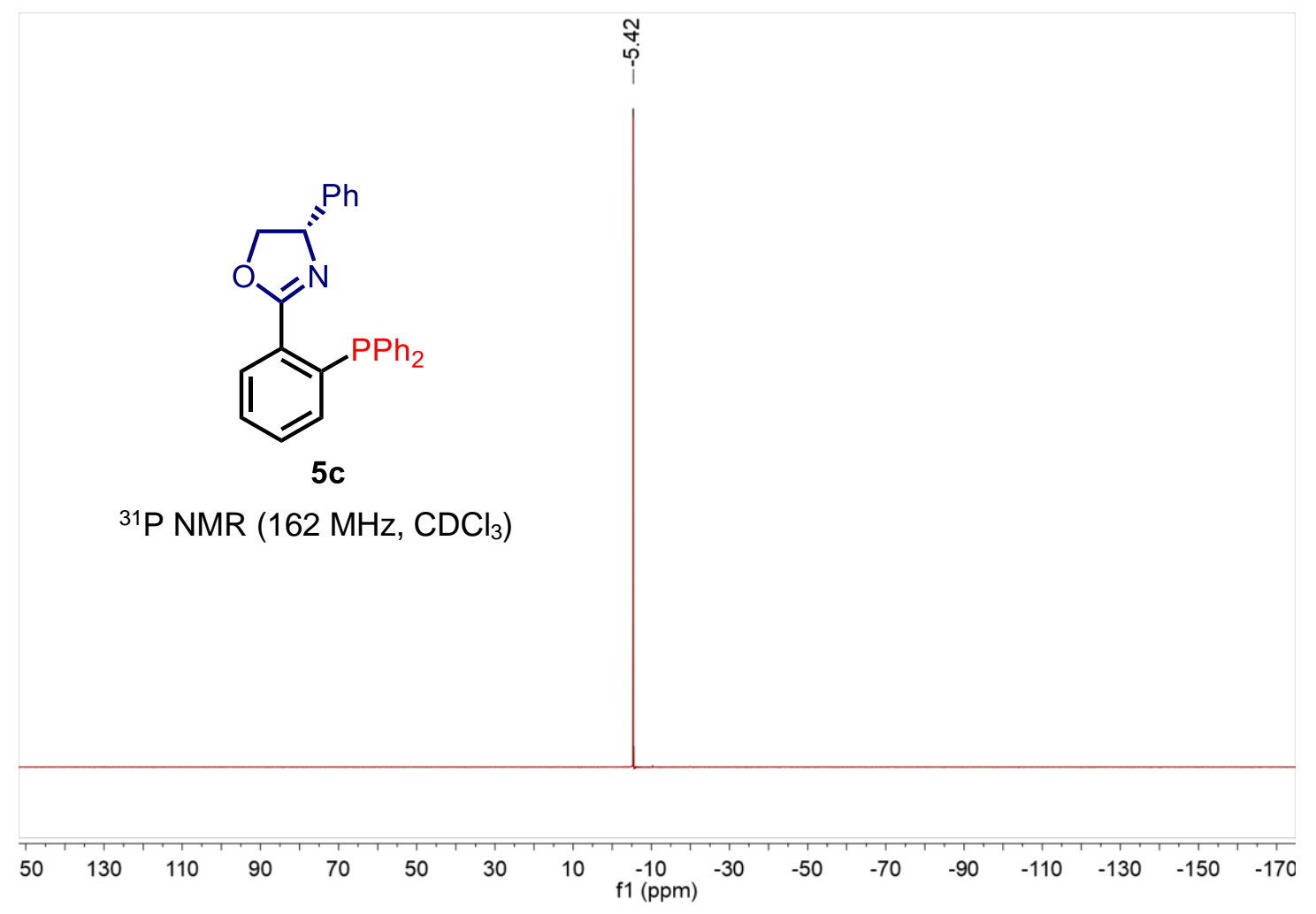




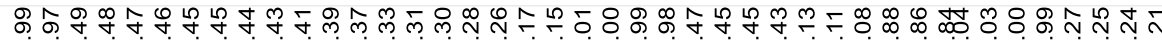

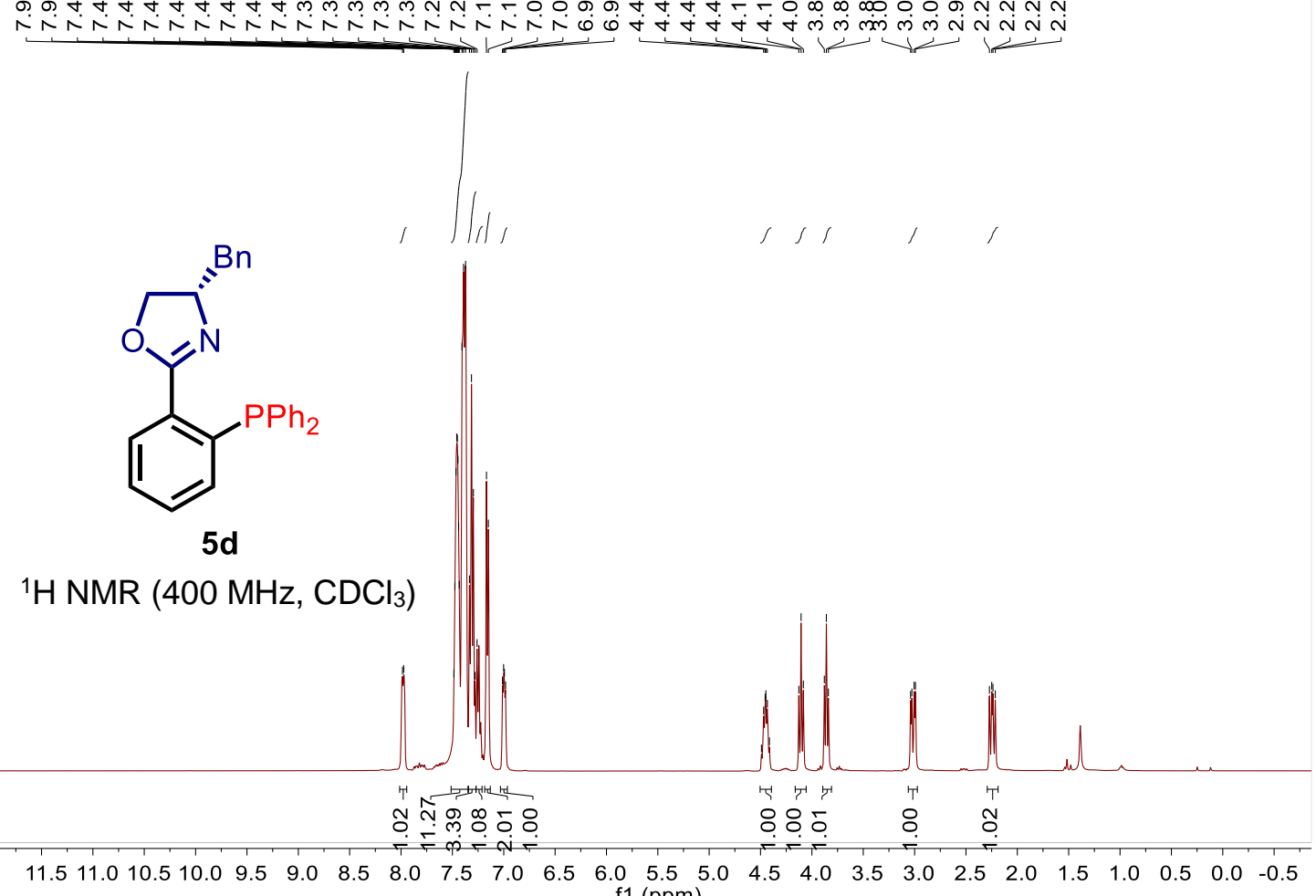

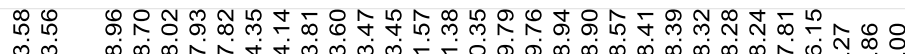

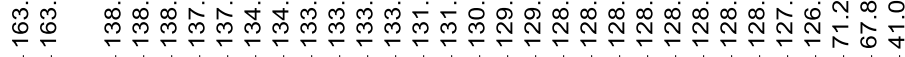

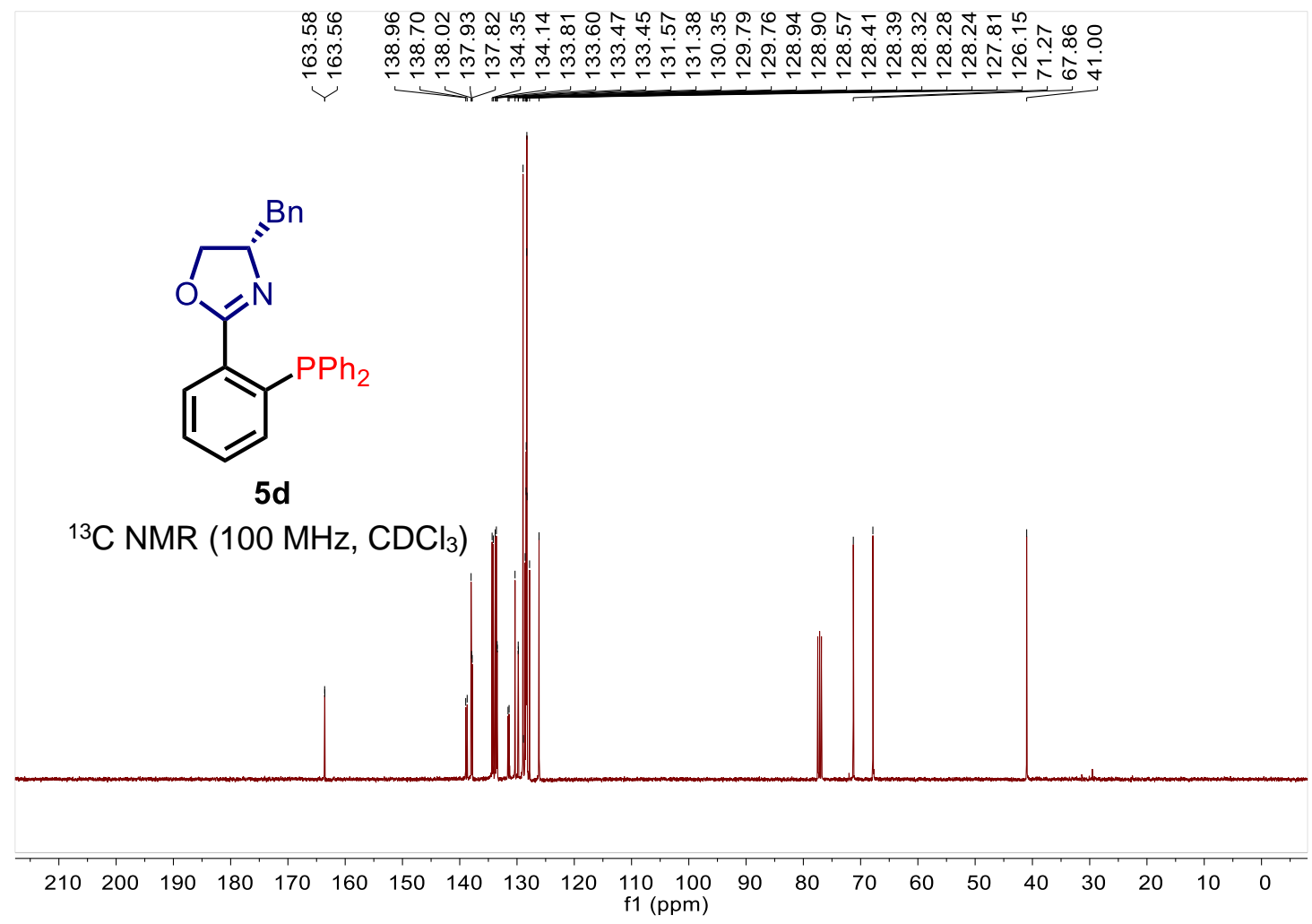




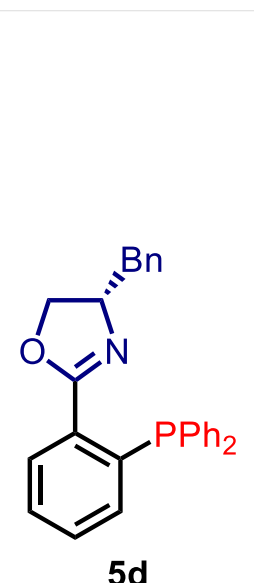

${ }^{31} \mathrm{P} \mathrm{NMR}\left(162 \mathrm{MHz}, \mathrm{CDCl}_{3}\right)$

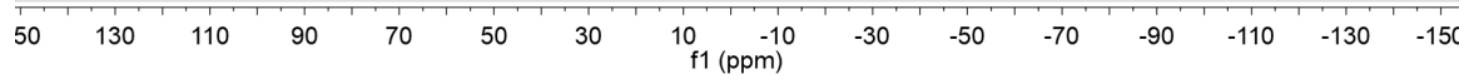

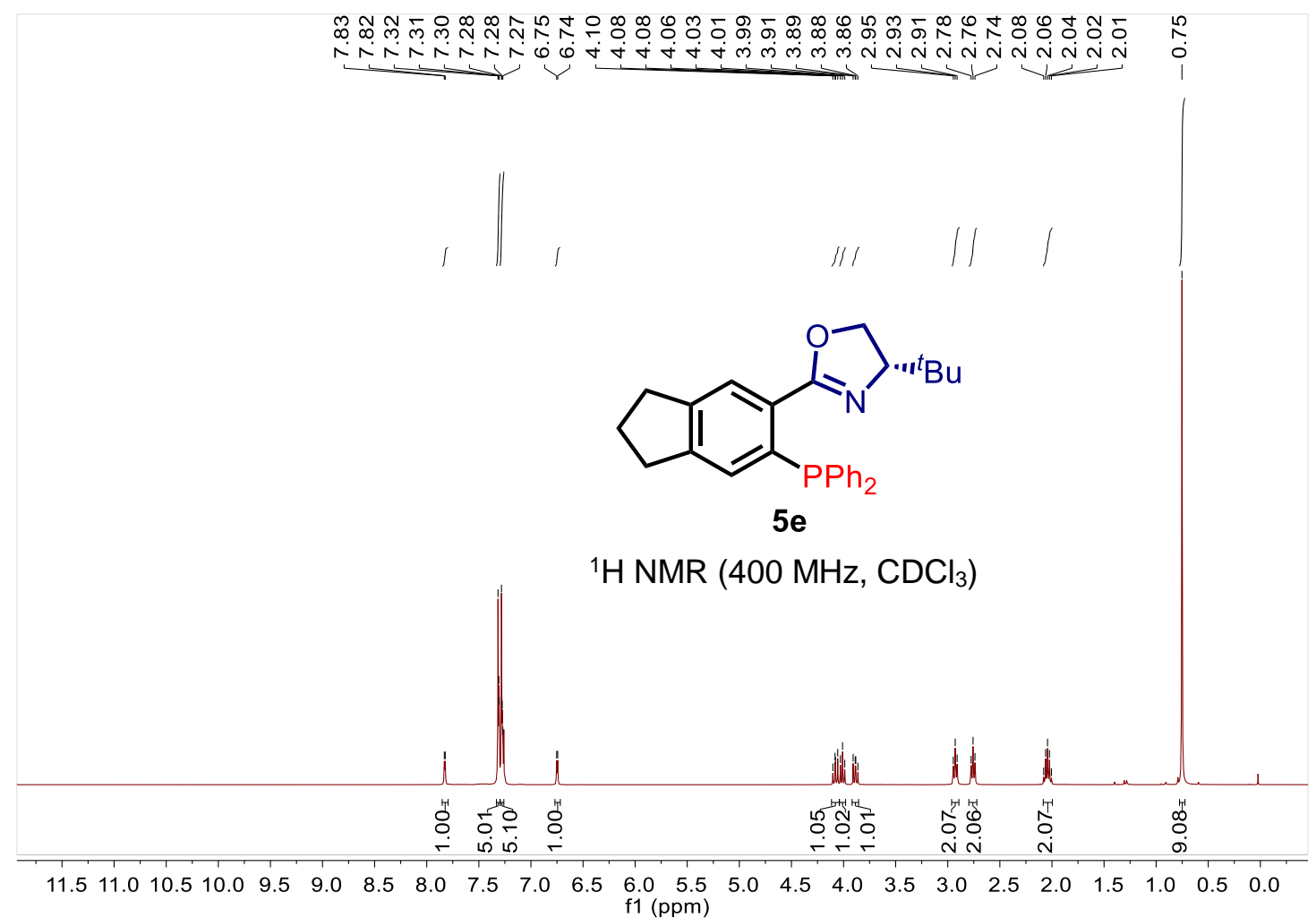




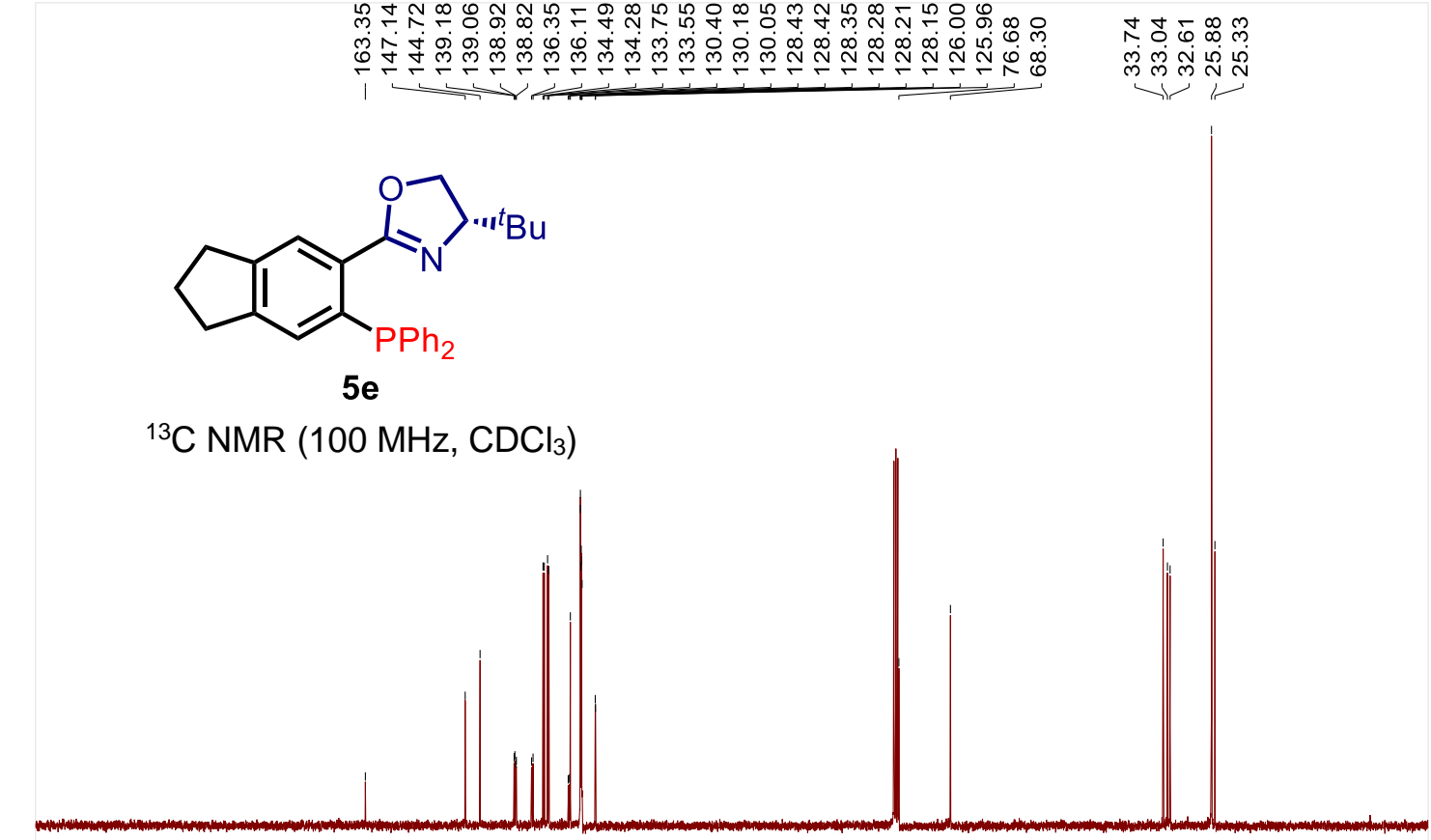

$\begin{array}{llllllllllllllllllllll}210 & 200 & 190 & 180 & 170 & 160 & 150 & 140 & 130 & 120 & 110 & 100 & 90 & 80 & 70 & 60 & 50 & 40 & 30 & 20 & 10 & 0\end{array}$

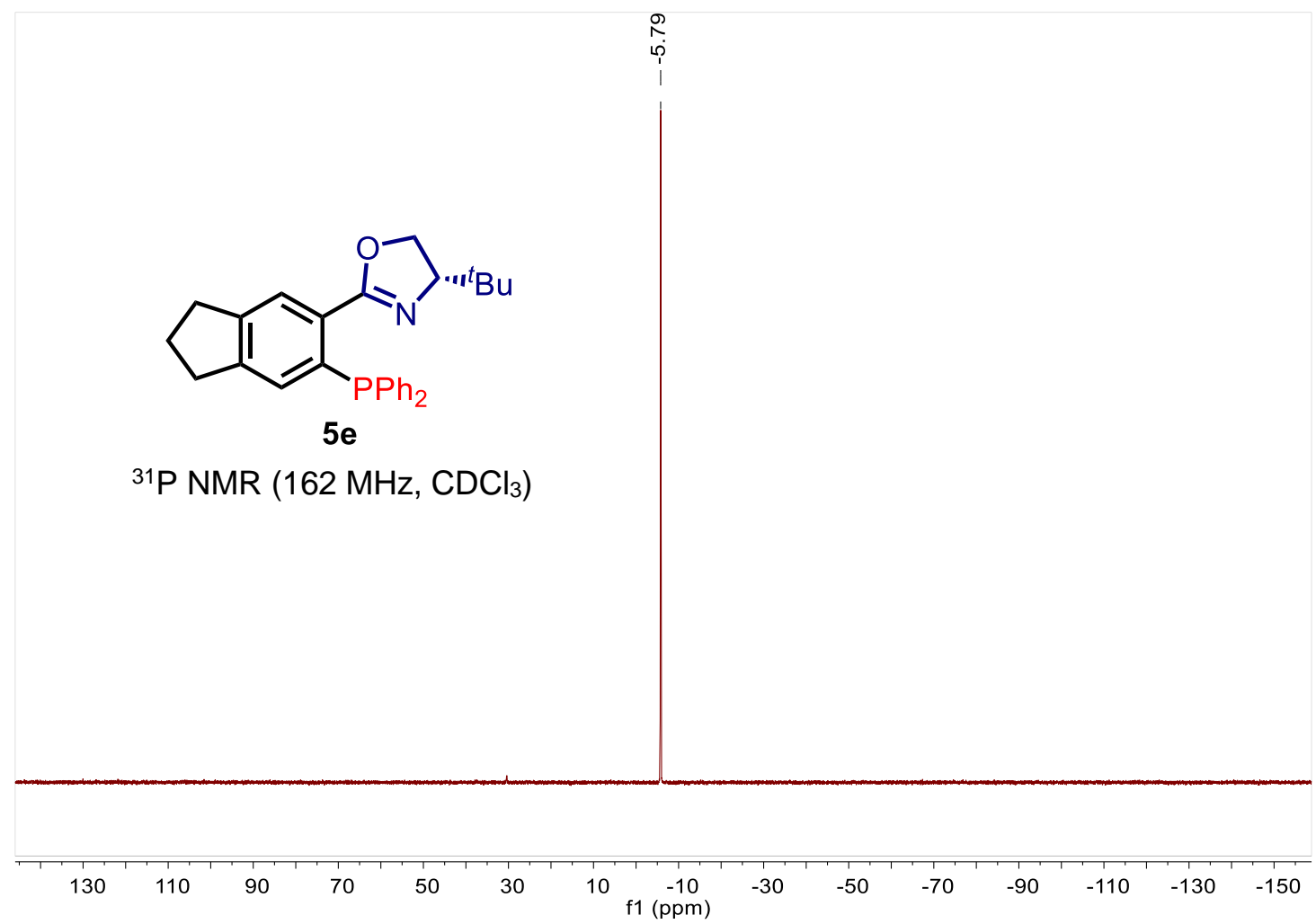




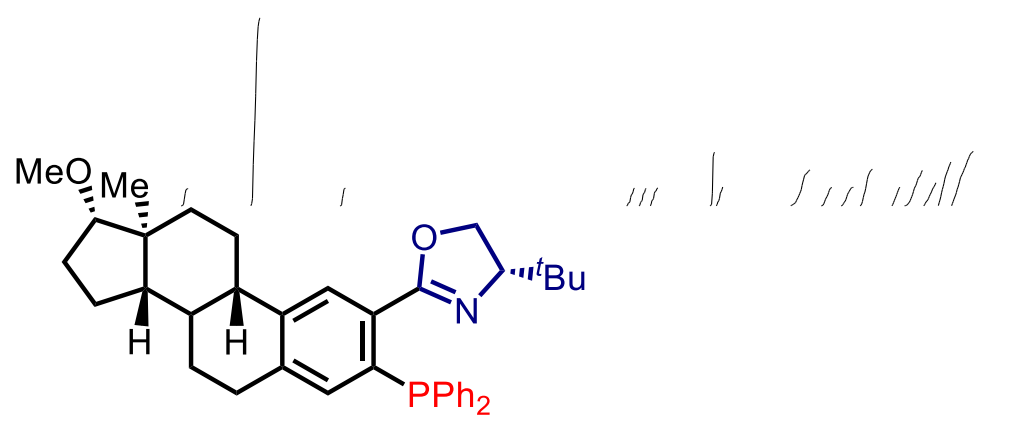

$5 f$

${ }^{1} \mathrm{H}$ NMR $\left(400 \mathrm{MHz}^{\mathrm{CDCl}}{ }_{3}\right)$

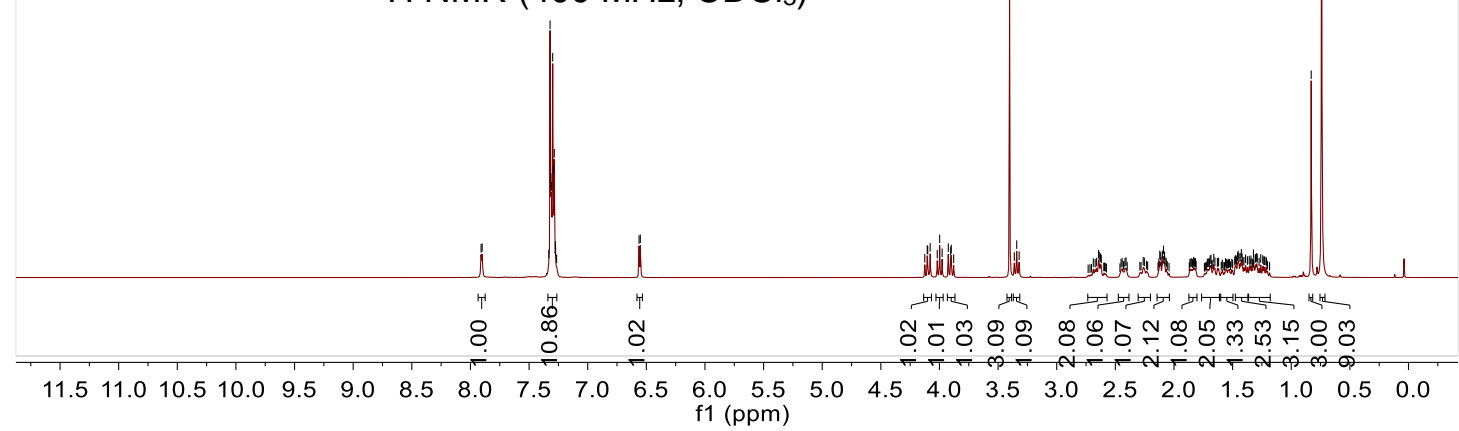

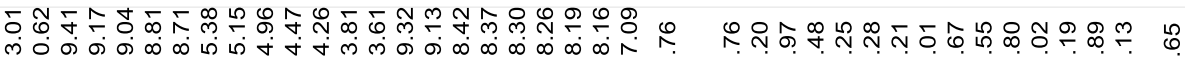

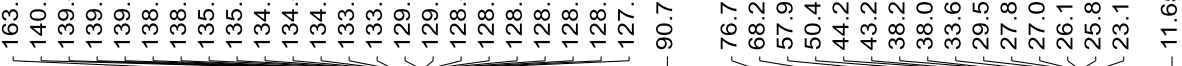<smiles>CO[C@H]1CC[C@H]2C3CCc4cc(P)c(C5=N[C@@H](C(C)C)CO5)cc4[C@H]3CC[C@]12C</smiles>

$5 f$

${ }^{13} \mathrm{C}$ NMR $\left(100 \mathrm{MHz}, \mathrm{CDCl}_{3}\right)$

$\begin{array}{lllllllllll}210 & 200 & 190 & 180 & 170 & 160 & 150 & 140 & 130 & 120 & \begin{array}{l}110 \\ \mathrm{f} 1(\mathrm{ppm})\end{array}\end{array}$

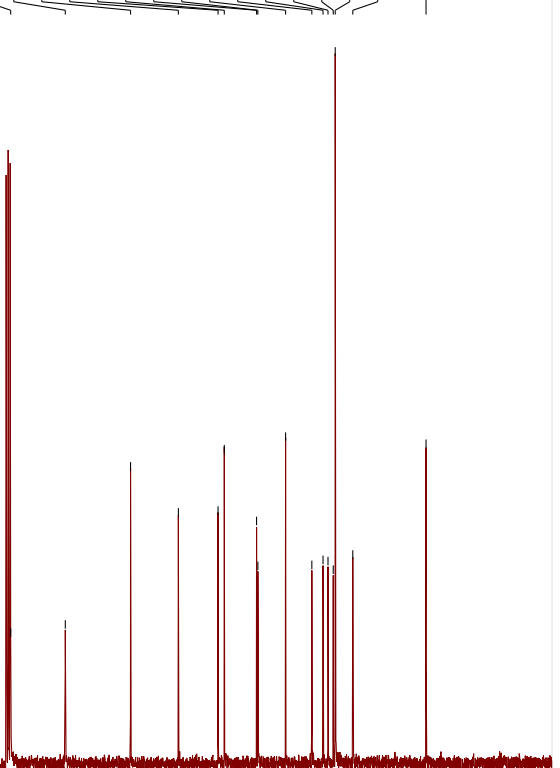



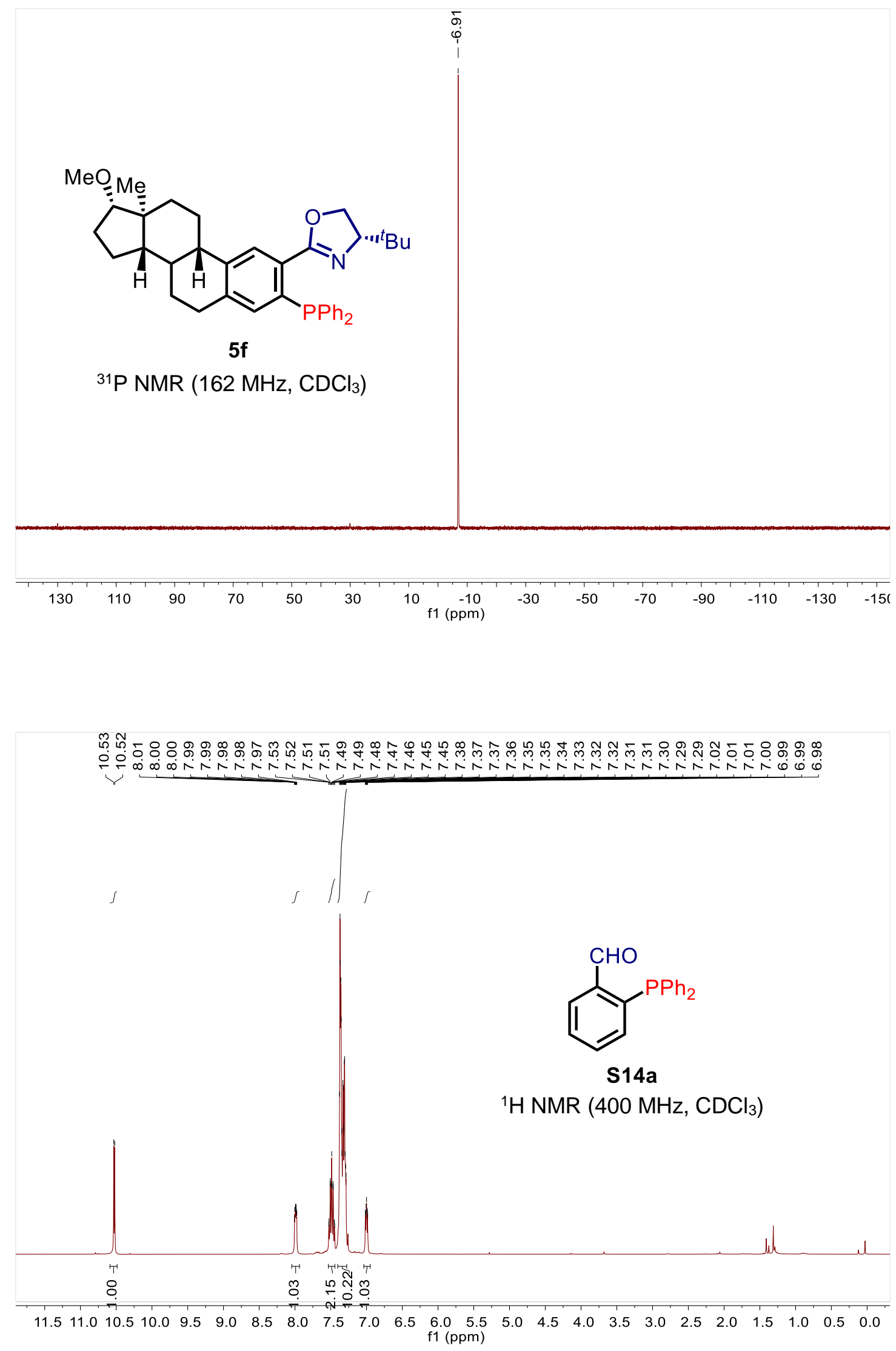


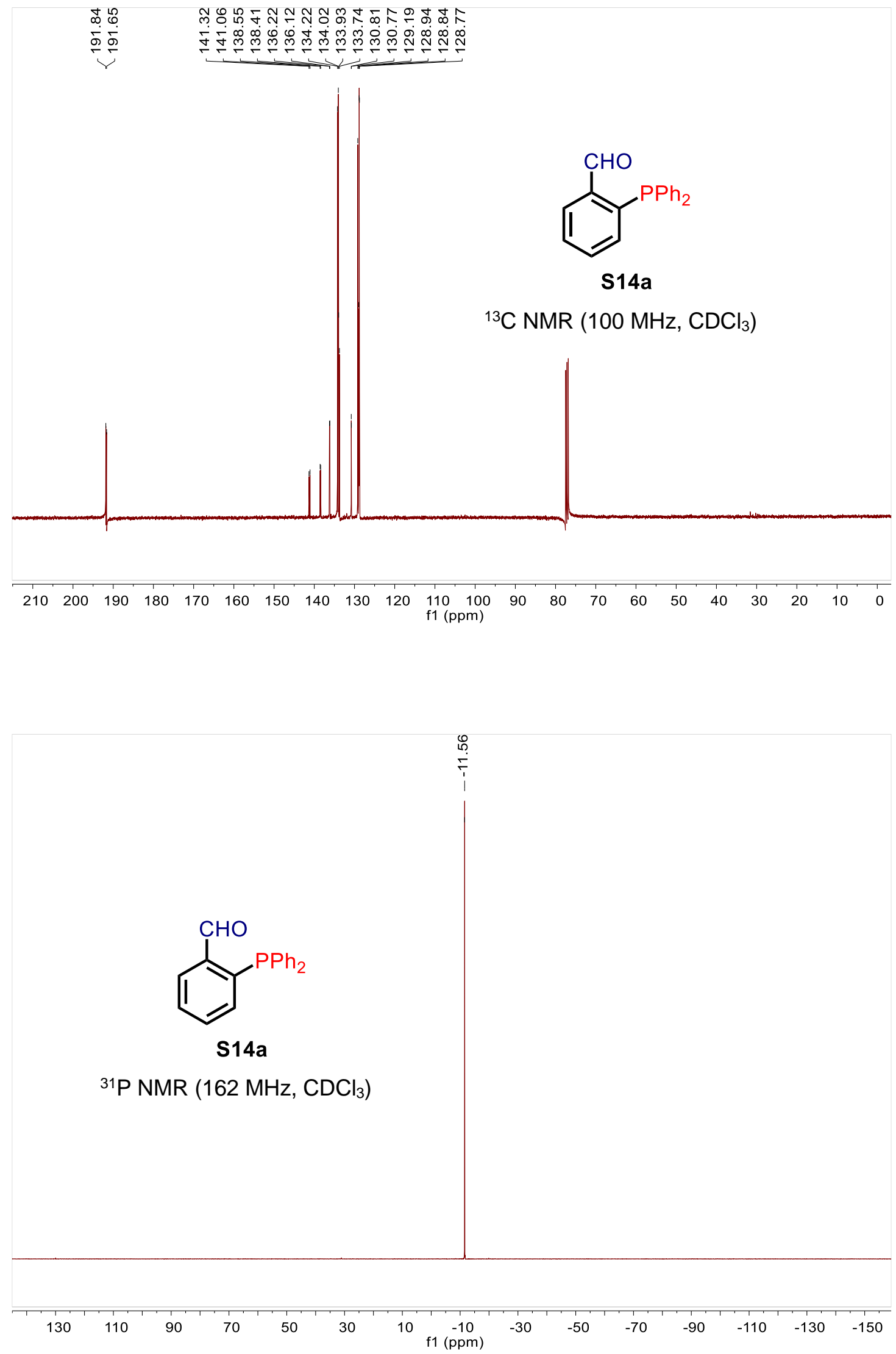



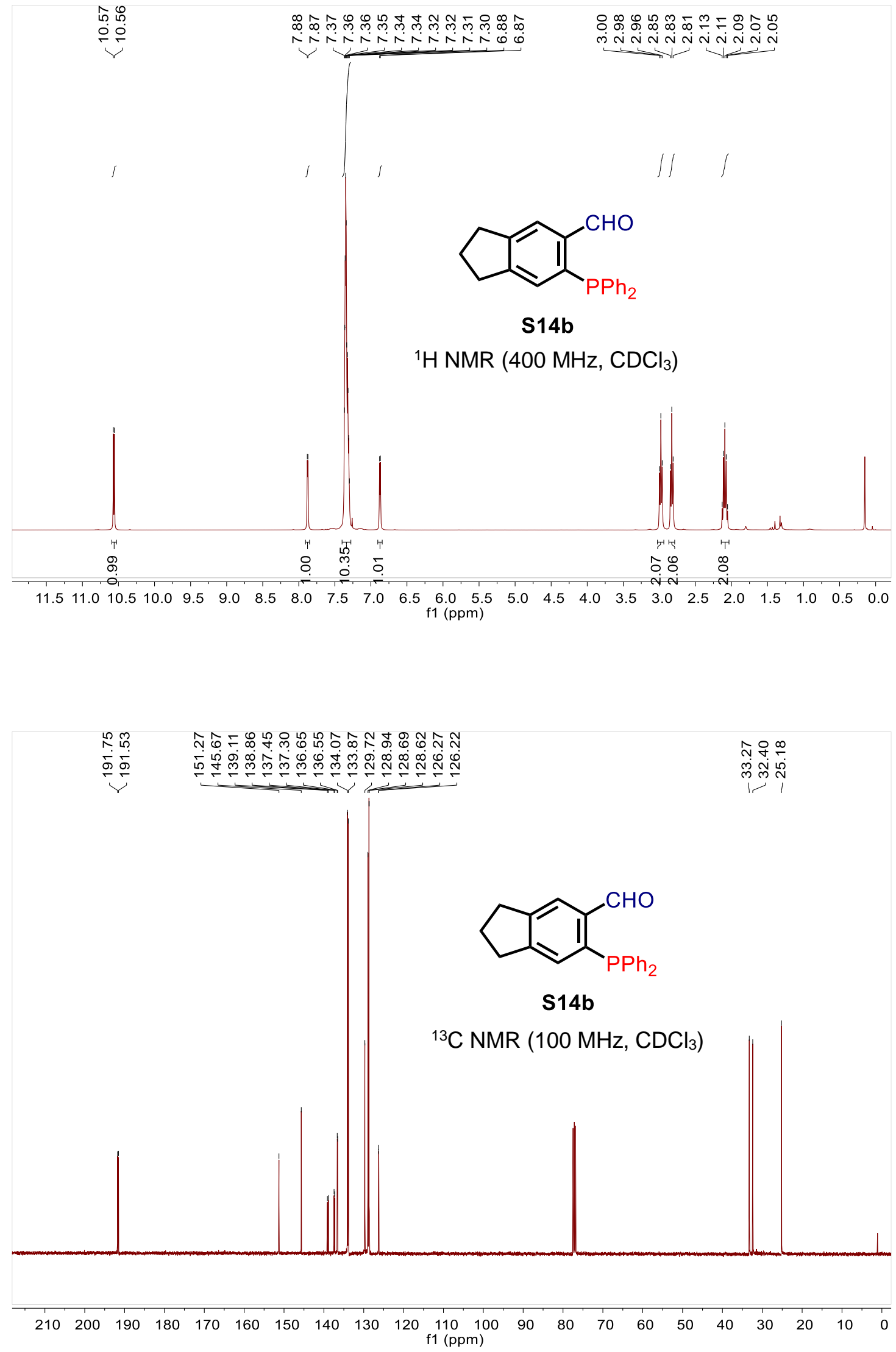


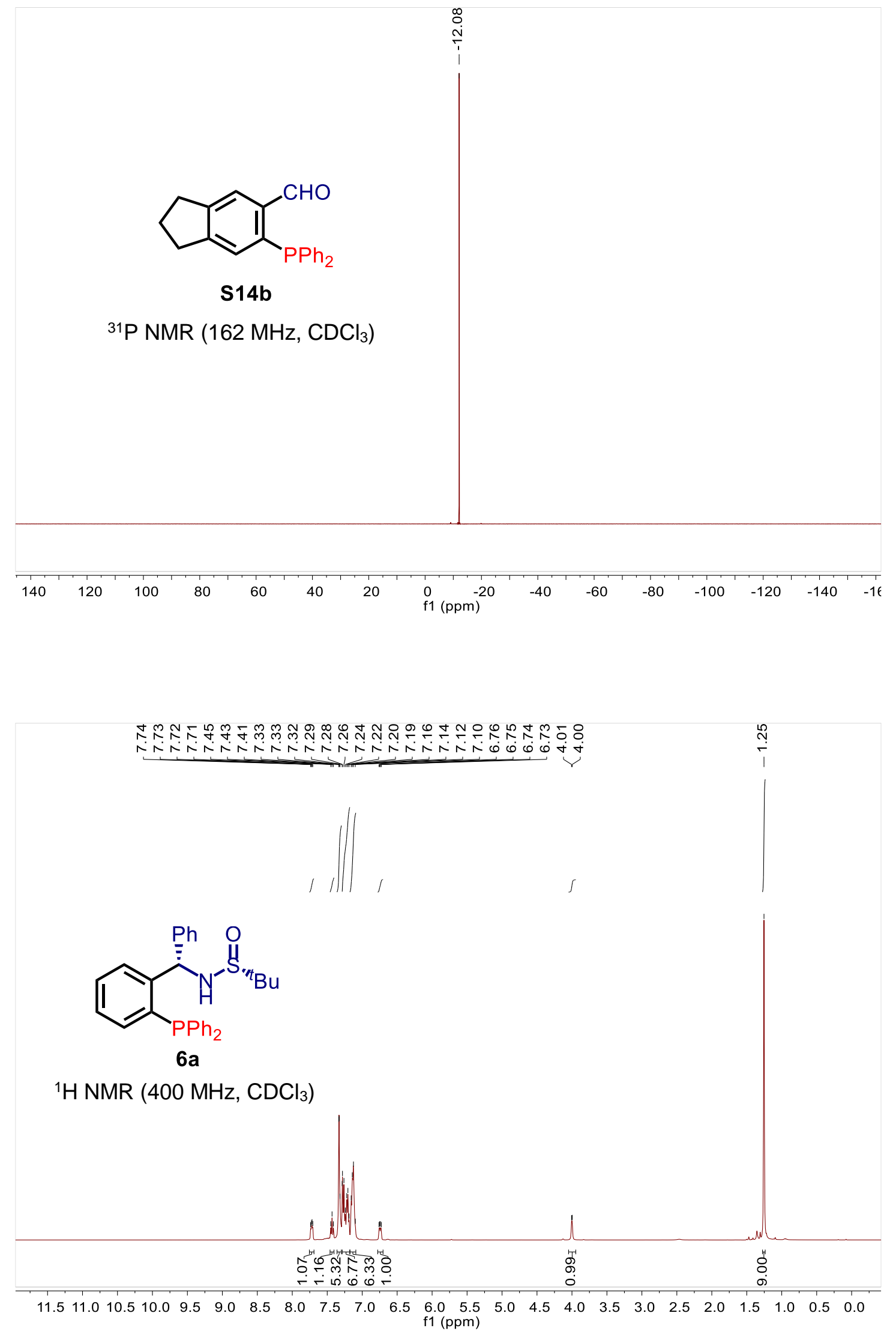




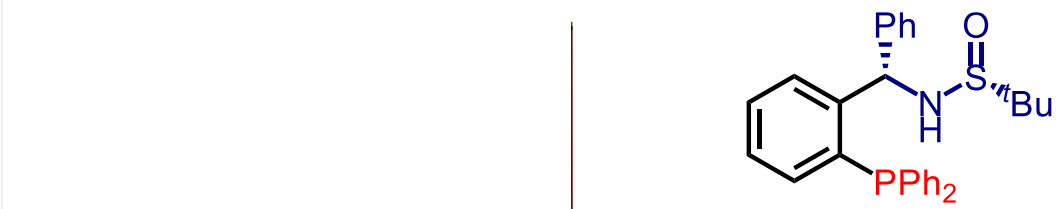

6a

${ }^{13} \mathrm{C}$ NMR $\left(100 \mathrm{MHz}, \mathrm{CDCl}_{3}\right)$

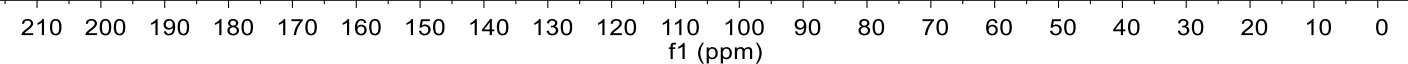

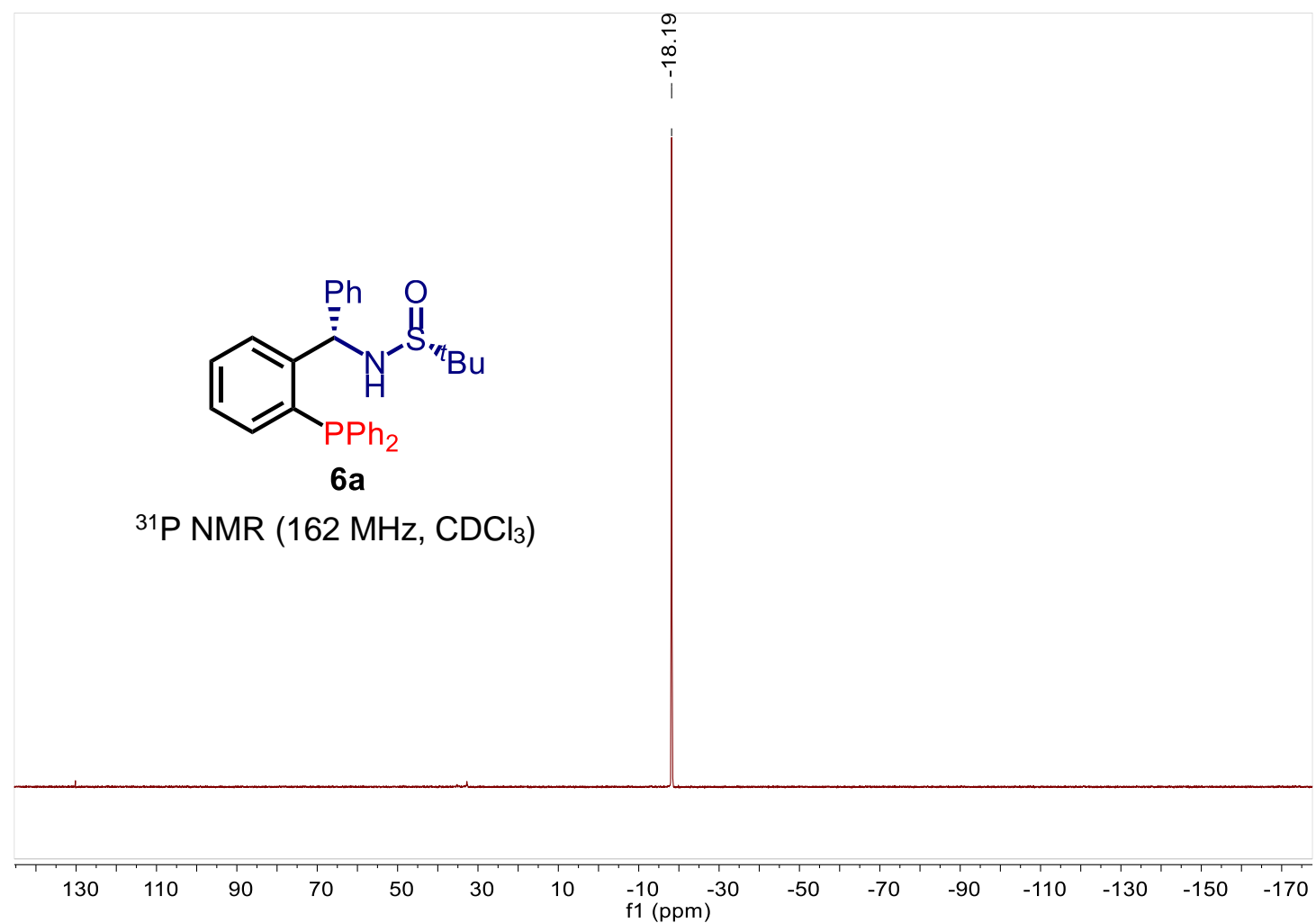


<smiles>CCCCCS(=O)N[C@@H](c1ccc(OC)cc1)c1ccccc1P</smiles>

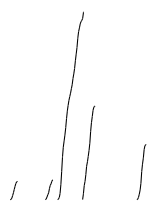

6b

${ }^{1} \mathrm{H} \mathrm{NMR}\left(400 \mathrm{MHz}, \mathrm{CDCl}_{3}\right)$

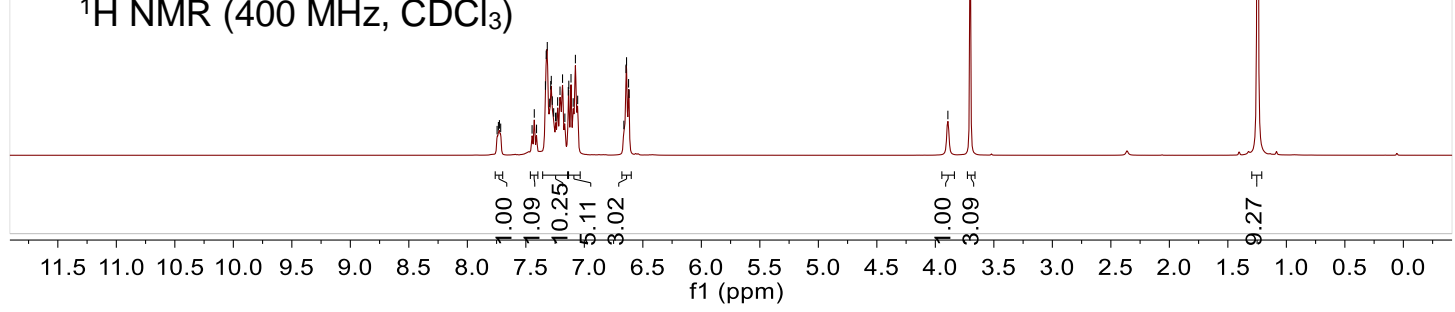

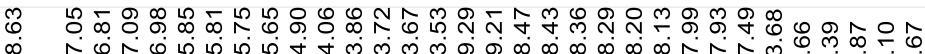

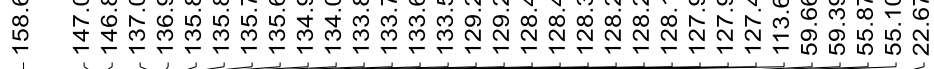<smiles>COc1ccc([C@H](NS(=O)CC(C)C)c2ccccc2P)cc1</smiles>

6b

${ }^{13} \mathrm{C} \mathrm{NMR}\left(100 \mathrm{MHz}, \mathrm{CDCl}_{3}\right)$

$\begin{array}{lllllllllllllllllllll}210 & 200 & 190 & 180 & 170 & 160 & 150 & 140 & 130 & 120 & \begin{array}{c}110 \\ f 1(\mathrm{ppm})\end{array} & 90 & 80 & 70 & 60 & 50 & 40 & 30 & 20 & 10 & 0\end{array}$ 


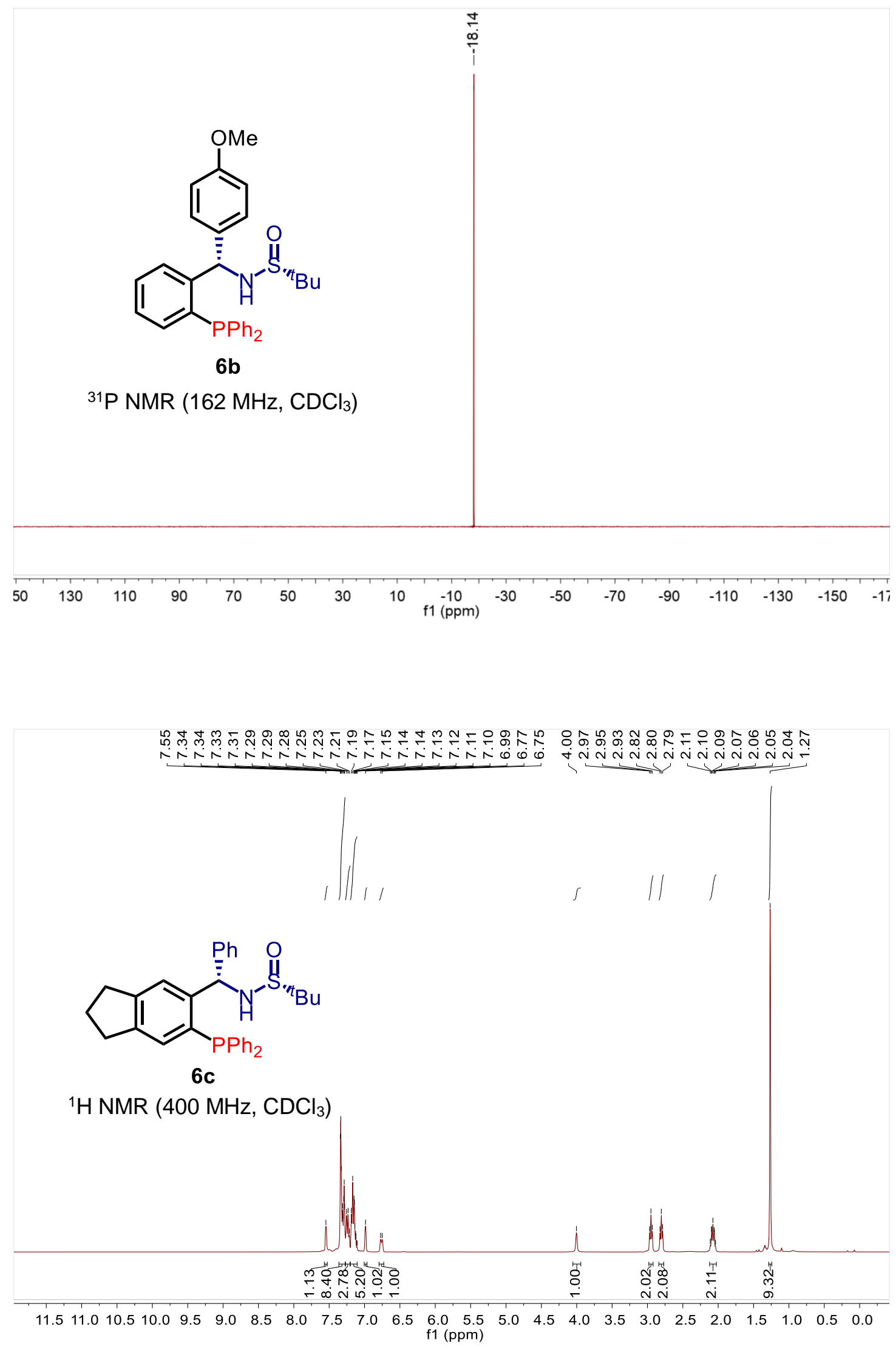




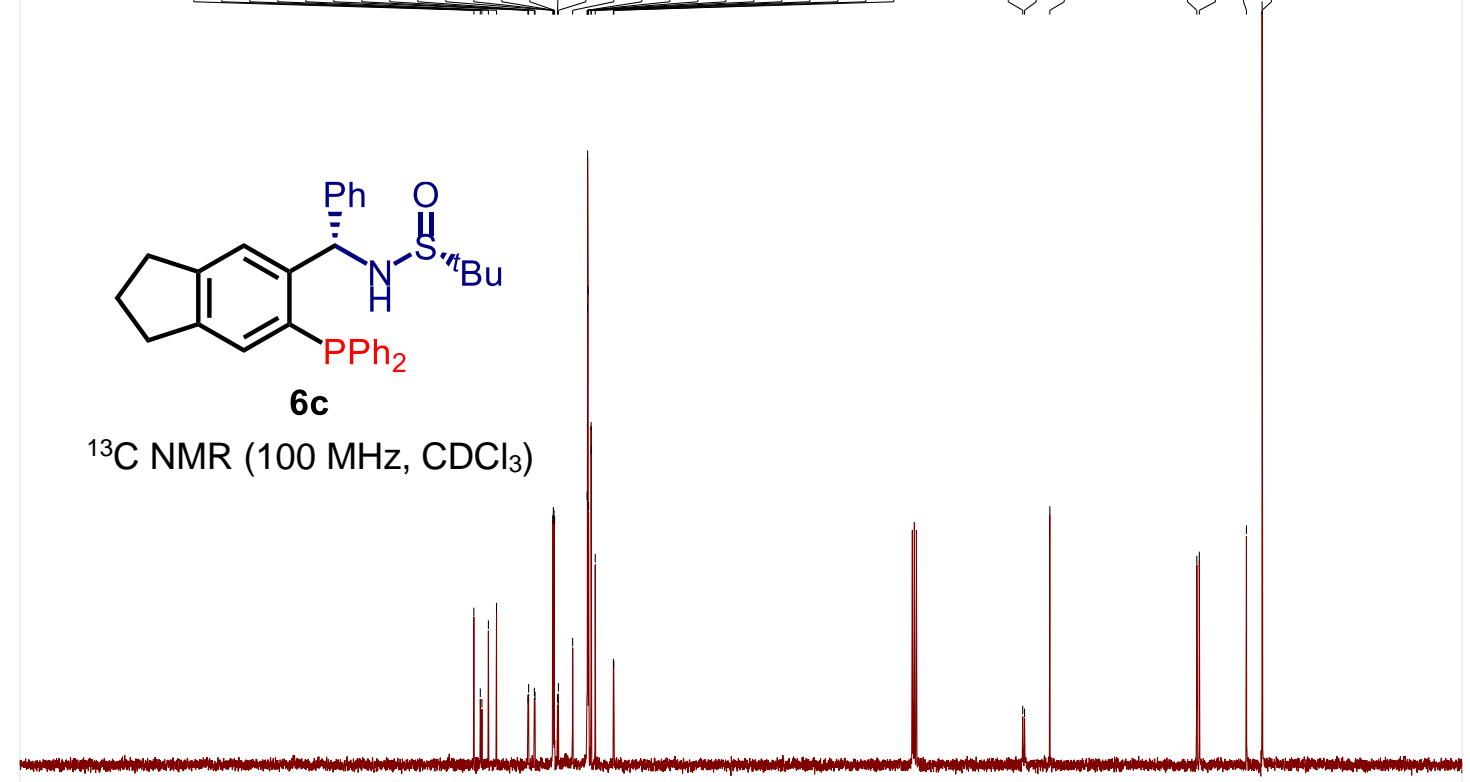

$\begin{array}{llllllllllllllllllllll}210 & 200 & 190 & 180 & 170 & 160 & 150 & 140 & 130 & 120 & \begin{array}{l}110 \\ \mathrm{f} 1\end{array}(\mathrm{ppm}) & 90 & 80 & 70 & 60 & 50 & 40 & 30 & 20 & 10 & 0\end{array}$
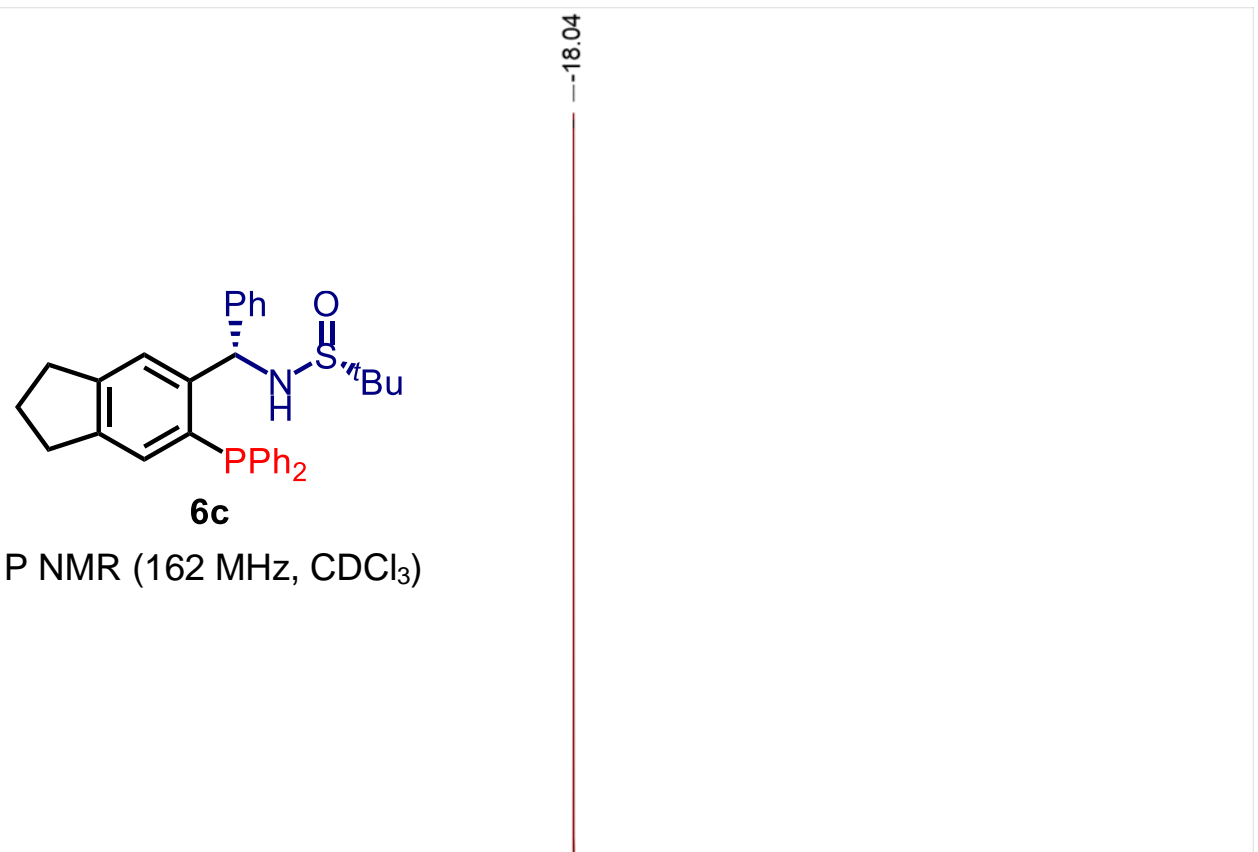

${ }^{31} \mathrm{P} \mathrm{NMR}\left(162 \mathrm{MHz}, \mathrm{CDCl}_{3}\right)$ 

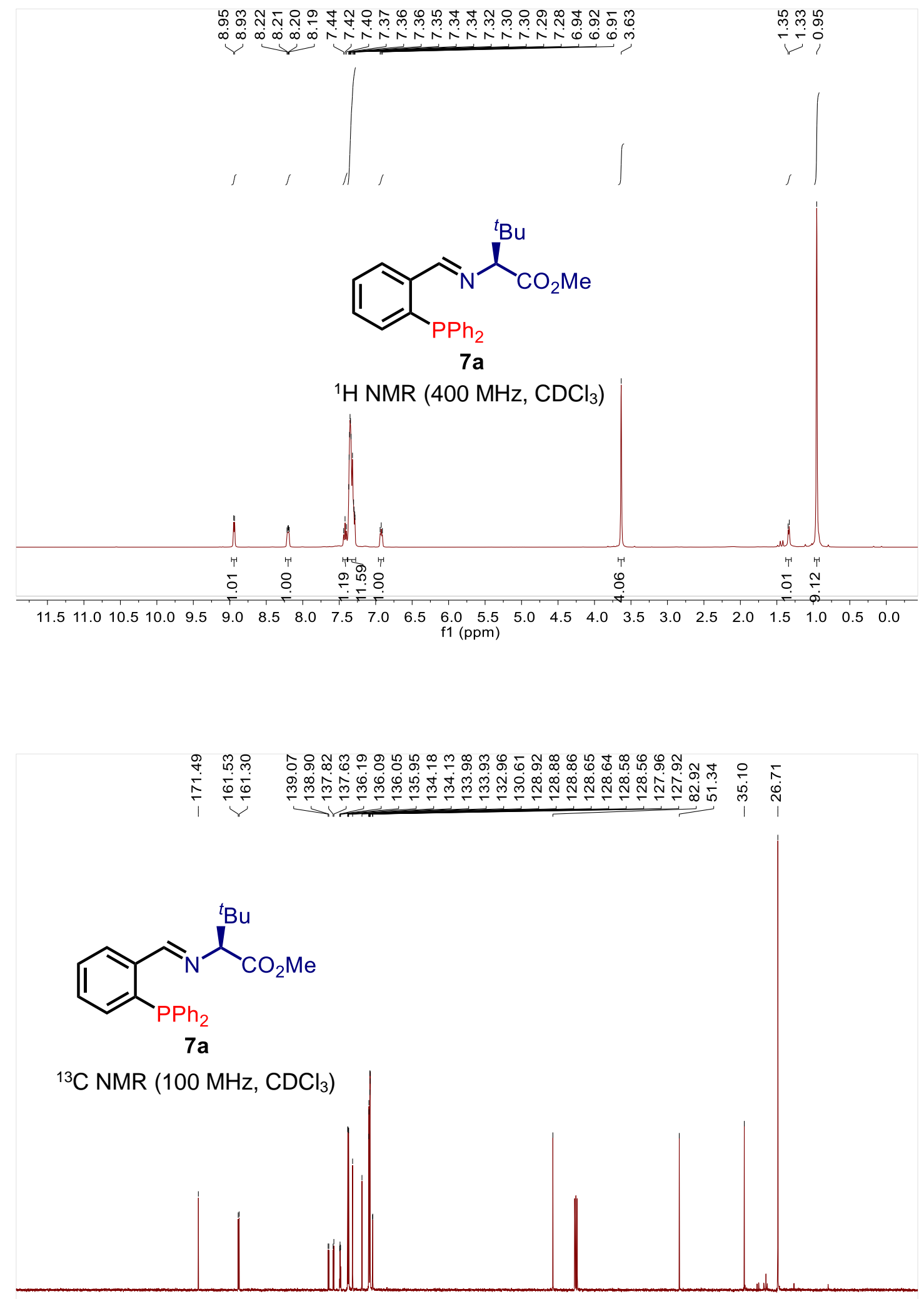

$\begin{array}{lllllllllllllllllllll}210 & 200 & 190 & 180 & 170 & 160 & 150 & 140 & 130 & 120 & \begin{array}{r}110 \\ \mathrm{f} 1(\mathrm{ppm})\end{array} & \begin{array}{l}100 \\ \mathrm{fl}\end{array} & 80 & 70 & 60 & 50 & 40 & 30 & 20 & 10 & 0\end{array}$ 


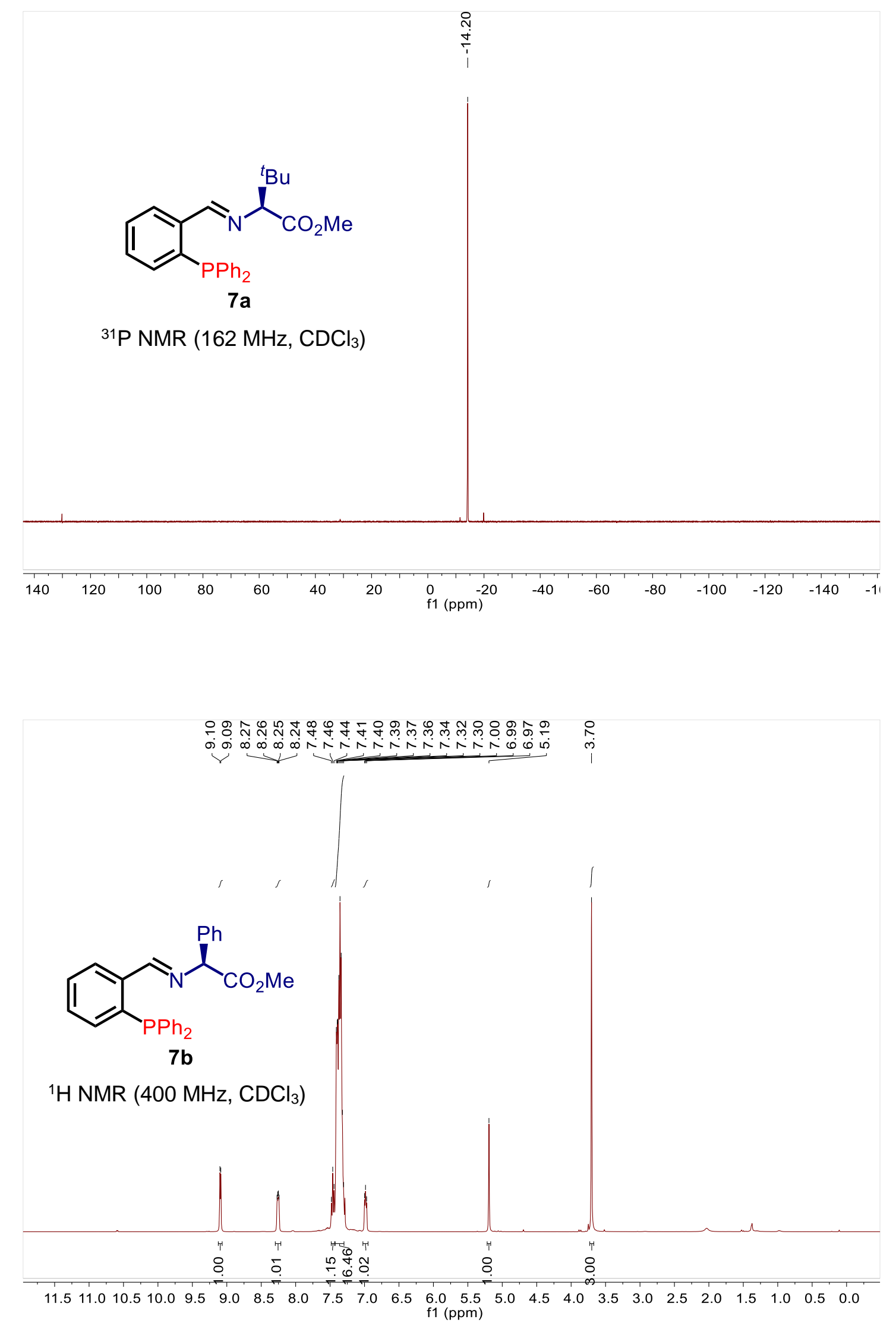




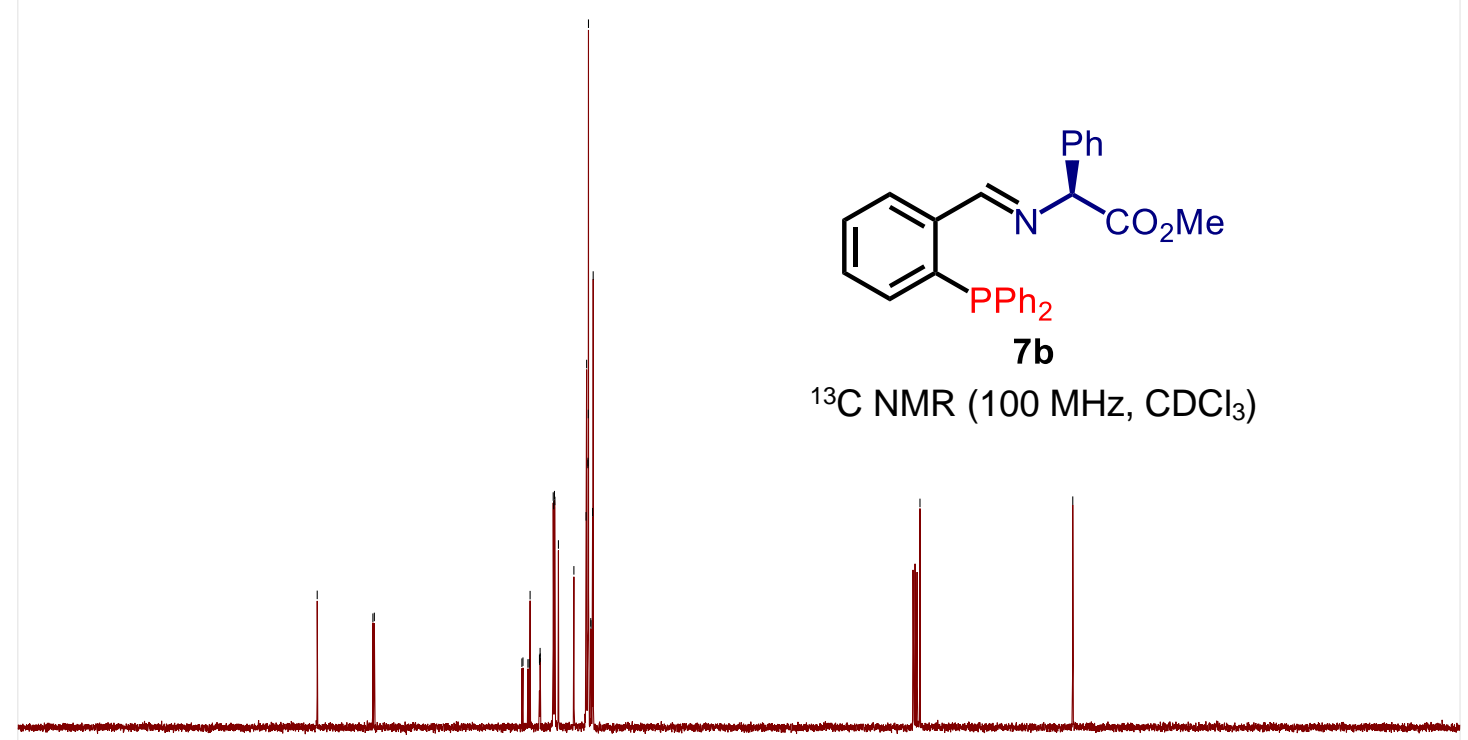

$\begin{array}{llllllllllllllllllllll}210 & 200 & 190 & 180 & 170 & 160 & 150 & 140 & 130 & 120 & \begin{array}{l}110 \\ \mathrm{f} 1(\mathrm{ppm})\end{array} & 100 & 80 & 70 & 60 & 50 & 40 & 30 & 20 & 10 & 0\end{array}$

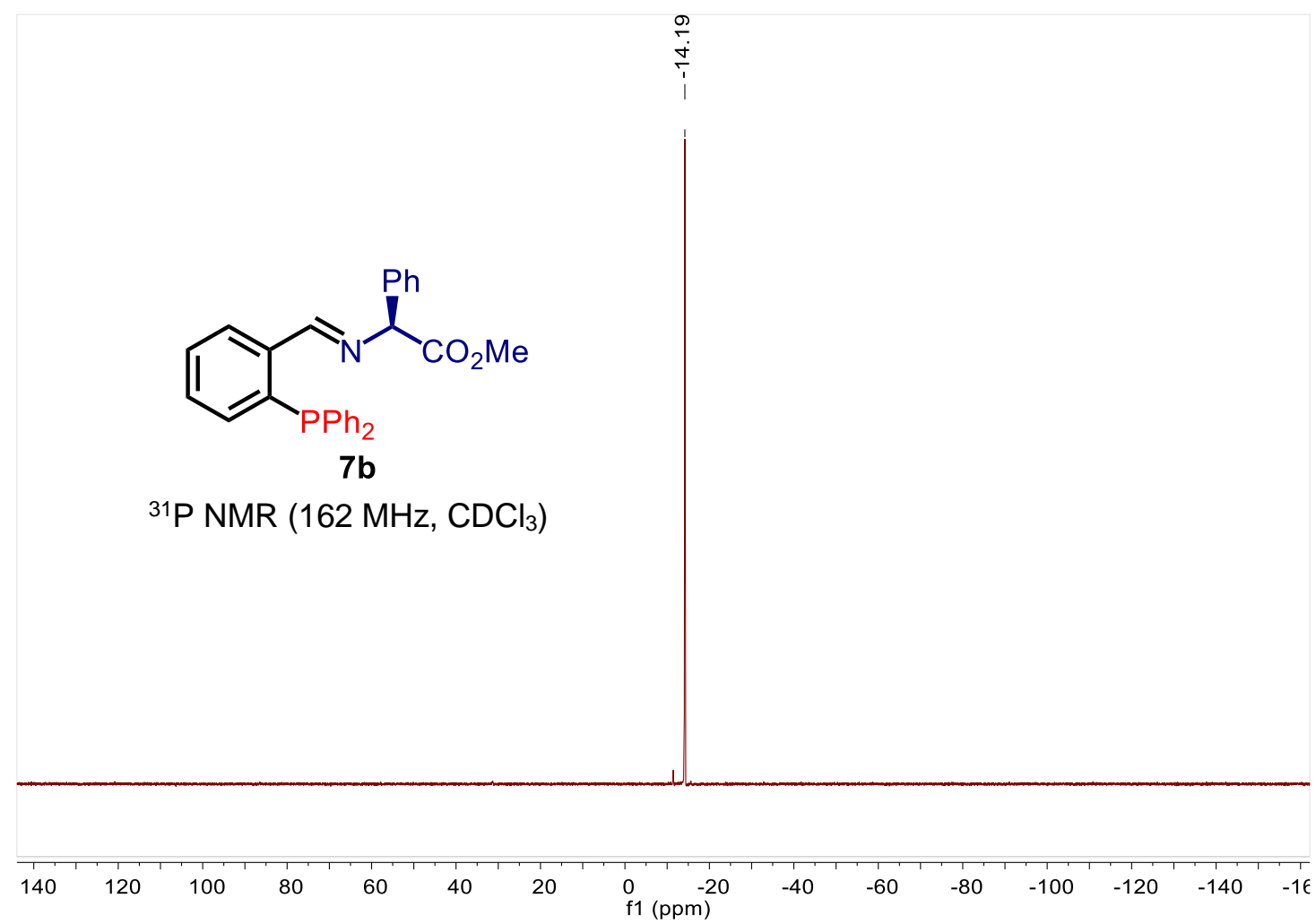




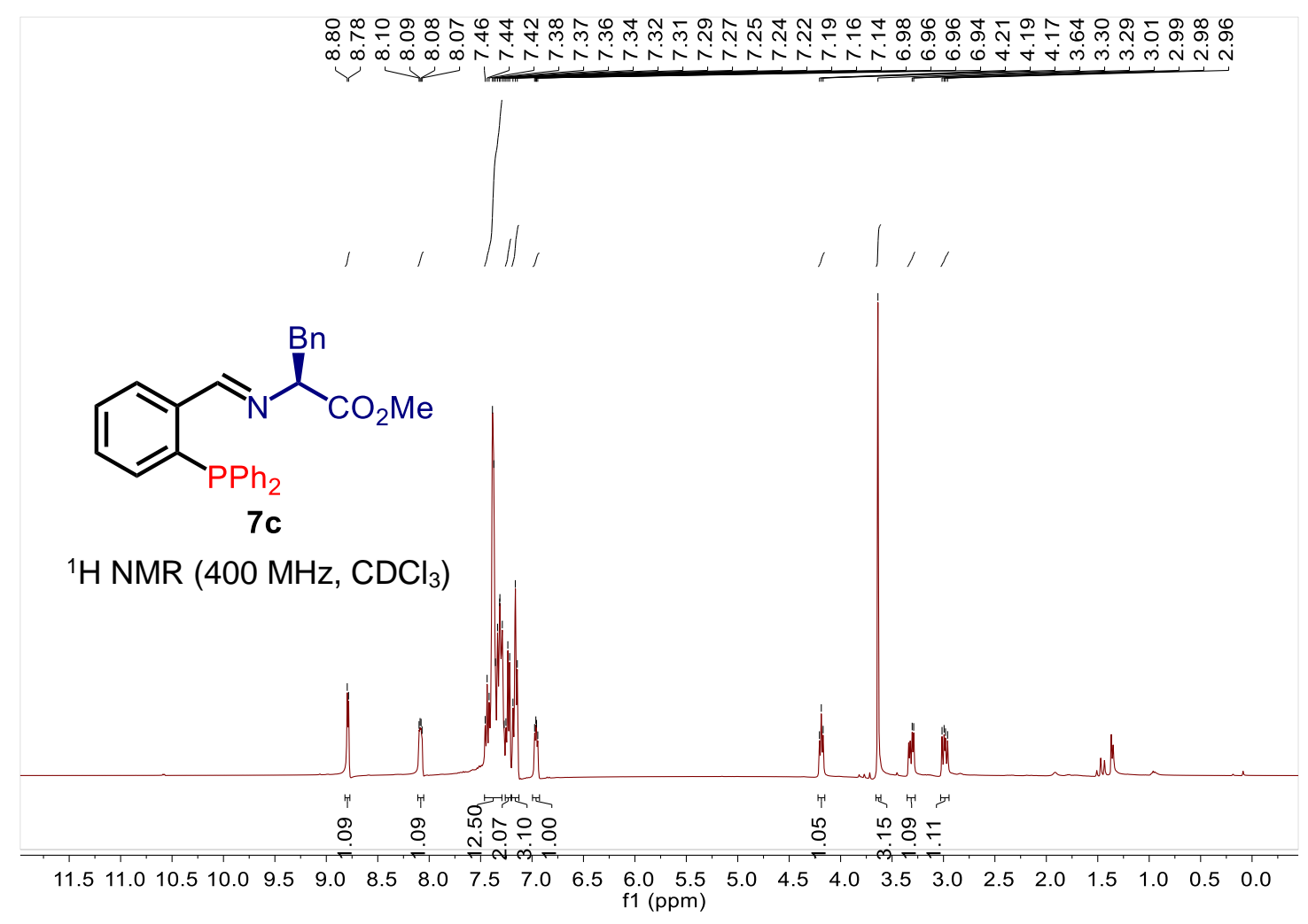

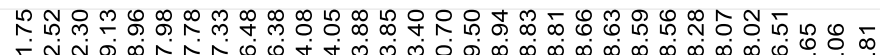

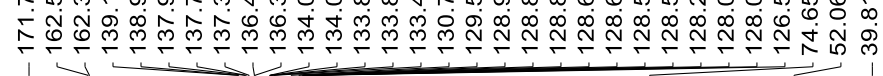

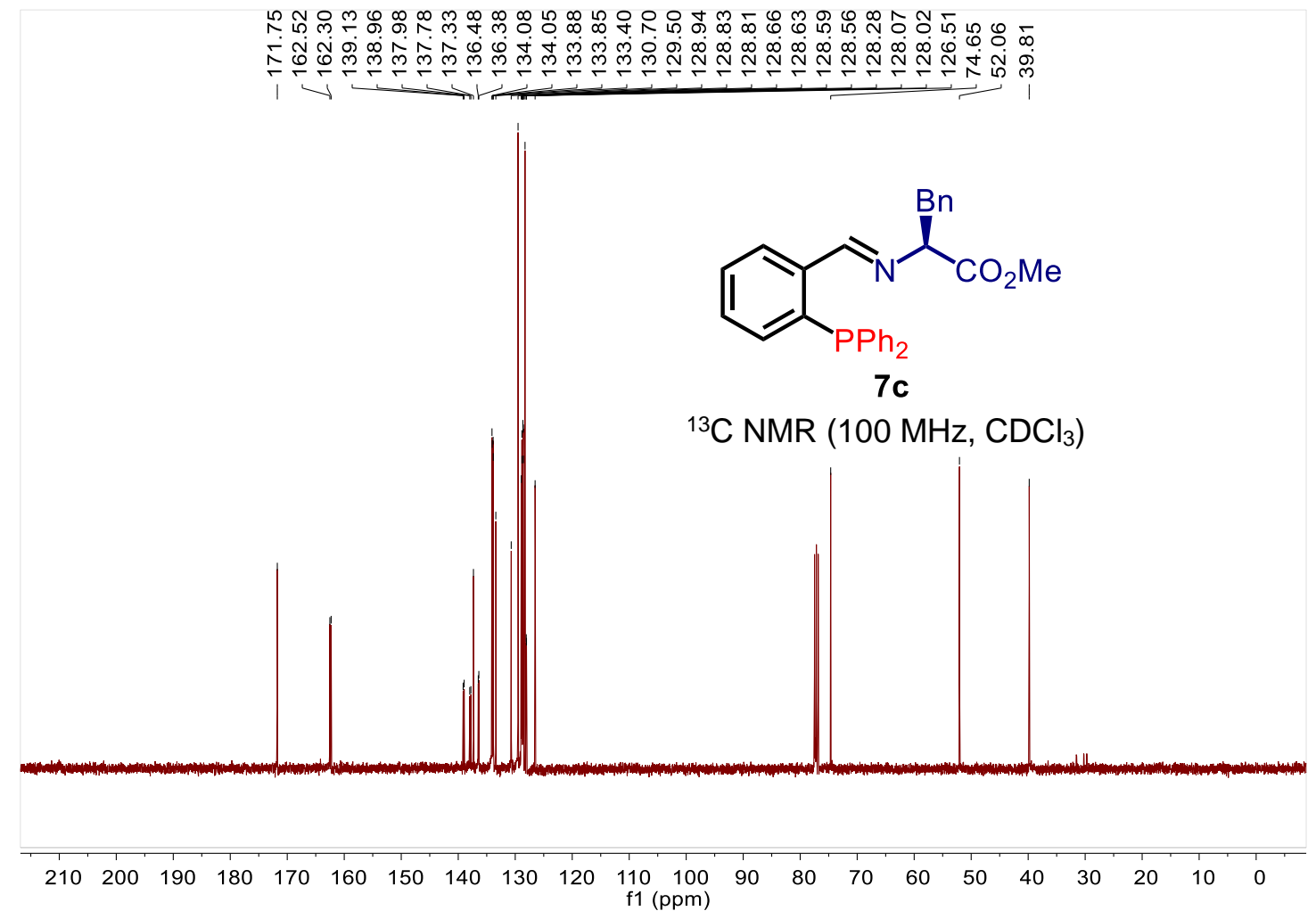




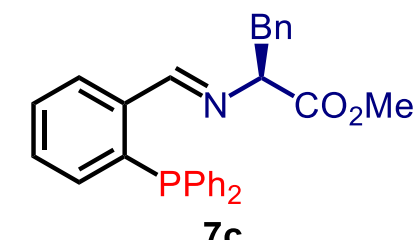

${ }^{31} \mathrm{P}$ NMR $\left(162 \mathrm{MHz}, \mathrm{CDCl}_{3}\right)$

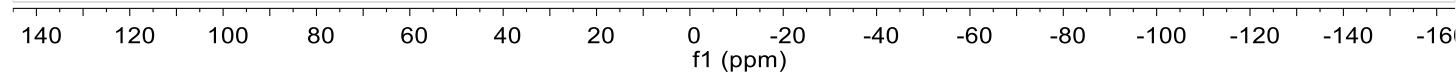

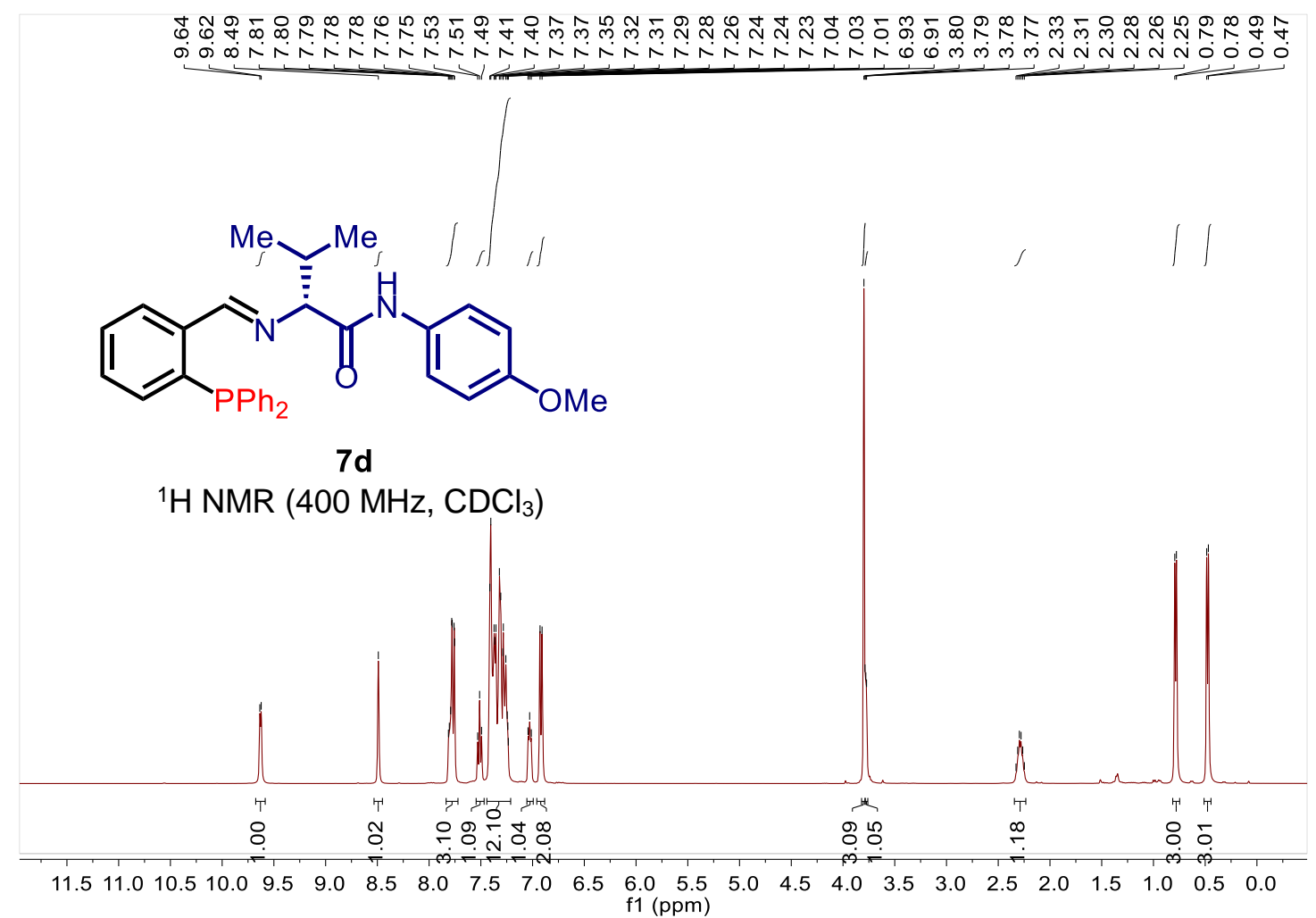




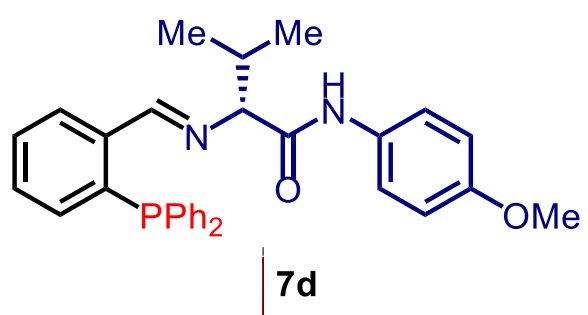

${ }^{13} \mathrm{C}$ NMR $\left(100 \mathrm{MHz}, \mathrm{CDCl}_{3}\right)$

$\begin{array}{llllllllllllllllllllll}210 & 200 & 190 & 180 & 170 & 160 & 150 & 140 & 130 & 120 & \begin{array}{c}110 \\ \mathrm{f} 1(\mathrm{ppm})\end{array} & 90 & 80 & 70 & 60 & 50 & 40 & 30 & 20 & 10 & 0\end{array}$

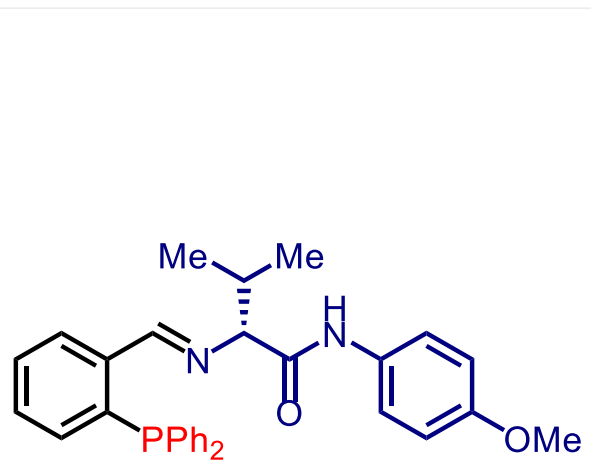

7d

${ }^{31} \mathrm{P}$ NMR $\left(162 \mathrm{MHz}, \mathrm{CDCl}_{3}\right)$ 


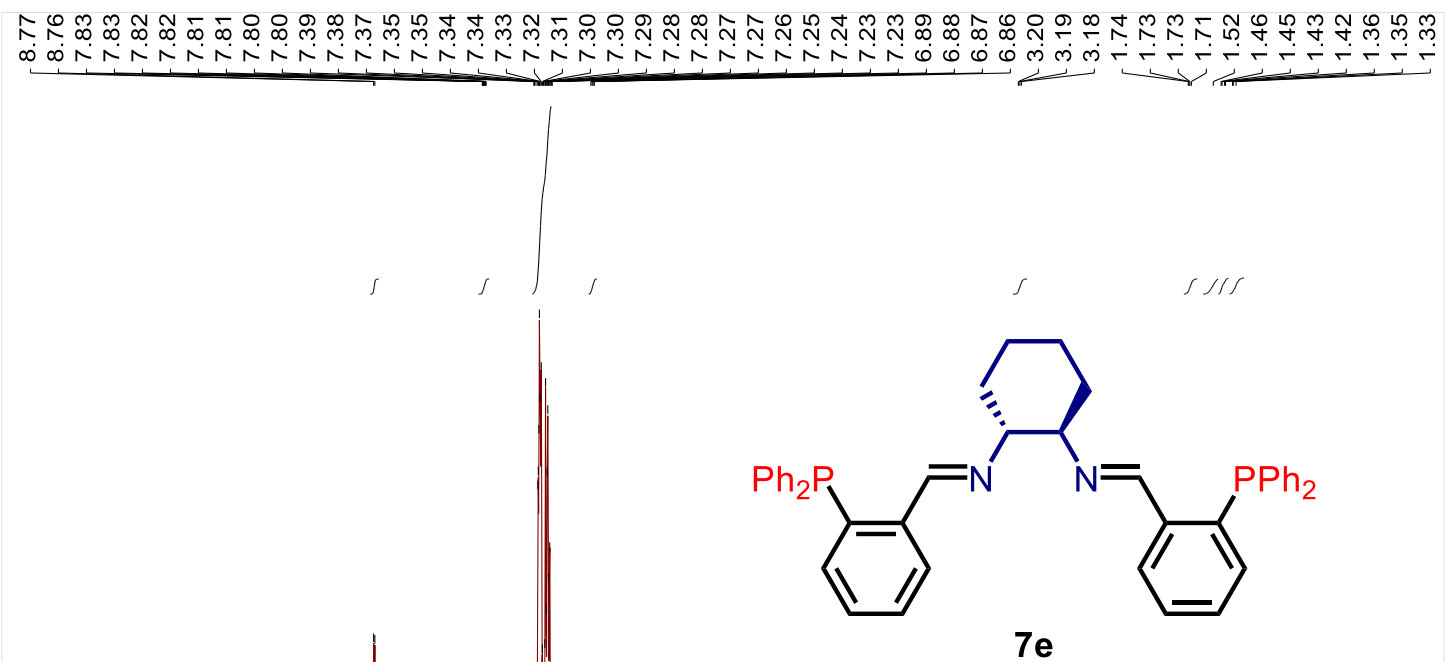

${ }^{1} \mathrm{H}$ NMR $\left(400 \mathrm{MHz}, \mathrm{CDCl}_{3}\right)$
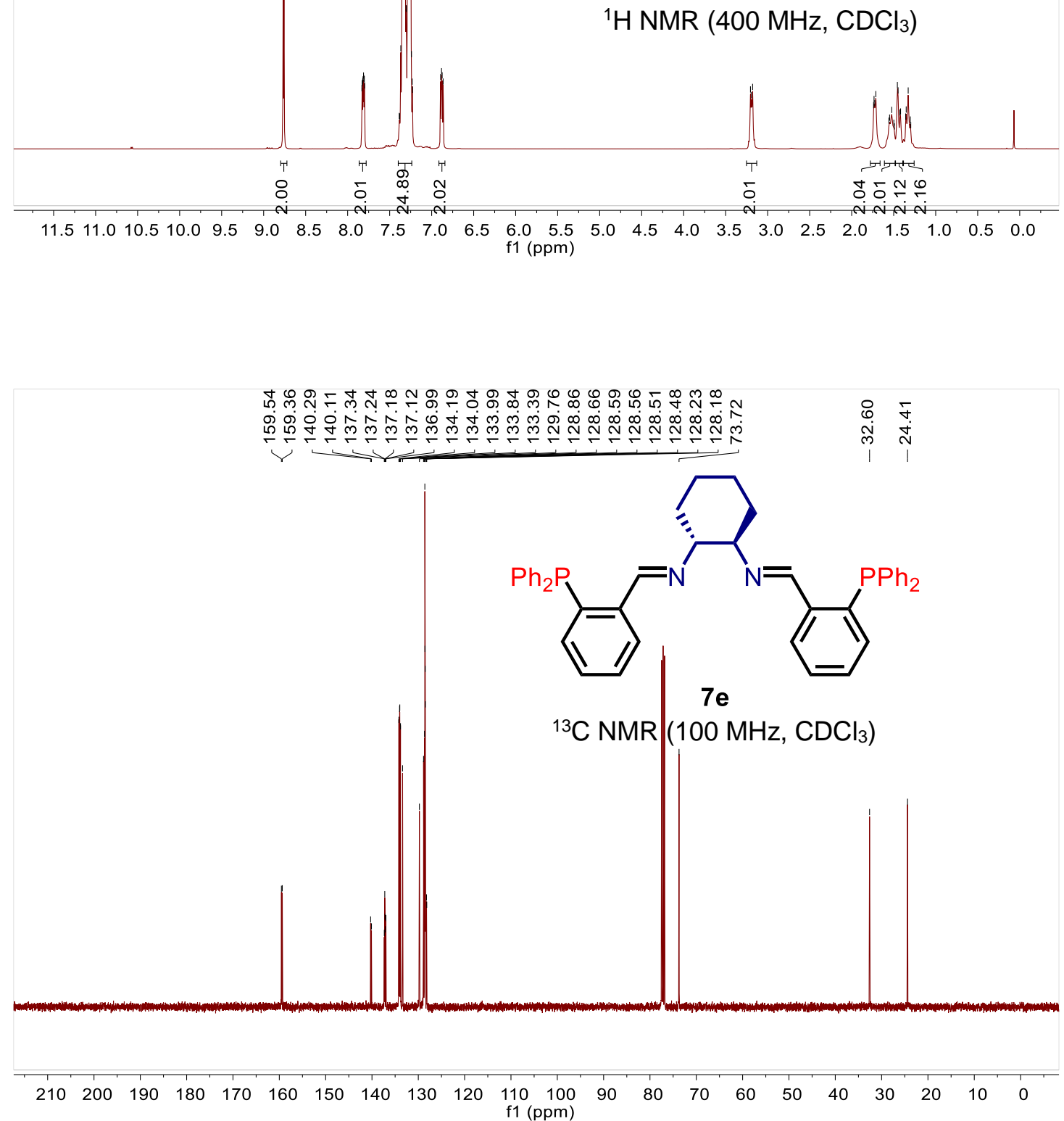


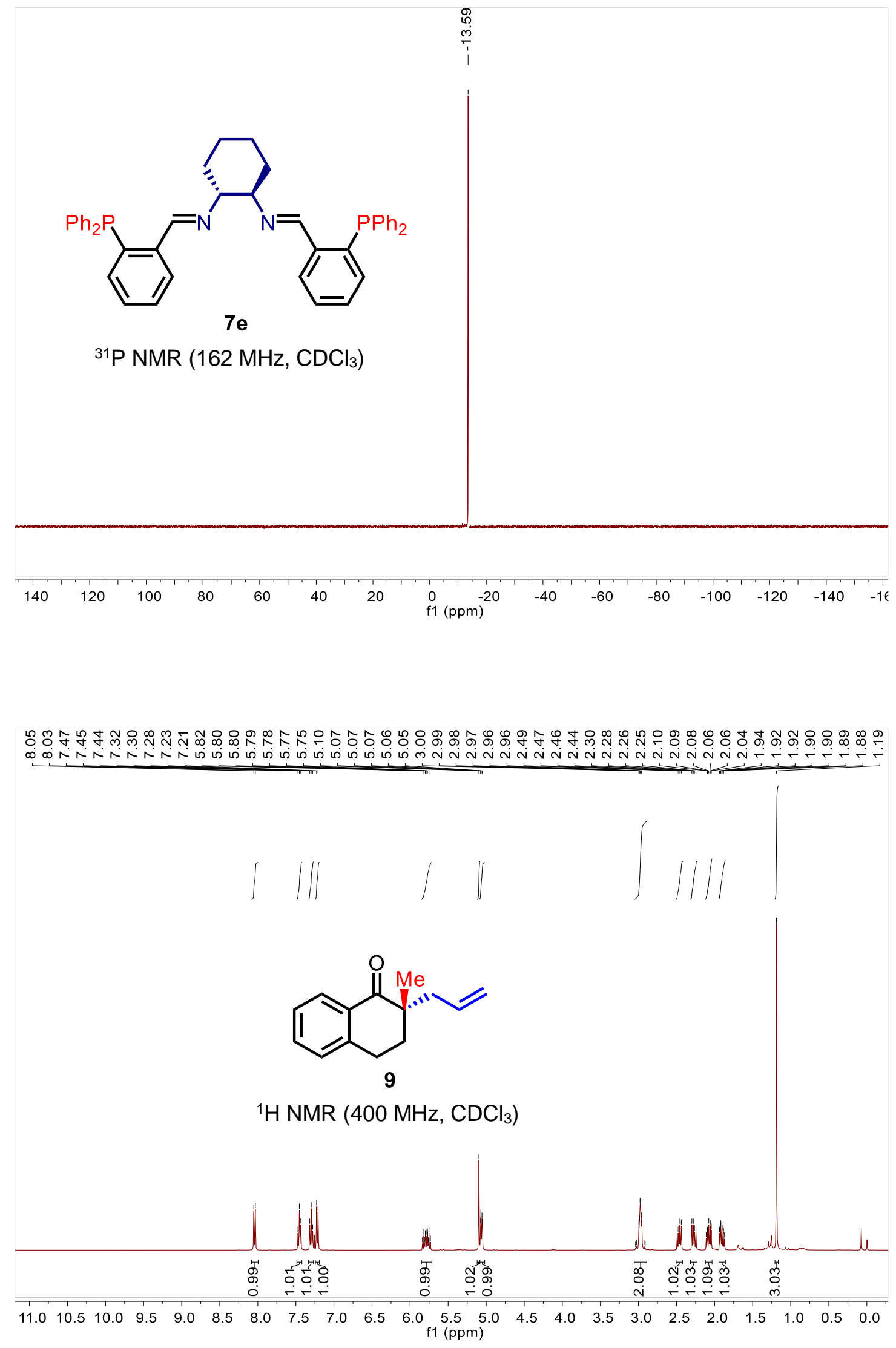




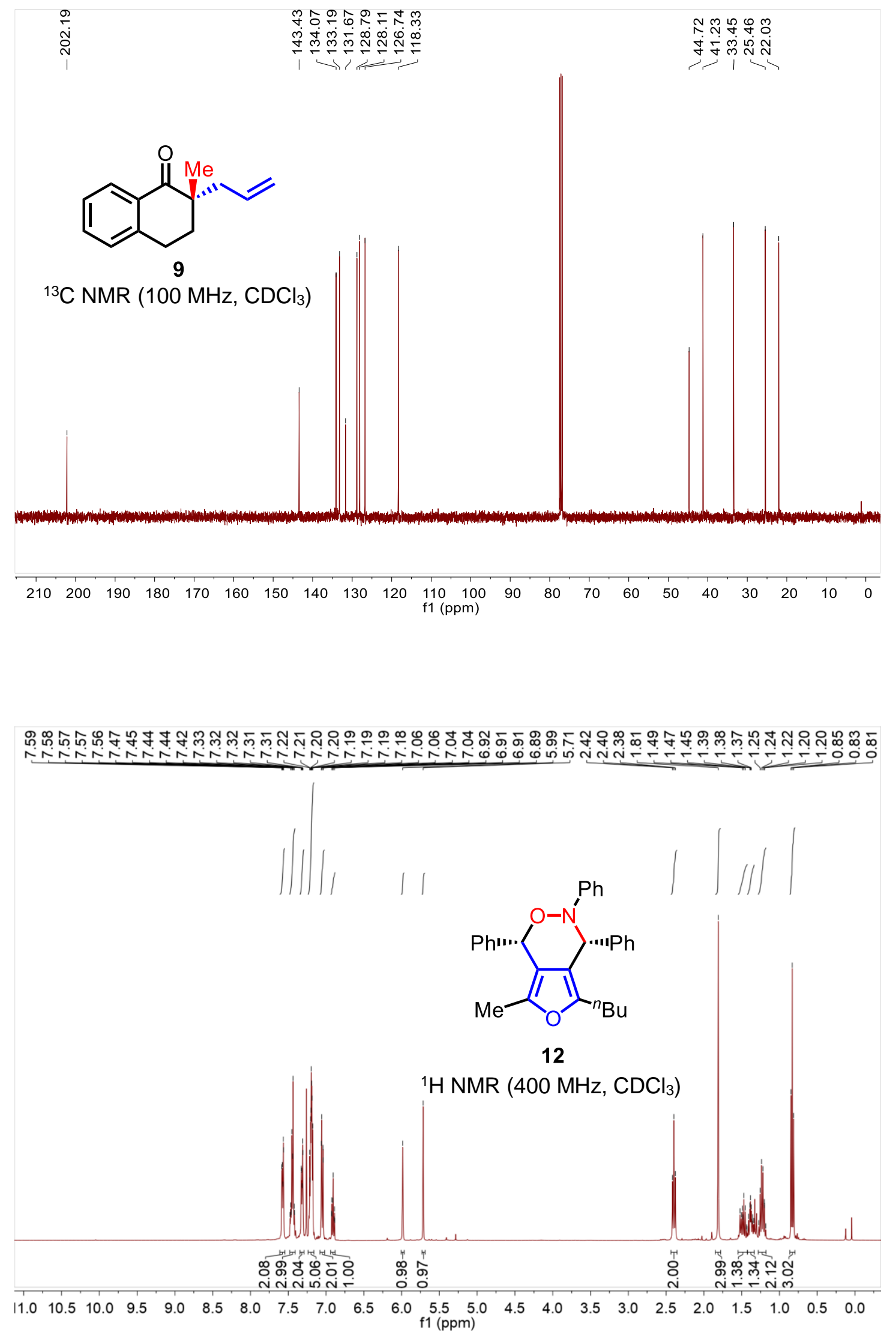




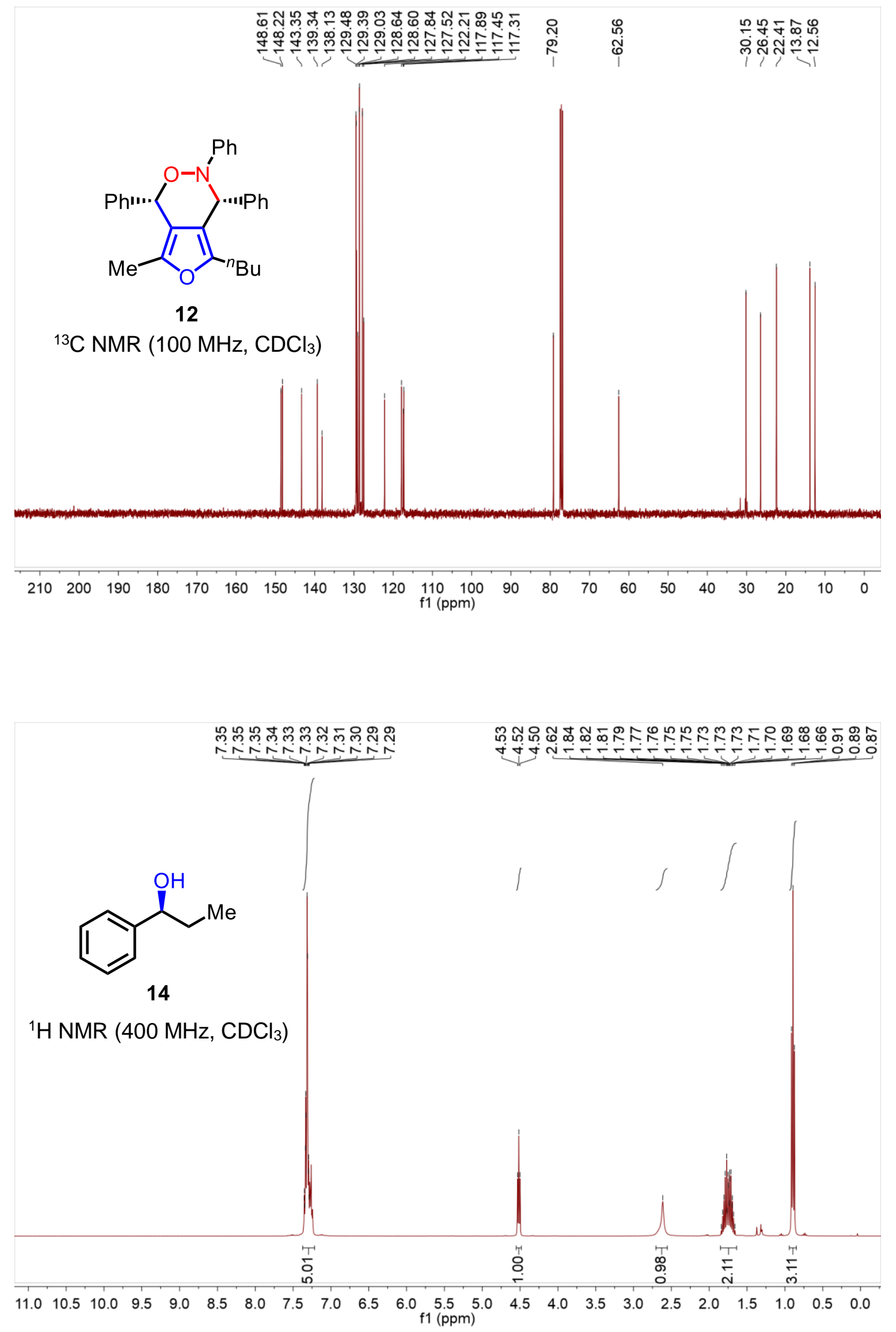




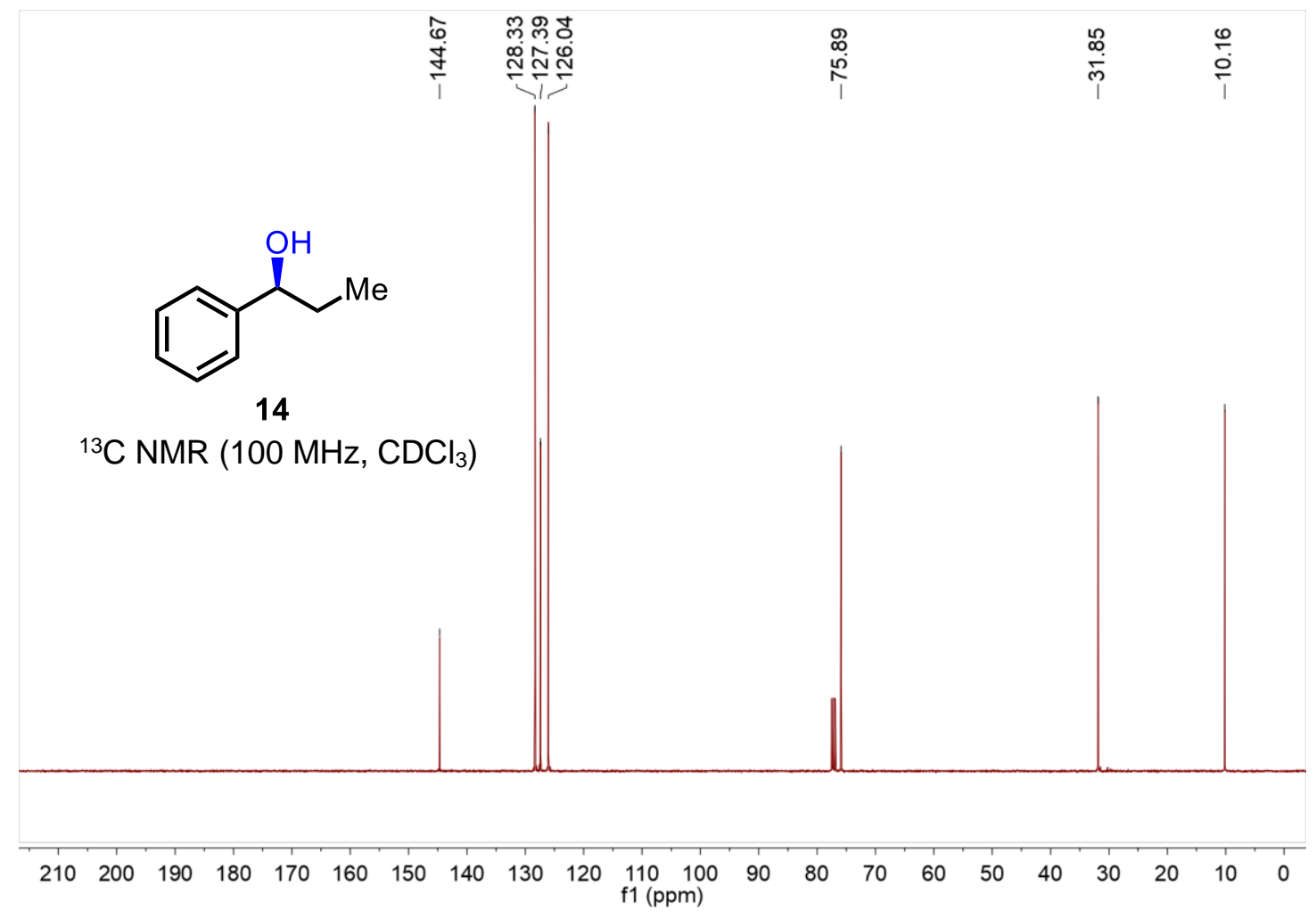

